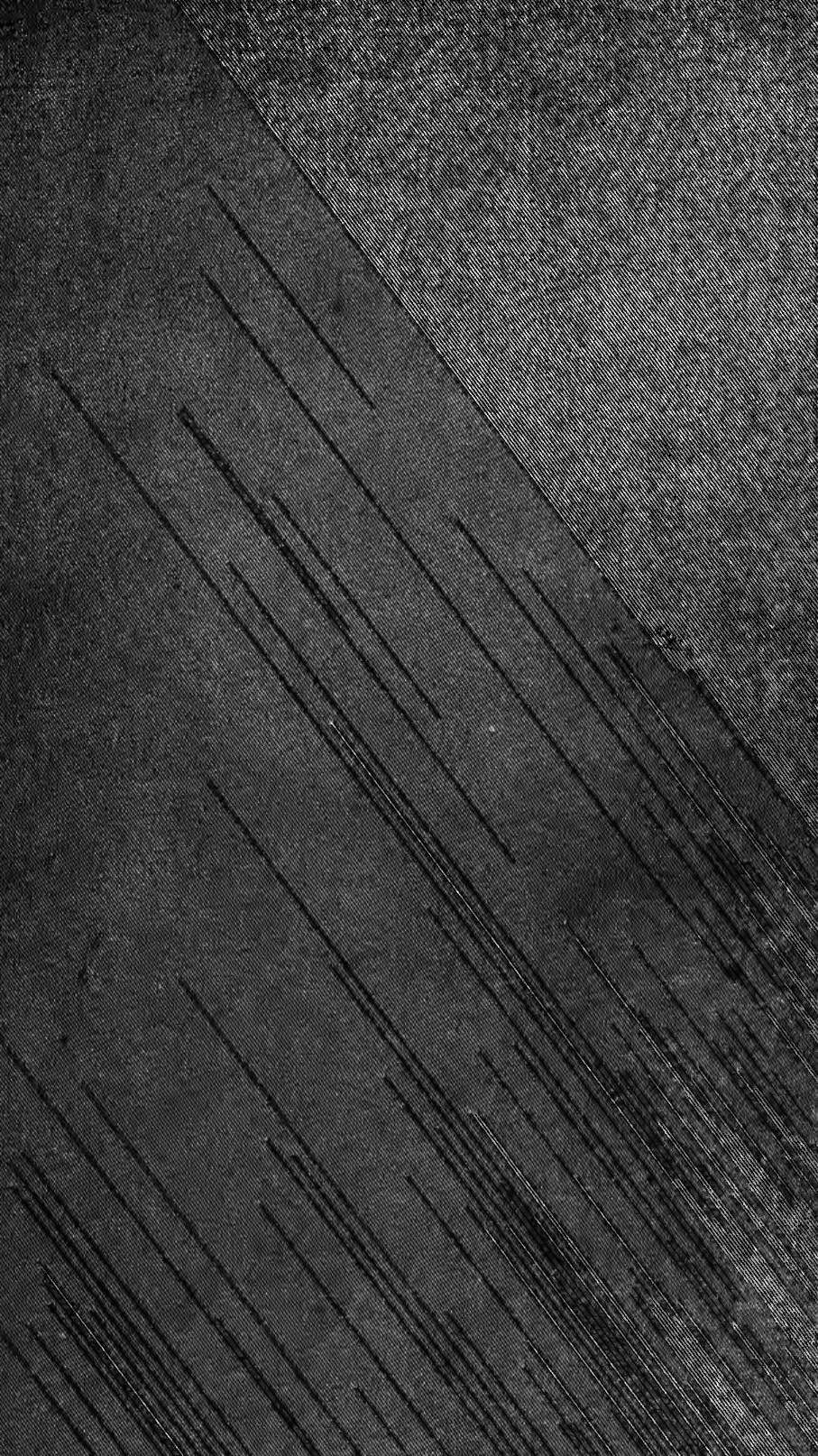





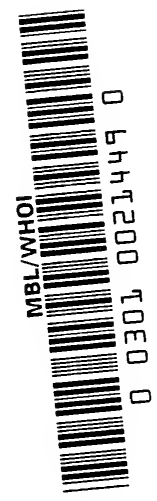





\section{THE BACTERIOPHAGE AND ITS BEHAVIOR}





\title{
THE BACTERIOPHAGE
}

\section{AND \\ ITS BEHAVIOR}

BY

F. D'HERELLE, M.D.

Directeur du Service bacteriologique du Conseil Sanitaire,

Maritime et Quarantenaire d'Egypte

\author{
TRANSLATED BY \\ GEORGE H. SMI'TH, PH.D. \\ Associate Professor of Bacteriology and Immunology, \\ School of Medicine, Yale University
}

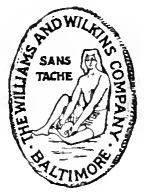

PUBLISHED BY

THE WILLIAMS \& WILKINS COMPANY

BALTIMORE, MD., U.S.A.

1926 
Copyright, 1926

\section{THE WILLIAMIS \& WILKINS COMPANY}

Made in United States of America

Published March, 1926

ALL RIGHTS RESERVED

COMPOSED AND PRINTED AT THE

WAVERLY PRESS

FOR

The Williams \& Wilkins Company

BALtimore, MD., U. S. A. 


\section{PREFACE}

The preliminary notes upon the phenomenon of bacteriophagy, beginning with those published in 1917, attracted the attention of scientists to but a moderate degree, despite the fact that the facts presented appeared to be contrary to all of that which had been described up to that time. Only after the publication of the first collected work in 1921 did investigators on all sides really undertake the study of this phenomenon, as interesting as it is complex. Four years have elapsed since then and during this time more than six hundred memoirs and notes have appeared, to what purpose the reader of the following pages will see.

The present text is divided into three parts.

It is evident, a priori, that by virtue of the principle that "there can be no effect without a cause" the phenomenon of bacteriophagy is induced by a "something"- by a "principle." This is necessarily true whatever may be the hypothesis advanced as to the state or nature of the causative agency.

A study of bacteriophagy in vitro is the first aspect of the problem which we will consider. In reality, this is simply a study of the respective behaviors of the unknown "principle,"the cause of the phenomenon, and of the bacterium which is subject to the phenomenon. Before studying the nature of a principle we must know something of how that principle behaves.

From the broader point of view all of the studies carried out on the phenomenon of bacteriophagy itself have confirmed my first observations. Thanks to the experiments of a great many authors certain details of the process have been more clearly defined, and it may be said that today the phenomenon itself is well understood. There remains, however, still another side to the question, a phase which opens a field of the greatest theoretical and practical importance, namely, the problems associated with the resistant forms of bacteria, with the infravisible forms in particular.

What are the properties of this "principle" which causes the phenomenon? What is its physical state? And more important yet, what is its biological state? These are the things that it is important for us to discover. These are the points which it is particularly important to bring to the attention of the trained scientific mind. 



\section{CONTENTS}

\section{INTRODUCTION}

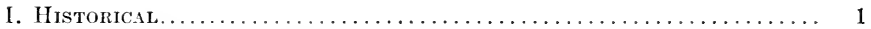

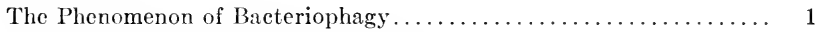

Bacterioclysis: The Twort Phenomenon.................. 13

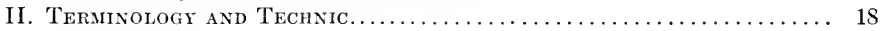

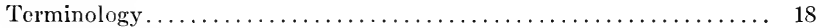

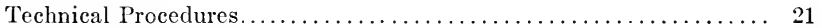

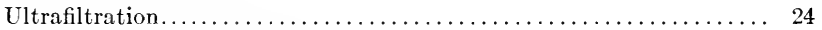

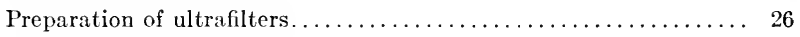

Assembling the ultrafilter........................ 29

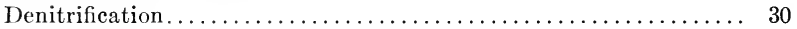

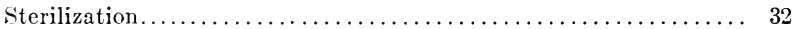

\section{PART I. THE PHENOMENON OF BACTERIOPHAGY}

Chapter I

BACTERIOPHAGY IN A FLUID MEDIUM

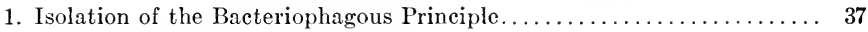

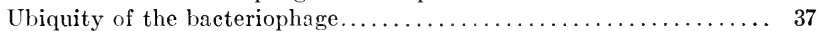

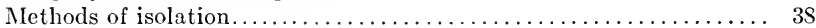

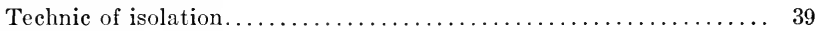

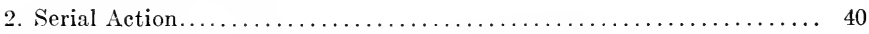

3. Environmental Conditions Favoring Bacteriophagy ............ 41

General conditions................................ 41

Reaction of the medium.......................... 43

4. Effect of the Condition of the Bacterium ................. 46

5. Effects of the Relative Concentrations of Bacteriophage and Bacteria.. 49

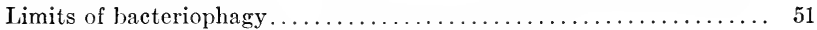

Limits of activity of the bacteriophage principle............ 55

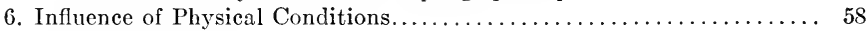

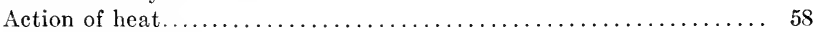

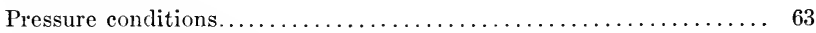

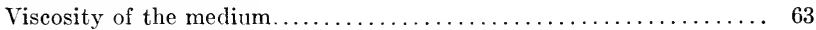

7. Effect of the Chemical Conditions of the Medium.............. 65

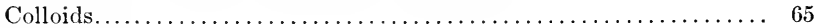

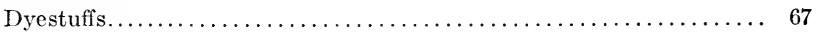

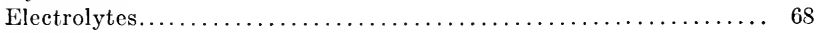

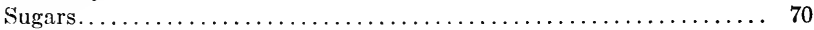

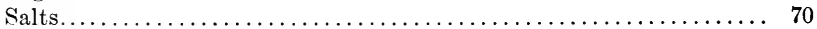

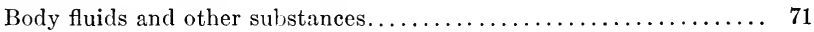

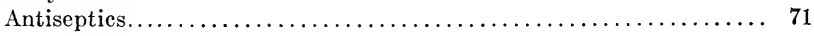

ix 
8. Phenomena Correlative with Bacteriophagy $\ldots \ldots \ldots \ldots \ldots \ldots \ldots \ldots \ldots$

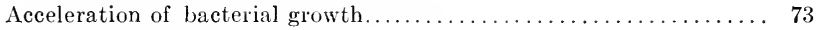

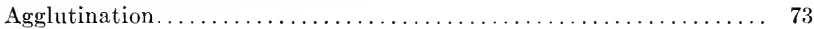

Résumé.......................................... 74

\section{Chapter II}

THE BACTERIOPHAGE CORPUSCLE

1. Bacteriophagy upon Solid Media..................... 76

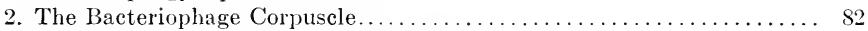

3. The Plaque: A Colony of Bacteriophage Corpuscles............ S7

4. Conditions Essential for Plaque Formation ................. 88

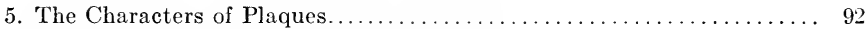

6. Enumeration of Bacteriophage Corpuscles................ 96

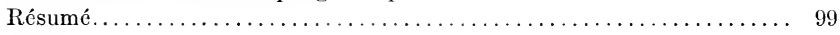

\section{Chapter III}

\section{THE MECHANISM OF BACTERIOPHAGY}

1. The Corpuscle: Obligatory Bacteriophage................... 101

2. Fixation of the Bacteriophage Corpuscle .................... 104

3. Penetration of the Corpuscle into the Bacterium . . . . . . . . . . . . 112

4. Multiplication of the Bacteriophage Corpuscle............... 115

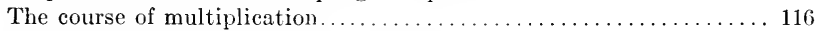

Influence of temperature upon multiplication . . . . . . . . . . 123

Multiplication as affected by the state of the bacteria........... 124

Multiplication in relation to the number of bacteria bacteriophaged. . 125

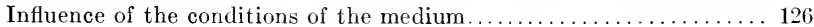

Cause of the arrest of multiplication..................... 127

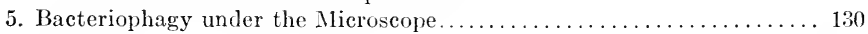

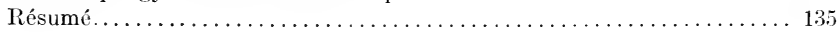

\section{Chapter IV}

THE VIRULENCE OF THE BACTERIOPHAGE

1. Variation in the Activity of Bacteriophage Corpuscles............ 137

2. Evaluation of the Virulence of a Bacteriophage .............. 141

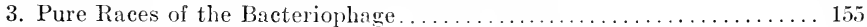

4. Variability in the Virulence of the Bacteriophage.............. 159

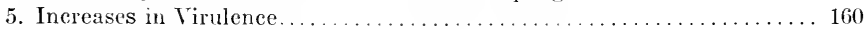

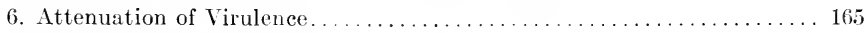

7. Homogeneous and Heterogeneous Bacterial species............. $16 \mathrm{~s}$

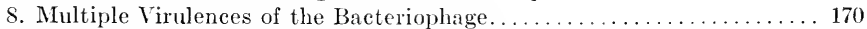

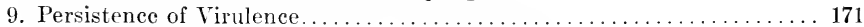

10. The Mechanism of the Persistence of Virulence.............. 173

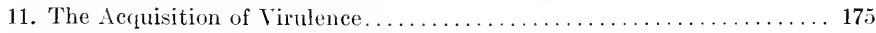

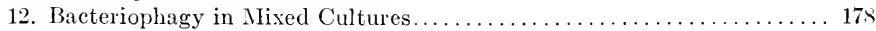

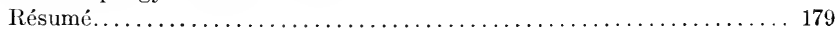




\section{Chapter V}

RESISTANCE OF THE BACTERIA

1. Secondary Cultures................................... 182

2. The Origin of Secondary Cultures ........................ 188

3. Variability in Acquired Resistance........................... 191

4. The Acquisition of Resistance............................. 194

5. The Behavior of the Bacteriophage in Secondary Cultures.......... 196

6. The Loss of Resistance.................................... 203

7. The Bacteria of Secondary Cultures......................... 206

8. Mixed Cultures....................................... 208

9. The Cause of Secondary Cultures......................... 215

10. The Resistant Bacterium................................ 217

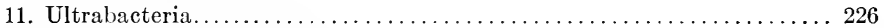

12. Natural Mixed Cultures................................. 230

13. The Purification of Natural Nixed Cultures................... 236

14. Isolation of the Bacteriophage from Naturally Mixed Cultures........ 237

Résumé........................................... 238

\section{Chapter VI}

SPECIES OF BACTERIA SUSCEPTIBLE TO BACTERIOPHAGY

1. Homogeneous Species...................................... 242

1. B. dysenteriae Shiga ............................. 242

2. B. dysenteriae Hiss.................................. 243

3. B. dysenteriae Flexner.................................. 244

4. Pseudo-dysentery organisms....................... 244

5. Bacilli of fowl typhoid............................ 245

6. Pasteurella bovis.................................. 246

7. B. pestis.................................... 247

2. Heterogeneous species............................... 248

1. B. typhosus...................................... 248

2. B. paratyphosus A ................................ 251

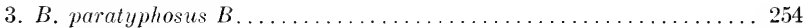

4. B. suipestifer.................................. 254

5. B. enteritidis................................... 254

6. B. typhi-murium................................. 254

7. B. coli......................................... 255

8. The Pneumobacillus of Friedländer................... 256

9. The Bacillus of Flacherie ........................ 256

10. B. proteus.................................... 257

11. The Bacillus of Swine Fever........................ 258

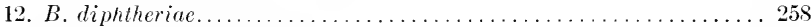

13. Nodule Bacteria of Plants............................. 259

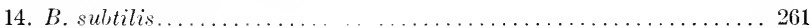

15. Vibrio eholerae ................................... 261

16. The Staphylococci............................... 264 
17. The Enterococcus............................... 266

18. Streptococcus pyogenes............................. 266

3. Phenomena Simulating Bacteriophagy $\ldots \ldots \ldots \ldots \ldots \ldots \ldots \ldots \ldots \ldots \ldots$

B. anthracis........................................ 266

B. pyocyaneus..................................... 267

Résumé......................................... 269

\section{PART II. THE BACTERIOPHAGE}

\section{Chapter I}

THE BEHAVIOR OF THE BACTERIOPHAGE TOWARD DIFFEREN'T AGENTS

1. The Physical State of the Bacteriophage .................... 273

Filtrability ........................................ 274

Diffusibility...................................... 277

Volatility....................................... 277

Sedimentation..................................... 280

Nature of the "Substance" of the bacteriophage corpuscle......... 281

2. Conservation of the Bacteriophage Corpuscle $\ldots \ldots \ldots \ldots \ldots \ldots \ldots 283$

3. Flocculation of Corpuscles.............................. 287

4. Adsorption of Bacteriophage Corpuscles...................... 288

5. Effects of Irradiation.................................. 291

6. Effect of Temperature.................................. 292

7. Action of Inorganic Chemical Substances..................... 300

8. Action of Organic Compounds............................ 302

9. Variability in the Resistance of the Bacteriophage.............. 306

Résumé........................................... 307

\section{Chapter II}

HYPOTHESES CONCERNING THE NATURE OF THE BACTERIOPHAGE

1. Possible Hypotheses....................................... 309

I. Hypothesis of a chemical principle foreign to the bacterium.... 309

II. Hypothesis of a principle derived from the bacterium ......... 311

A. Hypothesis of an abnormal inert principle.............. 311

B. Hypothesis of a living abnormal principle derived from the bacterium................................ 315

C. Hypothesis of a normal autolysin.................. 316

D. Hypothesis of a living principle, normally present in the bacterium................................... 325

III. The Bacteriophage is a Living Being, Foreign to the Bacterium.. 326 Résumé....................................... 327

\section{Chapter III}

THE NATURE OF THE BACTERIOPHAGE

1. Statement of the Problem.................................... 329

2. The Criteria of Life.................................... 330

3. The Autonomy of the Bacteriophage Corpuscle................... 333 
4. The Power of Assimilation of the Bacteriophage Corpuscle......... 341

5. The Power of Adaptation of the Bacteriophage Corpuscle.......... 341

6. The Faculty of Multiplication of the Bacteriophage Corpuscle........ 349

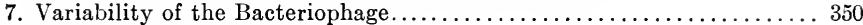

8. The Bacteriophage Corpuscle: A Living Ultravirus............. 354

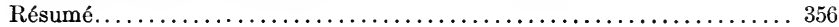

Chapter IV

THE UNICITY OF THE BACTERIOPHAGE PROTOBE

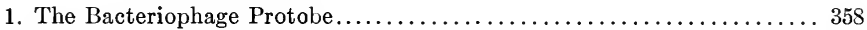

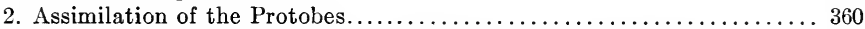

3. The Concept of Species Among the Microbes and the Protobes....... 365

4. The Unicity of the Species Protobios bacteriophagus................ 366

5. The Mode of Action of the Bacteriophage.................... 369

6. Consequences Resulting from the Living Nature of the Bacteriophage.. 374

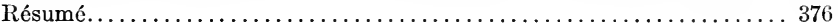

PAR'T III. THE BEHAVIOR OF THE BACTERIOPHAGE PROTOBE

\section{Chapter I}

THE BACTERIOPHAGE AS AN ANTIGEN

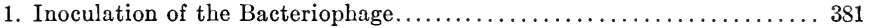

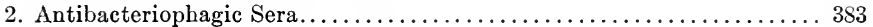

3. The Course of the Action of the Antibacteriophagic Serum.......... 386

4. Variability in the Behavior of the Bacteriophage............... 387

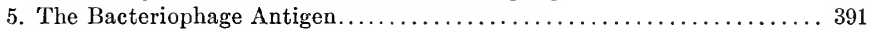

6. The Nature of the Antibacteriophagic Property............... 392

7. Complexity of the Antibacteriophagic Serum................. 397

8. The Anti-antibacteriophagic Serum ...................... 398

9. The Action of Antibacterial Sera....................... 400

10. The Phenomenon of Antiphylaxis..................... 403

11. The Opsonic Action of Bacteriophage Suspensions.............. 408

Résumé...................................... 414

\section{Chapter II}

\section{THE UBIQUITY OF THE BACTERIOPHAGE}

1. The Bacteriophage in the Intestinal Tract................. 416

2. The Bacteriophage in Healthy Man...................... 418

3. The Bacteriophage in Animals........................ 424

4. The Bacteriophage in the Horse...................... 426

5. The Bacteriophage in the Chicken and in the Goose............. 429

6. The Bacteriophage as Found in a Number of Different Species........ 430

7. The Virulence of the Bacteriophage in the Normal Animal......... 431

8. The Bacteriophage in the External Environment.............. 435

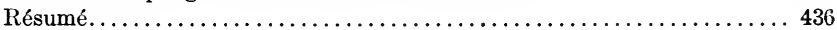




\section{Chapter III}

THE BEHAVIOR OF THE BACTERIOPHAGE IN DISEASE

1. Variations in the Virulence of the Bacteriophage.............. 437

2. The Bacteriophage in Bacillary Dysentery .................. 439

3. The Bacteriophage in Typhoid Fever................... 451

4. The Bacteriophage in Avian Typhosis.................... 479

5. The Bacteriophage in Staphylococcus and Streptococcus Infections... 483

6. The Bacteriophage in Colon Bacillus Infections................ 483

7. The Bacteriophage in Typhus Exanthematicus................ 485

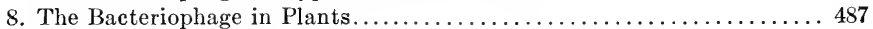

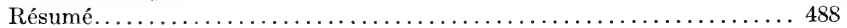

\section{Chapter IV}

THE BEHAVIOR OF THE BACTERIOPHAGE IN EPIDEMICS

1. Avian Typhosis.................................. 490

2. Hemorrhagic Septicemia of the Buffalo (Barbone) $\ldots \ldots \ldots \ldots \ldots \ldots \ldots 497$

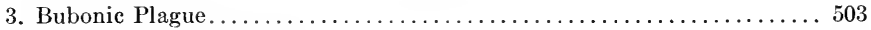

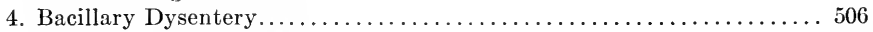

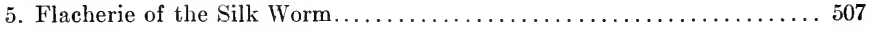

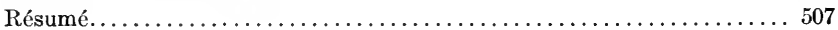

\section{Chapter V}

\section{IMMUNIZATION WITH BACTERIOPHAGE SUSPENSIONS}

1. The Problem of Prophylactic Immunization................. 509

2. Immunization against Avian Typhosis..................... 510

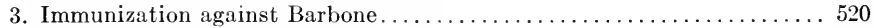

4. Immunization against Bacillary Dysentery ............... 533

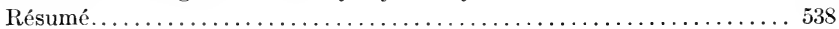

\section{Chapter VI}

SPECIFIC THERAPY WITH BACTERIOPHAGE SUSPENSIONS

1. The Specific Therapy of Bacillary Dysentery............... 540

2. Bacteriophage Therapy in Different Intestinal Disturbances......... 549

3. Bacteriophage Therapy in Typhoid and the Paratyphoid Fevers....... 549

4. Bacteriophage Therapy of Colon Bacillus Infections............. 553

5. Bacteriophage Therapy of Staphylococcus Infections........... 558

6. Bacteriophage Therapy of Infected Wounds.................. 564

7. Bacteriophage Therapy of Streptococcus Infections ............ 567

8. Bacteriophage Therapy of Bubonic Plague................. 567

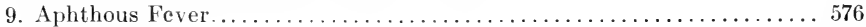

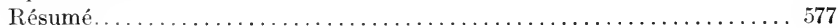

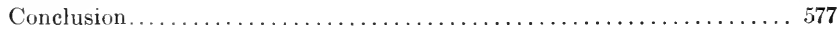

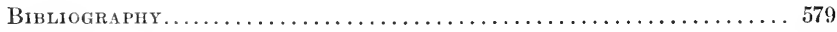

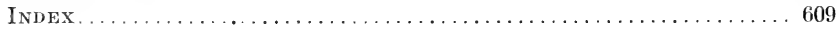




\section{INTRODUCTION}

\section{HistoricAL}

\section{THE PHENOMENON OF BACTERIOPHAGY}

Before considering the historical development of our knowledge concerning the phenomenon of bacteriophagy, let us consider the circumstances which offered a veiled suggestion that such a phenomenon existed, although none of the facts pertaining to the reaction were at that time known. Let us consider the initial observation which served as the starting point for the study of the phenomenon of bacteriophagy.

During the course of the investigation of a disease, bacterial in origin, affecting locusts, first noted in Mexico in 1909 where whole swarms of the insects succumbed to the infection* several new facts were observed. The disease, as it occurred naturally was primarily a septicemic condition, accompanied by intestinal disturbances, as revealed by the profuse diarrhea seen in the infected insects. The pathogenic microörganism, Coccobacillus acridiorum, was present in the intestinal fluids in great abundance.

Inasmuch as these locusts constitute an insect pest, causing enormous destruction of crops in many tropical regions, and indeed in some sub-tropical zones, it seemed that it might be possible and distinctly advantageous to artificially implant this natural epizoötic among the colonies made up of larval forms and thus destroy the harmful insects in large numbers. To this end, the virulence of the coccobacillus was enhanced by successive passages through locusts in the laboratory. Large quantities of a fluid culture medium were inoculated with the bacterium and this material, after incubation, was distributed from place to place among the masses of insects, still in the larval state. Under such conditions the disease developed rapidly. $\dagger$

* d'Herelle-Sur une épizootie de nature bactérienne sévissant sur les Sauterelles en Mexique. Compt. rend. Acad. sci., 1911, 152, 1413.

$\dagger$ Consult on this subject:

d'Herelle-Le Coccobacille des Sauterelles. Ann. Inst. Pasteur, 1914, 28, $280 ; 387$.

Beguet, M.-Deuxième campagne contre les sauterelles Stauronotus maroccanus Thun. en Algérie au moyen du "Coccobacillus acridiorum" d'Herelle. Ann. Inst. Pasteur, 1915, 29, 520. 
As has been said, the preparation of cultures for the mass infection of the colonies of insects involved the isolation of the virulent coccobacillus from the intestinal contents of locusts with an experimental laboratory infection. And in this procedure certain observations were made which now offer the point of greatest interest. On several occasions the culture tubes used for isolation, or for transplanting the cultures, yielded colonies which were of indented irregular contour, or, in the midst of a group of confluent colonies there were at times areas entirely free of growth. These cultural irregularities were sufficiently. pronounced to arouse my curiosity, and to explain them, and the phenomenon leading to their formation, an hypothesis was advanced, one which proved to be entirely false. Indeed, it must be added that for a very long time this hypothesis led me to perform many useless experiments.

In accord with this hypothesis it seemed that the coccobacillus could only be an "associated" organism, the true pathogenic agent necessarily being an ultramicroscopic organism, which, occasionally reaching the agar, inhibited the growth of the associated bacterium. This hypothesis appeared the more natural in view of the admirable work of de Schweinitz and Dorset upon hog cholera. But, after having demonstrated that the filtrate by itself contained nothing virulent for the locust the hypothesis was modified in accordance with the concept that the disease required that two agents, visible and invisible, be simultaneously present.

Throughout a long series of investigations attempts were made to determine if this hypothesis could be reconciled with the observed facts

Beguet, M.-Campagne d'expérimentation de la méthode biologique contre le Schistocerca peregrina en Algérie de Décembre 1914 a Juillet 1915, et en particular dans la région de Barika (Département de Constatine). Ann. Inst. Pasteur, 1916, 30, 225.

Velu, H., and Bouin, A.-Essai de destruction du Schistocerca peregrina au Maroc par le "Coccobacillus acridiorum" du d'Herelle. Ann. Inst. Pasteur, $1916,30,389$.

Velu, H.-Deuxième campagne d'expérimentation de la méthode d'Herelle au Maroc contre Schistocerca peregrina Olivier. Ann. Inst. Pasteur, 1917, 31, 277.

Revista del Ministerio de Industrias del Uruguay, 1918.

Revista del Ministerio de Obras publicos de Venezuela, Suppl., 1913.

Cheyssial, A.-Expérimentation de la méthode de d'Herelle en Guinée française pour la destruction des acridiens. Bull. Soc. path. exot., Par., 1922, 15, 762 . 
in some of the human diseases, particularly bacillary dysentery and typhoid fever. But here again, always under the domination of a false hypothesis, the studies were made upon intestinal contents of patients in whom the disease was at its height. To this end filtrates were prepared from a suspension of fecal material and these filtrates were combined with suspensions of the bacterium, B. typhosus or $B$. dysenteriae as the case might be, and these were next inoculated into laboratory animals, hoping thereby to induce symptoms resembling those seen in the human subject during the disease. At the same time, an agar culture medium was inoculated with the filtrate-bacterial suspension mixture in an attempt to reproduce the cultural "abnormalities" noted with the coccobacilli from the locusts.

It is true that this procedure occasionally revealed cultural irregularities, but the phenomenon was very inconstant and this fact prevented a solution of the question. One day, in reëxamining my experimental data my attention was attracted to the fact that when such cultural irregularities appeared it was never at the beginning of the disease but always when filtrates were used which were prepared from fecal material collected during convalescence. I then resolved, and logically this is where I should have commenced, to examine the fecal discharges of individual patients systematically, from the onset of the disease up to the time when convalescence was established.

In August, 1916, an adult with a severe bacillary dysentery (Shiga) was under treatment in the Pasteur Hospital. Each day about 10 drops of the stool were collected and placed in a tube of bouillon. After incubation over night the suspension was filtered through a Chamberland candle. Into some bouillon, previously inoculated with Shiga bacilli, about 10 drops of this filtrate were placed, and the material was returned to the incubator at $37^{\circ} \mathrm{C}$.

Throughout the duration of the disease, all of the tubes, prepared each day in the same manner, gave normal cultures of $B$. dysenteriae. One day, the tube prepared the day before remained sterile. Investigation showed that the patient gave evidence of notable improvement, and, as appeared later, this was shortly followed by definite convalescence.

To the bouillon thus inoculated and containing filtrate, and which had remained to all appearances sterile, a suspension of Shiga bacilli derived from a fresh agar culture was added to yield a marked turbidity. This tube was placed in the incubator. After about 10 hours it was again clear. 
This, of course, made it at once apparent that my first hypothesis was of necessity false, the truth of the matter being that the fecal material used in preparing the filtrate contained something which dissolved the dysentery bacilli. Nevertheless, my first hypothesis had one virtue, since, as it had led me for such a long time to consider the question of a virus pathogenic for the man or the animal it offered the suggestion that the dissolving principle might be a virus pathogenic for the bacterium. And because of this last hypothesis the following experiment was devised and carried out.

A drop of a dissolved culture was added to a fresh bouillon culture of Shiga bacilli. About 15 hours later the bouillon was clear, all of the bacilli originally present had been dissolved. Thus, several successive passages were effected in the same way, employing each time a drop of the culture previously dissolved added to a fresh culture of Shiga bacilli. In this repetition of the process, instead of becoming weaker the activity became more and more pronounced, that is, the disappearance of the bacilli was effected with greater and greater rapidity.

This time the guiding idea appeared to be correct. The principle present in the intestinal contents became regenerated at the expense of the Shiga bacilli. It behaved like a filtrable organism, parasitıc of bacteria.

The next step was an attempt to reproduce the cultural irregularities upon a solid medium. To do this, a very small amount, about $0.0001 \mathrm{cc}$. of one of the dissolved cultures was added to a young broth culture of Shiga bacilli and the mixture was subcultured immediately to an agar slant. Similarly, subcultures were made after incubation for 1, 2, and 3 hours, a drop from the inoculated tube being used in the transfers. After incubation of these agar subcultures the growth revealed some of the "abnormal" appearances which had formerly perplexed me. But this time, the characteristics of these abnormalities were so outspoken that their significance could not be overlooked.

In the first tube the surface of the agar was well-covered with a normal layer of $B$. dysenteriae, except that in the midst of the culture there were two little islands, two "plaques," perfectly circular in form, where the agar was bare, entirely free of all traces of culture. The second agar tube, planted 1 hour after the original combination was made, revealed 6 of these plaques. In the third, prepared after the dissolving principle had acted for 2 hours, there were about 100 of the plaques. The fourth tube remained without any evidence of bacillary growth. 
This, then, gave a new proof that the dissolving principle actually regenerated in the course of the action. Further, it demonstrated that the principle was condensed in the form of active particles.

It is to this principle that I have given the name Bacteriophage; the phenomenon of bacterial solution caused by it being termed Bacteriophagy.

With this simple statement of what is meant by the "phenomenon of bacteriophagy" in its broader aspects, let us see if the literature published prior to my papers on the subject contains data such as might be construed as dealing with the same phenomenon.

In the first work summarizing my communications on the subject ${ }^{321 *}$ mention was made particularly of a paper by Hankin dealing with a bactericidal property of the water of the Jumna and of the Ganges rivers. Since the text, "The Bacteriophage, Its Rôle in Immunity," was published various authors have made a study of the literature and many have sought to discover, in the many studies conducted upon the subject of "bacteriolysis," facts which bear upon the question of bacteriophagy.

Let us state immediately, and we will return to this question, that the term "lysis," which should always have been applied strictly in its sense of "a dissolution," has lost in biological usage all significance, and is in fact applied to phenomena which are without any effect upon the vitality of the bacterium regarded as undergoing a "bacteriolysis." This is the first source of error, as we shall see. The second cause of error is resident in a faulty logic on the part of those who have examined these earlier communications. Bacteriolysis, even true bacteriolysis, resulting in a dissolution of the bacterial cell, is not a phenomenon with but a single cause. Bacteriolysis is a syndrome, one might say, which can be provoked by different causes or diverse agents. A number of varieties of bacteria undergo dissolution when placed in water saturated with ether. May one speak here of bacteriophagy? The pneumococcus, like some other fragile bacteria, is dissolved within a few days if it is allowed to remain in the liquid culture medium where it has developed. Is this bacteriophagy? Certainly not. For, in neither case is the dissolution accompanied by the very special characteristics which delimit bacteriophagy.

Bacteriophagy, as we will see in the course of this text, is a phenomenon presenting very distinctive characteristics, such as permit it

* Referring to the bibliographic material appended to the text. 
to be clearly defined. To attempt to reconcile "bacteriolysis" and "bacteriophagy," making them identical and co-extensive is a scientific absurdity. Yet this is precisely what some authors, fortunately now becoming fewer in number, seem to have thought they must do.

In a lengthy review of the literature dealing with the bacteriophage Otto and Munter ${ }^{494}$ go into the historical aspect of the subject,* citing all of the more important contributions upon the question of bacteriolysis in general, beginning with that of Kruse and Pansini† upon the autolysis of the pneumococcus.

But bacteriolysis and bacteriophagy are by no means synonomous. Bacteriolysis, the general syndrome, is but an episode in bacteriophagy. It is not the event which distinctly characterizes the phenomenon. Bacteriophagy is certainly not involved in the autolysis of B. pyocyaneus, as observed by Emmerich and Löw, $\ddagger$ nor in the autolysis of $B$. anthracis, as noted by Gamaleia§ and studied by Malfitano.\|

The mechanism of the process here is not related to bacteriophagy any more than is the bacteriolysis of this same anthrax bacillus when subjected to the serum of certain animals. There is a similar lack of relationship as regards the inhibitory effects of old normal cultures upon the development of certain bacteria, as first reported by Eijk-

* Mention may be made of the fact that in presenting this historical review Otto and Munter did not seek to introduce any question of priority. Indeed, their paper is entitled "Bacteriophagy," bearing the sub-title "d'Herelle's Phenomenon," a fact in itself significant. Otto and Munter, like the other German investigators, have, in my opinion been perfectly logical in this respect. My sole criticism of the historical part of the review of Otto and Munter is one of a purely scientific nature; a criticism concerning the abuse of generalizations.

$\dagger$ Tíruse, W., and Pansini, S.--Untersuchungen über den Diplococcus pneumoniae und verwandte Streptokokken. Zeitschr. f. Hyg. u. Infektionskrankh., $1892,11,279$.

$\ddagger$ Emmerich, R., and Löw, O.-Bakteriolytische Enzyme als Ursache der erworbenen Immunität und die Heilung von Infektionskrankheiten durch dieselben. Zeitschr. f. Hyg. u. Infektionskrankh., 1899, 31, 1.

§ Gamaleia.-Bakteriolysine-bakterienzerstörenden Fermente. Abst. in:Centralbl. f. Bakt., I. Orig., 1899, 26, 661.

\| Malfitano, G.-La bactériolyse de la bactéridie charbonneuse. Compt. rend. Acad. sci., 1900, 131, 295.

Malfitano, G. and Strada, F.-Evaluation du pouvoir protéolytique des bactéridies du charbon. Compt. rend. Soc. biol., 1905, 59, 118;--Des influences qui peuvent faire varier le pouvoir protéolytique des liquides en contact avec des bactéridies du charbon. Compt. rend. Soc. biol., 1905, 59, 120;-Influence de l'aération des cultures sur le pouvoir protéolytique des bactéridies charbonneuse. Compt. rend. Soc. biol., 1905, 59, 197. 
man,* and later by Conradi and Kurpjuweit, $\dagger$ Rahn, $\ddagger$ and Faltin, $\S$ to cite only the more important studies.

All of these demonstrations of the bacteriolytic process can be repeated with the greatest ease, and none of them show the slightest resemblance to bacteriophagy. To even suggest a relationship is impossible. Indeed, it is but fair to state that none of the authors mentioned have endeavored to establish such a relationship. Recently Eijkman has conducted some experiments upon bacteriophagy and he states\| that he can not see any possible connection between the phenomenon which he formerly studied and that of bacteriophagy. More recently Hajós $\uparrow$ by the advice of Koranyi, has considered this question again, more particularly to determine if there is any relation between the inhibitory action of filtrates of old cultures and the phenomenon of bacteriophagy. His conclusions are identical with those of Eijkman,- the phenomenon of inhibition has no demonstrable connection with that of bacteriophagy.

These former studies are mentioned here simply because of the fact that certain authors (Pico ${ }^{511}$ ) have attempted to correlate all of the phenomena in which bacteriolysis represents a phase, establishing thus a certain degree of confusion, and because they are mentioned in the historical discussion of Otto and Munter.

There are, on the other hand, some experimental observations which may possibly be interpreted as revealing the intervention of the bacteriophage, but even here it is impossible to definitely affirm this, since some of the findings reported by the authors seem to oppose such a correlation.

Hankin** stated that the water of certain rivers of India possesses

* Eijkman, C.-Ueber thermolabile Stoffwechselprodukte als Ursache der natürlichen Wachstumshemmung der Mikroorganismen. Centralbl. f. Bakt., I. Orig., 1904, 37, 436.

$\dagger$ Conradi, H., and Kurpjuweit, O.-Ueber spontane Wachstumshemmung der Bakterien infolge Selbstvergiftung. München. med. Wchnschr., 1905, 1761.

$\ddagger$ Rahn, O.-Ueber den Einfluss der Stoffwechselprodukte auf das Wachstum der Bakterien. Centralbl. f. Bakt., I. Orig., 1906, 16, 417; 609.

$\S$ Faltin, R.-Studien über Hetero- und Isantagonismus, mit besonderer Berücksichtigung der Verhältnisse bei infektiosen Erkrankungen der Harnwege. Centralbl. f. Bakt., I. Orig., 1908, 46,$6 ; 109 ; 222$.

\| Reunion annuelle de la Societie des Bacteriologues Neerlandais, 1923.

T Hajós, K.-Beiträge zur Frage der wachstumshemmenden Wirkung von Bouillonkulturen. Centralbl. f. Bakt., I. Orig., 1922, 88, 583.

** Hankin, E.-L'action bactéricide des eaux de la Jumna et du Gange. Ann. Inst. Pasteur, 1896, 10, 511. 
an extremely marked antiseptic action for bacteria in general, and for the cholera vibrio in particular. Thus, the water of the Jumna, as it left the town of Agra, contained more than 100,000 bacteria per cubic centimeter, while some 5 kilometers further down the bacterial count was reduced to but 90 to 100 organisms.

Dealing more particularly with the cholera vibrio, his laboratory findings gave the results presented in the following table. In this table the first line shows the effect of the Jumna river water after filtration. The figures of the second line represent the action of the same filtered water after boiling. In both instances, the water had been inoculated with a culture of $V$. cholerae, and the rate of action is shown by the bacterial counts made after different intervals.

\begin{tabular}{|c|c|c|c|c|c|c|c|}
\hline & \multicolumn{7}{|c|}{ TIME } \\
\hline & 0 hour & 1 hour & 2 hours & 3 hours & 4 hours & 25 hours & 49 hours \\
\hline $\begin{array}{l}\text { Filtered, } \quad \text { unheated } \\
\text { water............. }\end{array}$ & 2,500 & 1,500 & 1,000 & 500 & 0 & 0 & 0 \\
\hline Filtered, boiled water. . & 5,000 & 4,000 & 6,000 & 10,000 & 6,000 & 10,000 & 36,000 \\
\hline
\end{tabular}

The germicidal action of the water of these rivers could always be detected, but it was not uniform in degree.

It is of interest that it is to this antiseptic action that Hankin attributes the fact that he never was able to demonstrate that the ingestion of the water of these rivers was responsible for the development of a single case of cholera. Certainly these rivers were never the carriers of epidemics; cholera always spreads from down-stream upwards.

Hankin showed that the antiseptic principle was destroyed by boiling, and, from his experiments, he deduced that it was volatile.

$\mathrm{Flu}^{220}$ denies that the bacteriophage was the agency responsible for this germicidal action, since Hankin had shown that a volatile substance was involved. It is certain that if this statement of Hankin is correct, it by itself suffices to prove that the bacteriophage was not involved. As a matter of fact the experiment on volatilization offers abundant chance for error, and we shall see that this error has contributed to the results of some investigators who have considered the question of the volatile nature of the bacteriophage. The error lies in the fact that when distillation is carried out at low temperatures, without special precautions, materials are carried over into the distillate, leading to the erroneous conclusion that a volatilization has 
taken place, when in reality it has not occurred and does not occur when the experiment is properly conducted.

It should be the duty of some bacteriologist in India to repeat the studies of Hankin. For if the antiseptic substance present in the water of these rivers is actually volatile the action can not be bacteriophagic in nature. While, on the other hand, if these waters reproduce the phenomenon with all of its characteristic features, the bactericidal action observed by Hankin must necessarily be referred to bacteriophagy.

There is another communication* where it is possible that the bacteriophage may not have been foreign to the results,-results which stimulated certain somewhat sarcastic remarks on the part of Metchnikoff. It must be admitted that before the era of the bacteriophage, these results might well excite wonder, and, indeed, certain recommendations of the authors of the memoir in question may, with reason, seem strange. For they state that bacteriologists should not attempt a confirmation of their experiments, most probably because they themselves could not interpret the phenomena observed and were unable to repeat them.

However that may be, here is the passage in the work of Metchnikoff $\dagger$ which refers to these investigations.

“. . . . Emmerich and Löw attribute acquired immunity to particular substances which they call "nuclease-immunoproteidine." In accordance with their supposition the bacterial products which are liberated within the body during the period of vaccination, the nuclease, combines with the protein substances of the blood and of the organs, yielding the substance designated by these authors by this very complex name. In their last publication Emmerich and Löw go so far as to describe a method for the production of this substance outside of the body, by causing beef blood, or better yet, ground-up spleen, to act upon the nuclease produced by the bacteria in old cultures. $\ddagger$ They attribute to this the property of dissolving diverse bacteria, of vaccinating against, and of curing, many infectious diseases. But these authors do not state whether this substance, so very remarkable, is identical or analogous to the antibacterial ferments com-

* Emmerich, R., and Löw, O.-Die künstliche Darstellung der immunisierenden Substanzen (Nucleasen-Immunproteïdine) und ihre Verwendung zur Therapie der Infektionskrankheiten und zur Schutzimpfung an Stelle des Heilserums. Zeitschr. f. Hyg. u. Infektionskrankh., 1901, 36, 9.

$\dagger$ Metchnikoff, E.-L'Immunité dans les Maladies Infectieuses. pp. 267-68.

$\ddagger$ These words are not underscored in the text of Metchnikoff. I have underlined them simply to attract the attention of the reader, for purposes which will become apparent shortly. 
posed as we know, of microcytase and of fixateur. It may be inferred that they believe it comparable to the alexin of Buchner, which is simply a mixture of the two substances already named. Unfortunately, all that this theory of Emmerich and Löw accomplishes is to confuse the reader, and in their publications no proof of their affirmations is to be found. In fact, many statements which they make are at variance with well established observations. Thus, they speak of a complete dissolution of the bacilli of swine erysipelas within the vaccinated animals by their soluble "immuno-proteidine-erysipelase." This has never been demonstrated by them and is indeed in complete contradiction to conscientious observations and to well established facts. On the other hand, they make statements in themselves contradictory. The "immuno-proteidine-pyocyanease" is a substance possessing an extraordinary bactericidal power, not only for the pyocyaneus bacillus but also for several other bacteria, such as the organisms of anthrax, of diphtheria, of typhoid, and of plague. This substance quickly dissolves these bacteria and cures experimental diphtheria and anthrax, but at the same time it is actually subject to contamination by even the most banal organisms, such as $B$. subtilis, from which it is necessary to protect it by the addition of antiseptics. To all of these contradictions, uncertainties, and inaccuracies it is still further necessary to add the advice, actually given to bacteriologists by Emmerich and Löw, that their experiments should not be repeated, for they are not to be successfully performed with ease. In this state of affairs I t elieve that despite the attractiveness in attributing to bacterial products a rôle in the elaloration of antibacterial substances, it is necessary to forbear from following further these authors.

Did the bacteriophage play a rôle in these experiments of Emmerich and Löw? It is indeed difficult to tell, but, if they are correct, or at least if the basis is correct, there can be no doubt that these authors have observed "something." There is hardly more than one possible explanation. One of the old cultures employed in obtaining their so-called "immuno-protein" must have been contaminated with the bacteriophage. We know, for example, that in working with about 100 different strains of the cholera vibrio $\mathrm{Flu}^{220}$ encountered one, and one only, which was contaminated with the bacteriophage. This bacteriophage caused within 3 or 4 hours the dissolution of a suspension of the cholera vibrio derived from any strain whatever.

Upon the basis of a single fact, accidentally observed, Emmerich and Löw doubtless made generalizations too hastily. Imbued with the theory of antibodies they were unable to comprehend what they had observed. Neither they themselves nor anyone else have been able to repeat their experiments, and because most certainly exaggerated from the standpoint of generalization their observation has remained sterile. On the other hand, if one wishes to hold the text of these authors to strict accountability and to take their statements literally 
it would be easy to demonstrate that it could not have been the bacteriophage which was involved, just as the bacteriophage could not have been the cause of the phenomenon observed by Hankin. But in the one case as in the other, making allowances for errors of commission and for generalizations which may have been prematurely drawn by the authors, over-enthusiastic concerning the facts accidentally observed, I can see a possible explanation only in the bacteriophage.

A third communication where the bacteriophage may have been the cause of the facts observed, and here the probability is somewhat greater, is that of Gildemeister.* In 1917, under the name of "Flattenformen" he described irregular aberrant colonies of certain bacilli (typhoid and coli). As a matter of fact it appears that what Gildemeister observed were simply bacterial colonies contaminated naturally by the bacteriophage. I attribute the formation of these aberrant colonies to bacterial mutations in the sense of deVries.

I have found only these three communications in which the facts disclosed might be explained as due to the action of the bacteriophage. In view of the violence, as it might be termed, with which bacteriophagy is often effected, it is indeed strange that it has not more often attracted attention forcibly, especially in view of the ubiquity of the bacteriophagous principle. I believe this can be explained in only one way. There must have been many bacteriologists who have witnessed the complete clearing of a broth culture which had been prepared with material derived from the body of a man or sick animal, or who have experienced the impossibility of subculturing to a solid medium an organism derived from the body, or again, who have observed on a solid medium the presence of "plaques," the bare spots mentioned in preceding paragraphs. Indeed, this is certain, since during the past few years many bacteriologists have told me that they have encountered such things.

In 1919, prior to my departure for Indo-China, that is, before I had discovered that resistance to the plague bacillus, in man and in the rat, was due to the presence in these animals of a bacteriophage virulent for this bacillus, Nageotte told me that in the course of a conversation with Haffkine upon the subject of my studies, the latter told him that he had observed repeatedly that bouillon tubes inoculated

* Gildemeister, E.-Weitere Mitteilungen über Variabilitätserscheinungen bei Bakterien, die bereits bei ihrer Isolierung aus dem Organismus zu beobachten sind. Centralbl. f. Bakt., I. Orig., 1916/17, 79, 49. 
with the contents of a plague bubo, after becoming immediately turbid through the development of $B$. pestis, became within the space of a few hours absolutely cleared. The phenomenon was known in his laboratory by the name of " $B$. pestis suicide." Unquestionably it was due to the bacteriophage; in these particular cases $B$. pestis and the bacteriophage being co-existent in the bubo developing toward recovery.

Pinoy has informed me that at the beginning of the late war, being in Morocco engaged in the preparation of anti-typhoid vaccine, he isolated a strain of $B$. paratyphosus $A$ which presented definite plaques, comparable in all respects to those which I have shown to be produced by the bacteriophage.

Also during the war, while stationed at Tiflis where he had charge of the sanitary control of the water of the Koura river, Eliava observed the following phenomenon. The water under examination was added to a peptone-water medium. After incubation for a few hours a specimen removed from near the surface of the medium showed microscopically an abundance of vibrios with a normal form. Transfers to agar gave a light dull layer of growth which was microscopically composed of a culture of the vibrios. Some 12 hours later, from both the peptone water and the agar all trace of the vibrios had disappeared. This observation was made several times, always giving a similar result and it was impossible to obtain a culture of a vibrio which had once commenced to develop and then disappeared a few hours later. This phenomenon was inexplicable up to the time when he noted the first communications dealing with the bacteriophage.

It is, therefore, certain that a large number of bacteriologists have accidentally demonstrated that such a strange phenomenon may take place in a solid or a liquid culture. But, because of the impossibility of reproducing the phenomenon at will, they have been unable to pursue the study and they have not ventured to publish such things since they appeared to be at variance with all known facts.

It is, indeed, simply to the strangeness of the phenomenon of bacteriophagy, to its character of being what might be called paradoxical that I owe the chance, so rare in contemporary science, of having been able to perfect the study of such an extremely complex phenomenon, to have had time to investigate its effects in nature, chiefly from the point of view of the cure of infectious disease, to study their experimental reproduction, even to make to this end a voyage and a year's stay in Indo-China, there to more readily observe human and animal 
contagious diseases, and all of this before anyone even attempted to prove that the phenomenon itself was real, a labor which might have required a half hour's time. This is the more unusual since I had published in some ten communications the facts observed and the results obtained. They must have considered the author as a dreamer; indeed, some have since admitted that this was the case.

\section{BACTERIOCLYSIS: THE TWORT PHENOMENON}

In 1915, almost two years before my first communication upon the subject of bacteriophagy, Twort described a phenomenon* which possesses a character in common with that which I have described, namely, it is reproducible in series. Aside from this common character, it offers other characteristics, not merely different but which preclude all possibility of identity, for the characteristics of the two phenomena are mutually exclusive. But inasmuch as some authors have tried, despite this, to attribute the two phenomena to a single cause, quite without any experimental demonstration it is true, it seems necessary to consider this subject at some length.

First, let me present that part of Twort's paper which describes the phenomenon which he observed. The transcription is literal.

Some interesting results, however, were obtained with cultivations from glycerinated calf vaccinia. Inoculated agar tubes, after 24 hours at $37^{\circ} \mathrm{C}$., of ten showed watery-looking areas, and in cultures that grew micrococci it was found that some of these colonies could not be subcultured, but if kept they became glassy and transparent. On examination of these glassy areas nothing but minute granules, staining reddish with Giemsa, could be seen. Further experiments showed that if a colony of the white micrococcus that had started to become transparent was plated out instead of being subcultured as a streak then the micrococci grew, and a pure streak culture from certain of these colonies could be obtained. On the other hand, if the plate cultures (made by inoculating the condensation water of a series of tubes and floating this over the surface of the medium) were left, the colonies, especially in the first dilution, soon started to turn transparent, and the micrococci were replaced by fine granules. This action, unlike an ordinary degenerative process, started from the edge of the colonies, and further experiments showed that when a pure culture of the white or the yellow micrococcus isolated from vaccinia is touched with a small portion of one of the glassy colonies, the growth at the point touched soon starts to become transparent or glassy, and this gradually spreads over the whole growth, sometimes killing out all the micrococci and replacing these by fine granules. Experiments showed

* Twort, F. W.-An Investigation on the Nature of Ultramicroscopic Viruses. Lancet, 1915, ii, 1241. 
that the action is more rapid and complete with vigorous-growing young cultures than with old ones, and there is very little action on dead cultures or on young cultures that have been killed by heating at $60^{\circ} \mathrm{C}$. Anaerobia does not favor the action. The transparent material when diluted (one in a million) with water or saline was found to pass the finest porcelain filters (Pasteur-Chamberland F. and B. and Doulton White) with ease, and one drop of the filtrate pipetted over an agar tube was sufficient to make that tube unsuitable for the grow th of the micrococcus. That is, if the micrococcus was inoculated down the tube as a streak, this would start to grow, but would soon become dotted with transparent points which would rapidly extend over the whole growth. The number of points from which this starts depends upon the dilution of the transparent material, and in some cases it is so active that the growth is stopped and turned transparent almost directly it starts. This condition or disease of the micrococcus when transmitted to pure cultures of the micrococcus can be conveyed to fresh cultures for an indefinite number of generations; but the transparent material will not grow by itself on any medium. If in an infected tube small areas of micrococci are left, and this usually happens when the micrococcus has grown well before becoming infected, these areas will start to grow again and extend over the transparent portions, which shows that the action of the transparent material is stopped or hindered in an overgrown tube; but it is not dead, for if a minute portion is transferred to another young culture of the micrococcus it soon starts to dissolve up the micrococci again. Although the transparent material shows no evidence of growth when placed on a fresh agar tube without micrococei it will retain its powers of activity for over six months. It also retains its activity when made into an emulsion and heated to $52^{\circ} \mathrm{C}$, , but when heated to $60^{\circ} \mathrm{C}$. for an hour it appears to be destroyed. It has some action, but very much less, on Staphylococcus aureus and albus isolated from boils of man, and it appears to have no action on members of the coli group or on streptococci, tubercle bacilli, yeasts, etc.

Such is the description given by Twort of the phenomenon which he observed.

The first remark, extremely important, is that there is no lysis, no dissolution of the bacteria. The final result of the transformation as described by Twort is a vitreous or transparent substance, formed of fine granules which take a red tint when stained with Giemsa. There is a fragmentation of the cocci, a phenomenon of bacterioclysis.

In the phenomenon of bacteriophagy a complete dissolution of the bacterial cell takes place. There is no residue.

But since Twort says nothing in any of his papers, neither in that of December, 1915, nor in that of $1922^{606}$ of what happens when he adds his "transparent material" to a broth suspension of staphylococci, it is not possible to establish a comparison. It is necessary, therefore, to restrict our comparison to the characteristics of the two phenomena as manifested on agar. 
Twort states that when a drop of a filtrate containing his active principle is spread upon the surface of an agar slant and this tube is next inoculated with a normal culture of the staphylococcus, a normal growth of the staphylococcus commences to develop. Then it undergoes a vitreous transformation, the change having its inception at certain points, to later spread throughout the whole extent of the bacterial layer. If the principle is but slightly diluted the glassy transformation occurs at the same time that the growth takes place.

In the phenomenon of bacteriophagy, if one spreads upon an agar slant a drop of filtrate containing a little of the bacteriophage principle, and if one then inoculates the surface by spreading over it a suspension of the staphylococcus, one obtains a culture for the most part absolutely normal in appearance, but here and there small islands, circular plaques, are found, where the agar is bare without any trace of growth in any form whatever. These plaques undergo no change, even after several days. They never invade the surrounding culture, nor are they ever covered by the bacterial growth. All about these plaques, the culture, retaining its normal appearance, is formed of cocci preserving their normal microscopic form. When one spreads upon the agar a filtrate containing a large quantity of the bacteriophage, and when one next seeds it with a normal culture of the staphylococcus, the agar surface, after incubation, remains naked, free of all evidences of bacterial growth or of anything visible.

If, says Twort, there remain in an infected tube some small regions where the culture is normal, these micrococci develop and invade the areas covered by the transparent material.

I have already stated that in bacteriophagy the plaques, isolated or confluent, where the agar is bare, remain unchanged and are not recovered by the normal surrounding culture, and this is true even if there be but a single plaque upon the entire surface of a culture, and even if this plaque is very small, having a diameter of but a fraction of a millimeter.

Twort states that if a normal culture of susceptible staphylococci is touched with a trace of the "transparent material" derived from a vitreous colony, the culture at the touched point becomes transparent, and the transformation next extends over the entire culture, the micrococci being replaced by granules.

If one touches an agar culture of staphylococci, young or old, in one or in many places, with a fluid containing the bacteriophage, or even if one touches a normal culture with a platinum wire previously 
touched to the surface of a plaque or to the periphery of the plaque, and then places the culture at any temperature whatever-room or incubator-one never observes any transformation. For any length of time whatever the culture as such retains its normal appearance. Microscopic examination shows, except at the point touched, that in all other portions of the culture the micrococci retain their normal form and are never transformed into granules. Indeed, granule formation occurs nowhere.

As is evident, the phenomenon observed by Twort and the phenomenon of bacteriophagy present two entirely different aspects. In the first there is the transformation of the bacteria into fine granules, a "breaking down" according to Twort himself, that is, a bacterioclysis. While when bacteriophagy takes place there is a total dissolution of the bacterial cells, leaving no solid residue visible under any magnification of the microscope.

Gratia $^{248,254}$ believed that he had proof of the similarity of the two phenomena when he stated that he had isolated from vaccinal pulp a principle causing the phenomenon of bacteriophagy with all of its characteristic manifestations, such as I have described them. As a matter of fact, Gratia proved precisely the opposite, that is, he showed that the two phenomena are necessarily different. One might admit a priori that the phenomenon of bacteriophagy might be manifested under different aspects in accord with the bacterium against which it is directed, that is to say, that it might be able to effect the detailed process as I have described it when it takes place in a culture of $B$. dysenteriae, B. typhosus, B. coli, B. pestis, B. proteus, Pasteurella, Vibrios, etc., and that it might occur under the form described by Twort when acting upon cocci. But Gratia has demonstrated that this is precisely what is not the case, but that on the contrary, under the influence of the bacteriophage, the staphylococcus undergoes a typical bacteriophagy, identical in all respects to that of other bacteria.

The sole conclusion to be drawn from the work of Gratia is that staphylococci (and undoubtedly other bacteria as well) are capable of presenting two "diseased states;" the one consisting of a fragmentation showing the characters described by Twort, the other expressing itself by a total dissolution. And for the latter, the distinctive manifestations are not simply different from those of the first, but quite exclusive. In the vaccinal lymph may be found, accidentally, the one or the other of the "principles" which cause these phenomena. There is, indeed, nothing impossible in the idea that they may co-exist in a 
single specimen. Quite commonly two principles, the causes of different phenomena, may be found together in the same medium. I have isolated from vaccinal pulp at two different times cocei presenting the phenomenon described by Fleming.* May one say that this phenomenon is bacteriophagy, simply because the susceptible coccus occurs in vaccinal lymph?

Incidentally, those authors who have likened the phenomenon of bacterioclysis of Twort to the phenomenon of bacteriophagy have restricted themselves to affirmations only, without offering any supporting proof whatever. I am certain that, with their attention being attracted to this point, should they wish to solve the question they will only have to read the paper by Twort on one hand, and on the other perform a few experiments upon the bacteriophagy of the staphylococcus, and for this a suitable bacteriophage is available to everyone. I believe that all will be in accord with me in the view that if the principle discovered by Twort and the bacteriophage are identical, when both are acting upon the same bacterium, the staphylococcus, and under identical conditions as to medium and temperature, they ought to incite identical phenomena. If these phenomena are different, as they actually are, it can only be because the principles differ.

When these authors have themselves made these observations, I trust that they will be willing to distinguish between the two phenomena, and to employ the term "phenomenon of Twort," or better the "phenomenon of bacterioclysis" to the bacterial fragmentation presenting the characteristics described by Twort, and the term "phenomenon of bacteriophagy" to the dissolution of bacteria presenting the characteristics which I first described.

In concluding, it may be remarked that from the very beginning I have considered the arguments which I have here mentioned and which show the dissimilarity of the two phenomena..$^{327}$ These considerations were further developed in the book "The Bacteriophage, Its Rôle in Immunity." They were again repeated at the meeting of the British Medical Association in 1922, a meeting attended by Twort also, who confined himself to simply repeating the statements of his paper of $1915,{ }^{606}$ affirming that the two phenomena were similar, but without discussing, or even alluding to, the arguments which I had advanced in opposition to his point of view. Again I returned to the

* Fleming, A., and Allison, V. D.-Further observations on a bacteriolytic element found in tissues and secretions. Proc. Roy. Soc., Lond., 1922/23, 94B, 142. 
question at the Scarborough Congress of the Institute of State Medicine in $1923 .{ }^{346}$ Twort was again present but he failed to discuss the arguments which I have advanced.

It is difficult to avoid the conclusion that if Twort refrains from such a discussion it is simply because the facts revealing the dissimilarity of the two phenomena are indisputable.

\section{Terminology and Technic}

\section{TERMINOLOGY}

Lack of precision in terminology is, in all branches of knowledge, a permanent cause of confusion. In biology it is the basis of the most unfortunate errors.

I have shown elsewhere* that the science of immunity has been held back for more than twenty years by the equivocation created in the subject by the term "lysis," which as a matter of fact has entirely lost its real significance. As it is, so to speak, impossible to re-establish the true etymological meaning to a word when it has once undergone deflection, and as I desire on the other hand to avoid all misunderstanding, I shall not employ the word "lysis" but will use the word "dissolution," or "to dissolve," and it is necessary to take these terms in their strict sense of "the passage of a solid body into a soluble state, without residue, macroscopically or microscopically visible."

I have applied the term "bacteriophagy" to the phenomenon, in reality very complex as we will see, which consists essentially in a "dissolution" of bacteria through the operation of a principle which I have termed "bacteriophage."

A bacterial suspension, or a culture in a liquid medium, in which complete bacteriophagy has taken place becomes a perfectly clear medium, all of the bacterial cells being dissolved. In it, under the highest magnification, either in the fresh state or after staining, will be found neither microörganisms nor granules.

Since the name "Bacteriophage" has been criticized, I may again state that obviously I have not used the suffix "phage" in its strict etymological sense of "to eat," but in that of "developing at the expense of," a sense which it bears very frequently in scientific nomenclature. Several examples of this might be eited, but I will only mention one, to which I will have occasion to return in the course of this

* Immunity in Natural Infectious Disease; Williams \& Wilkins Co., Balt., 1924. 
discussion. Dangeard* has described a Chitridinea, belonging to the group of Öomycetes, which parasitizes and develops in the nucleus of Ameba verrucosa Ehr. He has termed this Nucleophaga amebae. The word "phage" has exactly the same meaning in the two cases.

Up to the present time we have considered the term "sterile" as implying the freedom of a medium from visible or cultivable bacteria. Nevertheless a medium termed "sterile" is contaminated if it contains an ultravirus, whether this virus be the bacteriophage or the virus of rabies, of vaccinia, or of avian plague, even though the highest magnifications of the microscope fail to reveal the living agent or our artificial media fail to yield a growth. For it is only necessary to place this material in contact with a susceptible living being to demonstrate that "a something" is present which can be cultivated in viro. Consequently, although such a medium is termed "sterile" it is not so in reality. In the discussion to follow the term "sterile" will be employed in its usual sense, and we will designate as "ultrasterile" a medium which contains neither visible microörganisms nor an actually demonstrable ultravirus. I underscore the word actually for it is quite possible that we may sometime discover that there is no organic medium, natural or artificial, which is ultrasterile, at least, until after it has been subjected to an adequate amount of heat or treated with appropriate antiseptics. Only those ultraviruses which exercise a definite pathogenic effect upon another living being are demonstrable at the present time. Among the saprophytes only those can be detected which produce a demonstrable chemical transformation. $\dagger$

When one introduces a trace of bacterial culture into or upon a medium one says that this medium is "inoculated." Evidently the same word might be employed to designate the introduction of a trace of liquid containing the bacteriophage into a medium. It would be necessary to say "I inoculate bouillon with such and such a bacterium and again inoculate it with the bacteriophage," and such a statement in certain cases at least could readily lead to confusion. I shall employ then, the term "inoculate" in its usual sense, meaning the introduction of a bacteriophage into a medium, and when bacteria are introduced into a medium the terms used will be "plant," "implant," or "seed." Thus, I may say "I implant a medium with such and such a bacterium and then "inoculate" it with the bacteriophage."

* Dangeard, P. A.-Le Botaniste, 1894/95, No. 4, 199; 24 S.

$\dagger$ With reference to this subject see Immunity in Natural Infectious Disease, Williams \& Wilkins Co., Baltimore, 1924. 
By the words "normal suspension" as applied to bacteria should be understood "a suspension containing 250,000,000 bacteria per cubic centimeter" prepared from a young agar culture of the bacterium.

In the course of this text I will often have occasion to speak of "a strain" of such and such a bacterium and likewise of "strains" of the bacteriophage. In order to avoid repetition and possible confusion I will make use of the word "strain" in its usual sense as applied to bacteria, and in treating of the bacteriophage the word "race"will be used.

In order to avoid needless circumlocution, in designating the origin of a bacteriophage I shall precede the word bacteriophage by the name of the bacterium which this bacteriophage parasitizes within the body or from which it has been isolated, or against which it manifests its virulence. For example, a "Shiga-bacteriophage" is one which was originally isolated from a case of dysentery due to $B$. dysenteriae Shiga or it is a bacteriophage virulent for this organism. A "Staphylobacteriophage" was isolated from a lesion caused by the staphylococcus. A "Cholera-bacteriophage" was originally derived from a case of cholera. A "Plague-bacteriophage" was derived from a convalescent from plague or from an animal which had resisted this disease. Or, failing this immediate direct conneetion through origin, they may be races of the bacteriophage virulent for the staphylococcus, for Vibrio cholerae, or for $B$. pestis. This scheme will be followed in speaking of all races of baeteriophage.

I devote these few paragraphs to the matter of terminology in order to facilitate the discussion of the facts to be presented, and, I hope, to aid in their comprehension. It is impossible to be too careful in treating a subject of such extreme complexity, where we have to consider always the simultaneous actions and reactions of two living beings, and often of three, when we include the evolution of the bacteriophage in nature. And the subject is still further complicated in that it deals with rudimentary beings; with those whose adaptations to the conditions of the moment are rapidly effected. This fact already dominates the study of bacteria, since from our point of view as human beings, that which interests us most in these other beings is their "virulence" and this is inherently variable. But this faculty of adaptation, one of the principal characteristics of life, is even yet more marked in the bacteriophage, since this is an elementary living being. 


\section{TECHNICAL PROCEDURES*}

Every bacteriological laboratory possesses the materials necessary to conduct experiments upon bacteriophagy. I will only mention the apparatus needful for the isolation of the bacteriophage but I believe it pertinent to discuss the procedures usually employed in biology to effect filtration. $\dagger$

Under ordinary circumstances the bacteriophage passes through all of the usual filter candles, including those made of porcelain, of infusorial earth, of asbestos, etc. Thus, it is possible to use candles of all types, but simply because of economy, in view of the large number of filtrations which it is necessary to make, it is preferable to employ candles which can be sterilized and repeatedly used. Chamberland filters are of this type. A small candle, with an outside length of 7 to $8 \mathrm{~cm}$. may be obtained, which is particularly convenient for test filtrations carried out with small quantities of fluid. It is thus possible to filter the contents of a tube of bouillon containing about $10 \mathrm{cc}$. and to recover 7 to $8 \mathrm{cc}$. of filtrate.

Immediately after the filtration is completed, before the liquid saturating the filter has dried, I would suggest that the filter be boiled in water, as in a casserole, a procedure which is adequate to kill the pathogenic organisms which were present in the fluid subjected to filtration (in the case of spore-producing pathogens it is obviously necessary to subject the filters to autoclaving at $120^{\circ} \mathrm{C}$.). After boiling for about 10 minutes the candles can be removed from the boiling water with forceps and placed in the incubator at $37^{\circ} \mathrm{C}$. to dry. When a number of used filters have been collected they can be sterilized by heat, care being taken that the temperature does not go sufficiently high to damage the material (as the enamel) of which the filter is made. The small amounts of organic material present in the filters after boiling, provided the boiling process is carried out before the filters dry after the filtration, are thus consumed and the filter is restored to a condition comparable to a new candle. By proceeding in this manner it is possible to work for several years, carrying out from 6 to 8 filtrations daily, with 4 or 5 dozen filters, without having to supplement the supply. I emphasize this question a little because a number

* This section of the text is of interest only to those engaged in experimental work upon the subject of bacteriophagy.

$\dagger$ On this subject, of such extreme importance from the point of view of the study of all ultraviruses, consult the text already mentioned-Immunity in Natural Infectious Disease. 
of students located in countries where the rate of exchange is unfavorable have told me that it was impossible for them to make a study of bacteriophagy because of the price of filter candles. We will see in a moment, however, that even this difficulty can be circumvented.

With regard to porosity the most useful candles are those which correspond to the Chamberland $\mathrm{L}_{3}$ filters, which are impermeable to

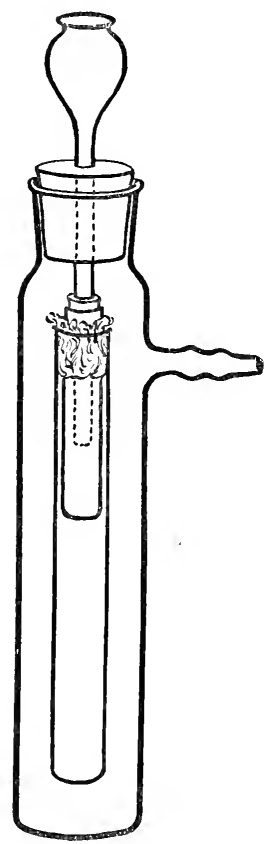

Fig. 1. Filter Assembled According to the Method of Martin

all bacteria. But let me state once more and finally, that a candle capable of heat sterilization and repeated use is perfectly suitable no matter what the make. I simply mention the Chamberland as indicating a type and because they are the filters which I have used heretofore.

Another question having a certain practical importance is the arrange- 
ment of the filtering candle. The best, certainly that which combines the greatest assurance of sterility with the highest degree of simplicity, is the scheme of Martin (fig. 1). This arrangement makes use of a cylinder with a neck, of a separating funnel and of a tube to receive the filtrate. The candle is mounted in the tube in advance by rolling a narrow band of non-absorbent cotton* about the varnished portion of the candle in such a manner that a plug is formed which fits firmly to the ground rim at the neck of the tube. In this way at one time a number of candles may be prepared and sterilized by dry heat.

For the filtration, upon the long tube of the funnel are placed, first, a large rubber stopper which fits into the neck of the cylinder, and then, a small stopper, also of rubber, which is introduced tightly into the opening of the candle. The apparatus being assembled, the fluid to be filtered is poured into the funnel, and in the cylinder a moderate vacuum (a few centimeters of mercury is adequate) is established.

When the filtration is complete, the large stopper is removed, lifting out in this way the candle adapted to its tube, and the funnel is removed. It only remains then to collect the filtrate with a pipette. If sufficient tubes are available, and if it is desired to preserve the filtrate, it is possible, instead of transferring the filtrate with a pipette, to remove the candle and to insert a sterile cotton plug withdrawn from a large test-tube.

I have tried a great many types of filtering apparatus, but of all of those which have been recommended, that of Martin is the most convenient and the most trustworthy. I have effected thousands of filtrations without a single filtrate being contaminated. The apparatus for assembling this type of filter is made in three sizes, the smallest utilizing the candles mentioned above, and serving well for the filtration of quantities of from 10 to $15 \mathrm{cc}$, the middle size is the usual laboratory size, and is adapted to the recovery of 40 to $50 \mathrm{cc}$. of filtrate, and the largest makes use of the same candles as the latter but is adapted to tubes having a capacity of about $150 \mathrm{cc}$. This last serves particularly well for the preparation of relatively large quantities of bacteriophage suspension intended for use in the treatment of patients.

* It is strange that a great many laboratories employ absorbent cotton for all purposes, even for plugging culture tubes. This is certainly illogical, since this cotton, quite true to its name, is hydrophile, i.e., it absorbs water. It is preferable to use a hydrophobe cotton, as a non-absorbent cotton, which has not undergone a special treatment converting it to the condition where it absorbs water. 


\section{ULTRAFILTRATION}

I have stated above that the porous candle usually allows the passage of the bacteriophage. When a liquid contains but a very few bacteriophage corpuscles these may, indeed, be absorbed by the candle and consequently the filtrate becomes ultrasterile.

As we will see, the bacteriophage corpuscle, like all ultraviruses, possesses the general properties of colloids. Indeed, this is a characteristic of all living things. For a long time the physical chemists have recognized that the porous candle can not be used for the filtration of colloids because of their adsorptive property which causes them to retain the substance fixed to the porous material.

Even colloids, the micellae of which possess dimensions infinitely smaller than the pores of the candles, may not pass through. As regards ultraviruses we know that for a long time bacteriologists have argued the question of the filtrability of the agents of rabies, of vaccinia, and of variola. And this difference of opinion has been quite legitimate, for, when filtered through porous candles the passage of the virus is but inconstantly observed. Under these circumstances there is always the possibility that when passage takes place it may have been because of a defective filtering apparatus. But if, instead of the candle an ultrafilter is employed the filtration experiment is invariably successful, even though the pores are, indeed, very considerably smaller. The reason for this is simple. Adsorption, very marked with porcelain, infusorial earth, asbestos, etc., and in general, with all of the mineral substances of which filter candles are composed, is reduced to a minimum with the ultrafilter membranes. Theoretically, only ultrafilters should be employed in microbiology. But, as I have said, in working with the bacteriophage, at least for ordinary studies, the convenient filter candle may be used. For special investigations such as seeking for the bacteriophage in body tissues, fluids, or products, it is essential to resort to ultrafiltration.

Since the matter of ultrafiltration scems to be, in general, rather poorly understood by bacteriologists, it may be well to describe the procedure which is, not only the simplest, but also the most satisfactory in all respects. I have tried all of the methods which have been proposed, and have decided that the method best suited to the work is that of the collodion sac.

J. C. Martin was the first to apply ultrafiltration, employing a silica jelly or gelatin. Shortly afterward Roux and Salimbeni devised the 
collodion sacs such as have been employed since for the introduction of bacterial cultures into the peritoneal cavity of laboratory animals. Borrel next utilized them for the filtration of toxins. But Malfitano should receive the credit for having applied them in 1904 to the systematic study of colloids. The method to be described is that devised by him. It is preferred to the method of Bechhold (or those of other investigators) devised two years later, which likewise makes use of collodion membranes, but in which the apparatus is far more complicated and an assurance of aseptic technic is lacking.

Collodion is a solution of nitrated cotton in a mixture of absolute alcohol and sulfuric ether.* The viscous liquid which results, spread out in a thin layer, becomes gradually impoverished in ether, and then in alcohol, as they evaporate, leaving a layer of nitrocellulose, homogenous and relatively resistant.

At the beginning of the drying the ether evaporates much more rapidly than does the alcohol, and, as the nitrocellulose is insoluble in alcohol alone a gel of sufficient toughness is obtained at a certain stage of the desiccation. At this moment it is necessary to interrupt the drying by plunging the membrane into water, for if drying becomes complete, the membrane is impermeable. Spongy membranes are thus obtained, permeated by an infinite number of ramifying invisible, or microscopic, pores, the latter being, according to the composition of the collodion employed, from some hundred-thousandths of a millimeter to one or two millionths of a millimeter in diameter.

The greater the amount of alcohol present in the collodion and the lower the content in nitrate of cotton, the larger the pores, and consequently the filters are the more porous and allow a more rapid filtration. It can be seen from this that to obtain all possible degrees of porosity it is only necessary to vary the relative proportions of the three clements which enter into the composition of the collodion. The most open membranes still retain bacteria with certainty and readily allow ultraviruses to pass through; the tightest retain both.

Membranes prepared from a collodion with the following formula permit the passage of the ultraviruses:

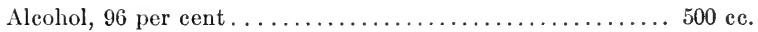

Ether, 65 per cent............................. $500 \mathrm{cc}$.

Nitrate of cotton......................... 20 grams

* The officinal collodion containing castor oil can not be utilized for it gives an absolutely impermeable film. As a general rule, to insure success, one must even prepare the collodion. 
As an indication of penetrability, a sac prepared from collodion of this composition will allow $55 \mathrm{cc}$. of water per hour per square decimeter to pass through when under a pressure of $50 \mathrm{~cm}$. of water (Duclaux). A very dense membrane, impermeable for ultraviruses of all kinds, is obtained by preparing a collodion according to the following:

Aleohol, 96 per cent. . . . . . . . . .

Ether, 65 per cent. . ...................... 750 cc.

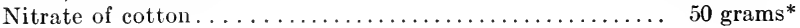

With a membrane prepared from this collodion a square decimeter filters only $5 \mathrm{cc}$. of water per hour under a pressure of 1 meter of water (Duclaux).

By varying the relative proportions of the constituents it is possible to have in reserve a series of collodions suitable for preparing membranes appropriate, as to porosity, to performing an experiment of any type, whatever its nature.

\section{Preparation of ultrafilters}

For the purpose of conducting investigations which must be carried out aseptically, as is always the case in microbiological work, the sole

* The following may be stated as practical suggestions. In order to obtain a quick and complete dissolution of the cotton it is necessary to insure the absorption of the alcohol by first saturating the cotton with a small portion of the alcohol, then add the ether little by little and when the mass has become transparent, add the rest of the alcohol. Another rather important point is that membranes prepared from a freshly made collodion are less homogeneous, and less tough, than are those made from a "ripened" collodion. It is wise, therefore, to pour the freshly made up collodion into a tightly stoppered flask and to keep it for a week in the incubator at $37^{\circ} \mathrm{C}$. Once ripened the collodion may be used indefinitely, provided it is properly preserved, at a low temperature by preference and in tightly stoppered containers. I have not found these recommendations definitely stated anywhere, yet they are "tricks" well-known in some laboratories and of considerable importance.

While on the subject of "tricks" I may mention one other. It is sometimes very difficult to obtain membranes free of air bubbles, yet these can very readily be avoided by placing the flask of collodion, still stoppered, in the incubator at $37^{\circ}$ for a few hours before it is to be used. When the mold which is dipped into the collodion to form the membrane has a temperature higher than that of the liquid (and this is usually the case, because it is necessary to carefully dry the mold with a soft cloth before immersing it in the collodion, and during this procedure the temperature of the hands heats the mold), the ether evaporating from contact with the walls forms bubbles. This does not occur if the temperature of the collodion is higher than that of the mold. 
practical method of assembling the ultrafilter is that which was first described by Malfitano. The ultrafilter, which has the form of a sac, is adjusted to the end of a glass tube of the same diameter.

It is first necessary to prepare a mold. Unless one has in view some special investigation necessitating a large quantity of ultrafiltrate (and then it is necessary to select a mold of appropriate size) filter sacs with a diameter of $15 \mathrm{~mm}$. and a height of from 6 to $8 \mathrm{~cm}$. are to be preferred. To make the mold a test-tube of the appropriate size is selected and by means of a blow-pipe a bulbous enlargement is
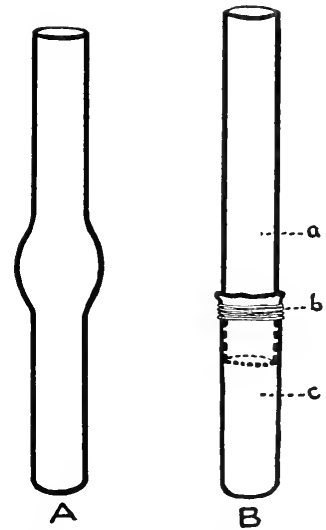

Fig. 2. A, Mold for Preparing the Ultrafilter; $B$, the Celloidin śac Adjusted to Supporting Tube

$a$, glass supporting tube; $b$, ligature; $c$, celloidin sac

formed near the middle of the tube (fig. 2). The mold being perfectly clean and thoroughly dry, and at a temperature slightly lower than that of the collodion (see the note on the preceding page), is plunged vertically into the collodion up to the point where the surface of the liquid reaches the middle of the bulb. It is then withdrawn very slowly and at a uniform rate.*

* It is well to have a watch available so that the same length of time can always be used for the different procedures, thus permitting the saes to be comparable as regards porosity. The greater the viscosity of the collodion the more slowly should the mold be removed, otherwise membranes are obtained which are too thick. As more specifically indicating the procedure the following may be of 
Immediately after removal from the collodion the mold should be held horizontally and should be rotated continually until the gel attains the requisite consistency. If the evaporation has not been sufficient the sac is likely to tear during removal from the mold; if drying has proceeded too far, the sac is liable to wrinkle and is removed from the mold with difficulty. With a little experience one is able to sense the opportune moment; if one employs the time intervals indicated in the preceding note a membrane of the requisite consistency is obtained after about a minute and a half. At this moment, plunge the mold into the collodion a second time, observing the same precautions as in the first immersion, and withdrawing at the same speed. Allow it to dry somewhat, holding it horizontally while continuously rotating it between the fingers. With the second layer permit the evaporation to continue for a slightly longer time, about two minutes.*

When the membrane thus prepared is of the requisite consistency, plunge the mold into water for a few seconds, then, separating with the finger nail the upper margin of the sac from the middle portion of the bulb, under a stream of running water, take hold of the loosened portion and turn it back on itself like the finger of a glove. The exterior surface of the membrane when the sac was upon the mold thus becomes, after removal, the interior. Place the sac in distilled water, or, if it is not to be assembled for use at once and if it is desired to prepare a supply of sacs ready for use, they should be immersed in an aqueous 20 per cent alcohol. $\dagger$

The reader must not assume from this detailed description that the preparation of ultrafilters is difficult. After a few minutes' practice one "gets the knack" if one has the least manual dexterity.

For sacs with a diameter greater than 4 or $5 \mathrm{~cm}$., that is, for sacs

interest. For the more fluid collodions the removal of the mold should require 10 to 15 seconds, for the more dense collodions this period should be extended to 30 to 45 seconds.

* Naturally the period of drying varies with the surrounding temperature, the presence or absence of air currents, etc., since anything which hastens evaporation shortens the time. In any case, the first sac made provides an index for further procedure. If the sac tears in the process of removal the period of drying must be extended; if it is wrinkled, stiff, and is detached with difficulty, drying has gone too far.

$\dagger$ If the proposed investigation is to demand a great many filtrations, it is well to prepare at one time enough sacs to meet all needs. In the first place this will save time, and in the second place, and this is of particular importance, it will tend to give greater uniformity in the series of sacs to be employed in the study. 
with a large capacity such as may be needed for special studies requiring a large amount of filtrate, the dipping method is not suitable. It is then necessary to proceed in the following manner.

Prepare a suitable glass mold as has been indicated above, and attach this mold, horizontally, to a shaft connected with a small motor. The centering should be as perfect as possible and the speed of rotation should be from one to two turns per second, according to the size of the mold. With the mold rotating, pour a fine stream of collodion upon the mold, beginning with the bulbous enlargement (the open end of the sac) and proceeding toward the closed end of the sac. Permit it to dry for an appropriate time, pour on some more collodion to give a second layer, repeat a third time, and even a fourth, thus increasing the toughness of the sac, which, being large, is proportionally fragile. Remove the sac as with the smaller sacs. The larger the sac, the more difficult is its construction.

\section{Assembling the ultrafilter}

Select a glass tube exactly* the same diameter as the exterior of the mold, and some 20 to $25 \mathrm{~cm}$. (or more in certain cases) in length. Long tubes facilitate filtration, for in filling this supporting tube with the fluid to be filtered sufficient pressure is obtained on the membrane to effect filtration quickly, at least this is the case when using membranes sufficiently porous to allow the passage of ultraviruses.

Draw the mouth of the sac upon the supporting tube, to a height of from 1.5 to $2 \mathrm{~cm}$. Tie the sac to the tube with a fine and strong string, introducing between the sac and the ligature a band of parchment paper to avoid tearing the sac (fig. 2-B).

At no time during the manipulation should the sac be allowed to dry, for with drying nitrocellulose membranes become impermeable, even if they are again immediately moistened. It is, however, relatively easy to keep them moist with water.

With the sac thus attached to its supporting tube, take a tube of large diameter and half fill it with distilled water. Also fill the adjusted sac with distilled water in such a way that the level of the water is some 4 to $5 \mathrm{~cm}$. above the ligature. Place the sac within the large tube and suspend it there at the desired height (the level of the water being the same in both tubes) by means of a strip of cotton rolled tightly about the supporting tube in such a way as to form a plug for the large tube.

* It is well to measure the diameter exactly, with calipers. 
Plug the opening of the small tube with cotton, as one would a culture tube (fig. 3).

If it is not essential to make the filtration aseptically, the ultrafilter is then ready for use. If sterility is requisite, the filter must be sterilized, either as it is or after denitrification.

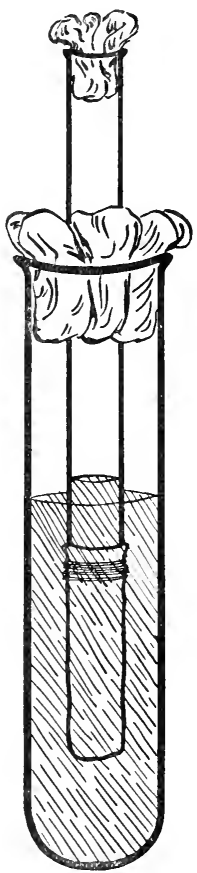

Fig. 3. The Ultrafilter Ready for Sterilization

\section{Denitrification}

This detailed description of the preparation of ultrafilters is here inserted because ultrafiltration must shortly become a common procedure in all experiments dealing with ultraviruses, the group of beings to which the bacteriophage belongs. That the description of the 
preparation may be complete, the method of denitrification, as it has been termed by Jacques Duclaux, should be included.

Membranes prepared from collodion are simply a gel of nitrocellulose. Such membranes possess very low adsorptive capacities, but this property can be diminished still further by decreasing the thick-

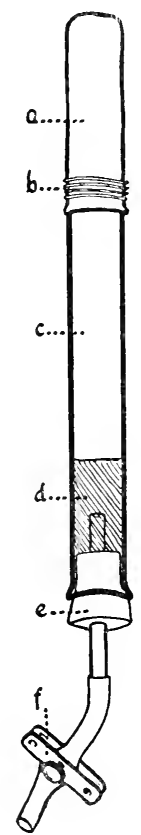

Fig. 4. Denitrified Ultrafilter Arranged for Drying

$a$, celloidin sac; $b$, ligature; $c$, supporting glass tube; $d$, column of water; $e$, rubber stopper; $f$, pinch-cock.

ness of the membrane, and this is what happens when the nitrocellulose is transformed into pure cellulose by denitrification. At the same time this procedure increases the toughness of the membrane.

For denitrification, mount the sac upon its supporting tube, adjusting it as has been described, but instead of immersing it in distilled water, substitute a 25 per cent solution of ammonium hydrosulfide in dis- 
tilled water. Place in a water-bath at $50^{\circ} \mathrm{C}$. for 45 minutes. Next wash the sac, outside and in, with a 10 per cent solution of ammonia, taking considerable care for at this stage the sac is very fragile. Finally wash with distilled water.

It is then necessary to completely dry the membrane, maintaining a positive air pressure upon the inside to prevent the sac from becoming deformed. The following has been found to be the most practical method. A rubber stopper (with one hole) which will fit the opening in the supporting tube is provided with a short glass tube, to the latter being attached a piece of rubber tubing provided with a good screw clamp (fig. 4).

Pour a few cubic centimeters of water into the sac and insert the stopper equipped with its tube and clamp. Blow through the rubber tube to distend the sac, taking care not to rupture the still fragile membrane. Close the clamp. When attached to a support, the sac uppermost, the few cubic centimeters of water placed in the sac will make an hydraulic seal and prevent the escape of air.

When dried, a denitrified sac will keep indefinitely. For use, it is placed in a large tube* in distilled water, after the manner indicated for sacs which have not been denitrified.

\section{Sterilization}

Such is the description of the preparation and assembling of sacs for ultrafiltration as given in texts designed for physical chemists. For us, as microbiologists, the procedure can not stop here, for we must work in an aseptic manner, that is, the ultrafilters must be sterilized. But, it is practically impossible to sterilize a collodion ultrafilter by moist heat in an autoclave. $\dagger$ The sac becomes deformed, the membrane is rendered opaque, and porosity is modified.

It is possible, as Duclaux has shown, to effect denitrification and once this is accomplished an ultrafilter can be sterilized by steam in the autoclave. Nevertheless, the deformation of the membrane, although very much less than with the original collodion membrane, is still very considerable.

After many trials I have adopted the following method, which has

* A more minute description of the details of ultrafiltration will be found in text-books dealing specifically with colloidal chemistry.

$\dagger$ It is easy to see the enormous errors attending the use of unsterilized or poorly sterilized ultrafilters, ${ }^{546}$ yet of all the proposed types of ultrafilters, the only ones that can be effectively sterilized are those designed by Malfitano, the preparation of which is here described. 
proved to be entirely satisfactory, since the ultrafilter undergoes no change, the membrane remains perfectly transparent, and sterility is assured.

Adjust the filtering sac as was directed in the section on "assembling," but instead of filling it, and the outside tube, with distilled water, use 80 per cent alcohol. On the other hand, in a small autoclave, replace the water by 90 per cent alcohol. Connect the air escape valve of the autoclave to a condenser by means of a rubber tube (simply to prevent the volatilized alcohol from escaping into the laboratory at the beginning of the procedure). Place the ultrafilters, mounted in alcohol, as indicated above, in the autoclave, as for an ordinary sterilization, and start the autoclave as would ordinarily be done. In brief, the sterilization is accomplished in a vapor of alcohol. When all of the air has been drawn off (this is shown by the fact that the alcohol will commence to distill over and with cooling will condense drop by drop in the condenser) close the exhaust valve, and regulate the source of heat in such a way that the autoclave will have a pressure of $\frac{1}{5}$ to $\frac{1}{4}$ of a kilogram per square centimeter. The sterilization being thus effected in hydrated alcohol, the temperature attained in this way is sufficient. Maintain this pressure for 30 minutes, allowing the autoclave to remain closed until after cooling is completed.

This method of sterilization can be applied equally well, whether the collodion ultrafilters are normal (nitrocellulose) or denitrified (pure cellulose). Except for particular investigations this method of sterilization renders denitrification unnecessary.

If, as I would advise, one prepares at one time enough ultrafilters for a whole series of experiments they can all be sterilized at one time, and preserved in alcohol just as they come from the autoclave.

At the time of use, empty the alcohol out aseptically, both from the interior of the sac and from the exterior tube, and replace it with distilled water. Allow the water to remain for 10 to 15 minutes, and then empty this out. Then introduce the fluid to be filtered into the sac (fig. 5).

With membranes of medium porosity, that is, those most commonly used, allowing ultraviruses to pass with certainty and just as surely retaining the smallest of the bacteria (Asterococcus of pleuropneumonia, for example), some ten cubic centimeters of ultrafiltrate can be obtained in a few hours (during a night), by simply filling the glass tube supporting the sac with the liquid to be filtered. The slight pressure thus exerted by the liquid in the tube and sac (15 to $20 \mathrm{cc}$., or even more if desired, since the volume can be increased by simply 
adjusting the sac to a longer tube) is adequate to accomplish the ultrafiltration unless the membrane is too dense. If it is necessary to use very dense ultrafilters (for example, when it is desired to retain the ultravirus and thus to obtain an "ultrasterile" ultrafiltrate, as is essential in carrying out studies upon the secretory products of the ultraviruses) pressure must be applied. For such purposes it is wise to prepare sacs, not with two layers of collodion, but with 4 or 5 , increasing thus the toughness of the membrane. They should be denitrified, and assembled as has been outlined, except that in the place
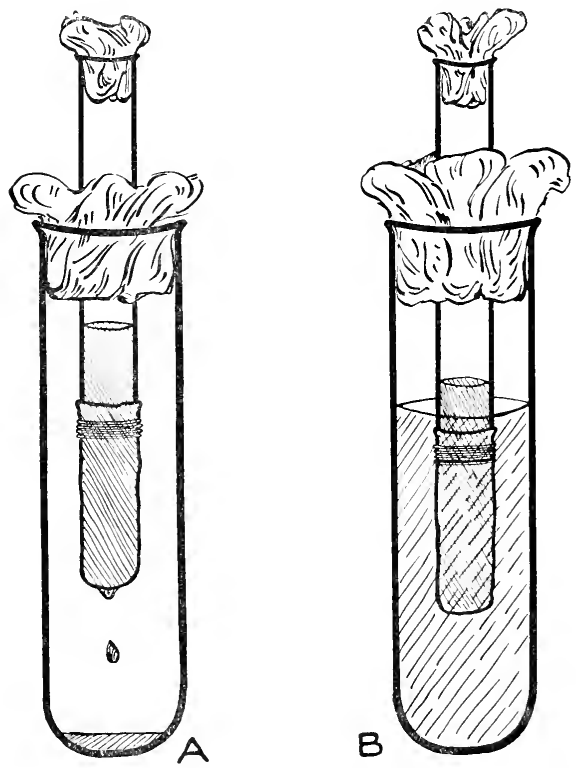

Fig. 5. The Ultrafilter Assembled as (A) an Ultrafilter and $(B)$ a DiALYZING SAC

of the large tube into which they are suspended, they should be introduced into a tube with a side neck. It is also obvious that it is then necessary to adapt the tube bearing the sac to the side-necked tube by means of a perforated rubber stopper. To the reader all of these manipulations may appear complicated, but as a matter of fact, a little experience will show that the preparation of ultrafilters, and ultrafiltration itself, are but simple procedures. 


\section{PART I}

THE PHENOMENON OF BACTERIOPHAGY

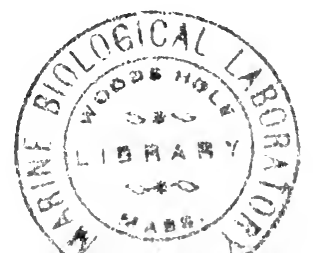





\section{CHAPTER I \\ Bacteriophagy in a Fluid Medium}

\section{ISOLATION OF THE BACTERIOPHAGOUS PRINCIPLE}

\section{Ubiquity of the bacteriophage}

The description of the fundamental experiment, as presented in the early pages of the Introduction, demonstrates that the phenomenon of bacteriophagy consists essentially in the dissolution of the bacterial cell.* This is accomplished through the action of a "principle" which passes through porcelain filters, and even, as we shall see, through ultrafilters whose pores are large enough to permit the passage of particles with a diameter as great as 30 millicrons. $\dagger$

Quite naturally the first question to arise is that of the isolation of this dissolving principle, that is, how can it be obtained in a pure state, in the bacteriological sense of the word. Where is it to be found in nature? What types of material must be examined to obtain it?

It is everywhere present, one might say. Up to the present time it has been shown to be present not only in the intestinal contents of the normal man and of healthy animals (d'Herelle $\left.{ }^{31 i}\right) \ddagger$ but particularly in those who are convalescent from a bacterial infection (d'Herelle ${ }^{310,316,322}$ ), in the urine of these convalescents (d'Herelle ${ }^{310}$ ), in their blood (d'Herelle ${ }^{313}$ ), in pus (d'Herelle ${ }^{321}$ ), in river water $\left(\right.$ Dumas $\left.^{188}\right)$, and in cultivated soil (Dumas $\left.{ }^{188}\right)$. Being, in fact, a constant inhabitant of the intestinal tract it may be encountered in everything which may be contaminated by fecal material.

Its constant presence in the intestine suggests indeed, a priori, the thought that certain bacterial strains may occasionally be found "contaminated" by a bacteriophage, and in fact, Otto and Munter ${ }^{492}$ have

* The word "cell" is used here simply because it is sanctioned by usage. As a matter of fact, it is very doubtful if a bacterium can be formed of a cell, in the strict sense of the word. This question is discussed in the next to the last chapter of "Immunity in Natural Infectious Disease."

$\dagger$ A millicron is one one-millionth of a millimeter, or one one-thousandth of a micron, i.e., $1 \mu \mu$.

$\ddagger$ Only the original papers are here cited. In a later chapter dealing in greater detail with the distribution of the bacteriophage we will review more extensively the findings of those who have investigated this question. 
isolated it from such cultures. Let us hasten to state, however, and we will return to this important point, that such contaminated cultures are rare.

\section{Methods of isolation}

The bacteriophagous principle is encountered but rarely in a substance which, in the usual sense of the word, is sterile. Usually it is necessary to isolate it from a medium in which bacteria are also present. Two methods may be followed to this end. Since the bacteriophage will pass through porcelain filters and through ultrafilters, the material under examination may be suspended in water or in bouillon and subjected to filtration through porcelain or ultrafilters (d'Herelle ${ }^{310}$ ). The bacteria are held back by the filter; the bacteriophage passes through and is thus found in a pure state in the filtrate. ${ }^{*}$ The bacteriophage resists temperatures of at least $60^{\circ} \mathrm{C}$. Thus, if it is present in a liquid medium containing non-spore-forming bacteria it is only necessary to heat at $58^{\circ} \mathrm{C}$. for a time sufficiently long to kill the bacteria. The bacteriophage will then be found in a pure state (Bordet and $\left.\mathrm{Ciuca}^{86}\right)$. The bacteriophage will resist three successive heatings made at this temperature $\left(58^{\circ} \mathrm{C}\right.$.) and consequently it is possible to isolate it by this means, even if the mixture contains spore-forming organisms, since they will be eliminated by the method of fractional sterilization (Tomaselli ${ }^{599}$ ). I have satisfied myself that the bacteriophage may be isolated from feces by this method.

In studying the influence of physical agents upon the bacteriophage we will see that temperatures, even below $60^{\circ} \mathrm{C}$., may cause some attenuation. It is therefore advisable to utilize filtration wherever possible and to resort to isolation through heating only in particular cases and as a last resort.

* This is true, naturally, only when the material does not contain, in addition to the bacteriophage and the bacteria, some other filtrable organism, such as the viruses of vaccinia, of rabies, of herpes, etc. In cases of this type a separation by filtration or ultrafiltration can not be effected, since, as we shall see, all ultraviruses, the bacteriophage included, possess comparable, or possibly identical, dimensions. This is the case, certainly, for the ultraviruses mentioned above, as has been shown by Levaditi. ${ }^{412}$ Where there is an associated ultravirus separation by heating may be employed, at least in those cases where the associated ultravirus is less resistant than the bacteriophage. In the opposite case (if, for example, the mixture contains an ultravirus of the mosaic group) separation can be accomplished by making several successive passages in the presence of a susceptible bacterium. Here, only the bacteriophage will multiply, and at the end of a few passages the associated ultravirus will have been eliminated by dilution. 


\section{Technic of isolation}

The isolation of the bacteriophage may be undertaken under one or another of the following circumstances:

1. The bacteriophage may be sought in a sterile fluid, for example, in normal blood or in an organic fluid collected aseptically. With such no treatment is necessary.

2. The material to be examined may be a clear, but not sterile, liquid. With this, filtration will eliminate the bacteria while the bacteriophage passes through into the filtrate.

3. The material may show a homogeneous turbidity; as a bacterial culture. Here, direct filtration results in an early occlusion of the pores of the filter candle. Thus, it is desirable to resort to a preliminary filtration. The following method of treatment is most satisfactory.

Provide a funnel with a folded filter paper sufficiently large to receive at one time the entire volume to be filtered. Fill the filter with water to which has been added a small amount of infusorial earth. When the water has passed through, the paper is left coated with a thin layer of the infusorial earth, thus rendering the paper less permeable. Through this the material to be examined is filtered prior to filtration through the candle.

4. The material may be a fluid holding in suspension organic particles, or it may be matter more or less solid in nature. This is the type of substance most frequently examined; such as fecal material, more or less fluid, pasty, or solid; or excreta admixed to a greater or less degree with earth, organic débris, etc. In such a case it is necessary to disintegrate as completely as possible the material to be examined.

To effect such a disintegration the most simple procedure consists in carefully suspending the material in bouillon, about 5 grams to 50 cc. of the medium, and incubating this suspension at $37^{\circ} \mathrm{C}$. for from twelve to eighteen hours. The bacterial fermentations which ensue, because of the diverse organisms introduced into the medium, lead to a sufficient disintegration. Upon removal from the incubator the material may be treated, as indicated above, by filtration through infusorial earth and a candle.

If the material under examination contains the bacteriophage and has been subjected to filtration, it will be found in the filtrate.

We will see that the bacteriophage possesses an activity manifested against a wide variety of bacterial species; without doubt against all. We will also see that against a given bacterium this activity is very 
variable. Races of the bacteriophage may be isolated which are extremely active, causing within a few hours a total dissolution of all of the bacteria contained in a culture or in a rather turbid suspension. On the other hand, other races may not cause any detectable dissolution, and it is only by spreading the mixtures of bacteriophage and bacterium upon an agar medium that their presence can be disclosed. More will be said upon this point in Chapter IV and those following.

Let us leave, for the moment, the study of these slightly active races and consider in these first three chapters only the typical phenomenon of bacteriophagy, that is, the phenomenon leading to a total dissolution of the bacteria of a young culture or a suspension of living organisms.

Whatever may be the bacterial species involved, under the action of an active bacteriophage the phenomenon of bacteriophagy manifests itself always in the same manner.

But the diverse races of the bacteriophage, active upon different bacterial species, are more or less frequent in nature and more or less easy to disclose. The bacteriophage which it is always easy to procure, in whatever place it may be found, is that which causes bacteriophagy of Shiga dysentery bacilli, and it is for this reason that, in the majority of the experiments to be recorded, I will take as types this race of the bacteriophage and this bacterium. One may isolate, with certainty, a race of the bacteriophage always very active against dysentery bacilli from the excreta of a convalescent from bacillary dysentery, often, indeed, from the fecal discharges in a case of any acute intestinal disease. Furthermore, such races are to be found in the excreta of the majority of horses and domestic fowls, even when in a normal state of health.

\section{SERIAL ACTION}

The basic experiment, as recorded in the Introduction, has shown that the bacteriophagous principle reveals itself through bringing about the dissolution of bacteria. But, and this is a distinctive peculiarity of this action, it is only in proportion as this dissolution is effected that the bacteriophagous principle, which is the cause of it, reproduces itself-multiplies.

To demonstrate this phenomenon of serial activity a small quantity of bacteria are removed from a young agar slant culture and suspended in bouillon in such a concentration as to produce an obvious turbidity. A platinum inoculating needle is then dipped in a "bacteriophage fluid" 
and the minute quantity adhering to the wire is inoculated into the prepared bacterial suspension. Within a few hours the suspension is clear; all of the bacteria have disappeared. To all appearances they have dissolved in the bouillon, just as sugar becomes dissolved in water. At this time, a bacterial suspension, prepared as was the first, is made and the tip of the platinum wire is immersed in the clear fluid which originally was the first suspension, and thus a minute quantity is transferred to the second turbid suspension. Again after a few hours, this second suspension will in turn have become limpid. A needle dipped in this second cleared suspension inoculated into a third results in a repetition of the process. In each successive suspension the bacteria become dissolved, and in this way it is possible to continue "serial passages" of the bacteriophage principle as long as may be desired. After some thousands of passages comparable to those described above the last suspension, once it has become clear, represents a "bacteriophage fluid" just as active as that originally employed to cause bacterial dissolution in the first passage. By this procedure I have maintained for almost ten years several particularly active bacteriophage races, certain of them having undergone several thousand passages at the expense of the appropriate susceptible bacterium.

The phenomenon of bacteriophagy is then, in reality, a double phenomenon. A dissolution of the bacterial cells takes place, and, in the course of this dissolution, the bacteriophage principle regenerates, reproduces itself.

It is unnecessary to give here experimental protocols supporting these statements. No one has questioned them, and, as a matter of fact, this entire text is simply an exposition of the manner and the results of this reproductive capacity of the bacteriophage.

\section{ENVIRONMENTAL CONDITIONS FAVORING BACTERIOPHAGY}

\section{General conditions}

Of the many culture media devised up to the present time none possess the composition requisite to the multiplication of the bacteriophage. The sine qua non for multiplication of the bacteriophage principle, thus permitting a dissolution of the bacteria, in itself a direct result of this multiplication, is the medium provided by living bacteria. It is further necessary, but this is evident a priori, that the bacteriophage principle which one opposes to this bacterium be active against the latter. 
These two basic conditions being satisfied, it may be said that, as a general rule, the most favorable medium, that in which bacteriophagy will take place in the most perfect fashion, is that which, because of its composition, provides best for the development of the particular type of bacteria selected to undergo the dissolution. This is not strange, for it is not in fact in the medium itself that the bacteriophage acts and multiplies, it is within the bacteria themselves. The interior of the bacterial cell is the true and sole medium for the multiplication of the bacteriophage ( $\mathrm{d}^{\prime} \mathrm{Herell}^{310}$ ). This is by no means equivalent to saying that the composition of the medium is of no consequence, for all conditions which modify the state of the bacteria are reflected in the phenomenon, one of whose manifestations and indeed the most obvious one, is the dissolution of these bacteria. For example, it is shown by many experiments that the "critical period" in the life history of the bacterial cell in the presence of the bacteriophage is the moment of its division. But it must not be assumed that only those bacteria in the process of division are subject to attack. It is then, because of its influence upon the development of the bacteria that the composition of the medium has a reflected effect upon the phenomenon of bacteriophagy.

All of the experiments presented in the first edition of this text* were carried out, except where stated to the contrary, with cultures or suspensions of bacteria prepared with the ordinary bouillon used in the Vaccine Laboratories of the Pasteur Institute. This is the so-called Martin's bouillon, made by mixing in equal parts a beef infusion (400 grams per liter) and a peptone solution, prepared in the laboratory by acid autodigestion at $50^{\circ} \mathrm{C}$. of pig stomach $(200$ grams of minced gastric mucosa per liter). The adjustment of the reaction was effected by the old method, using phenolphthalein as indicator, the final reaction being -6 to -8 , which corresponds very closely to a $\mathrm{pH}$ of 7.6 to 7.8. Preliminary control experiments had shown me that such a degree of alkalinity was best suited to the reaction ( $\left.\mathrm{d}^{\prime} \mathrm{Herell}^{321}\right)$, as the following indicates.

Peptone water (containing 25 grams of Chassaing peptone and 5 grams of NaCl per liter) is neutralized to phenolphthalein. The medium is then frankly alkaline to litmus. It is then distributed into tubes, 10 ec. to each. Hydrochloric acid is added to each tube in dilutions to form an increasing degree of acidity. All of the tubes are

* The Bacteriophage; Its Rôle in Immunity. Williams \& Wilkins Co., Baltimore, 1922 . 
planted with a concentrated suspension of Shiga bacilli, sufficient being added to give a normal suspension of 250 million per cubic centimeter. Finally, each tube is inoculated with $0.001 \mathrm{cc}$. of the bacteriophage fluid. After 24 hours the appearance of the suspensions shows a certain correlation to the reaction of the medium. The results are given in table 1.

At the beginning of my studies I stated that bacteriophagy could take place in an alkaline physiological salt solution ${ }^{310,331}$. Expressed in this manner, it may be that this statement may not be accurate, although assuredly under these conditions a very definite, sometimes complete, clearing of the medium may be observed. Others (Davison, ${ }^{171}$ Kabelik, ${ }^{385}$ ) have also observed this and have attributed, as I had

TABLE 1

\begin{tabular}{r|r|l}
\hline TUBE & $\begin{array}{c}\text { REACrION TO } \\
\text { PHENOLPHTHALEN }\end{array}$ & \multicolumn{1}{c}{$\begin{array}{c}\text { APPEARANCE OF THE SUSPENSTON } \\
\text { AFT 24 HOURS }\end{array}$} \\
\cline { 2 - 3 } 1 & 0 & Very slight clouding \\
2 & -2 & Very slight clouding \\
3 & -4 & Limpid \\
4 & -6 & Limpid \\
5 & -8 & Limpid \\
6 & -10 & Limpid \\
7 & -12 & Limpid \\
8 & -14 & Slight turbidity \\
9 & -16 & Turbid \\
10 & -18 & Turbid \\
11 & -20 & Turbid \\
12 & -22 & Turbid \\
\hline
\end{tabular}

done, the bacterial dissolution occurring in saline under the influence of the bacteriophage, to a typical bacteriophagy. We will return to this phenomenon later and consider the correct interpretation.

Maitland ${ }^{432}$ has, however, shown that bacteriophagy may take place in a medium very poor in food materials, such as physiological saline containing but 1 per cent of bouillon. I have substantiated this; under such conditions bacteriophagy undoubtedly occurs.

\section{Reaction of the medium}

Unquestionably, alkalinity of the medium affords the most favorable reaction for accomplishing the phenomenon of bacteriophagy. But if an attempt is made to define more precisely the exact degree of 
alkalinity that provides optimum conditions it becomes apparent at once that the conditions obtaining in one experiment are optimum conditions only for the bacterial species and the particular race of bacteriophage with which the experiment is performed. If another species of bacteria and a bacteriophage derived from another source are utilized the best conditions for effecting the phenomenon may be quite different from those of the first experiment.

Situations analogous to this will be observed repeatedly in the course of this study, and the reason for such a lack of fixed relationships is very obvious. Although in physics, or in chemistry, it is always possible to definitely fix the conditions of an experiment, to record these conditions by giving them invariable numerical expression, and to make the experiment entirely without regard to the past history of the chemical substances entering into the reaction, this is not possible in biology. Here, the past, the inheritance of the beings involved, constitutes a factor of prime importance. Moreover, this factor is always difficult, often impossible, to evaluate in advance. This is why two experiments in bacteriophagy, carried out under identical conditions, may yield different results, simply because in the two experiments the bacteria are of different species, or simply of different strains. Bacteriophagy is a disease of bacteria, and the aphorism, so true, "there are not diseases, there are patients," meaning that a single disease may have varied manifestations according to the individual affected, is just as true whether the patient be a man, or whether it be a bacterium.

In the last analysis, it is of course true, that disease represents the sum total of a series of purely chemical reactions, but whereas in chemistry a reaction can be predicted in its most minute details because of restricted conditions, all determinable in advance, in biology generally, and certainly in the case with which we are particularly concerned, the number of factors is so great, the majority of these factors being absolutely indeterminable, that it is impossible to predict the optimal conditions for a reaction. Only the general nature of the reaction can be foretold.

This concept must always be held in mind throughout the study of the phenomena caused by the bacteriophage. A great many authors have neglected these fundamental principles, with the result that many conflicting reports, with their attendant arguments, have appeared, each author assuming that he could generalize from his individual results. As a matter of fact, these basic facts are all-important, and generalization is particularly hazardous. 
To return to the subject under consideration, that of the effect of the reaction of a medium upon bacteriophagy, we will see that through a process of adaptation it is possible to so alter the bacteriophage that the processes of dissolution will take place in an acid medium. A further discussion of this is reserved for a later section. It is mentioned here simply to show the complexity of the conditions contributing to bacteriophagy and to emphasize the impossibility of stating in a definite manner conditions such as will provide for optimum activity regardless of the race of bacteriophage involved. It is certain, nevertheless, that in general an acid medium is but poorly suited to bacteriophagy. With the great majority of races of the bacteriophage the phenomenon does not take place readily when the reaction of the medium is acid (d'Herelle ${ }^{321}$ ). Scheidegger ${ }^{568}$ states that B. coli grows normally in a bouillon with a $\mathrm{pH}$ of 4.5 , even though the bacteriophage is present. But under such conditions the principle is not destroyed, for when such a medium is rendered slightly alkaline, bacteriophagy occurs.

While working with $B$. coli, investigating the process of bacteriophagy occurring under particular conditions (when a very small number of bacteria (simple seeding) in a peptone bouillon medium were mixed with an indeterminate, but very great (10 drops) quantity of a filtrate containing a but slightly active bacteriophage), Gratia ${ }^{249}$ noted that the degree of alkalinity most favorable was found in the neighborhood of $\mathrm{pH}$ 8.5. He states, however, that the phenomenon takes place in a slightly acid $\left(\mathrm{pH}\right.$ 6.8) medium. Scheidegger ${ }^{568}$ also records bacteriophagy in a medium of $\mathrm{pH} 6.5$.

These findings, together with other observations, show that the optimal reaction is not a constant, varying not only with the bacterial species and the race of the bacteriophage concerned, but differing also with the nature of the medium. In testing a Flexner-bacteriophage, acting upon its homologous bacterium, da Costa Cruz ${ }^{161}$ showed that bacteriophagy could take place in Martin's bouillon with a reaction of $\mathrm{pH} 6.5$, but that to obtain a reaction of equal intensity in peptone water it was necessary to adjust the reaction to $\mathrm{pH}$ 8.5.

Using a Shiga-bacteriophage in conjunction with its homologous organism, I have shown that in a peptone water (2.5 per cent), containing 0.5 per cent of salt, with the $\mathrm{pH}$ at 7.5 , a total dissolution of a normal bacterial suspension takes place in 14 hours at $37^{\circ} \mathrm{C}$. At pH 8.0 the dissolution is complete in 9 hours, and at pH 7.0 dissolution is only partial. 
A correlation of all of these findings shows, therefore, that the optimum $\mathrm{pH}$ varies $(a)$ with the bacterial species involved, $(b)$, with the race of the bacteriophage reacting upon it, and $(c)$ with the composition of the medium in which the reaction takes place. Nevertheless, it is possible to say that, in general, a slight alkalinity of the medium provides a favorable condition. Hence, unless one has in view some special investigation demanding particular conditions, the best medium for routine purposes is the ordinary peptone bouillon iwith a reaction of $\mathrm{pH} 7.8$.

Bacteriophagy will take place in synthetic media provided these permit the growth of the bacteria against which the bacteriophage is to act. Here the conditions as regards alkalinity are the same as in bouillon. The formula for such a medium, suited to the growth of $B$. coli, as well as to some, but not all, strains of $B$. dysenteriae, is:

Water..................................... $100 \mathrm{cc}$.

Sodium chloride ........................... 0.5 gram

Potassium phosphate ......................... 0.1 gram

Asparagine.................................. 0.5 gram

The reaction to be adjusted in accord with the experiment to be performed.

Another medium, suggested by Gratia ${ }^{259}$ for $B$ coli is:

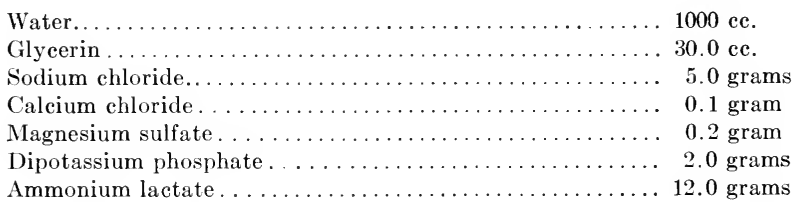

The reaction adjusted to $\mathrm{pH} 7.4$.

\section{EFFECT OF THE CONDITION OF THE BACTERIUM}

If we take, then, $10 \mathrm{cc}$. of this beef bouillon, containing peptone and salt, adjusted to $\mathrm{pH} 7.8$, and suspend some dysentery bacilli of the Shiga-Kruse type removed from an agar slant which had been planted some 18 to 24 hours previously, to provide a suspension containing approximately 250 million per cubic centimeter, and further, inoculate this suspension with a trace (0.001 ce., for example) of a filtrate containing a bacteriophage very active against $B$. dysenteriae, we will find, after incubation at $37^{\circ} \mathrm{C}$., that within a few hours, from 4 to 24 , 
according to the activity of the race of bacteriophage involved, all of the bacteria are dissolved, and the medium has again become limpid.

Instead of selecting Shiga-Kruse bacilli, we could have prepared in the same way a suspension from a bacterium of another species, and combined it with a race of bacteriophage of sufficiently high activity against this bacterium. The final result would have been the same; a complete dissolution of the bacterial cells and a clarification of the medium.

But such a complete dissolution does not take place under all conditions; the state of the bacterium exposed to the dissolving principle is of significance. Instead of taking a suspension prepared from a young freshly grown culture we may inoculate the bacteriophage into a fifteen-day old broth culture. A clearing of the medium, a partial dissolution, results, but a certain degree of turbidity remains. Nevertheless, it is possible to continue to use such a medium, making as many passages as may be desired. Some tube of the series when planted on agar or in bouillon will remain sterile, and a drop of this tube inoculated into a suspension of young bacilli will cause a perfect dissolution. In the old culture, then, the bacteriophage multiplies normally, although dissolution does not take place, at least, the solution is not complete. What is the explanation of this reaction? To answer this it is sufficient to compare the results of counting the total number of bacilli existing in an old culture (this can be done by the method of counting cells) with the results secured by counting the viable organisms only (done by the plating method). For a confirmation of this type, a Shiga culture in Martin's bouillon is made, incubated for 14 hours, and allowed to stand at laboratory temperature for 15 days. The total count of bacillary bodies will be about 625 millions; that of the viable bacilli, that is, those capable of yielding colonies when transferred to agar will be about 2 millions, in each half cubic centimeter of culture. Now, as we have seen, the bacteriophage is able to develop at the expense of living bacteria only, these being the ones which are dissolved. In the old suspension which we have mentioned, in which there is only about one organism in three hundred which is capable of being dissolved, it can readily be comprehended that if the dissolution of a suspension be taken as a criterion, the bacteriophage appears to be without action.

As a matter of fact, it is not necessary to resort to old cultures to find dead bacteria, for even in fresh bouillon cultures dead organisms will be found after as short a time as 24 hours. In a broth culture 
of Shiga bacilli, after only 24 hours of incubation about one-third of the organisms present are incapable of producing colonies when planted on agar. If, on the other hand, an agar slant culture is utilized, almost all of the bacteria are living after 24 hours at $37^{\circ} \mathrm{C}$. A 24-hour bouillon culture will, then, remain slightly turbid when the bacteriophagic process is accomplished, while a suspension made in broth from a young agar culture containing the same number of bacteria will be perfectly limpid when the dissolution is achieved. In this last case all of the bacteria were living and susceptible to the attack of the bacteriophage. It is for this reason that it is preferable to effect bacteriophagy in a suspension of bacteria rather than directly in a bouillon culture.

Certain bacteria give a homogeneous growth in a young culture in bouillon but when taken from agar they can be suspended only with difficulty. B. pestis is such an organism. When working with such bacteria it is preferable to have the bacteriophage act on a broth culture in the following manner. A bouillon tube is lightly seeded with the bacterium. When the culture has clouded, the bacteriophage active for this bacterial strain is introduced and at the same time the culture is diluted with an equal volume of sterile medium. This dilution should be made before the bacteriophage has had time to multiply sufficiently to parasitize an appreciable number of bacteria. Thus, the bacterial culture at the time of "departure" will consist almost entirely of young bacilli, readily subject to at,tack.

The following experiment demonstrates clearly that the products of bacterial growth as found in an old culture, products which, as is well-known, inhibit the development of bacteria (as in the so-called "vaccinated" media) are without effect upon the phenomenon of dissolution.

Two cultures of B. dysenteriae Shiga, the one aged 15 days, the other, 18 hours are centrifugalized. The sediment from the first culture is suspended in the supernatant fluid of the second, and the sediment of the second culture is combined with the supernatant fluid of the first. Both suspensions thus formed are inoculated with a drop of a bacteriophage filtrate. The suspension consisting of "old" bacilli and "young" medium remains turbid; that of "young" bacilli and "old" medium becomes perfectly clear after 7 hours.

But, although the products of bacterial metabolism are not inhibitory for the process of dissolution, the products of dissolution, as we will see, exert quite a different action. These products are the result of the activity of the bacteriophagic process, and, as such, they impede its activity. 
Although, as has been stated above, bacteriophagy will not take place with dead bacteria, this does not mean that it is essential that the bacteria be young. Various authors (Kuttner ${ }^{393}$ first, and later Bordet and Ciuca $^{93}$ ) have suggested that bacteriophagy can only be effected when the bacterium is in process of division. That the moment of division represents the most critical period for the bacterium, that bacteriophagy takes place much more actively when the bacteriophage acts on young bacteria, was stated among the very first of my reports. But it is none the less true that old bacteria, certainly no longer dividing, can undergo bacteriophagic dissolution, as has been shown in the experiments presented above. Moreover, this fact has been confirmed by Maitland,432 working with organisms of the typhoid-dysentery group, and more recently by collaborators of Bordet (Gratia and Rhodes ${ }^{275}$ ). Gratia combined a Staphylo-bacteriophage of a very high potency, using an extreme dilution of the filtrate, with a suspension of Staphylococcus aureus, and observed, under these conditions, that bacterial dissolution commenced only after about a week. The dissolution was, nevertheless, complete. He concluded quite rationally that bacteriophagy may take place with bacteria which are no longer reproducing.*

From these facts it may be deduced, in brief, that whatever the bacterial species, bacteriophagy may take place with a bacterium of any age, provided it be alive and normal. Yet, although all living unaltered bacteria are susceptible, the critical moment, the period when the bacterium is most vulnerable, is the moment of division.

\section{EFFECTS OF THE RELATIVE CONCENTRATIONS OF BACTERIO-}

PHAGE AND BACTERIA

Let us now consider the variable characteristics attending the phenomenon of bacteriophagy when it occurs under different concentration

* Gratia and Rhodes even add that the reaction occurs with bacteria in process of disintegration, since microscopic examination showed that after a week modified staphylococei were present. That these degenerating staphylococci had been dissolved is certain, since the medium ultimately became completely clarified, but to assume that the dissolution of these altered bacteria was brought about directly, by the bacteriophage itself, is another question. This possibility we will have occasion to treat at some length when we consider the mode of action of the bacteriophage. Here, let us simply say that the dissolution of modified bacteria, and dead cells as well, appears to be effected through the action of substances which become disseminated in the medium during the course of bacteriophagy. 
relationships, such as may be provided by varying within a given medium the proportions of the two antagonists involved, the bacteriophage principle and the bacterium to which it is opposed.

It must be recalled that in these first chapters we are dealing only with extremely active bacteriophage principles, that is to say, with those capable of completely and permanently dissolving all of the bacteria present in a medium, even though only an infinitely small quantity of the filtrate containing the bacteriophage principle (a millionth of a cubic centimeter, or less) is introduced into a definitely turbid suspension of bacteria.

Into a constant quantity of medium, or peptone broth with a $\mathrm{pH}$ of 7.8, we may introduce, either a very few bacteria (as about one to each centimeter) or a very great number (several billions). On the other hand, whatever the number of bacteria, we may inoculate the suspension with a very minute quantity of a filtrate containing the bacteriophage principle, as $0.000,000,000,1$, or $10^{-10} \mathrm{cc}{ }^{*}$ or with a considerable quantity, as 1 cc., or, indeed, with any intermediate amount.

Many of the experiments which I have published dealing with the effect of the relative concentrations in the medium of bacteria and bacteriophage in their bearing upon the nature and course of the phenomenon of bacteriophagy, have been repeated by many investigators, and in all cases their observations have been comparable to mine. It could hardly be otherwise; the facts are so clear-cut that doubt is impossible. As for the deductions inspired by these facts, questions bearing upon the mode of regeneration of the active principle, for

* Throughout the text the logarithmic notation, the most convenient for expressing such values, will be utilized. Despite the fact that this notation has been rather widely employed in recent publications, it may be unfamiliar to some. The following table of equivalents is therefore provided.

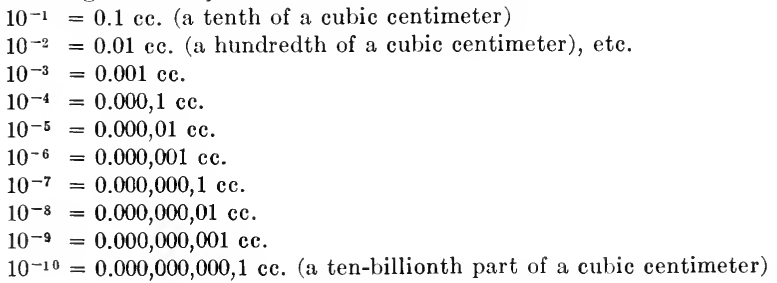

In brief, the negative exponent represents the number of figures at the right of the decimal point. It is likewise the characteristic of the $\log$ of the decimal fractional number expressing the quantity of liquid. 
example, discussion must be reserved for a later chapter. For the time being let us simply treat of the macroscopic appearance of the phenomenon; let us describe only those things that can actually be seen.

It may be well, however, to consider at once the significance of the distinction made by certain authors between what they term a "process of inhibition" and a "process of bacteriolysis." This distinction is based upon whether the bacteriophage principle is inoculated into a medium simply seeded with susceptible bacteria or whether it is introduced into a cloudy suspension of these bacteria. Such a differentiation implies that in the first case growth does not occur, the bacteriophage seems to "inhibit" multiplication, while in the second case the turbid medium becomes perfectly clear after the complete dissolution of the bacterial bodies.

As a matter of fact, it is difficult to conceive how the two cases can possibly be considered as distinct. For whatever may be the actual number of bacteria present in a medium the phenomenon is exactly the same, the course of the reaction is the same, and the end result, a complete dissolution of the bacteria present, is the same. It is very obvious that if the number of bacteria is so small that the medium appears elear from the beginning (as in a simple seeding) the medium will show no change, it will remain clear, for the few bacteria present will be dissolved. If, on the contrary, because of the enormous number of bacteria present, the medium was clouded or turbid at the outset, that is, at the time when the bacteriophage was inoculated, it becomes limpid only when all of the bacteria have been dissolved. In one case, just as in the other, the same phenomenon has taken place, the action of the bacteriophage has been of the same nature. A consideration of the different cases which we will present will leave no doubt upon this point; a basic and valid distinction between an "inhibition" and a "bacteriolysis" is impossible. Under both conditions as to quantity the phenomenon is qualitatively the same.

\section{Limits of bacteriophagy}

Let us repeat once more that all of the experiments presented in this first chapter deal with the typical phenomenon of bacteriophagy, that is, with that which takes place through the intervention of an extremely active bacteriophage. To enter upon a study of bacteriophagy using races of the principle having but slight activity is simply to willfully invite difficulties, both in experimental procedure and in interpretation. 
Having in mind the effects of variations in the relative concentrations of the factors involved in bacteriophagy, we may first consider the maximum concentration of bacteria permitting the return of the medium to a limpid state, in other words, what the maximal quantity of bacterial cells is which is capable of being dissolved in a given quantity of fluid.

The following experiments show the situation as regards $B$. dysenteria Shiga. Comparable results have been secured with Flexner and

TABLE 2

\begin{tabular}{|c|c|c|c|}
\hline \multirow{2}{*}{$\begin{array}{l}\text { INITIAL NUMBER OF } \\
\text { BACTERIA PER } \\
\text { CUBIC CENTIMETER }\end{array}$} & \multirow{2}{*}{$\begin{array}{l}\text { QUANTITY OF } \\
\text { BACTERIOPHAGE } \\
\text { FILTRATE } \\
\text { INOCDLATED }\end{array}$} & \multicolumn{2}{|c|}{ APPEARANCE OF THE MEDIUM AFTER } \\
\hline & & 24 hours & 48 hours \\
\hline & $c c$. & & \\
\hline 5,000 million & 0.1 & Turbid & \\
\hline 2,000 million & 0.1 & Cloudy & \\
\hline 1,000 million & 0.1 & Slightly cloudy & \\
\hline 500 million & 0.1 & Clear & \\
\hline 1,000 million & 0.01 & Cloudy (150) & Cloudy (100) \\
\hline 900 million & 0.01 & Cloudy (150) & Cloudy (100) \\
\hline 800 million & 0.01 & Cloudy (100) & Cloudy (50) \\
\hline 700 million & 0.01 & Cloudy $(50)$ & Clear \\
\hline 600 million & 0.01 & Cloudy (50) & Clear \\
\hline 500 million & 0.01 & Clear & Clear \\
\hline 400 million & 0.01 & Clear & Clear \\
\hline 300 million & 0.01 & Clear & Clear \\
\hline 200 million & 0.01 & Clear & Clear \\
\hline 100 million & 0.01 & Clear & Clear \\
\hline 50 million & 0.01 & Clear & Clear \\
\hline 25 million & 0.01 & Clear & Clear \\
\hline 10 million & 0.01 & Clear & Clear \\
\hline 5 million & 0.01 & Clear & Clear \\
\hline 1 million & 0.01 & Clear & Clear \\
\hline
\end{tabular}

with Hiss strains. The experiments presented in tables 2 and 3 are conducted in $10 \mathrm{cc}$. of a salt peptone bouillon at a $\mathrm{pH}$ of 7.8. The figures in parentheses indicate the opacity of the cloud in the medium, expressed in millions of bacteria per cubic centimeter, as determined by comparison with titrated control suspensions. The incubation is carried out at $37^{\circ} \mathrm{C}$.

The last three tubes, because of the small quantity of bacteria suspended in the medium, were clear at the beginning of the experiment. They remained so of necessity. 
When this experiment was repeated, under certain modifications, such as varying the quantity of bacteriophage filtrate within the limits between 1 and $0.001 \mathrm{cc}$, the results were identical, despite the fact that different strains of $B$. dysenteriae were used. Three different races of Shiga-bacteriophage, all of maximal activity, have likewise given results practically identical.

Results of the same nature as those recorded above are shown in the next experiment (table 3 ) in which a highly active Staphylo-bacteriophage acts upon Staphylococcus aureus. Here again, incubation is at $37^{\circ} \mathrm{C}$.

TABLE 3

\begin{tabular}{|c|c|c|c|}
\hline \multirow{2}{*}{$\begin{array}{l}\text { NUMBER OF STAPHYLOCOCCI } \\
\text { PER CUBIC CENTIMETER }\end{array}$} & \multirow{2}{*}{$\begin{array}{l}\text { QUANTITY OF } \\
\text { BACTERIOPHAGE } \\
\text { INOCULATED }\end{array}$} & \multicolumn{2}{|c|}{ APPEARANCE OF THE MEDIUM AFTER } \\
\hline & & 24 hours & 48 hours \\
\hline & $c c$. & & \\
\hline 1,000 million & 0.05 & Cloudy & Cloudy (100) \\
\hline 750 million & 0.05 & Cloudy & Cloudy $(50)$ \\
\hline 500 million & 0.05 & Cloudy (200) & Clear \\
\hline 250 million & 0.05 & Cloudy (100) & Clear \\
\hline 100 million & 0.05 & Cloudy $(50)$ & Clear \\
\hline 50 million & 0.05 & Clear & Clear \\
\hline 25 million & 0.05 & Clear & Clear \\
\hline 10 million & 0.05 & Clear & Clear \\
\hline 1 million & 0.05 & Clear & Clear \\
\hline 100 thousand & 0.05 & Clear & Clear \\
\hline 10 thousand & 0.05 & Clear & Clear \\
\hline 1 thousand & 0.05 & Clear & Clear \\
\hline 1 hundred & 0.05 & Clear & Clear \\
\hline ten & 0.05 & Clear & Clear \\
\hline
\end{tabular}

The suspensions containing less than 10 million staphylococci per cubic centimeter were clear from the beginning of the experiment, and they remained so indefinitely. After a month of standing at room temperature the tubes were returned to the incubator for 48 hours. No change in appearance resulted; the aspect remained as when originally removed from the incubator, the first two were cloudy, all of the others were clear.

The maximum number of bacteria capable of being dissolved in the medium is the same, whether the total number is introduced at one time at the beginning of the experiment, or whether the organisms are added in several fractions. This is shown by the following experiment, performed with $B$. dysenteriae. A salt peptone bouillon medium, adjusted to $\mathrm{pH} 7.8$ is used. Incubation at $37^{\circ} \mathrm{C}$. 
A suspension of 250 million of bacilli per cubic centimeter is inoculated with $0.0001 \mathrm{cc}$. of a bacteriophage filtrate. After 14 hours the dissolution of the bacteria is complete, the medium being clear. At this time, a concentrated suspension of young bacilli is added to this clear medium in such a way as to restore the titre to 250 million per cubic centimeter. Seven hours later the medium is again limpid, all of the bacteria having been dissolved. Another addition of a new quantity of concentrated suspension is made, again yielding a turbidity corresponding to 250 million bacteria to each cubic centimeter. This time, after 48 hours, the medium is still slightly cloudy.

To this medium, not entirely clear, a further addition of concentrated bacterial suspension is made, restoring the turbidity to the equivalent of 250 million bacteria per cubic centimeter. Eight days later the medium has cleared somewhat, but it is still definitely cloudy. However, plantings made from it upon agar and into bouillon remain sterile.

As is seen, whether the bacteria were present in the medium from the beginning, or whether they were introduced by fractions in the course of the action, the dissolution was complete only for quantities below approximately 700 millions per cubic centimeter of medium. Later we will have more to say about the cause which operates to hinder dissolution of more than a certain number of bacteria per unit volume of fluid.

These experiments reveal the fact that the bacteriophage principle is able to effect a complete dissolution of the bacterial cells in suspension in a medium propitious for bacteriophagy when the medium contains from one to 700 million bacterial cells per cubic centimeter. Within certain very wide limits the quantity of bacteriophage filtrate necessary to inoculate to cause this dissolution is a matter of no moment; the course of the action and the final result of the phenomenon is the same, whether the amount inoculated is $1 \mathrm{cc}$. or 0.001 , to $10 \mathrm{cc}$. of medium.

The total number of bacteria which may undergo a complete dissolution under the action of a very active bacteriophage appears to vary with different species of bacteria. As has been seen above, the maximum is about 700 million for $B$. dysenteriae, whether it be Shiga, Flexner, or Hiss, and for the staphylococcus, whether it be albus, aureus, or citreus. With B. coli, B. typhosus, and B. paratyphosus $A$ and $B$, a complete dissolution has been obtained with quantities of 350 million per cubic centimeter of medium but not of higher concen- 
trations. For B. gallinarum and the different Pasteurella organisms, the maximum titre is the same. With $B$. pestis, it has been impossible to go above a limit of 200 million per cubic centimeter. But here we must bear in mind that with $B$. dysenteriae and the staphylococcus, except for the extremely active races of the bacteriophage, the maximum number capable of complete dissolution is only 350 to 400 million per cubic centimeter, and it is quite possible that for B. coli, B. typhosus B. pestis, etc. a complete dissolution of more concentrated suspensions might be obtained with races of the bacteriophage still more active than those which I have isolated and worked with up to the present time.

In bacteriophagy all is relative; all depends upon the aptitudes, the qualities, of the race of bacteriophage with which one is working. Too many authors seem to forget this.

\section{Limits of activity of the bacteriophage principle}

What are the maximum and minimum quantities of the bacteriophage with which bacteriophagy can be effected?

The phenomenon takes place if bacteria are suspended in an undiluted bacteriophage filtrate. It is unnecessary to support this statement by citing experiments, particularly since those described upon the preceding pages demonstrate this adequately. They show that it is possible to add a new quantity of bacteria to a medium resulting from a complete dissolution of bacteria and that they are in turn entirely dissolved.

Experiments upon the lower limit are more interesting. Let me say once more that the condition portrayed here applies only to races of the bacteriophage having a high potency.*

* Although to some, these repetitions may appear quite uncalled for, experience shows that they are necessary. For many workers, carrying out their experiments under conditions other than those which I have indicated have obtained results differing from mine (it could hardly be otherwise) and have, upon this basis, felt warranted in contradicting my experimental findings. For example, and this example is selected from among many others, several investigators, early in their studies, have affirmed that the dissolution of bacteria is never complete, a conclusion reached simply because of the fact that they used a bacteriophage of but little activity. It is true that they have later recognized that a total dissolution occurs, a fact which today is unanimously accepted. But despite the fact that these authors have later revised their conclusions, it would have been somewhat more logical to have worked first under the conditions as I described them. This would have rendered unnecessary a subsequent retraction. 
We may consider first the reaction as it develops in suspensions of low bacterial content, for example, in a suspension containing 1 million Shiga bacilli per cubic centimeter. Let us inoculate a series of tubes, each containing $10 \mathrm{cc}$. of such a suspension, with decreasing quantities of the Shiga-bacteriophage as indicated in table 4 . The results, expressed by the macroscopic appearance of the tubes at different intervals will be as indicated.

The figures within the parentheses indicate the opacity of the cloud, by comparison with control tubes containing titrated suspensions of formolized Shiga bacilli. Further experiment showed that tube 11 did not contain any of the bacteriophage principle.

TABLE 4

\begin{tabular}{|c|c|c|c|c|c|}
\hline \multirow{2}{*}{ TUBE NUMBER } & \multirow{2}{*}{$\begin{array}{c}\text { QDANTITY OF } \\
\text { BACTERIOPHAGE } \\
\text { INOCULATED }\end{array}$} & \multicolumn{4}{|c|}{ APPEARANCE AFTER } \\
\hline & & 4 hours & 5 hours & 6 hours & 18 hours \\
\hline & $c c$. & & & & \\
\hline 1 & $10^{-1}$ & Clear & Clear & Clear & Clear \\
\hline 2 & $10^{-2}$ & Clear & Clear & Clear & Clear \\
\hline 3 & $10^{-3}$ & Clear & (?) & Clear & Clear \\
\hline 4 & $10^{-4}$ & $(?)$ & (25) & $(?)$ & Clear \\
\hline 5 & $10^{-5}$ & (?) & (25) & $(25)$ & Clear \\
\hline 6 & $10^{-6}$ & (?) & $(50)$ & (25) & Clear \\
\hline 7 & $10^{-7}$ & (?) & $(75)$ & $(50)$ & Clear \\
\hline 8 & $10^{-8}$ & (?) & $(75)$ & $(75)$ & Clear \\
\hline 9 & $10^{-9}$ & (?) & (75) & $(100)$ & Clear \\
\hline 10 & $10^{-10}$ & (?) & $(75)$ & $(100)$ & Clear \\
\hline 11 & $10^{-11}$ & (?) & $(75)$ & $(100)$ & Cloudy \\
\hline
\end{tabular}

(?) = clouding doubtful; if any, very slight.

Let us repeat this experiment, under the same conditions, except that we will combine the same series of dilutions of the bacteriophage filtrate with a suspension containing 100 million bacilli per cubic centimeter, that is to say, with a definitely cloudy suspension rather than with one having but 1 million organisms per cubic centimeter. As will be seen, the ultimate results are entirely comparable to those obtained with the less concentrated bacterial suspension (table 5).

The following experiment (table 6) shows that the general course of the process of bacteriophagy is the same if it is performed with a Staphylo-bacteriophage in decreasing amounts combined with a suspension of the staphylococcus. Here, each tube contains $10 \mathrm{cc}$. of a suspension of Staphylococcus aureus in a salt-peptone bouillon, adjusted to $\mathrm{pH}$ 7.8. Incubation is at $32^{\circ} \mathrm{C}$. 
The experiments presented above reveal the following facts:

The final result of the phenomenon, that is to say, the total dissolution of the bacterial cells present in the suspension, does not depend upon the quantity of the bacteriophage filtrate inoculated into the suspension (d'Herelle ${ }^{310}$ ). We shall see, however, that although

TABLE 5

\begin{tabular}{|c|c|c|c|c|c|c|}
\hline \multirow{2}{*}{$\begin{array}{c}\text { TUBE } \\
\text { NUMBER }\end{array}$} & \multirow{2}{*}{$\mid \begin{array}{c}\text { QUANTITY OF } \\
\text { BACTERIO- } \\
\text { PHAGE } \\
\text { INOCULATED }\end{array}$} & \multicolumn{5}{|c|}{ APPEARANCE AFTER } \\
\hline & & 4 hours & 5 hours & 6 hours & 24 hours & 36 hours \\
\hline & $c c$. & & & & & \\
\hline 1 & $10^{-1}$ & $(100)$ & $(75)$ & Clear & Clear & Clear \\
\hline 2 & $10^{-2}$ & (125) & (100) & (25) & Clear & Clear \\
\hline 3 & $10^{-3}$ & (125) & (125) & (25) & Clear & Clear \\
\hline 4 & $10^{-4}$ & (125) & (125) & $(100)$ & Clear & Clear \\
\hline 5 & $10^{-5}$ & (125) & (125) & (125) & Clear & Clear \\
\hline 6 & $10^{-6}$ & (125) & $(150)$ & $(150)$ & Clear & Clear \\
\hline 7 & $10^{-7}$ & (125) & $(200)$ & (175) & Clear & Clear \\
\hline 8 & $10^{-8}$ & (125) & $(200)$ & $(200)$ & Clear & Clear \\
\hline 9 & $10^{-9}$ & (125) & $(200)$ & $(250)$ & Clear & Clear \\
\hline 10 & $10^{-10}$ & (125) & $(200)$ & $(300)$ & $(100)$ & Clear \\
\hline 11 & $10^{-11}$ & (125) & $(150)$ & $(175)$ & Cloudy & Cloudy \\
\hline
\end{tabular}

TABLE 6

\begin{tabular}{|c|c|c|c|c|c|}
\hline \multirow{2}{*}{ TUBE NUMBER } & \multirow{2}{*}{$\begin{array}{c}\text { QUANTITY OF } \\
\text { BACTERIOPHAGE } \\
\text { INOCULATED }\end{array}$} & \multicolumn{4}{|c|}{ APPEARANCE OF THE SUSPENSIONS AFTER } \\
\hline & & 6 hours & 18 hours & 24 hours & 60 hours \\
\hline & $c c$. & & & & \\
\hline 1 & $10^{-2}$ & $(125)$ & Clear & Clear & Clear \\
\hline 2 & $10^{-4}$ & $(150)$ & $(25)$ & Clear & Clear \\
\hline 3 & $10^{-6}$ & $(150)$ & $(100)$ & $(2 \tilde{5})$ & Clear \\
\hline 4 & $10^{-8}$ & $(150)$ & $(250)$ & $(200)$ & Clear \\
\hline 5 & $10^{-10}$ & $(150)$ & $(350)$ & $(350)$ & Clear \\
\hline 6 & $10^{-11}$ & $(150)$ & $(350)$ & $(350)$ & Clear \\
\hline 7 & $10^{-12}$ & $(125)$ & $(250)$ & $(300)$ & Cloudy* \\
\hline
\end{tabular}

* Tests showed that this tube did not contain any of the bacteriophage principle.

quantity is a negligible factor, the quality of the bacteriophage is the most important feature of the phenomenon.

The only influence exerted by the quantity of the bacteriophage principle inoculated is in the time required for the completion of the phenomenon (Kuttner; ${ }^{398}$ d'Herelle $^{323}$ ). 
Between the smallest quantity of the bacteriophage filtrate capable of provoking bacteriophagy and the amount immediately below this a partial effect is not obtained. The action is complete or there is no activity at all (d'Herelle ${ }^{310}$ ).

The smallest quantity of bacteriophage filtrate, of a maximum activity, capable of causing bacteriophagy in a suspension of dysentery bacilli, is in the neighborhood of a ten-billionth of a cubic centimeter (d'Herelle ${ }^{310}$ ).

Against the staphylococcus, the smallest quantity to be active is even less; in the experiment given above, about one hundred-billionth of a cubic centimeter, that is, about 10 cubic micra (d'Herelle).

Ellis has stated, ${ }^{193}$ although he presents no protocols to demonstrate the fact, that if two bacterial suspensions are inoculated with the bacteriophage, one with a large quantity, the other with but a small amount, bacteriophagy will not take place equally in both, that is, dissolution will be only partial in the first, and complete in the second case. Even more recently Gohs has reported the following results obtained with a bacteriophage filtrate which had been preserved for a year. When the quantity of filtrate used varied between $\frac{1}{50,000}$ and $\frac{1}{10,000,000}$ of a drop bacteriophagy was complete; while when the quantity of filtrate used was from 5 drops to $\frac{1}{10,000}$ of a drop the bacteria remained alive. It is indeed difficult to reconcile results of this type with the many experiments which I have made; experiments in which the filtrates have varied in age from those used immediately after preparation to those which had been held for several months and even several years, and in which all of the other conditions contributory to the process of dissolution have been varied in all directions. It would seem that experimental error, something such as an interchanging of tubes, must have occurred.

\section{INFLUENCE OF PHYSICAL CONDITIONS}

\section{Action of heat}

As a general rule it may be said that bacteriophagy may always take place at that temperature which is the most favorable for the development of the bacterium subjected to the action of the bacteriophage. This is not equivalent, however, to saying that this temperature is that at which the phenomenon is effected at the greatest rate. Moreover, the phenomenon may occur at temperatures very remote from the optimum growth temperature of the bacterium. 
We may here state what has been demonstrated in this connection up to the present time for different bacteria when subjected to the action of homologous races of the bacteriophage.

B. typhosus. Bacteriophagy takes place at temperatures up to $41^{\circ} \mathrm{C}$.; it is even more active at this temperature than at $37^{\circ}$ (Kuttner ${ }^{398}$ ). The phenomenon does not take place at $45^{\circ} \mathrm{C}$. (Kuttner ${ }^{398}$ ).

B. dysenteriae. A complete dissolution of dysentery bacilli takes place at temperatures between $8^{\circ}$ and $41^{\circ} \mathrm{C}$. (d'Herelle ${ }^{321}$ ), but the time required for the end result to be attained is very variable.

Three tubes, each containing a normal suspension of Shiga bacilli* are inoculated with $10^{-7} \mathrm{cc}$. of a filtrate containing the bacteriophage. These tubes are placed, one at $8^{\circ} \mathrm{C}$., one at $22^{\circ}$, and the third at $37^{\circ}$. Subsequent examination shows that in the suspension held at $8^{\circ}$ the bacteria have been completely dissolved after 16 days. In the one held at $22^{\circ}$ dissolution is complete after 25 hours. In the one kept at $37^{\circ}$ the dissolution is complete after 13 hours.

$B$. pestis. Two races of the bacteriophage have been tested against a single strain of $B$. pestis. In both cases the maximum temperature limit for complete dissolution was $41^{\circ}$, with the optimum temperature at $37^{\circ} \mathrm{C}$.

Staphylococcus. Against a single strain of Staphylococcus aureus two different races of staphy'ococcus bacteriophage were tested; the first, race $\mathrm{H}$, caused a complete dissolution at temperatures up to $43^{\circ} \mathrm{C}$, the other, race $\mathrm{B}$, only up to $41^{\circ}$. The optimum for the first was at about $32^{\circ}$, for the second at about $36^{\circ} \mathrm{C}$.

$B$. coli. Doer' and Grüninger ${ }^{183}$ have reported that bacteriophagy of $B$. coli does not take place at $43^{\circ}$, despite the fact that the strain of $B$. coli used by them developed at this temperature. It seemed, indeed, from their experiments that the bacteriophage, acting under such conditions of temperature, totally disappeared from the medium at the end of 5 to 7 hours. In attempting to verify this experiment, the results of which appeared to be rather unusual, I obtained entirely different results. Details of these experiments follow. The B. coli, strain $\mathrm{H}$, was isolated from a case of cystitis. The bacteriophage,

* When, in the description of an experiment the volume of a suspension is not stated, it should always be understood to be $10 \mathrm{cc}$. In the same way, in order to avoid repetitions such as would make the explanation unnecessarily long, unless indicated to the contrary, the culture medium is a beef bouillon (400 grams of muscle extracted per liter) with 1 per cent of peptone and 0.8 per cent of salt. The reaction is adjusted to a $\mathrm{pH}$ of 7.8 . 
race $c$, was isolated from the stools of a patient convalescent from Asiatic cholera. The medium was prepared with Liebig's meat extract, containing 0.5 per cent of salt and 1 per cent of peptone. The reaction was adjusted to $\mathrm{pH} 7.6$. $45^{\circ} \mathrm{C}$.

I. The water-bath was regulated to maintain a temperature of

Ten cubic centimeters of a suspension of $B$. coli, 100 million per cubic centimeter, were inoculated with 0.02 cc. of bacteriophage filtrate.

After 1 hour the opacity was equivalent to 100 millions per cubic centimeter.

After 2 hours the opacity was equal to 75 millions.

After 3 hours the medium was clear; dissolution was complete.

Control 1; the same suspension, but uninoculated with the bacteriophage. After 1,2 , and 3 hours at the same temperature of $45^{\circ} \mathrm{C}$. there was no change. The opacity of the supension remained the same as at the beginning.

Control 2; bouillon simply seeded with $B$. coli. After 3 hours at $45^{\circ} \mathrm{C}$. the medium was clear; throughout a period of 3 hours at this temperature there was no obvious development.

II. The water-bath was regulated to maintain a temperature of $46^{\circ} \mathrm{C}$. The conditions were the same as for the preceding; $10 \mathrm{cc}$. of a suspension of $B$. coli, 150 million per cc., inoculated with 0.05 cc. of bacteriophage filtrate (a filtrate of the suspension dissolved at $45^{\circ}$ in the preceding experiment).

After 1 hour the opacity was equal to 150 millions per cubic centimeter.

After 2 hours the opacity was the same.

After 3 hours the opacity was equal to 100 million.

After $3 \frac{1}{2}$ hours the opacity corresponded to 50 million.

After 4 hours the medium was clear; dissolution was complete.

Control 1; bouillon simply seeded with $B$. coli, without the addition of the bacteriophage. No development occurred within 4 hours. The medium remained as it was at the beginning of the experiment, immediately after seeding.

Control 2; suspension of 150 millions per cubic centimeter, without bacteriophage. The turbidity was the same after 1, 2, 3, and 4 hours as at the beginning. The culture showed no evidence of bacterial multiplication.

III. The water-bath was regulated to maintain a temperature of $47^{\circ} \mathrm{C}$. The procedure was the same as that given above. 
After 6 hours the turbidity was the same as at the beginning. Bacteriophagy had not taken place. But the bacteriophage was not destroyed, for a drop of this suspension spread upon agar failed to yield a growth after incubation. Nor were the bacilli killed, for the inoculation of a drop of the control suspension, uninoculated with the bacteriophage, yielded a growth of $B$. coli.

These experiments, therefore, justify the conclusion that under the conditions under which they were performed bacteriophagy is effected up to temperatures of $46^{\circ} \mathrm{C}$., and that at this temperature it is very rapid indeed, even more rapid than at $37^{\circ} \mathrm{C}$.

It is difficult to explain the results obtained by Doerr and Grüninger. But one possibility suggests itself, namely, that their results were due to the unintentional use of an acid bouillon as culture medium, or, which would amount to the same thing, to the use of a medium containing sugar and rendered acid by the fermentation caused by the colon bacilli. This possibility is suggested to explain the destruction of the bacteriophage at this temperature. As for the absence of bacteriophagy at this temperature $\left(43^{\circ} \mathrm{C}\right.$.) it is readily explained by the fact that the temperature limit varies with the race of bacteriophage employed. For example, in so far as $B$. coli is concerned, using the same strain as that which was employed in the experiments previously cited, but using a bacteriophage of a different race (isolated from the feces of a convalescent from typhoid fever) the dissolution of the bacilli was not complete at $42^{\circ}$; at $43^{\circ}$ it did not take place at all.

In bacteriophagy it is vain to undertake to establish rigid rules (there are some who even speak of "laws") fixing with an air of finality the conditions of the phenomenon. What is true for one race of the bacteriophage is not necessarily true for another. "I have isolated several hundred strains of this bactericidal "principle," and I have not yet found two which were absolutely identical." These words appeared in one of my first publications, ${ }^{315}$ and it is indeed unfortunate that those who have since worked with the phenomenon have been unable to comprehend their true meaning, since had they done so they would have refrained from stating many "rules" which further investigation has not confirmed.

The single method to follow,--indeed, the sole logical procedure if one wishes to determine as exactly as possible any one of the conditions of bacteriophagy, -is to execute a large number of experiments, utilizing different bacterial species and even different strains of the same species, combining each one of them with several races of the bacterio- 
phage. The results thus obtained will show the extreme limits within which the phenomenon can occur, and it will show at the same time how vain it is to attempt, from a single experiment, to fix the limiting conditions or the optimal conditions for the reaction, since these vary for each race of the bacteriophage which acts, and for each bacterial strain subjected to its action.

As far as temperature relationships are concerned, experiment demonstrates that the minimal temperature permitting a typical bacteriophagy, that is to say, the lowest temperature at which the final result is a complete dissolution of all of the bacterial cells contained in a suspension subjected to test, is found at about $8^{\circ} \mathrm{C}$. (with $B$. dysenteriae, d'Herelle $\left.{ }^{321}\right)$. The maximum temperature observed up to the present time is $46^{\circ} \mathrm{C}$. (with $B$. coli, d'Herelle). This by no means implies that subsequent experiments will not extend these limits.

I have specifically stated that these limits are those which are compatible with a total dissolution of the bacterial cells. Above and below these limits bacteriophagy may still be effected, but the dissolution is only partial. Having available a race of bacteriophage, active against $B$. dysenteriae, whose upper limit for total dissolution was $41^{\circ} \mathrm{C}$., it has been possible to effect several serial passages at $44^{\circ}$, in spite of the fact that at this temperature a dissolution of the bacteria could not be detected macroscopically. But despite this apparent lack of activity bacteriophagy took place, since the active principle, the bacteriophage, regenerated itself, and this in itself affords proof that destruction had not taken place.

As regards the optimal temperature, that is to say, that at which the phenomenon manifests itself most quickly and most completely, basing my statements upon the results of my experiments with $B$. dysenteriae and B.typhosus, I had concluded that the temperature most favorable for bacteriophagy was that which was likewise most favorable for the development of the bacteria. The results of many subsequent experiments, performed with a variety of bacterial species, indicate that this conclusion may have been too restricted. As a general rule, the statement is certainly true for the bacteriophagy of $B$. dysenteriae and $B$. typhosus, but it is not equally true for all other species of bacteria. The optimum growth temperature for several different strains of the staphylococeus with which I have worked has been found to be at about 37 to $38^{\circ} \mathrm{C}$. With three different races of the bacteriophage the reaction was effected with these strains in a perfect manner at between 32 and $34^{\circ} \mathrm{C}$., with another race of bacteriophage complete dissolution occurred only at $37^{\circ} \mathrm{C}$. 
Several of my laboratory strains of $B$. pestis have an optimum growth temperature of 32 to $33^{\circ} \mathrm{C}$. Two races of the bacteriophage acted upon these cultures most vigorously at temperatures of 37 to $38^{\circ} \mathrm{C}$.

With $B$. coli, we have seen that, at least with certain races of the bacteriophage, an extremely rapid dissolution occurs at a temperature of $46^{\circ} \mathrm{C}$., under conditions where the development of the colon bacilli did not take place, or at least where it was so slow that it could not be detected in control suspensions not containing the bacteriophage.

\section{Pressure conditions}

According to experiments which I have carried out with $B$. dysenteriae, it would appear that bacteriophagy occurs as actively in vacuo as under normal atmospheric pressure. ${ }^{321}$ Various authors, Wollstein in particular, have reached this same conclusion.

\section{Viscosity of the medium}

Doerr ${ }^{178}$ has stated that in a bouillon medium the addition of an adequate amount of gelatin prevents dissolution of the bacteria by the bacteriophage. The experiment upon which he based this conclusion was performed with $B$. coli. In collaboration with Berger, ${ }^{181}$ Doerr reconsiders this statement, modifying considerably the rigor of his first conclusions. He observes that it is necessary that the medium contain a certain quantity of gelatin in order to prevent the dissolution of the bacteria, and that the dissolution is the less as the concentration of the gelatin is increased. Agar acts in the same way. On the other hand, the greater the concentration of the bacteriophage present, the higher must be the content in gelatin to prevent the dissolution. But despite this inhibitory effect the bacteriophage is still capable of acting upon the bacteria even in media with very high concentrations of gelatin, for, as he showed, the principle reproduced in such a medium. Consequently bacteriophagy must have taken place.

In a series of independent investigations Nakamura has studied the effect of substances having properties analogous to those of gelatin. ${ }^{48,499}$ He showed that gum tragacanth, or salep, exerted an influence upon bacteriophagy comparable to that of gelatin. The sugars also, but to a less degree, interfered with the process. It is significant that he observed that all races of the bacteriophage do not behave in the same way. With some the activity is but slightly modi- 
fied by the introduction of gelatin into the medium; with others, on the contrary, the same medium prevents multiplication completely. Brutsaert ${ }^{110}$ has confirmed this observation.

Again and again we are confronted by the same conclusion: the most important factor in bacteriophagy is the quality of the bacteriophage under consideration.

A final explanation for the inhibitory action of gelatin has been provided by Hauduroy..$^{292}$ He states, "It is solely the high viscosity of the medium containing gelatin which interferes with the production of the phenomenon." He showed, in fact, while working with several substances, such as the gums and egg albumin, having the property of augmenting viscosity, that the inhibition is a direct expression of the viscosity, quite unrelated to the chemical nature of the viscous substance. To attribute this inhibition of bacteriophagy to anything other than a reaction between colloids, allied to other reactions of this type, is impossible. In no case is the bacteriophage destroyed. After a period of contact of any duration it is only necessary to dilute the medium with bouillon, thus diminishing its viscosity, to permit the inception of the process of dissolution.

Certain experiments which I have made, confirming fully these conclusions of Hauduroy, may be mentioned.

To $10 \mathrm{cc}$. of a peptone bouillon containing 0.8 per cent salt, is added 20 per cent of gelatin. The $\mathrm{pH}$ is then adjusted to 7.8 . With the medium at a temperature of $35^{\circ} \mathrm{C}$., Staphylococcus aureus is introduced to give a concentration of about 200 million organisms per cubic centimeter. It is then inoculated with $0.05 \mathrm{cc}$. of a highly active bacteriophage filtrate, that is, with one capable of regularly causing a total and pe manent dissolution of a normal suspension in bouillon. After incubation for 6 days at $35^{\circ} \mathrm{C}$. the medium was perfectly limpid, dissolution was complete. In a control mixture in plain bouillon, without gelatin, dissolution was completed in 24 hours. The conclusion is obvious. When added in a sufficient quantity to a medium in which bacteriophagy should be effected, gelatin completely inhibits the action of races of the bacteriophage of low activity, while for more potent races the process is retarded, and the number of bacteria dissolved is the greater as the bacteriophage is the more powerful.* With

* With the acquisition of a resistance by a portion of the bacteria, an acquisition favored by the delay associated with the viscosity of the medium, we are not now concerned, yet in this connection the retardation is of considerable significance. This feature of the reaction will receive further attention when we deal with the behavior of the bacterium toward the bacteriophage. 
races possessing a maximum activity all of the bacteria are destroyed and dissolved. The rôle of gelatin, in the last analysis, consists solely in altering the rate of the phenomenon, that is, in extending the duration of the action.

All neutral substances, which possess the property of augmenting viscosity, affect the phenomenon of bacteriophagy as does gelatin, and the intensity of this effect is in direct proportion to the viscosity of the medium.

In the particular case under consideration the course of the phenomenon is regulated by variations in two factors, the degree of activity of the bacteriophage involved, and the viscosity of the medium.

\section{EFFECT OF THE CHEMICAL CONDITIONS OF THE MEDIUM}

\section{Colloids}

We have seen that gelatin, and this is also true for the gums and for egg albumin, have of themselves no effect upon bacteriophagy. It is only when such substances are added to the medium in a quantity sufficient to significantly augment the viscosity that they exert an effect, and even then this effect is simply a retardation of the action. Certainly this is the case when a potent bacteriophage is used.

It is of interest, in comparison with the above, to observe the effect exerted by a colloid which does not modify the viscosity of the medium to which it is added and whose action, if any, must be due to properties inherent in the colloid itself. For this purpose I have chosen colloidal silver (collargol, Clin, as provided in ampoules for purposes of injection).

The following experiment indicates the nature of the effects with such a colloid. To S ce. of Liebig's extract bouillon, made up with 1 per cent of peptone and 0.8 per cent of salt (reaction pH 7.6) collargol is added. Three tubes are prepared. The mixtures are implanted with a heavy suspension of Staphylococcus aureus derived from a young culture, the final concentration being 100 million organisms per cubic centimeter. Each of the suspensions is then inoculated with $0.02 \mathrm{cc}$. of a Staphylo-bacteriophage, and the tubes are incubated at $32^{\circ} \mathrm{C}$.

The appearance after 24 hours is as follows:

\begin{tabular}{c|c|c}
\hline TUBe Number & AMount of COllargol ADded & APPEArance AFter 24 Hours \\
\cline { 2 - 3 } 1 & $c c$. & \\
2 & 0.5 & Clear \\
3 & 1.0 & Clear \\
& 2.0 & Clear \\
\hline
\end{tabular}


Control tubes, lacking the bacteriophage filtrate, show that the staphylococcus develops normally in bouillon containing these quantities of collargol.

Under such conditions bacteriophagy proceeded normally, as rapidly and in as complete a manner, as in plain bouillon, even though the colloidal silver added $(2$ cc.) was sufficient to give the medium such a deep brown color that it was impossible to see and read print through the layer of fluid.

As an incidental finding, the following observation is interesting and is in some degree significant. In the control tubes, containing the same quantities of collargol and the same suspension of staphylococci, but without the bacteriophage, after incubation for 24 hours the colloidal silver was completely floceulated, forming a black precipitate on the bottom of the tube. In the suspensions inoculated with the bacteriophage no flocculation took place, the liquid remained perfectly clear after bacteriophagy, presenting the same aspect as a tube of sterile bouillon to which a comparable amount of collargol was added.

This experiment has been repeated with $B$. dysenteriae with the same result.

In another series of experiments I have used the dry colloidal silver of Heyden, bearing the trade name "Collargolum steril." To $10 \mathrm{cc}$. of culture medium (the same as that used above) $5 \mathrm{mgm}$. of the dry collargolum are added, and then enough of a thick suspension, prepared from a young agar growth, of cocci to yield a count of 250 million per cubic centimeter. Finally, 0.05 ce. of a Staphylo-bacteriophage filtrate is added. After 36 hours bacteriophagy is complete; the medium is absolutely clear and of a deep red-brown color. A drop of this spread upon agar yields no growth.

If performed with $B$. dysenteriae this experiment gives the same result.

As a check on these results, it is found that $B$. dysenteriae, and the staphylococcus as well, grow perfectly well in bouillon containing colloidal silver in the amount employed in the above experiment. The statement is very frequently made that colloidal silver is a powerful antiseptic. It would seem to be a fair question to ask how experiments should be conducted to demonstrate this fact. Unquestionably, several authors have published experiments tending to show that the bacteriophage is destroyed by the presence of negative colloids, of colloidal silver in particular, but, as a matter of fact, far from being destroyed, the bacteriophage develops normally in the presence of this substance. 
May not the erroneous conclusions reached by these authors be due to the fact that they have used commercial solutions of colloidal silver to which some antiseptic agent had been added, and have they not attributed to colloidal silver an effect which was due to some agent of whose presence they were unaware? Obviously, we can not tell. But in any case, a considerable number of experiments warrant the statement that pure colloidal silver (Collargolum siccum, Heyden,* for example) exercises no antiseptic action upon the colon-typhoiddysentery group of bacilli or upon the staphylococci, and that in its presence bacteriophagy occurs normally. $\dagger$

Colloidal sulfur, another electro-negative colloid, has also been tested. In its presence bacteriophagy takes place in an absolutely normal fashion. Indeed, with this colloid it would seem that bacteriophagy is, if anything, stimulated, as has been observed by Otto and Munter. ${ }^{494} \ddagger$

In brief, then, negative colloids, and these are the only ones which can be tested, since bacteriophagy will not take place in an acid medium, cause, of themselves, no effect upon the phenomenon of bacteriophagy.

\section{Dyestuffs}

Five-hundredths of a cubic centimeter of a Staphylo-bacteriophage is added to $10 \mathrm{cc}$. of a suspension (100 million cocci per cubic centimeter) of Staphylococcus aureus. To such mixtures the dyestuffs, as indicated in table 7 , are then added.

Control tubes are prepared, the bouillon containing the same quantities of the dyestuffs, and are simply seeded with the staphylococcus. These tubes demonstrate the possibility for growth in the dye-containing medium.

To all of these tubes which showed bacteriophagy a second implantation of a concentrated suspension of staphylococci was made, yield-

* A finely granular colloidal silver, yielding in water a very stable red pseudosolution.

$\dagger$ These results suggest grave doubts upon the validity of the experiments showing the so-called "oligodynamie" action of metals, of silver in particular. If silver in its colloidal form is not active there is the more reason to suspect that it may be inert when in its usual form.

$\ddagger$ We will see later that "secondary cultures" develop when the bacteriophage used is not of a maximum potency. It is significant that in the presence of colloidal sulfur secondary eultures are difficult to obtain, even though the bacteriophage used does not prevent their formation in normal bouillon. 
ing 200 million cocci per cubic centimeter. Again bacteriophagy was complete in all tubes after 24 hours.

From these results it is clear that when the substance, like fuchsin, has an antiseptic action bacteriophagy does not take place; if the dye is lacking in harmful effects upon the bacterium bacteriophagy is normal. Of itself, the dyestuff, like colloids, interferes in no way with the phenomenon (d'Herelle).

TABLE 7

\begin{tabular}{|c|c|c|c|}
\hline DYESTUFF & $\begin{array}{l}\text { QUANTITY } \\
\text { ADDED }\end{array}$ & $\begin{array}{l}\text { APPEARANCE } \\
\text { AFTER } 24 \\
\text { HOURS }\end{array}$ & $\begin{array}{c}\text { SEEDED } \\
\text { CONTROL TUBE }\end{array}$ \\
\hline & $c c$. & & \\
\hline Fluorescein (Grübler) ............. & $0.05^{*}$ & Clear & Growth \\
\hline Methylene blue (Grübler). . & $0.05 \dagger$ & Clear & Uiven \\
\hline Fuchsin (Grübler) .................. & $0.05 \dagger$ & Turbid & No growth \\
\hline Neutral red (Grübler) .......... & $0.05^{*}$ & Clear & Growth \\
\hline Acid fuchsin (Grübler). . & $0.05^{*}$ & Clear & Growth \\
\hline
\end{tabular}

* A saturated aqueous solution.

$\dagger$ A saturated alcoholic solution.

TABLE 8

\begin{tabular}{|c|c|c|c|c|}
\hline DATE & $\begin{array}{l}\text { TUBE NUMBER } \\
\text { CONTAINING } 8 \mathrm{cC} \text {. } \\
\text { OF MEDIUM }\end{array}$ & BACTERIOPHAGE ADDED & $\begin{array}{l}\text { B. DYSENTERIAE } \\
\text { FLEXNER, } 24 \text { HOUR } \\
\text { CULTURE }\end{array}$ & $\begin{array}{l}\text { APPEARANCE } \\
\text { AFTER } 24 \text { HOURS } \\
\text { AT } 37^{\circ} \mathrm{C} .\end{array}$ \\
\hline & & & $c c$ & \\
\hline $10 / 14$ & 1 (control) & 0 & 0.05 & Very eloudy \\
\hline $10 / 14$ & 2 & 0.05 & 0.05 & Clear \\
\hline $10 / 15$ & $3(\mathrm{co}$ & 0 & 0.05 (from & Very cloudy \\
\hline $10 / 15$ & 4 & 0.05 (from tube 2 ) & 0.05 (from & Clear \\
\hline $10 / 16$ & 5 & 0 & 0.05 (from tube 3 ) & Very cloudy \\
\hline $10 / 16$ & 6 & 0.05 (from tube 4$)$ & 0.05 (from & Clear \\
\hline $10 / 17$ & 7( & 0 & 0.05 (from tube 5 ) & Very eloudy \\
\hline $10 / 17$ & 8 & 0.05 (from tube 6 ) & 0.05 (from tube 5 ) & Clear \\
\hline
\end{tabular}

\section{Electrolytes}

In several communications da Costa Cruz has emphasized experiments which indicate that bacteriophagy will not take place in a medium very poor in electrolytes, a medium such as a 1 per cent Witte peptone solution in distilled water. When, however, he added various salts to this medium $\left(\mathrm{NaCl}, \mathrm{CaCl}_{2}, \mathrm{Na}_{2} \mathrm{SO}_{4}\right)$ the bacteria were dissolved. ${ }^{158,159}$

Although it may be true that in such a medium, which is incidentally 
rather unfavorable to the bacterium involved (B. dysenteriae Flexner), a complete dissolution of concentrated bacterial suspensions does not take place, it is equally true that bacteriophagy will take place, and in a complete fashion, when the suspension is less heavy, as the following experiments show ${ }^{343}$ (table 8 ).

The culture medium is a 1 per cent peptone water, having a $\mathrm{pH}$ of 7.0 .

This demonstrates that the bacteriophage is capable of multiplying in media poor in electrolytes, inasmuch as in such media bacteriophagy occurs.

Brutsaert ${ }^{110}$ arrived at the same conclusions, but, according to his observations certain races of the bacteriophage fail to develop under such conditions, while others, on the contrary, are unaffected and lead to a total dissolution of the bacterial cells present in the medium.

Here again, the same observation is pertinent. It is the quality of the bacteriophage which determines the course of the phenomenon.

Ciuca $^{145}$ lias also shown that bacteriophagy occurs normally in media poor in electrolytes, provided the active principle operates upon young bacteria. His media were alkaline, $\mathrm{pH} 7.8$ to 8.0.

In general, then, the conclusions seem to be that in media poor in electrolytes, that is, in salts, bacteriophagy does not take place when the medium is acid. In a neutral medium ( $\mathrm{pH}$ 7.0) bacteriophagy may or may not occur, depending upon the race of the bacteriophage concerned. In an alkaline medium, the phenomenon takes place with all races; and with the most active the dissolution of the bacteria is complete.

Instead of considering the effects of deficient quantities of electrolytes in the medium, the reverse situation offers a problem. What happens when the medium contains a large amount of salt? Bacteriophagy takes place normally in bouillon containing 2.5 per cent of salt (d'Herelle $^{321}$ ). Brutsaert ${ }^{109}$ has shown that $B$. coli does not grow in a medium containing more than 5 per cent of $\mathrm{NaCl}$, while staphylococci still develop freely in broth having 14 per cent of salt, yet in such media he observed that bacteriophagy took place normally. According to him, bacteria cultivated in hypertonic bouillon and then placed in contact with the bacteriophage are even more readily attacked than the same bacteria grown in a medium with a normal concentration of salt. 


\section{Sugars}

Asheshov ${ }^{27}$ has shown that with certain races of the bacteriophage (one only among those which he studied) bacteriophagy takes place more rapidly if glucose is added to the medium. With this particular race, active against $B$. dysenteriae Flexner, dissolution of the bacteria was complete after $4 \frac{1}{2}$ hours in the presence of glucose, while in ordinary bouillon it was only partial after a period of 7 hours. Further studying the reaction with this particular race he concluded that the acceleration must be due to the fact that $B$. dysenteriae in fermenting the glucose rendered the reaction acid. This, then, represents a particular distinctive race, with which the optimum activity occurs in a medium of increasing acidity. We will return to this observation.

Seiser ${ }^{584}$ has suggested that the addition of glucose to the medium favors bacteriophagy in all cases, but in agreement with Asheshov, I have not found this to be true. Indeed, it appears that the situation is quite the reverse.

The experiments which I have performed during the past few years have not modified my original conclusions in this respect. The addition of non-fermentable sugars* to a bacterial suspension subjected to the action of the bacteriophage has no effect upon the phenomenon. In the case of fermentable sugars, if the inoculation of bacteriophage has been massive, bacteriophagy takes place normally. If the inoculation has been weak (or if the race of bacteriophage is of low activity) bacteriophagy is incomplete or does not occur, the result depending upon the amount inoculated. The reason for such an effect is obvious. We already know that the great majority of races of the bacteriophage are very sensitive to the action of free $\mathrm{H}$ ions. With a minimal inoculation of bacteriophage the bacteria start to grow, they attack the sugars, and the medium becomes acid before the quantity of the bacteriophage, regenerating in the course of the action, is sufficient to effect a dissolution of the bacteria in the time available. In a word, the limit of acidity incompatible with the phenomenon is reached before the bacteriophage is able to become effective (d'Herelle ${ }^{321}$ ).

\section{Salts}

In previously reported experiments ${ }^{321}$ I have shown that calcium chloride interferes with the bacteriophagy of Shiga bacilli; that potas-

* This holds only when the quantity of sugar added is not sufficiently high to materially modify the viscosity of the medium. We have already seen that an increase in viscosity retards bacteriophagy. 
sium chloride retards the process; that magnesium sulfate and the phosphates of sodium and of potassium, in low concentrations, possess a stimulating action, especially with races of the bacteriophage of but weak potency. I have not observed these effects in bacteriophagy with other bacterial species. This apparently warrants the conclusion that when a salt, in small amounts, seems to exercise an inhibitory or a stimulating effect, it is because it favors or interferes with the development of the bacteria, rather than because it exerts any specific direct effect upon the process of dissolution.

\section{Body fluids and other substances}

When added to a bacterium-bacteriophage mixture, substances devoid of action upon the bacteria, have, in general, no effect upon the phenomenon. Thus, the process is not modified, for example, by normal serum, ascitic fluid, or urine (d'Herelle ${ }^{321}$ ). Bile is unquestionably inhibitory.

\section{Antiseptics}

Kabeshima ${ }^{388,389}$ has stated that the bacteriophagy of $B$. dysenteriae occurs even in the presence of antiseptic substances, for example, in media containing sodium fluoride or an excess of chloroform. If these observations are correct, they are quite at variance with the results which I had obtained. At my suggestion Bablet ${ }^{33}$ has repeated the experiments of Kabeshima. From his (Bablet) experiments, very carefully performed, and carried out as were those of Kabeshima upon $B$. dysenteriae, it appears that in a bouillon medium containing 1 per cent of sodium fluoride, the bacteriophage principle does not regenerate for after three passages in such a medium it has completely disappeared. Under such conditions, therefore, bacteriophagy does not take place.

The results were the same in the medium containing chloroform.

I have shown ${ }^{321}$ that bacteriophagy does not take place in media saturated with essences of thyme or of cloves, even though in such media the bacteria remain alive for at least 48 hours.

Wolff and Janzen ${ }^{628}$ have reported that bacteriophagy is not accomplished in the presence of different antiseptics, such as optochin, eucupin, vusin (these three substances being derivatives of quinin), chinosol yatren, trypaflavine, rivanol, and malachite green, even if these substances are added in quantities so small that the bacteria are killed only after an appreciable interval. Under these circumstances, although bacteriophagy is lacking, the bacteriophage is not destroyed; 
it simply remains inert. Their experiments were conducted with $B$. typhosus, B. coli, B. dysenteriae, and the staphylococcus.

That bacteriophagy does not occur in bouillon containing a quantity of methyl violet sufficient to affect the vitality of the bacteria has been reported by Tomaselli. ${ }^{599}$

A somewhat more interesting situation is revealed by those experiments in which the antiseptic agent is added to the medium in amounts so small as to allow bacterial development. Certain of my experiments were performed for the specific purpose of determining what happens under these conditions, sodium fluoride being selected as the antiseptic. Incidentally, in this series of studies I demonstrated that, contrary to what is stated in a number of physiological text-books, life is perfectly possible in media containing 1 per cent of salt. In such media $B$. dysenteriae or $B$. coli live perfectly well. In fact, multiplication, in agglutinated floccules, takes place provided the organisms have undergone a preliminary adaptation by a few passages through media of increasing salt concentration. But despite the fact that the bacteria are able to develop in this solution, bacteriophagy will not take place in such a medium.

A further fact revealed in these studies is that while $B$. coli develop freely, without a preliminary adaptation, in a bouillon containing 0.2 per cent of sodium fluoride bacteriophagy will not take place, even if the bacteriophage introduced is very active. If $10 \mathrm{cc}$. of this fluoride containing medium is seeded very lightly with $B$. coli and then $0.1 \mathrm{cc}$. of an extremely active bacteriophage filtrate is added, the colon bacilli develop as vigorously and as abundantly as in the same medium without the bacteriophage. Nevertheless, the bacteriophage is not destroyed; it simply remains inert (d'Herelle ${ }^{340}$ ).

By this experiment I had designed to show only the fact that the bacteriophage may remain inactive under conditions which permit the development of the bacterium. Brutsaert ${ }^{103}$ has extended this study and has sought to determine if the concentration of fluoride, compatible with the development of the bacterium, but inhibitory as regards bacteriophagy, is the same for all bacterial species. He found that the concentration limiting bacteriophagy varies for each species studied (from 0.25 to 0.05 per cent). Indeed, this might have been predicted, for although the bacteriophage remains passive it is certainly not because the fluoride has an effect upon it, but because it modifies the state of the bacterium. This conclusion is supported by the fact that in bouillon containing fluoride the bacteria do not grow normally but in agglutinated clumps. 
These observations may be summarized very simply, then, by stating that bacteriophagy is operative only with living and normal bacteria.

\section{8. phenomena CORRElative With baCteriophagy}

\section{Acceleration of bacterial grouth}

We have already seen, in the experiments described, that if a suspension of bacteria is inoculated with a relatively small amount of bacteriophage, the bacteria develop, as revealed by the increasing turbidity, and it is only after this initial growth that the bacteria commence to dissolve. But if the experiments described above be carefully watched something else may be observed, namely, that the growth is more rapid, often very much more so, in the suspensions where bacteriophagy is to take place than in the control suspensions uninoculated with the bacteriophage. It would seem that the presence of this dissolving principle applies a stimulus of some sort, as though the multiplication of the bacteria undergoes first a "speeding-up;" an acceleration of the processes of cellular division.

\section{Agglutination}

Very often it may be observed that dissolution of the bacteria is preceded by a very outspoken agglutination. This phenomenon is particularly marked when the bacterial suspension is inoculated with a relatively large amount of bacteriophage filtrate of average potency (d'Herelle ${ }^{321}$ ).

Sometimes this agglutination takes place very quickly, even within a few minutes after the bacteria come into contact with the bacteriophage. The cause of this agglutination does not appear to reside in the bacteriophage principle itself, but in "a something" which is associated with it in the filtrate. The following experiments show, in fact, that this agglutination takes place in physiological saline, and even if the bacteria are killed previously by heat. The agglutinating substance passes through colloidion membranes that are relatively open, and it resists ageing.

A bacteriophage filtrate, about 6 months old, active for $B$. dysenteriae Shiga, was diluted to 1:1000 in physiological saline and dialyzed, in a collodion sac, against physiological saline. After dialysis for 48 hours living Shiga bacilli, taken from a young agar culture, were suspended in the dialysate, yielding a suspension containing 100 million organisms 
per cubic centimeter. The bacilli were immediately completely agglutinated in the form of fine floccules, clearly visible macroscopically.

The same experiment was carried out with a bacteriophage active for the staphylococcus. When the dialysate was combined with organisms (Staphylococcus aureus) killed by heating for 30 minutes at $60^{\circ} \mathrm{C}$. a fine agglutinate immediately formed, the clumps being readily visible macroscopically.

There is present, then, in a filtrate, aside from the bacteriophage principle, which is inoperative upon killed bacteria, a substance capable of augmenting the surface tension of the bacteria, whether they be living or dead, against which the bacteriophage present in the filtrate possesses the power of bacteriophagy.

\section{RÉSUMÉ}

Summarizing the data presented in this chapter the following statements can be made.

There is a principle, very widely distributed in nature, normally occurring in the intestinal contents, which possesses the property of dissolving bacteria. This principle is present in a particularly active form in the intestinal canal and in the excreta during convalescence from a variety of infectious diseases (d'Herelle ${ }^{310}$ ).

The phenomenon which this principle causes, in vitro, in a liquid medium containing a suspension of bacteria susceptible to its action, consists essentially in a dissolution of the bacterial cells. This phenomenon of dissolution occurs only with living organisms, the medium best suited to the action being that where the bacterium under consideration develops best (d'Herelle ${ }^{310}$ ).

Dissolution of the bacteria is accompanied not only by a regeneration of the active agent but by a very pronounced multiplication of the principle.

The agency responsible for the phenomenon has been termed Bacteriophage, the phenomenon itself is called Bacteriophagy (d'Herelle ${ }^{312}$ ).

By virtue of the fact that in the course of its action the bacteriophage principle multiplies, the dissolving action can be carried out serially for an indefinite period (d'Herelle ${ }^{310}$ ).

Multiplication of the bacteriophage principle takes place whatever may be the number of bacteria present in the suspension, or in the culture into which it is inoculated (d'Herelle ${ }^{321}$ ).

Although multiplication of the bacteriophage is a process qualitatively independent of the number of bacterial cells exposed to its 
action, the dissolution of the bacterial cells, a direct result of this multiplication, is complete only within the limits of 1 and 700 million bacteria per cubic centimeter when the bacteriophage principle involved possesses a maximum activity. With a greater number of bacteria per cubic centimeter the dissolution is but partial (d'Herelle ${ }^{321}$ ).

The minimal quantity of bacteriophage principle necessary to obtain dissolution of a suspension or a bacterial culture is, in the more favorable cases, equal to a ten-billionth part of a cubic centimeter $\left(10^{-10}\right.$ cc. $)$; sometimes even a hundred-billionth part is sufficient $\left(\right.$ d'Herelle $\left.^{310}\right)$.

Bacteriophagy takes place under aerobiosis or anaerobiosis. The temperature extremes are 8 and $46^{\circ} \mathrm{C}$., but for all bacterium-bacteriophage systems the limits, as well as the optimum temperature are not identical. The temperature relationships vary, on the one hand, with the bacterial species, and even with the bacterial strain, and, on the other hand, with the race of bacteriophage (d'Herelle ${ }^{321}$ ).

Only living and normal bacteria are suited to the phenomenon of bacteriophagy. That is to say, the phenomenon does not take place in the presence of antiseptics, when the amount present is sufficient to modify, in any way, the state of the bacteria (d'Herelle ${ }^{310}$ ).

Bacteriophagy may be accompanied by accessory phenomena. The process of multiplication of the bacteria subjected to the action of the bacteriophage principle may exhibit an accelerated rate prior to the phase of dissolution. An initial phase of the reaction may be characterized by an agglutination. 


\section{CHAPTER II}

\section{The Bacteriophage Corpuscle}

\section{BACTERIOPHAGY UPON SOLID MEDIA}

The phenomenon of bacteriophagy can be demonstrated, not only in a liquid medium, but upon a solid medium as well, the presence of the active principle being readily revealed by the following simple procedure.

Take a young culture or a rather cloudy suspension of dysentery bacilli in bouillon. Also, dilute a drop of bacteriophage fluid, that is, a suspension which has been cleared through bacteriophagy, active for the dysentery organism, in a liter of sterile physiological saline. Inoculate the bacterial suspension with a drop* of this dilution of bacteriophage filtrate. The final mixture will then contain a great many bacilli and very little bacteriophage. Finally, remove a drop of this inoculated suspension and spread it over the surface of an agar slant or upon an agar plate. After incubation the agar will be covered by a layer of bacilli, but this layer presents a curious aspect, for throughout its extent there will be spots, perfectly circular in form, where the agar is bare, devoid of all apparent growth (d'Herelle, ${ }^{310}$ ).

What do these bare areas, these "plaques" as I have termed them, mean? In the first place it is certain that they bear some relation to the bacteriophage, for they never appear upon plates made with a normal culture of any species of bacteria. But they are always apparent, and always have an identical appearance, when the agar is planted with a culture or a suspension of any bacterial species previously inoculated with a very minute quantity of a bacteriophagic filtrate active for the bacterial species involved (d'Herelle ${ }^{315}$ ). The phenomenon is, then, not restricted to certain bacterial species. It is to be observed with any species provided the homologous dissolving principle is present.

This simple preliminary experiment shows clearly that there is a causal relationship between the presence of the bacteriophage in a culture or suspension and the appearance of the plaques in the layer of growth obtained by seeding such a suspension upon an agar medium.

* When speaking of a drop, it is to be understood that a normal drop, $0.05 \mathrm{cc}$.. is meant. 
Let us carry the experiment farther, and see if the nature of this relationship may be disclosed. Take a series of 12 tubes, each containing 9 cc. of bouillon to which a concentrated suspension of dysentery bacilli has been added to give a count of 250 million bacilli per cubic centimeter. With these 12 tubes, all containing like amounts of the same bacterial suspension proceed as follows:

Tube 1. Into one of these tubes, inoculate $1 \mathrm{cc}$. of a bacteriophage liquid, one very active for the dysentery bacillus. Each cubic centimeter of the tube will then contain 250 million bacilli and $0.1 \mathrm{cc}$. of bacteriophage fluid.

Tube 2. Into a second tube introduçe $1 \mathrm{cc}$. of the contents of tube 1, just prepared. This second tube will then contain, per cubic centimeter, 250 million bacilli together with the tenth part of the bacteriophage fluid transferred in the cubic centimeter of material from the first tube, that is, $0.01 \mathrm{cc}$.

Tube 3. Into a third of the 12 tubes of bacillary suspension introduce 1 cc. of the contents of tube 2 . This tube (no. 3) will then contain, as did the others, 250 million bacilli per ec., and the bacteriophage present in the cubic centimeter of fluid transferred from tube 2 , that is, to each cc., $0.001 \mathrm{cc}$. of the bacteriophage fluid.

Continue in this manner with eleven of the tubes of dysentery bacillus suspension, as first prepared. Each cubic centimeter of the fluid in all of these tubes will then contain 250 million bacilli per cubic centimeter, but each tube of the series will contain less of the bacteriophage fluid, since each successive tube will contain but a tenth of the amount in the tube preceding. We will have a series of tubes, all containing both bacteria and bacteriophage; but as is indicated in table 9 , the bacterial content is constant; the bacteriophage content is a variable.

Immediately after preparing these successive dilutions transfer a normal drop $(0.05$ cc.) of each of the 12 tubes to agar, either a slant or a plate, taking care to distribute the drop evenly over the surface, in other words, spread* it in a uniform layer. Place the 12 agar cultures in the incubator at $37^{\circ} \mathrm{C}$. After incubation, the following facts may be noted.

The tubes upon which the specimens removed from the dilutions $10^{-1}, 10^{-2}$, and $10^{-3}$ (tubes 1,2 , and 3 ) were spread show no trace of

* I shall of ten have occasion to speak of this operation. The word "spread" implying the even distribution of the liquid over all of the surface of the agar slant or the media of the Petri dish, will be employed. 
growth whatever; they are bare, just as though they had not been planted.

The tube corresponding to dilution $10^{-4}$ (tube 4) presents a few traces of $B$. dysenteriae growth; irregular fragments of a layer of bacterial growth being scattered over its surface.

The tube corresponding to dilution $10^{-5}$ (tube 5) shows a layer of bacillary growth studded with an infinity of confluent plaques.

The tube corresponding to dilution $10^{-6}$ (tube 6 ) is entirely covered by culture, but scattered throughout the layer there are some 20 plaques.

TABLE 9

\begin{tabular}{|c|c|c|}
\hline TUBE NUMBER & $\begin{array}{c}\text { BACTERIAL } \\
\text { CONTENT } \\
\text { PER CUBIC } \\
\text { CENTIMETER }\end{array}$ & BACTERIOPHAGE CONTENT \\
\hline & million & \\
\hline 1 & 250 & $+10^{-1}$ cc. of bacteriophage fluid \\
\hline 2 & 250 & $+10^{-2}$ cc. of bacteriophage fluid \\
\hline 3 & 250 & $+10^{-3}$ cc. of bacteriophage fluid \\
\hline 4 & 250 & $+10^{-4}$ cc. of bacteriophage fluid \\
\hline 5 & 250 & $+10^{-5}$ cc. of bacteriophage fluid \\
\hline 6 & 250 & $+10^{-6}$ cc. of bacteriophage fluid \\
\hline 7 & 250 & $+10^{-7}$ cc. of bacteriophage fluid \\
\hline 8 & 250 & $+10^{-8}$ cc. of bacteriophage fluid \\
\hline 9 & 250 & $+10^{-9}$ cc. of bacteriophage fluid \\
\hline 10 & 250 & $+10^{-10} \mathrm{cc}$. of bacteriophage fluid \\
\hline 11 & 250 & $+10^{-11}$ cc. of bacteriophage fluid \\
\hline 12 & \multicolumn{2}{|c|}{$\begin{array}{l}\text { This tube, a control, contains the bacillary suspension, } 250 \mathrm{mil}- \\
\text { lion per cubic centimeter, but is without bacteriophage }\end{array}$} \\
\hline
\end{tabular}

The tube representing dilution $10^{-7}$ (tube 7 ) is also covered with a culture of dysentery organisms, showing but 2 plaques.

The tubes corresponding to the dilutions $10^{-8}, 10^{-9}, 10^{-10}$, and $10^{-11}$ (tubes $8,9,10$, and 11 ) are covered with perfectly normal growth. They present an appearance exactly comparable to that found upon the control tube (tube 12), which contained only the bacillary suspension.

These cultures may be incubated for any length of time whatever, even up to the point where the medium becomes dried up, without causing any change in their appearance. Cultures which originally were free of growth (tubes 1,2, and 3) remain bare indefinitely, just as though they were sterile.* Those cultures containing plaques remain, like-

* I repeat once more that in the first three chapters of this text I am dealing only with the conditions found in typical bacteriophagy, that is, with what takes 
wise, as they first appeared; the plaques once formed undergo no modification, they never increase in size and they are never overgrown by the surrounding bacterial culture.

As for the cultures presenting a normal bacterial growth (tubes 8 , 9,10 and 11, and, of course, tube 12), they also undergo no further modification and if they be further examined by subculture it is found that they will yield indefinitely normal cultures of the dysentery bacillus.

If we observe the nature of the reaction which takes place in the suspensions themselves, that is, the tubes from which the agar slants were implanted we will find that after incubation for 24 hours bacteriophagy is complete in the tubes containing dilutions $10^{-1}$ to $10^{-5}$. After 48 hours it is also complete in tubes 6 to 10 , that is, in those containing dilutions $10^{-6}$ to $10^{-10}$, while the tube with the $10^{-11}$ dilution remains turbid. This last tube may be subcultured indefinitely with the result that the successive cultures are as normal as are those of the control tube (tube 12).

This experiment has been repeated a great many times with bacteria of different species: the staphylococcus, B. pestis, B. typhosus, B. gallinarum, etc., and has always given, from the broad point of view of the principle involved, analogous results. The course of the phenomenon is always the same. The only point subject to variation is the extent of the dilution, and such a quantitative difference is, of course, compatible with what we know of bacteriophagy. For example, in working with another race of bacteriophage, also active against the dysentery bacillus, the last active dilution may be $10^{-9}$, and in this case the agar tubes corresponding to clilutions $10^{-1}$ and $10^{-2}$ will be bare. The agar tube representing the dilution $10^{-3}$ will show shreds of growth. The tube representing $10^{-4}$ will be covered by a layer of culture studded with about 100 plaques, the one corresponding to $10^{-5}$ will present but 12 plaques, and those tubes corresponding to the remaining dilutions of the series will be covered with normal cultures of dysentery bacilli.

In another experiment carried out with Staphylococcus aureus and with a race of the bacteriophage active for this organism, the last active dilution may be $10^{-11}$. The agar tube corresponding to the dilution

place with races of the bacteriophage which are extremely potent, races which cause in a liquid medium a total and permanent dissolution of a normal suspension, the medium remaining limpid indefinitely. If there are any who have not been able to isolate such a race I will gladly send them one upon request. These races will permit them to carry out all of the experiments described in this text. 
$10^{-6}$ may be covered by a layer of culture eroded by confluent plaques. The agar slant prepared from the $10^{-7}$ dilution will present a layer of staphylococcus growth spotted by about 100 plaques, and the tube corresponding to $10^{-8}$ will give a culture where only about 8 plaques can be found.

The sole difference appearing in the results of these experiments is that the different bacteriophage filtrates each act up to a different dilution. Aside from this difference in "strength" the aspect of the phenomenon is always the same. A further fact revealed by these experiments is, and this is of extreme importance, that the number of plaques formed bears, in every experiment, a strict relationship to the quantity of bacteriophage liquid. When there are, for example, 20 plaques on the agar slant corresponding to a given dilution there will be, practically, one-tenth as many plaques on the agar tube corresponding to the dilution which is ten times greater.

As we know that the bacteriophage principle reproduces in the course of its action, since the phenomenon is indefinitely reproducible in series, let us take a suspension of dysentery bacilli and inoculate it with an extremely minute quantity of the bacteriophage liquid, for example, with 1 ce. of a $10^{-7}$ dilution of a bacteriophage filtrate. Place this bacterium-bacteriophage mixture in the incubator at $37^{\circ} \mathrm{C}$. and from hour to hour throughout the incubation spread one drop upon an agar slant or an agar plate. As the bacteriophage begins to multiply in the course of its action the plaques should increase in number, and in fact, after these agar subcultures have been incubated for 24 hours, we will find the following:

The agar tube planted from the mixture, after incubation for but one hour, shows no plaques; it is covered by a normal growth of dysentery bacilli. The agar tube prepared one hour later, that is to say, after the trace of bacteriophage has been in contact with the bacilli for two hours, will show about a dozen plaques. The agar tube seeded after the action has progressed for 3 hours is covered by a bacillary growth through which about 100 plaques are scattered. The appearance of this agar tube is then approximately the same as that which had received a drop of the $10^{-5}$ dilution of the preceding experiment. But in the first experiment the suspension was spread upon agar immediately after the inoculation of $10^{-5} \mathrm{cc}$. of bacteriophage liquid, while here the $10^{-7}$ cc. of liquid bacteriophage added to the suspension has acted during 3 hours and has, therefore, had time to multiply.

The agar tube planted after the suspension had remained for 4 hours 
in the incubator showed only a few traces of bacterial culture, the appearance resembling the agar tube representing the $10^{-4}$ dilution of the preceding experiment. As for the agar tubes upon which a drop of the suspension was spread after the bacteriophage had acted for 5 hours or more, none presented any evidence of growth. They were sterile. In appearance these tubes were just the same as were those of the other experiment where the plantings had been derived from the suspensions inoculated with a large amount of bacteriophage liquid. It seems that the bacteriophage was so abundant that the entire surface of the agar forms but a single plaque, or, to express it more correctly, the plaques were so numerous that they touched one another leaving no room for bacterial growth.

In brief, then, to summarize all of this, bacteriophagy in a liquid medium reveals itself macroscopically by a dissolution of the bacteria contained in the medium, the latter becoming as clear as sterile, uninoculated bouillon. Upon agar bacteriophagy takes place in the same manner. The agar, at the point where the phenomenon occurs, is bare, without any sign of growth, having the same aspect as a sterile agar slant. In this condition it remains indefinitely (d'Herelle ${ }^{310,321}$ ).

To mention all the authors who have confirmed these facts would be to enumerate, in fact, almost all who have investigated the phenomenon of bacteriophagy. These facts have not been disputed, although, as we will see, the interpretations of the facts differ very materially.

These experiments show that the number of "plaques" on agar is related to the quantity of bacteriophage contained in the fluid, but they suggest, in addition, an hypothesis of extreme interest, and one worthy of test for it is burdened with matters of the greatest concern.

We have seen that when a very great number of bacteria and a very small quantity of the bacteriophage principle are combined and spread upon agar immediately after the inoculation of the bacteriophage, the result, after incubation, is a culture formed by the development of the bacteria distributed upon the agar, and this is spotted with bare areas, or plaques. The small amount of bacteriophage present appears, then, to concentrate its action at particular points (d'Herelle ${ }^{310}$ ). Can the physical state of the bacteriophage be "discontinuous"? Can this principle exist, in what up to this time we have called a bacteriophage liquid or a bacteriophage filtrate, in particulate form, as corpuscles in suspension? 


\section{THE BACTERIOPHAGE CORPUSCLE}

The hypothesis which I have formulated in answer to the above questions is easy to verify. The experiment described in the preceding section showed that bacteriophagy occurred in the bacillary suspension containing $10^{-10} \mathrm{cc}$. of bacteriophage filtrate, while the phenomenon failed to take place in the suspension with the next higher dilution, $10^{-11}$.

If the bacteriophage exists in corpuscles this result is at once explained. What happens would then be exactly of the same nature as that which takes place when tubes of sterile bouillon are planted with serial dilutions, ever more and more dilute, of a bacterial culture. The tube of bouillon which receives one drop of a sufficiently high dilution will be implanted with but a single bacterium, but it will yield, after incubation, a culture as abundant as that of another tube seeded with several million of the same bacteria. On the other hand, the tube which receives one drop of the next dilution, will not yield a culture, for the drop introduced did not contain even a single bacterium. Either there will be growth, or there will not be growth. And, in the same way, bacteriophagy either occurs, or it does not occur. There are no intermediate gradations. Let us find out if this is the case; if experiment confirms theory.

Prepare again the same series of dilutions, in tens, as those described in the experiment of the preceding section, employing the same bacteriophage filtrate, but instead of making the successive dilutions in suspensions of $B$. dysenteriae, make them in sterile bouillon. The procedure consists simply in introducing $1 \mathrm{cc}$. of bacteriophage filtrate into 9 cc. of sterile bouillon, removing $1 \mathrm{cc}$. of this first dilution and placing it into a second tube containing 9 cc., then $1 \mathrm{cc}$. of this second dilution carried over into the next tube, and so on up to the tenth dilution. This last tube will then contain $10 \mathrm{cc}$. of bouillon in which there will be $10^{-9}$ cc. of bacteriophage filtrate, and each cubic centimeter of this tenth dilution will therefore contain $10^{-10} \mathrm{cc}$. of the filtrate.

Suspend in a flask containing $90 \mathrm{cc}$. of sterile bouillon some B. dysenteriae removed from a young agar slant, in such a way that each cubic centimeter of the medium will contain about 100 million bacilli. Distribute this $90 \mathrm{cc}$. of bacterial suspension among 10 tubes, $9 \mathrm{cc}$. to each. To each tube add $1 \mathrm{cc}$. of the bacteriophage principle previously diluted to $10^{-10}$. We now have 10 tubes of suspension, and in each of them an added 1 cc. of a $10^{-10}$ dilution of the bacteriophage filtrate. Place the 10 tubes in the incubator at $37^{\circ} \mathrm{C}$. 
After 48 hours, 3 of these suspensions are clear (this is the average of 7 experiments of 10 tubes each, made with the same bacteriophage filtrate). The other 7 are turbid, and repeated control tests show that they contain normal cultures of dysentery bacilli. The result is, then, complete bacteriophagy in 3 suspensions and complete absence of bacteriophagy in the other 7 (d'Herelle $\left.{ }^{318}\right)$.

This experiment settles the question. If the bacteriophage was to be found in a state of solution in the $10^{-10}$ dilution, it is obvious that each of the $10 \mathrm{cc}$. of this dilution would have contained a tenth part of it, that is, none of the $10 \mathrm{cc}$. would have been favored. Each would have contained a like quantity, and all of the 10 suspensions, each receiving one of these $10 \mathrm{cc}$., would have behaved in the same manner; they would have been the seat of a comparable phenomenon. But this is not the case, as shown by the fact that 3 suspensions undergo bacteriophagy, while 7 do not. There is, then, among the 10 cc. distributed in equal amounts among the ten suspensions, 3 portions of 1 cc. each which contained the bacteriophage principle. In the other 7 it was lacking. This is an absolute proof that the bacteriophage exists in discontinuous form, that is to say, in corpuscular form.*

Experiments of this type always yield the same result, regardless of the bacterial species involved, provided the bacteriophage principle be very active. The single difference that may appear among the different experiments is due solely to the fact that different filtrates do not all contain the same number of bacteriophage corpuscles per cubic centimeter. Sometimes the dilution will be $10^{-7}$, sometimes $10^{-8}$, often $10^{-9}$ or $10^{-10}$, and rarely $10^{-11}$, which, when distributed in equal portions among a number of bacterial suspensions, will provoke bacteriophagy in a certain number of these suspensions, leaving others untouched.

Inasmuch as the concept of the corpuscular nature of the bacteriophage dominates entirely the study of this principle, I believe it wise to introduce the protocols of a few other experiments, taken at random from among more than fifty which I have performed, and which, uniformly, have given the same results as regards the demonstration of the corpuscular state.

* During my residence at the University of Leiden, in discussing this question with my colleague, Professor Einstein, he told me that, as a physicist, he would consider this experiment as demonstrating the discontinuity of the bacteriophage. I was very glad to see how this deservedly-famous mathematician evaluated my experimental demonstration, for I do not believe that there are a great many biological experiments whose nature satisfies a mathematician. 
The following protocol deals also with $B$. dysenteriae, but with another race of the bacteriophage. It has already been published ${ }^{342}$ in reply to the communications of several authors, Bordet among others, who have affirmed that the bacteriophage principle exists in a soluble form, despite the proof of corpuscular nature which I had given from the time of my first publication ${ }^{310}$ and which has been re-stated at different times. Incidentally, none of these authors have discussed this experiment; they have all passed it over in silence. In an effort to make the matter clear, and to settle the point at issue I have presented the data several times, and have even offered, in case any doubt remained, to actually demonstrate the phenomenon. No one accepted the challenge, indeed, no one has since even mentioned the question.

In this experiment, $10 \mathrm{cc}$. of a $10^{-10}$ dilution of a bacteriophage principle active for $B$. dysenteriae were distributed, 1 cc. to each tube, into 10 supensions of dysentery bacilli. In 5 of these suspensions bacteriophagy was complete; in the other 5 the phenomenon did not take place; they remained cloudy.

Additional experiments of the same nature, carried out with a principle extremely active for the Staphylococcus aureus follow.

Ten cubic centimeters of a $10^{-10}$ dilution of this bacteriophage principle were divided, $1 \mathrm{cc}$. to each tube, among 10 tubes each containing a suspension of 100 million staphylococci per cc. After 72 hours of incubation at $32^{\circ} \mathrm{C}$. all 10 suspensions were clear. Ten cc. of the $10^{-11}$ dilution of the same principle were likewise distributed among 10 tubes of the same staphylococcus suspension. After 72 hours at $32^{\circ}, 2$ were clear, 8 were turbid.

In the same way, $10 \mathrm{cc}$. of the $10^{-12}$ dilution were distributed among 10 tubes of the suspension. After 72 hours all were turbid.

Each of the two dissolved suspensions, obtained by adding the material of the $10^{-11}$ dilution, was in turn diluted to $10^{-11}$. The $10 \mathrm{cc}$. of each of these two dilutions were distributed into 10 tubes of staphylococcus suspension. After 72 hours incubation at $32^{\circ} \mathrm{C}$, , of the 10 suspensions to each of which was added 1 ce. of one of the dilutions, 1 was clear, 9 were turbid; for the second dilution, 2 were clear, 8 were cloudy.

All of the suspensions which remained turbid were subjected to numerous control examinations and all yielded normal cultures of the staphylococcus. These turbid suspensions have been filtered serially, and in no case did the filtrates cause the slightest reaction which could be ascribed to bacteriophagy. 
Identical experiments have been performed with bacteriophage principles active for $B$. coli, for B. typhosus, and for B. pestis.*

With $B$. coli, the last active dilution, for the race under investigation was $10^{-9}$. This dilution divided among 10 tubes of suspension caused bacteriophagy in 8 , the other 2 remaining unattacked.

And, finally, the dilution $10^{-8}$ of the bacteriophage principle acting upon B. pestis, distributed among 10 suspensions of this organism caused bacteriophagy in 5 , the other 5 remaining turbid. Control tests showed that the turbid tubes did not contain a trace of the bacteriophage principle, even after repeated filtrations.

We have then, a definite proof that the bacteriophage exists in the form of corpuscles.

If further proof of the corpuscular nature of the bacteriophage is desirable, it has certainly been provided by the very beautiful demonstration presented by Eijkman at the meeting of the Society of Bacteriologists of Holland. His experiment was based upon the well recognized fact that if a drop of a liquid, which contains a substance in solution, is allowed to evaporate slowly, when it is completely dry the substance present will be found evenly distributed over the entire surface previously occupied by the drop; while, on the contrary, if the substance in the liquid is insoluble, in the form of corpuscles, as the drying proceeds the phenomenon of capillarity becomes operative and the corpuscles are attracted toward the periphery, so that when the evaporation is finished, the substance will be found in a circle indicating the circumference of the area previously occupied by the drop.

Eijkman placed on an agar plate a very dilute suspension of bacteriophage corpuscles, and allowed the fluid to evaporate slowly. When desiccation was finished he covered the surface of the plate with a suspension of the susceptible bacterium. After incubation, the plate revealed an appropriate number of plaques, and these were arranged in a circle, representing the contour of the drop of fluid which had been originally placed on the agar. Thus, making application of the phenomenon of capillarity, Eijkman has afforded further proof of the corpuscular nature of the active principle in a bacteriophage filtrate.

From the fact of the corpuscular nature, it becomes evident that the important thing permitting bacteriophagy to take place is not the concentration of the bacteriophage principle in the suspension, but the

* The bacteriophage filtrates utilized in these experiments were from suspensions of 200 million bacteria per cubic centimeter rendered limpid by bacteriophagy. 
extent of the dilution of the bacteriophage principle which is inoculated into the suspension. In other words, with a Staphylo-bacteriophage still causing bacteriophagy in the tube diluted to $10^{-11}$ (the dilutions being made by tens in volumes of $10 \mathrm{cc}$.) and not causing bacteriophagy in the tube with $10^{-12}$ dilution, it is not because this last tube has a dilution of $10^{-12}$ that bacteriophagy does not result, but simply because it has not received a single bacteriophage corpuscle.

To further emphasize this point, let us take, as did Gratia and deKruif $^{272}$ a liter of a staphylococcus suspension* and let us inoculate it with 1 cc. of a $10^{-10}$ dilution of Staphylo-bacteriophage. The dilution of the principle in this liter of suspension will be $10^{-13}$, yet bacteriophagy occurs. The reason is plain. We know that $1 \mathrm{cc}$. of the $10^{-10}$ dilution causes bacteriophagy when added to $9 \mathrm{cc}$. of bacterial suspension, clearly showing that it contains at least one bacteriophage corpuscle. And it makes no difference whether this corpuscle is inoculated into 10 ce. or into 10 liters of suspension. The result is the same, the principle multiplies and causes bacteriophagy.

In brief, then, the concentration of the bacteriophage principle in a suspension where bacteriophagy is to take place is of little importance. For the phenomenon to occur the necessary and sufficient condition is that at least one bacteriophage corpuscle be introduced into the suspension of organisms.

In conclusion, let me repeat just once more, that to be conclusively demonstrable, these experiments must be made with a very active bacteriophage principle, that is, with one capable of causing a complete and permanent dissolution of the susceptible bacterium when 250 million bacteria per cubic centimeter are present. $\dagger$

* These authors performed the experiment with a Coli-bacteriophage. I have repeated it with a Staphylo-bacteriophage, and the results have been identical in principle. They could not be otherwise. These authors stated that their experiment was open to several interpretations. This rather vague conclusion appears to have satisfied them, for the suggested interpretations have not yet appeared. As a matter of fact but a single interpretation is possible. The bacteriophage exists in the form of corpuscles. The experiment of Gratia agrees with experiments that I had published on several occasions, and this is all that Gratia could have said.

$\dagger$ In studying the phenomena of the resistance of the bacteria to the bacteriophage we will see the reasons why the experiment demonstrating the corpuscular form must be carried out with an extremely active bacteriophage principle. 


\section{THE PLAQUE: A COLONY OF BACTERIOPHAGE CORPUSClES}

Each plaque, scattered throughout the bacterial layer upon agar, represents, then, the point where, during the spreading of the suspension, a bacteriophage corpuscle was deposited. This being the case, the number of plaques must be strictly proportional to the number of corpuscles inoculated into the bacterial suspension. This is what the experiments presented in the first section of this chapter have already shown. The number of plaques, on the other hand, should be entirely independent of the number of bacteria in the suspension. This is shown by the following experiment (d'Herelle ${ }^{326}$ ).

Ten tubes, each containing $10 \mathrm{cc}$. of a suspension of Shiga dysentery bacilli in different concentrations - 100, 200, 300, 400, 500, 600, 700, 800, 900, and 1000 million bacilli per cc.,--are inoculated with a constant quantity of a bacteriophage filtrate, $5 \times 10^{-6}$. After shaking, 0.02 cc. is removed from each of the tubes and is carefully spread upon a corresponding agar slant. After incubation, each of the 10 slants shows a culture layer of bacteria, studded with plaques, and the number of plaques is practically the same in all of the tubes (the actual figures being-19, 25, 20, 21, 19, 19, 22, 20, 16, and 18).

Let us repeat this experiment, reversing the order of the factors. Let us use 10 suspensions of Shiga bacilli, all of the same concentration, 200 million bacilli per cubic centimeter, and let us inoculate these suspensions with an increasing quantity of a dilution of bacteriophage filtrate, in such a way that the first tube will receive a millionth of a cc., the second a 900 thousandth, the third an 800 thousandth, the fourth a 700 thousandth, and so on up to the tenth tube, which will get a 100 thousandth. Shake the tubes vigorously, and spread $0.02 \mathrm{cc}$. from each upon an agar slant. After incubation each of the agar slants has a layer of bacterial growth studded with plaques, but the number of the plaques varies with the quantity of bacteriophage filtrate inoculated into the tubes. Practically the proportions are: 1:10, 1:9, 1:8, 1:7, etc., up to $0.5: 1$; the actual numbers observed being, $4,4,5,6,8,10,10$, $14,19,42$ * $^{*}$

I have repeated this experiment with different races of the bacterio-

* The differences between the numbers observed and the numbers theoretically calculated are of the same magnitude as those which we find in counting bacterial colonies plated on agar from serial dilutions of a suspension. The numbers observed approach more and more closely to the calculated numbers as the number of tubes counted increases and average figures are obtained. 
phage, acting upon different bacterial species. It is needless to detail them here, for they are all comparable to the one described above.

The experiments all agree in showing that each plaque originates in a bacteriophage corpuscle which dissolves the bacteria in its environment. But this plaque occupies a certain area. With certain races particularly active for the dysentery bacillus each plaque may attain a diameter of $8 \mathrm{~mm}$., all of the bacteria found within a radius of $4 \mathrm{~mm}$. of the corpuscle being destroyed. Is this dissolution the result of a distant action of the corpuscle deposited on the agar at the time of spreading, or, does the original corpuscle multiply at the expense of the bacterial bodies as it proceeds with their dissolution? It is easy to subject this question to experimental proof and determine which of the two possibilities accords with the facts.

Touch the margin of a plaque with a platinum wire and then wash it off in a tube of sterile bouillon. Prepare also 10 tubes of a bacterial suspension susceptible to the action of the bacteriophage. Inoculate each of these tubes with a drop of the bouillon in which the needle has been washed off. After incubation, we will find that the 10 suspensions have undergone bacteriophagy. There must have been, then, at least one bacteriophage corpuscle in each drop of the bouillon with which these suspensions were inoculated. This proves, therefore, that the surface of the plaque is covered by bacteriophage corpuseles, and this, in turn, means that the initial corpuscle must have multiplied.

The plaque represents, then, a colony of bacteriophage corpuscles, the issue of the original corpuscle which was the origin of the plaque. Upon a solid medium, as in a liquid medium, the dissolution of the bacteria is accompanied by a multiplication of the bacteriophage corpuscles.

\section{CONDITIONS ESSENTIAL FOR PLAQUE FORMATION}

Before considering the characteristics of agar cultures of the bacteriophage corpuscles, let us note the conditions of the medium most favorable for their development.

First, let us state, but it is not necessary to emphasize this, that all of the conditions, as discussed in the preceding Chapter which bear upon the nutritive qualities of liquid media for the bacteria subjected to the action of the bacteriophage, such as the reaction $(\mathrm{pH})$, are entirely applicable to agar media. On solid media, as in liquid media, the state of the bacteria is important. Young bacteria are most readily attacked, and the critical period is the moment of division.

With regard to the consistency of the medium, I have shown ${ }^{321}$ that 
Martin's alkaline bouillon ( $\mathrm{pH} 7.6$ to 7.8) containing 2 per cent of agar serves perfectly well for the formation of plaques. More recently Nakamura ${ }^{4 ! 8}$ has studied comparatively the formation of plaques upon media containing different concentrations of agar. He found that the plaques were the larger as the concentration of agar was reduced. The most favorable medium was made up of bouillon containing 0.4 per cent of agar. Practically, in view of the semi-fluid state of such a medium which makes it rather difficult to work with, a medium composed of bouillon with $0 . S$ to 1 per cent of agar is the most convenient.

What is the cause of this reduction in the size of the plaque proportionate to the increase in the concentration of agar? Certainly it can not be, as Nakamura has suggested, a result of the inhibitory action of the agar colloid, for if this were the case it would hardly be clear why a medium containing 1 per cent would be very favorable, since the quantity of agar colloid here is sufficient to give the medium the character of a solid. Furthermore the fact that bacteriophagy takes place in a liquid medium in the presence of negative colloids, such as colloidal silver, and even in the presence of gelatin up to a certain concentration, shows indeed that this is not the real reason.

The stronger and stronger inhibition of bacteriophagy in media containing increasing quantities of agar is certainly related to the consistency of the substratum.

Products resulting from the dissolution of the bacteria exercise an inhibiting action upon the phenomenon, as is shown by the arrest of bacteriophagy in a liquid medium when a certain number of bacterial cells have been dissolved. Furthermore, it seems to be a general biological law that the accumulation of the products formed during a reaction inhibits a biological process. Such products not only limit the growth of organisms, but also impair the action of ferments. Whatever may be the nature of the bacteriophage corpuscle, an inhibition of the action which it causes, brought about through the accumulation in the medium of the products resulting from this action, is, then, in conformity with all that is known. It might even be said that if such an inhibition did not occur it would be unique, a new fact.

From this it is clear that the higher the amount of agar in the substratum the greater is its consistency, and consequently, the slower will be the diffusion into the substratum of the products formed during the dissolution of the bacterial cells. If the consistency is such that these products accumulate in the surface layer of the medium, there is, from the beginning, an arrest of the phenomenon, and, as a result, a reduction in the area of the plaque. Even its formation may be prevented. 
The following experiments permit of no doubt on this question.

Bail, ${ }^{45}$ Doerr and Berger, ${ }^{181}$ and Nakamura ${ }^{468}$ have observed that plaques do not form on gelatin, and they have invoked the intervention of colloidal reactions to explain this fact. The deduction of these authors is absolutely correct, and the following experiments provide the true explanation for the inhibition which they observed.

Prepare three series of Petri dishes, as follows: (a) containing an agar medium (bouillon with 2 per cent agar); $(b)$ containing a gelatin medium (bouillon with 15 per cent gelatin; $(c)$ containing agar (the 2 per cent agar of $(a)$ ) to a depth of $8 \mathrm{~mm}$. Immediately after the introduction of the agar the dishes of this series are placed in a horizontal position (obtained by means of levelling screws) and the agar is allowed to solidify. They are then placed in an incubator at $45^{\circ} \mathrm{C}$. to warm them somewhat, and over the surface of the agar is poured a quantity of gelatin (the 15 per cent gelatin mentioned above, in $(b)$ ), melted and cooled to about $60^{\circ} \mathrm{C}$., in such a way that by tipping the dish in all directions the entire surface is covered by a thin layer. Adjust the dishes with the levelling device so that they are perfectly horizontal and allow the medium to harden in the ice-box. Prepared in this way these dishes will contain a layer of agar upon which is superimposed a thin layer of gelatin. When planted the material is distributed solely upon the gelatin, and this medium will differ in no way from that present in the dishes containing gelatin alone (series $b$, above). The only difference will be that the $c$ series will have a substratum of agar into which those products resulting from reactions taking place on the surface may diffuse.

When these Petri dishes are thus ready, take 4 tubes, each containing a suspension of Shiga bacilli, 250 million per cubic centimeter. Inoculate the first with $0.1 \mathrm{cc}$. of a Shiga-bacteriophage; the second with 0.1 cc. of the first: the third with 0.1 cc. of suspension 2 ; and the fourth with $0.1 \mathrm{cc}$. of number 3 .

With these four suspensions the Petri dishes are implanted, 0.05 cc. of each of the individual suspensions being spread uniformly over the surface of a dish. When the results are read after 4 days, we find:

\section{Agar plates held at $37^{\circ} \mathrm{C}$.}

Suspension 1... Surface sterile

Suspension 2... A few scattered traces of growth

Suspension 3...Fragments of growth

Suspension 4 ... About 50 plaques, having a diameter of about $5 \mathrm{~mm}$. 


\section{Agar plates held at $30^{\circ} \mathrm{C}$.}

Suspension 1... Sterile

Suspension 2...Traces of growth

Suspension 3...Fragments of growth

Suspension 4 ... About 50 plaques, having a diameter of about $7 \mathrm{~mm}$.

III. Agar plates held at $18^{\circ} \mathrm{C}$.

Suspension 1... Sterile

Suspension 2... Sterile

Suspension 3... Scattered traces of growth

Suspension $4 \ldots$ Confluent plaques (about 50), with a diameter of about 13 $\mathrm{mm}$.

IV. Gelatin plates held at $18^{\circ} \mathrm{C}$.

Suspension 1... Continuous bacterial layer, absolutely like a normal culture Suspension $2 \ldots$ Continuous bacterial layer

Suspension $3 \ldots$ Continuous bacterial layer

Suspension 4 ... Continuous bacterial layer

\section{Gelatin plates with a substratum of agar}

Suspension 1...Sterile

Suspension 2... Sterile

Suspension 3...Culture débris

Suspension 4 ...About 50 plaques, with diameters of 3 to $4 \mathrm{~mm}$.

This experiment, repeated upon three different occasions, has always given similar results. By varying the depth of the gelatin layer I have observed that the size of the plaques varies inversely with the depth of the layer. When the layer was about $3 \mathrm{~mm}$. in depth the bacterial culture appeared normal.

Obviously it is not the gelatin as such which interferes with the process of bacteriophagy. It is simply because the gelatin is but slightly permeable to the products arising in the course of the phenomenon that an inhibitory effect is manifested. When the layer is very thin and is superimposed upon a permeable substratum bacteriophagy occurs just as it does on agar.

The experiment also shows some points with regard to the effect of temperature.

The dimensions of the plaque resulting, one might say, from a "race" between the rate of multiplication of the bacterium and that of the bacteriophage, it follows that at a given temperature the one or the other may be "handicapped." On the other hand, experiment definitely 
shows that the bacteriophage corpuscle is able, on a solid medium, to dissolve bacteria only if they are found in an extremely thin layer on the surface of the substratum. This is precisely the reason that the plaque, already formed when the bacterial growth has hardly become perceptible, no longer increases in size; the surrounding bacterial layer has become too thick.

To summarize all of this, the formation of the plaque, and its extent if its formation is possible, depends upon a whole series of factors, of which two appear to be; first, the greater or less facility with which the products resulting from the action diffuse into the substratum, and second, the respective powers of multiplication of the bacteriophage corpuscle and of the bacterium at the temperature at which the phenomenon takes place.

\section{THE CHARACTERS OF PLAQUES}

It is of interest to note further some of the characters of the colonies of the bacteriophage corpuscle on agar. Let us take, as an example, a very active race of Shiga-bacteriophage, although any other race could be taken, acting upon any bacterial species whatever, for in all cases, the formation and the behavior of the plaques are identical. The only variant is the extent; the diameter of the plaque.

Observation shows that in general, the size of the plaques formed with different bacterial species is the smaller the more rapidly the bacterium grows upon agar and the thicker the layer of growth becomes. Even with extremely active races of the Staphylo-bacteriophage the plaques are always small; their diameter (for the races studied) does not exceed $1.5 \mathrm{~mm}$. The plaque of the Shiga-bacteriophage, on the contrary, may reach a diameter of $8 \mathrm{~mm}$. But even here uniformity does not obtain, for against a single bacterial species the size of the plaque varies. With all other conditions the same, the size of the plaque is related to the race of the bacteriophage which is acting. We will see the cause for this in the next chapter.

When the surface of the agar remains bare because of the large number of bacteriophagous corpuscles and maintains this appearance indefinitely it has become unsuited for the cultivation of the Shiga bacillus. When inoculated at such a time with a culture of this bacillus, even in a very abundant sowing, not the slightest development can be detected. The medium is, however, normal for another bacterium. If inoculated with the cholera vibrio, for example, the growth will be as luxuriant as if planted upon fresh medium. Hence, if $B$. dysenteriae 
Shiga does not grow it is only because the bacteriophagous corpuscles remain on the surface of the agar and exercise their dissolving action on the bacteria deposited thereon. This is readily confirmed. If we take a tube of agar which has remained apparently sterile after having been inoculated with a suspension of the bacteria containing a bacteriophagous filtrate, and if the surface of the medium in such a tube is washed with a few drops of sterile bouillon and to this is added a fresh suspension of bacteria, this suspension will be dissolved within a few hours.

It sometimes happens, especially when using agar somewhat dried out, that a few colonies of Shiga are obtained, always located at the extreme edge of the layer of agar. We will return to this extremely interesting particular in the discussion of secondary cultures.

If, instead of spreading the bacteriophage filtrate over the entire surface the corpuscles are deposited in limited areas - and this is readily accomplished by placing drops of filtrate on the sterile surface of a tube of agar, or again, by drawing lines over the surface with a platinum loop dipped in the suspension of bacteriophage, and after the tubes have remained inclined for a few hours in the incubator to secure dryingwe find that the areas impregnated with the bacteriophagous filtrate remain free of Shiga bacilli, but that these organisms grow, on the contrary, perfectly well on the parts not covered by the bacteriophage.

When in the suspension planted upon agar the number of bacilli is infinitely great and the number of the corpuscles is sufficiently small, the bacteriophage principle as individual units is distributed over the surface of the agar, and under such circumstances the bacterial layer will appear studded with apparently sterile areas. These areas, or plaques, have a circular form varying in size from those spoken of as "pin-point" up to those with a diameter of $8 \mathrm{~mm}$. The plaques are in general of the greatest extent when the bacterial suspension is somewhat weak although sufficiently concentrated to give a continuous layer of growth rather than isolated colonies. On such a tube the areas are larger as the subjacent medium becomes thicker, that is, toward the bottom of the tube. Upon a Petri dish, where the agar layer is of essentially the same thickness throughout, all of the plaques of a given culture are of approximately the same diameter. As will be seen, the area of the plaque bears a relationship to the virulence of the bacteriophage which causes it.

If a tube or plate presenting plaques is held in the incubator at $37^{\circ} \mathrm{C}$., or at an entirely different temperature, no change occurs in the plaques; 
their diameter remains indefinitely what it was at first. They are never covered or encroached upon by the bacterial culture. At no time does there exist within the extent of the plaque, whatever its size may be, microscopically visible bacterial cells. The plaque is always rigorously sterile.

As soon as the culture is well developed, as after 18 to 24 hours of incubation, if the centre of such a plaque is touched with a platinum wire and this is immersed in a culture of Shiga bacilli the bacteriophage develops in this suspension and the latter is dissolved after a few hours. The plaque, although sterile, is not ultrasterile; it is in fact a colony of the bacteriophage corpuscles.

Furthermore, if a trace of the bacillary growth at the periphery of a plaque is taken with a platinum wire and seeded on agar it remains sterile and inoculation into a bacterial culture shows that the bacteriophage is present there also. But when the bacillary layer is taken, not at the immediate edge of the area, but at a distance of two millimeters from it, for example, and planted, the tubes show the growth of a normal culture. The bacteriophage is not found.

If the culture showing the plaques is returned to the incubator and the tests are repeated three or four days later, that is, culturing the bacillary growth at a distance of two millimeters from a plaque onto agar and into a suspension it will be found that the bacteriophage is there present at that time. The bacteriophage has, therefore, gradually invaded the bacillary layer. This invasion is always slow-proceeding more and more slowly as time progresses - so that the ring invaded, even after several months, amounts to a zone but a few millimeters wide. Beyond the limits of this zone the Shiga organisms remain cultivable just as long as they do in a normal control culture without the bacteriophage.

The question immediately arises as to why the bacteriophage does not invade the entire layer of bacterial growth. For this there are two reasons. The bacteriophage attacks the bacterial cell most readily when the bacterium is young. When placed upon agar the bacteriophagous corpuscles find themselves located in the immediate vicinity of bacilli which reproduce actively as soon as they are deposited upon a nutrient medium. They find then, within their range, very young bacilli distributed in a very thin layer over the agar. Dissolution is thus possible and the apparent sterility of the plaque results. But beyond this zone invaded by the bacteriophage during the first few hours the bacilli develop freely forming a layer of increasing thickness 
comprised of organisms of increasing age. In other words, a thicker and thicker layer of bacilli always becoming more and more resistant to dissolution develops. This can be readily demonstrated by direct experimental proof.

If the agar surface in a Petri dish is heavily seeded with a Shiga culture and at some point on this a drop of the bacteriophage filtrate is placed, and after a three-hour incubation period another drop of the bacteriophage is placed on the surface and this same process repeated after six, twelve and twenty hours, with continuous incubation of the plate during the intervals, it will be found fifteen hours later that the areas upon which the first three drops were placed have remained sterile-no bacillary growth has taken place. At the point where the fourth drop was placed, that is, after the culture had been incubated for twelve hours, there is a thin layer of growth composed of dead bacilli. The area where the drop of bacteriophage was placed after twenty hours presents an appearance practically normal. These five spots, then, represent the diverse aspects of an isolated colony of the bacteriophage, as from the centre to the periphery.

The second reason is of a more general nature, representing a phenomenon common to the majority of cultivable organisms. The colonies of the bacteriophage act absolutely like colonies of those bacteria which, except for organisms such as $B$. proteus, never progressively invade the surface of solid media. Thus, if the Shiga bacillus is planted upon agar in an amount suitable to yield isolated colonies, after 18 to 24 hours, each colony will be from two to four millimeters in diameter, the largest colonies to be found at the points where the medium has the greatest depth, that is, toward the bottom of the tube. Such colonies increase in size but very slowly, always more and more slowly as time progresses, and even after two months, the zone of increase will not be greater than a few millimeters. From the bacteriological point of view it is not peculiar, as has been suggested, that the bacteriophage does not invade the entire bacterial layer. Far from being dissimilar to other cultivable organisms, an isolated colony of the bacteriophage behaves exactly like a colony of bacteria.

Why does the bacterial colony fail to increase in size and invade the entire surface of the medium? Because the soluble substances resulting from the vital activity of the bacteria diffuse into the agar and these substances constitute a true specific antiseptic which limits the growth. The medium is "vaccinated" around the colony. The deeper the agar layer, or the farther the colonies are separated, the greater the volume 
of substratum capable of diluting this antiseptic substance, and the larger will be the colony. The situation is precisely the same with the bacteriophage; the more scattered the colonies and the deeper the substratum, the greater the diameter.

This is also the reason why the plaques are larger, other conditions being equal, when the medium contains less agar. We know that diffusibility in an agar substratum is diminished as the percentage of agar in the medium is increased.

\section{6. enUMeration of baCteriophage CORPUSCles*}

Since we have now presented the evidence proving the corpuscular nature of the bacteriophage we will no longer make use of such vague expressions as bacteriophage "liquid," "fluid," or "filtrate," but will employ the more precise term "suspension of bacteriophage corpuscles," or even more simply, "bacteriophage suspension." A bacterial suspension which has become limpid because the bacteria have disappeared through bacteriophagy, and in which, on the other hand, the bacteriophage corpuscles have multiplied from the beginning, has, then, become a "bacteriophage corpuscle suspension."

The evidence adduced above also shows that each plaque represents a colony of bacteriophage corpusclest and experiment shows that this colony originates in a single corpuscle deposited on the agar in the midst of the bacteria.

Obviously, this finding provides a method for the enumeration of the bacteriophage corpuscles to be found in a suspension (d'Herelle ${ }^{310,315,321}$ ).

The experiments described in a preceding section suggest, moreover, a second method. Since the limiting dilution of a suspension of bacteriophage distributed in equal fractions in bacterial suspensions, causes bacteriophagy in a certain number of them, the others remaining unattacked, it follows that each bacteriophaged suspension must have been

* We are considering here only the question of the enumeration of the corpuscles, not that which might be called the "titration" of the bacteriophage; a question much more complex, the study of which is reserved for a later chapter.

$\dagger$ No concept of the nature of the corpuscle is here involved. This is a question which we will approach when, having accomplished the study of bacteriophagy, we will consider the characters of this corpuscle. The word "colony" is employed because it signifies a "collection of individuals of the same type." As there is on agar a collection of corpuscles of the same type, having for their origin a corpuscle which has multiplied, it is evidently a colony, without predicating whether the corpuseles are living or not. 
inoculated with a single corpuscle (d'Herelle ${ }^{310,318,321}$ ). A simple calculation gives then the number of corpuscles to each cubic centimeter of the suspension. And these two methods of counting should agree with each other, yielding comparable results.

Applied to a specific case these two methods give the following values. A suspension of the Shiga-bacteriophage is titrated by successive dilutions, as in the experiments described previously. The last active dilution is found to be $10^{-10}$. Of this $10^{-10}$ dilution $10 \mathrm{cc}$. are distributed, in amounts of $1 \mathrm{cc}$. each, into 10 suspensions of Shiga bacilli. After incubation, we find that 5 of these suspensions have undergone bacteriophagy, the other 5 have not.

There were then, 5 bacteriophage corpuscles in the $10 \mathrm{cc}$. of the $10^{-10}$ dilution. These 5 corpuscles must have been introduced into this $10^{-10}$ dilution with the cubic centimeter of the $10^{-9}$ dilution which was combined with $9 \mathrm{cc}$. of bouillon to yield the $10^{-10}$ dilution. Manifestly, the $10 \mathrm{cc}$. of the $10^{-9}$ dilution contained 50 corpuscles. Continuing the same reasoning for the successive decreasing dilutions to the point of the undiluted suspension, we find that the latter contained 5,000 million bacteriophage corpuscles per cubic centimeter.*

Let us now take the $10^{-6}$ dilution of the series of dilutions above. Inoculate a normal suspension of dysentery bacilli with $1 \mathrm{cc}$. of this dilution. The suspension will then contain the same quantitiy of bacteriophage as the $10^{-7}$ dilution of the original bacteriophage. Spread immediately, on 20 agar tubes, one drop $(0.05 \mathrm{cc}$.) to each tube, $1 \mathrm{cc}$. of the inoculated suspension. After incubation we find that each agar

* More simply, the $10^{-9}$ dilution contained 5 corpuscles per cubic centimeter. The initial suspension undiluted contained then $5 \times 10^{9}$, or 5000 million per cubic centimeter.

The method for counting is the same, indeed, as that which was devised by Miquel for counting bacteris and this in turn was patterned after the procedure followed by Pasteur for the purification of bacterial cultures (the dilution method). Insofar as the bacteria are concerned, every tule of the medium which receives at least one bacterium gives a culture and every tube which does not receive one remains sterile. Witb the bacteriophage every bacterial suspension which receives at least one corpuscle undergoes bacteriophagy while the suspensions which receive none fail to reveal the phenomenon.

Obviously it is possible that the tubes which show a growth in the case of the bacterial counts or those which show bacteriophagy in the case of bacteriophage corpuscle counts may not have received a single element but, indeed, some of them may have received two or even more. The result obtained is, therefore, a minimum, a minimum which approaches more closely to the true number as the count is based upon a large number of tubes. 
tube is covered by a growth of $B$. dysenteriae spotted with plaques. The total number of plaques is 478 , distributed among the tubes in the following way:

$\begin{aligned} 1 \text { slant shows } 19 \text { plaques } & =19 \\ 3 \text { slants show } 20 \text { plaques } & =60 \\ 3 \text { slants show } 21 \text { plaques } & =63 \\ 4 \text { slants show } 23 \text { plaques } & =92 \\ 1 \text { slant shows } 24 \text { plaques } & =24 \\ 1 \text { slant shows } 25 \text { plaques } & =25 \\ 1 \text { slant shows } 26 \text { plaques } & =26 \\ 4 \text { slants show } 27 \text { plaques } & =108 \\ 1 \text { slant shows } 29 \text { plaques } & =29 \\ 1 \text { slant shows } 32 \text { plaques } & =32 \\ \text { Total } & =478\end{aligned}$

The 478 "formers of plaques" were found in $1 \mathrm{cc}$. of the $10^{-7}$ dilution. Calculation indicates that the initial suspension of the bacteriophage contained $478 \times 10^{7}$, or $4.78 \times 10^{9}$.

The second procedure, therefore, shows that each cubic centimeter of the undiluted suspension contained 4780 millions of "plaque formers," whereas the dilution method indicated that this suspension contained 5000 million corpuscles per cubic centimeter. To all intents and purposes these two figures agree, hence we can conclude that each plaque had its origin in a single bacteriophage corpuscle and that the method of counting the plaques forms a means of enumeration of the bacteriophage corpuscles present in a suspension.

I have repeated this experiment with two different races of Staphylobacteriophage. It is unnecessary to give the protocols of these experiments, since it would prolong this section needlessly, but both of them were comparable throughout with that which has just been described. I will only say that the undiluted suspension (that is, a suspension of staphylococci, 250 million per cubic centimeter, bacteriophaged and thus transformed into a suspension of bacteriophage corpuscles) of the more powerful race contained 200,000 million $\left(2 \times 10^{11}\right)$ corpuscles per cubic centimeter according to the dilution method and 121,000 million $\left(1.21 \times 10^{11}\right)$ according to the plaque method. For the second race the number of corpuscles was $6 \times 10^{9}$ by the first method and $8.1 \times 10^{9}$ by the second.*

* It is quite incorrect to assume that all suspensions of the same race of bacteriophage always contain the same number of corpuscles, indeed, the case is quite the contrary. The number of corpuscles present after bacteriophagy depends, as we will see in the following chapter, upon the number of bacteria bacteriophaged. The above experiments are given simply to show the agreement between the two methods of enumerating the corpuscles. 
In general, these two methods for the enumeration of bacteriophage corpuscles do not differ materially from those employed for counting the living bacteria present in a culture. The single difference resides in the fact that, for the bacteria the dilutions are planted in sterile bouillon or upon sterile agar, while for the bacteriophage corpuscles the counts can only be made in the presence of living bacterial cells. It could not be otherwise, for although the bacterium utilizes for its development the nutritive substances present in the medium itself, the bacteriophage corpuscle multiplies only at the expense of the living bacterium, which constitutes the medium within which it multiplies.

\section{RÉSUMÉ}

When a suspension of susceptible bacteria, inoculated with a relatively large quantity of bacteriophage, is spread upon an agar medium no further growth results: the medium remains bare indefinitely. Furthermore, the surface of this agar remains permanently unsuited to the development of these susceptible organisms, but if it be seeded with a bacterial species insusceptible to the bacteriophage involved a normal culture is obtained, just as though it had been spread upon sterile agar (d'Herelle, ${ }^{310}, 312,321$ ).

A suspension, or a culture, of a susceptible bacterium, inoculated with a minute quantity of the bacteriophage principle, spread upon agar gives a layer of bacterial growth studded with bare spots, circular in form, where the agar is free of all traces of growth. These bare spots or "plaques" once formed are unchanging; they do not increase in size nor are they ever covered by the surrounding bacterial growth. The area occupied by the plaque has become unsuited to the growth of susceptible organisms (d'Herelle ${ }^{310}, 312,321$ ).

The number of plaques is in direct proportion to the quantity of bacteriophage filtrate inoculated into the suspension spread upon the agar (d'Herelle ${ }^{310,321}$ ).

These facts suggest the hypothesis that the bacteriophage exists in the physical state of corpuscles (d'Herelle ${ }^{310}$ ).

The corpuscular state is demonstrated by the fact that dilutions at the limit of activity distributed in equal amounts among different suspensions of the susceptible bacterium induce bacteriophagy in certain of these suspensions while the phenomenon fails to take place in others. Either bacteriophagy occurs, or it does not occur; there is no intermediary stage. This proves, not only from the biological point of view, but from the point of view of physics as well, that the bacteriophage is 
found in the liquid in a "discontinuous" state, that is to say, in the form of corpuscles (d'Herelle ${ }^{318}$ ).

A bacteriophage filtrate is then, simply a suspension of bacteriophage corpuseles (d'Herelle ${ }^{310}$ ).

Each plaque is a colony of bacteriophage corpuseles derived from a single corpuscle (d'Herelle $\left.{ }^{310},{ }^{318}\right)$.

The phenomenon of bacteriophagy leads to the same results upon solid media as in fluid media. There is a dissolution of the bacterial cells and a multiplication of the bacteriophage corpuscles (d'Herelle ${ }^{310,}{ }^{321}$ ).

As a result of the mode of action of the bacteriophage corpuscles, the enumeration of the corpuscles present in a suspension may be accomplished in two different ways, by the method of dilutions and by that of counting the plaques (d'Herelle, ${ }^{310}, 312,318$ and following). 


\section{CHAPTER III}

\section{The Mechanism of Bacteriophagy}

\section{THE CORPUSCLE: OBLIGATORY BACTERIOPHAGE}

Whatever the nature of the medium, in the absence of a susceptible bacterium the bacteriophage corpuscles do not multiply. Nor is multiplication to be observed even though the medium be favorable for the phenomenon if the corpuscles are placed in contact with killed bacterial cells. The method of killing the bacteria is without significance: the reaction does not take place with bacteria killed by aging, heat, chloroform, essences of thyme, cinnamon or mustard, by alcohol, mercuric chloride, or by carbolic, sulfuric or hydrochloric acids.*

The living bacterial cell is indispensible for the multiplication of the bacteriophage corpusele ( $\left.\mathrm{d}^{\prime} \mathrm{Herell}^{310}\right)$. Indeed, it is even essential that the living cell be "normal," that is, not exposed to the action of substances which may modify its characters appreciably even though they do not kill it. It is unnecessary to repeat the experiments bearing upon this point, since they have been considered in Chapter I in the section entitled "Influence of Chemical Conditions on the Phenomenon of Bacteriophagy."

It can readily be shown that the phenomenon fails to take place solely because of the disturbing influence of the medium upon the bacterium, for, if the unattacked bacteria are separated by centrifugation from the medium and suspended in a pure bouillon they undergo bacteriophagy. With such organisms the reaction is, however, more or less delayed; a fact entirely in keeping with the idea that the "abnormal" bacteria, now multiplying in a pure medium, become again "normal" and when this happens, become subject to the attack of the bacteriophage.

What is the nature of this anomalous condition which renders the bacteria unattackable? We will be able to interpret and reply to this

* This applies only to the multiplication of the bacteriophage corpuscles. A dissolution of the bacterial cells may be effected even if the bacteria are dead. But this last reaction is not in reality bacteriophagy. We will return to this fact, since in several instances the dissolution of dead bacterial cells has led to the erroneous conclusion that bacteriophagy had taken place. ${ }^{321,348}$ 
question when we have studied the effect of an antibacterial serum upon the phenomenon of bacteriophagy. Here let us simply say that it is probable that this anomaly consists in a modification of the surface tension of the bacterial cell.

We have seen above that the bacteriophage corpuscle multiplies only in the presence of living and normal bacteria. While this is in general true, it is likewise true that under very particular conditions, as observed by Wollman, ${ }^{632}$ a certain amount of bacteriophage development appears to take place in the absence of intact bacterial cells. This investigator prepared a series of collodion sacs of different densities, thus regulating the permeability.* After sterilization he filled the sac, having a capacity of $6 \mathrm{cc}$, with a bouillon implanted with ShigaKruse bacilli. In the outer tube, within which the sac was suspended, he placed $20 \mathrm{cc}$. of bouillon containing bacteriophage filtrate. The concentration of bacteriophage in this external fluid was such that when $10 \mathrm{cc}$. of a suspension of $B$. dysenteriae was inoculated with 1 drop of the fluid and 1 drop of the resulting mixture was spread upon agar but two plaques would form.

In this way he prepared a series of collodion sacs of increasing permeability, all arranged in the same manner, with a suspension of $B$. dysenteriae within the sac and a bouillon suspension of the bacteriophage in the tube into which the sac was immersed.

After incubation he observed the following:

Tery permeable sacs. It will be recalled that, as I had shown previously, ${ }^{312}$ the bacteriophage corpuscle passes through collodion membranes which are sufficiently permeable to permit the passage of the molecule (perhaps it would be better to say micella) of serum albumin. In this experiment of Wollman the same fact appears, for he found that the corpuscles passed through the permeable membranes, penetrating into the sac. There coming into contact with the dysentery bacilli they caused bacteriophagy, as would be expected.

Less permeable saes. With these sacs the bacilary culture within the sac remained normal, but the number of corpuscles inoculated into the bouillon surrounding the sac increased. A preliminary test showed, as we have seen, that a drop of the bouillon outside gave two plaques on agar. After incubation, a test conducted in the same manner with the same amounts, yielded from 20 to 30 plaques. There were, then, from 10 to 15 times as many corpuscles after incubation as be-

* For the methods of preparation and arrangement of collodion sacs see the section "Ultrafiltration" in the introduction. 
fore. Nevertheless, no bacteria had been introduced into the bouillon and obviously none of the bacteria present in the sac had been able to reach the external medium, since the bacteriophage corpuscles, themselves much smaller than the bacteria, did not pass through the sac. This last fact is the more certain since the culture in the sac did not undergo bacteriophagy.

Sacs of least permeability. Here no change took place after incubation. The number of corpuscles in the bouillon did not increase nor did the bacterial culture within the sac show any evidence of bacteriophagy.*

Wollman concluded that even though bacteria were not present the bacteriophage commenced to develop because of the presence of certain diffusible bacillary products. These substances passed through the membranes impermeable to the bacteriophage corpuscles. He compared this behavior of the bacteriophage corpuscle to that of Dictyostelium mucoroides. With this, a Myxomycete, Pinoy $\dagger$ has shown, by means of a technic comparable to that of Wollman, that development starts because of the presence of diffiusible products of $B$. fuorescens, although normally, the Myxomycete develops only in the presence of living bacteria.

Asheshov has personally told me that he has repeated Wollman's experiment with the same results.

It is necessary, then, to conclude that the multiplication of bacteriophage corpuscles can take place, at least to a certain degree, in the absence of the bacterial cell, and that for this development the corpuscles utilizes certain diffusible products present in the culture of susceptible bacteria.

In any case, it is certain that these diffusible products can be utilized by the bacteriophage corpuscle only immediately after their derivation from the bacterium, for on several occasions, employing a variety of procedures, I have tried to cultivate the corpuscles in filtered bacterial cultures or in autolysates, always unsuccessfully. This fully agrees with further observations made by Pinoy on the Myxomycete, which, although developing to some extent in bacterial products diffusing through a collodion membrane never multiplies in autolysates or in culture filtrates.

* This last statement is based upon a verbal communication; it does not appear in the paper of Wollman. ${ }^{632}$

$\dagger$ Pinoy, E.-Rôle des bactéries dans le développement de certaines Myxomycètes. Ann. Inst. Pasteur, 1907, 21, 622; 686. 


\section{FiXATION OF THE BACTERIOPHAGE CORPUSCle}

In view of the fact that it is possible to enumerate the bacteriophage corpuscles present in a liquid, we can now go somewhat further into the mechanism of the phenomenon of bacteriophagy.

One of the first questions to consider bears upon the sphere of activity of the bacteriophage. If, in an appropriate liquid, we combine bacteriophage corpuscles and susceptible bacteria, do the corpuscles act at a distance or must they first come into immediate contact with the bacterial cell?

The following experiment (d'Herelle ${ }^{321}$ ) answers this question.

The following suspensions are prepared:

1. One hundred cubic centimeters of a suspension of the Shiga bacillus containing 250 million bacilli per cubic centimeter. This is inoculated with $0.25 \mathrm{cc}$. of bacteriophage suspension.

2. One hundred cubic centimeters of a suspension of the cholera vibrio, containing 250 million bacilli per cubic centimeter. This also is inoculated with $0.25 \mathrm{cc}$. of the same suspension of Shiga-bacteriophage.

3 . One hundred cubic centimeters of bouillon containing only 0.25 cc. of the same bacteriophage.

The material of all three flasks is incubated at $37^{\circ} \mathrm{C}$. Immediately after the inoculation, after 30 minutes, and again after 1 hour, 20 cc. are taken from each of the three flasks and centrifuged at 4000 revolutions per minute for 10 minutes.

There are thus 9 tubes which have been centrifuged. From the supernatant fluid of each of these, $0.02 \mathrm{cc}$. is taken and introduced into other tubes containing suspensions of the shiga bacillus, and counts of the corpuscles are made by plating 0.02 cc., of each of these 9 tubes on six plates of medium. In this way an average of the counts can be obtained, and the results of the counts indicate the number of corpuscles remaining in the medium, since those which have penetrated the bacterial cells before the centrifugation have been thrown down with the cells during this procedure and, as a result are to be found in the sediment.

The results of the counts are as follows:

Tube 1. Shiga suspension plus bacteriophage.

a. Counts of the material made immediately after the preparation are $214,193,187,221,229$, and 183 plaques. The average is 204, representing 5,000,000 corpuscles per cubic centimeter in the original suspension immediately after inoculation. 
$b$. Counts on the suspension after incubation for 30 minutes are 3 , $7,4,6,6$, and 3 plaques. The average is 5 . This indicates that there are 125,000 bacteriophagous corpuscles in the suspension 30 minutes after the inoculation. That is, of each 41 corpuscles inoculated, 40 have disappeared from the fluid.

A count made directly upon the suspension, without centrifugation, gives 5,000,000 elements per cubic centimeter. It is therefore certain that the corpuscles which have disappeared from the fluid during the centrifugation have gone down with the bacteria. And, as we will see in the two control experiments, in the absence of Shiga bacilli this sedimentation of the bacteriophage does not occur (at least, when centrifuged at a speed of 4000 revolutions).

c. After 1 hour, the count, made as before upon the supernatant fluid gives an average of 8 plaques, or 200,000 corpuscles per cubic centimeter; a number essentially the same as that secured after 30 minutes. At this time a count of a suspension which has not been centrifuged gives $6,500,000$; a number very close to that secured immediately after the inoculation.

d. Counts made upon the suspension with and without centrifugation after one and one-quarter hours give the same number of corpuscles-about 90 million. The inoculated corpuscles have therefore increased from 5 to 90 millions; the increase being in a proportion of about $1: 18$. And this increase has taken place in apparently a very abrupt manner, only to be explained as a result of the liberation of actual colonies containing an average of about 18 corpuscles. We will see by ultramicroscopic examination that the dissolution of a parasitized bacterium takes place brusquely, by bursting.

Tube 2. Control. Suspension of $V$. cholerae plus the Shiga-bacteriophage.

Counts made immediately after inoculation of the bacteriophage give; for the centrifuged material, 201; for the non-centrifuged, 211 plaques.

After 30 minutes the counts are: for the centrifuged, 210; for the non-centrifuged, 216.

After 1 hour the counts are: for the centrifuged, 203; for the noncentrifuged, 199.

After one and one-half hours the non-centrifuged suspension gives 207.

Tube 3. Control. Sterile bouillon plus Shiga-bacteriophage.

The counts immediately after the inoculation are: for the centrifuged, 206; for the non-centrifuged, 210. 
After 30 minutes the corresponding counts are; 201 and 211.

After 1 hour the counts are: 203 and 206.

After one and one-half hours the non-centrifuged medium contains 198.

As is evident, in the absence of bacteria capable of being attacked nothing happens. The corpuscles remain inert in the medium.

The nature of the multiplication taking place in the presence of the Shiga bacillus does not permit of any doubt on the following points:

1. After a contact of 30 minutes at $37^{\circ} \mathrm{C}$. the corpuscles have almost entirely disappeared from the fluid; they are fixed by the bacteria. After 1 hour the situation is essentially the same.

2. The fixation is elective. It does not occur with $V$. cholerae, for example, for which the bacteriophage in question is without action (d'Herelle ${ }^{321}$ ).

A complementary experiment, conducted in the same fashion, but centrifuging the suspension at 10-minute intervals during the first half-hour, has shown that very few of the bacteriophage corpuscles are fixed during the first 10 minutes, although they are almost all fixed after 20 minutes. The union, therefore, requires about a quarter of an hour.

Additional data ohtained with the staphylococcus may be introduced as bearing upon this subject of fixation. Ten cubic centimeters of a normal suspension of cocei are combined with $0.1 \mathrm{ce}$. of the bacteriophage suspension. The temperature is held throughout at $30^{\circ} \mathrm{C}$. With this particular race of bacteriophage, of relatively high but not of maximum potency, the fluid still contains 97 per cent of the introduced corpuscles after 45 minutes. After 1 hour 62 per cent are present; after 75 minutes, but 8.5 per eent remain.

On the other hand, with two races of the bacteriophage of maximum potency the fixation was extremely rapid. When $0.1 \mathrm{ec}$. of a suspension containing 20,000 million corpuscles per culic centimeter was introduced into $10 \mathrm{cc}$. of bacterial suspension (250 million cocci per cubic centimeter) and the material was filtered through a candle after 10 minutes there was not a single corpuscle in $0.05 \mathrm{cc}$. of the filtrate. The fixation was complete.

This experiment shows particularly well how the activity of the bacteriophage race influences the speed of fixation.

The fixation of the bacteriophage corpuscle to the susceptible bacterium constitutes then, the first act of bacteriophagy (d'Herelle ${ }^{317},{ }^{321}$ ). This fact is accepted by all authors. The only difference to appear in 
different experiments is the time requisite for the fixation, and this varies with the bacterial species and with the race of the bacteriophage, as well as with the reaction of the medium, the temperature, and other environmental conditions.

With a single strain of bacteria the fixation is the more rapid and complete the more active the bacteriophage. It is particularly pertinent in this connection to remember that the mechanism of bacteriophagy can be studied only with races of the bacteriophage of maximum activity. With races of less potency the phenomenon of bacterial resistance masks to a greater or less degree the processes of attack.

By a series of experiments performed with different bacterial species I" am convinced that with bacteriophage races of low potency it is impossible to demonstrate the process of fixation to the bacteria. At least, it is impossible to demonstrate that the corpuscles disappear from the liquid. The reason for this is obvious, for with such races fixation takes place slowly and instead of the process occurring almost simultaneously with practically the entire number of inoculated corpuscles, as is the case when the bacteriophage is very active, the time of fixation varies enormously among the different corpuscles. It thus happens that a great number have not been fixed when those which are first fixed have already commenced to reproduce. Consequently, the fixation remains undetected.

Carrying out experiments analogous to those which I have described but employing filtration instead of centrifugation, Janzen and Wolff ${ }^{373}$ obtained the following results in two experiments with B. typhosus using different races of the Typhoid-bacteriophage.

Number of free corpuscles per cubic centimeter, unfixed to the bactcria

I. Immediately after inoculation . . . . . . . . . . . . 18,000,000 After 15 minutes. . . . . . . . . . . . . . . . . . . . . . 280,000

II. Immediately after inoculation . . . . . . . . . . . . 30,000,000 After 15 minutes......................... 4,000,000

It is probable that the number of bacteriophage corpuscles fixed would have been still greater if the counts had been made some 5 or 10 minutes later.

The process of fixation is lacking when a bacteriophage is placed in contact with a bacterium insusceptible to bacteriophagy by the particular bacteriophage involved.321 With but one exception all of those who have studied this question agree on this point. Later we will 
see that a Typho-bacteriophage, and this is also true for a Coli-bacteriophage or a Staphylo-bacteriophage, is as a rule not active upon all strains of B. typhosus; certain strains being susceptible while others are naturally resistant. This is what I have meant when a bacterial species has been termed heterogeneous toward a bacteriophage. To return to the exception mentioned above, Janzen and Wolff ${ }^{374}$ have reported that a Typho-bacteriophage may be fixed to typhoid bacilli of strains against which this Typho-bacteriophage is inert. This must be regarded as an exceptional case, for Jaumain and Meulemans ${ }^{378}$ have shown with different races of Coli-bacteriophage and Staphylobacteriophage that fixation does not occur with bacteria belonging to insusceptible strains. My own observations agree with those of Janzen and Wolff, for I have observed a fixation, although but partial (it is, however, the same when these races act upon susceptible bacteria, it being simply a question of degree), with insusceptible staphylococei. We will see shortly that this is also what happened in some experiments reported by Flu.

As a modification of the above experiments da Costa Cruz ${ }^{153}$ has shown that fixation takes place upon heat-killed bacteria, provided they were of a susceptible strain. Working with a Flexner-bacteriophage he has seen that the corpuscles are fixed to dysentery bacilli which have been killed by heating at $60^{\circ} \mathrm{C}$., but that they are not fixed to staphylococci, also killed at the same temperature. This has been confirmed by several authors.

Prausnitz and Firle ${ }^{539}$ have seen that fixation took place with susceptible bacteria after they had been heated at 60, 70, 80, 90 and $100^{\circ}$, but that it did not take place when the bacteria had been exposed to a temperature of $120^{\circ} \mathrm{C}$.

These observations warrant the conclusion that fixation proceeds in the same manner whether the susceptible bacteria are living or dead, although the bacteriophage corpuscles can develop only at the expense of the former.

These facts make clear the reason for the delay in bacteriophagy caused by the viscosity of the medium, whether this viscosity is due to gelatin, to a gum, or to any other substance which is, of itself, without action upon the phenomenon. When we place bacteria and bacteriophage corpuscles within a liquid it is evident that inasmuch as the first act of bacteriophagy consists in a fixation of the corpuscles to the bacterial cells, these corpuscles must first of all traverse the distance which separates them from the nearest bacteria. This necessitates 
the assumption that there be a positive chemotaxis of the bacteriophage corpuscle for the susceptible bacterium, or, if one prefers (it is of no consequence at the moment), of the bacteria for the corpuscle. Whatever may be the nature of the force which leads to the union, the attraction exists, and it is evident that anything which augments the viscosity of the liquid tends to interfere with the approach of the corpuscle to the bacterium, and, as a result, with its fixation. If the viscosity is sufficiently high the number of corpuscles attaining fixation will be so small that the phenomenon will be incapable of detection macroscopically because of the small number of bacteria dissolved. Only the proof provided by a demonstration of the multiplication of the corpuscles will show that the bacteriophagy of a few organisms has taken place.

Doerr and his collaborators ${ }^{181,183}$ have attempted to draw a parallelism between the fixation of the bacteriophage corpuscle to the bacterium and the fixation of an antibody. Such a comparison is inadinissible, for the characteristics of the two phenomena are entirely different.

We know that the agglutinin content of a serum can, to all intents and purposes, be completely exhausted by "saturation" with homologous bacteria, and that the saturation required is in direct proportion to the agglutinating potency of the serum; that is to say, fewer bacterial cells are required to exhaust a weakly agglutinating serum than to exhaust the same quantity of a strongly agglutinating serum. With the bacteriophage the situation is exactly the reverse. In a later chapter we will see that races of the bacteriophage may be isolated which differ widely in their activity for a single bacterium, some being weakly active, others possessing extreme activity. This difference does not involve a difference in the number of corpuscles, but is due rather to a difference in the activity of the corpuscles. But the particular point as regards fixation is precisely this, that the greater the activity of the bacteriophage the fewer bacterial cells are requisite to give fixation. This fact has been disclosed consistently by several experiments made with both $B$. dysenteriae and the staphylococcus and their homologous bacteriophages.

With a bacteriophage of maximum activity, using 0.1 ce. of a suspension containing more than 10,000 million corpuscles per cubic centimeter in conjunction with $10 \mathrm{cc}$. of a bacterial suspension (250 million per cubic centimeter), the fixation is complete with the staphylococcus, and almost complete with $B$. dysenteriae, within a period of 20 minutes. 
Employing a less active bacteriophage, $0.1 \mathrm{cc}$. of a suspension with 1000 million corpuscles per cubic centimeter, added to $10 \mathrm{cc}$. of a bacterial suspension, shows very little fixation after contact for 30 minutes. Even with the contacts repeated for 5 successive times, the material being centrifuged between each contact and fresh organisms added each time, a complete fixation is not attained.

In brief, therefore, the course of fixation is exactly opposite to that observed in the fixation of agglutinins. We must regard the fixation of bacteriophage corpuscles to the bacteria as a phenomenon of colloid nature-and nothing could be more legitimate since all of the reactions of living matter are colloiclal reactions-and it would be strange indeed if bacteriophagy formed an exception.

It is assuredly true that as yet we do not know the intimate mechanism of the process, yet we may unquestionably affirm, without appearing too radical, that it is a colloidal process. This, however, means but little since this is true for all of the phenomena of life. Beyond this statement all we can definitely say is that the first phase of bacteriophagy consists in the approach of the bacteriophage corpuscle to the bacterium, and that this is followed by its fixation to the bacterial cell.

Unquestionably, the actual fixation is elective, as all available experimental data indicate. But with regard to the process which leads to the contact between the bacteriophage corpusele and the bacterium there is the question as to whether it is a passive phenomenon or whether it is a true chemotaxis.

It might be assumed that the corpuscles, in violent motion because of the brownian motion which animates them, become fixed only when they come into contact with a susceptible bacterium. With these the fixation is then elective, but it takes place only after contact is effected. In this connection Kabelik ${ }^{384}$ has recently published some experiments which enforce the conclusion that a real chemotaxis exists between bacteriophage corpuscles and susceptible bacteria.

The statements of Kabelik, embodying his results, are here inserted.

Bacterial migration may readily be studied in glass U-tubes which contain the nutritive fluid and in which the bottom of the tube is filled with sterile sand. For our tests these simple U-tubes are not quite adequate, and we have therefore devised an apparatus which is, in effect, a combination of several of these tubes. A horizontal tube, $9 \mathrm{~mm}$. in diameter, and $21 \mathrm{~cm}$. long, is provided with seven vertical arms, about $8 \mathrm{~cm}$. in length, spaced about $3 \mathrm{~cm}$. from each other. In the bottoms of all of these vertical tubes some very fine sand, thoroughly washed and 
sterilized, is placed, in such a way that migration through the material and the fluid is rendered very difficult. These tubes are then filled with bouillon to a height of 3 or $4 \mathrm{~cm}$. above the sand. (They may be used for several purposes, particularly for separating $B$. typhosus from $B$. coli.)

In an apparatus of this type (no. $1^{*}$ ) we inoculated a drop of a virulent bacteriophage suspension (strain $\mathrm{H}$ of d'Herelle) in the first vertical arm. Tests were made after $2,4,6,8,12$, and 24 hours to see if the bacteriophage had penetrated to the other vertical arms. These tests consisted simply in seeding a loopful of the contents of each arm on to agar previously implanted with Shiga bacilli. These agar cultures were allowed to incubate for 24 hours, and then the number of plaques, that is, the number of bacteriophage corpuscles appearing were recorded (see the table). When the plaques were completely fused together, resulting in a large sterile area covering the entire surface of the medium the result was expressed as infinity $(\infty)$.

In another apparatus (no. 2), under the same conditions, we inoculated the bacteriophage into the first upright arm after this had been filled with bouilon seeded with Shiga bacilli. In a third apparatus (no. 3) the bacteriophage was inoculated into pure bouillon in the first arm, and the last, or seventh, we implanted with Shiga bacilli. In a fourth apparatus (no. 4) only Shiga bacilli were implanted; this set to test (as a control) the rapidity with which bacterial infiltration into the neighboring arms occurred. This control showed that after 4 hours the bacilli were to be found in the sccond arm only. Obviously, the absolute rate of penetration of bacilli and bacteriophage depends, ceteris paribus, upon the size of the grains of sand.

The results of these tests are summarized as follows:

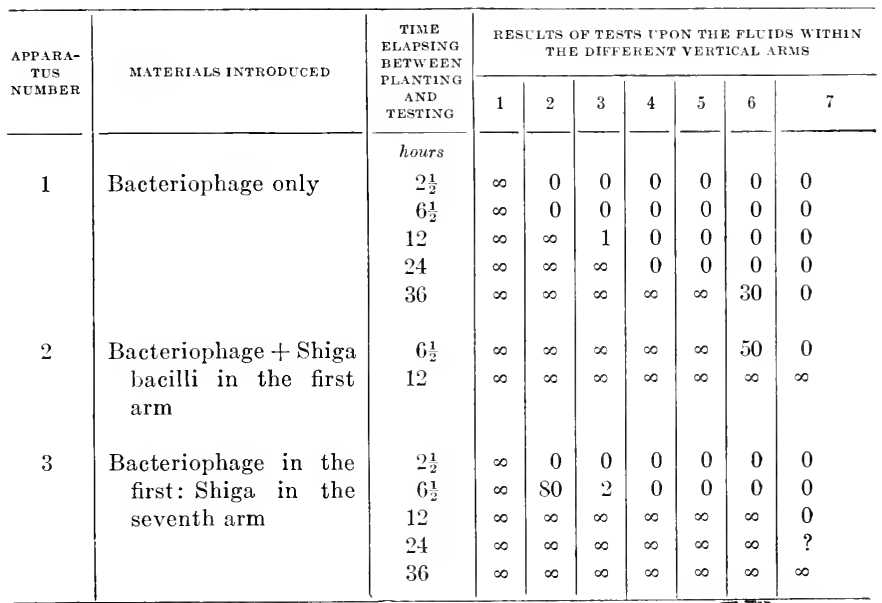

* See the table below. 


\begin{tabular}{|c|c|c|c|c|c|c|c|c|c|}
\hline \multirow{2}{*}{$\begin{array}{l}\text { APPARA- } \\
\text { TUS } \\
\text { NUMBER }\end{array}$} & \multirow{2}{*}{ MATERIALS INTRODUCED } & \multirow{2}{*}{$\begin{array}{l}\text { TIME } \\
\text { ELAPSING } \\
\text { BETWEEN } \\
\text { PLANTING } \\
\text { AND } \\
\text { TESTING }\end{array}$} & \multicolumn{7}{|c|}{$\begin{array}{l}\text { RESULTS OF TESTS CPON THE FLUIDS WITHIN } \\
\text { THE DIFFERENT VERTICAL AKMS }\end{array}$} \\
\hline & & & 1 & 2 & 3 & 4 & 5 & 6 & 7 \\
\hline 4 & Shiga bacilli only & $\begin{array}{l}\text { hours } \\
6 \\
24\end{array}$ & $\begin{array}{l}\operatorname{gr} . \\
\operatorname{gr} .\end{array}$ & $\begin{array}{l}\text { gr } \\
\mathrm{gr}\end{array}$ & $\begin{array}{l}\mathrm{gr} . \\
\mathrm{gr} .\end{array}$ & $\begin{array}{c}0 \\
\mathrm{gr} .\end{array}$ & $\begin{array}{c}0 \\
\mathrm{gr} .\end{array}$ & $\begin{array}{r}0 \\
\mathrm{gr} .\end{array}$ & $\begin{array}{l}0 \\
20 \text { colo- } \\
\quad \text { nies }\end{array}$ \\
\hline 5 & Bacteriophage only & $\begin{array}{l}6 \frac{1}{2} \\
24\end{array}$ & $\begin{array}{l}\infty \\
\infty\end{array}$ & $\infty$ & $\begin{array}{l}0 \\
2\end{array}$ & $\begin{array}{l}0 \\
0\end{array}$ & $\begin{array}{l}0 \\
0\end{array}$ & $\begin{array}{l}0 \\
0\end{array}$ & $\begin{array}{l}0 \\
0\end{array}$ \\
\hline 6 & $\begin{array}{l}\text { Bacteriophage + Shiga } \\
\text { bacilli in the first } \\
\text { arm }\end{array}$ & $\begin{array}{l}6 \frac{1}{2} \\
24\end{array}$ & $\infty$ & $\infty$ & $\infty$ & $\infty$ & $\infty$ & $\begin{array}{c}0 \\
\infty\end{array}$ & $\begin{array}{c}0 \\
\infty\end{array}$ \\
\hline 7 & $\begin{array}{l}\text { Bacteriophage in the } \\
\text { first: Shiga in the } \\
\text { seventh arm }\end{array}$ & $\begin{array}{l}6 \frac{1}{2} \\
24\end{array}$ & $\infty$ & $\infty$ & $\begin{array}{c}100 \\
\infty\end{array}$ & $\begin{array}{l}0 \\
2\end{array}$ & $\begin{array}{l}0 \\
0\end{array}$ & $\begin{array}{l}0 \\
0\end{array}$ & $\begin{array}{l}0 \\
0\end{array}$ \\
\hline
\end{tabular}

Tests 1 to 4 were made in bouillon; 5 to 7 were made in physiological saline. gr. = bacterial growth.

In view of these results it would seem that we must assume that a true chemotactic influence is operative in the behavior of the bacteriophage corpuscle.*

\section{PENETRATION OF THE CORPUSCle INTO THE BACTERIUM}

With the bacteriophage fixed to the bacterium, does it remain adherent to the surface or does it penetrate to the interior of the cell? "Macroscopic" experiments are inadequate to determine this point, but "microscopic" observation, chiefly by means of the dark-field method, provides some information.

Inoculate $0.1 \mathrm{cc}$. of a very active Shiga-bacteriophage suspension into 10 cc. of a suspension of 250 million Shiga bacilli per cubic centimeter. During the period when bacterial dissolution is taking place most vigorously remove a drop of the suspension and examine it under the dark-field. Not a single bacterium will be seen which appears to be undergoing disintegrative changes. The only visible abnormality is that in the midst of the normally appearing bacteria some few will be seen presenting an "inflated" form. Those departing farthest from the typical cell are completely spherical, with a diameter of 3 to

* In this preliminary note, adequate however to allow of a decision, Kabelik announces the publication of a more extended memoir on the question. 
$5 \mu$, and between the normal forms and the spherical forms are to be found all intermediates. But despite this variable morphology all of the cells have a sharply outlined contour.

If the spherical cells are observed with care it is seen that after a variable length of time, sometimes amounting to only about 10 minutes, an actual bursting takes place; a process consuming only a fraction of a second. Immediately afterward, in the place of the spherical cell there remains a slightly cloudy floccule which slowly dissolves. These spherical cells are particularly abundant at the time when the dissolving process is at its maximum rate. There can be no question concerning the nature of these cells; they are bacilli which, operated upon by a force exerting its effects from within, take at first a globoid form and later rupture. This is the more certain since at times one can witness the rupture of the swollen bacilli, even before they have assumed a completely spherical contour. This observation provides direct proof that the corpuscle develops and exerts its action within the bacterial cell. Destruction of the bacilli would be an entirely different process if the dissolving action were exerted on the exterior. The spherical form and the bursting process prove beyond doubt that the operating force is internal (d'Herelle $\left.{ }^{321,}{ }^{323}\right)$. $^{*}$

Although, as stated above, it is best to make microscopic observations with a suspension in the process of being dissolved under the action of a powerful bacteriophage, this does not mean that the rupture of the bacterial cell takes place in this case only, for the phenomenon has occurred in the same manner with all of the races of the bacteriophage which I have isolated. It is, however, more readily observed when bacteriophagy is intense. I am convinced, because of various experiments with bacteria of varied species and with bacteriophage races of different types, that the destruction and the dissolution of the bacteria occurs always by bursting. But in the case of a slightly active bacteriophage the process may pass unobserved, for the number of ruptures occurring at a given moment is then extremely small, and it is pure "chance" if the rupture of a cell takes place at a given time within the extremely minute quantity of material under the objective of the microscope. With such materials it may at times

* The bursting phenomenon was first observed by P. Jeantet, Chief of the Laboratory of Microphotography at the Pasteur Institute. There are few who have the capacity for microscopic observation as highly developed as he. Moreover, he has the habit, as rare as it is original, of not publishing the things which he observes. Instead of taking to himself the credit for those things disclosed in the studies in which he takes an interest, there are many who have benefited from his powers of observation. 
require an hour of continuous search and observation before the first rupture is seen. It is for this reason that those who desire to witness this curious phenomenon should use Shiga bacilli in contact with a highly potent bacteriophage. When they have once observed the reaction, recognizing the manner in which it takes place, they can then investigate other cases where the phenomenon is less conspicuous.

Personally, I have observed the rupture of bacteria contaminated by bacteriophage corpuscles with $B$. dysenteriae Shiga and Flexner, with $B$. typhosus and the paratyphoid strains, with $B$. pestis, and with the staphylococcus. As a matter of fact, I have never failed to see it when I have sought for it. With the staphylococcus the individual coccus undergoing rupture will have a diameter 2 to 3 times as great as that of a normal coccus.

The rupture of bacteriophaged bacteria has also been observed by da Costa Cruz, ${ }^{153}$ Pondman, ${ }^{530}$ as well as by Hauduroy ${ }^{299}$ and by Flu.

Naturally, a quite logical question is, How can the bacteriophage corpuscle penetrate the bacterium? The following observations permit an hypothesis, although they do not give a clear and complete picture of the mechanism.

At the moment when bacteriophagy is most intense we see by darkfield observation that the single unusual feature presented by the bacteria is a more or less outspoken swelling. None of the bacteria appear damaged, and with the exception of the "floccules" which follow the bursting and which disappear after a few minutes there is no bacterial débris. If a drop of the suspension in which the process of rupture is taking place is removed and spread upon a slide, dried, and stained, it will be seen that along with the normal bacteria there are some swollen organisms and some amorphous material which certainly represents stained floccular material. In such a stained smear the background is not colorless as in an ordinary preparation of a suspension of normal young bacteria, but is tinted. All of the bacteria are sharply defined, even those which are distended.

Another interesting situation develops if we take a drop of the suspension when bacteriophagy is at its maximum and place it between a slide and cover-glass (selecting a thick cover-glass to avoid breakage) exerting strong pressure upon the cover, as though a crushing of the bacteria was desired. Allow the preparation to dry in the incubator, then, after removal of the cover-glass, fix the smear and stain with any of the ordinary dyes (Loeffler's blue, carbol-fuchsin). Examination will reveal many of the bacteria presenting a curious appearance, for about some of the well-stained bacterial cells may be seen one, two, 
three, or sometimes several, stained "discharges." The material gives a definite impression that a portion of the contents of the bacterium have escaped by means of one, or of several, apertures.

This observation suggests that the wall of the bacterial cell (formed beyond doubt of condensed protoplasm) has been perforated by one, or several, bacteriophage corpuscles, and that the avenue of entrance has remained open.

However this may be, the single fact that the phenomenon of rupture is "explosive" in nature shows that the bacteriophage corpuscle certainly penetrates to the interior of the bacterium, and that it is in this location that the multiplication takes place.

\section{MULTIPLICATION OF THE BACTERIOPHAGE CORPUSCLE}

At the very beginning of this section I believe it wise to state once more that when I cite an illustrative experiment it is not equivalent to stating that the rate of the reaction of all experiments which may be carried out upon the same subject must be identical. Such an erroneous deduction has been reached by a number of authors. For example, the experiment presented in the second section of the present chapter, and to which we will return, shows that the first increment in the bacteriophage was in a ratio of $18: 1$. This numerical ratio holds necessarily for this experiment only. In other cases it is possible to have ratios of increase anywhere between $6: 1$ and 60:1. Everything depends upon the conditions of the experiment, chiefly upon the "virulence" of the bacteriophage with which one is working.

Bacteriophagy always takes place in the same manner; the sequence of events is always the same. The bacteriophage corpuscle must invariably become fixed to the bacterium to exercise its action. Destruction of the bacterium is always accomplished by bursting. The bacteriophage corpuscles always multiply within the bacterial cell and are always liberated with the rupture of this cell. But the time required for the fixation to take place, the time necessary for the bacterium to undergo rupture, the number of young bacteriophage corpuscles developing within the bacterium to be liberated with its rupture, all vary in each particular case, according to a multitude of conditions which vary from one experiment to another.

Having again emphasized this, let us consider the manner in which multiplication of the bacteriophage corpuscle takes place, and the nature of the conditions which exercise an effect upon their development. 


\section{The course of multiplication}

The experiment described in the second section of this chapter shows that:

After 30 minutes of contact at $37^{\circ} \mathrm{C}$. the bacteriophage corpuscles have almost entirely disappeared from the liquid.

After 60 minutes the situation is the same.

After 90 minutes the corpuscles have suddenly reappeared in the liquid, and their number is 18 times greater than was that of the inoculated corpuscles. In other words, each inoculated corpuscle has yielded 18.

Another experiment may be inserted, likewise showing the sudden multiplication of the bacteriophage corpuscles. Six tubes of Shiga bacillus suspension are inoculated with a bacteriophage suspension (containing 3000 million per cubic centimeter) in such a way that each tube receives one six-millionth of a cubic centimeter. When incubated, four give normal cultures of $B$. dysenteriae and all subcultures on agar yield normal growths. These are, therefore, without interest for us. The other two, each of which received probably one, certainly not more than two corpuscles, show the following picture: The suspensions become more and more turbid. After 2 hours at $37^{\circ} \mathrm{C}$. the opacity is about 2 times as great as at the beginning. After 3 hours it is about $2 \frac{1}{2}$ times as great, and after 4 hours, about 3 times. It then begins to diminish, so that after 5 hours the density is about twice as great as at the beginning of the incubation. This clearing continues gradually, so that after 14 hours the culture is almost entirely clear. If immediately after the inoculation with the bacteriophage, and then every 30 minutes, $0.02 \mathrm{cc}$. of each of these two suspensions is transferred to agar slants, these tubes will show, after incubation, the following:

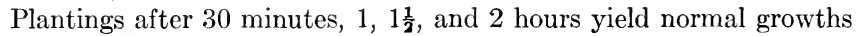
of $B$. dysenteriae. After $2 \frac{1}{2}$ hours the subcultures show 3 plaques in one tube and 5 in the other (average, 4). Therefore, after $2 \frac{1}{2}$ hours the inoculated suspension contains 2000 bacteriophage corpuscles.

After 3 hours the subcultures show 5 and 4 respectively. There has been no material increase between $2 \frac{1}{2}$ and 3 hours.

The $3 \frac{1}{2}$ hour plantings show 9 and 5 plaques (average, 7 ). The number of bacteriophagous elements has slightly increased.

After 4 hours, the agar tubes show 101 and 111 plaques respectively (average, 106). After 4 hours, therefore, the number of corpuscles is between 50 and 60 thousand. 
After $4 \frac{1}{2}$ hours, the counts are 145 and 160 (average, 152), indicating that the suspension contains 75,000 ; a number but slightly higher than the count after 4 hours.

After 5 hours the agar tubes remain sterile. When diluted to 1:1000 in a suspension of Shiga bacilli and transferred immediately to agar in the same way, the tubes give 4 and 6 plaques. Thus, it appears that after 5 hours the suspension contains about 1,500,000 bacteriophage corpuscles per cubic centimeter.

Although all authors are virtually in agreement with me upon the question of the specific fixation of the bacteriophage to the bacteria, several have denied that the multiplication occurs through successive sudden increments. Thus, Doerr found that the increase in "lytic substance" was very rapid but took place gradually, the titre increasing by about $10^{\circ}$ times every 15 minutes. After having carried out many tests upon a variety of bacteria with different races of the bacteriophage I adhere definitely to my previous statement, ${ }^{321}$ namely, that the increase in the number of corpuscles does not take place in a continuous progressive fashion, but by successive liberations. It may be pertinent to observe that in order to clearly observe this phenomenon it is essential that the experiments be performed in such a way that the course of the reaction is not obscured. To effectively demonstrate the phenomenon it is necessary to observe the following conditions: (1) To work with a bacterial species which undergoes a rapid bacteriophagy. Such a one is the Shiga bacillus. (2) To work with a bacteriophage of maximum activity for the bacterium in question. (3) To utilize a very small number of bacteriophage corpuscles, acting upon a large number of bacteria.

The reasons which make these conditions essential if the phenomenon is to be observed distinctly can readily be understood. If one uses a bacteriophage of weak activity the corpuscles of the successive generations become fixed very slowly and at a very unequal rate. Those fixed at first have already formed a colony and have caused the rupture of the bacterium before the other corpuscles are even fixed. Under such circumstances it can be understood that it is impossible to observe the true course of multiplication of each corpuscle, and Doerr is then apparently correct, for the total course of the multiplication is indeed progressively continuous.

Even in the case of a bacteriophage of maximum activity the fixation of all of the corpuscles does not take place with mathematical precision within the same interval of time. Obviously, for this there are several 
reasons. The first step in the process is the approach of the corpuscle to the bacterium (it can not be otherwise, since we know that the corpuscle can only act after it is fixed), and the more distant a corpuscle from the nearest bacterium the greater will be the time required for the fixation. Furthermore, we will see that even in the case of a bacteriophage of maximum potency all of the corpuscles do not have an equal virulence. The rapidity of fixation to a given bacterium, all of the other conditions being equal, is in direct proportion to its "virulence." This represents a further reason why the fixation of all of the corpuscles may be distributed over a certain period of time. Recognizing these facts, it is clear that the best condition for observing the true nature of the reaction consists in inoculating at the beginning only a very small number of corpuscles so that complete fixation can be accomplished in a minimum of time. As a result of this all of the ruptures, and consequently the liberations of young corpuscles, will occur after a like interval of time. This will permit one to observe that multiplication, the increase in the number of corpuscles, takes place suddenly.

Another requisite condition, already mentioned, involves the presence of a large number of bacteria. The significance of this factor is evident, for if there is only a small number each bacterium will be found at a considerable, and very variable, distance from the nearest corpuscle. This again means that fixation will occur in a very irregular manner.

With due regard to the conditions mentioned anyone may demonstrate readily that the multiplication of bacteriophage corpuscles takes place by means of successive jumps and not in a gradual progressive fashion. But even here, this fact can be shown definitely only for the first liberation, as is quite natural, since as bacteriophagy progresses the greater will become the number of corpuscles, and the virulence of each of them, as individuals, being different, the time of fixation, and consequently, the speed of multiplication, will proceed in an irregular manner.* The multiplication by successive jumps, extremely clear-cut in the beginning of the process, becomes with time less and less sharply defined. The first series of liberations of the young corpuscles is accomplished within a short time, while for the

* This fact is not astonishing. We have known since the days of Pasteur that in a bacterial culture each of the organisms presents individual characteristics, chiefly in those attributes dealing with its virulence. In the chapter devoted to "The Virulence of the Bacteriophage" we will see that the situation is exactly the same with the bacteriophage. 
following liberations this interval of time becomes progressively greater, and finally, the ruptures of the last cells of a series take place only when the first burstings of the following series have commenced. That is, at this stage and under these circumstances the multiplication of the culture as a whole is in effect a continuous process.

The phenomenon of bacteriophagy is biological in nature. Therefore, it is impossible for its course to have the simplicity of a strictly chemical reaction.

Since the publication of my experiments upon this question many students have studied the course of the multiplication of the bacteriophage. It also appears from the work of Maitland ${ }^{132}$ that the sudden multiplication takes place only after an incubation period. I am not able to insert here his experiments for he has adopted a method of "titration" of the bacteriophage which does not allow of the exact enumeration of the corpuscles. I shall return to this later, when treating of the different methods proposed for measuring the activity of the bacteriophage. Let it suffice here to say that from his experiments it seems, as he has remarked, that at $37^{\circ} \mathrm{C}$. there is no multiplication during the first hour; sometimes during an even longer period. Following this period of latency there is a period of rapid augmentation occurring between the second and the third hours after the mixture is made. During this increase the "titre" of the bacteriophage may attain 10,000 times the original titre. The rate of the increase then diminishes and the maximum concentration is reached at about the fifth hour.

These results agree, in a general way, with those which I have published. But the great majority of those who have worked with the bacteriophage have wished to generalize from their results and in this tendency is to be found one of the causes of confusion in the study of the bacteriophage. All that one may correctly conclude from a given experiment is that under the conditions of this experiment such and such a result has been obtained. Beyond this nothing is permissible. Warranty for generalization is afforded only when a constant effect is produced, whatever may be the race of the bacteriophage and whatever may be the bacterial species under investigation.

Nevertheless, it may be stated that with a given bacterial species the rapidity of fixation and consequently the rate of multiplication is a function of the total "virulence," that is, an expression of the average virulence of the different corpuscles which are acting upon the bacteria of the suspension. Each of the corpuscles present fixes itself 
and multiplies the more rapidly as its "virulence" is the greater. But it is equally important to observe that against different bacterial species bacteriophage races of the same degree of virulence may become fixed and may multiply at different rates.

From the experiments which I have carried out upon this subject the following may be cited as giving the maximum and minimum rates observed.

With the most active race of Shiga-bacteriophage which I have isolated the fixation amounted to 94 per cent after 12 minutes ( 10,000 corpuscles per cubic centimeter in the presence of $250,000,000$ bacteria per cubic centimeter) at a temperature of $30^{\circ} \mathrm{C}$. Inoculating a single corpuscle into $10 \mathrm{cc}$. of a suspension containing 50,000,000 bacteria per cubic centimeter there were, after 11 hours, 12,000 million corpuscles per cubic centimeter.

With a Shiga-bacteriophage of low activity, in inoculating 100,000 corpuscles into $10 \mathrm{cc}$. of a suspension containing $250,000,000$ bacteria per cubic centimeter after 20 minutes only 10 per cent had been fixed. Here also the temperature was $30^{\circ} \mathrm{C}$. After 60 minutes only 34 per cent had been fixed. Inoculating a single corpuscle into $10 \mathrm{cc}$. of a suspension containing 250,000,000 bacteria per cubic centimeter there were, after 24 hours, only $17,000,000$ corpuscles present in the liquid.

With an extremely potent race of the Staphylo-bacteriophage, working under the same conditions as those described for the active Shigabacteriophage, the fixation was complete 20 minutes after the inoculation. Inoculating a single corpuscle into $10 \mathrm{cc}$. of a suspension containing 50,000,000 bacteria per cubic centimeter* the maximum value was reached only after 44 hours, and at this time there were 98,000 million corpuscles per cubic centimeter.

Working under the same conditions as those of the preceding experiment, but using another race of Staphylo-bacteriophage, one much less potent, the fixation after 75 minutes amounted to 54 per cent. A single corpuscle inoculated into a suspension of staphylococci containing 50,000,000 per cubic centimeter yielded, after 48 hours, only $780,000,000$ corpuscles per cubic centimeter.

In bacteriophagy the result of a single experiment always depends upon the conditions of the experiment, the most important of these

* We have seen in Chapter I that in inoculating a very small amount of bacteriophage the initial number of bacteria is of little consequence, for the latter develop abundantly up to the moment when the multiplication of the bacteriophage is sufficient to effect bacteriophagy of all of the bacteria. 
conditions being resident in the "characters" of the bacteriophage involved. Each bacteriophage presents, as we will discover from every page of this text, particular distinctive characteristics.

Among the published experiments bearing upon the multiplication of the bacteriophage may be mentioned one presented by Doerr and Grüninger $^{183}$ carried out with a Coli-bacteriophage.

These investigators have adopted a method of titration proposed by Applemans, concerning which we will offer certain criticisms later, where we will see that it gives an approximation of such a crude nature (and even absolutely fails in certain instances) that it can not serve for the enumeration of corpuscles. However this may be, Doerr and Grüninger have concluded from this experiment that an intense "bacteriolysis" corresponds to a stabilization in the titre of the bacterio-

TABLE 10

\begin{tabular}{c|r|r}
\hline TIME OF INCUBATION & $\begin{array}{c}\text { TITRE OF BACTERIOPHAGE } \\
\text { PER CUBIC CENTIMETER }\end{array}$ & $\begin{array}{c}\text { NUMBER OF BACTERIA } \\
\text { PER CUBIC CENTIMETER }\end{array}$ \\
\hline minutes & 100 & $10,000,000$ \\
0 & 100 & $16,000,000$ \\
30 & 100 & $20,000,000$ \\
60 & 100 & $35,000,000$ \\
90 & 100 & $45,000,000$ \\
105 & 1,000 & $60,000,000$ \\
120 & 10,000 & $130,000,000$ \\
150 & $10,000,000$ & $600,000,000$ \\
180 & $1,000,000,000$ & $200,000,000$ \\
210 & $1,000,000,000$ & $20,000,000$ \\
270 & & \\
\hline
\end{tabular}

phage. This can hardly be the case, since their experiment indicates that the number of bacteria diminish from $600,000,000$ to $200,000,000$ within the interval between 180 and 210 minutes, while in this same period the bacteriophage increases from $10,000,000$ to $1,000,000,000$. It would appear that this demonstrates precisely that the maximum increase in the bacteriophage corresponds to the greatest destruction of the bacteria. As for the assumed stabilization in the titre of the bacteriophage, which remains at 1000 million while the number of bacteria diminish from $200,000,000$ to $20,000,000$, this results simply from the method adopted for the titration of the bacteriophage. In fact, the so-called "dilution method" does not allow one to say that there are 1000 million corpuscles per cubic centimeter; it simply permits the statement that there are more than 1000 million and less than 
10,000 million. Such being the case, the conclusion of Doerr is not supported by experimental proof, indeed, the contrary interpretation is more logical, for the experimental data indicate that the period of increase in the bacteriophage corresponds to the period of destruction of a great many bacteria.

In this same communication Doerr and Grüninger ${ }^{183}$ state that the dissolution of the bacteria contained in the suspension occurs when the concentration of "lysin" equals $e_{L} 5$, according to the notation of Werthemann. ${ }^{43}$ This is simply equivalent to saying that when the concentration of the bacteriophage is such that $1 \cdot 10^{-5}$ cc. of a suspension in process of being bacteriophaged is added to a fresh suspension of the same bacteria bacteriophagy of the latter ensues. In other words, and to state it somewhat more precisely, dissolution of the bacteria takes place when the number of corpuscles is between 100,000 and 1,000,000 per cubic centimeter. Again it is necessary to repeat that to attempt to establish precise rules as governing the reaction is an illusion. The statement of Doerr is the more remarkable in that I have never seen a macroscopically detectable dissolution of bacteria with such a small number of corpuscles per cubic centimeter. Obviously, if the number of bacteriophage corpuscles is very small, very few of the bacteria are attacked; they remain normal and multiply normally. On the other hand, the corpuseles multiply, proliferating at the expense of the bacteria, but the number of bacteria destroyed at the beginning of the process is infinitely smaller than the number which reproduce. If one bacterium is bacteriophaged while 100 reproduce, macroscopically it will be impossible to detect this destruction. Only when the number of bacteria destroyed by bacteriophagy exceeds those bacteria which remain normal and which continue to multiply is it possible to perceive the change, and then macroscopic clearing of the medium begins.

The facts that the bacteria undergo destruction through bursting and that the increase in the number of bacteriophage corpuscles is intermittent, as may be clearly observed at the beginning of the process, as well as the fact that the greatest multiplication of the bacteriophage coincides with the moment when rupture of the bacterial cells occurs at the greatest rate can hardly leave a doubt concerning the mechanism of the liberation of the young corpuscles at the time of the bursting of each bacterium. 


\section{The influence of temperature upon multiplication}

In the communication already mentioned Doerr and Grüninger have also stated that the bacteriophage does not multiply at a temperature of $43^{\circ} \mathrm{C}$; ; a temperature at which, nevertheless, the bacteria concerned in the experiment $\left(B\right.$. coli) reproduce perfectly. Prausnitz ${ }^{539}$ has carried out an experiment which shows clearly the error committed by Doerr in his effort to generalize from the results of one experiment. I have reserved discussion of this experiment of Prausnitz until this time and it is here presented since it affords certain clues concerning the manner in which the bacteriophage multiplies at different temperatures. In the experiment here given a Flexner-bacteriophage was used. The culture medium was a bouillon with a pH of 7.6. The results obtained at different temperatures are given in table 11.

TABLE 11

\begin{tabular}{|c|c|c|c|c|c|c|c|c|c|}
\hline \multirow{3}{*}{$\begin{array}{l}\text { TIME OF } \\
\text { INCU- } \\
\text { BATION }\end{array}$} & \multicolumn{3}{|c|}{ TEMPERATURE $43^{\circ}$} & \multicolumn{3}{|c|}{ TEMPERATURE $45^{\circ}$} & \multicolumn{3}{|c|}{ TEMPERATURE $47^{\circ}$} \\
\hline & \multicolumn{2}{|c|}{$\begin{array}{c}\text { Bacteria }+ \\
\text { bacteriophagc }\end{array}$} & \multirow{2}{*}{$\begin{array}{c}\text { Bacteria } \\
\text { alone }\end{array}$} & \multicolumn{2}{|c|}{$\begin{array}{c}\text { Bacteria }+ \\
\text { bacteriophage }\end{array}$} & \multirow{2}{*}{$\begin{array}{c}\text { Bacteria } \\
\text { alone }\end{array}$} & \multicolumn{2}{|c|}{$\underset{\text { bacteriophage }}{\text { Bacteria }}$} & \multirow{2}{*}{$\begin{array}{l}\text { Bacteria } \\
\text { alone }\end{array}$} \\
\hline & $\begin{array}{l}\text { Bacteri- } \\
\text { ophage }\end{array}$ & Bacteria & & $\begin{array}{c}\text { Bacteri- } \\
\text { ophage }\end{array}$ & Bacteria & & $\begin{array}{c}\text { Bacteri- } \\
\text { ophage }\end{array}$ & Bacteria & \\
\hline hours & & & & & & & & & \\
\hline 0 & 100 & 380 & 110 & 0.1 & 116 & 265 & 12 & 180 & 120 \\
\hline 1 & 100 & & 180 & 0.7 & 110 & 260 & 17 & 185 & 88 \\
\hline 2 & 600 & 172 & 195 & 1.5 & 186 & 228 & 15 & 150 & 80 \\
\hline 3 & 500 & 193 & & 1.8 & 149 & 238 & 23 & 220 & 74 \\
\hline 4 & 700 & 226 & 236 & 1.4 & 140 & 214 & 12 & 160 & 62 \\
\hline 6 & 900 & 160 & 206 & 1.1 & 97 & 230 & 13 & 115 & 58 \\
\hline 8 & 1300 & 170 & 210 & 1.2 & $10 \tilde{3}$ & 220 & 3 & 106 & 34 \\
\hline 10 & 3400 & 185 & 18.5 & 2.0 & 90 & 111 & 2 & 22 & 20 \\
\hline 24 & 6200 & 95 & $13 S$ & 0 & 37 & 58 & 0.1 & 3 & 4 \\
\hline
\end{tabular}

The number of bacteriophage corpuscles are determined upon the basis of $10^{-2} \mathrm{cc}$, that of the bacteria on $10^{-5} \mathrm{cc}$. Prausnitz calls attention to the fact that in this experiment, at $43^{\circ} \mathrm{C}$., the number of bacteria increases two times and the number of bacteriophage corpuscles 62 times. At $45^{\circ} \mathrm{C}$. the number of bacteria did not increase while at the tenth hour the corpuscles were 20 times as numerous as at the beginning. Even at $47^{\circ} \mathrm{C}$. the corpuscles appear to begin to multiply during the early hours of the experiment.

We have seen elsewhere (Chapter I) that in working with the Colibacteriophage a complete dissolution of a suspension containing 200 
million $B$. coli per cubic centimeter was obtained within a very short time ( $3 \frac{1}{2}$ hours) in an incubator at $46^{\circ} \mathrm{C}$. In this experiment I did not measure the rate of increase of the corpuscles but it is certain that at the moment when bacteriophagy was complete the multiplication must have been considerable.*

\section{Multiplication as affected by the state of the bacteria}

Doerr and Grüninger ${ }^{183}$ have suggested that when the bacteriophage is inoculated into an actively growing bacterial culture the bacteriophage develops immediately, without a latent period. It has been impossible for me to verify this, although I have made many experiments to this end with bacteria of different species and with races of the bacteriophage of diverse activities. Data on 21 such experiments are at hand, all carried out in the same manner, inoculating the bacteriophage corpuscles into cultures containing about $50,000,000$ bacteria per cubic centimeter, (faintly turbid) and incubated at $36^{\circ} \mathrm{C}$. for 5 hours before the introduction of the bacteriophage. Titrations of the bacteriophage made every 15 minutes have shown the minimum time before which the first increase was to be observed was 45 minutes, and indeed this was obtained in only one of the experiments. On this occasion although present, the increase was slight ( 3 -fold). In this same experiment the bacteriophage had increased 39 -fold after 60 minutes; 41 -fold after 75 minutes. In this single case the first rupture had taken place after 45 minutes. The first series of ruptures was complete after 1 hour. In 12 other experiments of this same type the first increase took place after 60 minutes (4 races of Coli-bacteriophage, 5 of Shiga-bacteriophage, and 3 of Typhoid-bacteriophage; all very virulent races). In the other $\delta$ experiments the first increase could be detected only after 90 minutes. And in all of these, races of the Coli-bacteriophage of relatively high activity were used.

If we compare these experiments with the other results which have been mentioned it appears that although multiplication may not start immediately, the rate of reproduction may be accelerated when the bacteriophage is inoculated into an actively developing culture. While superficially this fact might appear to be significant, in reality the result is simply due to the fact that the temperature is favorable $\left(37^{\circ} \mathrm{C}\right.$.) for the process at the moment of inoculation. If bacteria are suspended in a bouillon previously warmed to $37^{\circ} \mathrm{C}$. and this is inocu-

* But they are weakened, as we will see. 
lated at once with the bacteriophage multiplication will take place just as promptly and as vigorously as in an actively developing culture.

\section{Multiplication in relation to the number of bacteria bacteriophaged}

Working with $B$. dysenteriae Shiga and with B. coli, Meuli ${ }^{452}$ reached the conclusion that the final "lytic" titre is independent of the initial titre. This deduction is true or false according to the conditions of the experiment; it all depends on the total number of bacteria available and suitable for serving for the multiplication of the bacteriophage corpuscles. If we combine in a medium a very few bacteria together with a very small number of bacteriophage corpuscles, the corpuscles which find a bacterium in their immediate vicinity readily available will be relatively few and thus the opportunity for multiplication will be restricted. Little by little, progressively, the number of corpuscles will augment, but before the number becomes sufficiently great for all of the bacteria, which meantime have had time to develop, to be "parasitized," a culture equivalent to several hundreds of millions of bacteria per cubic centimeter will have had time to mature. From this it is apparent that when but very few corpuscles are inoculated the initial titre of the bacteriophage is to a degree immaterial and has no great effect upon the final titre. Incidentally this view of Meuli is in some respects in accord with what I stated in the first edition of my collected papers, ${ }^{321}$ namely; "In a word, whatever may be the original titre of the suspension at the time when it is inoculated with a limited number of bacteriophagous organisms the latter must always operate on a suspension of about 650 million bacilli per cubic centimeter, since in all cases the bacilli reproduce until they attain this number."

But Meuli has gone further, he has generalized, and his conclusions are entirely false when the conditions are changed. If the medium contains a small number of bacteria and a relatively large number of bacteriophage corpuscles, the final titre depends upon the initial titre in the sense that it varies but little, and solely in proportion to the number of bacteria implanted. This is to be interpreted in this way: each of the bacteria present at the moment of inoculation is in close proximity to one of the corpuscles, since the latter are very numerous, and all of the bacterial cells are parasitized and dissolved before they have had time to multiply to any appreciable extent.

To state the situation correctly, it may be said that the final number of corpuscles depends upon the number of susceptible bacteria sub- 
jected to bacteriophagy. This is true whether the process takes place solely with the bacteria implanted when the number of corpuscles inoculated is sufficiently great for all of the bacteria present to be bacteriophaged at the beginning, or whether, because of the small number of corpuscles present at first, the bacteria implanted have had time to multiply before they are subjected to bacteriophagy. Between these two extremes, -1 corpuscle to 650 million bacteria, and 10,000 million corpuscles (and even more with the Staphylobacteriophage) to 1 bacterium,--by varying the relative concentrations of the two factors there is an infinite number of combinations and of differing situations. But in every case the final number of corpuscles is determined by the number of bacteria susceptible to bacteriophagy, and, consequently, capable of serving for the multiplication of the corpuscles.

\section{Influence of the conditions of the medium}

And yet, the statements made in the preceding section are true only when the conditions of the medium are optimum for the process of bacteriophagy. For example, if we vary the reaction of the medium the final result of the multiplication of the corpuscles will vary, even though in all cases bacteriophagy may be complete. The following experiment clearly demonstrates this fact.

A peptone water (peptone 25 grams, $\mathrm{NaCl} 5$ grams, water $1000 \mathrm{cc}$.) is rendered neutral to phenolphthalein. The medium is definitely alkaline to litmus. After it has been distributed in $10 \mathrm{cc}$. amounts into tubes, $\mathrm{HCl}$ is added in appropriate amounts to provide a series of tubes having an increasing scale of acidity. All of the tubes are implanted with a concentrated suspension of Shiga bacilli to give a normal suspension, that is, 250 million per cubic centimeter. Then each tube is inoculated with $0.001 \mathrm{cc}$. of the bacteriophage. After an incubation period of 24 hours simple observation of the tubes indicates varying degrees of turbidity, and appropriate counts indicate the final number of corpuscles present in the individual tubes. In tabulated form the results of such an experiment are as shown in table 12 .

Here are, for example, two strictly comparable experiments which show that this is indeed the case. Inoculate $10 \mathrm{cc}$. of a suspension of staphylococci containing 50 million bacteria per cubic centimeter with but a single bacteriophage corpuscle. A count made after 3 days shows that although the medium is perfectly clear there are present 81,000 million corpuscles. Inoculate a like suspension $(20 \mathrm{cc}$. of 
suspension was originally prepared and divided into two equal portions, one part being used in the test presented above) with 500 million corpuscles. After 3 days (bacteriophagy was complete in less than 24 hours) the number of corpuscles was 7000 million.

\section{Cause of the arrest of multiplication}

It may be asked why the bacteriophage ceases to multiply when the medium contains a certain number of them, even though bacteria are still present. Quite as logically it might be asked why bacteria stop multiplying even though food materials are left in the medium. The

TABLE 12

\begin{tabular}{c|c|l|r}
\hline TUBE & $\begin{array}{c}\text { REACTION TO } \\
\text { PHENOLPHTHALEIN }\end{array}$ & $\begin{array}{c}\text { MACROSCOPIC APPEARANCE OF THE } \\
\text { SUSPENSION AFTER 24 HOERS }\end{array}$ & $\begin{array}{c}\text { NUMBER OF } \\
\text { BACTERIOPHAGE } \\
\text { CORPUSCLES PER } \\
\text { CUBIC CENTIMETER }\end{array}$ \\
\cline { 1 - 3 } 1 & 0 & Very slight clouding & $400,000,000$ \\
2 & -2 & Very slight clouding & $500,000,000$ \\
3 & -4 & Clear & $500,000,000$ \\
4 & -6 & Clear & $1,250,000,000$ \\
5 & -8 & Clear & $2,750,000,000$ \\
6 & -10 & Clear & $1,000,000,000$ \\
7 & -12 & Clear & $1,000,000,000$ \\
8 & -14 & Slight clouding & $250,000,000$ \\
9 & -16 & Turbill & 500,000 \\
10 & -18 & Turbid & $1,000,000$ \\
11 & -20 & Turbid & 500,000 \\
12 & -22 & Turbid & None \\
\hline
\end{tabular}

answer is the same in both cases. Multiplication stops when the products resulting from the "vital reaction" reach a certain concentration.

Insofar as bacteriophagy is concerned, let us note first that with all of the conditions best suited to bacteriophagy the final number of corpuscles differs with the bacterium attacked. With the most active races of the Shiga-bacteriophage $I$ have never obtained a final titre greater than about 10,000 million per cubic centimeter. With the Staphylo-bacteriophage the final titre often goes above 100,000 million.

In the first chapter the statement was made that the substances resulting from the distinctive activity of the bacterium, that is, those substances which "vaccinate" the medium against the bacterium, do not exert an inhibitory effect upon bacteriophagy. The experiments carried out with Shiga bacilli leading to this conclusion ${ }^{* 21}$ have been confirmed by Maitland. ${ }^{432}$ 
Another experiment, performed with the staphylococcus, may be cited since it substantiates further this conclusion. A flask containing 250 cc. of bouillon ( $\mathrm{pH} 7.8$ ) is seeded with a strain of Staphylococcus aureus. After incubation for 15 days at $27^{\circ} \mathrm{C}$. the culture is filtered through a Chamberland candle. Two series of tubes are then prepared as follows:

\section{Series $I$}

Tube 1. 10 cc. of fresh bouillon

Tube $2 . \quad 7.5$ cc. of fresh bouillon +2.5 cc. of the filtrate

Tube 3. 5 cc. of fresh bouillon +5 cc. of the filtrate

Tube $4.2 .5 \mathrm{cc}$. of fresh bouillon $+7.5 \mathrm{cc}$. of the filtrate

Tube 5. 10 cc. of the filtrate, undiluted with bouillon

To this series of tubes a suspension of the staphylococcus is added, the strain being the same as that used for the preparation of the filtrate. After 24 hours all of the tubes are turbid, but the turbidity in tube 5 is about half as great as that in tube 1. After 48 hours all tubes show the same degree of turbidity.*

\section{Series II}

The initial mixtures of fresh bouillon and of filtrate are the same as those in series I. To the 5 tubes a suspension of the staphylococcus is added to provide approximately 125 million organisms per cubic centimeter. All of the tubes are then inoculated with $0.001 \mathrm{cc}$. of Staphylo-bacteriophage. After 24 hours, the dissolution is complete in tubes 1 and 2, partial in the other three. After 48 hours it is complete in all. The number of corpuscles, per cubic centimeter at this time is:

$\begin{array}{ll}\text { Tube 1. } & 52,000 \text { million } \\ \text { Tube 2. } & 46,000 \text { million } \\ \text { Tube 3. } & 38,000 \text { million } \\ \text { Tube 4. } & 44,000 \text { million } \\ \text { Tube 5. } & 50,000 \text { million }\end{array}$

I have not been able to determine the cause of these differences, but in spite of this variation it is possible to conclude that the products resulting from the distinctive activity of the bacterium itself have no effect upon the phenomenon of bacteriophagy, nor upon the multiplication of the bacteriophage corpuscles.

\footnotetext{
* According to this experiment the staphylococcus, at least the strain under examination, has but little "vaccinating" activity.
} 
The situation is quite different as regards the effects of the products resulting from bacteriophagy. The following experiment is illustrative.

A bouillon suspension containing 250,000,000 bacilli per cubic centimeter is inoculated with $0.001 \mathrm{cc}$. of bacteriophage suspension. The next morning, that is, after 14 hours, dissolution is complete. A count shows that there are 1600 million corpuscles per cubic centimeter. At this time a concentrated bacterial suspension is added to the dissolved suspension to again yield 250 million bacteria per cubic centimeter. Seven hours later the medium is again clear, and a count shows that there are in each cubic centimeter 2100 million corpuscles. This second dissolution being completed the bacterial count is again restored. This time the dissolution is not quite complete after 48 hours; the medium still shows a slight clouding. The count is 2400 million. At this time, then, the medium contains in each cubic centimeter the dissolved substance of 750,000,000 bacteria. For the fourth time the suspension is made up to a bacterial count of 250 million. After incubation for $\delta$ hours the clearing is slight. The count now is 2600 million corpuscles. Inoculations upon agar or into broth remain sterile. From this it is clear that the more concentrated the medium becomes in dissolved substances the more marked becomes the inhibition and the less effective the process of bacteriophagy.

As a matter of fact, such a result is not unexpected. Bacteriophagy and the resulting multiplication of corpuscles follow a general biological rule. Whether it be a bacterial culture, whether it be an enzyme reaction, whether it be bacteriophagy, the products resulting from all biological reactions first retard, then prevent, the reaction from continuing indefinitely in the same medium.

In concluding this section mention may be made of a statement by Bail and Matsumoto ${ }^{51}$ to the effect that there should be produced, in the course of bacteriophagy, as many bacteriophage corpuscles as bacteria that have been destroyed. Nothing is less true. All experimental work demonstrates that the proportion of bacteriophage corpuscles which are formed, in proportion to the number of bacteria destroyed, may be 100 to 1 , and even more. In order to demonstrate the error of these authors it is only necessary to count the corpuscles after bacteriophagy of the staphylococcus. With potent races of the bacteriophage one may readily find 100,000 million corpuscles per cubic centimeter. Simply start with a staphylococcus containing 100,000 million cocci per cubic centimeter and see if the statement of Bail and Matsumoto is correct. 


\section{BACTERIOPHAGY UNDER THE MICROSCOPE}

We have already seen how the destruction of the bacteria takes place through the action of the bacteriophage. Here are a few other observations made in studying the course of bacteriophagy with $B$. dysenteriae. ${ }^{321}$

We know that if the inoculation of the bacteriophage has been massive, all of the bacteria are attacked at the outset; the fixation of the corpuscles takes place immediately. If a very active race of the bacteriophage is used, within 2 or 3 hours the medium commences to clear little by little, and becomes completely limpid after a short time. If, on the contrary, the inoculation is minimal, the few corpuscles inoculated only affect an equal number of bacteria; the great majority remain unaffected and multiply as they would in a normal medium. But the corpuscles likewise multiply, following a progression more rapid than that pursued by the bacteria, so that within a few hours their number becomes equal to, or greater than, that of the bacteria. This is the time when macroscopic dissolution becomes evident.

Let us consider the first case, that of the massive inoculation. If we take from time to time a drop of the suspension up to the point when dissolution is complete, spread these drops on slides and stain, either with the Gram stain, with carbol-thionin, or by the RomanowskyGiemsa method (all staining methods give essentially the same picture), results such as the following are secured.

A suspension of Shiga bacilli, 250,000,000 per cubic centimeter is inoculated with $0.1 \mathrm{cc}$. of a suspension of the bacteriophage and incubated at $37^{\circ} \mathrm{C}$.

After fifteen minutes it appears as a culture of normal bacilli.

After thirty minutes it appears essentially the same, except that a few of the bacilli are poorly stained.

After forty-five minutes about 10 per cent of the organisms stain poorly.

Between one and two hours, the number of bacilli which stain badly continues to increase, and after 2 hours only a rare cell can be found which has taken the stain normally. At the same time, amorphous débris and granulations, derived most certainly from the bacteria already dissolved are seen. Similar material is seen very abundantly in old normal cultures of the Shiga bacillus. These granulations dissolve more slowly than the remaining portions of the bacterial protoplasm. Finally, and this is a most important point, spherical forms, more or 
less ellipsoidal, of variable dimensions, always rare, measuring 4 to 7 by 3 to $5 \mu$ may be detected. We will see in a moment to what they are due. There are occasional bacillary forms, well-stained, having a length of from 8 to $12 \mu$.

Between the second and third hours the amorphous débris considerably augments and the bacillary forms rapidly disappear. A few spherical forms are still to be seen.

After four hours, solution becomes more and more complete. Only a single poorly stained bacillus will be found in two or three fields.

Gradually the formless débris disappears, and, in turn, the granules. After thirty-six hours nothing whatever can be distinguished in stained preparations.

With the ultramicroscope at no time can there be seen elements other than the bacilli (whose number gradually diminish, to disappear entirely in about two hours) and the extremely fine granules. It can hardly be said that the latter represent formed elements. At the beginning the bacilli present a normal appearance. After forty-five to sixty minutes fine granules are seen, ever becoming more and more abundant within the interior of the bacterial cells. The number of bacterial cells containing granules also rapidly increases with a corresponding diminution in the number of normal bacilli.

Not all of the amorphous material seen in the stained preparation is to be seen under the ultramicroscope. Apparently, strongly imbibing water, it assumes the same refractile index as the medium. This amorphous débris is certainly composed of the "floccules" which remain after the rupture of the bacteria, floccules which hydrate gradually and which thus become invisible under the microscope even though they still take the stain. Neither in the stained preparation nor under direct examination can corroded bacteria be observed.

At the stage of the process when the number of refractile granules is the greatest the swelling of the bacteria, of which we have spoken, is particularly noticeable, and it is interesting to note that these distended bacteria are the ones which contain the greatest number of refractile corpuscles. The number of corpuscles reaches its maximum within those bacteria which are spherical and ready to burst.

What do the fine granules that can be seen under the ultramicroscope represent? While nothing can be affirmed with absolute assurance there is nothing to preclude the supposition that they represent the corpuscles of the bacteriophage, basing this upon the comparative examination of suspensions in which the number of corpuscles has 
previously been counted. By such a procedure it is found that in taking two cultures presenting a great difference in count, a parallelism is always to be noted between the counts and the number of granules observed.

It would likewise be well to recall what we have already seen with reference to the multiplication of the corpuscles, namely, that this multiplication appears to take place in successive jumps (which correspond to the rupture of a large number of parasitized bacilli) in which the number of corpuscles liberated after $1 \frac{1}{4}$ to $1 \frac{1}{2}$ hours corresponds to about 18 to each single one inoculated. And we will see that the number of granules consequent upon the rupture of a cell amounts to between 15 and 25. There is, therefore, a great probability that the granules are actually the ultramicroscopic bacteriophagous corpuscles.

We may consider a second case, that of a minimal inoculation. In this case the medium becomes more and more turbid before dissolution actually commences.

A suspension of Shiga bacilli, containing 250,000,000 per cubic centimeter is inoculated with $0.0001 \mathrm{cc}$. of a suspension of the bacteriophage, a very active race being selected.

After 30 minutes the medium has its original turbidity; essentially that of a normal culture of the Shiga bacillus.

After one hour the original turbidity is still maintained. When smeared and stained all the bacilli are of normal shape, but an occasional form stains poorly.

After two hours the culture is about twice as turbid as at first. There is amorphous débris in the bottom of the tube. All of the bacilli appear to stain normally. Many of the bacilli (about two in every three) are about four times the normal length, that is, of the bacilli used to seed the culture, and there are all intermediary forms. Oval and spherical forms are relatively numerous, but they are always fewer than would be expected from a comparative ultramicroscopic examination. These forms are indeed very fragile and are particularly liable to destruction during fixation upon the slide so that their demonstration in stained preparations requires great care.

After three hours the suspension is slightly cloudy. The bottom of the tube is covered with fine clébris without definite form, with, from place to place, great amorphous masses and numerous granules resembling those encountered in very old cultures of normally grown Shiga bacilli. Only a single spherical form can be detected in a ten- 
minute search. Each field may contain a dozen large bacilli, well stained.

After four hours the turbidity is very slight. There is somewhat less material in the bottom of the tube, and this shows only a single poorly stained bacillus to a field.

After six hours the medium is limpid. There is still less deposit in the bottom of the tube and it is with difficulty that a single poorly stained bacillus may be found in searching 25 fields.

After eighteen hours nothing at all can be seen in the preparation.

As is evident, the aspect of this preparation differs but little from that seen in the former case, the only departure being that the bacilli which have grown immediately after inoculation, before the action of the bacteriophage becomes operative, present abnormally large forms.

A comparable ultramicroscopic examination in the two cases shows that in the last, where the inoculation was made with a bacteriophage which was extremely active, at the time when dissolution occurs with greatest intensity, that is, between two and three hours after the inoculation, the spherical forms were present in greatest numbers. There were as many as two to three to a field, and their rupture was readily observed. When the bacteriophagic process is once terminated the most careful search fails to reveal such forms.

It is here fitting to recall an observation already made which should be noted by those wishing to investigate the subject. When a simple fermentative action is operative it proceeds with uniform rhythm when under identical conditions. This is not the case here. Up to the present time more than a hundred different races of the Shiga-bacteriophage have been isolated and no two of them have been found to conduct themselves in an exactly identical manner. The final result is always as has been indicated, the phases of the phenomenon always progress in the same order, but the time of the reaction will vary. With one race of the bacteriophage complete dissolution is obtained in three hours, with another, only after twelve hours. The phases follow each other in one case four times more quickly than in the other.

Another point which should be remembered is that all that which has been said up to the present time has been in reference to bacteriophagous races which were extremely active; that is to say, races capable of producing a complete dissolution of a normal suspension of bacteria.

A summary of the foregoing shows that, in so far as the microscopic observations are concerned, there is no time when one can distinguish 
in stained preparations, whatever the magnification, microörganisms other than B. dysenteriae.

We have already stated that corroded or partially destroyed bacteria, such as would necessarily occur if the dissolution was made from the outside inward, are never seen. Destruction always takes place by rupture. This is true not only for the dysentery bacillus but for all bacteria which undergo bacteriophagy. Furthermore, for all species the "microscopic" picture of the phenomenon is the same.

Examination under the ultramicroscope clearly indicates, then, that the bacteriophage corpuscles multiply within the interior of the bacterial cell, and it is possible that the very fine refractile granules, so small as to approach the limits of visibility with the dark-field, observed within the interior of the bacteria in process of being bacteriophaged represent these corpuscles. These granules are still visible in the floccules which float in the liquid for some time after the rupture of the bacteria. They cease to be visible when the floccules are completely dissolved.

This part of the discussion is, evidently, only an hypothesis, for as yet it is impossible to affirm that these fine corpuscles may not be due to changes in the bacteria. Nevertheless, the "coincidences" argue in favor of their bacteriophage nature.

The fact that these granules are only visible when within the bacteria and that they cease to be so when they are free in the medium is not a basic objection to this view. That the bacteriophage is of corpuscular nature is undoubtedly true; indeed the fact is no longer questioned.* We will see that its dimensions are essentially the same as those of the protein micella. Its diameter has been determined by Prausnitz in one way and by von Angerer in another, and both methods agree in placing the size at between 20 and 30 millicrons. If it is not visible under the ultramicroscope it is most certainly because of its strong power of imbibition; the same thing that prevents the protein micella from being visible, $\uparrow$ namely, because their refractile indices are essentially the same as that of the liquid in which they are suspended. But the index of refraction of the bacterium is certainly different from that of the liquid medium, as is shown by the fact that they are perfectly visible without staining. It follows therefore that the index of refrac-

* Doerr is the only author who is not quite convinced upon this point, although he does not deny it.

$\dagger$ Metallic micella, even those whose diameter is much less, are visible because the index of refraction differs from that of the liquid. 
tion of the bacteriophage corpuscle must be different from that of the substance of the bacterium within which it multiples. And it violates no fundamental principle to assume that the corpuscle can be visible when found enclosed within the substance of the bacterium or even in the floccules before they dissolve, and that it may cease to be visible just as soon as these floccules are dissolved. However this may be, the visibility of the bacteriophage corpuscle within the bacterium is only an hypothesis, but it is a plausible and possibly a probable hypothesis.

In concluding this section we may call attention to two facts which tend to show that, under the action of the bacteriophage, the electrical potential of the bacteria (which are, as we know, negatively charged) is diminished. In the first place it can readily be shown that their affinity for basic dyes is reduced, and in the second place, very frequently an agglutination takes place; the bacteria flocculate under the action of the bacteriophage. Flocculation results from an increase in the surface tension, associated with a reduction in charge.

\section{RÉSUMÉ}

The bacteriophage corpuscle is unable to multiply in any medium in the absence of living and normal bacteria. The bacterial cell constitutes the sole culture medium for the bacteriophage (d'Herelle ${ }^{310}$ ). An experiment of Wollmann ${ }^{632}$ suggests that development, to some degree, may occur in the presence of diffusible bacterial products.

The first act of bacteriophagy consists in the approach of the bacteriophage corpuscle toward the bacteria, then in the fixation of the corpuscle to the latter (d'Herelle $\left.{ }^{317}\right)$. The rapidity with which fixation takes place depends upon various factors; principally upon the degree of activity of the bacteriophage. Fixation is the more rapid the higher the virulence of the bacteriophage corpuscle.

The fixation is specific, that is to say, that it takes place only with susceptible bacteria (d'Herelle ${ }^{321}$ ), and it may occur even if the bacteria are dead (da Costa $\mathrm{Cruz}^{153}$ ). There is, however, an exception to this; the bacteriophage corpuscle fixes itself upon a bacterium naturally refractory to the action of this bacteriophage provided the latter attacks other strains of bacteria belonging to the same species (Janzen and Wolf $\left.{ }^{374}\right)$. This is not true for bacteria with an acquired resistance.

The bacteriophage corpuscle penetrates into the interior of the bacterial cell. When, as a result of its faculty of multiplication, the bacteriophage corpuscle which has penetrated into the bacterium forms 
a colony of a number of elements, the bacterium ruptures suddenly, liberating into the medium the young corpuscles which are then ready to continue the action (d'Herelle ${ }^{317,321}$ ).

The extent to which the bacteriophage may multiply in the course of the process of bacteriophagy, that is, the final titre of the suspension, depends upon various factors, but the factor having by far the greatest importance is the total number of bacteria capable of being bacteriophaged, and consequently available for serving as a "cuiture medium" for the bacteriophage corpuscles (d'Herelle ${ }^{321}$ ). 


\section{CHAPTER IV}

\section{The Virulence of the Bacteriophage}

\section{VARIATION IN THE ACTIVITY OF BACTERIOPHAGE CORPUSCLES}

Among the very first of the facts revealed by my early studies ${ }^{310,314}$ was the observation that bacteriophage principles, as isolated from natural sources, presented very considerable differences. Subsequent study has afforded abundant confirmation of this. With regard to their action upon a single bacterial strain different races of the bacteriophage possess differing degrees of activity. Just as there are races which provoke within a few hours a total dissolution of all of the bacteria to be found in a turbid suspension, so also there are other races of so low an activity that their presence can be detected only by the demonstration of the rare and minute plaques which they form upon agar.

Early in the first chapter the technic for disclosing the presence of the bacteriophage in different types of material was described. If, following this technic, a series of studies are undertaken for the purpose of isolating races of the Shiga-bacteriophage, for example, it will quickly become apparent that when bacterial suspensions, identical except for bacteriophage material, are inoculated with equal quantities of different filtrates a complete dissolution of the bacteria is not always obtained. The following experiment is ample to demonstrate this.

Inasmuch as the intestinal contents of animals,-- horses and fowl, in particular -almost always contain a bacteriophage active against $B$. dysenteriae Shiga, ${ }^{31{ }^{3}}$ procure a dozen specimens of feces from animals of these species. Prepare filtrates according to the method described (Chapter I) for working with such materials. At the same time prepare 12 tubes from a young agar culture of $B$. dysenteriae Shiga, each tube containing $10 \mathrm{cc}$. of a broth suspension having 75 million bacteria per cubic centimeter.* The turbidity of such a suspension is slight, yet the broth is definitely clouded. Add to each of the tubes 5 drops of one of

* I have shown that for such a study it is preferable to use suspensions containing but 75 to 100 million bacteria per cubic centimeter instead of bouillon cultures. 
the 12 filtrates. Place them in the incubator at $30^{\circ} \mathrm{C}{ }^{*}$ After incubation for 24 hours it will be found, as a usual thing, that some of the suspensions are limpid, indicating thus the presence of a very active bacteriophage. Other tubes are but slightly less clouded than the control suspension without added filtrate. Others are as turbid as the control, sometimes even more so. $\dagger$ Filter these cloudy, or turbid, suspensions through candles. Add 1 cc. of each of the filtrates to a tube containing 10 cc. of a suspension ( 250 million per cubic centimeter) of Shiga bacilli, and immediately spread 0.05 cc. of the mixture upon a plate or an agar slant. After incubation, some of these agar cultures will appear sterile; others will show confluent or isolated plaques. In some tubes the plaques will be large; in others small, even pin-point in size.

This experiment shows that different bacteriophage races, although active for a single strain of bacteria, present a whole range of potencies. Some of the races cause a prompt and complete dissolution of heavy suspensions; others can be detected only by the formation of minute plaques upon the agar. Between these extremes are all intermediate degrees of activity. Indeed, it is quite possible that there are still weaker races which escape detection because of an insufficiently delicate technic.

But, it may be said, we know that the bacteriophage principle is formed of corpuscles. May it not be that the differences in activity as manifested by different filtrates are due, not to a qualitative difference among the corpuscles, but rather to a difference in the number of corpuscles present within a given volume of the different filtrates? Is it not possible that the very active filtrates contain a large number of corpuscles, while those which are weak contain but few?

Two observations already recorded suffice to show that there is indeed a qualitative difference among the corpuscles. We have seen that as a matter of fact bacteriophagy may be complete in some instances if but a single very active corpuscle is introduced into the bacterial suspension. And yet in other cases, in the suspensions which remain turbid, there may be a great many corpuscles, as shown by the fact that a single drop

* At first ${ }^{312}$ I stated that the temperature should be $37^{\circ} \mathrm{C}$. More recently Hauduroy has suggested that it is preferable to allow the tubes to remain at room temperature. Taking into consideration the results obtained in all of the experiments performed it would seem that with weak races of bacteriophage the results are best when the temperature is held at $30^{\circ} \mathrm{C}$.

$\dagger$ In general, it appears that the feces of animals contain a more aetive bactcriophage in summer than in winter, and that the feeal bacteriophage is more aetive in hot countries than in cold regions. We will return to this subject in Part III. 
planted upon agar yields many plaques. This can only mean a qualitative difference. The many corpuscles present in the second case are not as powerful as the single very active corpuscle.

The second observation bearing upon the idea of a qualitative difference deals with plaque formation. Each plaque has its origin in a single corpuscle. The plaques vary in diameter with different races of the bacteriophage, and we know definitely that the formation of large plaques corresponds to races of the bacteriophage which cause a complete dissolution of the suspension, while those which do not dissolve the bacterial suspension completely yield little plaques. The more potent the corpuscle, the greater the area of the plaque (d'Herelle ${ }^{312}$ ).

The idea of qualitative variation among races of the bacteriophage receives additional support from the fact that everyone who has studied the phenomenon is in agreement upon this point.

But what is the real reason for this variation in activity among different races of the corpuscular bacteriophage? The following experiment contributes the answer. ${ }^{321}$

$A$. Ten cubic centimeters of a suspension of Shiga bacilli are inoculated with $1 \mathrm{cc}$. of a filtrate made directly from the feces of a patient with dysentery. The suspension is held at $37^{\circ} \mathrm{C}$. Counts of the corpuscles, made at different times during the incubation, give the following results when 0.01 cc. is plated on agar.

When plated immediately, 16 plaques develop, representing 1600 corpuscles per cubic centimeter. The filtrate from the feces therefore contained 16,000 per cubic centimeter.

After one and one-quarter hours, the count is 40 plaques, or 4000 per cubic centimeter.

After two and one-half hours, a 1:10 dilution gives 42 plaques, or 42,000 per cubic centimeter.

After three and three-quarter hours, a 1:100 dilution gives 18, or 180,000 per cubic centimeter.

After five hours, a 1:1000 dilution gives 4, or 400,000 per cubic centimeter.

After fourteen hours, the dissolution is not complete, the medium is cloudy and becomes more and more turbid, so that after forty-eight hours it is very turbid. Here there is an abundant culture, but the solution is never complete. The bacteria have, then, acquired a certain resistance which has allowed them to reproduce in spite of the presence of the bacteriophage.

A result of this kind is usual when the filtrate is prepared from a stool taken shortly before the manifestations of convalescence appear. 
B. Ten cubic centimeters of the Shiga suspension are inoculated with $1 \mathrm{cc}$. of the filtrate prepared from the feces from the same dysentery patient, but collected 24 hours later, the patient now being convalescent. Counts of this mixture give:

When plated immediately, no plaques, or less than 100 corpuscles per cubic centimeter. Thus, the filtrate contained less than 1000 per cubic centimeter.

After one and one-quarter hours the plating shows no plaques.

After two and one-half hours there are 9 plaques, or 900 corpuscles per cubic centimeter.

After three and three-quarters hours, in a 1:10 dilution, there are 27 plaques, or 27,000 per cubic centimeter.

After five hours, a 1:1000 dilution shows 13 plaques, representing $1,300,000$ per cubic centimeter.

In this last experiment (B) the corpuscles were present in the filtrate in very small numbers, certainly less than 1000 per cubic centimeter, that is, there were less than one-sixteenth as many as in the filtrate of the first preparation (A). Nevertheless, the suspension was completely dissolved in ten hours and the fluid remained sterile indefinitely.

It is unnecessary to insert here the many experiments made for the purpose of proving that the multiplication of the bacteriophage corpuscles is always proportionate to their activity. All have given results comparable to those presented above: The more active the bacteriophage the greater the multiplication of corpuscles. We have already seen in the preceding chapter that with a Shiga-bacteriophage of low activity a single corpuscle yielded only 17 millions after 24 hours, while under the same conditions, a single corpuscle of a very active race gave, in the same length of time, 12,000 million per cubic centimeter. With these two races the increase with the second is 700 times that of the first. With the Staphylo-bacteriophage I have observed an increase from 1 corpuscle to 780 millions with a race of average activity, and from 1 to 98,000 millions with a race that is very active. Here, with two races acting under the same conditions one is 125 times more active than the other. For but slightly active races figures still lower have been observed.

From these results it may be concluded that activity in the bacteriophage corresponds to the vigor with which it multiplies at the expense of susceptible bacteria (d'Herelle ${ }^{315}$ ).

This conclusion leads to an interesting deduction: the facts disclose a curious coincidence, not without significance. What do we mean by the 
virulence of a pathogenic bacterium? Simply the power to develop within and at the expense of the host, and we consider the degree of virulence to be the higher as this development is the more rapid. Logically, then, if these definitions are correct that which we have termed "activity" in a bacteriophage corpuscle represents a "virulence" in the strictest sense of the word (d'Herelle $\mathrm{e}^{310,313}$ ). It is evident, and not without interest, that the term "virulence" as applied to the bacteriophage is employed in this same sense by those authors (Otto, for example $)^{493}$ who still consider the bacteriophage to be a ferment.

\section{EVALUATION OF THE VIRULENCE OF A BACTERIOPHAGE}

The virulence of the bacteriophage being variable from one race to another, it is desirable to be able to express numerically the virulence of a given race. Let us consider the methods which have been proposed, and evaluate them with regard to their precision and their utility.

From the very first of my studies I have advocated and employed solely the method involving an enumeration of the corpuscles. To this end I have carefully spread upon an agar slant in a $22 \mathrm{~mm}$. tube, 0.02 cc. of a suspension of the susceptible bacterium containing 250 million bacteria per cubic centimeter inoculated with a dilution of the bacteriophage of such a titre that, after incubation, the plaques are isolated. $^{321}$ In some instances, as an alternative procedure, I have used Petri dishes, and in this case I have spread 0.05 cc., or 0.02 cc. of the suspension, according to the size of the plate. ${ }^{321}$ This method is the only one which should be employed for the study of the phenomenon of bacteriophagy if false interpretations, associated with poor methods of evaluating the bacteriophage, are to be avoided.

When it is not essential to obtain results of the greatest accuracy, for example, when it is desired simply to observe the variations in virulence shown by the bacteriophage as isolated from the body at different stages of a disease and during convalescence, a more simple and rapid method may be employed, based upon the development of bacteriophagy in a liquid medium and on the general appearance of agar sub-cultures. ${ }^{321}$

Since it will be necessary, in many cases throughout this discussion to indicate the relative degree of virulence possessed by a given race of the bacteriophage, it may be well to indicate here a method to express this virulence. This system is somewhat arbitrary, but it meets all practical needs, and will facilitate expression.

$0=$ no virulence toward a given bacterium. Normal cultures of the bacterium develop in bouillon or on agar, 
whatever the quantity of the filtrate from the feces which had been added.

$+=$ weak virulence. The growth in bouillon of the bacterium to which the filtrate has been added is apparently normal. Transfer of this culture to agar gives, after incubation, a culture layer showing a few minute plaques. Some of the bacteriophagous corpuscles have therefore attacked the bacteria and have formed colonies.

$++=$ medium virulence. The culture of the bacterium to which the filtrate has been added is almost normal in bouillon. Transfers of this culture to agar give, after incubation, either a culture layer of the bacterium studdled with very numerous colonies of the bacteriophage, presenting an appreciable surface area, or of fragments of bacterial culture because of the very great number of bacteriophage colonies.

$+++=$ high virulence. Dissolution of a bacterial suspension is obtained but secondary cultures constantly develop. The reinoculations on to agar remain sterile or give only rare colonies of the bacterium.

$++++=$ extreme virulence. The bouillon suspension shows complete, and, in general, permanent dissolution. Inoculations on to agar always remain sterile.

Obviously, it would be possible to establish a more detailed scale of virulence. In fact, this has been done in the curves which will be given in Part III of this text, where the interval between no virulence and extreme virulence has been subdivided into ten steps, in accordance with the aspect of the cultures, the number of colonies of the bacteriophage, and the size of the plaques, which bear a relation to its virulence. Practically, the appreciarion is adequate with four steps, particularly in view of the fact of the extreme variability of virulence in the bacteriophage in the body of a single individual from one time to another.

Perhaps the first method for determining virulence which we should consider is that of Appelmans. ${ }^{13}$ Objecting to the method of plaque counting on the grounds that colonies of the bacteriophage upon agarthe plaques -are sometimes difficult to see (an ill-founded objection) and that plaques may be confused with bare spots on the agar due to the method of distribution of the material during the spreading (a difficulty encountered only when the technic is poor) he has proposed a method 
analogous to that devised by Miquel for counting bacteria, that is, the procedure known as the "method of successive dilutions."

In accordance with this procedure $1 \mathrm{cc}$. of the bacteriophage suspension to be "titrated" is added to 9 cc. of bouillon. This gives an initial dilution of $1: 10$, each cubic centimeter containing $1 \cdot 10^{-1} \mathrm{cc}$. of the original suspension.* One cubic centimeter of this first dilution is removed and introduced into $9 \mathrm{cc}$. of bouillon. This second dilution is $1: 100$, containing per cubic centimeter $1 \cdot 10^{-2} \mathrm{cc}$. of the original suspension. One cubic centimeter of this dilution is then carried on into $9 \mathrm{cc}$. of bouillon, giving a third dilution, $1: 1000$, each cubic centimeter containing $1 \cdot 10^{-3} \mathrm{cc}$. of the original suspension. Continuing thus, by tens, the dilution up to the twelfth tube, a series of dilutions is obtained in which in each cubic centimeter of the individual tubes there is $1 \cdot 10^{-1}$, $1 \cdot 10^{-2}, 1 \cdot 10^{-3}, 1 \cdot 10^{-4}, 1 \cdot 10^{-5}, 1 \cdot 10^{-6}, 1 \cdot 10^{-7}, 1 \cdot 10^{-8}, 1 \cdot 10^{-9}, 1 \cdot 10^{-10}$, $1 \cdot 10^{-11}$, and $1 \cdot 10^{-12} \mathrm{ce}$. of the original suspension.

Having prepared these dilutions Appelmans next seeds each of them with a culture of the susceptible bacterium and allows bacteriophagy to proceed. The greater the dilution with which bacteriophagy takes place the more active was the original bacteriophage suspension.

This method has proved very attractive, doubtless because of its appearance of "mathematical" precision. In reality it has exerted an unfortunate influence upon the study of bacteriophagy, for this procedure is, in great part, responsible for the errors which have been committed by those who have employed it. Several circumstances render results obtained by this method invalid, among which we may mention the following.

1. The method can not be employed for measuring the virulence of a race of bacteriophage of weak virulence. The difficulty here rests in the fact that such a bacteriophage in no way inhibits the development of bacteria. The presence of such races in a fluid medium can be disclosed only by the development of plaques when this fluid is spread upon agar. ${ }^{315}$ I am aware that many authors (Otto, ${ }^{493}$ and later Doerr ${ }^{185}$ and their collaborators) have supported the reverse opinion, namely, that a bacteriophage of weak virulence may be detected by "lysis" in bouillon when plaques are lacking. But these authors have fallen into a double error, as will be pointed out shortly.

* Obviously, one might take $4.5 \mathrm{cc}$. of boullion and add to it $0.5 \mathrm{cc}$. of the bacteriophage suspension. Then proceding by removing $0.5 \mathrm{cc}$. of this first dilution and introducing it into $4.5 \mathrm{cc}$. of bouillon, a second dilution would be obtained. Continuing thus a series could be prepared in all respects comparable to the series described. 
2. With bacteriophage races of average activity "secondary cultures" (of which we will speak in the next chapter) develop, and where this is the case the method of dilutions gives results which are entirely false.

3 . For very virulent bacteriophages the precision of the method is in inverse proportion to the degree of dilution. Yet, with such races, it is in the high dilutions that precision is most essential. As illustrating this, here is a titration of a suspension of Staphylo-bacteriophage made by the dilution method. Duplicate titrations are made.

First titration. The last dilution in which bacteriophagy takes place is $1 \cdot 10^{-10}$. From this one might conclude that in $1 \cdot 10^{-10} \mathrm{cc}$. there is but one corpuscle, and that the original suspension contained, therefore, 10,000 million per cubic centimeter.

Second titration. In this the last dilution to show bacteriophagy is $1 \cdot 10^{-11}$. Applying the same reasoning, this time the suspension should contain 100,000 million per cubic centimeter. Obviously these two determinations present a very considerable discrepancy.

By means of counts of the colonies of the bacteriophage developing on agar we find an explanation of the difference. As a matter of fact these counts show that the original suspension contained 81,000 million corpuscles per cubic centimeter. There were in reality 8 corpuscles in the $10 \mathrm{cc}$. of the $1 \cdot 10^{-10}$ dilution. Consequently, the $1 \cdot 10^{-11} \mathrm{dilu}$ tion shows, or does not show, bacteriophagy, depending upon whether the particular fraction removed from the $10^{-10}$ tube contained, or did not contain, a corpuscle.

In the first dilutions, the error resulting from this fact is negligible. It would be a maximum of 90 corpuscles between $10^{-1}$ and $10^{-2}$, of 900 corpuscles between $10^{-2}$ and $10^{-3}$. But it becomes enormous in the higher dilutions. The error may amount to 900 million between $10^{-8}$ and $10^{-9}$; to 9000 million between $10^{-9}$ and $10^{-10}$; and to 90,000 million between $10^{-10}$ and $10^{-11}$.

This error of technic makes it readily apparent that errors of interpretation of considerable significance may result from the use of such a method, a fact all the more unfortunate since the procedure has an atmosphere of precision. We have mentioned in the preceding chapter one of the mistakes made by Doerr in relation to the multiplication of bacteriophage corpuscles.

With reference to this method it is interesting to cite an observation of Gratia and de Kruif ${ }^{272}$ which, to them, seemed "curious," although in reality it is not so strange. These authors prepared a series of increasing dilutions of a suspension of Coli-bacteriophage, and found the 
last active dilution to be $10^{-5}$. Working with these dilutions in a volume of $5 \mathrm{cc}$., they removed $4 \mathrm{cc}$. from the last active dilution and distributed it as follows:

a. One cubic centimeter into a sterile tube.

b. One cubic centimeter into a tube with $10 \mathrm{cc}$. of bouillon.

c. One cubic centimeter into $100 \mathrm{cc}$. of bouillon.

d. One cubic centimeter into $1000 \mathrm{cc}$. of bouillon.

The four specimens were then seeded with a culture of $B$. coli. Bacteriophagy took place in all of them. It seemed strange to Gratia and de Kruif that bacteriophagy should occur in the flask containing 1000 cubic centimeters, where the "concentration of the lytic principle," according to their expression, was only $10^{-11}$ when it did not take place in the $10^{-9}$ dilution when working with 5 cc. quantities. Inasmuch as a long time before this I had shown that bacteriophagy takes place if but a single corpuscle is added to a bacterial suspension, and that bacterial dissolution does not occur if this corpuscle is lacking (a condition recalling the "all-or-none law"), the results of Gratia and de Kruif should not have appeared so unusual.

Incidentally, the term "concentration" as applied by these authors to the bacteriophage principle is an expression without significance, and it can only lead to confusion when used in this connection.

Werthemann ${ }^{626}$ has proposed a scheme of notation designed to express the "concentration" of the bacteriophage principle. According to this scheme "concentration" is indicated by the exponent of the last active dilution. For example, in the experiment of Gratia and de Kruif already mentioned, the Coli-bacteriophage would be termed active up to the concentration $1 \cdot 10^{-8}$, or $e_{L} S$ according to the scale of Werthemann. Since concentration is not the dominating factor in the phenomenon of bacteriophagy one might well question the significance of such a notation. Not only does it aggravate the defects inherent in the method of titration by dilution, but it adds a false conception. To ascertain how invalid such a method is I would recommend that those who employ the procedure repeat the experiment of Gratia.

Beckerich and Hauduroy ${ }^{57}$ have proposed the following method. Each of a series of tubes receives 1 cc. of a young culture of the susceptible bacterium. The bacteriophage suspension whose activity is to be measured is then added in decreasing quantities - perhaps 1:3 in the first tube, $1: 5$ in the next, up to a dilution equal to 3 parts in $10,000,000,000$. A portion of the contents of each tube is spread over an agar plate, while the remainder is retained in the tube to indicate what takes place in a fluid medium. 
After incubation at $37^{\circ} \mathrm{C}$. they note the presence or absence of plaques on the plates, and designate the result by $\mathrm{P}_{1}, \mathrm{P}_{2}, \mathrm{P}_{3}$, etc., according to the number of plaques. If plaques are abundant they use the terms many, or very numerous. As for the results in the fluid media; $\mathrm{L}_{0}$ indicates no dissolution, $\mathrm{L}_{1}$ means a slow and partial dissolution, $\mathrm{L}_{2}$ is a partial dissolution, and $\mathrm{L}_{3}$ implies a complete solution.

Here is an example cited by them, showing that a weak bacteriophage can not be measured by the dilution method. Indeed, it may not even be detected. Expressed according to this scheme, using a bacteriophage very weakly active for $B$. paratyphosus $B$ the results were:

$$
\begin{aligned}
& \text { Dilution, } 1: 3 ; \mathrm{P}_{\text {many }}, \mathrm{L}_{0} \\
& \text { Dilution, } 1: 30 ; \mathrm{P}_{\text {rare }}, \mathrm{L}_{r} \\
& \text { Dilution, } 1: 700 ; \mathrm{P}_{0}, \mathrm{~L}_{0}
\end{aligned}
$$

This method of Beckerich and Hauduroy, based upon the principle which I have advocated ${ }^{321}$ determines simultaneously both the dissolving power in a fluid medium and the formation of plaques upon a solid medium, and it permits an approximately accurate titration, disclosing bacteriophage races which would remain undetected by the dilution method alone. Nevertheless, there is one criticism which may be made to the work of Beckerich and Hauduroy. In certain of their titrations with potent Shiga-bacteriophages it appears that the formation of plaques does not always follow when a quantity of suspension, most certainly containing corpuscles, is spread over the agar. I have carried out some thousands of titrations, and I have never observed this sort of a result. It must be due to some error in their experiments which remains undetected.

Janzen and Wolff ${ }^{374}$ effect a titration employing the following technic.

1. One drop of the suspension of bacteriophage to be titrated is introduced into a culture of the susceptible bacterium.

2. A tube of bouillon lightly seeded with culture is also inoculated with 1 drop of bacteriophage suspension.

3. Immediately after the mixture is made 1 drop of culture no. 1 is spread over an agar plate.

After incubation the results with culture no. 1 are recorded as ++++ (complete dissolution),,+++++ , or + (partial dissolution), or finally as \pm if the dissolution is hardly perceptible. With culture no. 2, (which is a seeded bouillon, not a turbid suspension) no growth is expressed by ++++ , very weak, and weak growth by +++ and ++ , and growth but slightly less than the control by + . Culture no. 3, designed to show plaque formation on agar, gives results 
recorded as ++++ , meaning sterility, +++ , meaning confluent plaques, ++ , many plaques, or + , indicating that plaques are few.

This is an excellent method for approximating quickly the degree of virulence of a bacteriophage, but it is not sufficiently precise for a study of the detailed features of the process of bacteriophagy. The authors who devised the method have used it for studies to which we will give considerable attention, and for which the method yields fully adequate results.

Pfreimbter, Sell and Pistorius ${ }^{503}$ follow a procedure which consists in adding a drop of the bacteriophage suspension to be titrated to a suspension of the susceptible bacterium and spreading a definite quantity of this mixture upon agar plates, immediately after preparing the mixture, and again after intervals of 3, 6, and 24 hours. After the plates are incubated the presence of plaques and the number found provide the significant data.

This is a very good method, especially for comparing the virulences of races that are weak. With highly active races it is necessary to work with dilutions.

According to the technic of Otto and Munter, ${ }^{496}$ a culture of the susceptible bacterium is spread over the surface of an agar plate and upon this surface drops of the suspension to be titrated, diluted to different degrees, are deposited. After incubation the areas where the drops of bacteriophage were deposited are observed and the results are recorded as 0 , meaning a normal bacterial culture, that is, no bacteriophage, \pm , indicating a few plaques, ${ }^{*} t_{1}$ and $t_{2}$ implying that plaques are numerous or confluent, $t_{3}$ means that only isolated bacterial colonies develop, and $+_{4}$ denotes that the surface of the asar is sterile. A record of such a titration, employing this notation, is:

$$
\begin{aligned}
& \text { Dilution, } 1: 1000,+_{4} \\
& \text { Dilution, } 1: 1,000,000,+_{2} \\
& \text { Dilution, } 1: 100,000,000 \pm
\end{aligned}
$$

In effect, this procedure is simply a modification of the method of counting colonies of the bacteriophage. But it lacks accuracy, and it is precisely this defect which has caused those who devised the method to arrive at certain erroneous deductions. ${ }^{493}$ For example, they observe that when greater and greater dilutions of a fluid containing the bacteriophage are made, a degree of dilution is reached where spreadings no longer show plaques, although in this dilution active principle is un-

* A rather curious notation, for the sign \pm usually indicates a doubtful result. If there are plaques, there is no chance for doubt, the bacteriophage is present. 
doubtedly still present, since it may be revealed by passages. The cause for this difficulty is readily explained. Let us suppose that the limiting dilution is one where there is but a single corpuscle in the whole volume of the dilution. This is, as we know, sufficient to cause bacteriophagy. Let us remove a drop of this dilution and spread it upon agar. Either the corpuscle will be in the drop taken or it will remain in the rest of the fluid in the tube, and naturally, the second possibility is the greater. What will happen? In the first case we will have a plaque when the spreading is made on the agar, and as no bacteriophage corpuscles will be left in the dilution bacteriophagy will not take place there. The deduction here would be that bacteriophagy in the fluid medium does not take place when but few corpuscles are present, even though they are very active. In the second case, it may be concluded, as Otto and Munter decided, that bacteriophagy in a fluid medium takes place, even when plaques do not appear after the material is spread upon agar. Both conclusions are equally false, and a defective method of titration is responsible for the errors.

Maitland ${ }^{432}$ has proposed a method of titration also based upon the principle of counting the plaques found on agar. He prepares 6 dilutions, from $10^{-1}$ to $10^{-\beta}$, in suspensions of the susceptible bacterium. A fixed and uniform quantity of each of these dilutions is spread upon agar, and after incubation he denotes the appearance of the agar cultures by numbers, as follows:

$$
\begin{aligned}
& 1=1 \text { to } 10 \text { plaques } \\
& 2=10 \text { to } 20 \text { plaques } \\
& 3=\text { numerous plaques } \\
& 4=\text { confluent plaques } \\
& 5=\text { culture broken up into irregular masses } \\
& 6=\text { traces of culture growth } \\
& 7=\text { culture débris } \\
& 8=\text { isolated colonies } \\
& 9=\text { but a single colony, or medium sterile }
\end{aligned}
$$

Here is an example of such a titration, showing how, by repeated platings he observes the multiplication of the bacteriophage in the course of bacteriophagy. The titration is performed with a culture of $B$. dysenteriae 24 hours old, to which is added an appropriate quantity of the filtrate prepared from the feces of a patient convalescent from bacillary dysentery. From time to time (as indicated) specimens of the mixture are removed, dilutions are made, and agar plates are spread. The results obtained are given in table 13 . 
This method is certainly a good one, but it would be still more accurate and much more simple if the number of plaques, that is to say the number of bacteriophage corpuscles, was plainly stated.

Such are the different methods proposed. Can it be assumed that any of them permit a precise evaluation of the virulence of a bacteriophage? A logical method of titration must take the facts into consideration; it must recognize the physical state of the bacteriophage as well as its behavior. We know several facts which should afford a basis for a proper method of titration. Thus, we know (1) that the bacteriophage exists in the form of corpuscles, and (2) that it is possible to enumerate these corpuscles. We also know (3) that the corpuscle possesses a "virulence" in the true sense of the word, that is, it possesses

TABLE 13

\begin{tabular}{|c|c|c|c|c|c|c|}
\hline \multirow{2}{*}{ SPECIMEN REMOVED } & \multicolumn{6}{|c|}{ DILUTIONS PLATED } \\
\hline & $10^{-1}$ & $10^{-2}$ & $10^{-3}$ & $10^{-4}$ & $10^{-5}$ & $10^{-6}$ \\
\hline Immediately $\ldots \ldots \ldots \ldots \ldots \ldots \ldots \ldots$ & 3 & 0 & 0 & 0 & 0 & 0 \\
\hline 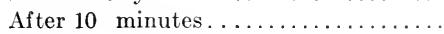 & 1 & 1 & 0 & 0 & 0 & 0 \\
\hline After 1 hour............... & 1 & 0 & 0 & 0 & 0 & 0 \\
\hline 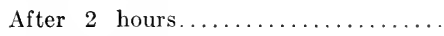 & 2 & 0 & 0 & 0 & 0 & 0 \\
\hline After $3_{4}^{\frac{3}{4}}$ hours......... & 6 & 5 & 1 & 1 & 0 & 0 \\
\hline After $5 \frac{1}{2}$ hours..... & 8 & 7 & 5 & 3 & 1 & 1 \\
\hline After $8 \frac{1}{4}$ hours....... & 8 & 8 & 4 & 1 & 1 & 0 \\
\hline After $11_{4}^{\frac{3}{4}}$ hours $\ldots \ldots \ldots$ & 8 & 8 & 4 & 3 & 1 & 1 \\
\hline After $25 \frac{1}{2}$ hours......... & 8 & 8 & 7 & 5 & 3 & 1 \\
\hline After 7 days.......... & 8 & 8 & 6 & 5 & 2 & 1 \\
\hline
\end{tabular}

the faculty of multiplying at the expense of bacteria; and (4) that the virulence of bacteriophage corpuscles is variable, the degree of virulence of different corpuscles being strictly proportional to the rapidity with which each race multiplies.

From these facts, based upon the experiments detailed in the preceding chapters, it follows that if we inoculate a series of tubes containing identical suspensions of a susceptible bacterium with equal quantities of bacteriophage suspensions derived from different sources, we will find, after a given time, the conditions throughout being the same, that the resulting bacteriophage suspensions will each contain a different number of corpuscles. These numbers will be strictly proportional to the virulence of the different bacteriophages.*

* It may be noted that if it were possible to effect the same operation with a pathogenic bacterium we could likewise obtain a numerical expression of viru- 
In view of the nature of the bacteriophage and considering the principles governing its reproductive activity it would seem that the following technic should serve best for determining the rate of multiplication of a race of the bacteriophage.

Take a series of 12 tubes, each containing $4.5 \mathrm{cc}$. of the bacterial suspension with respect to which the virulence of the bacteriophage is to be measured. This suspension should be prepared from a fresh 24hour agar culture of a known susceptible strain, and should contain 100 million bacteria per cubic centimeter. Introduce $0.5 \mathrm{cc}$. of the bacteriophage suspension (whether it be fecal filtrate, bacteriophage culture, or what not) into the first tube of the series. Shake thoroughly. This will give an initial dilution in which each cubic centimeter will contain $1 \cdot 10^{-1} \mathrm{cc}$. of the original suspension. Remove* $0.55 \mathrm{cc}$. of this $10^{-1}$ dilution and introduce $0.5 \mathrm{cc}$. into a second suspension, giving thus a dilution of $10^{-2}$. Spread the 0.05 cc. remaining in the pipette over an agar plate. $\dagger$

After carefully shaking the second dilution remove 0.55 cc. and spread

lence. Thus, with 2 strains of $B$. pestis we could inoculate 2 guinea pigs with a like quantity of the 2 cultures. After a given time, for example, 48 hours, if it were possible to determine the exact number of plague bacilli present in each of the 2 pigs, we would have 2 figures which would represent the relative virulences of the strains. Such a determination would be infinitely more exact than the usual method, based upon the time factor or upon the lethal dose.

* Various authors have observed that it is necessary to use a fresh sterile pipette for each successive dilution. This is self-evident, and I have always followed this procedure, for it is obvious, since the bacteriophage is corpuscular, that if but one single corpuscle remains adherent to the wall of the pipette it will suffice to completely falsify the result. This would be much less significant if the bacteriophage were soluble, like an antibody, for example.

$\dagger$ It is essential that neither the surface of the agar nor the cover of the Petri dish contain excess moisture which will mix with the drop of suspension and thus form in effect a fluid medium in which the bacteriophage can develop. This would give an entirely erroneous result. To avoid such an excess of water of condensation the agar should be cooled to 50 to $55^{\circ}$ before it is poured into the plates, and as additional precautions, when the agar has solidified the covers of the plates should be replaced by fresh sterile covers, and the plates then placed in the incubator until the next day. In this way the agar becomes well dried out and the drop of fluid placed upon the surface evaporates rapidly, thus preventing growth of the corpuscles in the drop of liquid which otherwise would persist for a considerable time before evaporation. It is well to bear in mind the fact that a liberal amount of agar should be used; the layer in the plates should have a depth of at least $3 \mathrm{~mm}$. If the agar is too thin the plaques will be too small, and will form poorly. The reason for this has been discussed. 
$0.05 \mathrm{cc}$. on another plate, inoculating the $0.5 \mathrm{cc}$. into a third suspension. This will give a $10^{-3}$ dilution. If there is reason to believe that the race is very active continue in this same way up to the twelfth tube (dilution $10^{-12}$ ). If the race is of but moderate potency it is unnecessary to go beyond the tenth dilution. It is also unnecessary to prepare plates corresponding to the last two dilutions, $10^{-11}$ and $10^{-12}$, or, if the race is of less virulence, those corresponding to the $10^{-9}$ and $10^{-10}$ dilutions. In these extreme dilutions the number of corpuscles is too small for them to appear, except by accident, in the $0.05 \mathrm{cc}$. spread over the agar.

I would recommend placing the suspension dilutions in the incubator at $32^{\circ} \mathrm{C}$. To obtain plaques of the largest possible diameter, and for this reason more readily counted, even in the case of weakly active bacteriophage races* it is preferable to hold the plates at a temperature of $20^{\circ}$ to $22^{\circ} \mathrm{C}$., in an incubator such as is used for gelatin media.

After incubation the series of dilutions will permit an evaluation of the intensity of bacteriophagy in relation to the dilution of the active principle. But this is but a tentative measure, the less trustworthy in that in many cases "secondary cultures" associated with the phenomenon of bacterial resistance, develop, rendering such a titration completely false and obscuring the true result. The exact measure of virulence can be obtained only by counting the colonies of the bacteriophage. In the series of plates there are always two, sometimes three, plates upon which the plaques are separated and easy to count. Since the quantity of suspension spread upon the surface and also the degree of dilution are known, it is easy to calculate the number of corpuscles contained in the original suspension.

This method, so well suited to virulence determinations, is also the most practical procedure for studying the various phases of the phenomenon of bacteriophagy. If one wishes to follow, for example, the course of the phenomenon under definite determined conditions, it is only necessary to remove at suitable intervals $0.5 \mathrm{cc}$. of the medium in which bacteriophagy is taking place and to carry out the technic which has been outlined in order to know the number of corpuscles present at the moment when the specimen was removed.

Can the method be used to measure the relative degrees of virulence of different bacteriophages, when by serial passages we know that the materials in question contain the active principle? Here is a typical

* We have seen that the diameter of the plaque is proportionate to the virulence, at least when the bacteriophage is acting upon strains of the same bacterial species. 
example, involving virulence determinations of three Shiga-bacteriophages derived from different sources; the first isolated from the feces of a fowl, the second from the feces of a horse, both having undergone but a single culture passage ( + in potency), the third isolated in 1916 from the stools of a convalescent from dysentery, and since that time subjected to about 2000 culture passages.

Take 3 tubes, each containing $10 \mathrm{cc}$. of a suspension of Shiga bacilli with 250 million organisms per cubic centimeter. Inoculate $0.01 \mathrm{cc}$. of the suspension of bacteriophage No. 1 into the first, 0.01 cc. of bacteriophage No. 2 into the second, and the same quantity of bacteriophage No. 3 into the third. After 24 hour's at $32^{\circ} \mathrm{C}$. examination shows that suspension No. 1 is cloudy, suspension No. 2 is slightly cloudy, and suspension No. 3 is clear. Filter the contents of the three tubes through Chamberland $\mathrm{L}_{3}$ candles, yielding thus 3 bacteriophage suspensions. Remove $0.55 \mathrm{cc}$. of suspension No. 1 and make a series of 10 dilutions, with 8 platings. This should be adequate to measure the activity for this race is of but low potency. For races Nos. 2 and 3, make the larger series of 12 dilutions and 10 spreadings. After incubation the results are as follows:

I. Dilutions. After incubation at $32^{\circ} \mathrm{C}$. for 24 hours the tubes containing fluid media with successive dilutions of the filtrates show:

Bacteriophage No. 1; the first 2 dilutions are cloudy, the others turbid.

Bacteriophage No. 2 ; the first 4 dilutions are clear, the others cloudy.

Bacteriophage No. 3; the first 5 dilutions are clear, the remainder cloudy.

After incubation for 48 hours:

Bacteriophage No. 1 ; all tubes are turbid.

Bacteriophage No. 2; the first 5 are strongly clouded, the others turbid.

Bacteriophage No. 3 ; the first 9 are clear, the last 3 turbid.

It is obvious that these findings do not express the virulences of the 3 races in a precise manner.

II. Platings. These are incubated for 3 days at $20^{\circ} \mathrm{C}$., at the end of which time the readings can readily be made, and are as follows:

Bacteriophage No. 1.

Plating of dilution $10^{-1}$; bacterial layer abnormal, roughened.

Plating of dilution $10^{-2}$; bacterial layer abnormal, roughened.

Plating of dilution $10^{-3}$; bacterial layer abnormal, roughened. 
Plating of dilution $10^{-4}$; bacterial layer smooth, with 141 small plaques.

Plating of dilution $10^{-5}$; layer with 13 plaques (diameter about $0.25 \mathrm{~mm}$.).

Plating of dilution $10^{-6}$; normal culture without plaques.

Platings of dilutions $10^{-7}$ and $10^{-8}$; normal culture without plaques.

Computation upon the basis of the above would indicate that the original suspension contained 26,000,000 corpuscles per cubic centimeter.*

Bacteriophage No. 2.

Plating of dilution $10^{-1}$; isolated colonies.

Plating of dilution $10^{-2}$; scattered traces of growth with isolated colonies in the open spaces.

Plating of dilution $10^{-3}$; the same, except that the fragments of culture are somewhat more extensive.

Plating of dilution $10^{-4}$; the same, still more culture evident. Plating of dilution $10^{-5}$; culture layer with numerous plaques. Plating of dilution $10^{-6} ; 27$ plaques (diameter, $3 \mathrm{~mm}$.).

Plating of dilution $10^{-7} ; 3$ plaques.

Plating of dilution $10^{-8} ; 1$ plaque.

Plating of dilution $10^{-9}$; normal culture without plaques.

Plating of dilution $10^{-10}$; normal culture without plaques.

The original suspension of bacteriophage No. 2 contained therefore, $540,000,000$ corpuscles per cubic centimeter.

Bacteriophage No. 3.

Platings of dilutions $10^{-1}$ to $10^{-3}$; plates sterile.

Platings of dilutions $10^{-4}$ and $10^{-5}$; traces of culture.

Plating of dilution $10^{-6}$; confluent plaques.

Plating of dilution $10^{-7} ; 38$ plaques $(7 \mathrm{~mm}$. in diameter).

Plating of dilution $10^{-8} ; 5$ plaques.

Platings of dilutions $10^{-9}$ and $10^{-10}$; normal culture without plaques.

The original suspension of this race contained, then, $7,600,000,000$ corpuscles per cubic centimeter.

The number of corpuscles in the three suspensions being respectively 26, 540, and 7600 million per cubic centimeter after bacteriophagy has continued in identical suspensions for the same length of time obviously means that these figures represent the power of multiplication of the

* $0.05 \mathrm{cc}$. of dilution $10^{-5}$ containing 13 corpuscles, 1 ec. would contain 20 times as many, or 260 . The original suspension would then contain $260 \times 10^{5}=$ $26,000,000$. 
corpuscles of each of the three races. And since virulence, by common consent, is considered as an expression of the power of multiplication in a foreign host, the respective virulences of the three bacteriophage races above could be represented by the ratios:

$$
\frac{26}{26}: \frac{540}{26}: \frac{7600}{26}=\text { approximately } 1: 20: 300
$$

If the increase in the number of bacteriophage corpuscles taking place during bacteriophagy is determined for several bacteriophages of different virulences it becomes obvious that the numbers derived will be proportionate to the rate of bacteriophagy with each. Such a deduction is, of course, obligatory. The contrary could not be comprehended, since insofar as the study of bacteriophagy is concerned the single method permitting the recognition of the behavior of the bacteriophage consists in determining the virulence by means of the increase in the number of corpuscles. From the practical point of view it is convenient to designate the intensity of the virulence of a bacteriophage by the rate of the phenomenon which it causes. Such a procedure is, indeed, quite logical since there is always a parallelism between the rate at which the corpuscles increase and the rate of bacteriophagy.

Different degrees of virulence may be designated by the following terms: the determinations being made always in bouillon having a $\mathrm{pH}$ of 7.6 to 8.0 , and the temperature being between 30 and $32^{\circ} \mathrm{C}$.

Maximum virulence. Single corpuscles inoculated into 10 cc. of a normal suspension (each cubic centimeter containing 250 million bacteria derived from an 18 to 24 hour culture on agar) cause complete bacteriophagy. The suspension of the bacteriophage resulting remains clear indefinitely provided the suspension is held at a temperature lower than $32^{\circ} \mathrm{C}$. This virulence corresponds to a multiplication of such an order that for each corpuscle inoculated the final number is greater than 10,000 million per cubic centimeter. It may be mentioned that this number is apparently fixed, that is to say, it does not apply to bacteriophagy of any particular bacterial species. Whatever the race of bacteriophage this titre of multiplication is that of a race of maximum virulence.

Very high virulence. Here the phenomenon is the same as in the above case, except for the fact that the suspension of corpuscles resulting from bacteriophagy when a normal suspension is inoculated with a single corpuscle becomes clouded again through the development of a secondary culture. On the other hand, secondary cultures do not usually 
form in suspensions where bacteriophagy results from the dissolution of a normal suspension by a large number of corpuscles (a million or more). Here the virulence corresponds to a multiplication such that for each corpuscle inoculated into the $10 \mathrm{cc}$. of normal suspension, there is an end result between 5000 and 10,000 million per cubic centimeter.

High virulence. Here again the phenomenon is the same, with the exception that with races of this type secondary cultures are the rule, quite without regard to the number of corpuscles inoculated. The virulence corresponds to an increase whereby a single corpuscle in the course of the action becomes between 1000 and 5000 million per cubic centimeter.

Moderate virulence. Bacteriophagy is never complete when a single corpuscle is inoculated. When the inoculation involves a million or more corpuscles complete bacteriophagy of a normal suspension results. The development of secondary cultures is constant. The virulence here represents an increase in the number of corpuscles to such an extent that for each one originally present there are at the end of the process between 100 and 1000 million per cubic centimeter.

Weak virulence. Bacteriophagy is never complete whatever may be the number of corpuscles inoculated, even though the suspension contains very few bacteria. The opacity of the suspension may be reduced to a greater or less degree. If complete bacteriophagy by any chance takes place the number of corpuscles is always below 100 million per cubic centimeter.

Very weak virulence. With such strains no clearing of the medium can be observed. The presence of the bacteriophage can be disclosed only by the development of plaques on an agar medium. The rate of increase of corpuscles is very low; after 24 hours only a few hundred per cubic centimeter are to be found.

\section{PURE RACES OF THE BACTERIOPHAGE}

Different races of the bacteriophage may be isolated which possess virulences for bacteria belonging to different species. On the other hand, the degree of virulence for each of the bacteriophages possessing an activity for a given bacterium is in its very nature variable. As a matter of fact, among the hundreds of bacteriophage races which I have isolated I have yet to find two which are exactly alike. ${ }^{315}$ It is, therefore, evident that there are under natural conditions an infinite number of different races of bacteriophage corpuscles.*

* This, of course, has no direct bearing upon the question of the unicity of the bacteriophage; a question which will be discussed in a later chapter. 
When we filter a fecal suspension, a specimen of river water, or even tap-water, we obtain a filtrate which is a suspension of bacteriophage corpuscles, but there is no reason for affirming that all of the corpuscles present belong to the same race. Indeed, it is certain that quite the contrary is true. Therefore, it is essential, especially for a study of the processes of bacteriophagy, to isolate the corpuscles so that one may work with strictly pure races, the issue of a single corpuscle.

In order to accomplish such an isolation we have only to imitate bacteriological methods. For example, assume that we have a material containing, aside from a variety of banal bacteria, a very few organisms pathogenic for a given animal, such as the guinea pig. How would we obtain a pure culture of this pathogen? We would inoculate a guinea pig with some of the material, the pathogenic bacterium would multiply within the body of the animal and at the death of the animal we would find a pure culture of the bacterium in the blood or in some lesion. But it is evident that if the initial material contained two bacteria pathogenic for the particular animal used the two organisms might be recovered in a mixed culture. If we wish to separate all of the bacteriophages which may be found in a stool filtrate virulent for a given bacterial species, B. typhosus for example, eliminating all of the races which do not possess a virulence for this bacterium, we have only to adopt an analogous method.

Inoculate $0.1 \mathrm{cc}$. of this filtrate into $10 \mathrm{cc}$. of a $B$.typhosus suspension. After 24 hours, bacteriophagy having taken place and the Typhobacteriophage corpuscles having developed, filter through a candle. Inoculate $0.01 \mathrm{cc}$. of this filtrate into a fresh suspension of B. typhosus. Incubate, filter, and continue such passages. After a sufficient number of these have been carried out we may be sure that, because of the extreme dilution brought about through the passages, no trace of the original filtrate from the stool will remain. At this time all of the corpuscles present will obviously have reproduced at the expense of the typhoid bacillus; that is to say, there will be present only those resulting from the development of corpuseles present in the initial filtrate virulent for B. typhosus. None of the corpuscles of the original filtrate which were virulent for other bacterial species and non-virulent for B. typhosus will be recoverable after a certain number of transfers. At whose expense could they have multiplied? And if they have not multiplied they have necessarily disappeared when the degree of dilution has reached a certain limit, readily calculable.*

* Determination of the limiting dilution in which there no longer remains a 
This method of purification by successive passages is the first one that I employed. ${ }^{315}$ But this method permits, however, the reasonable objections that although it may be true that all of the bacteriophage corpuscles avirulent for the bacterium at the expense of which the passages are made become eliminated it is none the less true that not all of those which are virulent and which have multiplied necessarily belong to the same race. As a matter of fact it is quite possible to assume that in a filtrate of feces or of water not all of the corpuscles virulent for a given bacterium are derived from a single corpuscle. According to this, then, in order to be sure of working with a pure race it is necessary, as is the case in bacteriology, to start with a single corpuscle. There are two methods which will permit this; the method of dilution in liquid media of Pasteur, and the method of colony isolation of Koch.

We have seen that when we make serial dilutions, by tens, of a suspension of the bacteriophage and add susceptible bacteria to each of these dilutions bacteriophagy occurs up to a certain dilution. In the higher dilutions it does not take place. If we prepare a second series of comparable dilutions in sterile bouillon and distribute the 10 cubic centimeters of the last active dilution among 10 suspensions of the susceptible bacterium, adding $1 \mathrm{cc}$. to each suspension, bacteriophagy will take place in some of these 10 suspensions, wherever a single bacteriophage corpuscle had been present. To be more certain of attaining the desired end we may filter one of these suspensions and repeat the same operation. This can be repeated even a third time. Such is the method of "successive dilutions" which I have most frequently employed. I prefer it to that of plaque isolation, for reasons which will be explained later. Let us observe that with very virulent bacteriophages determination of the presence or absence of bacteriophagy is simple, inasmuch as when it is present the suspension becomes completely cleared. With less virulent bacteriophage races the formation of "secondary cultures" frequently masks the clearing of the medium. Here, in order to ascertain whether bacteriophagy has occurred it is necessary to spread the suspected suspension on agar and the culture developing there will provide the answer.*

finite amount of the original filtrate involves the calculation of that degree of dilution where the volume of the original material present is less than the volume of one electron. The electron being the smallest possible mass of matter, to say that as the result of the successive dilutions there remains in the liquid a quantity of material less than one electron, is to say that there remains a hypothetical but not an actual amount.

* The characters of secondary cultures will be discussed in a following chapter. 
The dilution method can be applied even in the case of races of low virulence, provided bacteriophagy in the limiting suspensions is controlled by spreading them on agar. However, with races of low virulence a purification by means of isolation through plaques is certainly less complicated.

We have seen that it is only necessary to touch the centre of a plaque with a sterile platinum wire and then immerse this wire in a suspension of the susceptible bacterium to cause bacteriophagy of this suspension. Each plaque on the agar being a colony of bacteriophage corpuscles resulting from the multiplication of a single corpuscle, we have here a method of isolation absolutely analogous to that discovered by Koch for the isolation of bacteria. But we may mention the fact that as is often the case with bacteria, the colony developing at a given point on the agar may be derived, not from a single corpuscle, but from a group of two or more. This being the case, it is unwise to rely upon a single isolation. Certainty demands that the process of isolation be repeated two or three times.

The method of isolation by plaques has been employed especially by Bail and his collaborators ${ }^{53}$ who were the first to call attention to the possibility that a filtrate possessing activity for a single bacterial species could contain a mixture of bacteriophage races. Wolff and Janzen ${ }^{630}$ have likewise made this observation in studying different races of Typho-bacteriophage.

It may be well to emphasize the fact that if we would effect isolation by the plaque method it is necessary to proceed in the following manner. Inoculate a heavy suspension, $250,000,000$ bacteria per cubic centimeter, with a very small quantity of the raw suspension of bacteriophage. When this mixture is spread immediately upon agar the surface should show, after incubation, but very few plaques. If too many corpuscles are inoculated into the bacterial suspension it is possible that several will become attached to a single bacterium and the plaque will be formed of elements not the issue of a single corpuscle. Furthermore, for this purpose Petri dishes should not be used. Agar slants are much better, since they may be placed in a vertical position immediately after planting in such a way that it will insure that none of the liquid remains on the surface. The presence of fluid facilitates the development of the corpuscles, as in a liquid medium, and if this occurs the isolation will be fictitious. 


\section{VARIABILITY IN THE VIRULENCE OF THE BACTERIOPHAGE}

From the time of Pasteur we have known that in a single bacterial culture each of the bacteria possesses certain characters which are peculiar to itself and differentiate it from the associated organisms, even though all of the organisms present the general characters belonging to the species. Such qualities as vitality, resistance to heat and to antiseptics differ for every organism within a culture. This fact is particularly striking as regards virulence. For example, let us take a culture of $B$. pestis and implant it on an agar slant in such a way as to secure isolated colonies. By the inoculation of susceptible animals we can show that cultures of some of these colonies kill the animal in an infinitely small amount while others are inoffensive in very large quantities.

The bacteriophage, just like the bacterium, is of corpuscular nature and different races of the bacteriophage, just like different strains of bacteria, possess different virulences. One might, indeed, ask if the analogy does not go still further and if in a given suspension of bacteriophage each corpuscle does not possess a particular virulence. It is very easy to determine that this is indeed the case.

Isolate, by means of dilutions, an extremely active Staphylo-bacteriophage, such a race as I have described in the preceding section. Distribute $10 \mathrm{cc}$. of the limiting dilution among 10 suspensions of the staphylococcus, 1 cc. to each. The results of such an experiment will be as follows:

After 36 hours: 1 suspension will be clear

1 suspension will be clouded

8 suspensions will be turbid

After 48 hours: 2 suspensions will be clear

2 suspensions will be clouded

6 suspensions will be turbid

After 72 hours: 5 suspensions will be clear

1 suspension will be cloudy

1 suspension will be very cloudy

3 suspensions will be turbid

After 7 days: 5 suspensions will be clear

2 suspensions will be very cloudy

3 suspensions will be turbid

Spreadings made upon agar, and repeated passages will show that the 3 turbid suspensions are free of bacteriophage; that is, bacteriophage 
corpuscles were not present in the $1 \mathrm{cc}$. quantities which each received. As for the 2 cloudy suspensions, they contain a bacteriophage whose virulence is much less than that of the suspensions which had undergone complete bacteriophagy.*

It is unnecessary to state that the race of Staphylo-bacteriophage utilized was derived from a single corpusele; as a matter of fact it had passed through multiple isolations carried out at different times either by the dilution method or by the plaque method.

This experiment shows that, considering the end result as well as the rate of the reaction, among 7 corpuscles each possessed a different degree of virulence. We must, therefore, conclude that in a suspension of a race of the bacteriophage every corpuscle possesses a special degree of virulence. The same experiment carried out with Coli-, Typho-, and Shiga-bacteriophages has given in every case comparable results. This finding indicates the reason for the differences in virulence of different races of the bacteriophage as they are found under natural conditions.

\section{INCREASES IN VIRULENCE}

We have seen that a bacteriophage suspension, originating from a single very virulent corpuscle, contains corpuscles possessing a virulence equal to that of the original corpuscle which served as the source of the race, together with others whose virulence is much lower. This simply means that virulence may be attenuated, as is the ease with bacteria. But Pasteur showed that the virulence of a bacterium is capable of being increased by successive passages within the body of a susceptible animal. Is this likewise true for the bacteriophage?

Again the analogy is complete. The virulence of a bacteriophage may be exalted by successive passages in suspensions of a susceptible bacterium (d'Herelle ${ }^{310,315}$ ). There are several methods by means of which a bacteriophage but slightly active at the time of its isolation may be increased in virulence. ${ }^{321}$ For example, the following procedure will result in such a change in virulence.

When an agar inoculation has shown that a bouillon suspension contains an active bacteriophage this suspension is filtered through infusorial earth and then through a bougie. A slightly turbid suspension is prepared, using the bacterial strain against which the bacteriophage has shown some activity, and into this suspension are introduced some four or five drops of the filtrate. After incubation, if dissolution has not been produced, this second bacterial suspension is filtered as before and

* They contain secondary cultures also. 
a third suspension is inoculated with four or five drops of the filtrate. Such transfers are continued until evident dissolution occurs. During the process it is easy to verify the presence of the bacteriophage in each passage, and to detect any increase in virulence, simply by spreading the successive cultures on agar slants. Comparison of the cultures secured with each passage reflects the degree of virulence. For example, the agar growth obtained from the first passage shows a culture growth with ten plaques, the second passage shows 100, with the third the layer of bacillary growth is broken up with an abundance of the areas, while with the fourth passage only a few isolated colonies of bacteria are seen. It can be readily seen that the virulence of the bacteriophage, that is, its ability to develop at the expense of the bacteria, increases with each transfer until a point is reached where complete dissolution of the suspension is obtained.*

Usually it is relatively easy to increase the virulence of a weak race of the bacteriophage, but at times it may become very difficult, particularly when working with races active against the Gram-positive cocci. In such cases it is necessary to effect a great number of passages, and there is considerable risk of losing the bacteriophage altogether, particularly during the first few passages. I might cite as an example an anti-staphylococcic race with which Eliava was forced to make passages during four months in order to obtain sufficient virulence to induce complete dissolution of a suspension containing 500 million staphylococci per cubic centimeter.

It may be well to emphasize here a point of some importance. We will see that the virulence of the bacteriophage becomes considerably weakened when it is held in contact with bacteria which resist its action. For this reason it is wise to avoid, in each passage, contact with those organisms which have had time to acquire a resistance. To accomplish this it is best at first to conduct the passages at a relatively low temperature; $30^{\circ} \mathrm{C}$. is adequate to permit the active development of the corpuscles and it retards somewhat the acquisition of a resistance to the process of bacteriophagy by the bacterium. Bacterial resistance de-

* The bacteriophage is not destroyed until a temperature of about $76^{\circ} \mathrm{C}$. is reached. Bordet and Ciuca ${ }^{86}$ have proposed to utilize this fact to avoid filtration. They hold the bacteriophage suspension at $58^{\circ} \mathrm{C}$. for an hour during which time the bacteria are killed while the bacteriophage resists. This method of isolating a virulent bacteriophage should never be employed during the course of a series of passages designed to increase virulence. For although heating may not kill, it attenuates the virulence of the bacteriophage with the result that the advantage gained by each passage is lost through the heating. 
velops somewhat more rapidly at a temperature of $37^{\circ} \mathrm{C}$, but even at the lower temperature of $30^{\circ} \mathrm{C}$. the bacterium reacts vigorously. For this reason it is essential to restrict the period of contact to that just sufficient to afford the corpuscles opportunity to multiply. And at the same time a limited period of contact will prevent the bacteria from acquiring a resistance through processes of adaptation. As a matter of fact practical experience shows that this end is obtained by effecting two passages every day; one in the morning upon arrival at the laboratory, the other in the afternoon just before leaving.

An excellent procedure which provides through serial passages for the increase in the virulence of the bacteriophage by adaptation and at the same time for the selection of those corpuscles most apt at acquiring such a virulence consists in carrying out a series of isolations by the dilution method. The following scheme indicates how this can be effected.

Among 10 suspensions, each inoculated with $1 \mathrm{cc}$. of the last active dilution of a bacteriophage, a certain number undergo bacteriophagy. Select the tube in which the phenomenon has been the most marked.* This suspension is filtered through a candle and the isolation procedure is begun again. Once more that suspension in which bacteriophagy is the most intense is filtered. This procedure is repeated up to the point where the maximum virulence has been obtained.

The only difficulty with this resides in the determination of the limiting dilution, especially with very weak races of the bacteriophage, or where bacteriophagy can only be disclosed by agar cultures. Moreover, in carrying out the passages one can not take time to make the preliminary studies necessary to ascertaining the limiting dilution. In order to obviate this difficulty the increase in virulence may be started by a few simple passages and later, when a degree of virulence has been obtained sufficient to cause bacteriophagy such as can be detected by microscopic examination, the limiting dilution may be determined.

When this is once determined the filtrate is utilized and an isolation by dilution is made. The choice from among the bacteriophage suspensions falls on the one where the phenomenon occurred most intensely. This one is filtered, and the filtrate serves for a new isolation, arbitrarily choosing as the limiting dilution the same as that disclosed by the pre-

* Needless to say, if for any reason it is desired to make a selection from among a very great many corpuscles, in the place of utilizing $10 \mathrm{cc}$. of the limiting dilution in 10 suspensions, one might use $20 \mathrm{cc}$. of the limiting dilution, or even more, distributing it among 20 or more suspensions. 
ceding titration. If this time it is found that the 10 suspensions inoculated are bacteriophaged, that one where the process occurred most vigorously is selected and is filtered. For the next passage, as is obvious, the next higher dilution is selected. Simple observation of the 10 suspensions here will show the dilution to be used for the following passage. If there are only one, two, or three of the 10 suspensions bacteriophaged, it may be well to use the same limiting dilution as that previously used. If there are 8,9 , or 10 suspensions bacteriophaged it is desirable to take the next higher dilution for the next passage.

At first sight this method appears rather complicated but it gives excellent results. In this way I have been able to obtain bacteriophage races causing regularly a complete and invariably permanent dissolution of normal bacterial suspensions. Those who have worked with the bacteriophage realize how difficult it is to attain a virulence sufficiently high to preclude the development of secondary cultures.

A number of authors, among whom may be mentioned Izar, ${ }^{367}$ and Janzen and Wolf ${ }^{374}$ have observed that all races of the bacteriophage do not acquire virulence with equal facility. This is quite true. And in this same connection I have found ${ }^{331}$ that certain races of very low virulence may lose their activity with the first passage, and for this we will see the reason later. But it is likewise true that the mode of procedure has a very great influence upon the result. Just to indicate the importance of the matter of the bacterial strain selected I may state that upon several occasions, when working with a single race of bacteriophage, I have made two parallel series of passages for the purpose of increasing the virulence. One series was made at the expense of one strain of the bacterium, the second at the expense of another strain of the same species. It was found frequently that virulence increased rapidly with one strain and but very slowly with the other. Further study showed that this difference in result was due to the fact that one of the two bacterial strains developed a resistance far more readily than the other.

With certain strains which possess the faculty of developing resistance in a high degree, the result of serial passages is not an increase in virulence but the bacteriophage is overcome and sometimes it disappears even in the first passage. We will study the phenomenon of bacterial resistance in the next chapter so that we will not enter into further detail here, resting content with the statement that the choice of the bacterial strain at the expense of which the passages are to be made is of extreme importance. It might be well to add, however, that once the increase in 
virulence for one strain is obtained (when working with organisms belonging to homogeneous races - we will see later the significance of this) virulence is likewise enhanced for strains which were unsuited to the development of this virulence. The reason for this fact is that when the virulence is once increased, the resistance of the bacterium, which previously would have been able to withstand a low virulence, is overcome by a higher virulence. Upon this point again the story of pathogenic bacteria provides us with ample examples of facts of the same nature.

Before ending this section I would like to call attention to an error of fact which has been made by Seiffert. ${ }^{578}$ This author has assumed that the increase in the virulence of the bacteriophage effected by successive passages at the expense of bacteria is due to an "adaptation" on the part of the bacteria and not to an adaptation of the bacteriophage. With regard to "theories" of this type Pasteur stated, "they are under no particular obligations as regards the facts."

Just how valid such a theory is may be shown by the following, which indicates exactly the sequence of events leading to the adaptation. Inoculate a drop of filtrate containing bacteriophage corpuscles of low virulence into a suspension of bacteria. After an appropriate time filter through a candle. This effectively discards all of the bacteria which have been subjected to contact with the bacteriophage corpuscles. Introduce this filtrate, containing no bacteria whatever, into a second suspension of young bacteria-bacteria which have never yet been in contact with the bacteriophage. When bacteriophagy has occurred filter again and discard once more all of the bacteria which have been in contact with the bacteriophage corpuscles. Carry out in this manner a series of passages, introducing a filtration to eliminate the bacteria between each passage. None of the bacteria of the suspension in which one passage is made can be carried over into the suspension of the following passage. Under such conditions, since the bacteria are not involved in the passages, how can they undergo adaptation? Such an adaptation as a being may acquire can be transmitted only when there are descendents.

It is easy to understand that the fact that the virulence of the bacteriophage is subject to increase is disturbing to the partisans of certain theories which we must discuss later. Nevertheless, if objections are raised to the concept which I have evolved, the opposing arguments should, at least, take the facts into consideration. 


\section{6. attenuation of Virulence}

Just as it is possible to produce experimentally an increase in the virulence of the bacteriophage, so also is it possible to cause an attenuation of virulence.

Exposure to high temperatures is one of the methods of causing such an attenuation (d'Herelle and Pozerski ${ }^{360}$ ). The effect of heat is clearly indicated by the data which follow.

In the following experiments the suspension of bacteriophage under test, previously filtered through a bougie, is taken up in capillary pipettes, sealed at both ends, and completely submerged in a waterbath maintained at the temperatures indicated in each experiment. In each series of experiments 8 tubes with the suspension are maintained for thirty minutes at temperatures of $60,62,64,66,68,70,72$, and $75^{\circ} \mathrm{C}$.

\section{Anti-Shiga bacteriophage}

Two drops of the suspension from tubes maintained at 60, 62, 64, and $66^{\circ} \mathrm{C}$., when introduced into suspensions of Shiga bacilli, cause complete dissolution in less than fourteen hours. The tests repeated with a second strain of Shiga bacilli give identical results. The bacteriophage heated to 68 and $70^{\circ} \mathrm{C}$. causes dissolution with one strain of Shiga bacilli but not with the other. When heated to 72 and $75^{\circ} \mathrm{C}$. the bacteriophage fails to dissolve the organisms.

One drop of each of these suspensions, which had received the bacteriophage previously maintained at $68,70,72$ and $75^{\circ} \mathrm{C}$., and which had not been submitted to bacteriophagy is planted on slant agar. After incubation, all of the cultures, except the last, which is normal, show plaques characteristic of the presence of the bacteriophage.

Serial passages may be effected, thus permitting the enhancement in virulence of the bacteriophage attenuated by the action of temperature. After two such passages, with the corpuscles heated to 68 and $70^{\circ} \mathrm{C}$., and after three passages with those heated to $72^{\circ} \mathrm{C}$., dissolution in liquid media is obtained.

Comparable experiments have demonstrated that the bacteriophagous corpuscles active for B. dysenteriae Flexner, B. dysentcriae Hiss, B. coli, and $B$. paratyphosus $B$, act in a quite similar manner. With the bacteriophage active for $B$. paratyphosus $A$ attenuation begins at about $64^{\circ} \mathrm{C}$. (at least with the strain tested). With that virulent for $B$. typhosus attenuation is already apparent at about $62^{\circ} \mathrm{C}$. In all cases, when heated to $75^{\circ} \mathrm{C}$. the bacteriophage is completely inactive, either 
actually destroyed or attenuated to such an extent that its presence can no longer be detected. In all these instances the bacteriophage shows a recuperative power, the virulence being restored when the temperature to which the corpuscles have been subjected is not higher than $72^{\circ} \mathrm{C}$.

\section{Anti-staphylococcus bacteriophage}

Attenuation of this bacteriophage is already manifest after heating to $60^{\circ} \mathrm{C}$. Subcultures of suspensions which have not been dissolved show that it is a simple attenuation, for, even with suspensions inoculated with a bacteriophage previously held at $72^{\circ} \mathrm{C}$. for thirty minutes, plaques are obtained characteristic of the presence of an active bacteriophage. Moreover, two passages suffice to restore the original virulence to corpuscles heated to $62,64,66$, and $68^{\circ} \mathrm{C}$. After heating at 70 and $72^{\circ} \mathrm{C}$. the attenuation of virulence does not disappear until after six passages. When heated to $75^{\circ} \mathrm{C}$. the bacteriophage is deprived of all activity.

It may be concluded from these experiments that all races of the bacteriophage react to temperature in the same manner. When heated above $60^{\circ} \mathrm{C}$. they are attenuated more or less rapidly, the speed depending to some extent upon the bacterial species for which they are active. But all are completely killed, or at least paralyzed, at a temperature of about $75^{\circ} \mathrm{C}$.

The results observed in these experiments can not be ascribed to a reduction in the number of corpuscles, for the Shiga-bacteriophage used, like the Staphylo-bacteriophage, had caused complete bacteriophagy in a unit volume before exposure to the high temperature, and it will do the same after the virulence is again increased.

My experiments have also shown that bacteriophage races of low virulence are attenuated at lower temperatures than are races possessing a more outspoken virulence for the same bacterial strain.

Time also exerts an effect upon virulence, but here there is a very great difference in the effects, depending upon the race of bacteriophage, and especially upon its virulence. Bacteriophage races possessing a high virulence are in general but slightly modified while those of low virulence are much more sensitive.

Asheshov ${ }^{29}$ was the first to observe that certain races of the bacteriophage may lose their virulence very rapidly. A Flexner-bacteriophage which I isolated, and which had a relatively high virulence, manifested a very considerable attenuation even within a period of two months, 
in spite of the fact that it was preserved in sealed ampoules. Various observations made in the course of my studies contribute additional data upon this question of spontaneous attenuation.

One race of the Shiga-bacteriophage, originally very virulent, has been held for a period of 9 years in a sealed tube, and throughout this period it has remained almost as active as it was at the beginning. The sole change consisted in the number of corpuscles, which diminished from 2400 million to 110 million, but despite this reduction in numbers those which survived retained their virulence without change and this virulence was equal to that of corpuscles having the same origin but which had undergone, throughout this time, between 1500 and 2000 passages. Other races of Shiga-bacteriophage from various sources, but all very virulent, likewise maintained their activity unchanged during periods of 3 to 5 years. A very active Staphylo-bacteriophage, held in a sealed ampoule, retained its potency completely for a period of 3 years. A second race, somewhat less virulent, was attenuated after 4 years to such an extent that it was lost in the second passage when an attempt was made to restore its virulence. Two very active races of Colibacteriophage maintained their virulence for 6 years. Three races which originally showed a strong virulence, although somewhat less than that of the two races just mentioned, were dead or totally avirulent after the same interval of time.*

A Barbone-bacteriophage of high virulence when sealed up in an ampoule had lost a large part of its virulence after 11 months, but with 3 passages the virulence was restored. After 26 months this particular race was dead or completely avirulent in some of the ampoules whereas in others it was still present, although attenuated to such a degree that it was lost during the first passage.

A Cholera-bacteriophage tested after it had been preserved for 8 months was completely avirulent. During the attempt at rejuvenation a few minute plaques could be seen in the first passage, but with the second passage it disappeared.

A Plague-bacteriophage (of rodent origin) retained almost all of its activity throughout a period of 26 months. Another race (of human origin), somewhat less virulent at the beginning, was very markedly attenuated in the same length of time. After 40 months the first race was still very virulent; the second was dead or avirulent.

* The bacteriophage can be recognized only through its virulence for a given bacterium. Consequently it is not possible to tell whether the bacteriophage as such has disappeared or whether it has become avirulent, for in either case it would not be possible to disclose its presence. 
It is obvious that it is impossible to state any specific rule as applying to the phenomenon in all cases. Each bacteriophage behaves in a particular manner. The most that can be said is that in general races with a high virulence retain virulence for a long time, while a low virulence disappears relatively quickly.

Antiseptics likewise exert an effect upon virulence, but we will reserve a study of this subject until we consider the properties of the bacteriophage corpuscle.

Virulence is weakened through contact with resistant bacteria. This aspect of the subject will be considered in detail in the next chapter.

\section{HOMOGENEOUS AND HETEROGENEOUS BACTERIAL SPECIES}

Different bacterial species behave in a different manner as regards susceptibility to bacteriophagy. With certain species it is found that when one strain is susceptible to a given race of the bacteriophage all other strains are also susceptible.*

The Shiga dysentery bacillus is typical of those species which I have termed homogeneous insofar as the bacteriophage is concerned. During the past 10 years I have isolated several hundred races of bacteriophage active for this bacterium and I have never yet found a strain of the Shiga bacillus which was not attacked by any of these races. The degree of virulence is the only variable. A given bacteriophage of maximum activity for a single strain may possess only a moderate virulence for others, but a few passages are always sufficient to intensify the virulence for those strains which were at first but weakly attacked.

$B$. pestis is another bacterial species which I have found to be homogeneous as regards bacteriophagy.

I have shown ${ }^{313}$ that certain races of Typhoid-bacteriophage have an extremely specific action, attacking but a single strain of B. typhosus to the exclusion of all other strains. Bordet and Ciuca ${ }^{86}$ have confirmed this finding, and they have shown that with B. coli a comparable specificity may be demonstrated with some races of bacteriophage.

My experiments have disclosed the fact that this non-susceptibility of certain strains of a bacterium when others are attacked, is limited to certain bacterial species, and these I have termed heterogeneous.

* Naturally, this is true only insofar as it deals with typical strains, that is to say, with those presenting all of the characters of the species. In general, atypical strains (those which are arbitrarily classified in a species to which they do not conform in all characters) are found to be resistant to the action of the bacteriophage. We will return to this subject later. 
The strains which are not attacked possess a true natural immunity, but this immunity is limited, not absolute. As a matter of fact, Janzen and Wolff, ${ }^{374}$ working with $B$. typhosus, have shown that those strains of this bacterium not attacked by a certain bacteriophage are attacked by others.

It is, however, quite essential that we do not confuse this natural immunity, possessed by certain bacterial strains toward some races of the bacteriophage, with the immunity acquired by a susceptible bacterium in reacting to the action of a bacteriophage.

Each race of the bacteriophage which has a virulence for a single bacterial strain belonging to a heterogeneous species possesses an individual range of virulences. This range varies from one race to another. Certain bacteriophage races will attack only a few strains of the heterogeneous bacterium; other races will attack a large number, and there are some which will attack all. With respect to the last, the bacterial species will be homogeneous.

Furthermore, each susceptible strain will be attacked with greater or less intensity, that is to say, the violence of the reaction will vary. Nevertheless, in these cases it is always possible to inerease the virulence by means of passages with the strain that was at first but slightly attacked.

Since all combinations of these variables are encountered and since there are all degrees of virulence for cliverse strains, and since also there are differences in the intensity with which each strain is attacked it is obviously practically impossible to isolate two races of the bacteriophage possessing identical characters (d'Herelle ${ }^{315}$ ).

A very typical case is that of the Staphylo-bacteriophage, where races may be isolated which are virulent for but a single strain of the staphylococcus. It is of some significance that the races of Staphylobacteriophage isolated from vaccinal lymph* are of this type. On the other hand I have isolated races of Staphylo-bacteriophage from the pus of abscesses, undergoing resolution, which were virulent for a number of staphylococcus strains. Gratia ${ }^{257}$ has isolated a race (race $\mathrm{H}$ ) which is virulent for all strains of the staphylococcus, including strains of the

* As we will see, in some specimens of vaccinal lymph a bacteriophage-bacterium symbiosis between a staphylococcus and a bacteriophage may be found. The symbiotic organisms are continuously reinoculated at the same time as the ultravirus of vaccinia from calf to calf. This represents a true final adaptation of the bacteriophage to parasitism of the staphylococcus with which it has been associated in a parasitic relationship for a great many generations. 
several species, albus, aureus, and citreus. As a matter of fact, with this particular race I have not yet found a typical staphylococcus which is not attacked, even Micrococcus tetragenus is dissolved.

We will return to this question of homogeneous and heterogeneous bacterial species when we study the peculiarities of the phenomenon of bacteriophagy with the different bacterial species. We will then review the studies of Janzen and Wolff, particularly those dealing with the bacteriophagy of the heterogenous $B$. typhosus.

\section{MULTIPLE VIRULENCES OF THE BACTERIOPHAGE}

We have seen that certain races of the bacteriophage are virulent for only a single bacterial strain, while others are virulent for all strains of a given species. Furthermore, we have stated that still other races are virulent not only for all strains of a single species but also for strains of bacteria belonging to different species, sometimes to species rather remotely related (d'Herelle ${ }^{315}$ ). As a matter of common observation it has been found that a given race of the bacteriophage, when derived from the organism, is rarely active for but a single bacterial species. Usually at this time it attacks a number of species and possesses for each of them a varying degree of virulence (d'Herelle ${ }^{313}$ ).

Such a race of the bacteriophage might have, for example, a very high virulence for the Hiss strain of $B$. dysenteriae, a moderate virulence for some strains of $B$. coli, and a low virulence for other strains of $B$. coli as well as for the Shiga bacillus. For $B$. paratyphosus $B$ the action may be very weak, and for other species no virulence whatever can be demonstrated. Another race may be very active for certain strains of $B$. coli and of B. typhosus, less virulent for other strains of these organisms, and at the same time it may show but little activity for $B$. dysenteriae Flexner with no virulence for all other bacterial species. But provided an activity, even though weak, is manifested by such a race it is always easy, by passages, to enhance the virulence for one of these organisms.

We have seen further that because of variations in virulence a given race of the bacteriophage may vary materially at different times. All of the combinations of virulence, in quality as well as in quantity, being possible, that is to say, in the range of the action against various bacterial species and in the intensity of the action for each of the strains of these different species, one can readily understand, in view of the infinite number of possible combinations, that there can be no two races of the bacteriophage which can be absolutely identical (d'Herelle ${ }^{315}$ ). 
All of those investigators who have studied the bacteriophage, with the exception of Bail and of Wagemans, ${ }^{613}$ have confirmed the fact that a given race of the bacteriophage may show multiple virulences, and this conclusion is reached whatever may be the individual opinion as regards the nature of the bacteriophage itself. The two authors mentioned above as exceptions have advanced the suggestion that when a bacteriophage filtrate causes bacteriophagy with different bacterial species the phenomenon is due to the fact that the material contains a mixture of several races of the bacteriophage. The experiments which will be detailed in the following paragraphs show that such a conclusion is inadmissible, simply because it is contrary to demonstrated experimental facts. Foreseeing this objection I had refuted it before it was advanced $^{321}$ but the authors named above have not even mentioned the experiments which I had reported as bearing upon this point.

\section{PERSISTENCE OF VIRULENCE}

A race of the bacteriophage possesses the faculty of regaining its virulence for a given bacterium, and this capacity persists throughout a great many passages effected in vitro with a bacterium belonging to another species. In $1916 \mathrm{I}$ isolated a bacteriophage extremely active for B. dysenteriae Shiga. At that time, as it was derived from the body it also showed a moderate virulence for one strain of $B$. coli and a low virulence for different strains of $B$. typhosus and the paratyphoids $\mathrm{A}$ and $\mathrm{B}$. This bacteriophage was used in many experiments throughout the years 1916,1917,1918, and 1919, and during this time was subjected to a very great many passages, always at the expense of $B$. dysenteriae Shiga. As a matter of fact the number of passages somewhat exceeded 1200. Early in 1920 I showed that, without a preliminary adaptation, it still possessed a moderate virulence for the strain of $B$. coli and a slight virulence for the strain of B.typhosus toward which it was active 4 years earlier. By means of passages with these bacteria the virulence was increased, up to the point where it caused complete dissolution. ${ }^{315}$

Having preserved this race of Shiga-bacteriophage I carried out, in 1923, three successive purifications by the method of selection described above, without causing a loss in the virulences for B. coli and B. typhosus. This experiment has been repeated at different times with several races of the bacteriophage, the result being that the multiple virulences persisted after three successive isolations. Inasmuch as the suspension 
of the bacteriophage which manifests these multiple virulences is most certainly derived from a single corpuscle, the effects can hardly be said to be due to a mixture of races.

The fact that multiple virulences persist despite a great many passages made at the expense of a single bacterial strain, is, however, the most valid evidence that these multiple virulences are attributes of a single bacteriophage corpuscle. Let us assume that a stool filtrate causing bacteriophagy of several bacteria of different species contains several races of the bacteriophage. If, with this filtrate, we carry out passages at the expense of a single bacterium we must admit that only those corpuscles should multiply which possess a virulence for this bacterium. We must likewise admit that all corpuscles avirulent for this bacterium should disappear gradually in the course of the passages. And the number of passages necessary to eliminate all of the avirulent corpuscles within the initial filtrate is readily calculable. Physicists have determined that the smallest possible quantity of matter is the electron, whose mass is $1 \cdot 10^{-27}$ grams. When, as the result of successive dilutions such as take place from passage to passage, we introduce into a suspension a quantity of the initial filtrate less than $1 \cdot 10^{-27}$ grams we may be very sure that there no longer remains any of the original filtrate, and, a fortiori, none of the bacteriophage corpuscles which were in this filtrate. All of these corpuscles present at this time result from a multiplication of virulent corpuscles. In carrying out the series of passages by introducing into the first suspension 0.001 cc. of filtrate, into the second $0.001 \mathrm{cc}$. of the first suspension after it has undergone bacteriophagy, and continuing thus, introducing each time $0.001 \mathrm{cc}$. of the last suspension dissolved into $10 \mathrm{cc}$. of fresh bacterial suspension, it can be readily calculated that in the seventh passage there can be but $1 \cdot 10^{-28}$ grams of the original filtrate, that is to say, a virtually nonexistent quantity, since it is smaller than an electron. This seventh suspension can not contain any of the bacteriophage corpuscles avirulent for the bacterium at the expense of which the passages were made, even though such corpuscles were found in the original filtrate. If the corpuscles found in the last filtrate manifest a virulence for a bacterium of a species other than that with which the passages were made the only inference is that these corpuscles possess the property of causing a bacteriophagy of different bacterial species at one and the same time.

But experiment shows that, not only after the seventh, but after more than 1000 passages, a race of the bacteriophage still possesses the property of causing bacteriophagy with bacteria belonging to different 
species. This affords, then, mathematical proof that multiple virulences are really attributes of bacteriophage corpuscles.*

\section{THE MECHANISM OF THE PERSISTENCE OF VIRULENCE}

A bacteriophage which has received more than a thousand passages with $B$. dysenteriae has a relatively weak action upon the typhoid bacillus. This can be demonstrated by spreadings made on agar, with subsequent observation of the formation of characteristic plaques. If we introduce into a tube of bouillon about 10 drops of an anti-dysentery bacteriophage and then a small amount of typhoid culture we secure, after incubation for 18 to 24 hours, an apparently normal culture of $B$. typhosus, but if it is spread upon agar a few plaques are obtained.

This finding provides us with additional information as to the mechanism of the virulence of the bacteriophage. For here we have introduced into the suspension of $B$. typhosus several thousand million bacteriophage corpuscles, all virulent for $B$. dysenteriae. Each plaque to develop on the agar, upon which the suspension of B. typhosus inoculated with the Shiga-bacteriophage is spread, represents a colony derived from a corpuscle virulent for B. typhosus. And this shows that among several billions of corpuscles virulent for $B$. dysenteriae there are only a few which are also virulent for B. typhosus.

Furthermore, by such a procedure it can be shown that the number of corpuscles having a virulence for $B$. typhosus hardly varies throughout the series of passages with $B$. dysenteriae, for the number of plaques obtained by spreading a suspension of typhoid bacilli inoculated with the suspension of bacteriophaged dysentery bacilli is approximately the same, whether the bacteriophage has undergone $50,100,500$, or 1000 passages, always with $B$. dysenteriae. Nevertheless, the number of corpuscles active for $B$. typhosus diminishes, although the aptitude for the bacteriophagy of $B$. typhosus is lost but very slowly. After 1500 passages the virulence was so weak that spreadings upon agar no longer showed plaques, yet plaques appeared after only two passages. After 17 passages with B. typhosus the dissolution of a normal suspension of typhoid bacilli was complete.

* At least, if it is understood that the bacteriophage is not derived from the bacterium itself. This is a point which we will examine in another chapter, but I believe it wise to state here that there is very conclusive evidence which has not been discussed or contradicted by anyone, which demonstrates that the bacteriophage corpuscle is autonomous, independent of the bacterium which is subject to its action. The evidence supporting this will be presented when we discuss the question of the nature of the bacteriophage. 
It is probable that by continuing the passages the aptitude for reacting with $B$. typhosus would have gradually diminished and finally would have been completely lost. This would have represented, then, a strict adaptation to the bacteriophagy of $B$. dysenteriae.

As I have said above, I have repeated at different times, with diverse races of the bacteriophage, experiments which show that after a series of passages followed by three successive isolations the virulence of a bacteriophage for several bacterial species persists. Here is such an experiment.

A race of Typhoid-bacteriophage, isolated in 1918 from the stools of a convalescent from typhoid fever, manifested at its origin a strong virulence (complete bacteriophagy of a normal suspension but with almost always the development of secondary cultures) for many strains of $B$. typhosus and B. coli, for the paratyphoids A and B, and for all strains of B. dysenteriae, Shiga, Flexner, and Hiss. In 1924 I made two or three hundred passages with $B$. coli, B. typhosus, and B. dysenteriae, alternating the bacterium with which the bacteriophage was placed in contact. A series of a dozen passages were then made with $B$. dysenteriae to eliminate avirulent corpuscles if any were present (in each passage 0.001 cc. of the suspension previously dissolved was introduced into $10 \mathrm{ec}$. of a fresh suspension). And finally, the race was carried through three successive isolations by selection. This procedure assured two things; first, that all of the corpuscles avirulent for $B$. dysenteriae must have been eliminated by dilution, and second, that the derived suspension represented the progeny of a single corpusele. With the final suspension the virulences were the same as those shown immediately after the isolation of the race in 1918 . This can only mean, therefore, that a single bacteriophage possesses multiple virulences.

In all of the experiments performed, where consideration was given to this point, it has been possible to show that among the corpuscles comprising a race of the bacteriophage, there are some-many or few, as the case may be - which show a virulence for a bacterium of a species other than that at the expense of which the passages have been made. The persistence of a latent virulence is a function of certain particularly apt corpuscles. In view of the very great number of corpuscles present after bacteriophagy, with the law of large numbers applying to each passage, it follows that the number of corpuscles presenting a latent virulence for a bacterium of another species continues practically the same. It is only after a very great many passages, always at the expense of a single bacterium, that the aptitude for bacteriophagy with 
other organisms becomes lost. In this connection we will see later many examples showing that the characters pertaining to each race of the bacteriophage are very stable.

When, after a number of passages at the expense of a given bacterium, the bacteriophage corpuscle is placed in contact with a bacterium of another species for which some of the corpuscles possess a latent virulence, a selection occurs. Only the corpuscles having this virulence multiply; the virulence for the new bacterium is thus increased so that after a few passages the virulence reaches such a degree that complete bacteriophagy is effected. It is necessary, however, to state that this tendency toward an increase in virulence differs very markedly for different races of the bacteriophage. Given two races of the bacteriophage, both presenting a latent virulence for a single bacterial strain, one may obtain, after a few passages, a high virulence with one of the races while it may require a considerable number of passages before the second race attains a comparable degree of virulence. Indeed, with certain races this may never be accomplished.

We have seen in the experiments recorded above that even in the case of a bacteriophage having a maximum activity for a given bacterium each corpuscle possesses its own individual virulence. Certain of them are high in virulence, others are less active. We will see that this is also true for the latent virulences which these corpuscles may manifest. This behavior of bacteriophage corpuscles is identical to that of pathogenic bacteria. Since the time of Pasteur we have known that in a pure bacterial culture each individual cell, although possessing the general characteristics of the species, presents "variations" which are peculiar to it. A bacterium may be considered, according to the expression of Maurice Nicolle, as a "mosaic" of properties, each of these propertiesvitality, resistance to such and such an agent, virulence for such and such an animal-being subject to continual variation. And the variations in each of these factors follow different lines with each cell division. Everything would indicate that the situation is precisely the same for bacteriophage corpuscles. Each race of the bacteriophage possesses characters which distinguish it from other races, and each of the corpuscles of a given race possesses characters which are subject to continual variation.

\section{THE ACQUisition OF VIRULENCE}

From the very first of my communications upon the subject I have insisted upon the fact that a bacteriophage can acquire, experimentally, 
by passages, a virulence for a bacterium toward which it previously manifested no activity. In the first edition of my collected papers ${ }^{321}$ experiments were presented supporting this idea. Since that time a great many authors have confirmed this fact. The contributions of Otto and his collaborators in particular present many experiments demonstrating the truth of this idea. But I will mention here only the following, for these experiments are extremely interesting from the theoretical point of view since they show how the virulence of the bacteriophage is acquired under natural conditions, and from the practical point of view they are significant in that they indicate a procedure particularly well adapted to bring about, experimentally, such an acquisition of virulence.

TABLE 14

\begin{tabular}{|c|c|c|c|c|c|c|}
\hline \multirow{2}{*}{$\begin{array}{l}\text { STRAINS OF } \\
\text { B. TYPHOSUS }\end{array}$} & \multicolumn{3}{|c|}{$\begin{array}{l}\text { VIRULENCE DETERMINATIONS } \\
\text { IMMEDIATELY AFTER ISOLATION }\end{array}$} & \multicolumn{3}{|c|}{$\begin{array}{l}\text { VIRCLENCE DETERMINATIONS AFTER A } \\
\text { SERIES OF PASSAGES WITH STRAIN SM }\end{array}$} \\
\hline & Dissolution & Inhibition & Plaques & Dissolution & Inhibition & Placues \\
\hline Wi & 0 & 0 & 0 & 0 & 0 & 0 \\
\hline 1 & 0 & $+t+$ & $++t$ & 0 & $+t+t$ & ++++ \\
\hline 24 & 0 & 0 & 0 & 0 & \pm & $+++t$ \\
\hline 27 & 0 & 0 & 0 & 0 & 0 & 0 \\
\hline 29 & 0 & 0 & 0 & $+t$ & +++ & $++++^{*}$ \\
\hline
\end{tabular}

* The method employed by Janzen and Wolff for measuring virulence has been described. In the tables given here, dissolution refers to bacteriophagy in a fluid medium; inhibition to a retardation of growth when the bacteriophage is added to a seeded medium; and plaques, of course, refers to the formation of these areas upon agar.

Janzen and Wolff were the first to show that a race of Typhoid-bacteriophage, manifesting at its isolation a virulence for certain strains of $B$. typhosus and at the same time completely avirulent for others, was able to acquire a definite virulence for some of the latter strains, and this simply by means of passages at the expense of certain susceptible strains. I am including here two of their experiments. ${ }^{375}$

The virulences of the bacteriophage involved were determined for 5 different strains of B. typhosus immediately after its isolation from the intestinal tract and again after a series of passages with a single strain of the typhoid bacillus. The results obtained with bacteriophage race $R e$, are shown in table 14.

Additional data are given for bacteriophage, race Wi (table 15).

The results are essentially these: bacteriophage Re, by virtue of passages at the expense of typhoid strain Sm has acquired a virulence 


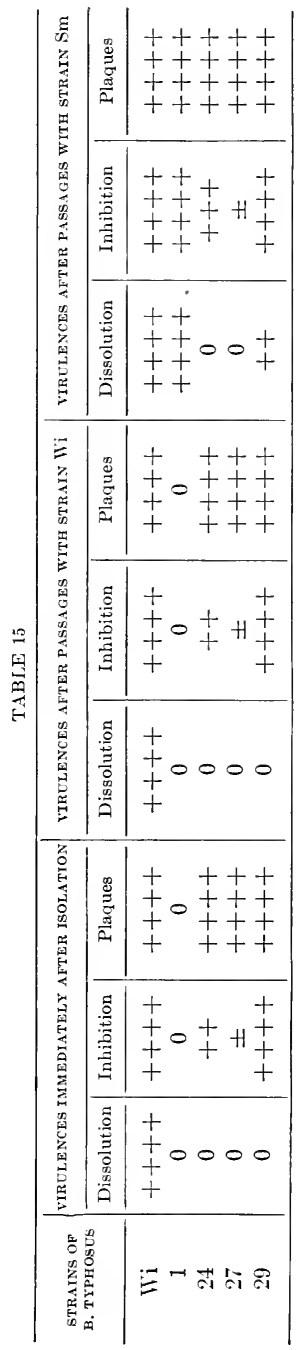


for strains 24 and 29, while bacteriophage $\mathrm{Wi}$, through passages with this same strain Sm, has acquired a virulence for strain 1.

Da Costa $\mathrm{Cruz}^{153}$ has applied the same procedure to develop a race which would give bacteriophagy of a strain which was originally not attacked. When isolated this bacteriophage had but a slight virulence for strain 1 of $B$. typhosus, but it was very active for strain 18 . With these two strains as extremes, the virulence for a number of other strains was more or less marked. The first passages were made with strain 18, then with strains which originally were less susceptible. Contacts were thus made involving successive passages with strains which were less and less susceptible. When the series of contacts was completed the race showed a very marked virulence for strain 1 .

It would appear, therefore, that contacts with species more and more remote from the normal host may, in reality, be the device whereby a given race of the bacteriophage acquires a virulence for a bacterium previously insensitive to its action.

Eliava and myself ${ }^{321}$ have adapted a Staphylo-bacteriophage to bacteriophagy of $B$. dysenteriae Shiga, but thus far it has been impossible to accomplish the reverse change.

McKinley, ${ }^{458}$ working with a race of Shiga-bacteriophage* quickly obtained a high virulence for the meningococcus by means of four passages in a bouillon containing glucose and blood. This bacteriophage, after its adaptation to the meningococcus, was found to be virulent for Staphylococcus albus, aureus, and citreus, for M. tetragenus, for Streptococcus hemolyticus, and for pneumococci of Groups I, II and III.

This same Shiga-bacteriophage, without the passages with the meningococcus was avirulent for all of these different organisms.

The virulence of a race of bacteriophage is not fixed. No race is immutable; all vary continually. Some of them lose virulence, although very slowly, and others acquire virulences by adaptation. We will see that this behavior of the bacteriophage, here demonstrated experimentally, is but a reproduction of processes occurring in the same way in nature.

\section{BACTERIOPHAGY IN MIXED CULTURES}

If one introduces a bacteriophage possessing multiple virulences into a bacterial suspension prepared by mixing cultures of several different

* A race which I sent him; indeed, one of the races which is mentioned here in a number of the experiments. It had undergone, at the time when it left my laboratory, about 1200 passages with $B$. dysenteriae. 
susceptible bacterial species, bacteriophagy occurs, but not all of the organisms are attacked with the same intensity. Bacteriophagy is complete for the species toward which the virulence is maximal or very high, it is partial for others, and is, indeed, the less marked as the virulence for the organisms is the less pronounced. As a matter of fact, whether the bacteriophage acts upon different susceptible organisms separately or whether it acts upon mixtures of these bacteria, the attack occurs in just the same manner.

It is rather interesting that it appears that the virulence for bacteria which are attacked but weakly may be increased more rapidly in combined cultures, as is indicated by the following experiment.

Three tubes of bouillon receive respectively $0.01,0.1$, and $1 \mathrm{cc}$. of a known Shiga-bacteriophage. The three tubes are then lightly planted with $B$. coli. Normal cultures develop in the three tubes. Platings on agar give few plaques. Each of the three cultures is transferred to fresh bouillon. Normal B. coli cultures develop. Transfers to agar give two plaques for the first tube, none for the other two. The culture yielding the two plaques is again re-inoculated. A normal culture develops. The bacteriophage has been eliminated.

This strain of Shiga-bacteriophage possesses, therefore, an extremely feeble virulence for the strain of $B$. coli under test.

To 10 cc. of bouillon is added 1 drop of a concentrated suspension of Shiga bacilli (this should give a slight turbidity equal to about $50,000,000$ bacilli per cubic centimeter) and 1 drop of an equally concentrated suspension of B. coli. This double suspension is then inoculated with $0.01 \mathrm{cc}$. of the Shiga bacteriophage used in the above experiment.

After twenty-four hours there is a slight turbidity. A new passage into a double Shiga-Colon suspension is made. Perfect dissolution takes place after eleven hours.

The dissolved suspension is then introduced, in a quantity of 0.04 cc., into a simple suspension of $B$. coli. Dissolution is complete in seven hours.

The corpuscles have developed at the expense of the Shiga bacilli, and thus being maintained in the medium they have gradually acquired a virulence for $B$. coli (d'Herelle ${ }^{321}$ ).

\section{RÉSUMÉ}

The multiplication of bacteriophage corpuscles belonging to different races does not take place with the same intensity, even though the conditions for the process are identical for all. The activitv-the 
"force"- of a bacteriophage is always strictly proportional to the intensity of its power of multiplication. The activity of a bacteriophage corresponds, then, to a "virulence" in the strict sense of the word (d'Herelle $\left.{ }^{315}\right)$.

A method of "titrating" the activity of a race must take the attributes of the bacteriophage into consideration, in particular, the fact that it is corpuscular in nature and that the corpuscle possesses a virulence. Inasmuch as the activity of a bacteriophage for a particular bacterium is causally related to the intensity of its power of multiplication at the expense of this bacterium the only correct method of numerically expressing virulence consists in a determination of the number of corpuscles per cubic centimeter to be found in a suspension after bacteriophagy is complete. Results are comparable only if the figures representing the intensities of multiplication are derived through experiments carried out under comparable conditions. The larger the number of corpuscles found the more intense has been the development, and the higher is the virulence of the race (d'Herelle). Particular emphasis is placed upon this point because of its very great importance; because of its lack of precision the so-called "dilution method" of counting bacteriophage corpuscles can not be used in the study of bacteriophagy. It represents the greatest single cause of error appearing in the results of those who have, unfortunately, employed it.

Inasmuch as different races of the bacteriophage show varying degrees of virulence it is necessary, in experimental procedures, to work with pure races, that is to say, with a race derived entirely from a single corpuscle (Bail ${ }^{53}$ ). There are two methods of purification which permit one to oltain a pure race. The first consists in the removal of corpuscles from a colony or plaque arising from the multiplication of a single corpuscle (d'Herelle ${ }^{310,318}$ ); the second is the method where dilution is carried to the point where but a single corpuscle per unit volume is present (d'Herelle 318,342 ).

In a given suspension of the bacteriophage not all of the corpuscles are endowed with exactly the same properties. Virulence, in particular, is variable for each one of them (d'Herelle ${ }^{321}$ ). The method of isolation by the limiting dilution permits the selection of the most virulent corpuscles as the origin of pure races. This procedure is at one and the same time a method of isolation and of selection (d'Herelle).

The virulence of a bacteriophage is variable. It can be increased experimentally. Increase in virulence may be ohtained by serial passages with a bacterium toward which one desires the virulence to be increased 
(d'Herelle, $\left.{ }^{310}\right)$. It is also possible to produce, experimentally, an attenuation in the virulence of a bacteriophage. Attenuation occurs when it is subjected to an appropriate degree of heat (d'Herelle and Pozerski ${ }^{360}$ ). It may occur spontaneously with the passage of time (Asheshov ${ }^{29}$ ).

The action of certain races of the bacteriophage is strictly specific; they act upon but a single strain of a given bacterium. Examples of this are afforded by certain races of the Typhoid-bacteriophage (d'Herelle $\left.^{313}\right)$. Certain bacterial species are homogeneous with regard to the bacteriophage, that is to say, when a race of the bacteriophage is virulent for one strain it is also virulent for all other strains. B. dysenteriae Shiga and B. pestis are examples of homogeneous species. Other bacterial species are heterogeneous, that is to say, certain strains may be attacked while others are insusceptible (d'Herelle $\left.{ }^{321}\right)$. This natural resistance of certain strains is not absolute inasnuch as a strain may be unattacked by one race of the bacteriophage although it is attacked by others (Janzen and Wolff ${ }^{374}$ ).

Ordinarily a bacteriophage is virulent, not only for bacteria of a given species, but also for bacteria belonging to different species, sometimes to organisms very distantly related (d'Herelle ${ }^{315}$ ). The range of virulence and the intensity of virulence may differ for each race of the bacteriophage and as the result of this it is impossible to conceive of two races as having absolutely identical characteristics (d'Herelle ${ }^{315}$ ).

The manner in which virulence for different bacterial species persists in a bacteriophage indicates that virulence is a fairly stable property. Virulence will persist throughout a very great number of passages, all carried out at the expense of a single strain (d'Herelle ${ }^{315}$ ). Multiple virulences persist in spite of the fact that the race is subjected to purification procedures, indicating that they are, therefore, an inherent property of a race derived from a single corpuscle (d'Herelle).

The persistence of virulence for a bacterium other than that with which the passages are made is not an attribute of all corpuscles but of certain ones only, and the number of these varies but slightly during a series of passages (d'Herelle ${ }^{321}$ ).

It is possible, by experimental adaptation procedures, to cause a race of the bacteriophage to acquire a virulence for an organism against which it was originally completely inactive (d'Herelle $\left.{ }^{310,321}\right)$. 


\section{CHAPTER V \\ Resistance of the Bacteria}

\section{SECONDARY CULTURES}

'I'hroughout the first three chapters we have studied the typical phenomenon of bacteriophagy as it is brought about through the action of powerful races of the bacteriophage. Here the bacteriophage corpuscles develop in proportion as the bacteria become dissolved and when the process is once completed that which was a few hours previously a suspension of bacteria has become a suspension of bacteriophage corpuscles, free of all bacteria. We have also seen that the power of provoking bacteriophagy varies among races of the bacteriophage. For some of them the power to reproduce at the expense of the bacteria is considerable, for others it is less, and this reproductive capacity corresponds exactly to a virulence more or less great. As a rule each race of the bacteriophage will attack bacteria belonging to different species and will have for each of them a different degree of virulence, capable of being increased by adaptation.

But there is another aspect of the phenomenon. In biology there is a general law to the effect that all living beings react against an agent which attacks them. If we consider the bacteria, endowed with virulence for some animal species, we do not know of any whose virulence can be such that an opposing immunity may not possibly be acquired.* The natural law of resistance comes into play in the same way when a bacterium is attacked by a bacteriophage. Bacteria have the capacity to resist, and may thus acquire an immunity to the bacteriophage corpuscles which attack them (d'Herelle ${ }^{317}$ ).

There are races of the bacteriophage of such virulence that under certain determined conditions of temperature and of medium the susceptible bacterium is always overcome. Resistance is impossible. Bacteriophagy is then complete and the medium becoming clear remains so indefinitely.

I have obtained, by repeated selection of the most apt corpuscles,

* This has occurred and will occur in the future. The inescapable result of the possession by a bacterium of an "absolute virulence" for an animal species means the disappearance of this animal species within a short period of time. 
races of the Shiga-bacteriophage capable of effecting a complete dissolution of normal suspensions and with such a race it is not necessary to filter the dissolved suspension through a candle to insure that the medium remain clear and sterile indefinitely. With such races bacteriophagy can be continued throughout an unlimited series without filtration between the successive passages. After a process of selection it is the same for the Staphylo-bacteriophage described by Gratia as race $\mathrm{H}$, but in order to obtain this result it is necessary that bacteriophagy take place at a temperature of $32^{\circ} \mathrm{C}$. and that the bouillon have a $\mathrm{pH}$ greater than 7.5. If these conditions are not satisfied a number of the suspensions, after a complete clearing, again become cloudy after an interval of time, and it is of interest that the number of suspensions to become turbid increases as the conditions become more divergent from the optimum.

The following experiments were carried out with Staphylo-bacteriophage, race H. Normal suspensions, containing 250 million cocci per cubic centimeter, prepared from a 24-hour agar culture were used. Ten cubic centimeters of the suspension were inoculated with 0.001 cc. of the bacteriophage. Each of the experiments comprised 24 tubes, each containing $10 \mathrm{cc}$., subjected to the same treatment at the same time.

Experiments 1 to 5 illustrate the effect of temperature. In all of these the reaction of the bouillon was 7.8.

1. Temperature, $25^{\circ} \mathrm{C}$. After 32 hours bacteriophagy was complete in all 24 tubes. After 7 days, at $25^{\circ} \mathrm{C}$., the 24 suspensions were limpid. After 2 months at laboratory temperature the 24 suspensions remained unchanged,--perfectly clear.

2. Temperature, $31^{\circ} \mathrm{C}$. After 19 hours bacteriophagy was complete in the 24 tubes. After 7 days, at $31^{\circ} \mathrm{C}$. the 24 suspensions were clear. After 2 months at laboratory temperature they still remained clear.

3. Temperature, $36^{\circ} \mathrm{C}$. After 18 hours bacteriophagy was complete in the 24 tubes. After 72 hours at $36^{\circ} \mathrm{C}$. the 24 suspensions were limpid. After 4 days at $36^{\circ} \mathrm{C}$., 23 were limpid; one was cloudy. After 5 days at $36^{\circ} \mathrm{C}$., 22 were limpid and 2 were cloudy. After 7 days at $36^{\circ} \mathrm{C}$. the result was the same. After 2 months at room temperature the results still remained the same; 22 tubes being clear, 2 being cloudy.

4. Temperature, $40^{\circ} \mathrm{C}$. After 18 hours bacteriophagy was complete in all 24 tubes. After 28 hours, 11 tubes were clear, 13 were cloudy. After 72 hours all 24 tubes were cloudy.

5. Temperature, $31^{\circ} \mathrm{C}$. After 24 hours all 24 of the suspensions were clear. After 6 days they still remained clear. At this time 20 of the 
tubes were placed in the incubator at $41.5^{\circ} \mathrm{C}$., the other 4 remained at laboratory temperature. After 24 hours at $41.5^{\circ} \mathrm{C}$., 7 of the 20 suspensions were clear, 13 were cloudy. After 48 hours at this temperature all 20 of the suspensions were cloudy showing an abundant secondary culture. The four suspensions held at laboratory temperature were clear and remained so for 2 months.

A second part of this experiment, designed to show the effect of the reaction of the medium was carried out at a temperature of $31^{\circ} \mathrm{C}$.

6. The bouillon used had a pH of 7.0. Bacteriophagy of all 24 tubes was complete after 24 hours. After 48 hours the 24 tubes were turbid. This, compared with part 5 above shows clearly the importance of the hydrogen ion concentration.

Experiments carried out with a potent Shiga-bacteriophage have given results of the same nature; the conditions of temperature and of reaction exerting a comparable influence.

It may be well to state, and later we will consider this point further, that the optimum temperature may vary through some degrees. Furthermore, we have seen that the optimum temperature is not the same for all races; it is simply a question of adaptation as may be readily demonstrated by experiment. But it is none the less true that insofar as the bacteriophagy of $B$. dysenteriae and of the staphylococcus is concerned, once an adaptation has been accomplished, a temperature of $32^{\circ} \mathrm{C}$. is optimum - the critical temperature. Above this, whatever may be the virulence of a bacteriophage and although it may be adapted to induce bacteriophagy at a higher temperature, permanent sterility of unfiltered suspensions of the bacteriophage is not uniformly obtained.

Experiment 5 (above) is of significance, for it shows that although media in which bacteriophagy has been complete will remain sterile indefinitely if conditions favorable to the bacteriophage are maintained, they will yield secondary cultures if placed under conditions favorable for the bacterium, although the media were perfectly clear and no bacteria could be found even by examination of the sediment secured by centrifugation. From where then, in these cases, are the resistant bacteria derived? This is a question which we will consider in a later section entitled Ultrabacteria.

Macroscopic examination and biological reactions show that the turbidity which occurs in bacteriophaged suspensions is due to the development of bacteria of the same kind as those which formed the suspension prior to bacteriophagy. These cultures which develop under these circumstances, i.e., after bacteriophagy, I have termed secondary cultures. ${ }^{317,324}$ 
Once bacteriophagy is completed with the suspensions perfectly clear; those tubes which are to give a secondary culture can not be distinguished macroscopically or microscopically in any way from those which are to remain clear indefinitely. Transfers to bouillon and on to agar of bacteriophaged suspensions in which a secondary culture is to develop later remain sterile up to the time that the secondary culture appears. This does not often occur until 5 or 6 days after the dissolution, sometimes even later.

A suspension of Shiga bacilli, containing 250 million bacilli per cubic centimeter, is inoculated with $0.001 \mathrm{cc}$. of a culture of the bacteriophage. Normal bacteriophagy takes place in 5 hours, with the medium perfectly limpid. The dissolved suspension is planted on agar and in bouillon $1,2,3,4,5,6$, and 7 days after the dissolution is complete. All of the plantings remain sterile. On the eighth day the dissolved suspension is slightly clouded. On the ninth day a drop is introduced into broth and drops are spread over 3 tubes of agar. Two of the agar tubes remain sterile, the third shows 4 small colonies. The broth tube gives an agglutinated, sedimented culture. ${ }^{321}$

The following experiments show that the number of secondary cultures to develop diminishes as the virulence of the bacteriophage is increased. The most potent race of the bacteriophage which I have yet isolated (these experiments were carried out in 1918) was combined with 2 strains of the Shiga bacillus. One of these bacterial strains has been for a long time under artificial cultivation, being used by the Pasteur Institute for the inoculation of horses in the production of anti-dysentery serum (type strain). The other was recently isolated from the stool of a patient with dysentery (strain Jerv.).

(A) Twelve tubes of the suspension of the type strain of the Shiga bacillus are each inoculated with 0.001 cc. of a culture of the bacteriophage. This latter has been carried on for a great number of generations always at the expense of a single bacillary strain. In all twelve tubes dissolution is perfect, with complete clearing in four hours. After three days at $37^{\circ} \mathrm{C}$. one of the tubes is slightly cloudy, the others are clear. (Five other experiments, each consisting of 12 tubes, with the same race of the bacteriophage and the same bacillus give the following results:- tubes showing secondary cultures in each set, $0,2,0,3$ and 1. There develop, then, 7 secondary cultures in the 60 tubes, or 12 per cent.)

(B) Twelve tubes of suspension were prepared with the strain Jerv., a strain with which the bacteriophage in question had never been in 
contact. Each of these tubes is inoculated with 0.001 cc. of the same culture of bacteriophage as that used in the preceding experiment (A). Seven of the 12 tubes give secondary cultures. The results from five other experiments with the same strains are, $9,5,10,5$, and 6 secondary cultures, or 70 per cent. A week later 12 cultures of the Jerv. bacillus are inoculated from one of the previous tubes that had remained clear. From these, 5 secondary cultures are secured.

A further passage made after another week, gives 4 secondary cultures in the 12 suspensions. After another week, a fourth passage, still taking the bacteriophage from a perfectly limpid culture, yields but one secondary culture among the twelve inoculated.

$(C)$ At the beginning of convalescence in the dysentery case (Jerv.) a bacteriophage was isolated which was tested in the same manner both on the type Shiga strain and on the Jerv. strain. This last was derived from the patient early in the infection at a time when the intestinal bacteriophage had manifested no activity for this organism.

With the bacteriophage Jerv. on the type bacillus 4 secondary cultures develop among the 12 suspensions dissolved.

With the bacteriophage Jerv. on the bacillus Jerv., there are no secondary cultures among the 12 tubes dissolved. When repeated upon an additional 12 suspensions a single secondary culture develops.

To state the situation briefly, the frequency of secondary cultures is strictly related to the virulence of the bacteriophage. With the bacteriophage of maximum virulence they do not occur when the conditions are optimum for the development of the bacteriophage corpuscles. If the conditions are not best suited to the development of the bacteriophage, secondary cultures may develop and the number to appear becomes increasingly large as the conditions are more remote from the optimum. With bacteriophage races but slightly below a maximum virulence a certain number of secondary cultures can always be obtained even though the conditions are optimum. A number of the bacteriophage suspensions, many or few as the case may be, will remain limpid indefinitely while other tubes will give secondary cultures. With races still less virulent, but yet capable of causing a total dissolution of the bacteria of the suspension, secondary cultures always develop. And finally, for races where the virulence is only moderate or weak, the bacteria acquire, within the first few hours, a resistance sufficient to enable them to develop in spite of the presence of bacteriophage. Under such conditions clearing of the medium can not take place. Coincident with the dissolution of those bacteria which are least capable of developing a 
resistance there occurs a multiplication of the bacteria which are the more apt to acquire a resistance (d'Herelle ${ }^{317}$ ).

This makes it clear why it is absolutely necessary, during a series of passages made to enhance the virulence of a bacteriophage, to separate the bacteriophage corpuscles in the process of increasing their virulence from the bacteria which have acquired a resistance. As we know this can be done either by filtration or by heating. By causing in each new passage the corpuscles whose virulence is gradually being increased to react upon fresh, normal bacteria which have never yet been in contact with the bacteriophage, the phenomenon of gradually increasing resistance does not intervene to counterbalance the progressive acquisition of virulence.

We have already refuted the rather peculiar theory of Seiffert ${ }^{578}$ according to which it is not the bacteriophage which increases its virulence but rather the bacterium which adapts itself to the secretion of a "lysin." An adaptation can not be transmitted to non-existent descendants. As a matter of fact the result of the adaptation of the bacterium to the bacteriophage is exactly the opposite to the production of a "lysin," for the bacterium adapts itself to resist the agent which provokes its dissolution.

Bacteriophagy is in reality a very complex phenomenon. The two cardinal factors which come into play are the bacteriophage corpuscles on the one hand with their virulence and on the other, the bacterium with its capacity to resist. Each of these factors is by its nature a variable, subject to the conditions of the moment. The virulence and the resistance fluctuate continually; they are increased or they are diminished. The macroscopic result of bacteriophagy, that is to say, the dissolution of the bacterial cells, is the resultant of the two factors which operate in opposition to each other (d'Herelle ${ }^{317}$ ).

An experiment of Gratia ${ }^{249}$ exteriorizes, one might say, this struggle between virulence and resistance. Working with $B$. coli he has shown that in an acid ( $\mathrm{pH} .6 .8$ ), neutral (7.0), or even slightly alkaline (7.2) medium one may observe a succession of waves of growth and of dissolution of the bacteria exposed to the action of a bacteriophage. With each wave the growth is a little more accentuated and the dissolution following is less complete. With the same bacteriophage but under conditions which were more favorable, that is, in an alkaline medium ( $\mathrm{pH}$ 7.5), the phenomenon of bacteriophagy occurred normally.

If the conditions are still more unfavorable to the bacteriophage, for example, if the medium is definitely acid, the bacteriophage no longer 
attacks the bacterium. Under these circumstances the latter does not acquire a resistance since it is not attacked. Thus Scheidegger ${ }^{568}$ working with $B$. coli, has shown that in a bouillon having a $\mathrm{pH}$ of 4.5 , in which this organism is still able to develop, the bacteriophage remains inert, and the bacterium acquires no resistance. But if such a medium is neutralized bacteriophagy takes place and the colon bacillus develops a resistance. The optimum conditions for development are not the same for bacteriophage corpuscles and for bacteria. Consequently the conditions favoring the one or the other of the two antagonists determines whether the first or the second will finally win out.

\section{THE ORIGIN OF SECONDARY CULTURES}

What is the intimate mechanism of the process that results in the formation of secondary cultures? A priori two hypotheses can be formulated. Two factors are present, a bacteriophage whose virulence may be attenuated, and a bacterium whose resistance may be augmented. Thus, are secondary cultures due to a weakening of the activity of the bacteriophage, or, do there exist in the bacterial suspension certain individual cells which acquire an immunity to the bacteriophage, thus leading to the development of a resistant race? The following experiments clearly settle the question in favor of the last hypothesis.

In the section treating of the isolation of the bacteriophage we have seen that in the large majority of cases the races which are freshly isolated are of too low activity to effect a complete dissolution of a bacterial suspension; cases where the presence of the corpuscles could only be detected by the presence of plaques upon the agar slants. These same races were able to acquire, by successive passages, a very high activity, a potency which enabled them to bring about dissolution of very heavy suspensions. This method of serial passages of the bacteriophage, in which it is forced to develop in vitio at the expense of a given bacterium, corresponds exactly with the method of Pasteur for effecting an enhancement in virulence of a bacterial strain by repeated passage through a given animal species.

This single experiment, repeated a considerable number of times, in fact, each time that a bacteriophage of low virulence is isolated from the body - shows that secondary cultures are not produced by a simple diminution in the virulence of the bacteriophage. Indeed, there is, on the contrary, an enhancement with each passage, even if macroscopic dissolution is not to be seen. For this the following experiment offers direct proof : 
The contents of a tube that gave a secondary culture is filtered through infusorial earth and a bougie. Twelve tubes of a Shiga suspension are inoculated, each receiving $0.001 \mathrm{ce}$. of the filtrate. Perfect dissolution is seen in all tubes, and in all but one the dissolution is permanent. This single tube again becomes turbid after 4 days.

From this it is clear that the bacteriophage has not lost in virulence, and that secondary cultures can not be ascribed to a change in that direction. The bacteriophage remains virulent, coexisting with bacteria which have become resistant. The secondary cultures, then, are the result of an adaptation undergone by the bacterium which acquires an immunity to its parasite.

It has already been shown that the number of corpuscles inoculater is without influence on the appearance of secondary cultures. The

TABLE 16

\begin{tabular}{c|c|c}
\hline TUBE & $\begin{array}{c}\text { AMOUNT } \\
\text { OF BACTERIOPHAGE } \\
\text { FILTRATE } \\
\text { INOCULATED }\end{array}$ & \multicolumn{1}{c}{ RESUlts } \\
\cline { 2 - 3 } 1 & $c c$. & Normal dissolution, secondary cultures \\
2 & 0.1 & Normal dissolution, no secondary cultures \\
3 & 0.02 & Normal dissolution, no secondary cultures \\
4 & 0.004 & Normal dissolution, secondary cultures \\
5 & 0.002 & Normal dissolution, no secondary cultures \\
6 & 0.0002 & Normal dissolution, no secondary cultures \\
\hline
\end{tabular}

conflict is not one of numbers; it is rather a struggle in which the significant factors are virulence on one side and ability to resist on the other.

A suspension of B. dysenteriae, 250,000,000 per cubic centimeter, is distributed into 6 tubes and these are inoculated with variable quantities of the same bacteriophage filtrate. The results obtained are given in table 16.

The tubes yielding secondary cultures are distributed at random throughout the series, showing no fixed relationship to those tubes in which the dissolution was permanent.

Another experiment may be presented, indicating as it does, the random manner in which secondary cultures may develop.

This experiment shows the serial activity of the bacteriophage together with the appearance of secondary cultures. Each tube of the series is prepared with a suspension of B. dysenteriae, 250,000,000 per cubic centimeter, and into each is introduced $0.001 \mathrm{cc}$. of the dis- 
solved suspension of the preceding tube. Transfers are made after twenty-four hours, that is, at a time when dissolution is complete. (See table 17.)

Certain salts, when added to the suspension in very minute quantities, $0.1 \mathrm{mgm}$. to $10 \mathrm{cc}$. of culture, favor the development of secondary cultures. The salts of lead (nitrate and acetate) and of silver (nitrate and sulfate) act in this way. The soluble phosphates and magnesium sulfate appear to be without action. With a single race of bacteriophage and a given strain of bacillus the development of secondary cultures is, in

TABLE 17

\begin{tabular}{|c|c|c|}
\hline DATE & $\begin{array}{l}\text { A FRESH SUSPENSION RECEIVED THE } \\
\text { MATERIAL INDICATED }\end{array}$ & RESULT \\
\hline July 8 & $0.001 \mathrm{cc}$. of bacteriophage suspension & Permanent dissolution \\
\hline July 9 & $\begin{array}{l}0.001 \text { cc. of suspension bacteriophaged on } \\
\text { July } 8\end{array}$ & Permanent dissolution \\
\hline July 10 & $\begin{array}{l}0.001 \text { cc. of suspension bacteriophaged on } \\
\text { July } 9\end{array}$ & $\begin{array}{l}\text { Secondary cultures in } 3 \\
\text { days }\end{array}$ \\
\hline July 11 & $\begin{array}{l}0.001 \text { cc. of suspension bacteriophaged on } \\
\text { July } 10\end{array}$ & Permanent dissolution \\
\hline July 12 & $\begin{array}{l}0.001 \text { cc. of suspension bacteriophaged on } \\
\text { July } 11\end{array}$ & Permanent dissolution \\
\hline July 13 & $\begin{array}{l}0.001 \text { cc. of suspension bacteriophaged on } \\
\text { July } 12\end{array}$ & Permanent dissolution \\
\hline July 14 & $\begin{array}{l}0.001 \text { cc. of suspension bacteriophaged on } \\
\text { July } 13\end{array}$ & $\begin{array}{l}\text { Secondary cultures in } 4 \\
\text { days }\end{array}$ \\
\hline July 15 & $\begin{array}{l}0.001 \mathrm{cc} \text {. of suspension bacteriophaged on } \\
\text { July } 14\end{array}$ & Permanent dissolution \\
\hline July 16 & $\begin{array}{l}0.001 \text { ce. of suspension bacteriophaged on } \\
\text { July } 15\end{array}$ & Permanent dissolution \\
\hline July 17 & $\begin{array}{l}0.001 \text { ce. of suspension bacteriophaged on } \\
\text { July } 16\end{array}$ & Permanent dissolution \\
\hline
\end{tabular}

general, more frequent when the suspension is prepared from agar cultures several days old than when made from fresh cultures.

At first thought it appears strange that when secondary cultures develop with a race of bacteriophage of high potency, they appear in some tubes and not in others. The following experiment offers an explanation for this.

Two flasks, each containing 200 cc. of a $B$. dysenteriae suspension $(250,000,000$ per cubic centimeter) are inoculated with 0.04 cc. of a culture of the bacteriophage (the same race as that used in the preceding experiments). Immediately after inoculation the contents of the 
first flask is distributed into 20 tubes, $10 \mathrm{cc}$. to each. In all of these dissolution takes place normally, being permanent in 19, showing a secondary culture in 1 . The second flask is portioned out the next day, that is, after dissolution is completed, $10 \mathrm{ec}$. being placed in each of 20 tubes. None of these become turbid. When this second part of the experiment is repeated, 18 remain clear, and 2 tubes yield secondary cultures.

Each flask of suspension contained 50,000 million bacilli, and the above experiments show that of this number but one or two were capable of aequiring an immunity to the very active bacteriophage. It is these "immune" bacilli which give rise to organisms that enjoy the same degree of resistance.

Secondary cultures, then, have their origin in the operation of the phenomenon of natural selection, whereby some bacilli show a greater aptitude than others to the acquisition of a resistance to the bacteriophage.

The phenomenon of secondary culture formation is governed by the individual properties of the bacterium and bacteriophage. Against a single strain of bacterium the less virulent the bacteriophage the greater will be the proportion of secondary cultures, or, in other words, the greater is the number of bacilli in the suspension capable of acquiring a resistance.

Gratia $^{250}$ has suggested that resistance to the action of the bacteriophage may not consist in the acquisition of resistance by the bacterium but in a selection of those bacteria naturally endowed with this property prior to the action of the bacteriophage. That a selection takes place is precisely what $I$ had shown previously ${ }^{317}$ by means of an experiment which has been presented in this present section. But it operates not through a selection of the bacteria naturally endowed with a resistance, but through a selection of those susceptible bacteria which are the more apt at acquiring resistance. This experiment shows definitely that what takes place is really an acquisition, in the strictest sense of the word, of resistance to the action of the bacteriophage. This is emphasized by the fact that a resistant bacterium gradually loses resistance in the absence of virulent bacteriophage corpuscles. If resistance can be lost, obviously it is only because it has been acquired. We will consider further this phenomenon in a later section.

\section{VARIABILITY IN ACQUIRED RESISTANCE}

Appelmans and Wagemans ${ }^{16}$ have published some experiments designed to show that bacteria may become resistant to one race of the 
bacteriophage and remain susceptible to another and this quite without regard to the virulence of the bacteriophage against which the resistance is acquired.

Unquestionably this observation is correct, but it is not legitimate to base too broad generalizations upon it. Everything depends upon the respective characters of the race of bacteriophage and the strain of bacteria. In my experience, it has seemed that the matter of virulence usually exerts the greater influence, but to this there are many exceptions, particularly when the bacterium involved belongs to a heterogeneous species. A bacterium which has acquired a resistance to a bacteriophage of low virulence may remain susceptible to another bacteriophage of high virulence. But a bacterium which has acquired a resistance to a bacteriophage of very high virulence is usually resistant to bacteriophagy by other races which are of lower virulence.

In cases where two races of the bacteriophage have approximately the same degree of virulence a bacterium may become resistant to one of them and remain susceptible to the attack of the other. But even here, the matter of virulence often plays a rôle as is proved by the fact that if a bacterium acquires a refractory state toward a bacteriophage of maximum virulence this bacterium usually resists the action of all other races of the bacteriophage even if they are likewise endowed with a maximum virulence.

An experiment illustrative of these facts may be given. The Staphylo-bacteriophage $v$ has a maximum virulence for but a single strain of the staphylococcus, Staphylococcus albus $V$. Staphylobacteriophage $h$ also possesses a maximum virulence, but its action is exercised indiscriminately upon all strains of the staphylococcus. By carrying out the process of bacteriophagy in a bouillon having a $\mathrm{pH}$ of 6.8 , leading thus to secondary cultures, I have obtained a strain of staphylococcus resistant to bacteriophage $h$. With this bacterium a series of cultures were made in the presence of increasing amounts of bacteriophage $h$, first incubating them at $37^{\circ} \mathrm{C}$, and then at $30^{\circ} \mathrm{C}$. Finally a strain of Staphylococcus $V$ was derived which was refractory to bacteriophage $h$, that is to say, at this time the staphylococcus developed in a normal manner, macroscopically, in a pure suspension of bacteriophage $h$ corpuscles in a medium with a $\mathrm{pH}$ of 7.8 and at a temperature of $30^{\circ} \mathrm{C}$.

At this time this staphylococcus was found to be refractory to the action of race $v$ also, developing normally insofar as macroscopic observation could reveal in a pure suspension of corpuscles of bacteriophage $v$ with the medium of a pH of 6.8 and the temperature at $30^{\circ} \mathrm{C}$. 
These two races of the bacteriophage are, however, as different from each other as it is possible for them to be. As I have already stated, one of them, $v$, is virulent only for the $V$ strain of the Staphylococcus albus. The other race, $h$, is virulent for all staphylococci, whether they are classed as albus, aureus, or citreus. *

I have obtained the same results with three races of the Shiga-bacteriophage. A Shiga bacillus, having become resistant to one of the races was also refractory to the action of the other two.

These experiments warrant the conclusion that the resistance of a bacterium is by no means limited to the action of a single race of the bacteriophage, but that a bacterium having acquired a resistance against one race of the bacteriophage may manifest a resistance to the action of any other race whatever. It is only in the cases where the resistance acquired is relative that this resistance may be lacking with respect to the action of a bacteriophage of another race.

It is, indeed, quite impossible to state any fixed rules governing the phenomenon of resistance to the bacteriophage. All that can be said is that usually such and such is true, but that there are many exceptions. For example, in an experiment reported by Bail ${ }^{46}$ dealing with a strain of $B$. coli which was naturally resistant to one race of the bacteriophage but susceptible to another, by suitable treatment this strain was induced to acquire a resistance to the last race of bacteriophage, and when this had developed the strain was found to be susceptible to the race for which it previously was resistant.

It would be just as unwise to generalize from the outcome of this experiment as to draw sweeping conclusions from the fact that under certain circumstances resistance appears to be specific.

Let us bear clearly in mind, in order to avoid confusion such as has occurred with certain authors, that the natural resistance to a bacteriophage presented by strains belonging to a heterogeneous species bears no relation to the acquired resistance developing in a susceptible bacterial strain. These two distinct phenomena are comparable to those processes which are designated in immunology by the terms "natural immunity" and "acquired immunity."

* As we know, an acquired resistance is gradually lost during successive transfers. In this experiment, the resistance of the $V$ strain of the staphylococcus to bacteriophage $v$ is lost after 7 transfers; resistance to bacteriophage $h$ is lost only after 19. 


\section{THE ACQUISITION OF RESISTANCE}

How can this acquisition of immunity by a bacterium be explained? Numerous experiments have shown that if a certain quantity of a slightly active suspension of a bacteriophage is introduced into a relatively heavy (1000 to 2000 million per cubic centimeter) suspension of bacilli, the corpuscles, readily demonstrated at first by the presence of plaques on plantings on agar, disappear from the medium after an interval of time varying from one hour to two or three days, and that they can not later be demonstrated. Subcultures give normal cultures of bacteria. On the other hand, we have seen that with a very virulent bacteriophage the corpuscles disappear from the fluid between ten and twenty minutes after introduction into a suspension, but that they reappear in about twenty times as great a number in from one to one and a half hours later - they have multiplied within the interior of the bacteria. In the case of a bacteriophage of low virulence it seems, therefore, that penetration of the bacteria takes place but that multiplication can not be effected. The bacterium resists and the corpuscle is actually destroyed in vivo. These parasitized bacteria which "recover" acquire by this an immunity.

Another fact has been sometimes observed which shows that certain bacteria are able to become "carriers." As has been said, heavy suspensions which are inoculated with a filtrate containing a relatively avirulent bacteriophage give after a few hours absolutely normal cultures on agar, free of plaques. If serial transplants are made of these cultures, the plantings being made in such a manner as to yield an even layer of growth, it sometimes happens that after a certain number of transplants, two to four, a very definite plaque appears, which is indeed a colony of the bacteriophage corpuscles. This is evidenced by the fact that successive passages from this plaque yield a very active bacteriophage. From where could this corpuscle have so suddenly come? The corpuscle had remained alive within a bacillus, and at a given moment, it overcame the resistance of the latter and multiplied. Its virulence being increased, the young corpuscles were able to parasitize the neighboring bacilli and form a colony. Any other explanation seems impossible, since, immediately after the inoculation of the bacteriophage, seeding upon agar shows plaques characteristic of the presence of virulent bacteriophagous corpuscles, then these corpuscles completely disappear, the bacteria ,however, remaining sensitive to the action of a more active bacteriophage, for perfect dissolution is secured if the suspen- 
sion is inoculated with a trace of a very active race of the bacteriophage, and finally, the active bacteriophage reappears after a series of subcultures on agar in the course of which all the bacillary cultures have been normal. This corpuscle can only be one of those which had disappeared. The fact, demonstrated by experiment, of the penetration of virulent bacteriophage corpuscles into the bacteria, warrants us in thinking that this corpuscle (but slightly virulent) has been preserved in a latent living state within the interior of the bacterium. At a given moment the resistance of the bacterium is broken down and infection results (d'Herelle ${ }^{321}$ ).

The fact that a bacteriophage corpuscle may penetrate a bacterium and be destroyed there has been confirmed by Flu ${ }^{211}$ who has carried out the following admirable experiment.

Flu found, among the cultures of the Institute of Tropical Medicine at the University of Leiden, a bacterium (isolated from the stools of an individual affected with sprue) which presented all of the cultural, biochemical, and serological characteristics of B. dysenteriae Flexner, except that it was inagglutinable. This bacillus was refractory to all races of bacteriophage virulent for $B$. dysenteriae Flexner. Nevertheless, suspensions of this bacillus, whether living or killed by heat, fixed the bacteriophage corpuscles of these races.

By a method of grinding with anhydrous sodium sulphate, a method which will be described in a later section, Flu was able to recover the corpuscles which had been fixed to the killed bacteria. When they had been fixed to living bacilli he was unable to recover them. As he has observed, this fact can only be explained by a destruction of the bacteriophage corpuscles by the protoplasm of the bacillus.

I have since proved by this same method that a bacterium rendered experimentally refractory behaves in the same manner. As a matter of fact, this experiment was carried out with the refractory strain of staphylococcus $V$, mentioned in a preceding paragraph. Destruction of the bacteriophage occurred not only with the corpuscles of race $v$, but also with those of race $h$. The destruction was, as a rul ; complete when the number of corpuscles in proportion to the number of cocci was not too great, for example, when there was not more than 1 corpuscle to 100 cocci. When the ratio was higher very frequently unfixed corpuscles remained.

A bacterium possessing a moderate degree of resistance may destroy bacteriophage corpuscles of low virulence. A refractory bacterium may destroy corpuscles of maximum virulence. 


\section{THE BEHAVIOR OF THE BACTERIOPHAGE IN SECONDARY CULTURES}

The way in which the bacteriophage behaves in secondary cultures involves a number of very interesting peculiarities.

We have seen that a single bacteriophage corpuscle, provided it is endowed with a high virulence, may cause complete bacteriophagy in a normal bacterial suspension and dissolution is still more certain if the suspension is somewhat less dense. Dissolution of the bacterial cells is complete. If one simply seeds the bacterium into bouillon inoculated with a single very virulent corpuscle there is a simultaneous development of a culture of the bacteria and of the corpuscles. When the latter have become sufficiently abundant to parasitize each of the young bacteria, dissolution occurs rapidly and the medium becomes clear.

Under all circumstances, then, the introduction of a single corpuscle of maximum virulence into a merlium containing from 1 to $250,000,000$ susceptible bacteria per cubic centimeter learls to a complete bacteriophagy, and secondary cultures never appear, provided the conditions are optimum for bacteriophagy, that is to say, provided the medium has a $\mathrm{pH}$ greater than 7.6 and the temperature is lower than $32^{\circ} \mathrm{C}$. Furthermore, in all such cases the corpuscles derived from the single corpuscle also have a maximum virulence.

With a bacteriophage of high, but not maximum, virulence the inoculation of a single corpuscle causes a bacteriophagy manifesting itself in the same manner as the preceding, but with further incubation a secondary culture always develops in the medium which had previously become completely cleared.*

Let us, then, observe the course of bacteriophagy as it takes place when a single corpuscle of high virulence is inoculated into a series of tubes each containing $10 \mathrm{cc}$. of a normal suspension of susceptible bacteria. $\dagger$ When bacteriophagy is complete and secondary cultures appear separate these suspensions in which the phenomenon has taken place into two groups. Filter the first group immediately while the medium is still clear. With the second group allow secondary cultures to form and filter them after a few days.

Comparing the two filtrates it will be found that the number of bacteriophage corpuscles is practically the same in both, but while those of the first filtrate have a very high virulence, equal to that of the original corpuscles, the virulence of the second filtrate is attenuated. If pas-

* See the section "Evaluation of Virulence."

$\dagger$ Following the technic which has been described, distributing $10 \mathrm{cc}$. of the last active dilution into 10 suspensions, $1 \mathrm{cc}$. per tube. 
sages are continued in this same manner, allowing the filtrate which has been in contact with the resistant bacteria to remain unfiltered in each passage until secondary cultures form, it will be found that a gradual attenuation of virulence takes place.

Hadley ${ }^{281}$ has reported that a Shiga-bacteriophage (one which I sent him) produced, at the beginning, plaques with a diameter of about 6 $\mathrm{mm}$. During a series of passages continued throughout a period of 2 years the diameter of the plaques became progressively less, up to the point where they ceased to be larger than $1 \mathrm{~mm}$. At this time, on going back to the original suspension which had been preserved for 2 years in a sealed tube, he found that the plaques formed were just as at the beginning, i.e., about $6 \mathrm{~mm}$. in diameter. And since the diameter of the plaques, all conditions being equal, is directly proportional to the virulence of the bacteriophage (d'Herelle ${ }^{312,331}$ ), Hadley has concluded that by successive cultures the bacteriophage has degenerated.

But I have maintained this same race of the bacteriophage* and it has undergone in my laboratory a great many passages and the plaques still actually measure between 5 and $6 \mathrm{~mm}$. in diameter. This would seem to prove that the degeneration observed by Hadley is a result of the conditions under which bacteriophagy took place throughout his experiments. Whatever may have been the unfavorable conditions, there was most certainly a formation of secondary cultures and these must always be avoided if it is desired to maintain intact the virulence of a bacteriophage. To accomplish this it is only necessary to filter the material just as soon as bacteriophagy is complete. This is the best means of avoiding, even from the beginning, the development of second. ary cultures which macroscopically might pass undetected.

A bacteriophage, then, becomes attenuated during the same process which leads to an acquisition of resistance by the bacterium. Even a bacteriophage of maximum virulence may be "overcome." To bring this about it is only necessary to inoculate it into a very concentrated suspension of bacteria. $\dagger$ It can be shown that under these conditions the virulence of the bacteriophage becomes weakened. After a series of passages is made in very heavy suspensions ( 8000 to 10,000 million

* Intentionally every time that I have given out a Shiga-bacteriophage I have always selected this same race.

$\dagger$ We have seen that if a suspension contains more than 700 to $800,000,000$ bacteria per cubic centimeter the dissolution of the bacteria is incomplete, even under the action of a bacteriophage of maximum virulence. With a bacteriophage of less virulence the number of bacteria capable of being dissolved is less. 
per cubic centimeter) the virulence is attenuated to such a degree that after a few passages the bacteriophage is lost.

In a word, each time that a secondary culture develops the virulence of the bacteriophage concerned becomes attenuated and this attenuation is the more pronounced when, on the one hand, the initial virulence was low and when, on the other, the number of resistant bacteria in proportion to the number of bacteriophage corpuscles present was high.

This is precisely the experiment performed by Bordet and Ciuca ${ }^{95}$ who inoculated a relatively large amount (a twenty-millionth of a cubic centimeter) of a Coli-bacteriophage suspension of average virulence* into a tube of bouillon which they then seeded with a culture of $B$. coli. After a few days in the incubator at $37^{\circ} \mathrm{C}$., that is to say, after a long contact of the corpuscles with a heavy culture of bacilli which had acquired a resistance, they heated the mixture at $57^{\circ} \mathrm{C}$. in order to kill the bacilli (a further cause of the attenuation of the virulence). They demonstrated that the fluid contained a bacteriophage attenuated both quantitatively and qualitatively.

These authors have attempted to explain this attenuation by assuming that a degeneration of the "lytic principle" occurred. As a result of its low concentration each bacterium in the suspension could fix but a very small amount and being thus but very slightly stimulated it could regenerate only a weak "lytic principle."

Such an explanation is not admissible for it does not take into account the fact that the bacteriophage exists in the form of corpuscles. Following this publication of Bordet and Ciuca, I offered to give a demonstration of the experiment proving the corpuscular nature of the principle, ${ }^{342}$ but the offer was not accepted. As a matter of fact, shortly after Bordet had published his experiment one of his collaborators, Gratia, published an experiment confirming the fact that the "concentration" of the bacteriophage principle plays but a secondary rôle. ${ }^{272} \dagger$ It is very evident that the explanation of Bordet can not be correct since if one inoculates the smallest active dilution of a suspension of bacteriophage into $1,10,100$, or $1000 \mathrm{ec}$. of bacterial suspension bacteriophagy takes place in the 4 suspensions in the same manner.

As a final proof let us prepare from a young agar culture of the staphylococcus 2 cc. of suspension each cubic centimeter containing 10,000 million cocci. Remove $1 \mathrm{cc}$. and introduce it into $99 \mathrm{cc}$. of sterile bou-

* As indicated by their experiments.

$\dagger$ The experiment of Gratia and DeKruif has been referred to in the section dealing with "Evaluation of Virulence." 
illon. We will thus have on the one hand $1 \mathrm{cc}$. of bouillon containing 10,000 million cocci and on the other hand a flask containing the same number of cocci suspended in $100 \mathrm{cc}$. of bouillon. Inoculate the two suspensions with the same quantity $\left(1 \cdot 10^{-8}\right)$ of a bacteriophage of maximum virulence. After incubation it will be found that the liquid in the flask is clear and remains so indefinitely, and the corpuscles present show a maximum virulence. At this same time the cubic centimeter quantity is extremely cloudy and after filtration it will be found that only attenuated corpuscles are present, their virulence being not even moderate.

It is possible to demonstrate this same fact in a still more conclusive manner. The cubic centimeter of suspension is inoculated with 0.1 cc. of a bacteriophage suspension; the hundred cubic centimeters with a hundred-millionth of this amount of the same suspension. After incubation the results are absolutely comparable to those of the preceding experiment. Corpuscles of a maximum virulence are found in the 100 cc. quantity and corpuscles of a low virulence in the cubic centimeter. In view of the fact that the number of bacteria was the same in both cases, ${ }^{*}$ the attenuation of virulence can not, then, be explained by assuming that the bacteriophage disseminates its action through too large a number of bacteria. This is, however, a priori certain since we know that the bacteriophage acts as a unit; a single bacteriophage inoculated into $10 \mathrm{cc}$. of a normal suspension, that is, placed in contact with 2500 million bacteria, effects a complete bacteriophagy.

What, then, can be the cause of the attenuation in virulence? The single point which differentiates the process of bacteriophagy as it occurs in one suspension from that taking place in the other is that in the 100 cc. of suspension a secondary culture does not develop. None of the bacteria there acquire a resistance. In the $1 \mathrm{cc}$. the bacteria become resistant. All of the other conditions such as the number of corpuscles and the number of bacteria, the state of these bacteria and of the corpuscles combined with them, the nature of the medium and the temperature are alike in the two suspensions. This being the case, it seems necessary to conclude that the single difference observed, that is, the acquisition of a resistance by the bacteria, is responsible for the attenuation of the bacteriophage corpuscles.

Of interest in this same connection are the observations of Bordet, ${ }^{80}$ as well as those of Gratia and de Kruif. ${ }^{273}$ They have found that if

* One might even accomplish the experiment by placing three or four times as many bacteria in the $100 \mathrm{cc}$. as in the cubic centimeter. 
bacteriophage corpuscles are removed from the center of a plaque on agar, the corpuscles are virulent while in the periphery of the plaque, at the margin of the bacterial growth an attenuated bacteriophage is found.

The reason for this attenuation is still the same. Let us recall the manner in which the plaque is formed. A corpuscle is deposited upon the surface of the agar in the midst of many bacteria. This corpuscle parasitizes the bacterium in its immediate vicinity and multiples, and young corpuscles are liberated by the destruction of the parasitized organism. These freed corpuscles in their turn parasitize the bacterial cells with which they come in contact and the process thus continues in this manner. But during this time those bacteria which are found beyond the reach of a corpuscle multiply. The bacterial layer becomes thicker and thicker and consequently more and more difficult to attack. If the corpuscles are very virulent, that is to say, if they are reproducing actively the plaque has reached a diameter of several millimeters by the time the critical period is reached, when the bacterial layer becomes sufficiently dense to "suffocate" the bacteriophage corpuscles." If the bacteriophage is of low virulence this period is reached when the plaque is small, simply because of the slowness with which the corpuscles have multiplied. When the layer of growth has reached a certain thickness the products resulting from the activity of the bacteriophage corpuscles can no longer diffuse into the agar. $\dagger$ We have seen already that it is precisely because of the non-diffusion of the products resulting from bacteriophagy that the process on agar is limited. The activity of the corpuscles becomes paralyzed and the bacteria become resistant and acquire an immunity. This is precisely what takes place at the periphery of the plaque. The same conditions are to be found there as when corpuscles are inoculated into extremely dense bacterial suspensions,

* We have seen that a number of factors limit the multiplication of bacteriophage corpuscles on agar. The first of these is the thickness of the layer of medium which to some extent regulates the rapidity with which the products which result from the activities of the bacteriophage and which impede its action, diffuse. Furthermore, the critical moment for the bacterium is that time when it is dividing. Division is intense when the layer on the agar is very thin, but it is much less active when the layer becomes somewhat thicker for it then contains a number of old bacteria but slightly susceptible to attack.

$\dagger$ The fact that bacteriophagy takes place perfectly well on gelatin provided that the layer is very thin on a substratum of agar shows beyond possible contradiction the effect of diffusible products upon the activity of the bacteriophage corpuscles. 
and the result on the corpuscles is the same in both cases,- an attenuation of virulence.

Bordet $^{80}$ has further shown that if a Petri dish is seeded with a culture of B. coli (and all other bacteria behave in the same manner) and if, then, a drop of bacteriophage of moderate or weak* potency is deposited upon the surface the bacteria do not develop in this region, but after some time colonies of resistant bacteria appear. If these mixed colonies, which, as we will see in a later section, contain both bacteria and bacteriophage corpuscles the latter possess an attenuated virulence. The reason is always the same; virulence becomes attenuated through contact with bacteria which have acquired a resistance. Bordet and Ciuca ${ }^{95}$ have stated that a bacteriophage attenuated in one or the other of these experiments which have been cited, is no longer able to increase in virulence by passages with susceptible organisms. Brutsaert ${ }^{10 t}$ has shown that this is not the case. After 12 to 20 passages he obtained an increase in virulence to such a degree that it became equal to the virulence of the bacteriophage prior to its attenuation. I have confirmed this fact entirely.

The erroneous conclusion reached by Bordet may be ascribed to the fact that the virulence of a bacteriophage attemuated through contact with resistant bacteria becomes increased only very slowly during the first passages with susceptible organisms. It is only after 7 or 8 passages that the virulence begins to be increased to an appreciable degree. Once it has started to augment the increase is rapid.

One might conclude from these facts that the bacteriophage corpuscles become increased in virulence by passages with susceptible bacteria, and that virulence is attenuated by passages with bacteria which have acquired a resistance, that is, an immunity. Bacteria which have acquired a completely refractory state may even destroy the corpuscles. Here again the manner in which the bacteriophage corpuscles and the susceptible bacterium which has acquired an immunity behave is exactly like that of the pathogenic microörganism and the susceptible animal which has aequired an immunity. $\dagger$ It is, on the other hand,

* If the bacteriophage is of maximum virulence or is very active no resistant bacterial colonies develop. Bordet did not state, in his paper, the virulence of the bacteriophage with which he worked, but it is obvious that the virulence was weak because of the fact that resistant colonies developed.

$\dagger$ Among all of the experiments which might be mentioned and which have been of interest to all biologists there is one which is of particular interest because the conditions, experimentally, are almost identical with those of corpuscles attenuated through contact with resistant bacteria.

Wollmann inoculated a few drops of an attenuated culture of $B$. anthracis into 
evident that the attenuation of the virulence of a bacteriophage takes place only when the resistance of the bacteria dominates the virulence. In the opposite case, when the resistant bacteria are overcome there results, naturally, an increase in virulence. A bacteriophage, when overcome, is attenuated. A bacteriophage when overcoming is enhanced and the increase in virulence is in direct proportion to the resistance of the bacterium overcome. Whether these events take place in the scale of beings that involve the susceptible animal and the virulent bacterium or the susceptible bacterium and the virulent bacteriophage is of no fundamental significance. The result is exactly the same.

Some experiments of Gratia and of Wollstein illustrate particularly well the increase in virulence resulting from the contact of a victorious bacteriophage with bacteria which have acquired a resistance. ${ }^{246} \mathrm{~A}$ bacteriophage at its time of origin presented a specific activity, limited to a single strain of $B$. coli. Successive passages were made at the expense of resistant bacilli of this same strain. The results are summarized in table $18(++++=$ complete bacteriophagy, no resistant colonies forming when the material is spread upon agar; $+++=$ almost complete dissolution, less than 12 colonies developing when spread upon agar $++=$ partial dissolution, many resistant colonies appearing $;+=$ no dissolution, a few plaques forming when spread upon agar; $-=$ no bacteriophagy $; \mathrm{S}=$ the susceptible strain, $\mathrm{R}=$ the resistant strain).

the peritoneum of a guinea-pig. After a few hours he removed the peritoneal exudate and centrifuged it at moderate speed, the leukocytes, together with the phagocytized bacteria, collecting in the sediment. The free bacteria, i.e., those which had resisted phagocytosis, remained suspended in the supernatant fluid. With these materials he inoculated two guinea-pigs intraperitoneally. To one he gave a few drops of the supernatant fluid, containing, of course, "victorious" bacteria, and to another he gave a portion of the sediment containing phagocytized bacteria, that is to say, "conquered" organisms. He continued these passages in a double series and demonstrated that the virulence of the strain resulting from the selection of the "victorious" bacteria increased with each passage and ended by being very high. On the contrary the strain resulting from the selection of "conquered" bacteria became more and more attenuated. It is only necessary to substitute in the experiment of Wollmann the word "guinea-pig" or better yet "leukocytes of the guinea-pig" by "bacterium" and the word "bacterium" by "bacteriophage corpuscle" in order to realize how completely the facts observed by him conform to the behavior of the bacteriophage. 
These experiments are of great interest for they indicate, as Gratia himself has remarked, a method of increasing the virulence of a bacteriophage and they show how virulence toward diverse bacterial species may be acquired.

\section{THE LOSS OF RESISTANCE}

Bordet and Ciuca ${ }^{88}$ were the first to show that the serum of an animal which had received a series of injections of a bacteriophage suspension possesses the property of inhibiting the action of this bacteriophage. We will return to these experiments in another chapter. They distributed $^{92}$ over the surface of an agar slant a few drops of an anticolibacteriophage serum, and allowed the agar to become impregnated with this by holding the tube for a few hours in the incubator. They then seeded the tube with a resistant $B$. coli admixed with a bacteriophage. The latter was unable to develop and consequently they obtained an

TABLE 18

\begin{tabular}{|c|c|c|c|c|c|c|c|c|c|c|}
\hline \multirow{3}{*}{ PASSAGES } & \multicolumn{4}{|c|}{ B. COLI } & \multicolumn{3}{|c|}{ B. DYSENTERIAE } & \multirow{3}{*}{$\begin{array}{c}\text { B. } \\
\text { typho- } \\
\text { sus }\end{array}$} & \multirow{2}{*}{\multicolumn{2}{|c|}{$\begin{array}{l}\text { B. para- } \\
\text { typhosus }\end{array}$}} \\
\hline & \multirow{2}{*}{ Type } & \multirow{2}{*}{$\underset{1 R}{\text { Type }}$} & \multirow{2}{*}{$\begin{array}{l}\text { Com- } \\
\text { munis }\end{array}$} & \multirow{2}{*}{$\begin{array}{l}\text { Com- } \\
\text { munior }\end{array}$} & \multirow{2}{*}{ Shiga } & \multirow{2}{*}{ Flexner } & \multirow{2}{*}{ Hiss } & & & \\
\hline & & & & & & & & & $A$ & $B$ \\
\hline 0 & +++ & $+t$ & - & - & - & - & - & - & - & - \\
\hline 1 & $+t+$ & $+t$ & - & - & + & + & + & - & - & - \\
\hline 2 & ++++ & +++ & +++ & - & +++ & +++ & +++ & - & - & - \\
\hline 3 & ++++ & +++ & $+t+$ & - & ++++ & ++++ & ++++ & + & - & - \\
\hline 4 & +++ & ++ & + & - & ++ & $++t$ & +++ & +++ & - & ++ \\
\hline
\end{tabular}

ultra-pure culture of $B$. coli refractory to bacteriophagy. By a series of subcultures they showed that the resistance was gradually lost, but it was only after 21 consecutive passages carried out in the complete absence of the bacteriophage that its sensitivity was restored to a degree corresponding to that of the original B. coli.

Bruynoghe ${ }^{112}$ did not succeed in purifying a contaminated strain of B. dysenteriae Shiga with an antiserum. He demonstrated that bacterial "recovery" and the return to sensitivity is the more difficult to obtain when bacteria have been for a long time and during a very considerable number of generations, in contact with bacteriophage corpuscles.

Eliava and Pozerski ${ }^{191}$ have noted that if the material resulting from the complete dissolution of a suspension of dysentery bacilli by a bacteriophage of high virulence (but not maximal for then the agar 
remains completely sterile) is spread over an agar surface the latter remains sterile except for a very limited growth of fine colonies. This restricted growth takes place at the extreme upper margin of the agar at the point where the substrate forms only a very thin layer.* With the tip of a very fine glass needle they removed the center of one of these minute colonies and seeded it upon an agar slant which had been prepared for a few days and was, therefore, dry. After 2 or 3 comparable transfers, that is to say, after removing daily for seeding the center of a fine colony located at the margin of the agar they obtained an ultrapure bacterial culture, one which no longer contained bacteriophage corpuscles. $\dagger$ The ultrapure culture thus obtained is composed of refractory bacilli insusceptible to the bacteriophage even if the latter is of maximum virulence. A suspension of such bacilli inoculated with any quantity whatever of bacteriophage suspension develops as in a pure bouillon.

They transplanted the ultrapure culture every day. At the end of 8 transfers they showed that the bacteria were still resistant but no longer completely refractory for the inoculation of a bacteriophage into suspensions caused bacteriophagy although the dissolution was only partial. During the course of subsequent cultures upon agar resistance gradually diminished but it was still demonstrable after 15 transplants.

In brief, then, the conclusion of these authors confirms that of Bordet and Ciuca insofar as it deals with the gradual loss of resistance.

I have repeated this experiment, effecting the purification in another manner, simply by the classical procedure of isolated colonies on agar, operating on the basis of the fact which I had previously noted ${ }^{317}$ that by implanting secondary cultures on agar one might obtain ultrapure colonies. I have, therefore, very readily effected repeated purifications by isolated colonies on agar following the old method described by E. Roux. $\$$ Each of the tubes during the isolation should remain for 3 days

* The formation of these colonies in spite of the presence of bacteriophage corpuscles is due to the thinness of the agar layer. The products resulting from bacteriophagy can not diffuse into the substrate and the action of the bacteriophage is inhibited.

$\dagger$ In what follows I will designate colonies, or a culture or a bacterial strain which is free of bacteriophage corpuscles by the qualifying word "ultrapure."

$\ddagger$ This is a procedure which I can not recommend too highly for the isolation of pure strains, not only in the case of a contamination by bacteriophage corpuscles but also as a general procedure for purifying bacterial strains. The materials needed are a tube of sterile bouillon (6 or $7 \mathrm{ec}$.) and an agar slant. The only condition is that the agar slant contain some water of condensation,--if the amount 
in the incubator, thus providing for the development of sufficiently large colonies. At each isolation material is removed from the margin of a colony in such a way as to obtain the youngest organisms, that is to say, those organisms belonging to the generations most distant from the bacillus from which the colony originated. After 18 subcultures the bacilli have lost all resistance. They undergo bacteriophagy within exactly the same time and in the same manner as do bacilli of the same strain which have never been in contact with the bacteriophage. Simple calculation (after having determined the number of bacilli composing a colony produced under the conditions of the experiment) has shown that in the case of $B$. dysenteriae Shiga which had acquired a refractory state through cultivation in the presence of bacteriophage corpuscles of maximum virulence and which were then cultivated in the absence of corpuscles, the loss in resistance was complete only after 400 to 500 generations, that is to say, after 400 or 500 divisions.

In comparable experiments carried out with a staphylococcus complete loss of resistance was obtained after 150 to 200 generations of the ultrapure culture.

All of these experiments agree in showing that in the absence of bacteriophage corpuscles, resistance, that is to say, the immunity acquired by a bacterium, is transmitted hereditarily throughout a great many generations but that it diminishes gradually with successive generations. After a larger or smaller number of generations, the loss of this immunity is complete. The bacterium has again become as susceptible as it was prior to its conflict with the bacteriophage.

present is not sufficient, that is, 6 or 8 drops, it should be supplemented by the addition of a few drops of sterile bouillon. With a fine platinum wire remove from an agar slant a very small quantity of the culture which is to be purified. Introduce this trace of culture into the bouillon tube removing the material by rubbing the culture off on the wall of the tube at the surface of the liquid. Shake the medium vigorously in order to suspend the bacteria introduced. Sterilize the platinum wire and dip the end into the bouillon. Carry the trace of liquid over into the condensation water of the agar tube. Shake this and then incline the tube and distribute the implanted condensation water over the entire surface of the agar. Place the tube vertically in the incubator. After a few attempts it is possible to carry out the two procedures (that of seeding the agar and then the transfer to the water of condensation) in such a way that from 5 to 10 colonies on the agar slant can regularly be obtained. This method of isolation, which I believe has not been published, was shown me be Dr. Roux. He employed the procedure for the isolation of pure strains of $B$. diphtheriae in connection with his wellknown studies leading to the discovery of diphtheria toxin. 


\section{THE BACTERIA OF SECONDARY CULTURES}

Spread a drop of a secondary culture upon the agar of a Petri dish prepared in such a way that the agar layer has a depth of some 7 to 8 mm.* The result of seeding differs in accord with the virulence of the bacteriophage present in the secondary culture, and consequently with the resistance of the bacterium, since in a secondary culture there is an equilibrium between the distinctive properties of the two antagonists.

A secondary culture of $B$. dysenteriae Shiga or of the staphylococcus obtained at $38^{\circ} \mathrm{C}$. seeded upon a thin layer of agar usually gives no colonies in spite of the presence of a bacteriophage of maximum virulence. The agar is sterile (d'Herelle ${ }^{317}$ ) although the microscope shows the turbidity of the secondary culture was due to the presence of bacteria.

We have seen that on agar the inhibitory products, resulting from the attack on the bacteria by the bacteriophage, diffuse into the substrate. $\dagger$ In a liquid medium these products freely exercise their inhibiting action. Upon deep agar they diffuse as quickly as they are formed and no longer interfere with the bacteriophage corpuscles so that the latter are able to overcome the bacteria in spite of an acquired resistance. That this is the true explanation is very well shown by the fact that on a thin agar layer having a depth of only 1 or $2 \mathrm{~mm}$. confluent colonies of resistant bacteria develop when it is seeded with the same secondary culture which gives no colonies whatever upon a thick layer of the medium.

This question of the thickness of the substratum is also of significance in cases where the spreading is made upon an agar slant; the lower part of the medium may remain sterile while the upper portion is covered by colonies, the latter being the more numerous as the layer of the agar becomes thinner.

If the bacteriophage has a high virulence, but not a maximal, the thick layer of agar may still remain sterile although at times a few colonies may be observed (d'Herelle ${ }^{317}$ ).

With a bacteriophage still less virulent the bacterial colonies are always present and their number is the greater as the virulence of the bacteriophage is the less.

* We have seen that bacteriophagy does not take place upon solid media when the products resulting from the action of the bacteriophage are not able to diffuse into the medium. If a secondary culture is spread upon gelatin or even upon a thin layer of agar a continuous layer of bacteria, quite naturally, develops.

$\dagger$ We have likewise seen that these inhibiting products can not be the normal secretory products of the bacteria since bacteriophagy takes place in filtrates of old cultures. 
Experimenting with $B$.dysenteriae, I was the first to show ${ }^{317}$ that all of the colonies which appeared on agar were ultrapure, that is to say, contained only bacteria with a complete absence of bacteriophage corpuscles. Bordet and Ciuca ${ }^{8 i}$ working with $B$. coli were, on the other hand, the first to find that all of the colonies obtained after seeding a secondary culture were contaminated with the bacteriophage. Kuttner ${ }^{398}$ working with B. typhosus has shown that in reality one may find two types of colonies, ultrapure colonies and contaminated colonies. And shortly after this, Bordet and Ciuca ${ }^{91}$ reported that there are three types of colonies: (a) ultrapure colonies formed of resistant bacteria; (b) contaminated colonies containing resisting bacteria and the bacteriophage, and $(c)$ ultrapure colonies which contain only susceptible bacteria.

It is probable that these last are in reality colonies formed by bacteria with a very low resistance but it is doubtful if they are entirely devoid of resistance. Bruynoghe, ${ }^{112}$ has, as a matter of fact, shown that one may find a whole scale of resistance, from colonies composed of refractory bacteria up to those in which the resistance is so weak that it is only manifest by a very insignificant retardation in bacteriophagy. The proportion of contaminated colonies in relation to the number of ultrapure colonies is extremely variable. It varies on the one hand with the degree of resistance of the bacterium and on the other with the duration of the symbiosis. Bruynoghe and Maisin ${ }^{119}$ report that with a secondary culture of $B$. coli seeded upon agar they did not find a single contaminated colony among the 30 which they examined. In another experiment performed with Shiga bacilli 9 of 10 colonies were ultrapure, one only was contaminated. We will see on the other hand that when the symbiotic association has continued for a long time it may be very difficult to obtain ultrapure colonies. In this last case it is necessary to adopt special methods of isolation.

If we make a preparation for procuring isolated colonies from a colony of low resistance we will find that all of the colonies which are derived from it are likewise either lacking in resistance or present a very low resistance, usually somewhat below that of the bacteria of the original colony. On the contrary, if we secure, also by the method of colonies, ultrapure colonies possessing a high resistance the result is exactly the same as though we had made the planting directly from the secondary culture, that is, the majority of the colonies are formed of bacteria having little or no resistance while some few are made up of bacteria of high resistance. This shows us that in a colony, which tested as a whole appears to be resistant, the resistance actually belongs to some few of the 
bacteria. If one suspends all of such a colony and inoculates this suspension with bacteriophage corpuscles the more highly resistant bacteria will not be attacked, but will multiply while the less resistant bacteria are parasitized and this permits a simultaneous multiplication of the bacteriophage corpuscles.

As for the resistant colonies contaminated by corpuscles, colony isolation shows that, like the secondary culture from which they were derived, they form ultrapure colonies of varied resistance as well as contaminated colonies.

We have seen that in a suspension of bacteriophage corpuscles each one possesses its own virulence, one corpuscle differing from another. We also know that in a suspension of susceptible bacteria each organism presents a capacity for resistance which varies from one bacterium to another. We now find that in a secondary culture each of the bacteria have a special resistance, varying from one to another. It is these variations which render biological phenomena so complex and which render their study so difficult.

\section{MiXed CULTURES}

We have seen what takes place if a secondary culture is subcultured by means of isolated colonies. What happens if the subculturing is made "in toto," that is to say, if a drop of a secondary culture is removed and implanted into sterile bouillon, or if a portion of a contaminated colony is transferred to either a tube of bouillon or to an agar slant?

When one inoculates bouillon with a secondary culture "en bloc" or with a contaminated colony from agar the medium frequently remains sterile. In such a transfer the bacteriophage is introduced and bacteriophagy takes place with the young bacteria derived from the multiplication of the bacteria implanted. Sometimes on the agar slant or in the bouillon it is the bacteria which prevail. Due to their resistance they develop in spite of the bacteriophage corpuscles which are present and under these conditions the cultures can be recultivated indefinitely (Bordet and Ciuca ${ }^{83}$ as well as Kuttner. ${ }^{398}$ ) It is, indeed, possible to so arrange things that the bacterium will always prevail. To accomplish this it is only necessary to modify the conditions in such a way as to provide an unfavorable environment for the corpuscles. This is what happens in a liquid medium when one introduces the bacteriophage into a medium containing a sugar fermented by the bacteria. Acid is formed, and as is known, bacteriophagy takes place with difficulty in such a medium. Upon a solid medium an inhibitory influence is provided 
by reducing the thickness of the substrate thus interfering with the diffusion of those products which are inhibitory.

I have applied the term "mixed cultures" to cultures derived from secondary cultures when resistant bacteria and virulent bacteriophage corpuscles co-exist. The appearance of mixed cultures varies in accordance with the degree of resistance of the bacterium.

With the bacteria possessing but relatively little resistance, and as a consequence having a bacteriophage of but low virulence, the appearance upon a solid medium or in a liquid medium very closely approaches that of a normal bacterial culture. The isolated colonies are often irregular (Kuttner;"398) the so-called "flattenformen" of Gildemeister.

When the degree of resistance on the one hand and the virulence on the other are somewhat higher the bacteria develop in the sediment, the medium remaining more or less clear $\left(\mathrm{Gratia}^{259}\right)$.

When the resistance and the virulence are very high, the bacteria grow in the form of very dense agglutinated masses impossible to dissociate by shaking. None of them float in the medium; all are collected on the bottom and on the walls of the tube. Growth is very slow but after some 15 days each of the agglutinated masses may have the size of a small pin-head (d'Herelle ${ }^{321}$ ). I have observed this appearance of mixed cultures, always with refractory bacteria, with the organisms of the colon-typhoid-dysentery group and with staphylococci.

According to Brutsaert, ${ }^{108}$ secondary cultures always present this appearance in a hypertonic bouillon.

This very distinctive appearance, characteristic of resistance and maximal virulence, is retained but rarely throughout a series of successive transfers in bouillon. I have found this appearance to be permanent in only two cases and these two were mixed cultures of Shiga bacilli and the bacteriophage. Usually the virulence of the bacteriophage weakens rapidly, beginning with the second or third transplant and one then finds the ordinary type of mixed culture in the sediment while the supernatant fluid is more or less cloudy. In the two cases of permanently agglutinated mixed cultures it was, however, only necessary to render the conditions favorable for the bacterium (increase in temperature, in acidity, etc.) in order to obtain mixed cultures in the sediment.

Upon agar, mixed colonies containing bacteria of very high resistance and bacteriophage corpuscles of high virulence are usually small, viscous, and of slow growth (d'Herelle ${ }^{321}$ ).

Bordet and Ciuca ${ }^{86}$ have obtained mixed colonies of $B$. coli which 
assumed the form of $B$. aerogenes colonies. I also have observed colonies of this type. Their occurrence is very irregular and up to the present it has been absolutely impossible for me to determine the conditions governing their appearance.*

As a matter of fact, there is hardly anything which presents a wider variation in appearance than do mixed cultures. With the staphylococcus, for example, Gratia and Jaumain ${ }^{268}$ have seen mixed cultures on agar which were thick and opaque; others were discrete, like cultures of the streptococcus; some were rich in pigment, others poor; some grew with a homogeneous growth in bouillon, others gave a sedimented growth with a clear supernatant fluid. Apparently almost any type of growth may occur.

We have just seen that in certain mixed cultures a perfect equilibrium is established. It is, however, rare that these mixed cultures in the form of agglutinated masses become indefinitely cultivable in bouillon in this form. Yet, I have preserved throughout a period of three years, with weekly transfers, such mixed cultures of $B$. dysenteriae and the bacteriophage and after this time the two antagonists were always present. The multiplication of bacteriophage corpuscles here is extremely slow.

One of these mixed cultures is centrifuged after about 100 passages. The sedimented material is washed several times with sterile bouillon. The bouillon of the sixth washing filtered through a candle contains no corpuseles, for when it is inoculated into a suspension of B. dysenteriae bacteriophagy does not take place. A trace of the sedimented washed bacilli is implanted into bouillon. It is only after the fourth day that the inoculation of $0.05 \mathrm{cc}$. of fluid, freed of the agglutinated material by filtration, causes bacteriophagy with a suspension of normal dysentery bacilli.

This is, however, an extreme case. When the resistance of the bacteria of a mixed culture is not as great corpuscles will be found in the liquid after a few hours. The higher the resistance of the bacteria of the mixed culture the slower is the multiplication of the bacteriophage corpuscles.

* Gory ${ }^{242}$ has obtained them regularly in working with a normal strain of $B$. coli and tap-water, which always contains a large number of bacteriophage corpuscles virulent for this bacterium. After a number of passages in the media containing the sewage the transformation of $B$. coli into $B$. aerogenes or into a bacterium which could not be distinguished from the latter took place. It would appear that the $B$. aerogenes resulted from a mutation of the $B$. col $i$ subjected to the action of the bacteriophage. 
A mixed culture results from the establishment of a state of equilibrium between the virulence of the bacteriophage corpuscles and the resistance of the bacterium. In such cultures a true symbiosis obtains in the true sense of the word; parasitism balanced by the resistance to infection.* What is, in the last analysis, the mechanism leading to the formation of mixed cultures? If one inoculates into an ultrapure suspension of bacteria, which have acquired a refractory state, some bacteriophage corpuscles of low virulence or even of moderate or high, but not maximal, virulence these corpuscles are unable to parasitize the bacteria and are eliminated after a few passages. At different times I have even observed a complete destruction of the corpuscles by refractory bacteria. ${ }^{321}$ This fact has been confirmed by Flu. ${ }^{211}$ Indeed, a destruction of the corpuscles must always occur when corpuscles having a virulence below the maximum value are combined with susceptible bacteria which have become refractory, for one may observe regularly, after a few hours, a greater or smaller reduction in the number of corpuscles. But it is only very infrequently that the destruction is complete.

If the resistance of the bacterium is limited and the virulence of the bacteriophage is very high or maximum a fortiori bacteriophagy occurs and the bacteria are destroyed.

If the resistance of the bacteria is balanced by the virulence of the corpuscles, and this is true whatever may be their respective values, a symbiosis is established. We know that in a mixed culture each bacterium possesses its own more or less outspoken resistance, and in a similar way each bacteriophage corpuscle possesses its own peculiar virulence, more or less exalted. The more virulent corpuscles are able to parasitize the bacteria of least resistance; but the bacteria of the greatest resistance multiply even though the corpuscles have a very high virulence. There occurs, then, under these conditions a continual selection with the indefinite co-existence of the two antagonists. Such mixed cultures, symbiotic in nature, can be subcultured indefinitely.

* The respective behaviour of the bacterium and the bacteriophage is exactly that of the seed of the orchid and of the fungus. Before the days of Noël Bernard we were not familar with the processes of germination in the orchids, but through his work we now know that fertilization takes place only when the seed is parasitized by a fungus. The fungus penetrates into the seed and one of three results may ensue. The resistance offered by the seed may overcome the virulence of the parasite and the latter is then destroyed, or the virulence of the fungus outweighs the resistance of the seed and here the seed is destroyed, or finally, the virulence of the one and the resistance of the other balance, a symbiosis results, which in effect is a limited parasitism. 
One finds by experiment that the stability of mixed cultures is the greater as the symbiosis is of longer duration. In the course of the first passages in symbiosis it is frequently found that the one or the other of the antagonists is overcome, either because the resistance and the virulence were not balanced or because changes in the experimental conditions favored one or the other. Without a modification in the growth conditions in the case of a mixed culture formed by a slightly virulent bacteriophage and a normal bacterium it is usually the bacterium which survives. In the course of the subcultures the resistance of the more vigorous bacteria increases gradually, they survive and multiply. Sooner or later the corpuscles no longer find bacteria susceptible to attack, or they cease to multiply because of the lack of susceptible organisms, or they are destroyed. In any case after a certain number of subcultures they become eliminated.

Sometimes a perfect symbiosis develops and it is then permanent. When one finds that after a dozen subcultures (effected each time by introducing $0.001 \mathrm{cc}$. of the preceding mixed culture into $10 \mathrm{cc}$. of sterile bouillon) the symbiosis continues, it is rare that it can not be maintained indefinitely. Where the symbiosis is stable there is a perfect equilibrium between the virulence and the resistance.

is As we have seen such symbiotic cultures may occur whatever the absolute degrees of resistance and of virulence, provided the two are balanced. We will see the considerable importance of this fact, since it is not solely a test tube experimental phenomena, but one which occurs also in nature.*

* Arkwright ${ }^{17}$ has claimed that from all cultures of intestinal bacilli it is possible to isolate upon agar colonies of different appearance, some susceptible to the bacteriophage, others resistant. The many experiments which I have carried out upon this subject warrant me in contradicting definitely such a statement. Indeed, the contribution of Arkwright is absolutely incomprehensible and it would stem that he has no idea of what is in reality the phenomenon of bacteriophagy.

He says that among the strains of B. dysenteriae Shiga only those which develop in the sediment and agglutinate spontaneously in physiological saline are susceptible to attack by the bacteriophage. I am perfectly ready to conduct a demonstration showing that all normal strains of $B$. dysenteriae Shiga are susceptible to attack by any race of Shiga bacteriophage whatever having a high virulence; races which it is easy to isolate. By normal strains I mean those which present the characters assigned to Bacillus dysenteriae Shiga by the Society of American Bacteriologists.

The rare strains naturally contaminated by a bacteriophage develop exactly in the form of sedimented growth. They are composed of resistant bacilli and 
It is interesting to see what happens when one seeds symbiotic cultures into a suspension of susceptible bacteria or into pure suspensions of virulent corpuscles. When dealing with a mixed culture of coexistent bacteria of low resistance and corpuscles of low virulence the implantation of the mixed culture into a suspension of susceptible bacteria has no effect; the result is a mixed culture of the same nature. If, on the contrary, one inoculates a suspension of the bacteriophage into such a mixed culture everything depends' upon the degree of virulence. If the virulence of the corpuscles is very high or is maximum bacteriophagy takes place; when the virulence is not high nothing is changed.

In the case of mixed cultures where very virulent corpuscles and very resistant bacteria co-exist things take place differently.

When a mixed, agglutinated culture is inoculated into a pure culture of the bacteriophage, that is, into a suspension previously inoculated and which has undergone complete dissolution, the growth consists of an agglutinated culture, just as though the inoculation had been made into fresh sterile bouillon.

If some of the agglutinate, even if washed, is introduced into a sus-

the bacteriophage and they are refractory to bacteriophagy. This is precisely contrary to the statements of Arkwright. These occasional, naturally contaminated strains are, however, easy to purify. Ultrapure colonies from them give in subculture ultrapure cultures indefinitely, presenting upon agar but a single type of colony. After a number of transplantings, when the resistance to bacteriophagy has disappeared these ultrapure colonies show all of the characters of normal strains and all of the colonies on agar are susceptible to bacteriophagy.

In this same contribution Arkwright says that I have classified the Shiga dysentery bacilli as a homogeneous species as regards bacteriophagy but that my experiments contradict this statement for I have found strains which, when derived from the body, were refractory to bacteriophagy. Manifestly this author has not comprehended the difference between natural resistance and acquired resistance. After a few passages in ultrapure culture the strains which were not attacked at the time of their recovery from the body because of a resistance acquired in the body become susceptible and subject to attack. As a matter of fact, such a resistant state is extremely rare insofar as the dysentery bacillus is concerned. But even if it occurred very frequently it would have nothing to do with the question of homogeneity. A species is homogeneous with reference to the bacteriophage when all of the strains presenting the characters of the species are subject to attack by any race whatever of a bacteriophage which is virulent for any strain whatever of this species. The only refractory strains of such a species are those which possess an acquired resistance, and this is lost by a series of transfers in ultrapure culture. They then undergo bacteriophagy in exactly the same manner as a susceptible strain. 
pension of $B$. dysenteriae dissolution takes place and the suspension becomes perfectly clear within five or six hours. Four or five days later, however, the agglutinated masses begin to appear and gradually increase in size. The corpuscles contained in the agglutinate used as inoculum provoke the dissolution of the normal bacilli of the suspension, bacilli which are non-resistant, and then later the resistant agglutinated bacilli in their turn reproduce and the result is that which would have been secured had they been inoculated into fresh sterile bouillon.

All stages intermediary between these two extremes may be obtained; cloudy mixed cultures presenting the appearance of a normal bacterial culture where the equilibrium is essentially unstable; cultures in agglutinated form in the presence of a perfectly limpid fluid, representing a state of stable equilibrium. The medium may be more or less cloudy with the bacterial masses nore or less compact, sometimes resembling a coagulum. The type of the mixed culture bears a relationship to the virulence of the bacteriophage and to the resistance of the bacterium. Hence, the appearance of the mixed culture may be as variable as is the variability in the properties of the two organisms which are present.

I have spoken above of mixed colonies on agar formed by very resistant bacteria and very virulent corpuscles. These colonies are small, mucous, and difficult to dissociate.

Even when abundantly seeded upon agar these colonies never give a smooth layer of growth but always isolated colonies, more or less abundant, and always of variable size. Among the bacteria of the inoculum but few are able to form colonies. There is always a state of unstable equilibrium between the two elements present: the bacterium with its resistance, and the bacteriophage with its virulence. The bacterium forms, or does not form, a colony according to the accidental predominance of one or the other of these factors. This is especially to be observed when agar is seeded with the agglutinated masses, for however abundant may have been the planting only very rare isolated colonies, all of the mucous type, develop.

The cultures secured by the inoculation of the mucous colonies on different media show the following reactions:

In agar stabs: small lenticular colonies about the needle track.

In gelatin: as in agar, the resistant bacteria remain alive and cultivable for at least eleven months. In the case of the Shiga dysentery organisms this represents a viability at least ten times as great as that of the normal bacillus.

In gelatin stabs: large opaque colonies with opaque centers. 
On glycerin potato (prepared as for the cultivation of $B$. tuberculosis): very rare colonies on the potato, very abundant growth in the fluid at the bottom of the tube.

In milk: no coagulation in ten days.

In litmus milk: becomes a mauve color after two months.

On coagulated serum: no growth.

In neutral red: no change in two months, either on agar or in bouillon.

In litmus milk (Petruschky): acid after ten days and remains acid.

When the mucous colonies are suspended and heated to $60^{\circ} \mathrm{C}$. they are not cultivable, for then the culture contains only the living very virulent corpuscles which are not killed until a temperature of about $75^{\circ} \mathrm{C}$. is reached. Reinoculated into bouillon, the refractile, mucous, mixed colonies yield two types of culture, $(a)$ mixed cultures showing changes in turbidity, and (b) agglutinated cultures, which, as we know, always depend upon the degree of virulence of the bacteriophage and the capacity of resistance of the bacterium, factors which regulate the appearance of the culture.

We have seen that if an agglutinate, taken from a mixed culture in stable equilibrium, is introduced into a suspension, a dissolution of the suspension is followed by a growth of the agglutinate. The same thing transpires if an abundant seeding is made on tubes of slant agar having a growth of the Shiga bacillus. First, plaques appear, and then after three or four days a mucous colony develops in the center of each plaque. In both instances the bacteriophage acts upon the normal non-resisting bacteria and dissolves them, then the refractory bacilli multiply as they would have done on sterile agar or in bouillon (d'Herelle ${ }^{321}$ ).

\section{THE CAUSE OF SECONDARY CULTURES}

When a secondary culture in bouillon of any bacterium whatever is spread upon agar, the appearance of the agar after incubation may vary greatly, the aspect depending upon the virulence of the bacteriophage found in the secondary culture. When the bacteriophage is very virulent, transfers to agar usually remain sterile, in spite of the fact that the liquid medium may be cloudy and microscopically the material may show many bacterial forms.

To what may we ascribe this lack of growth on agar when the resistant bacterium develops perfectly in bouillon? We must admit that in a liquid medium the bacteriophage is inhibited, while upon agar it manifests its action. In other words, in bouillon the resistance of the 
bacterium prevails, on agar, the virulence of the bacteriophage. But this inhibition on agar certainly has a cause. What is it? The thought that comes immediately to mind is that some products having an inhibitory effect upon the bacteriophage are liberated in the liquid, and that on agar, although they must necessarily be present also, they may diffuse into the substratum; they do not accumulate on the surface where the conflict between the bacteria and the bacteriophage corpuscles takes place. This hypothesis can be proved very readily, for if it is a correct explanation, it is only necessary to conduct the experiment in such a way that these products can not diffuse into the substratum. This should inhibit bacteriophagy on the solid medium, just as in bouillon.

Spread $0.1 \mathrm{cc}$. of a secondary culture of $B$. dysenteriae which has developed at $40^{\circ} \mathrm{C}$. in spite of the presence of a bacteriophage of maximum virulence upon each of the following media:

(a) Upon an agar layer, about $10 \mathrm{~mm}$. in depth, in a deep Petri dish.

(b) Upon an agar layer (same medium as the above) that is only 2 to $3 \mathrm{~mm}$. thick.

(c) Upon another agar layer (again the same medium) that is only about $1 \mathrm{~mm}$. in depth.*

After incubation, we find that no colonies have appeared on the deep agar layer, that a few isolated colonies are on the agar of moderate depth, and that on the thin layer the colonies are confluent.

The same results are obtained if this experiment is performed with any other bacterium, the staphylococcus in particular. If, instead of taking secondary cultures which have developed despite the presence of a potent bacteriophage, secondary cultures consequent to bacteriophagy by races of strong or even moderate virulence are spread over the agar layers of different depths, a similar result will be obtained: A few colonies on the thick layer, many colonies on the agar of moderate thickness, a continuous layer of bacterial growth on the thin layer.

Since in such experiments only the thickness of the substratum is varied, the only deduction is that the cause of the inhibition is some product which is effective if it remains in contact with the bacteriophage corpuscles. Furthermore, it is evident that the harmful action is most pronounced when its concentration is high.

In the section dealing with "Bacteriophagy on Solid Media" I have demonstrated experimentally that although bacteriophagy does not take place on gelatin it is by no means because of the nature of the

* To avoid rapid drying in the incubator, invert the plate, and place 1 or 2 cc. of sterile water in the cover. 
medium, but rather owing to the fact that the gelatin does not permit the diffusion of the products formed during bacteriophagy, products which inhibit the action of the bacteriophage.

If we repeat these experiments by spreading a drop of a secondary culture over the surface of $(a)$ a gelatin medium (bouillon containing 15 per cent gelatin), and $(b)$ the same gelatin medium spread out in a thin layer over a substratum of agar, we will find that after incubation at $22^{\circ} \mathrm{C}$. the gelatin is covered by a continuous bacterial layer composed of a mixed culture-resistant bacteria-virulent bacteriophage corpuscles. Upon the thin layer of gelatin spread over the agar there will be, either no colonies at all, or only isolated colonies, depending upon the virulence of the bacteriophage. Here again, this result can be explained only as a result of the concentration at the surface of the first medium of products inhibiting the action of the bacteriophage. In the second medium these products diffuse into the deep substratum.

It seems to me that these experiments leave no doubt but that products inhibiting the action of the bacteriophage are formed during bacteriophagy, and that these products are, in the last analysis, the cause of secondary cultures.

What is the origin of these inhibiting substances? Certainly they are not products resulting from the normal metabolism of the bacteria, for bacteriophagy takes place normally in filtrates of old cultures. This being true it must be that these substances are secreted by the bacteria in their reaction to the bacteriophage. They are then true bacterial immune substances. A bacteriophage is, indeed, most highly virulent when it resists the action of these inhibiting substances.

The degree of virulence of a bacteriophage corpuscle for a given bacterium represents, therefore, two factors, one being an ability to multiply at the expense of the substance of this bacterium, the other, its power to resist the action of the inhibiting substances secreted by the bacterium which is reacting to its action.

\section{THE RESISTANT BACTERIUM}

It is necessary first of all to point out the difference between a resistance acquired to the action of the bacteriophage and the natural resistance possessed by a bacterial strain. We are concerned here with acquired resistance only. It may be stated, however, that the natural resistance which occurs in a bacterium belonging to a heterogeneous species is not characterized by any peculiar sign. Such a bacterium is normal both morphologically and in its biological properties. As for 
bacteria possessing an acquired resistance it is necessary to differentiate the case where the resistance is active, that is to say, where it manifests itself in the presence of a bacteriophage, from a latent resistance, a resistance transmitted through inheritance in the absence of bacteriophage. We already know that a latent resistance is gradually lost.

The acquisition of resistance by a bacterium is accompanied by various biological modifications and these modifications are, indeed, the more out-spoken as the degree of resistance is increased. The loss of resistance is accompanied by a return to the usual form and to normal properties.

The most constant change shown by a bacterium in a state of active or latent resistance is a loss in agglutinability with specific antisera $\left(d^{\prime} H e r e l l e^{317}\right)$. The loss of agglutinability seems to be related to the degree of acquired resistance, for the refractory state is accompanied by a complete inagglutinability and there is only a diminution if the resistance is partial. I have shown that such a loss or reduction in agglutinability occurs with the following bacteria when they are refractory or resistant: B. dysenteriae Shiga, Flexner, or Hiss, B. coli, B. typhosus, $B$. gallinarum and $B$. pestis.

This loss in agglutinability correlative with the acquisition of resistance has been shown by several others who have studied this question. I might mention Gratia in particular ${ }^{259}$ for he has tested the agglutinability of different strains of $B$. coli presenting different degrees of resistance.*

I have shown that typhoid bacilli which are inagglutinable when derived from the patient are also resistant to the action of the bacteriophage, which later attacks them when, after a series of passages agglutinability is restored..$^{321}$

The vitality of bacteria in a state of active resistance is a great deal higher than that of normal bacteria. For example, the Shiga dysentery bacillus has a relatively low vitality (there are few strains which may be subcultured after a month; of the many cultures with which I have worked none could be transplanted after two months on agar) but the small, mucous, mixed colonies can be transplanted successfully after 18 months, the tube being sealed during the interval with a rubber stopper.

Bordet and Ciuca ${ }^{8}$ have shown that a resistant B. coli does not produce the change in color of neutral red, that it is less readily phagocytized than the $B$. coli of the same normal strain and that its virulence

* The table given by Gratia appears later in this section. 
for laboratory animals is higher. Gratia ${ }^{24}$ has noted the same thing. Davison ${ }^{171}$ has shown that a resistant Shiga dysentery bacillus kills rabbits in a quantity one-fifth of that required with susceptible bacteria of the same strain. Insofar as B. pestis is concerned, working with a slightly virulent strain recently isolated from a bubo in a benign case, I have shown that while with the susceptible strain it was necessary to inject 0.1 ce. of a 24 -hour bouillon culture in order to kill a guinea-pig of 400 grams in 52 to 58 hours, a dose of $0.0002 \mathrm{cc}$. of bacilli of the same origin, but rendered experimentally refractory to the action of a very virulent bacteriophage, regularly caused death in 46 to 50 hours.

It is, however, quite important to bear in mind that all of these new characters associated with the acquisition of resistance to the bacteriophage by a bacterium are not uniformly distributed among the different colonies derived from a single secondary culture. This is particularly true as regards the loss of agglutinability and the increase in virulence. For example, with $B$. pestis (taken as an illustration because of its high virulence for laboratory animals), associated with colonies of increased virulence I have found others completely avirulent. We are not dealing, therefore, with an absolute rule. What actually happens is that through the influence of the resistance to the bacteriophage sudden mutations occur. These are almost always of a temporary nature, disappearing when the resistance is lost. But sometimes they are irreversible, as we will see. These mutations do not involve all of the characters of the bacterium, but only a certain number of them, varying from one bacterium to another, even in a single culture. With one bacterium the character "agglutinability" will be modified, with another, the character "virulence," and with a third, both of these will be changed at the same time. All that may be predicted is that, usually, there is a reduction in agglutinability and an increase in virulence.

The changes in the bacterium endowed with an acquired resistance extend to its morphology also. The bacilli frequently assume a coccobacillary form; they may even take the coccus form. The loss of resistance is accompanied by a return to normal form. I have obtained this reversion on several occasions, with resistant colonies constituted solely in the beginning of cocci, as is shown by the following experiment:

A Petri dish is heavily seeded from an agar culture of $B$. dysenteriae and is placed in the incubator at $37^{\circ} \mathrm{C}$. for about four hours. A drop of the bacteriophage filtrate is then placed in the centre of the plate. The race of bacteriophage should be one of average activity, that is, one capable of regularly causing complete dissolution of a bacterial suspen- 
sion but with which secondary cultures usually develop. (With too virulent a race the area where the drop was placed remains sterile indefinitely.) The plate is returned to the incubator. After eighteen to twenty-four hours a layer of culture composed of normal dysentery bacilli develops, showing in the centre a spot devoid of growth, apparently sterile. After thirty-six to forty-eight hours, the spot becomes covered with extremely fine colonies, which, when examined microscopically are composed of cocci only. These cocci are of different sizes, from 1 to $4 \mu$ in diameter, arranged in irregular forms,-in diploand in tetrad groupings. Two days later microscopic examination still shows cocci, but among them are bacillary forms in great number. Subcultures on to agar always give isolated colonies, each colony always reproducing with the same appearance and with the same sequence of forms,-first a coccoid culture, then a mixture of cocci and bacilli. These cultures always contain, moreover, bacteriophage corpuscles.

I have not been able to determine the conditions leading to the formation of these colonies made up solely of the coccus forms. Coccoid organisms are always to be found in mixed colonies, that is to say, in cultures where the bacilli are in a state of active resistance. This renders it certain that a state of resistance contributes one of the factors. But the appearance of these very fine colonies formed entirely at the beginning, of cocci is not constant, indeed, it is rare. Therefore there must be another unknown factor. This unknown factor does not reside in a distinctive property of certain races of the bacteriophage or in certain strains of bacilli for the appearance of these colonies is not constant with a given race or with a given strain, even if one works under conditions which appear to be identical.*

There is, however, one other modification, a fixed mutation for which it has thus far been impossible to control the conditions determining its appearance. There also, a resistance is one of the conditions, but there are others, for the occurrence of this mutation is very irregular even if one works under conditions which appear to be uniform. This is the

* I have observed ${ }^{321}$ another form of colony, formed only of cocci, but ultrapure. The coccus form is here permanent, maintaining itself through passage on agar or in bouillon. I have obtained colonies of this type only about 20 times, and always with secondary cultures transplanted on agar, the tube being sealed. They appear in from 2 to 6 weeks at laboratory temperature, growing very slowly, and attain a diameter of 10 to $12 \mathrm{~mm}$. after a year. They are always situated at the top of the agar slant. It would appear that they are a fixed mutation. I have observed them in mixed cultures on agar of B. dysenteriae, B. typhosus, and B. coli. 
transformation which a colon bacillus subjected to the action of a bacteriophage sometimes undergoes. It was noted first by Bordet and Ciuca. ${ }^{85}$ The growth is dense, shining, and even fluent. I have shown that this is a true mutation of B. coli into B. aerogenes and it is impossible to distinguish such cultures from those of typical bacilli of this species. They have the same morphology, the same growth characteristics, and give characteristic fermentative reactions. Moreover, Gratia ${ }^{259}$ has shown that cultures of this type are ultrapure. Here is, then a mutation, effected through the action of the bacteriophage, which persists after the elimination of the latter. It seems to be permanent.

TABLE 19

\begin{tabular}{|c|c|c|c|c|c|c|}
\hline TYPE & $\begin{array}{l}\text { DEGREE OF } \\
\text { RESISTANCE }\end{array}$ & MOTILITY & $\begin{array}{c}\text { MUCOID } \\
\text { CHARACTER }\end{array}$ & $\begin{array}{l}\text { TENDENCY } \\
\text { TO ASSEME } \\
\text { THE MUCOID } \\
\text { CHARACTER } \\
\end{array}$ & $\begin{array}{l}\text { FLUORES- } \\
\text { CENCE }\end{array}$ & $\begin{array}{l}\text { SERUM } \\
\text { AGGLUTI- } \\
\text { NATION }\end{array}$ \\
\hline $\mathrm{O}$ & + & 0 & 0 & + & $t+t+$ & 0 \\
\hline OR1 & $+t+t$ & 0 & $++t+$ & & 0 & \\
\hline OR2 & $+t+t$ & 0 & 0 & 0 & 0 & \\
\hline S & + & 0 & 0 & 0 & ++ & $++t$ \\
\hline SR & $+t+t$ & 0 & 0 & 0 & 0 & $+t+$ \\
\hline $\mathrm{R}$ & ++ & $+t+t$ & 0 & 0 & ++ & $++t+$ \\
\hline $\mathrm{RR}$ & $+t+t$ & $+t+t$ & 0 & 0 & 0 & $++t+$ \\
\hline M1 & $+t+$ & $+t+$ & $+t+t$ & & $+t+t$ & $+t+t$ \\
\hline Mla & + & 0 & 0 & + & $+t+t$ & 0 \\
\hline Mib & $+t$ & $+t+t$ & 0 & 0 & $+t$ & $++t+$ \\
\hline M2 & $+t+$ & $+t+$ & 0 & $+t+t$ & 0 & $++t+$ \\
\hline
\end{tabular}

$\mathrm{O}=$ Original undifferentiated culture.

In the fifth column is expressed the tendency to assume the mucoid form when exposed to a Coli-bacteriophage.

With regard to mutations of $B$. coli occurring under the action of the bacteriophage, Gratia ${ }^{259}$ has succeeded in isolating 10 different strains derived from a single culture of $B$. coli $^{*}$ Table 19 , taken from the paper of Gratia, gives the characteristics of these mutations.

Table 20 summarizes the experiments of $\mathrm{Gratia}^{259}$ on the crossed agglutination of these different mutations when subjected to the action of three antisera prepared by the injection of rabbits with strains "Original," "S" and "R."

* This history of this strain is interesting. It came from my collection and was given to Ciuca during my absence in Indo-China. It was a mixed culture (a transplant on agar of a secondary culture). The strain was originally derived from a $B$. coli maintained among the stock cultures of the Pasteur Institute. 
These mutations of B.coli have been studied quite extensively, thanks to the work of Bordet, and of Gratia especially. It is certain that a study of the mutations of other bacterial species brought about through the action of the bacteriophage will reveal many surprising things. It is, indeed, probable, as various investigators have suggested, that all of the fixed mutations occurring among bacterial species are produced through the action of the bacteriophage.*

The formation of a capsule through the action of the bacteriophage appears to be a most constant phenomenon accompanying the state of

TABLE 20

\begin{tabular}{|c|c|c|c|c|c|c|c|c|c|c|}
\hline \multirow{2}{*}{$\underset{\text { SERUM }}{\text { ANTI- }}$} & \multirow{2}{*}{ TITRE } & \multicolumn{9}{|c|}{ STRAINS } \\
\hline & & 0 & $\mathrm{~s}$ & SR & $\mathrm{R}$ & $\mathrm{RR}$ & M1 & M1a & M1b & M2 \\
\hline \multirow{5}{*}{$\mathrm{O}$} & $1: 10$ & - & ++ & + & +++ & +++ & +++ & - & +++ & ++ \\
\hline & 00 & - & + & - & +++ & +++ & ++ & - & ++ & - \\
\hline & $1: 500$ & - & - & - & ++ & ++ & + & - & + & - \\
\hline & 0 & - & - & - & - & - & - & - & - & - \\
\hline & 5000 & - & - & - & - & - & - & - & - & - \\
\hline \multirow{5}{*}{ S } & $1: 10$ & - & +++ & +++ & ++ & ++ & +++ & - & ++ & ++ \\
\hline & $1: 100$ & - & +++ & +++ & ++ & ++ & +++ & - & ++ & ++ \\
\hline & 500 & - & ++ & ++ & + & ++ & +++ & - & ++ & ++ \\
\hline & 1000 & - & + & - & + & + & +++ & - & + & ++ \\
\hline & $1: 5000$ & - & - & - & - & - & +++ & - & + & ++ \\
\hline \multirow{5}{*}{$\mathrm{R}$} & & + & +++ & +++ & +++ & +++ & +++ & + & +++ & ++ \\
\hline & $1: 100$ & + & +++ & +++ & +++ & +++ & +++ & + & +++ & ++ \\
\hline & $1: 500$ & - & +++ & +++ & +++ & +++ & +++ & - & +++ & ++ \\
\hline & $1: 1000$ & - & +++ & ++ & +++ & +++ & +++ & - & +++ & ++ \\
\hline & $1: 5000$ & - & ++ & - & +++ & +++ & +++ & - & ++ & ++ \\
\hline
\end{tabular}

high resistance to the bacteriophage. It is particularly clear-cut in the bacteria composing the small mixed colonies which can be observed after spreading upon agar a secondary culture in which very virulent corpuscles and very resistant bacteria co-exist (d'Herelle) ${ }^{324}$ The capsule usually disappears in the first transplant in the ultrapure colonies. However, it appears that under conditions not yet known a stable muta-

* We must not forget to mention the kindly and too little known Noël Bernard when the question of mutations occurring because of symbiosis are considered, that is to say, when they occur through the effects of a mitigated parasitism. This scientist has shown that in the plants symbiosis is the primary eause of fixed mutations. 
tion may occur and that the formation of a mucous capsule may become a permanent characteristic, as is indicated by the experiments of Bordet and Ciuca, and by those of Gratia, who have obtained ultrapure strains of $B$. coli cultivable indefinitely as $B$. aerogenes.

But here is another experiment which tends to show that under the action of bacteriophage a mutation of another type may occur.

Flu ${ }^{211}$ has kept in his stock cultures a bacillus (KB) isolated from a patient with sprue. This bacillus presents all of the characters of a Flexner dysentery organism, except in the matter of its agglutinability with anti-Flexner sera. That it is actually an inagglutinable Flexner bacillus is shown by its absorption of the corresponding agglutinins, by the fixation reaction, and by its agglutinogenic character. A serum prepared with this organism agglutinated different strains of B.dysenteriae Flexner in a dilution of 1:7000 but the serum remained inert upon the KB bacillus itself.

Flu then found that this bacillus is completely refractory to the action of a Flexner-bacteriophage although the Flexner dysentery bacilli are a homogeneous species, and although the races of the bacteriophage against which the KB bacillus proved to be refractory, caused, nevertheless, bacteriophagy with various normal strains among the cultures of Flu.*

Here is, then, an abnormal strain of the Flexner bacillus (a species which is homogeneous), endowed with the refractory state toward the bacteriophage and this refractory state is due to the fact that the protoplasm of this bacterium destroys bacteriophage corpuseles. It has, then, a true immunity. It is very probable that this refractory state was acquired $\dagger$ in the body of the patient. Ordinarily in the absence of virulent bacteriophage corpuseles the refractory state is gradually lost. In the case of bacillus "KB" it appears that a fixed mutation has occurred, the refractory state being permanent in the absence of the bacteriophage. If the fact of such a mutation, here probable, but obviously subject to discussion, were experimentally demonstrable beyond all possible doubt, it would be of considerable importance in

* This is the bacillus "KB" of which we have already spoken with regard to destruction of the bacteriophage corpuscles in the bacterial protoplasm. Flu has been able to show that this refractory bacillus "phagocytizes" the corpuseles.

$\dagger$ I have isolated from a patient a Flexner bacillus which was refractory to the bacteriophage and inagglutinable, but in this case the refractory state gradually diminished during cultivation in the absence of the bacteriophage. 
biology, not only from the point of view of bacteriophagy, but especially in that it would show a possible origin of a natural immunity.*

Bacterial metabolism is also profoundly modified by the acquisition of resistance, and as a rule the reëstablishment of the original metabolism coincides with the loss in resistance, but here also fixed mutations may occur.

It has already appeared from the preceding section that such changes take place in mixed cultures, a condition due, as a matter of fact, to resistant bacteria.

Here again it is a case of repeating what has already been said with regard to the agglutinability and the virulence of resistant bacteria. Changes in the metabolism can not be precisely described, for they vary from one bacterium to another, even in a single culture of resistant bacteria. From the beginning of my studies I have been interested in ascertaining the fermentative characteristics of Shiga dysentery bacilli resistant to the bacteriophage, but despite some hundreds of tests this has been impossible, for every colony developing from seeding a mixed culture on agar shows different fermentation reactions.

Very recently Fejgin ${ }^{198}$ has described 3 different strains isolated from a single mixed culture of Shiga bacilli. These three strains possessed the following characters:

Strain 1. The fermentation reactions were those of $B$. dysenteriae Shiga. Inagglutinable with an anti-Shiga serum. The homologous antiserum sgglu tinated the Shiga bacillus, but not its own strain.

Strain 2. Fermentation, with no gas production, takes place in glucose, maltose and mannite. A Shiga antiserum agglutinates the organism to a titre of $1: 800$, and this serum also agglutinates its homologous strain.

Strain 3. With this strain fermentation, with gas formation, occurs in lactose, glucose, maltose, arabinose, levulose, mannite, and dulcite. It is agglutinated to a titre of $1: 1600$ by an anti-Shiga serum and by its homologous serum.

In brief, then, we have as mutations, first, an inagglutinable Shiga bacillus, second, a Flexner bacillus, and third, a colon strain which does

* For example, the Algerian sheep are refractory to spontaneous anthrax. This, also, must be a fixed mutation produced through the effects of the parasite, $B$. anthracis. If all sheep could enjoy the same immunity we would say that the sheep species is naturally refractory to anthrax and that we had here an acquired immunity transformed into a natural immunity as the result of the mutation. 
not produce indol. This third strain, as a matter of fact, aside from its fermentation reactions, changes a neutral red medium to a canary yellow, coagulates milk, reddens litmus milk very strongly, but does not produce indol.

Fejgin has isolated similar strains from the stools of patients, showing particularly the same agglutinative characters.

This work is of the greatest interest. but it is not a question of the possibility of isolating three strains from a mixed Shiga-bacteriophage culture; there are thousands. All of the characters of the Shiga species (and it is the same for any bacterium whatever) form, one might say, a "puzzle" of which each of the fragments represents a character capable of varying from zero to infinity. The character "glucose fermentation" may be zero or it may be an energetic fermentation with gas, or it may be an intermediate stage. The "agglutinability" character may be anywhere from zero to a titre of 1:50,000, or even higher. The character "toxicity" may lie anywhere between zero and a high potency. The character "form" extends from coccus to bacillus. And the same possibility for variation exists with respect to each character. But experiment shows that through the influence of the bacteriophage, each of these characters may be changed independently of the others. Consequently the number of possible mutations is, in reality, unlimited, and although the tendency for certain changes is undoubtedly far greater than for others, it seems that a very great many are possible.

It may be added, with reference to the staphylococcus, that when a mixed culture, grown out at a temperature of $40^{\circ} \mathrm{C}$. in spite of the presence of a bacteriophage of maximum activity, is planted in gelatin no liquefaction occurs. From this it appears that a staphylococcus with a maximum resistance no longer secretes a proteolytic enzyme. In addition the capacity to produce pigment is lost.

Biologists who have not kept in touch with the new facts disclosed by our knowledge of bacteriophagy may well be astonished at these statements, and they may even remain somewhat skeptical. But the experimental procedure is so extremely simple that they can readily become convinced of the truth of all these statements. Moreover, a deeper study of these mutations is sure to completely revise our present concept of the fixity of species.

In summary, then, the most important fact to be derived from all of these studies is that, exposed to the action of the bacteriophage, bacteria undergo mutations, usually unstable ones, but that these may become fixed under conditions as yet undetermined. These mutations are associated with a state of resistance acquired by the bacteria. 
We have seen in a previous chapter that the first phase of bacteriophagy consists in the fixation of bacteriophage corpuscles to the bacterium. Fixation takes place in the same way with bacteria which have acauired a refractory state $(\mathrm{Flu}) .^{211}$

\section{ULTRABACTERIA}

Working with a Coli-bacteriophage Izar" ${ }^{3}$ has seen that "if the clear liquid obtained by filtering a culture of $B$. coli dissolved under the action of the bacteriophage is seeded in bouillon or upon agar immediately after filtration it does not cause either turbidity or growth if it is protected from the air in sterile tubes which are completely filled and sealed in the flame. But if the filtrate is held in sterile tubes closed with cotton or in tubes which are only partly filled, in many cases coccobacilli develop after a period varying from 48 hours to 11 days."

I also have found that suspensions obtained by the filtration, through Chamberland $\mathrm{L}_{2}$ or $\mathrm{L}_{3}$ candles, of bacteriophage suspensions of $B$. dysenteriae Shiga, Flexner, or Hiss, of B. typhosus, of B. pestis, of B. gallinarum and of $B$. coli may become cloudy again. The period of time required for the appearance of this phenomenon varies and I have found that the cause of the turbidity is the growth of resistant bacteria. This observation is recorded in a note appearing in the first edition of the "Bacteriophage." It reads as follows:

The following suggests that under the influence of the bacteriophage nonspore-forming bacteria may give rise to filterable forms. I have noted, although rarely, that a filtrate obtained by passing a secondary culture through a Chamberland bougie $\left(\mathrm{L}_{2}\right.$ and even $\left.\mathrm{L}_{3}\right)$ becomes turbid after some days. Each time that this has been noted the turbidity has been due to the growth of a resistant bacterium such as was present in the secondary culture prior to the filtration. The conditions under which this phenomenon occurs have not been ascertained, thus the observation is simply mentioned without emphasis being placed on its interpretation.

In what follows I shall term these filtrable forms of bacteria "ultrabacteria."

Tomaselli ${ }^{599}$ has studied the conditions leading to the appearance of these ultrabacteria and has made an important contribution to our knowledge of this curious phenomenon.

He has shown that the difficulty which is sometimes experienced in increasing the virulence of a bacteriophage for certain bacterial strains is due to the fact that resistant ultrabacteria pass through the candle.

We have seen, as a matter of fact, the mechanism of the increase in virulence of the bacteriophage. Corpuscles having a weak virulence 
for a given organism are introduced into a suspension of these bacteria. A double phenomenon occurs. The corpuscles, as the result of the passages from bacterium to bacterium increase in virulence, but certain particularly apt bacteria resist and serve as the origin of resistant lines. The elimination of such organisms is what makes it necessary to filter through a candle between each of the passages from suspension to suspension of susceptible organisms. One may observe very frequently, however, that the virulence of the corpuscles, after starting to increase, becomes fixed in spite of multiple passages and Tomaselli has shown that in these cases precisely, a production of filterable forms of ultrabacteria leads to the development of normal resistant forms. From this one can see that filtration through a candle is of no avail. Both the bacteriophage corpuscles and the ultrabacteria pass through, and the latter, developing in the following passage yield resistant bacteria.

The importance of this phenomenon warrants inserting here a summary of the experiments of Tomaselli. He worked with a Coli-bacteriophage in combination with 5 strains of $B$. coli. He showed that at the beginning, before any passages were made, the virulence of this race for these different strains was as follows:-weak $(+)$ for strains 1, 2, and 3; moderate $(++)$ for strains 4 and 5 . Invariably the filtrates obtained after the bacteriophagy of strains 3 and 4 again became turbid, while on the contrary, the filtrates from 1, 2, and 5 remained clear indefinitely.

With this bacteriophage he carried out serial passages with each of these 5 strains, following the usual method, that is to say, inoculation of the filtrate into a suspension of normal bacteria and filtration through a candle after 24 hours. The passages were continued without interruption up to a point where the virulence ceased to increase. He obtained thus a maximal increase $(++++)$ for strains 1 and 2 ; a moderate virulence $(++)$ for strains 3 and 5 ; while the virulence remained weak $(+)$ for strain 4 .

After each passage the filtrates obtained with strains 3 and 4 became turbid after some time, meaning that with these two strains ultrabacteria formed.

In further experiments Tomaselli sought to determine whether the formation of ultrabacteria was limited as to time. He found that they no longer formed when the corpuscles and the bacteria had remained in contact for about 20 days. As a matter of fact, if, instead of filtering the suspension where bacteriophagy had taken place 24 hours after the inoculation of the bacteriophage, he permitted the contact to be pro- 
longed and filtered samples after 5, 10, 15, and 20 days, ${ }^{*}$ the filtrates obtained after 5 and 10 days always contained ultrabacteria, those filtrates prepared after 15 days sometimes contained them, while those filtered after 20 days never contained them.

Instead of making passages every 24 hours one has only to filter the suspensions which have resulted from bacteriophagy after 20 days. The result is that which has been described. From the first passages the virulence of the bacteriophage for strain 4 , from weak $(+)$ becomes maximal $(++++)$. The same result is obtained for strain 5 after 3 passages.

It must be then the passage through the filter of resistant ultrabacteria which interferes with the increase in virulence.

As for strain 5, although the passages had been carried out under the same conditions, the virulence of the bacteriophage could not be increased to its maximum, nor could Tomaselli demonstrate the formation of ultrabacteria with this strain.

Tomaselli has demonstrated the same facts with $B$. dysenteriae Shiga and Flexner.

Aside from the conclusions which this author has drawn from his experiments concerning the increase in the virulence of the bacteriophage the following may be stated: 1 . The formation of ultrabacteria takes place especially through the action of bacteriophages having a weak virulence. 2. Not all of the strains of a single bacterial species possess the same faculty of assuming the ultrabacterial form. 3. The vitality of the ultrabacteria (at least insofar as bacteria of the colontyphoid-dysentery group are concerned) is relatively weak. They do not remain viable for more than 15 to 20 days.

Hauduroy ${ }^{298,299}$ has also observed the formation of resistant ultrabacteria in connection with the bacteriophagy of $B$. dysenteriae, $B$. typhosus, and B. coli. He has noted the frequency with which it occurs under the action of bacteriophage races of relatively low virulence. He has found that in the filtrates the ultrabacteria do not appear to be very numerous, for in distributing $200 \mathrm{cc}$. of a filtrate among 20 tubes with $10 \mathrm{cc}$. to each tube he has observed the appearance of cultures in only some of the tubes.

Let us note, however, that it is just as easy to believe that the ultrabacteria may be very abundant in the filtrates but that only a very few

* He did not prolong the contact beyond this for he found that the attenuation of the bacteriophage through contact with the resistant bacteria was then very pronounced. 
of them are capable of regaining a normal, visible form under the conditions of the experiment. Certain observations which I have made incline me to accept this last hypothesis.

However this may be, Hauduroy has studied the morphology and the characters of the resistant bacteria derived from these ultrabacteria.

In bacteriophage suspensions filtered through a candle the cultures may assume one of two forms. In both cases the turbidity is at first very slight, resembling rather an opalescence similar to that of an eggwhite suspension, but in a certain number of cases the turbidity gradually increases up to the appearance of the clouding which one observes in normal cultures of bouillon. In other cases the medium clears and one may observe the appearance of small and large agglutinates.

I have proved that these two aspects correspond to a difference in virulence and as a result to differences in the resistance of the bacteria derived from the ultrabacteria. In the first case the bacteriophage is always of relatively low virulence, whereas when agglutinates form, the virulence of the bacteriophage is very high.

Hauduroy has followed with the microscope the formation of these cultures. His conclusions on this subject may be quoted.

To these different macroscopic appearances there are corresponding microscopic peculiarities. The tubes which have a homogeneous turbidity of the "egg-white type" contain minute granules which do not take the Gram stain. They are very abundant; isolated, or arranged as on a filament which may at times be seen. There are occasional bacilli which do not take the Gram stain. There are masses without definite structure of variable size, having a mucous character and in which granules can be detected. In tubes containing the agglutinates we may also find granules which do not stain with the Gram stain. They are very fine, extremely numerous and are found in masses which appear to be formed of a reticulum containing granules and very rare bacilli.

These elements are living. They may, as a matter of fact, reproduce and give birth to elements comparable to themselves or to typical dysentery bacilli.

The cultural characters and the biochemical reactions of these elements are ordinarily different from those of the Shiga bacilli from which they are derived. Several more or less distinctive types can be differentiated, which might be characterized as follows: 1 . Elements, which, as a rule, may be readily transplanted serially into the usual liquid media and yield there either homogeneous cultures, or agglutinated cultures, or a sedimented culture which can be removed from the bottom of the tube only by shaking. At times these cultures do not appear until after several days' incubation at $37^{\circ} \mathrm{C}$. 2. These elements can be cultivated only exceptionally on solid media under aerobic conditions. After many attempts I obtained once a growth on ordinary agar slants, and once a culture on lactose agar. 3. The fermentation reactions of these elements are, as a rule, different from those of the normal Shiga bacillus. They may have a 
well developed fermentative power producing gas but they do not give indol and do not change the color of litmus lactose agar. After a few transplants one finds either a return to the typical reactions or a persistence of the abnormal characters. The sugar fermentation reactions can be observed only by implanting the sediment into sugar-containing agar and it sometimes requires three or four days for the fermentation to reach its maximum.

The morphological characters of the elements which form secondary cultures after filtration may persist throughout a series of transplants. The elements are comparable to those which have been described above: isolated granules, masses supported on a network, occasional bacilli, etc. In certain cases there is a return to the bacillary form.

To summarize all this, the granules described by Hauduroy are certainly the coccus forms such as I have observed in a simple secondary culture and of which I have spoken in preceding paragraphs. It is, in fact, certain that in ordinary secondary cultures obtained after complete bacteriophagy, the formation of ultrabacteria takes place in the same manner as in the filtrates. It is even probable that all secondary cultures developing after a complete bacteriophagy with a total clearing of the medium result from the formation of ultrabacteria which then reproduce in the form of cocei and later pass into the normal form.

In reality, what are these ultrabacteria? In the second part of this text I will give the hypothesis which I have formed ${ }^{349}$ and which seems to me to be the only one to accord with the facts. At the moment I will only state that I have shown these filterable bacterial forms to be ultra-filterable, that is, they pass through collodion filters ${ }^{349}$ provided the pores are sufficiently open to allow the serum albumin micella to pass. Their dimension is, therefore, that of a protein micella. This renders it apparent that the filterable forms of bacteria which develop through the influence of the bacteriophage are in reality ultraviruses of the same size as those of variola, of encephalitis lethargica, or of rabies. We will see later the importance of the fact that there are ultrabacteria.

\section{NATURAL MIXED CULTURES}

Bacteriophagy is a phenomenon occurring under natural conditions. We will see that the bacteriophage is a normal inhabitant of the intestinal tract throughout the animal series from the insects up through man. It is found in everything which is exposed to contamination by excreta; in the water of rivers and of the sea, in the soil and in the dust. Within the animal it does not remain confined to the intestine but it may pass into the circulation and through this channel it may be con- 
veyed into any region of the body whatsoever. It may be found in plants, for in the nodules of leguminous plants the nitrifying bacteria are not in pure culture but in a culture mixed with the bacteriophage. None of the bacteria are necessarily protected from the bacteriophage. Wherever the bacterium may be found the bacteriophage intervenes and a struggle takes place. But the bacteria resist and symbiotic cultures or mixed cultures result. Without treating at length all of these facts, which will not be emphasized here, it may be well to remark that the fact that mixed cultures are found among laboratory stock cultures is not particularly incomprehensible.

We know that unless the bacteriophage possesses a maximum virulence the formation of secondary cultures after bacteriophagy is the usual thing. It would be indeed strange if this were not also true under natural conditions. To assert the contrary would be to affirm that bacteriophagy is solely a laboratory phenomenon. If we transplant on to agar an artificial mixed culture we know that certain colonies are ultrapure, while others, more or less numerous, are contaminated by a bacteriophage. If the secondary culture forms in nature, by starting with any natural product whatever and culturing this on agar we should obtain both ultrapure colonies and mixed colonies. Transplanting one of these ultrapure colonies the strain isolated will remain ultrapure indefinitely, but if by chance we select for transplanting a mixed colony the strain may perpetuate itself in the form of a mixed culture contaminated by bacteriophage corpuscles.

I have said "may perpetuate" for experiment shows that a mixed culture prepared artificially frequently undergoes an auto-purification through the elimination of the bacteriophage corpuscles in the course of successive transfers. The same thing certainly takes place with naturally mixed strains and I have noted upon two different occasions a purification of this type during the series of transplants. Both of these instances occurred with two strains of $B$. coli isolated from cases of cystitis. It would seem that if this ultrapurification by elimination did not take place the number of mixed strains to be found in laboratories would be far greater than it actually is.

Let us bear in mind that it may be very difficult to prove whether a bacterial strain is ultrapure or contaminated. Experiments on artificial mixed cultures show that after a certain number of transfers there is not only no further elimination of corpuscles, but a perfect equilibrium is established between the capacities of the two antagonists to attack and to react. No plaques are to be found on agar and the appearance of 
the culture in a liquid medium hardly differs from that of ultrapure cultures of bacteria of the same species. When dealing with bacteria belonging to a homogeneous species it is easy to prove ultrapurity, for it is only necessary to filter a bouillon culture of the bacterial strain in question and to combine this filtrate with a normal organism of the same bacterial species. If bacteriophagy takes place the culture is an artificially mixed one; if it does not, the strain is certainly ultrapure. But with regard to bacterial strains belonging to heterogeneous species the proof may be far more difficult, although we will see that it is still possible.

Gildemeister seems to have been the first to notice that certain strains of intestinal bacteria might give, on agar, abnormal colonies which he termed "Flattenformen,"-of variable aspect. He noticed that subcultures made by the isolated colony method from such "Flattenformen" gave colonies of different appearances. He concluded that certain strains of intestinal bacilli were subject to mutations, without, however, recognizing that these mutations took place through the influence of a principle separable from the bacterium.

Bail $^{43}$ has succeeded in isolating bacteriophages from 3 strains of Flexner dysentery bacilli.

Moreover I have observed that in certain cases of cystitis the bladder contains, not a pure culture of $B$. coli, but a mixed culture composed of resistant $B$. coli and a bacteriophage. Platings of the urine upon agar yield ultrapure colonies and mixed colonies from which it is possible to isolate a bacteriophage. ${ }^{321}$

Otto and Munter, ${ }^{492}$ Weinberg and Aznar ${ }^{623}$ and Seiffert ${ }^{577}$ have also isolated bacteriophages from different strains of intestinal bacilli, but these authors have attempted to generalize and have affirmed that all bacterial strains contain a dissolving principle. Jötten ${ }^{381}$ has expressed the same opinion although he admits that "the experiment is not always successful." As for Gildemeister and Herzberg ${ }^{232}$ they admit that many strains appear to be free of bacteriophage but they suggest the hypothesis that when filtrates of old cultures appear to be free of all "lysogenic" action this must be because they are not acting upon a susceptible bacterium. If all bacterial species were heterogeneous with regard to the bacteriophage such an hypothesis would be open to discussion but the existence of homogeneous species obviously renders it inadmissible. If we take a secondary culture or a mixed culture of dysentery bacilli and filter it through a candle and then combine the filtrate with a normal strain of any $B$. dysenteriae whatsoever, bacteriophagy takes place. 
If the hypothesis of Gildemeister were correct, that is to say, if all bacterial strains contained a "lytic principle" it would only be necessary to introduce a filtrate of any old culture of Shiga bacilli whatsoever into a suspension of young bacilli in order to bring about bacteriophagy. But this is not the case. Indeed, the fact that it is possible and easy to purify any strain contaminated by the bacteriophage likewise renders the hypothesis of Gildemeister inadmissible.

It may not be out of place to mention the fact that the majority of those who, at the beginning of their experiments upon the subject, suggested that it was easy to isolate a bacteriophage from an old bacterial culture are not as emphatic on this point in their more recent publications. Thus Otto and Munter ${ }^{493}$ now state that the nature of the bacterial strain employed plays a rôle of the greatest importance. This is to implicitly recognize that there are pure strains and that there are contaminated strains.

Immediately after the publications of Otto and of Weinberg appeared I showed ${ }^{338}$ that in naturally mixed cultures the dissolving principle represents an impurity in the bacteriological sense of this word, since it is only necessary to carry out purifications upon an agar medium according to the usual technic of colony isolation to obtain ultrapure colonies cultivable indefinitely as such and from which it is henceforth impossible to isolate a bacteriophage. This finding, since confirmed by a number of workers, proves that the bacteriophage is foreign to the bacterium inasmuch as a culture which contains it is divisible into two fractions, the bacteriophage corpuscles on the one hand and the ultrapure bacteria on the other.*

But are contaminated bacterial strains as common as some authors assumed, especially at the beginning of their studies? The following experiments afford an answer.

Beckerich and Hauduroy ${ }^{59}$ isolated, as I had done, a mixed culture of bacteriophage and B. coli from a case of cystitis. They, also, succeeded in purifying this contaminated strain by a single isolation, and from that time on it was impossible for them to obtain from the ultrapure strain a principle having an activity suggesting in any way the presence of a bacteriophage.

Tomaselli ${ }^{599}$ likewise encountered 5 cases of pyelocystitis yielding mixed cultures of bacteriophage and $B$. coli. With the first 4 he readily

* It seems, indeed, that a number of authors at the beginning of their studies confused a simple temporary inhibition of growth with bacteriophagy. This caused them to think that the bacteriophage was to be found everywhere. 
obtained upon agar a separation into mixed colonies and ultrapure colonies. With the fifth it was necessary for him to resort to the other method which I have indicated, namely, that of growth on glucose agar. $^{321}$ In this way he obtained ultrapure colonies of this strain when isolation on ordinary agar had been unsuccessful. Having then of these 5 strains both mixed colonies and ultrapure colonies he showed that although the first were indefinitely cultivable in the form of mixed cultures from which it was possible to isolate a bacteriophage, the second, on the contrary, continued indefinitely as ultrapure cultures, and from these it was henceforth impossible to isolate a bacteriophage. This makes it obvious that in mixed cultures the bacteriophage represents an impurity.

From two other cases of uncomplicated cystitis Tomaselli isolated ultrapure cultures with no bacteriophage from the urine.

Gildemeister and Herzberg, ${ }^{232}$ working on old cultures, tried in vain to derive a bacteriophage, although they applied very drastic treatment. They subjected the bacteria to trituration and extracted the bacterial juices under a pressure of 4000 atmospheres.

Pondman ${ }^{530}$ studied systematically the 13 strains of $B$. dysenteriae found among the stock cultures of the Institute of Tropical Medicine at the University of Leiden. He cultivated these different strains in bouillon, and filtered them after an interval varying from 24 hours to 30 days. Among these 13 strains one only (Y-14) contained a bacteriophage. In additional experiments he dissolved the bacteria with trypsin or with pyocyanase and in other tests he substituted heating for filtration through a candle. The final products obtained in these different ways were combined with the bacteria. He did not succeed by any of these procedures in demonstrating any "lytic" principle whatever in cultures of 12 of these strains. But with strain $\mathrm{Y}-14$ he was able to isolate a bacteriophage by all of these methods. Twelve of these strains were, therefore, ultrapure; 1 was a mixed culture.

During a series of experiments, representing several months' work, Flu ${ }^{212}$ systematically examined 43 different bacterial strains: 13 of Vibrio cholerae, 6 of B.typhosus, 4 of B. dysenteriae Shiga, and 20 of the Flexner or Hiss dysentery bacillus.

With each strain he performed a double series of tests. A flask containing $50 \mathrm{cc}$. of bouillon was seeded with a loopful of a fresh culture of the strain under examination. After incubation at $37^{\circ} \mathrm{C}$. for 10 days the material was passed through a candle. To the filtrate he added an equal volume of sterile bouillon and seeded it again with a loopful of a 
fresh culture of the same strain. The seeded medium was returned to the incubator at $37^{\circ} \mathrm{C}$. and allowed to remain there for 6 weeks. After this time the old culture was filtered and the filtrate was tested systematically on different strains of susceptible bacteria, either in bouillon in order to see if any "lytic" phenomena took place, or on agar in order to detect the presence of plaques. This same procedure was repeated for each strain examined. Furthermore, the test was repeated with each strain substituting heating for 1 hour at $58^{\circ} \mathrm{C}$. for the filtration. Finally he made serial passages with the filtrates (or with the heated suspensions) with different bacteria, for the purpose of increasing the virulence of the bacteriophage if any was present.

These experiments forming a double series;-one with a filtration between each passage and the other with heating,---were carried out for each of the 43 strains. The results showed that only one was contaminated by a bacteriophage and this strain was the Y-14 strain of the Hiss bacillus, the same strain that had proved to be contaminated when studied by Pondman.

Flu then purified this Y-14 strain by means of colony isolation, obtaining ultrapure colonies. The cultures derived from these ultrapure colonies were subjected to the same treatment that permitted him to isolate a bacteriophage from the original strain. It was impossible for him to isolate one. Cultures derived from the ultrapure colonies were indefinitely ultrapure; the bacteriophage had been eliminated. It was, then, an impurity.

In another series of investigation ${ }^{220} \mathrm{Flu}$, who as has just been stated, examined 13 strains of Vibrio cholera, tested the ultrapurity of 10 other strains. This second series of studies, conducted as methodically as was the first, showed one of the 10 strains to be a mixed culture and from it he isolated a bacteriophage.

In all, then, of 53 strains of different types of bacteria taken at random from the cultures kept at the Institute of Tropical Medicine at the University of Leiden, 51 were ultrapure. None of the methods applied permitted the detection of a principle exerting any action in any way resembling bacteriophagy. Two were contaminated by races of the bacteriophage which Flu isolated. After a series of passages with susceptible bacteria, the first brought about complete bacteriophagy of a suspension of the Hiss bacillus, the second, complete bacteriophagy of a suspension of Vibrio cholerae.

Reichert ${ }^{550}$ examined 11 strains of dysentery bacilli; 9 were ultrapure, 2 were contaminated by a bacteriophage. He purified these two strains 
by colony isolations and the new strains yielded henceforth ultrapure colonies free of the bacteriophage.

Bürgers and Bachmann ${ }^{131}$ carried out 200 tests on strains of Grampositive organisms and succeeded once in isolating a bacteriophage from an old culture of the organism of swine fever. In spite of the fact that they were only once successful these authors certainly employed a method which should have enabled them to detect a bacteriophage had any been present, for they employed the procedure of Buchner for the extraction of zymase. The bacteria were triturated with sand and then subjected to a pressure of 400 atmospheres.

From all of these experiments it appears definitely that there are bacterial strains containing bacteriophage corpuscles, but that these strains, naturally contaminated, are relatively rare; more rare even than one would have a priori supposed in view of the ubiquity of the bacteriophage and the frequency under natural conditions of the bacteriophage-bacteria symbiosis in natural mixed cultures.

As I have shown, ${ }^{331}$ in accord with the present state of our knowledge, if bacteriological studies are to be conducted under properly controlled conditions, it must first be shown that the bacterial strains involved in the study are, not only pure, but also ultrapure.

Strains contaminated by a bacteriophage may be recognized in that they give mutant colonies, while all the colonies of an ultrapure strain are identical. The bacteria of contaminated strains are but slightly or not at all agglutinable by a specific antiserum. The bacteria are resistant to the action of bacteriophages which attack homologous strains. Every time that one or the other of these characters is encountered there is reason to suspect contamination by a bacteriophage.

\section{THE PURIFICATION OF NATURAL MIXED CULTURES}

Purification of natural mixed cultures is the more readily accomplished when the symbiosis between the bacteriophage and the bacterium has not been of long duration. In ordinary cases the usual method of colony isolation upon agar permits the selection of ultrapure colonies. It may be added that it is always well to repeat this purification procedure through 2 or 3 consecutive platings. The method of Roux, in connection with the purification of artificial mixed cultures is particularly well adapted to this purpose.

Bruynoghe ${ }^{112}$ has discovered that mixed cultures may be purified more readily if the mixed condition is of recent origin, and that when the symbiosis is of longer duration the simple procedure of colony isolation 
on ordinary agar may not suffice, for all of the colonies may then be contaminated. I would recommend in such a case ${ }^{321}$ the use of an agar containing sugar, that is to say, a medium containing some sugar fermented by the bacterium which is undergoing purification. Here it is necessary to resort to several successive purifications, each time taking the material for the new isolation from the margin of a colony as close as possible to the edge.

If the mixed culture can not be purified by this method it may be necessary to carry out a series of cultures in liquid media, acid in reaction and at the limiting temperature of the culture. In other words, it may be necessary to make a series of 8 to 10 cultures at the highest temperature in media containing the greatest amount of acid compatible with the development of the bacterium involved. After this ultrapure colonies may be obtained by cultivation upon a sugar-containing agar. These methods are based upon the fact that the bacteriophage may not develop in a medium whose acidity still allows the development of bacteria, especially at the temperature limit for the development of the bacterium.

An additional method for isolation, proposed by Prausnitz, ${ }^{539}$ depends upon the use of gelatin as a culture medium.

Still another method, applicable to certain cases, consists in the use of the antiserum. Such an antiserum is prepared by giving a rabbit a series of injections of the natural mixed culture. It has been shown by Bordet that the serum of an animal prepared by injections of mixed cultures possesses antibacteriophage properties. Isolations can then be made upon an agar medium in which this antiserum is incorporated. From the point of view of efficiency this procedure is inferior to the other methods described.

It should be stated that in those very rare cases where isolation on ordinary agar does not permit the selection of ultrapure colonies, isolation upon sugar-containing agar has always permitted me to isolate such a strain from naturally mixed cultures, except in one instance (among about 100 experiments), where it was necessary to employ passages in an acid medium at the temperature limit.

\section{ISOLATION OF THE BACTERIOPHAGE FROM NATURALLY MIXED} CULTURES

When dealing with bacteria belonging to homogeneous species it is usually only necessary to filter a bouillon preparation of the mixed culture and to combine the filtrate with an ultrapure suspension of 
bacteria of the same species. But the situation may some times be much more complicated. I might cite as an example an experiment of Flu, ${ }^{211}$ for in cases comparable to this the method devised by him may be very conveniently employed.

Everyone who has noted that it is possible to derive from certain bacterial strains among stock culture collections a bacteriophage active for normal bacteria of the same species has indicated that the isolation was possible only from old cultures and not from cultures recently transplanted. But, as Flu has stated, if the bacteriophage is really an impurity it is evident that the corpuscles must have been present in the previous culture from which the transfer was taken. This being the case they must be introduced into the bouillon when the bacteria are implanted, and consequently the corpuscles must have been present in the young cultures. He has been able to demonstrate this.

Flu centrifuged a 24 hour bouillon culture of the Y-14 strain, of which we have spoken in a preceding paragraph. He mixed the sediment resulting from the centrifugation with four times its weight of anhydrous sodium sulphate, ${ }^{*}$ and ground the dry mass resulting from this mixture in a mortar. The dry powder was dissolved in water and filtered through a candle. This filtrate contained virulent corpuscles which caused bacteriophagy in a suspension of normal Flexnerbacilli. The corpuscles, therefore, were actually present in the young cultures of a naturally mixed strain.

It should be stated that the sodium sulphate solution must be filtered for the bacteria resist this treatment as do the bacteriophage corpuscles, and if the resistant bacteria are not eliminated they will develop in the suspension to be used in the test for bacteriophagy and the latter process may then be undetected.

I have also succeeded in isolating a bacteriophage from young mixed cultures by dissolving the bacteria by pyocyanase. The filtrate contained the corpuscles.

The fact that a mixed culture, either natural or artificial, can be separated into two parts shows beyond possible contradiction that in such cultures bacteriophage corpuscles represent an impurity, in the bacteriological sense of the word.

\section{RÉSUMÉ}

1. The behavior of bacteria with regard to bacteriophage corpuscles does not escape a general biological law: one which might be termed the

* This is the method devised by Rowland for the extraction of the toxin of Shiga dysentery bacilli. 
"law of reaction." Bacteria attacked by bacteriophage corpuscles react and may acquire a resistance such as to afford a true immunity. According to the virulence of the corpuscles, to the tendency to resist on the part of the bacteria, and to the conditions of the moment, the one or the other of the two antagonists finally predominates. If it is the bacteriophage which is victorious the medium in which bacteriophagy takes place remains clear indefinitely, it being a pure suspension of bacteriophage corpuscles. If the bacteria succeed in acquiring an immunity the medium again becomes cloudy, as a result of the development of resistant bacteria. Here there is a secondary culture (d'Herelle $\left.{ }^{317}\right)$.

2. The secondary cultures result from a phenomenon of selection, not of bacteria naturally endowed with resistance but of certain bacteria which possess a faculty for the acquisition of resistance. In a given suspension the number of bacteria capable of acquiring resistance is the greater as the virulence of the bacteriophage is the less (d'Herelle ${ }^{317}$ ).

3 . The resistance acquired by a bacterium does not manifest itself solely toward the race of the bacteriophage in contact with which the resistance has been acquired but against other races as well. A bacterium which has once become refractory to the action of one race of bacteriophage is refractory to the action of all other races.

4. Experiment shows that the protoplasm of bacteria which have acquired a resistance is able to destroy bacteriophage corpuscles (d'Herelle, ${ }^{321} \mathrm{Flu}^{211}$ ). This experimentally demonstrated fact renders plausible the hypothesis that the acquisition of resistance by a bacterium takes place after "recovery," that is to say, as the result of the destruction of the corpuscles within the protoplasm of this bacterium.

5. Bacteriophage corpuscles which are enhanced by passages with susceptible bacteria are attenuated during the process of the acquisition of resistance by the bacteria.

6. If one purifies a secondary culture, that is to say, a culture containing bacteria endowed with an acquired resistance and virulent bacteriophage corpuscles, either by the use of an antibacteriophage serum (Bordet and Ciuca ${ }^{92}$ ) or by the fishing of ultrapure colonies (Eliava and Pozerski ${ }^{191}$ ) it will be found that in the course of a series of transfers on agar the acquired resistance gradually diminishes, and then disappears, and at this time the bacteria have again become susceptible (Bordet and Cinca $\left.{ }^{92}\right)$.

7. When planted upon agar secondary cultures give a variable result, depending upon the virulence of the bacteriophage. With very viru- 
lent corpuscles the agar often remains sterile $\left(d^{\prime} H_{e} e^{317}\right)$. When colonies form some are ultrapure, others are contaminated by corpuscles (Kuttner ${ }^{398}$ ). Among the ultrapure colonies some are composed of susceptible bacteria, others of resistant bacteria (Bordet and Ciuca ${ }^{91}$ ), and the degree of resistance is in its nature variable from one colo $y$ to another (Bruynoghe ${ }^{112}$ ).

8. The transfer of a secondary culture to bouillon yields, in cases where growth occurs, a mixed culture in which resistant bacteria and virulent corpuscles co-exist (Bordet and Ciuca, ${ }^{86}$ Kuttner $^{398}$ ). The appearance of mixed cultures in bouillon differs in accord with the virulence of the bacteriophage which they contain. In order that there may be a permanent mixed culture, that is to say, one indefinitely transferable in the form of a mixed culture without either of the two antagonists predominating, it is necessary that a state of equilibrium be established between the resistance of the bacteria and the virulence of the bacteriophage corpuscles. If, as the result of circumstances, this equilibrium is not attained one or the other of the two antagonists will be eliminated.

9. Secondary cultures result from an inhibition of the bacteriophage corpuscles by substances secreted by the bacteria which are reacting to the action of the bacteriophage. Inhibition is most outspoken when the corpuscles are of low virulence (d'Herelle).

Through the influence of the reaction opposed by the bacteria to the bacteriophage mutations are produced. These are almost always transitory, disappearing along with the cause which produced them (Eliava and Pozerski ${ }^{191}$ ), but occasionally permanent. These mutations may involve all of the characters of the bacterium; among others, morphology (d'Herelle ${ }^{317}$ ), biochemical characteristics (d'Herelle ${ }^{321}$ ), agglutinability, which is usually diminished (d'Herelle ${ }^{317}$ ), vitality, which is usually increased (d'Herelle ${ }^{321}$ ), or virulence, also usually increased (Bordet and Ciuca ${ }^{86}$ ) but sometimes abolished (Fejgin ${ }^{201}$ ).

With regard to morphology, bacilli in a state of active resistance take a cocco-bacillary form, even assuming a definite coccus form. They become surrounded by a capsule (d'Herelle $\left.{ }^{324}\right)$. When the bacteriophage is eliminated there is usually a return to normal form (Eliava and Pozerski $\left.{ }^{191}\right)$. Nevertheless it appears that under certain undetermined conditions a fixed mutation involving morphology may take place.

10. Under the influence of the acquisition of resistance there occurs under certain conditions infra-visible forms of resistant bacteria capable of reproducing the ordinary visible form (Izar $\left.{ }^{367}\right)$. These 
infra-visible, filterable bacterial forms are ordinarily the cause of the failure occasionally experienced in attempting to increase the virulence of certain bacteriophage races by the method of passages (Tomaselli ${ }^{599}$ ).

11. The ubiquity of the bacteriophage in nature and the frequency of mixed cultures in the animal body (d'Herelle ${ }^{321}$ ) makes it a priori certain that there are mixed cultures among laboratory stock cultures. That such cultures exist is a fact (Bail $\left.{ }^{13}\right)$. Bacterial strains in mixed cultures are, however, relatively rare as has been shown by the careful investigations of several workers. The studies of $\mathrm{Flu}^{212,220}$ are particularly interesting because of the number of strains examined and the manner in which he carried out his tests. Of 53 strains of different bacteria he found 51 to be ultrapure. Two only contained a bacteriophage.

12. These natural mixed strains are "contaminated" by bacteriophage corpuscles in the same way as are artificial mixed cultures. This is proved by the fact that it is easy to purify them by the method of colony isolation on agar as usually employed in bacteriology (d'Herelle ${ }^{338}$ ). Strains derived from ultrapure colonies are henceforth ultrapure and it is impossible to derive from them any "lytic principle" whatsoever.

13. On the other hand it is also possible to isolate bacteriophage corpuscles from a naturally mixed strain and this can even be done with young cultures $\left(\mathrm{Flu}^{211}\right)$.

The fact that from all naturally mixed cultures it is possible to obtain, on one hand, bacterial strains henceforth ultrapure, that is to say, not admixed with the bacteriophage, and on the other hand, bacteriophage corpuscles free of the bacteria, shows beyond any possible doubt that in such strains the bacteriophage corpuscles represent an impurity in the bacteriological sense of the word. 


\section{CHAPTER VI}

\section{Species of Bacteria Susceptible to Bacteriophagy}

\section{HOMOGENEOUS SPECIES}

\section{B. dysenteriae Shiga (Eberthella dysenteriae)}

The Shiga dysentery bacillus is the first bacterial species which I recognized as being susceptible to bacteriophagy. ${ }^{310}$ It is also the bacterium for which it is most easy to isolate virulent races of the bacteriophage; races frequently of sufficient virulence to overcome the organisms without the necessity of enhancing virulence by passages.

Up to the present time I have isolated several hundred races of the bacteriophage virulent for B. dysenteriae Shiga and I have yet to find two which at the time of their isolation are identical, not only with regard to the intensity of their virulences for this bacterium, but also as to the extent and the degree of their activity for other bacterial species..$^{315}$ All of the races which I have isolated were virulent for all of the strains of $B$. dysenteriae Shiga against which I have tested them,--strains selected at random either from among stock cultures or obtained from cultures derived from plates spread directly from dysentery cases. A race very virulent for a given strain of the Shiga bacillus may be less active for another strain but after a few passages at the expense of the latter it acquires a virulence comparable to that exhibited toward the first (d'Herelle ${ }^{321}$ ).

Among all of the races which I have studied I have encountered but a single one whose virulence was directed solely toward the Shiga bacillus. All of the others were virulent for one or another of the dysentery bacilli, Flexner or Hiss, and many were active against the two at the same time..$^{321}$ Very few races attack the Strong bacillus.

A great many races are at one and the same time virulent for a greater or less number of strains belonging to the heterogeneous bacterial species; B. coli, B. typhosus, B. paratyphosus $A$ and $B$, and other species of this group, and this may be the case immediately after isolation (d'Herelle $\left.{ }^{315}\right)$.

I will not return to the possibility of acquiring a virulence for bacterial species very distantly related, such as B. pestis, the staphylococcus, etc., for this question has been treated in detail. 
Races of the bacteriophage virulent for $B$. dysenteriae sthiga have been isolated up to the present from a variety of sources, including the stools of convalescents from bacillary dysentery (d'Herelle ${ }^{310}$ ); the stools of individuals effected with mild intestinal disturbances (d'Herelle ${ }^{312}$ ); the feces of normal men (d'Herelle ${ }^{312}$ ), particularly in epidemic periods $\left(\right.$ d'Herelle $\left.^{31^{\prime}}\right)$; the feces of domestic animals (d'Herelle ${ }^{31}$ ); from drinking water and from rivers (Dumas $\left.{ }^{188}\right)$; from cultivated soil (Dumas ${ }^{188}$ ); from sea water at the mouths of rivers; and from tap-water (d'Herelle ${ }^{321}$ ). In brief, races of bacteriophage virulent for $B$. dysenterice may be encountered in everything that is exposed to contamination by excreta.

\section{B. dysenteriae Hiss (Eberthella paradysenteriae)}

Everything that has been said in this text upon the subject of bacteriophagy with the Shiga dysentery bacillus and of the behavior of this organism toward the bacteriophage may be applied without change to B. dysenteriae Hiss.

We have seen that a bacteriophage virulent for $B$. dysenteriae shiga is also virulent for the bacillus of Hiss. As a general rule the virulence for a bacterium of any kind within the colon-typhoid-dysentery group frequently extends to the Hiss bacillus.

Reichert ${ }^{550}$ has observed an incompatability between the virulence of a bacteriophage for $B$. coli and for B. dysenteriae Hiss. He has found that if, by passages, a bacteriophage acquires a virulence for the first it loses virulence for the second, and the reverse is likewise true. According to my own observations the situation described by Reichert is a fortuitous coincidence rather than a general law for I have often encountered the simultaneous existence of these two virulences. In this connection I have even effected the following experiment. After preparing a mixed suspension by adding to bouillon a drop of a very concentrated suspension of $B$. coli, one demonstrated to be susceptible, and then one drop of a very heavy suspension of $B$. dysenterice Hiss, a strain also susceptible, complete bacteriophagy in about 12 hours occurred through the action of a bacteriophage isolated from a dysentery convalescent.

Beckerich and Hauduroy ${ }^{59}$ have found that a bacteriophage virulent for the enterococcus (isolated by them from the feces of a horse) was likewise virulent for $B$. coli and for $B$. dysenteriae Hiss.

Secondary cultures reinoculated into litmus sugar media do not ferment the sugars in the same way as do normal bacilli. Media containing glucose, maltose, and mannite become acid after ten days; those 
containing lactose, levulose, saccharose, and also glycerine remain alkaline. After a month the lactose, saccharose and levulose media remain alkaline. Secondary cultures, and also mixed cultures, give the indol reaction but do not react on either neutral red or lead acetate. The resistant bacilli are inagglutinable, have a high viability, and are more virulent for man. In Part III of this monograph we will consider a case of $B$. dysenteriae $H i s s$ septicemia in which the bacillus was resistant to the action of the bacteriophage (d'Herelle ${ }^{321}$ ).

Races of the bacteriophage virulent for B. dysenteriae Hiss have been isolated from the intestinal contents of dysentery convalescents (d'Herelle $^{310}$ ). They may be encountered under the same conditions as are those races virulent for the Shiga bacillus.

\section{B. dysenteriae Flexner (Eberthella paradysenteriae)}

Races of the bacteriophage virulent for B. dysenteriae Flexner may frequently be encountered under the conditions noted for the preceding.

All of the races which I have isolated were active for certain strains of B. coli; although with some, activity for other varieties of dysentery bacilli was lacking.

With reference to the bacteriophage, Flexner bacilli constitute a homogeneous species. Resistant bacilli ferment glucose, levulose, maltose, and mannite. They do not ferment lactose, do not blacken lead acetate in an agar medium, and do not react on neutral red. They form indol. They are inagglutinable by a specific serum and possess a high viability.

The atypical character of certain strains of $B$. dysenteriae when freshly isolated from the organism may surely be ascribed to their resistance to the bacteriophage. Elsewhere we will consider a typical case. Furthermore, this observation is of general significance, applicable not to dysentery bacilli alone (d'Herelle ${ }^{321}$ ).

A Flexner-bacteriophage was isolated for the first time from the stools of dysentery convalescents (d'Herelle $\left.{ }^{310}\right)$.

\section{Different intestinal bacilli (para- or pseudo-dysentery organisms)}

In cases of enteritis, bacilli may often be found which do not present all of the distinguishing characters of B. dysenteriae, B. typhosus, or the paratyphoids. Some of these strains may deviate from the descriptions a great deal. Nevertheless, it would appear that they may be the cause of the disease. 
At different times I have isolated these unidentified bacilli and I have always found that at the time of the disappearance of the more or less severe disturbance, the stools contained a bacteriophage virulent for the bacillus isolated from the intestinal tract at the beginning of the disease. Generally these bacteriophages are also virulent for the typical dysentery types, but the reciprocal of this is not always true, for the bacilli may often be insusceptible to the action of bacteriophages having a maximum virulence for $B$. dysenteriae.

During the summer of 1919 , I studied about 100 cases of diarrhea in infants on the service of Dr. Hutinel at the Hôpital des Enfants-malades. With the exception of the cases which progressed rapidly and died (cases to which we will return) I constantly isolated from the stools a bacillus which $I$ have also found in a few cases of gastro-enteritis in adults. This organism I have termed $B$. dysenteriae $X{ }^{321}$

When inoculated on litmus sugar agar media it fails to ferment any of the sugars tested (lactose, glucose, levulose, saccharose, maltose, mannite, galactose). It causes no change in lactose and maltose Barsiekow medium, but this medium containing glucose and mannite is turned red. It is agglutinated by convalescent serum in titres of $1: 100$ to $1: 500$, is not agglutinated by anti-Flexner or anti-Shiga sera. With a serum which agglutinates the Hiss strain to 1:2500 the " $\mathrm{X}$ " strain is agglutinated in dilutions of $1: 200$. It is non-motile, is morphologically like the other dysentery organisms, is Gram-negative, and is toxic for rabbits.

Several races of bacteriophage active for this bacillus have been isolated. This bacteriophage is constantly present in the intestine in convalescents who have shown $B$. dysenteriae " $\mathrm{X}$ " in their stools during the infection. Races have also been recovered from the intestinal tracts of healthy animals, both man and other animals. The "X" bacillus constitutes a homogeneous species as regards the bacteriophage.

Certain races of the bacteriophage active for B. dysenteriae " $\mathrm{X}$ " were likewise active for other species of dysentery bacilli, others were virulent for only one or two among them. When maintained for several generations at the expense of $B$. dysenteriae " $\mathrm{X}$ " they almost completely lose their activity for other dysentery organisms (d'Herelle ${ }^{321}$ ).

\section{Bacilli associated with fowl typhoid (Eberthella sanguinaria, pfaff, Rettgeri, Jeffersonii, Salmonella pullora)}

Eberthella sanguinaria is a very homogeneous species. During the course of the epizoötic in France occurring most extensively during the 
years 1918 and 1919, I isolated many strains of this bacillus and also many races of virulent bacteriophages. All of these bacteriophages caused bacteriophagy with all of the strains isolated, including some American strains sent me through the kindness of Dr. Hadley.

I have isolated, uniformly, races of the virulent bacteriophage from the excreta of domestic animals which were resisting the infection on the farms where the typhoid raged, even among those which were naturally immune. The bacteriophage is extremely virulent in those animals which have recovered from the typhoid.

Outside of the epizoötic foci, in spite of a great many examinations, I have never been able to isolate a bacteriophage possessing any action whatsoever for Eberthella sanguinaria from the excreta of chickens or any other domestic animals. ${ }^{314}$

I have also isolated races of the bacteriophage virulent for the related bacilli,-E. pfaffi, E. jeffersonii and Salmonella pullora. ${ }^{321}$ The number of experiments carried out with these organisms is not, however, sufficiently great to permit me to say whether these bacterial species are homogeneous or heterogeneous. Nevertheless the following fact is sufficient to suggest that E. pfaffi is heterogeneous and E. jeffersonii on the contrary may be homogeneous. Some races of the bacteriophage virulent for Eberthella sanquinaria are virulent for $E$. pfaffi, others are not. This would not be the case if $E$. $p f a f f i$ were a homogeneous species. On the contrary all of the races of the bacteriophage virulent for Eberthella sanguinaria are also virulent for E. jeffersonii.

With regard to the question of crossed virulences among these different bacteria the following may be stated. One race of the bacteriophage presented the following virulences: for Eberthella sanguinaria, ++++ ; for E. jeffersonii, ++++ ; for S. pullora $A,++$; for S. pullora $B$, + ; for E. pfaffi, 0 ; and for E. rettgeri, $0 .{ }^{*}$

Another race isolated from the excreta of a chicken which had resisted an epizoötic due to $E$. jeffersonii showed the following virulences; Eberthella sanguinaria, $0 ;$ E. jeffersonii, $0 ;$ S. pullora A, $0 ;$ S. pullora $B$, + ; E. pfaffi, ++++ ; and E. rettgeri, 0 (d'Herelle ${ }^{321}$ ).

\section{Pasteurella bovis}

I have isolated about 30 races of bacteriophage virulent for this organism. About 12 of these were, when isolated, endowed with a very

$*++++=$ very strong virulence; $+++=$ strong virulence; $++=\bmod -$ erate virulence, $+=$ weak virulence $0=$ no virulence. 
high virulence but I have not yet found one of maximum virulence (giving complete and always permanent bacteriophagy).

All of the races tested presented about the same virulence for the different strains of bacteria isolated from the blood of buffaloes dead of barbone (pasteurellosis of buffaloes) in Indo-China; as well as for a strain found in Italy and tested after frequent passages through rabbits; for a strain isolated from the mud of a water-hole frequented by buffaloes, some of which contracted the disease shortly afterward.* On the other hand in two instances I have isolated from the excreta of sick buffaloes refractory strains of the bacterium, and it is of interest that although presenting all of the morphological, cultural, and fermentative characters of the bacterium they were inagglutinable by an antiserum. These two strains were not normal, then, and the fact that they were not attacked does not prove that Pasteurella bovis is not a homogeneous species.

Races of the bacteriophage virulent for Pasteurella bovis are to be found, during an epizoötic period, in the intestinal contents of buffaloes who escape infection (d'Herelle ${ }^{322}$ ).

\section{B. pestis (Pasteurella pestis)}

Two races of the bacteriophage virulent for $B$. pestis have proved to be active for all strains of $B$. pestis with which they have been tested. Of the plague bacillus two cultures were obtained in Indo-China, two in France (Marseilles, 1919, and Paris, 1920), seven from the Dutch Indies, and five from Egypt. B. pestis is certainly, then, a homogeneous species as regards the bacteriophage.

After about the thirtieth passage one of these two races proved to be virulent for $B$. dysenteriae also and this virulence, at first weak, suddenly increased at the 10 th passage with $B$. dysenteriae and became very strong.

Races virulent for $B$. pestis are likewise virulent for the bacillus causing pseudo-tuberculosis in guinea-pigs.

The colonies of $B$. pestis which are resistant to the bacteriophage are very viscous, and are made up of polymorphous bacilli. Some of the resistant colonies are composed of bacilli inagglutinable by an antiserum, and they are extremely virulent for the guinea-pig.

Races of the bacteriophage virulent for $B$. pestis may be found in the intestinal contents of rats which have survived a plague epizoötic,

* With barbone raging in the neighborhood, buffaloes which were sick had undoubtedly frequented this previously. 
and in man in the pus of buboes at the period of suppuration (d'Herelle $\left.^{321,322}\right)$.

\section{HETEROGENEOUS SPECIES}

\section{B. typhosus (Eberthella typhi)}

Among the heterogeneous species B. typhosus is the one which is really the best known insofar as its behavior toward the bacteriophage is concerned, thanks to the work of Janzen and Wolff.

It may be stated, however, that insofar as the phenomenon of bacteriophagy itself is concerned, including the nature of the resistance of the bacterium, there is nothing to be added nor need we change anything that has been said in the preceding chapters. In these respects $B$. typhosus behaves in exactly the same manner as B. dysenteriae.

We have seen that for homogeneous species any bacteriophage race virulent for one strain is likewise active upon all others provided the strains are normal, that is, provided they present all of the characters of the species. With regard to heterogeneous species it not the same for with the latter a given race of the bacteriophage may prove to be virulent for a number of strains while other strains are absolutely refractory to it. Nevertheless these insusceptible strains may be subject to the action of other races..$^{321}$

Some races of the bacteriophage exercise an action limited to a small number of strains, sometimes to but a single one, while other races of the bacteriophage bring about bacteriophagy with a very large number of strains.

Table 21, taken from the work of Janzen and Wolff, ${ }^{372,374}$ shows better than any explanation the behavior of the heterogeneous species $B$. typhosus toward the bacteriophage.

I would state again (see "Evaluation of Virulence") that Janzen and Wolff determined the virulence of a bacteriophage in the following ways: I. By the dissolution of a suspension of young bacilli in bouillon. II. By inhibition or the interference with growth when sterile bouillon, inoculated with the suspension of the bacteriophage, is planted with the bacteria. III. By plaque formation on agar, obtained by spreading a drop of suspension I (above). Four degrees are employed in expressing the results of each of these tests: Weak $(+)$, moderate $(++)$, strong $(+++)$, and very strong $(++++)$. The results are read after incubation at $37^{\circ} \mathrm{C}$. for 24 hours.

Although maintaining its characters through successive passages (we will later discuss this question at length) a race of the bacteriophage 


\begin{tabular}{|c|c|c|}
\hline \multirow{3}{*}{ 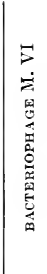 } & $\exists$ & 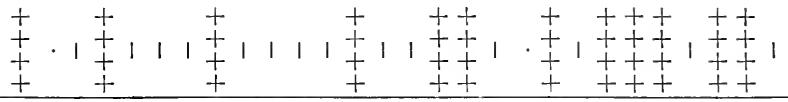 \\
\hline & $\exists$ & $\begin{array}{l}+ \\
+ \\
+ \\
+\end{array}$ \\
\hline & -1 & 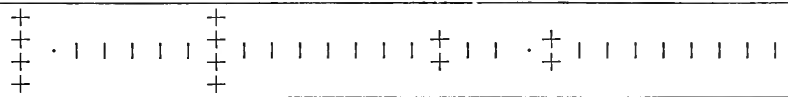 \\
\hline \multirow{6}{*}{ 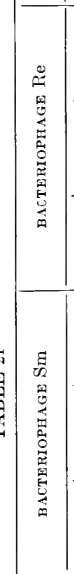 } & $\Xi$ & 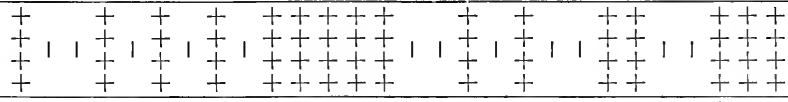 \\
\hline & $\exists$ & 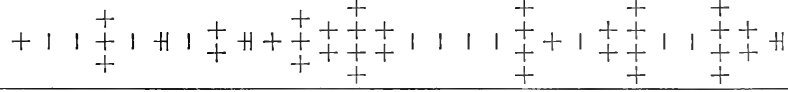 \\
\hline & $r$ & 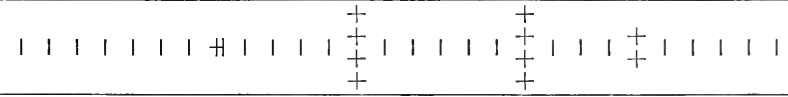 \\
\hline & $\Xi$ & 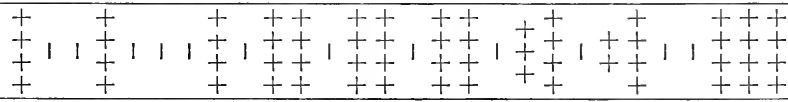 \\
\hline & $\exists$ & 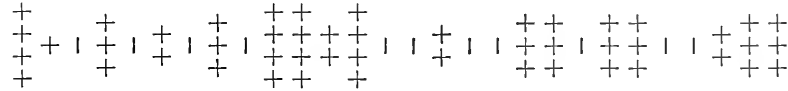 \\
\hline & -1 & $\begin{array}{l}+ \\
+\end{array}$ \\
\hline \multirow{3}{*}{ 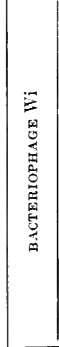 } & $\Xi$ & 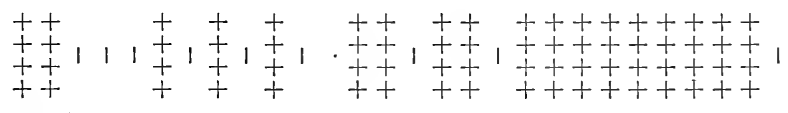 \\
\hline & $\exists$ & 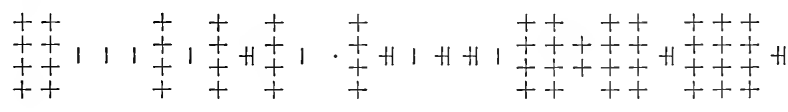 \\
\hline & -1 & 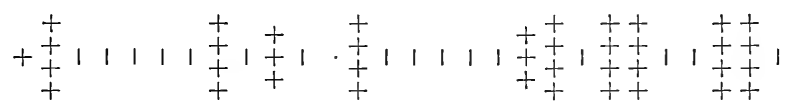 \\
\hline 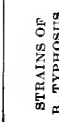 & & 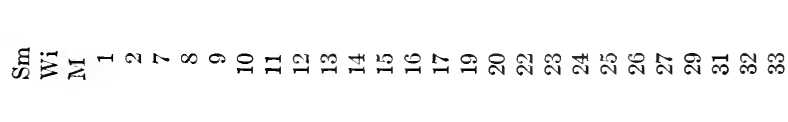 \\
\hline
\end{tabular}


may acquire new virulences. We have already seen that by repeated contacts it is possible to cause a bacteriophage to acquire a virulence for new species. Here the case is the same, for insofar as the bacteriophage is concerned, different strains of a heterogeneous species behave like distinct species.

Janzen and Wolff ${ }^{372,375}$ present some additional data dealing with the virulence of Bacteriophage $\mathrm{Wi}$ when tested upon 5 strains of $B$. typhosus. When this bacteriophage was tested immediately after its isolation from the intestinal tract the results were as shown in table 22 . When tested again after a series of passages at the expense of the Wi strain the results were as given in table 23 .

TABLE 22

\begin{tabular}{c|c|c|c}
\hline STrain & I & II & II \\
\cline { 2 - 3 }$W \mathrm{Wi}$ & ++++ & ++++ & ++++ \\
1 & - & - & - \\
24 & - & ++ & ++++ \\
27 & - & + & ++++ \\
29 & - & ++++ & +++ \\
\hline
\end{tabular}

TABLE 23

\begin{tabular}{c|c|c|c}
\hline STRAIN & I & II & II \\
\cline { 1 - 3 } Wi & ++++ & ++++ & ++++ \\
1 & - & - & - \\
24 & - & ++ & ++++ \\
27 & - & + & ++++ \\
29 & - & +++ & +++ \\
\hline
\end{tabular}

From this it appears that with this bacteriophage the reactions were exactly the same when tested immediately after its recovery from the body where it had undergone passages at the expense of strain IVi which was isolated from the patient, and when tested later after passages in vitro at the expense of this same typhoid bacillus. Whether the passages had taken place in vivo or in vitro the behavior of the bacteriophage was the same.

Janzen and Wolff then subjected this bacteriophage to passages at the expense of strain $\mathrm{Sm}$. After 10 passages the reactions were as shown in table 24 . This shows definitely that by a series of passages at the expense of strain $\mathrm{Sm}$ the race had acquired a virulence for strain 1 , and the virulence for strain 29 had been increased.

Several authors have obtained comparable results. Of those who 
have confirmed this observation Reichert, ${ }^{550}$ who has especially studied this question, may be mentioned. This author has found, indeed, that the opposite effect may also take place, namely, that through passages at the expense of one strain a virulence for another strain may be lost.

Typhoid bacilli which have acquired a resistance are inagglutinable or but slightly agglutinable by an antiserum.* They kill laboratory animals in smaller doses than do normal bacilli. $\dagger$

The virulence of a bacteriophage is rarely limited to B. typhosus, but usually extends to one or several related species. This is, indeed, a rather general phenomenon and it will be unnecessary to repeat this comment in connection with each bacterial species considered. A bacteriophage is rarely virulent for but a single bacterial species.

Occasionally it is possible to isolate from the intestines of normal men and of animals virulent races of the bacteriophage but as a rule, when derived from these sources the virulence is weak. On the con-

TABLE 24

\begin{tabular}{c|c|c|c}
\hline strain & I & II & III \\
\cline { 2 - 3 } Wi & ++++ & ++++ & ++++ \\
1 & ++++ & ++++ & ++++ \\
24 & - & +++ & ++++ \\
27 & - & + & ++++ \\
29 & ++ & ++++ & ++++ \\
\hline
\end{tabular}

trary the virulence is very high in races derived from the stools of typhoid convalescents and it was from such a source, that is, a convalescent from an acute infectious intestinal disease, that it was first isolated (d'Herelle ${ }^{313}$ ). Races may also be found in the urine of convalescents (d'Herelle ${ }^{310}$ ), and are constantly present in the blood at the termination of the septicemia (Hauduroy ${ }^{293}$ ). They have been isolated from the water of a number of rivers (Beckerich and Hauduroy ${ }^{58}$ ).

\section{B. paratyphosus A (Salmonella paratyphi)}

Everything which has been said with regard to B. typhosus applies to paratyphoid $A$, although the latter bacillus has received much less study.

* Complete inagglutinability is only observed, as we have seen in connection with dysentery bacilli, when the resistance is absolute. Agglutinability is restored gradually as the resistance is gradually lost.

$\dagger$ When the term resistant bacteria is used without qualification it should be understood that it invariably refers to "bacteria possessing an acquired resistance, in ultrapure culture." 
A bacteriophage virulent for this organism may be isolated under the same conditions as was the case for B. typhosus. I have found it, possessing a very high virulence, in the stools of patients convalescent from paratyphoid fever. In normal individuals it is found less frequently than are races virulent for $B$. typhosus and especially for $B$. paratyphosus $B$. The last is the most frequently encountered of the three (d'Herelle).

Bachmann and de la Barrera ${ }^{41}$ have reported that extremely interesting mutations of this bacillus occur through the action of the bacteriophage.

Aside from colonies showing a strongly modified morphology, they say, others are to be found which are small and transparent, and which are very different from the normal type.* The bacilli composing these colonies showed an outspoken increase in agglutinability by an anti-typhoid serum and this agglutinability increased during the course of successive transfers. With the anti-typhoid serum used by these authors the following agglutinations were obtained:

With the homologous B. typhosus.................... 1:6000

With the original $B$. paratyphosus $A \ldots \ldots \ldots \ldots \ldots \ldots \ldots \ldots$............ 1:1000

With $B$. paratyphosus $A$, mutant $\left(\mathrm{F}_{1}\right) \ldots \ldots \ldots \ldots \ldots \ldots \ldots \ldots$. $1: 2000$

With the same after the 4 th generation $\left(\mathrm{F}_{4}\right) \ldots \ldots \ldots \ldots \ldots \ldots \ldots \ldots \ldots$

With the same after the 6 th generation $\left(F_{6}\right) \ldots \ldots \ldots \ldots \ldots 1: 6000$

This by no means represents an increase in the general agglutinability of the original para A organisms, for while this original para A bacillus was agglutinated by its homologous serum in a dilution of $1: 3200$ the para A mutant of the 6 th generation $\left(\mathrm{F}_{6}\right)$ was agglutinated only in a dilution of $1: 1600$.

But here is a fact which is still more strange. The anti- $\mathrm{F}_{4}$ serum, that is to say, the serum of an animal prepared by injections of the mutant form in the 4th generation, agglutinated the homologous $\mathrm{F}_{4}$ organism at a titre of $1: 3200$, agglutinated $B$.typhosus, $1: 800$, and agglutinated the original para A bacillus, 1:800.

The anti-serum to the $F_{6}$ generation agglutinated its homologous organism, as well as $B$. typhosus and the $\mathrm{F}_{4}$ strain, to the limit, but it did not agglutinate to any degree the original $B$. paratyphosus $A$ from which the mutants $F_{4}$ and $F_{6}$ were derived.

To summarize this, Bachmann and de la Barrera have observed with

* Unfortunately these authors did not show whether they were working with mixed colonies or not, or if ultrapure, whether the colonies were resistant or susceptible. According to their description, however, it would appear that they were dealing with partially resistant, ultrapure colonies. 
regard to the mutant form, 1 . A reduction in the agglutinability by an anti-A serum. 2. An increase in the agglutinability by an anti-typhoid serum. 3. The production of antisera which agglutinate B.typhosus more strongly than they do the original paratyphoid A organism, this property becoming more manifest during the course of successive transfers.

These authors have confirmed these findings by agglutinin absorption experiments. Weil has shown that the bacillus of Gaertner, which is agglutinated to the titre by an anti-typhoid serum, may have its coagglutinating action increased and at the same time retain intact its specific agglutinating power. But the anti-typhoid serum used by Bachmann and de la Barrera, deprived of its partial agglutinins by saturation with the bacillus of Gaertner, also lost its activity for the original para A strain, although it still agglutinated B. typhosus at 1:3200, and the mutant form of para A at 1:1600.

By exhausting the anti-typhoid serum with the original paratyphoid A heated to $100^{\circ} \mathrm{C}$. they obtained a serum which no longer had any action upon the original para $\mathrm{A}$, but it agglutinated $B$. typhosus at $1: 3200$ and the mutant form at $1: 1600$.

As for the anti-serum produced to the $F_{6}$ mutant, absorption with heated bacilli removed all action for the original para $\mathrm{A}$ but did not impair its activity for B. typhosus and for the $\mathrm{F}_{4}$ and $\mathrm{F}_{6}$ mutants.

These facts can only mean that there has been a transformation of the antigenic properties brought about through the action of the bacteriophage.

"We have witnessed, then," say Bachmann and de la Barrera, "a progressive evolution, in which the properties of these organisms become increasingly unlike the original para $\mathrm{A}$ bacillus and approximate more closely those of B. typhosus."

One might go still further and question whether the bacterial species belonging to the colon-typhoid-dysentery group do not result from mutations brought about through the intervention of the bacteriophage. Baerthleins obtained in one case the transformation of a $B$. paratyphosus $B$ into a $B$.typhosus, and one immediately wonders if, in this successful case, he was not working with a mixed strain of $B$. paratyphosus $B$. Some experiments to be deseribed later seem to substantiate this interpretation. At the very beginning of my experiments I emphasized the fact that the question of the bacteriophage is certainly a dominating one in the study of bacteria and the communications published during the last three years seem to show, indeed, that this statement was 
warranted. As a matter of fact we are only at the beginning of this phase of the subject.

\section{B. paratyphosus B (Salmonella Schottmülleri)}

But few studies have been made of this organism with regard to its behavior toward the bacteriophage, but it would appear that it is somewhat less heterogeneous than is B. typhosus, for the races of the bacteriophage which I have isolated have been, in general, virulent for all of the strains with which I have tested them.

A bacteriophage virulent for $B$. paratyphosus $B$ is frequently found in the intestinal tracts of man and animals, a virulence for this bacillus being extremely common, being exceeded in frequency only by that for B. dysenteriae (d'Herelle ${ }^{315}$ ).

Upon a few occasions I have obtained resistant colonies assuming the viscous and flowing form of the bacillus of Friedländer.

\section{B. suipestifer (Salmonella suipestifer)}

I have isolated a bacteriophage virulent for this bacillus from the stools of a normal man. With a moderate virulence for a strain of $B$. suipestifer it was without virulence for organisms of the colontyphoid-dysentery group. I have also isolated bacteriophages virulent for $B$. suipestifer from the intestinal contents of normal horses, but these races have shown an accessory virulence for $B$. dysenteriae. ${ }^{315,321}$

\section{B. enteritidis (Salmonella enteritidis)}

Upon several occasions I have found bacteriophages virulent for either B.typhosus or the paratyphoids $A$ or $B$, to be virulent for certain strains of $B$. enteritidis also. Frequently $I$ have isolated such races from the excreta of animals..$^{315}$

\section{B. typhi murium (Salmonella typhi-murium)}

Races of the bacteriophage virulent for B. paratyphosus $B$ occasionally possess some degree of virulence for this bacillus. I have isolated very active strains from the intestinal tracts of white and gray rats which were resistant to the experimental clisease caused by the ingestion of cultures of B. typhi murium.

Certain resistant colonies are composed of bacilli which are very virulent and they may be, under certain circumstances, utilized for the destruction of gray rats, which, as a rule, resist the ingestion of the 
ordinary virus. On the other hand, it is conceivable that human infection might occur because of this enhanced virulence.*

I have demonstrated the presence, very transitory however, of a virulent bacteriophage for this organism in the blood of several white rats which resisted a laboratory infection. ${ }^{321}$

\section{B. coli (Escherichia coli)}

Under normal conditions races of the bacteriophage virulent for B. coli will be found in the intestinal tract of all animals (d'Herelle ${ }^{311}$ ). $B$. coli normally occurs as a mixed culture in the intestine, always possessing a resistance at the time of its isolation. It is necessary then, after purification by colony isolation, to carry it through a series of subcultures in order that it may lose this acquired resistance.

On several occasions we have seen that bacteria undergo mutations through the influence of the bacteriophage. Symbiosis with the bacteriophage being a normal state for $B$. coli in the intestine, the species of $B$. coli in its entirety is a mutant species. It is for this reason that $B$. coli is the most heterogeneous of all bacterial species and that each strain possesses its own peculiar characteristics as is shown, among other things, by the serum reactions.

After a series of subcultures and the loss of the acquired resistancet

* As I have already stated ${ }^{349}$ a really effeetive campaign against rats ean not be carried out by means of a bacteriuni. It is necessary to utilize an ultravirus and that for the following reasons, which have been discussed in detail in the work cited. Bacteria never cause an experimental contagious disease, even when the natural disease attributed to this organism is fatal in the highest degree. Experimental eholera of fowls for example, is not contagious. On the eontrary all of the experimental diseases eaused by ultra-viruses have the same degree of contagiousness as the natural disease. A reently disclosed faet offers some support to my idea. Avian plague has heen, up to the present time, unknown in America. A baeteriologist there received from the Pasteur Institute in Paris some of the plague virus and contaminated some elickens. The lisease did not remain loealized to the infeeted farm-yard, but extended rapilly, and after a few months it had actually beeome disseminated over several states. American bacteriologists had an excellent opportunity to study the propagation of an epizoötie. They should have profited by this, for such an opportunity is encountered but rarely.

But to return to the question of rats, an experiment would be easy to carry out since Novy has succeeded in isolating an ultra-virus pathogenie for this animal.

$\uparrow$ We know that at the time of isolation a mixed eulture, cither natural or artificial, gives three kinds of colonies; mixed colonies, ultra-pure susceptible colonies, and ultra-pure resistant colonies, the degree of resistance passing, ac- 
bacteriophagy with $B$. coli takes place in a manner identical to that with B. dysenteriae.

The resistant forms have been studied in particular by Bordet and Ciuca, and by Gratia, and their papers have been analyzed atlength in the chapter dealing with resistance of the bacteria. They will not be considered again here.

\section{The pneumobacillus of Friedländer (Encapsulatus pneumoniae)}

Caublot $^{141}$ having isolated a Friedländer bacillus from a blood culture and having shown that it presented all of the characters of the species attempted to find in the stools of the recovered patient (one month after his discharge from the hospital) a bacteriophage virulent for the organism isolated from the circulation. As a matter of fact he found a bacteriophage having at first a moderate virulence but after a few passages causing complete bacteriophagy of a bouillon suspension. When spread upon agar it gave characteristic plaques. Its virulence extended to other strains. The author states, without any further description, that the secondary colonies on agar are formed of atypical bacterial elements.

\section{The bacillus of Flacherie*}

Races of the bacteriophage virulent for this bacterium are frequently encountered in the intestinal tracts of healthy worms found in infected establishments (d'Herelle ${ }^{321}$ ). The activity of such a bacteriophage has been found to be the same for 3 strains of the bacillus isolated from the intestines of sick worms. Nevertheless I would classify this bacterium among the heterogeneous species for the tests have not been sufficiently numerous with regard to the number of strains involved to affirm that it is homogeneous. The same may also be said with regard to the bacillus of Friedländer.

cording to the colonies, from a refractory state to a state of low resistance. From the first isolation one may obtain, then, susceptible colonies but they are hardly different in appearance from ultra-pure resistant colonies and in order to avoid error it is always wise to make a series of subcultures in order that a possible resistance may be lost.

* Flacherie of silk worms 'does not seem to be a single disease. Several bacterial species appear to produce diseases manifesting the same characteristics. The flacherie which I observed occurred in the silk worm establishments of IndoChina. 


\section{B. proteus (Proteus vulgaris)}

I have found two races of bacteriophage which were very virulent for this organism at the time of isolation from the stools of two infants who had died of infantile cholera. ${ }^{315}$ We will later say more with regard to this point. When tested against a dozen strains of $B$. proteus only 3 were found to be susceptible and they were the same ones for each of the two races. Two strains of $B$. proteus $\mathrm{X}_{19}$ were refractory.

A bacteriophaged suspension, freed of bacilli by filtration, is extremely toxic for rabbits immediately after the dissolution of the bacilli. The subcutaneous injection of 0.5 cc. causes death within a few hours. After about 10 days the toxicity has diminished to almost nothing, and at this time the rabbit survives the intravenous injection of 2 ce. (d'Herelle ${ }^{321}$ ).

In the urine of a patient suffering from typhus fever Otto, Munter and Winkler ${ }^{49}$ found a bacteriophage virulent for B. typhosus, which, after two passages, acquired a virulence for $B$. proteus $X_{19}$. In another case, also of typhus fever, they found a race of the bacteriophage which, after a few passages with the Flexner dysentery strain, was virulent for $B$. dysenteriae Shiga, for the Hiss strain, and for B. proteus $X_{19}$. Unquestionably these races had a virulence for the proteus strain when they were isolated, and the virulence was enhanced for B. proteus $X_{19}$ simultaneously with that for B. typhosus in the first case and for the dysentery bacilli in the second.

Fejgin ${ }^{200,201,202}$ has studied bacteriophagy and the bacterial forms isolated from secondary cultures of $B$. proteus $X_{19}$. Unfortunately she did not determine whether she was dealing with contaminated resistant strains or with ultrapure strains, either resistant or susceptible.

Fejgin first isolated a bacteriophage from an old culture of proteus $\mathrm{X}_{19}$ obtained from Weil's laboratory. After about 15 passages it brought about the dissolution of a suspension within 6 to 8 hours. The filtrate contained from 3 to 6000 million bacteriophage corpuscles per cubic centimeter.

Plantings of secondary cultures on agar yielded five strains, with which the colonies were respectively irregular, round, opaque, white, and chromogenic.

Among the white strains she observed two types: short rods with very little motility and rod-shaped organisms that were very motile.

Of three chromogenic cultures two were a bright yellow, 1 a canary yellow. All three strains liquefied gelatin slowly along the line of puncture but they caused no reaction with sugars nor on milk, and did not produce indol. 
For the first two strains, both bright yellow, she found in smears, along with the fine bacilli, some long, tortuous filaments, but all were motile and were Gram-positive. With the canary yellow strain growth was luxuriant and spreading, extending rapidly over the agar. This culture was formed of minute bacilli only slightly motile. All of the organisms were alike: they were Gram-positive. With all three strains, the pigment was soluble in alcohol.

The three chromogenic strains were agglutinated by the sera of rabbits prepared by injections of cultures of proteus $\mathrm{X}_{19}$. With one of the two bright yellow strains agglutination occurred in a dilution of $1: 1600$, with the other strain of this type and with the canary yellow strain the titre was only 1:200. Fejgin prepared rabbits by injections of these three strains. Each of the three sera agglutinated the original proteus $\mathrm{X}_{19}$ strain to a titre of $1: 3200$. The antigenic power, therefore, remained intact although agglutinability was strongly diminished, at least for two of the three strains. In any case these interesting experiments indicate that there are actually mutant forms of $B$. proteus.

Fejgin had previously isolated different mutant forms from guineapigs infected by injeetions of proteus $\mathrm{X}_{19}$. With these strains positive serological reactions were obtained with the sera of rabbits prepared by injections of the mutant strains obtained in vitio through the action of the bacteriophage. The Castellani reaction demonstrated an almost complete identity for the mutants produced in vivo and those obtained in vitro.

In agreement with this author we must eonclude that through the aetion of the bacteriophage abrupt mutations take plaee and that for B. proteus $\mathrm{X}_{19}$ these mutations are stable and heritable.

\section{The bacillus of swine fever}

A bacteriophage virulent for this organism has been isolated by Bürgers and Bachmann ${ }^{131}$ from an old, naturally mixed culture. They have observed plaques on agar but the bacteriophage was lost in the course of a series of passages.

\section{B. diphtheriae (Corynebacterium diphtheriae)}

I have isolated two races of the bacteriophage, virulent solely for atoxic strains of this bacillus, from the excreta of two horses immunized by the injection of cultures of diphtheria bacilli. ${ }^{312}$

Blair $^{70}$ has isolated races of the baeteriophage virulent for Corynebacterium diphtheriae by different methods. He gave a guinea-pig an 
intraperitoneal injection of a culture of a diphtheria bacillus of low toxicity. After 4 days he isolated from the intestinal contents a bacteriophage which proved to be avirulent for the strain injected and for three other toxic strains, but virulent for one atoxic strain. From another guinea-pig, which died 40 hours after a subcutaneous injection of a very toxic bacillus, he isolated a bacteriophage which was but slightly active on 7 strains and inactive with 3 , among which was the strain injected.

He procured on 3 different occasions races from the stools of an infant convalescent of diphtheria. One of these filtrates contained a bacteriophage active for the strain isolated from the patient and for 7 other strains, of which 3 were toxic. A second filtrate was active upon 5 strains and inactive with three others. The third filtrate was devoid of all activity.

He also isolated virulent bacteriophages from the peritoneal exudates of guinea-pigs which had previously received an intraperitoneal injection of a diphtheria bacillus culture. Here the intestinal contents also contained the bacteriophage. And, finally, attempting to isolate races of the bacteriophage from old cultures of Corynebacterium diphtheriae Blair found one strain which yielded a filtrate possessing a weak activity.

The majority of the bacteriophages isolated caused bacteriophagy also with different organisms of the colon-typhoid-dysentery group.*

\section{Nodule bacteria of the Leguminosae (Rhizobium radicicolum)}

Gerretsen, Gryns, Sack, and Söhngen, ${ }^{228}$ in a remarkable contribution which has, it would appear, passed unnoticed, have described their experiments upon bacteriophagy with Bacterium radicicolum.

They worked upon nodules derived from the roots of clover, of lupin, and of sarrandella. These nodules were first of all washed in a solution of sublimate, 1:1000, and then they were passed successively through

* Blair refers in his paper to a note of Botez ${ }^{96}$ in which this author pretends to have induced the formation of bacteriophages by the action of methyl violet on cultures of various bacteria, Corynebacterium diphtheriae among others. I do not know what Botez regards as bacteriophagy but in any case $\mathrm{I}$ am able to affirm, on the basis of many experiments, that his conclusions are erroneous. As a matter of fact several investigators, of whom Polettini Bruno ${ }^{529}$ and Tomaselli ${ }^{599}$ were among the first, have refuted the work of Botez and the latter has not substantiated his claim. It is extremely unfortunate that such errors should pass from paper to paper. It would seem to me that the first thing to do when one encounters a contested point, and especially when the author does not support this point, is to verify the finding before holding it established. 
alcohol and through sterile distilled water. Fragments of these nodules, cut up with sterile instruments, were then distributed over solid media which had previously been seeded with a culture of $B$. radicicolum, derived either from clover, lupin, or sarrandella.

They found, after incubation, a zone of clarification attaining a diameter of from 1 to $2 \mathrm{~cm}$. around the fragments of nodule. This activity was specific, that is to say, the nodule fragments from lupin were the center of a plaque with the culture of $B$. radicicolum derived from lupin, while the culture was normal when the bacterium was derived from clover; mutatis mutandis.

They also ground up these nodules with sterile saline in a mortar and filtered the material through a candle. By this procedure they isolated very virulent bacteriophages, usually specific. The filtrates brought about a dissolution of the bacteria, but rather slowly, requiring about 10 days at ordinary temperature. Nevertheless, it was complete, while after this same interval the controls were very turbid. When drops of the filtrate were placed on agar no colonies developed within the area covered by the drop. When suspensions, to which a small quantity of the filtrate had been added, were spread over an agar surface plaques appeared after incubation. It may be added that the paper is accompanied by photographs which show beyond question that the reaction was a typical bacteriophage phenomenon.

Among the races isolated they found some whose action extended to strains of $B$. radicicolum other than those isolated from nodules of the same plant. A race of bacteriophage isolated from clover nodules possessed a weak but nevertheless definite virulence, for a $B$. radicicolum strain isolated from the bean. A bacteriophage isolated from lupin was weakly virulent for strains of $B$. radicicolum isolated from the clover and from the bean. On the contrary B. coli, Azotobacter chromaceum, Radiobacter, B. fluorescens liquefaciens and B. violaceus were absolutely insusceptible to any of the races of the bacteriophage isolated.

In three attempts out of seven they isolated bacteriophages from the stalks of the clover virulent for the $B$. radicicolum present in the nodules of the roots. All attempts to isolate them from the leaves were unsuccessful. Working with 100 gram samples of garden soil and of cultivated earth they obtained virulent races of this bacteriophage but with forest earth and with uncultivated soils their results were uniformly negative. 


\section{B. subtilis}

I have isolated a race of the bacteriophage virulent for $B$. subtilis from the stools of a patient sick with dysentery. The virulence was high. After 6 hours at a temperature of $37^{\circ} \mathrm{C}$. dissolution was complete when it was added to a slightly turbid suspension prepared from a bacillus grown from 12 to 14 hours on agar. Within 12 to 15 hours after the dissolution was complete a secondary culture began to cloud the medium. When spread upon agar characteristic plaques developed. ${ }^{321}$

\section{Vibrio cholerae (Vibrio comma)}

Of about 100 cases of cholera which I studied in Indo-China I have seen only one which recovered.

In none of the fatal cases have I been able to demonstrate a bacteriophage virulent for the cholera vibrio. In the single convalescent which I have seen a filtrate of a stool taken at the moment when the symptoms improved added in the amount of $0.25 \mathrm{cc}$. to $10 \mathrm{cc}$. of a suspension of cholera vibrios did not "apparently" cause bacteriophagy, but when spread on agar immediately after the addition of the filtratethere appeared, after incubation, about 50 plaques which had a diameter of about $2 \mathrm{~mm}$. and which were perfectly characteristic. The bacteriophage was therefore present in the stools. In spite of numerous attempts I have not been able to cultivate it serially. ${ }^{321}$ It is interesting to discover the reason for this failure.

Jötten $^{379}$ has isolated a bacteriophage from an old culture of the cholera vibrio.

Meissner, ${ }^{448}$ working in the laboratory of Prausnitz, has isolated a bacteriophage virulent for Tibrio cholerae and for the Tor vibrio from the peritoneal exudate of a guinea-pig which had previously received an intraperitoneal injection of a mixture of Vibrio cholerae, the Tor vibrio, and an anti-cholera serum.

In another attempt Meissner obtained a bacteriophage by the same method injecting the Tor vibrio alone. At the beginning the virulence was weak but after a few passages a complete dissolution of a suspension was obtained with a dilution of $10^{-4}$. Dissolution was complete after 12 to 15 hours, but at the seventeenth hour a secondary culture appeared which rapidly clouded the medium. At the twenty-fourth hour the turbidity was practically the same as that of a control culture.

This author has also obtained a virulent bacteriophage by combining in vitro a mixture of the peritoneal exudate of a guinea-pig (obtained 
by the previous injection of sterile bouillon) and an anti-cholera serum together with a culture of the cholera vibrio.

Meissner states that the bacteriophage must be present in the intestinal tract of the guinea-pig and pass through into the peritoneal exudate because of the irritation caused by the injection. $\mathrm{Flu}^{220}$ has suggested that we must not overlook the possibility that the strain of cholera vibrio used in the experiment may have been naturally contaminated by a bacteriophage, that is, that it was a naturally mixed culture. I am in accord with this view of Flu, particularly because of my experience during 1918 and 1919, when, being very anxious to obtain a Cholera-bacteriophage I subjected a great many guinea-pigs to this test and always failed. The technique which I employed resembled that of Meissner but the strains of vibrio which I used were ultrapure.

Flu ${ }^{220}$ has isolated a bacteriophage from a naturally mixed strain. Here is the summary given by him in his paper.

The author has isolated an anti-cholera bacteriophage from a pure culture of Vibrio cholerae. The bacterial strain had been isolated in 1915, from the stools of a patient in Java.

In order to isolate the bacteriophage the strain was cultivated upon an agar slant and the growth was suspended in a small amount of bouillon. The suspension was combined with an equal quantity of anhydrous sodium sulphate, thus forming a dry mass. This mass was ground for a half-hour and suspended in 100 cc. of bouillon ( $\mathrm{pH} 7.5)$. The suspension was then heated for an hour at $58^{\circ} \mathrm{C}$. and distributed in $10 \mathrm{ec}$. portions among 10 flasks, each of which contained 100 cc. of bouillon and one loopful of an 18-hour culture of a strain of cholera vibrio differing from the ground up strain. After incubation for 1 or 2 weeks the material was filtered through a candle and the filtrate was tested for a bacteriophage against 10 strains of the cholera vibrio.

Of the 10 strains examined one was found to be infected by the anti-cholera bacteriophage.

The anti-cholera bacteriophage is very sensitive and can not be cultivated with lysogenic strains.

In order to cultivate and preserve this bacteriophage it is necessary to inoculate bouillon with strains which are readily lysible and to use but a very small amount of an 18-hour culture. This should be placed in the incubator and should be watched continuously from hour to hour. When the tube containing the bacteriophage is clear it should be filtered through a candle and a new inoculation of bouillon with the filtrate should be made.

The bacteriophage became very active, still giving lysis in dilutions up to $10^{-7}$ but it remained entirely inactive for the strain from which it had been isolated.

For the cultivation of an anti-cholera bacteriophage from the feces of a cholera patient, it is suggested that the feces be dihted with saline $(0.9$ per cent), that the matcrial be heated for an hour at $5 \mathrm{~s}^{\circ} \mathrm{C}$., and that the cooled liquid be 
implanted with laboratory strains of the cholera vibrio. After an incubation period of from 2 days to 1 week the liquid should be examined for its action upon the laboratory strains of the vibrio.

It might be added that after some 10 passages according to the protocols given by Flu, he obtained a complete dissolution of a suspension containing 200 million cholera vibrios per cubic centimeter within a period of from 3 to 5 hours at a temperature of $37^{\circ} \mathrm{C}$. Within 1 to 2 hours after the liquid became completely cleared, the medium again began to become clouded because of the formation of a secondary culture and a few hours later the medium was as turbid as was the culture control.

In a word, then, with the race isolated by Flu dissolution was very rapid; as rapid as is the bacteriophagy of $B$. dysenteriae under the action of a bacteriophage of maximum virulence. But secondary cultures began to develop almost immediately. If one waits about 12 hours (as I had done in Indo-China where I observed the suspensions inoculated in the afternoon on the following morning) in order to determine if bacteriophagy has occurred, the secondary culture already developed will lead one to think that he is dealing with a normal culture.

It does not appear, however, that with all races of the bacteriophage virulent for the cholera vibrio secondary cultures develop quickly. Eliava has informed me that in 1918, before he had seen any published work upon the subject of bacteriophagy he had observed during an epidemic period, that certain specimens of water from the river Koura which passes Tiflis brought about a complete and permanent dissolution of cultures of the cholera vibrio. In these cases, then, secondary cultures did not occur. Whether they do, or do not develop is simply a question of the virulence of the race of bacteriophage. Moreover I have noted that as a usual thing the virulence of bacteriophage races isolated from old naturally mixed cultures is never strongly enhanced by passages with a susceptible bacterium. Usually the virulence becomes fixed at a moderate value, rarely very high, beyond which it is impossible to increase it. There are, indeed, few races of the bacteriophage which are capable of acquiring a maximal virulence. This is, as a matter of fact, a general law applicable to the virulence of pathogenic bacteria as well as to the bacteriophage; not all strains or races are capable of becoming enhanced to the same degree.

When a suspension inoculated with a few corpuscles is spread upon agar characteristic plaques appear after incubation.

The virulence of the bacteriophage isolated by Flu extended over a great many strains of vibrio, but he found, nevertheless, ultrapure, naturally resistant strains. 
In 1924, during the return of the pilgrims from Mecca, while they were quarantined at the lazaretto at Tor, I isolated from the stools of one of these pilgrims (although he showed none of the symptoms of cholera) a vibrio which agglutinated with an anti-cholera serum to its titre. It is significant that there was no epidemic* at this time. While held in the lazaretto the stools of this pilgrim were examined every 48 hours. After a time the stool revealed a non-agglutinating vibrio, and after a further 48 hours all of the vibrios had disappeared. $\dagger$

The vibrio susceptible to agglutination was bacteriophaged perfectly by the bacteriophage isolated by Flu; while the vibrio which was inagglutinable, isolated 48 hours later, was refractory. At this time the intestinal contents contained a bacteriophage having a high vurulence for the agglutinable vibrio. This virulence was very specific, for when it was tested against six strains definitely known to be Vibrio cholerae, it was without action.

\section{The staphylococcus}

There are many authors who attribute to Twort the discovery of a bacteriophage virulent for the staphylococcus. Unquestionably Twort should receive the credit of having discovered the first bacterial transformation to take place serially but it is quite impossible that this process is bacteriophagy, for according to his own description the phenomenon which he observed was not a dissolution of the bacterial bodies but simply a fragmentation. The culture layer on agar became transformed into a vitreous layer formed of granules, staining red by Giemsa. I do not understand how it is possible that such a phenomenon, termed by Twort the "breaking down of bacteria," that is, a "bacterioclysis," can be likened to bacteriophagy which is a phenomenon characterized by a "dissolution without residue." No one has advanced the least explanation to reconcile these facts. Everyone has been satisfied with affirming that there is a likeness without offering any proof and without any discussion.

In a word, for Twort to claim priority insofar as the observation that a serial phenomenon occurs is absolutely just, but that he should affirm that what he observed was bacteriophagy is absolutely contradicted

* For more than 10 years no case of cholera has been found at the quarantine station at Tor nor has any case been observed in the Hedjaz.

$\dagger$ Crendiropoulo has noted that the same thing takes place in the course of epidemics where he observed the phenomenon on numerous occasions. A carrier would show an agglutinable vibrio; then a vibrio that agglutinated less vigorously, then one which was inagglutinable, and finally the vibrios all disappeared. 
by his own description. I would refer the reader to the introduction of the present volume where I have incorporated, in extenso, the description of the phenomenon given by Twort.

The only objection which might be made is this: that the phenomenon of bacteriophagy, that is to say, the attack of the bacterium by the bacteriophage, may manifest itself with Gram-negative bacteria by a dissolution and with the Gram-positive bacteria by a simple fragmentation. Gratia (without questioning this, however) has shown that this objection can not be considered for he has shown that a true bacteriophagy of the staphylococcus occurs in absolutely the same way as bacteriophagy with other bacteria, with the Shiga dysentery bacillus for example.

It is, then, Gratia ${ }^{248}$ who unquestionably should be credited with first demonstrating a bacteriophage virulent for the staphylococcus and who, at the same time, clearly proved that bacteriophagy manifests itself in an absolutely identical fashion for both Gram-positive cocci and for Gram-negative bacilli.

Gratia $^{258}$ has the further merit of discovering a race of the bacteriophage attacking all strains of the staphylococcus;-albus, aureus, and even citreus, and as I have shown, M. tetragenous also. With such a bacteriophage, a bacterial species so heterologous as are the staphylococci, behaves exactly like a homologous species.

I will not describe here bacteriophagy with the staphylococcus; the characteristics of the phenomenon are identical with those observed with the bacilli. Furthermore, in the majority of instances I have used the dysentery bacillus and the staphylococcus as examples in the experiments illustrating the facts presented in Part I of this text.

The staphylococcus may acquire a resistance just as do the other bacteria. But with staphylococci this phenomenon presents a single interesting peculiarity. In artificially mixed cultures (that is to say, in those composed of staphylococci with an acquired resistance and bacteriophage corpuscles) along with typical staphylococcus forms isolated cocci, diplococci, and chains of a few elements may be seen. By the serial cultivation of a Staphylococcus aureus possessing an acquired resistance in pure suspensions of the bacteriophage (the polyvirulent strain $\mathrm{H}$ of Gratia) I have obtained cultures formed of fine sedimented agglutinates, the medium remaining clear. Under microscopic examination one sees only isolated cocci, diplococci, and streptococcus-like chains, the longest being composed of 11 elements, but there is no arrangement of cells resembling a typical staphylococcus grouping. Transfers of these cultures to agar remain sterile. 
This is not, however, a fixed mutation, for transplants into bouillon result in the growth of the ordinary forms characteristic of mixed cultures,-isolated cocci, diplococci, chains of 4 to 5 elements, and small masses. These mixed cultures strongly suggest a mixture of staphylococci and streptococci.

This observation suggests the following possibility: Is not the "streptococcus" the result of an irreversible mutation of the staphylococcus, a mutation brought about through the action of a bacteriophage?

\section{The enterococcus}

A bacteriophage virulent for this organism has been isolated by Beckerich and Hauduroy ${ }^{59}$ from the excreta of a horse. The race isolated was also virulent for some strains of B. coli and for B. dysenteriae Hiss.

\section{The streptococcus (Streptococcus pyogenes)}

Piorkowski ${ }^{526}$ has isolated a bacteriophage virulent for this organism. I have also found one in the excreta of a horse. When isolated it possessed a virulence, although weak, for a strain of the streptococcus of strangles. It was impossible for me to enhance the virulence of this race and it was finally lost in the course of passages. McKinley, ${ }^{460}$ as well as Eichhorn, have also isolated races active for the streptococcus.

Such are the bacteria which, up to the present time, have been recognized as susceptible to bacteriophagy. Is the list completed? It is hardly probable.

\section{Phenomena simulating bacteriophagy (pseudo-bacteriophagy)}

In finishing this chapter I may mention two phenomena reported as being due to bacteriophagy, but which, as a matter of fact are entirely different.

Lemos Monteiro, ${ }^{467}$ then Kraus and Gomez, ${ }^{395}$ and later Pico ${ }^{517}$ have described as bacteriophagy the partial clearing observed in bouillon cultures of $B$. anthracis. Upon several occasions I have observed this phenomenon (while attempting to obtain a virulent bacteriophage for the anthrax bacillus) and have obtained it by adding to a young bouilon culture of $B$. anthracis a large amount of filtrate from a culture of this same organism. The clearing is strictly proportional to the quantity of filtrate added. If, to $10 \mathrm{cc}$. of a culture, less than a cubic centimeter of filtrate is added no appreciable clearing can be detected. Further- 
more (and this was recognized by Lemos Monteiro and by Kraus) such a culture containing added filtrate spread upon agar yields nothing having the appearance of plaques.

This phenomenon does not present any of the characters of bacteriophagy, but is a reaction which has been studied for a long time by many authors and was first described by Gamaleia. As a matter of fact, Pesch ${ }^{501}$ has dealt with the erroneous interpretation of this phenomenon in a recent paper entitled "The Anthrax Pseudo-bacteriophage."

A number of authors, of whom Quiroga was the first ${ }^{548}$ have described a phenomenon taking place in agar cultures of B. pyocyaneus, and again, this reaction has been likened to bacteriophagy. Some of those who have observed this change appear, however, to have some doubt on this point, for example, Combiesco and Magheru ${ }^{149}$ speak of it as a phenomenon "resembling bacteriophagy."

Hadley, ${ }^{281}$ in particular, has studied this transformation in pyocyaneus cultures, and it has been found to take place with a very large number of B. pyocyaneus strains. When cultured on agar spots appear having an irridescent lustre. These for a time gradually extend and finally end by being re-covered by the surrounding culture. Within the area of the spots the agar is not bare but is covered by a layer which is vitreous, transparent, and greenish in color. Examined under the microscope this vitreous material is found to be composed of fine granules which stain red by the Giemsa method. The vitreous material, filtered through a candle, appears to induce the formation of irridescent spots. I say "appears to," for strains spoken of as "susceptible" produce these same areas spontaneously without the addition of filtrate.

This is by no means a phenomenon of dissolution but is rather a phenomenon of fragmentation.* It appears, comparing this description with that of the Twort phenomenon, that it is due to the phenomena of bacterioclysis discovered by Twort.

But, one might ask, can not the two phenomena be caused by the same principle? We have seen that this objection can not be raised with regard to what takes place with the staphylococcus since Gratia has shown that this organism shows, when acted upon by the bacteriophage, not the phenomenon of bacterioclysis of Twort, but the phenomenon of bacteriophagy and this proceeds in a manner absolutely identical with that which occurs when the bacteriophage reacts upon

* Those writing upon this subject, Hadley among others, naturally speak of "lysis," a term which no longer has any precise meaning in biology. Certainly this term is very convenient, partly because of its lack of precision. 
B. dysenteriae. There are, then, two "diseases" affecting staphylococci, the one probably "organic" and caused by a change of an enzymatic nature,- the phenomenon of bacterioclysis of Twort. The other is certainly of an infectious nature, as is proved by the data to appear in a later chapter,- this is the phenomenon of bacteriophagy.

Do both of these phenomena take place with $B$. pyocyaneus? It is very probable. But however that may be, insofar as the phenomenon of bacterioclysis of $B$. pyocyaneus is concerned, we have absolute proof that it is not due to a bacteriophage. We will see, as a matter of fact, in a later chapter, that all bacteriophages, whatever may be the race to which they belong and whatever may be the bacterium at the expense of which they reproduce, pass through collodion ultra-filters if the pores are sufficiently open to permit the passage of the serum albumin micella.* But the agent which causes the phenomenon of irridescent spots on B. pyocyaneus cultures does not pass through collodion filters, as shown first by Otto and Munter. ${ }^{494}$ These authors have not, however, drawn any deductions from this fact. I have investigated the question and I have shown that the diameter of the particles which are responsible for this phenomenon is far greater than that of the bacteriophage corpuscles. Taking as test material a mixture of races of the bacteriophage active for B. dysenteriae, B. coli, B. typhosus, B. pestis, and the staphylococcus, all of the races pass through or all do not pass through, depending on the size of the pores. I have then added to this mixture of bacteriophage races the "principle" responsible for the phenomenon of bacterioclysis with $B$. pyocyaneus and I have never found this to pass through. Seven strains of $B$. pyocyaneus, all giving these irridescent spots, have given uniformly negative results.

Therefore, the phenomenon observed with $B$. pyocyaneus can not be bacteriophagy. Its manifestations are entirely different and it is caused by a principle which lacks the characteristics of the bacteriophage. The principle is certainly associated in some way with the function of pigment production, for all strains which produce pyocyanin show these iridescent spots upon agar, their number, and their extent as well, being related to the quantity of pigment produced.

* Some authors do not appear to realize that the size of the pores depends upon the mode of preparation. They speak of membranes without specifying the size of the pores. To say, as certain authors have said, that "the bacteriophage does not pass through collodion membranes" is exactly the same thing as saying "mice can not pass through holes." In one case, just as in the other, it is necessary to specify the size of the hole which restricts the passage. 
Recently Lisch ${ }^{425}$ has made a careful study of this question and has concluded that the phenomena observed with $B$. pyocyaneus, and described by so many authors under the name of a bacteriophagy, has, in reality, nothing whatever to do with the true bacteriophagic process.

\section{RÉSUMÉ}

The studies made up to the present time show that the following bacterial species are susceptible to bacteriophagy:

B. dysenteriae Shiga

B. dysenteriae Hiss

B. dysenteriae Flexner

Various intestinal bacilli

B. gallinarum and related organisms

Pasteurella bovis

B. pestis

B. typhosus

B. paratyphosus $A$

B. paratyphosus $B$

B. suipestifer

B. enteritidis

B. typhi-murium

B. coli

Bacillus of Friedländer

Bacillus of Flacherie

B. proteus

Bacillus of swine fever

B. diphtheriae

Nodule bacteria of leguminosae

B. subtilis

Vibrio cholerae

Staphylococcus

Enterococcus

Streptococcus
(d'Herelle $^{310}$ )

(d'Herelle $^{310}$ )

(d'Herelle $^{310}$ )

(d'Herelle ${ }^{321}$ )

(d'Herelle 314,321 )

(d'Herelle $^{322}$ )

(d'Herelle ${ }^{322}$ )

(d'Herelle $^{313}$ )

(d'Herelle ${ }^{310}$ )

(d'Herelle $^{313}$ )

(d'Herelle ${ }^{315}$ )

(d'Herelle ${ }^{315}$ )

(d'Herelle ${ }^{321}$ )

(d'Herelle ${ }^{311}$ )

(Caublot ${ }^{141}$ )

(d'Herelle ${ }^{321}$ )

(d'Herelle ${ }^{315}$ )

(Bürgers and Bachmann ${ }^{131}$ )

(d'Herelle ${ }^{321}$ )

(Gerretsen, Gryns, Sack, and Söhngen ${ }^{228}$ )

(d'Herelle ${ }^{\text {i21 }}$ )

(d'Herelle ${ }^{321}$ )

$\left(\right.$ Gratia $\left.^{248}\right)$

(Beckerich and Hauduroy ${ }^{59}$ )

(Piorkowski ${ }^{526}$ ) 

PART II

THE BACTERIOPHAGE 



\section{CHAPTER I}

The Behavior of the Bacteriophage Toward Different Agents

\section{THE PHYSICAL STATE OF THE BACTERIOPHAGE}

I will not revert here to the question of the corpuscular nature of the bacteriophage, since this subject has been discussed at length in Part I. Moreover, the demonstration that I have given has not been disputed. Everyone has accepted this fact, whatever their concept as to the intimate nature of the corpuscle itself.*

A suspension containing more than 10,000 million corpuscles per cubic centimeter is perfectly limpid, not only after it has been passed through a filter candle but even immediately after bacteriophagy, provided dissolution is brought about through the action of a bacteriophage of maximum virulence.

When a ray of light is passed through a perfectly clear suspension of the bacteriophage the Tyndall phenomenon may be observed.

At first I believed that the fine granules, appearing as brilliant points when a bacteriophage suspension is observed under the dark-field, must be the bacteriophage corpuscles. Exceptions were taken to this view, and apparently quite correctly, for further study indicates that these granules are certainly derived from the dissolved bacteria. If a suspension is allowed to sediment for a few weeks, the supernatant fluid becomes free of granules capable of detection by dark-field examination, despite the fact that this fluid contains an abundance of bacteriophage corpuscles.

Additional evidence is afforded by the repeated examination of a bacterial suspension which has undergone bacteriophagy, for it is found that although these brilliant points are very abundant immediately after the dissolution is finished, they diminish rapidly. After three days only one or two can be found in each field. Thus, they are most

* Doerr alone, as I have already stated, has hesitated to commit himself. He has, however, shown that the bacteriophage principle does not pass through tight collodion membranes. If a body does not pass through such a membrane it is obviously because it is not in solution, but is in suspension, for it must be in either one or the other of these states. And if it is found as a suspension it is formed of corpuscles. 
certainly granules derived from the bacteria, granules which are as yet undissolved.

As for the granules which are to be seen within the bacteria during the process of bacteriophagy, many observations but add to my belief that they are bacteriophage corpuscles, or masses of corpuscles, visible because of the difference in refraction between the substance of the corpuscles and that of the bacterium.

\section{Filtrability}

Because of the following experiment, I believe ${ }^{312}$ the bacteriophage corpuscle to be about the same size as the molecule, or perhaps it would be better to say, the micella, of serum globulin.

To $100 \mathrm{cc}$. of physiological saline $10 \mathrm{cc}$. of a shiga-bacteriophage filtrate and $2 \mathrm{cc}$. of an anti-tetanus serum were added. A few cubic centimeters of this mixture were placed in each of a number of sacs, prepared of collodion in such a way that the sacs of the series possessed an increasing scale of permeability. Each sac was dialyzed against physiological saline, the level of the fluid within the sac being 3 to $4 \mathrm{~cm}$. higher than that of the external fluid in order to provide a slight pressure. Tests made under these conditions showed that with all of the sacs through which the antitoxin passed, the bacteriophage passed also, and that the bacteriophage was retained wherever the antitoxin failed to dialyze.

Subsequent experiments of this same type have been performed with races of the bacteriophage virulent for $B$. coli, B. typhosus, Pasteurella pestis, Pasteurella bovis, and for the staphylococcus and in all instances the results have been similar to those detailed above. It is interesting to note, however, that on many occasions I found that the bacteriophage passed through the walls of the sac which, in the series of increasing permeability came immediately before the first one which allowed passage of the globulins. This would suggest that the bacteriophage corpuscle may be slightly smaller than the micella of serum globulin.*

* Let us note in passing, that to state, as eertain authors have done, that the bacteriophage "passes," or "does not pass" through eollodion membranes, without specifying the degree of porosity of the membrane with which they were working, is without meaning. It is the same as saying that "a mouse can not pass through a hole," which is true if we are considering the eye of a needle, but is manifestly false if we have in mind a railroad tunnel.

These author's seem to be ignorant of the fact that collodion membranes may be prepared in which the pores are greater than $100 \mu \mu$ in diameter, while other membranes may have pores of but $1 \mu \mu$. It is unfortunate that the published results warrant such comments. 
Wollman ${ }^{636}$ has also found that the bacteriophage will pass through open membranes and that it is retained by tight membranes.

Prausnitz ${ }^{536}$ has gone a step further in solving this question of size. Working with a Flexner-bacteriophage he has carried out filtrations using as a measure of comparison colloids in which the size of the granules has been determined. Making use of a series of Hahn membranes, he compared the passage of bacteriophage corpuscles with that of the granules of colloidal silver ( 0.001 per cent solution), of gelatin ( 1 per cent) and of hemoglobin ( 1 per cent).

His results are summarized in table 25 .

TABLE 25

\begin{tabular}{|c|c|c|c|c|}
\hline & $\begin{array}{l}\text { BACTERIO- } \\
\text { PHAGE* }\end{array}$ & COLLARGOL $\dagger$ & GELATIN & $\begin{array}{l}\text { HEMO- } \\
\text { GLOBIN }\end{array}$ \\
\hline Before filtration ....... & 230,000 & & & \\
\hline Passed by filter No. $20 \ddagger$. & 129,000 & $\begin{array}{c}\text { Slight re- } \\
\text { duction }\end{array}$ & $\begin{array}{l}\text { Very slight } \\
\text { reduction }\end{array}$ & No loss \\
\hline Passed by filter No. 50 . & 27,000 & $1 / 10$ & $\begin{array}{c}\text { Very slight } \\
\text { reduction }\end{array}$ & No loss \\
\hline Passed by filter No. 100 . & 2,500 & $1 / 1000$ & $\begin{array}{l}\text { Slight reduc- } \\
\text { tion }\end{array}$ & No loss \\
\hline Passed by filter No. 200 . . & 0 & Traces & $\begin{array}{l}\text { Marked re- } \\
\text { duction }\end{array}$ & No loss \\
\hline Passed by filter No. 400 . & 0 & 0 & $\begin{array}{c}\text { Very strong } \\
\text { reduction }\end{array}$ & No loss \\
\hline
\end{tabular}

* By counting plaques.

$\dagger$ Quantity present in the filtrate, determined colorimetrically.

$\ddagger$ The numbers assigned to the Hahn filters represent the number of minutes required for the filtration of $100 \mathrm{cc}$. of water.

Prausnitz concluded that the diameter of the bacteriophase corpuscles is approximately that of the micella of the collargol used in his experiment, about $20 \mu \mu$.

By an entirely different procedure (refraction) von Angerer ${ }^{11}$ derived results indicating that the diameter of the corpuscle must be about $30 \mu \mu$.

The figures obtained by Prausnitz and by von Angerer are, therefore, remarkably near the same.*

Levaditi and Nicolau have found, also by means of collodion mem-

* For purposes of comparison, it may be said that the Asterococcus of pleuropneumonia of cattle, the smallest organism visible under the microscope. has a diameter of between 100 and $200 \mu \mu$. 
branes of different permeabilities, that the ultraviruses of rabies, of vaccinia, of encephalitis lethargica, and the bacteriophage all have, within the limits of the method of measurement, the same diameter. When one of the four passes through a membrane they all pass, when one is retained, all are held back.

Experiment seems to show that the bacteriophage corpuscle undergoes a shrinking with ageing. As a matter of fact, when comparative filtrations through membranes of approximately the same porosity are made it is found that corpuscles which are 20 days or more old pass through membranes which hold back young corpuscles. An experiment bearing on this point may be cited. It has been repeated on two different occasions, and the results of both tests were alike.

A suspension of Shiga-bacteriophage filtered immediately after the completion of bacteriophagy, was mixed with an equal quantity of a suspension of the Staphylo-bacteriophage which had not been filtered through a candle until one month after bacteriophagy had taken place. At the same time a second mixture was prepared of equal parts of a suspension of Staphylo-bacterioplage filtered through a candle immediately after bacteriophagy, and a suspension of Shiga-bacteriophage, filtered 20 days after the dissolution. Two series of collodion sacs were prepared, the sacs of each series progressing from very tight sacs to those more porous. Into the sacs of one series the first mixture was introduced, the second mixture being placed in the second series. The materials of all the sacs were dialyzed against bouillon, a pressure of 3 to $4 \mathrm{~cm}$. of bouillon being established in each sac.

After 48 hours the external bouillon was examined to ascertain which, if any, of the bacteriophages had passed through. These tests showed the following:

Series 1. With the four sacs that were least porous there was no passage. Through sac No. 5 the Staphylo-bacteriophage corpuscles had passed, unmixed with the Shiga-bacteriophage. From sac No. 6 a great many Staphylo-bacteriophage corpuscles had passed and also a very few Shiga-bacteriophage corpuscles, the ratio being 100:7. With the two most porous sacs the corpuscles of the two races of bacteriophage had passed in essentially the same proportions.

Series 2. Nothing had passed through the first 5 sacs, that is through those sacs which were least porous. Through sac No. 6 a great many Shiga-bacteriophage corpuscles had passed as well as a very few Staphylo-bacteriophage corpuscles, the ratio being 100:2. Through sacs Nos. 7 and 8, both races had passed. 
These results indicate, then, that for some undetermined reason, the size of the corpuscles diminishes with age.

This variation in filtrability can not be a question of the union of the young bacteriophage corpuscles into agglutinates for in this case comparative countings of the plaques on agar, made from a recent filtrate and from an old filtrate, would reveal this change, since each agglutinate would yield only one plaque. But this is not the case, for comparable spreadings on agar show rather a diminution in the number of corpuscles in the old filtrates, even if they are aged but a few days.

\section{Diffusibility}

Jötten ${ }^{379}$ has implied that the bacteriophage diffuses into a 3 per cent agar. With reference to such experiments it should be stated that tests of diffusibility offer many opportunities for error.

Prausnitz and Firle ${ }^{539}$ carried out a series of studies for the purpose of determining whether or not such a diffusion of the bacteriophage corpuscles takes place. All of their tests gave results indicating that this question must be answered in the negative. They were unable to obtain a filtration through a layer of 3 per cent agar deposited on a Berkefeld candle, even when the pressure amounted to $26 \mathrm{~cm}$. of mercury and the test was prolonged for 3 or 4 hours.

$\mathrm{Flu}^{221}$ reached the same conclusion, after a series of experiments, all giving negative results. In his tests the bacteriophage did not diffuse through a centimeter of 3 per cent agar.

As a matter of fact, the uniformly accepted conclusion, that the bacteriophage will not pass through tight collodion membranes renders it highly improbable that it would diffuse through a 3 per cent agar. For we know that the interstices found in a gel are much smaller than those of membranes. Thus, if there were a diffusion it would necessarily have followed that the passage of the material took place by virtue of some power of motion resident within the corpuscle. Manifestly, such a condition is possible but it would be strange in view of the dimensions of the corpuscles.

\section{Volatility}

It is also because of an imperfect technic, an error, that Olsen and Yasaki $^{485}$ have been led to believe that the bacterjophage is volatile. Apparently they had forgotten the experiments of Flügge who showed a long time ago that bacteria as large as $B$. tuberculosis, may be carried over by the droplets of the liquirl which leave a fluid boiling under reduced pressure. 
I have shown that ${ }^{351}$ it is only necessary to carry out the distillation in such a way that these bubbles (droplets of Flügge) can not form to secure a distillate free of all bacteriophage, even if in the process the filtrate is evaporated to dryness. Such a result is, indeed, inevitable, for, in view

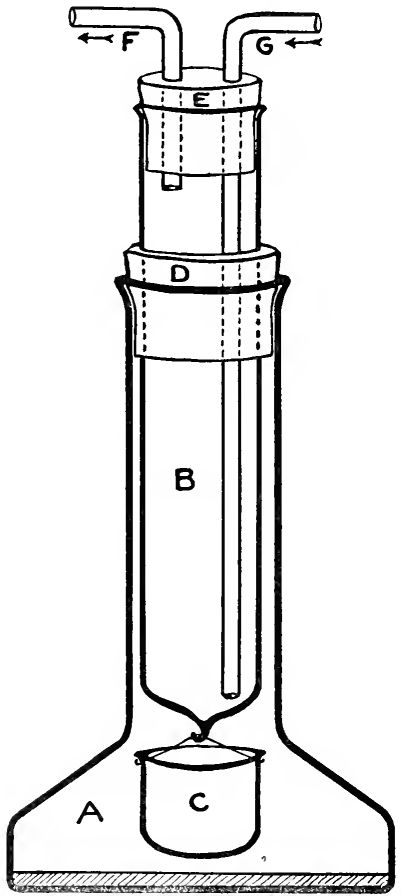

Fig. 6. Apparatus for Distilling at Low Temperatures

$A$, receptacle within which the fluid to be distilled is placed; $B$, cooling tube; $C$, receptacle for collecting the distillate; $D$, rubber stopper closing $A$ and supporting $B ; E$, rubber stopper closing cooling tube $B ; F$, out-flow tube for the cooling fluid; $G$, in-flow tube for the cooling fluid.

of the fact that the bacteriophage is corpuscular in nature, volatilization is physically impossible.

Because of the disputed nature of this fact, it may be well to insert an 
illustrative experiment. Incidentally, it may be well to describe the apparatus which $I$ have devised for such a test for it may serve for experiments of a wholly different nature. I believe it to be the only apparatus which permits, under normal barometric pressure, or even under a higher pressure, a fluid to be distilled at a low temperature with the recovery of all of the volatile products and without the possibility of any loss. With aqueous materials, such as a fluid culture medium, distillation can be effected at $30^{\circ} \mathrm{C}$.

The apparatus (fig. 6) is composed of a conical glass receptacle provided with a neck having a diameter of 5 to $6 \mathrm{~cm}$. and a length of $25 \mathrm{~cm}$. The opening is closed with a rubber stopper having a hole about $4 \mathrm{~cm}$. in diameter. Through this stopper a glass tube (a test-tube) with a diameter of $4 \mathrm{~cm}$. and a length of $40 \mathrm{~cm}$. is passed. On the bottom of this tube is fused a small glass hook, to which is attached by means of a fine platinum wire forming a loop, a small glass bucket, $40 \mathrm{~mm}$. in width and $60 \mathrm{~mm}$. deep, made from a test-tube.*

The figure presented herewith shows the apparatus as assembled and ready to operate. The liquid to be distilled is placed in the bottom of the long-necked flask which is tightly closed with the rubber stopper, and the flask is placed in a water bath at the desired temperature. The flask should be immersed in the water up to within a few centimeters of the top in order to avoid a condensation of the vapor upon the walls. The interior of the tube which passes through the rubber stopper is cooled, either by allowing a stream of cold water to flow through it, or by filling the tube with cracked ice which may be renewed from time to time, or by filling it with a refrigerating mixture of cracked ice and salt. The liquid in the bottom of the flask evaporates, the vapor condenses on the outer surface of the refrigerating tube, and the drops find their way to the bucket suspended below. When the cooling is effected by a stream of water cooled to $15^{\circ} \mathrm{C}$. distillation can be satisfactorily effected at a temperature of 43 to $45^{\circ} \mathrm{C}$., with cracked ice, at about $35^{\circ} \mathrm{C}$., and with a mixture of ice and salt, at about $30^{\circ} \mathrm{C}$.

Twelve tests on the behavior of the bacteriophage during distillation have been carried out with this apparatus, each time evaporating 30 cc. of bacteriophage filtrate to dryness, at temperatures varying between 35 and $48^{\circ} \mathrm{C}$. Uniformly these tests have failed to show bacteriophage

* Obviously these dimensions may be varied in accordance with the use to be made of the apparatus. If it is desired to distill some cubic centimeters of a fluid the apparatus can be made with an Erlenmeyer flask with thick walls, or of a Kitasato flask, with the lateral arm closed. 
in the distillate. This result is not peculiar to one race alone, for absolutely identical results were secured with races virulent for Shiga, B. pestis, B. coli, B. typhosus, and for the staphylococcus.

Gildemeister and Herzberg, ${ }^{235}$ Borchardt, ${ }^{76}$ Spät, ${ }^{588}$ Bronfenbrenner and Korb, ${ }^{99}$ and Meissner ${ }^{49}$ have obtained the same results. The last used a simple distillation apparatus of the open model as ordinarily used in laboratory work. She found that the bacteriophage passed over in two experiments out of eight when she took no precautions to prevent the passing over of droplets. It was only necessary to suspend a small disc of rubber over the opening of the tube emitting the vapor to prevent entirely the passage of the droplets and of the bacteriophage corpuscles as well. This offers additional proof that the droplets are in reality the vehicles which carry over the corpuscles.

\section{Sedimentation}

The following experiments ${ }^{321}$ show that the bacteriophage corpuscles sediment to the bottom of a vessel. This deposition occurs either spontaneously with the passage of time, or mechanically, through centrifugation.

A suspension of an anti-dysentery bacteriophage is filtered through a bougie and allowed to stand without moving in a cupboard for eleven months. At the end of this time, specimens from the surface and from the bottom of the tube are taken with capillary pipettes.

The count of the superficial layers showed $280,000,000$ per cubic centimeter.

The count of the deeper layers showed 2,900,000,000 per cubic centimeter.

That the corpuscles can be sedimented, although incompletely, by centrifugation at very high speed is shown by the following.

Twenty-five cubic centimeters of the bacteriophage (antidysentery) are filtered through a bougie and are centrifuged in a Jouan apparatus for 30 minutes at 12,000 revolutions per minute. Counts show the following:

$$
\begin{aligned}
& \text { per cubic } \\
& \text { centimeter } \\
& \text { Before centrifugation . . . . . . . . . . . . . . . . . . } 1,750,000,000
\end{aligned}
$$

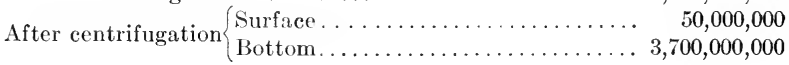

These results have been contradicted by a number of authors, but nevertheless I am still convinced of their accuracy, for in recent experi- 
ments I have obtained comparable results. However, it is only fair to state that those who have questioned the correctness of my findings have generally performed their experiments under conditions differing from mine, using slower speeds for the centrifugation.* It is evident that an enrichment of the lower layers may be obtained, although it is never possible to render the upper layers entirely free of corpuscles. Levaditi and $\mathrm{Nicolau}^{412}$ have shown that the same thing happens with the ultraviruses of rabies and of vaccinia. As a matter of common experience, it is well known that it is extremely difficult to obtain a sterile supernatant fluid by centrifugation of a bacterial suspension. There is all the more reason to encounter this same difficulty with ultraviruses.

\section{Nature of the "substance" of the bacteriophage corpuscle}

We know that the colloidal state depends solely upon two factors,insolubility and the size of the particles. In reality there is but one factor determining the colloidal state, namely, the mass of the granule, for if a substance suspended in a liquid remains in a granular condition it is insoluble.

The colloidal state does not begin suddenly with a certain mass, to end as suddenly when an upper limit is reached. There is a gradual transition of the properties of the molecular state (solutions) into those of the solid state (precipitates), and between these two extremes the colloidal state is to be found.

Considering only those substances immersed in water, which is the general case in biology, one might say that the colloidal state becomes manifest when the mass of the particle immersed is such that it is subject to brownian motion. With organic particles this takes place when the particles reach a diameter of from 1 to $2 \mu$, and this property becomes accentuated as the diameter diminishes, being particularly marked when the diameter reaches a point between $100 \mu \mu$ and $1 \mu \mu$.

It may be well to state that the nature of the substance forming the granules is of no consequence, nor is it of significance whether the particle is living or dead. The sole factor is the mass; if it is sufficiently small the particle becomes a colloidal "micella." $\dagger$

* I know, however, of some experiments, as yet unpublished, made at the Institute of Delft (Holland), involving centrifugation at high speeds, that have given results which confirm those reported here.

$\dagger$ The water containing these mineral or organic bodies in suspension is the intermicellar fluid. Fluids other than water are not considered here for they are of no interest to biologists. 
The bacteriophage corpuscle having a diameter of 20 to $30 \mu \mu$, and being insoluble in water, necessarily possesses colloidal properties. Within a culture medium it is a colloidal "micella" whatever may be the intimate nature of the substance of which it is formed.

The bacteriophage is either a living being or a product derived from a living being (bacterium); and such being the case the "substance" of the bacteriophage corpuscle must be either of lipoid nature or of protein nature.*

If it is a lipoid it should be soluble in the solvents for these bodies,ether, chloroform, or petroleum ether.

Kabeshima ${ }^{388}$ has affirmed that the bacteriophage is soluble in both ether and chloroform. I have shown ${ }^{318}$ that this is not true, for if one mixes equal parts of ether and bacteriophage suspension, it is found after prolonged shaking that the proportion of corpuscles present in the ether and in the subjacent fluid is as 1:40,000. This clearly indicates that there has been a commingling and not a dissolution, for it is only necessary to shake this ether with bouillon to remove the greater part of the corpuscles, and by repeating the extraction with bouillon it is possible to remove them all.

De.Poorter and Maisin ${ }^{532}$ have effectively answered this question of ether solubility. They mixed 1 ce. of a bacteriophage suspension with 2 cc. of ether. Then they superimposed 10 cc. of ether, taking care through all of these procedures not to wet the walls of the tube, for if this is done corpuscles may remain adherent to the walls and give erroneous results. After allowing it to stand for 24 hours at laboratory temperature they removed a few cubic centimeters of the supernatant ether, evaporated it at a low temperature and looked for the bacteriophage in the residue. No trace of it could be found.

f. They performed the same experiment with petroleum ether and with chloroform, with the last by first introducing $10 \mathrm{cc}$. of chloroform into a burette and then a well shaken mixture of bacteriophage suspension and chloroform. The results were the same; there was nothing to indicate that the bacteriophage was dissolved.

These authors concluded that the substance of the bacteriophage is not a lipoid, and this being the ease it follows, therefore, that the bacteriophage corpuscle is made up of a protein substance.

Wollman ${ }^{633}$ has stated that the bacteriophage is destroyed by tryptic

* Glycogen occurs also in the form of colloidal granules, but the bacteriophage is not glycogen, for a liquid which contains the bacteriophage, boiled after the addition of a strong acid, does not reduce Fehling's solution. 
digestion. In a more recent communication ${ }^{936}$ he admits that the destruction is only partial, and that it is more or less complete depending on the race of the bacteriophage used.

With reference to the many publications, announcing the discovery of the bacteriophage in enzyme-containing products, a subject to which we will return, I have performed a variety of experiments to determine the nature of the action of papaine and of trypsin on the bacteriophage. I have never been able to find any manifest changes when a suspension of one or the other of these ferments was combined with the bacteriophage. On the contrary, when I mixed trypsin or papaine with a bacterial suspension inoculated with the bacteriophage, I observed a very sharp inhibition, but, in view of the former experiments, I am not able to attribute this inhibition to a destruction of the corpuscles by the ferment, but rather to an action of the ferment on the bacterium, an action which interferes with bacteriophagy. Among other experiments having a similar significance, we will see that an antibacterial serum has no action whatever upon the bacteriophage, but that it exercises an inhibitory action upon bacteriophagy with the homologous bacterium. The surface of the bacterial body must be modified in some way, both by the antiserum and by the proteolytic enzymes, so that it is not susceptible to the adhesion of the bacteriophage corpuscles. It may be mentioned, however, that in any case the inhibition is relative only; bacteriophagy is retarded but it still takes place.

\section{CONSERVATION OF THE BACTERIOPHAGE CORPUSCLE}

Before undertaking an analysis of this phase of the subject of bacteriophagy it may be well to call attention to a very important point, which applies as well to the effect of the passage of time as to that of any agent, physical or chemical. We find that a suspension of the bacteriophage contains a number of corpuscles which we may designate as "x." We subject this suspension to the action of any agent whatsoever and when the action is completed we find, for example, that the number of corpuscles has become $\mathrm{x} / 10$. We conclude immediately that $9 / 10$ of the corpuscles have been destroyed. Is this deduction beyond question legitimate? By no means. IVe can detect the bacteriophage only because of the fact that it causes bacteriophagy, that is, we detect its "virulence." Death of the corpuscle or the loss of its virulence would lead to the same effect, the absence of bacteriophagy. Let us observe that through the action of time, through increases in temperature, or 
through exposure to certain antiseptics many bacteria* become avirulent. Inasmuch as we find that they still multiply we conclude that they are not destroyed, as we would most certainly do if the bacteria were infravisible. Experiment shows us that it is certainly the same for the bacteriophage. Under the action of a harmful agent the virulence is lost first, destruction follows later. This comment applies to all ultraviruses generally.

In general, the vitality of bacteriophage corpuscles is very high. A Shiga-bacteriophage which has been preserved in a sealed ampoule for nine years has lost none of its virulence, although the number of the corpuscles has diminished very considerably. When first prepared there were 2000 million corpuscles per cubic centimeter, after four years the number was reduced to only 100 million, after nine years to but 40 millions. From this it seems that a very great many corpuscles are destroyed during the first months. Those which remain are the more resistant ones and consequently the reduction in number occurs more and more slowly. With another race the number of corpuscles fell within nine years from 2400 million to 110 millions. Such a diminution in numbers is not peculiar to the bacteriophage, for precisely the same thing takes place with the bacteria.

The vitality of a single bacteriophage differs according to the bacterial species which has contributed to its development. Tests made upon this same bacteriophage which we have just considered, after it had been subject to a few passages at the expense of B. typhosus, showed that it became totally inactive when held for 52 months.

It will be recalled that we have already considered the attenuation of virulence which takes place with the passage of time. It is unnecessary to repeat it here.

Confirming what has been said above, namely, that the bacteriophage first becomes avirulent, and then "dies," although we can not tell when, is the fact that the apparent vitality of the bacteriophage is often proportionate to its virulence. But this is not a general fact; it is not uniformly true as the following experiment shows. ${ }^{321}$

Typhoid patient Mor. . . . . Examination of the stool was made at the beginning of convalescence. On August 20th, 1918, the stool was treated according to the method described for securing the bacteriophage. The filtrate was distributed in $0.5 \mathrm{cc}$. amounts in suspensions of the following bacteria: B. dysenteriae Shiga, B. typhosus, B. para-

* Among others, B. anthracis, which beeomes avirulent when it is cultivated at a temperature of $42^{\circ} \mathrm{C}$. (Pasteur) or in the presenee of phenol (Roux). 
typhosus $A, B$. paratyphosus B, and B. coli. After 24 hours of incubation these suspensions were planted on agar with the following results:

B. dysenteriae Shiga..................... Sterile

B. typhosus ............................ Sterile

$B$. paratyphosus $A \ldots \ldots \ldots \ldots \ldots \ldots \ldots$. . . . . . . . . . . . .

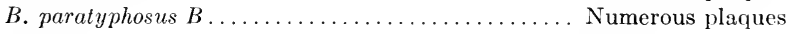

B. coli ............................. Sterile

Specimens of the feces and of the filtrate were preserved in sealed tubes. On January 22nd, 1919, that is, after 5 months, these materials were examined again, with the results shown in table 26.

In this material the virulence of the bacteriophage for $B$. dysenteriae and for $B$. paratyphosus $B$ remained unaltered during the five months, it diminished for B. coli, and disappeared entirely for B. typhosus and B. paratyphosus $A$.

TABLE 26

\begin{tabular}{|c|c|c|}
\hline \multirow{2}{*}{ SUSPENSION } & \multicolumn{2}{|c|}{ RESULT } \\
\hline & Freshly prepared filtrate & Original filtrate \\
\hline B. dysenteriae Shiga. & Sterile & Sterile \\
\hline B. typhosus...... & Normal culture & Normal culture \\
\hline B. paratyphosus $A$.. & Normal culture & Normal culture \\
\hline B. paratyphosus $B \ldots$ & Numerous plaques & Numerous plaques \\
\hline 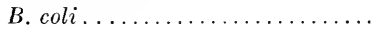 & Numerous plaques & Numerous plaques \\
\hline
\end{tabular}

It should be noted that the result was the same whether the bacteriophage was preserved directly in feces or in the filtrate, that is, in bouillon. Likewise, in this instance, it is significant that the degree of virulence has no influence on the preservation or the disappearance of the virulence. It was strong for $B$. typhosus and became negative, it was weak for $B$. paratyphosus $B$, yet this remained intact.

In the absence of passages, simply as an effect of old age, the virulence of the bacteriophage varies then with time, and indeed in a different manner for the diverse bacteria attacked. It becomes attenuated more quickly for some than for others, and for this no general rule can be fixed. We have seen elsewhere that after four years and in spite of passages in contact with the dysentery bacillus, the virulence for $B$. typhosus persisted. The last experiment cited is not only interesting then, in that it shows an attenuation of virulence associated with the lapse of time, but also in that it gives evidence that the loss does not occur in equal degrees for all of the bacteria attacked by one and the same bacteriophage. 
At all events, it is certain that, as a general rule, the weakest virulence disappears first, but we should not overlook the fact that there are many exceptions to this rule and it is well to consider that each race of the bacteriophage may behave in its own individual way.

If a bacteriophage suspension is allowed to evaporate slowly at room temperature it is found that living corpuscles may be found in the few drops of syrupy fluid remaining in the bottom of the tube. Indeed, certain bacteria act in the same way. On the contrary, living corpuscles are no longer to be found after twelve months in glucose bouillon cultures, although they may still be alive in lactose bouillon.

From fecal material preserved at room temperature in sealed tubes for thirty-four months (September, 1915, to July, 1918) it was possible to recover the living bacteriophage, as active as at the beginning. This experiment has been performed successfully with four specimens of feces from convalescent cases of dysentery.

In a mixture of equal parts of neutral glycerol and of bacteriophage suspension, held for five years in sealed tubes, the virulence of the bacteriophage corpuscles (Shiga-bacteriophage) remained unimpaired.

It is noteworthy that the liquid in which the corpuscles are suspended exerts a considerable effect upon their preservation. As for the reaction of the liquid, the effects of acid are once more evident, for if the fluid is ever so little acid the period of the conservation will not extend beyond a few weeks, when the tubes containing the corpuscles are held at laboratory temperature.

On the question of the preservation of the bacteriophage in the dry state I have several apparently contradictory facts.

In 1916 I placed some drops of filtrate (Shiga-bacteriophage) upon some sheets of filter paper. These were dried and then aseptically transferred to glass tubes which were sealed in the flame. These tubes were left in the laboratory, exposed to the light, for 6 months. At the end of this period a fragment of the filter paper, introduced into a tube containing a suspension of Shiga bacilli, caused bacteriophagy. ${ }^{321}$

Seiffert ${ }^{581}$ has taken exception to this, and quite correctly, for it has been impossible for me to repeat this experiment, although I do not yet see any possible cause for error which has not been considered. Did the nature of the filter paper play any rôle? In this same connection it may be added that Gerretsen, Gryns, Sack and Söhngen ${ }^{228}$ have found that their bacteriophage, virulent for $B$. radicicola, remained active for two months when dried on filter paper.

If a suspension of the bacteriophage is allowed to dry slowly at room 
temperature, it is possible to recover the bacteriophage, still virulent, from the few drops of syrupy liquid remaining in the bottom of the tube. ${ }^{3<1}$ The fluid may, indeed, be evaporated to dryness, particularly in the apparatus which I have described, and in the pulverized residue virulent corpuscles may still be found.

\section{FLOCCULATION OF CORPUSCLES}

The bacteriophage corpuscle being, from the physical point of view, a colloidal "micella" it should possess an electric charge, and in order to determine the sign of this charge it is only necessary to ascertain whether these micellae are flocculated by $\mathrm{H}^{+}$or $\mathrm{OH}^{-}$ions. In the first case the charge would be negative, in the second it would be positive.*

We know that the bacteriophage usually multiplies in an alkaline medium, and that for the overwhelming majority of races, the corpuscles remain inactive in an acid medium. Furthermore, as da Costa Cruz has shown by direct experiment, the corpuscles flocculate under the influence of acids.

This being the case the corpuscle possesses a negative charge, and consequently the bacteriophage is what is termed a "negative-colloid." Incidentally, we may add that according to many investigations the great majority of bacteria also possess a negative charge, and they also are flocculated by acids.

But we have seen that certain races of the bacteriophage may bring about bacteriophagy in an acid medium (Asheshov ${ }^{27,28}$ ). Da Costa Cruz has isolated a race of the bacteriophage which is active in a medium with a $\mathrm{pH}$ of 5.1 , that is, in a medium definitely acid. It would appear then, that flocculation by acid does not take place at the same $\mathrm{pH}$ for all races.

Maisin $^{429}$ has found that the corpuscles are completely precipitated by saturation with ammonium sulfate and that they may be recovered, still virulent, from the precipitate. Precipitation is only partial with half saturation with the sulfate. Magnesium sulfate acts in the same way (de Poorter and Maisin ${ }^{52}$ ). Here again the corpuscles react like "protein micellae."

Levaditi and Nicolau ${ }^{412}$ have seen that the virus of vaccinia behaves in the same way when subjected to these conditions.

Bacteriophage corpuscles are carried down only in part by the precipitate formed with tricalcium phosphate $\left(\mathrm{Maisin}^{429}\right)$, and I have shown that there is but little removal of the corpuscles by the precipitate of

* Sender and Picton, Hardy, J. Perrin, etc. 
calcium carbonate brought about by the addition of a concentrated lactate solution.

Kabeshima ${ }^{388}$ has stated that the bacteriophage is precipitated by alcohol and by acetone. This is true,,$^{318}$ but, contrary to the additional statement of this author, the bacteriophage is very quickly destroyed if the contact is prolonged, either with alcohol or with acetone. We will revert to this point later.

\section{ADSORPTION OF BACTERIOPHAGE CORPUSCLES}

The bacteriophage corpuscle being a negatively charged colloidal micella, at least, when in an alkaline medium, it should be subject to the phenomenon of adsorption. Those who have studied this phase of the question are a long way from being in accord. From what does this disagreement result? Unquestionably two fundamental causes contribute to the difference in opinion.

A great many bacteriologists who undertake to deal with physicochemical questions are not too familiar with this science. They forget, for example, that the conditions of the medium, the pH in particular, exert a very definite effect upon colloidal phenomena. We have, for example, studied the phenomena of adsorption with bacteria, and we have found that apparently insignificant differences in the reaction of the medium exercise a great influence on their ability to pass through filters. We know, in fact, that filters are permeated by pores whose diameter is far greater than that of the bacteria. If the bacterial cells are retained, it is not because they can not pass through these pores, but because they are adsorbed by the walls. This phenomenon of adsorption is not observed solely with filter candles, but even with the grains of sand which form the filter beds of the commercial filters used for the purification of drinking water. But even here, the passage of bacteria is found to be, in great part, determined by the reaction of the medium.

The phenomena of adsorption are the more intense as the colloidal properties are the more outspoken, that is to say, as the particles of the colloid become smaller. If granules as voluminous as bacteria are capable of being adsorbed, the adsorption being modified by the reaction of the medium, there is the greater reason that this phenomenon may be more intense with bacteriophage corpuscles whose diameter is but one-twentieth or one-fiftieth as great.

But there is a second possible cause of error depending on the inherent variability of the bacteriophage, since each race presents its own 
peculiar properties. We have seen that certain races cause bacteriophagy in definitely acid media, at a pH of 5.1. Should the phenomena of adsorption be the same for corpuscles capable of causing bacteriophagy in a medium of $\mathrm{pH} 6.5$ and for those capable of causing bacteriophagy at $\mathrm{pH} 5.1$ ? It is quite within the bounds of experience to doubt if the behavior of the two races will be the same, for it is very probable that these differences in properties correspond to quantitative differences in the electric charge.

However this may be, the greatest cause for the lack of concordance in the results reported may certainly be attributed to differences in the reaction of the medium. There is the less reason to be surprised at divergent results when we consider that some workers have used suspensions of the bacteriophage corpuscles diluted with distilled water or physiological saline neutral in reaction (often even slightly acid) while others have worked with pure suspensions, that is, with undiluted, alkaline, bacteriophage filtrates.

From the beginning of my studies I have held that adsorption of the corpuscles by porous candles (Chamberland) was very slight. Prausnit $^{53}$ i has to a degree confirmed this conclusion by showing that it is necessary to repeat the filtration of a suspension eight times in order to cause a measurable reduction in the number of corpuscles.

It may also be recalled that I have recommended ${ }^{321}$ as the best technic for the isolation of a bacteriophage from turbic organic fluids a preliminary filtration through a filter paper covered with a layer of infusorial earth. This procedure clarifies the material prior to its filtration through a candle. And this technic was recommended because I had demonstrated that such a preliminary filtration does not lead to a diminution in the number of the corpuscles, a proof that they are not adsorbed by contact with the infusorial earth. In all of my experiments I have worked with the bacteriophage suspended in a fluid having an alkaline reaction, - $\mathrm{pH} 7.6$ to 8.2 .

In opposition to this conclusion Seiffert ${ }^{581}$ has affirmed that the corpuscles are adsorbed by infusorial earth and by kaolin.*

Gildemeister and Herzberg ${ }^{235}$ have observed a considerable adsorption of the corpuscles by infusorial earth (Kieselguhr). But they have added

* This author has deduced from this that the bacteriophage is an electro-positive colloid, apparently forgetting that the electro-positive colloids are flocculated by $\mathrm{OH}$ ions, that is to say, in an alkaline medium. This is not the case for the bacteriophage, since alkaline media are quite uniformly best suited for work with the bacteriophage and for the process of bacteriophagy. 
that if the infusorial earth after adsorption is washed with some water rendered alkaline with ammonia, the corpuscles are freed into the liquid. This would indicate that the adsorption occurring with this material takes place only if the medium is acid, or at most, neutral. It is significant that these authors have further noted that filtration through a Berkefeld candle is more readily effected if the medium is alkaline in reaction.

A similar set of experiments authorized Prausnitz and Firle ${ }^{539}$ to state that the amount of adsorption with infusorial earth is practically zero, even if the earth is very fine, and the test is performed with the materials in an alkaline medium. These experiments of Prausnitz are noteworthy, moreover, for their precision, which places them above all criticism.

Arloing, Langeron, and Sempé ${ }^{21}$ have also found that the degree of adsorption to infusorial earth and to kaolin is essentially negative. In comparison they also report that the corpuscles are adsorbed almost completely by sheep cells freed of plasma, and that the bacteriophage may be found in the sediment after centrifugation.

According to de Poorter and Maisin ${ }^{532}$ there is a partial adsorption to animal charcoal and to the serum colloids.

It is well known that when suspensions of colloids of opposite signs are mixed a reciprocal flocculation takes place. Since the bacteriophage bears a negative charge, ${ }^{*}$ a flocculation should not take place when a bacteriophage suspension and a negative colloidal suspension are mixed, but when mixed with a positive colloid flocculation should occur.

Otto and Munter ${ }^{494}$ have seen that the corpuscles retain all of their properties in the presence of colloidal sulfur (a negative colloid), and certain facts suggest that when in contact with this colloid bacteriophagy is favored. Some of my experiments confirm entirely the observations of these authors.

De Necker has suggested ${ }^{477}$ that the bacteriophage corpuscles are partially adsorbed by various negative colloids (colloidal preparations of iodine, of manganese, of selenium, of silver, of copper, and of rho-

* Without going into detail on this point, it may be said that a "micella" is composed of a granule which bears the charge represented by one or two ions, while a layer of ions of the opposite sign is found in the layer of liquid which surrounds the granule. The sign of the colloid is conferred by the nature of the granular charge. For the bacteriophage corpuscle, for example, the granule must bear negative ions $\left(\mathrm{OH}^{-}\right)$and in the layer of liquid which surrounds the granule, there must be the opposite, i.e., positive ions $\left(\mathrm{H}^{+}\right)$. 
dium). From my own work I feel warranted in definitely contradicting this. It is probable, as I have stated above, that de Necker carried out his experiments with the materials in an acid medium.

Contributory evidence on this point is afforded by some experiments with colloidal silver. For example, I have added $10 \mathrm{mgm}$. of "Collargolum siccum" of Heyden to $10 \mathrm{cc}$. of a suspension of Staphylo-bacteriophage. Titrations of these corpuscles made 13 months later showed that the bacteriophage had not lost its virulence. Determinations of the same kind made with a Shiga-bacteriophage, yielded the same result.

Moreover, some of the experiments reported in Chapter I may be recalled, showing that bacteriophagy takes place normally in bouillon, at a $\mathrm{pH}$ of 7.8 , containing $5 \mathrm{mgm}$. of dry collargol to each 10 cc.*

As for the so-called inhibitory action of the colloid gelatin, I think that I have shown by the experiments detailed in Part I, that the inhibitory effect manifested is of a wholly different nature. In a liquid medium gelatin is inhibitory because of its viscosity (Hauduroy), on a solid medium because the gel does not permit the diffusion of the products resulting from bacteriophagy, products which impede the activity of the bacteriophage itself.

For purposes of comparison it is interesting to note that Valée and Carré have observed that different ultraviruses, that of aphthous fever in particular, are strongly adsorbed by red blood cells, and that Levaditi and Nicolau ${ }^{412}$ have reported that the ultravirus of vaccinia is adsorbed by animal charcoal and by the serum globulins.

\section{EFFECTS OF IRRADIATION}

Appelmans ${ }^{14}$ states that a suspension of the bacteriophage subjected to ultraviolet rays is inactivated by an exposure of ten minutes.

According to Gildemeister, ${ }^{230}$ the sensitivity of the bacteriophage toward ultraviolet rays is the same as that of the bacteria.

Zoeller $^{646}$ observed a complete inactivation after 15 minutes of exposure.

Gerretsen, Gryns, Sack, and Söhngen, ${ }^{228}$ working with a bacteriophage virulent for $B$. radicicola, have exposed at a distance of $30 \mathrm{~cm}$., either

* De Necker also states that aluminium hydroxide causes a flocculation of the bacteriophage. When the precipitate is dissolved by the careful addition of acetic acid the bacteriophage reappears in the liquid. Flocculation was never complete. A possible error in this experiment is the occlusion of the pores of the candle used to filter the precipitated hydroxyl-bacteriophage. 
plaques of the bacteriophage on agar, or suspensions contained in quartz tubes, to a Heraeus lamp operating under 4 amperes at 220 volts.

Although under these conditions, $B$. radicicola was killed after exposure for 15 minutes, the bacteriophage was intact after 30 minutes. Indeed, it was still alive after 2 hours, but was completely destroyed after $2 \frac{1}{2}$ hours.

Brutsaer $\mathrm{t}^{105}$ has investigated the effect of radium emanations. In his experiments the radio-active energy, measured as $\gamma$ rays, was equivalent to $0.914 \mathrm{gr}$. of radium. He found that after a prolonged exposure, the bacteriophage remained intact. A Coli-bacteriophage, of average virulence, was but slightly attenuated after an exposure of 24 hours, for its virulence was fully restored by a single passage.

The effects of irradiation may be summarized briefly by stating that the bacteriophage behaves in essentially the same way as does the bacterium.

\section{EFFECT OF TEMPERATURE}

Among the very first of my reports on bacteriophagy and the bacteriophage itself there appeared the statement ${ }^{315}$ that the Shiga-bacteriophage resisted a temperature of $65^{\circ} \mathrm{C}$. when exposed for a half-hour. Kabeshima ${ }^{388}$ reported it was not destroyed at $70^{\circ} \mathrm{C}$., but that destruction occurred only when it was heated for a half hour at $75^{\circ} \mathrm{C}$. Kuttner ${ }^{398}$ made the same observation in determinations with the Typhobacteriophage.

In collaboration with Pozerski ${ }^{360}$ additional consideration was given to this question of heat-resistance and from these studies the reason for the divergent reports became apparent. It was found that the destruction of the corpuscles is preceded by an attenuation in the virulence, the attenuation being the more pronounced as the temperature to which the corpuscles are heated is increased. For all corpuscles, of whatever virulence and of whatever race the temperature of apparent destruction, that is to say, of complete inactivation* is about $75^{\circ} \mathrm{C}$. The following experiments demonstrate this.

A bacteriophage suspension, previously filtered through a candle, is taken up in a series of capillary pipettes, and the pipettes are carefully sealed at both ends. The pipettes are then completely immersed

* I repeat that we can only recognize the bacteriophage through its virulence. Loss of virulence or destruction of the corpuscle as such would lead to the same phenomenon,-absence of bacteriophagy. After exposure to $75^{\circ} \mathrm{C}$. the bacteriophage is completely avirulent. It may not be destroyed until the temperature reaches 80 or $90^{\circ} \mathrm{C}$., but this we can not determine. 
in the water-bath, adjusted to maintain the temperature desired, as indicated in each of the following experiments. In each part of the experiment, 8 tubes containing the suspension are held for 30 minutes at a constant temperature. The temperatures selected were 60,62 , $64,66,68,70,72$, and $75^{\circ} \mathrm{C}$.

\section{Shiga-bacteriophage}

From each of the tubes held at each of the temperatures $60,62,64$, and $66^{\circ} \mathrm{C}$. two drops of suspension were inoculated into bouillon suspensions of Shiga bacilli. In all instances a complete dissolution of the bacilli took place within 14 hours. When these tests were repeated with a second strain of susceptible Shiga organisms the results were identical in every respect. With the suspensions which had been heated to $68^{\circ}$ and to $70^{\circ} \mathrm{C}$. the material caused a complete dissolution when added to one of the Shiga strains, but it failed to induce a dissolution of the other. The material heated to $72^{\circ} \mathrm{C}$, as well as that heated at $75^{\circ} \mathrm{C}$. failed to cause dissolution with either strain.

For control purposes, plantings on agar were made; one drop of the bacterial suspensions receiving the bacteriophage corpuscles heated to $68,70,72$, and $75^{\circ} \mathrm{C}$. being spread over agar prior to the incubation. Later examination of these cultures, after incubation, showed that all, except those receiving the bacteriophage heated to $75^{\circ} \mathrm{C}$. showed characteristic plaques; the growths obtained from the $75^{\circ} \mathrm{C}$. tubes were normal bacterial cultures.

When the virulence of a bacteriophage is attenuated by heating, the activity may, within limits, be restored by effecting serial passages. For example, with the corpuscles heated at 68 and at $70^{\circ} \mathrm{C}$. two passages were required, with those heated at $72^{\circ} \mathrm{C}$. three passages were necessary to restore the virulence to such an extent that a dissolution of the bacteria would take place in a fluid medium.

Experiments comparable to the above in every respect showed that an entirely similar effect occurs with bacteriophage races active for other organisms: B. dysenteriae Flexner, B. dysenteriae Hiss, B. coli, and $B$. paratyphosus $B$. It is interesting that with the strain of $B$. paratyphosus $A$ tested, the attenuation of virulence began at a temperature of $64^{\circ} \mathrm{C}$., while with the race virulent for $B$. typhosus an attenuation was evident at a temperature as low as $62^{\circ} \mathrm{C}$. However, despite these individual racial variations, with all races exposure to $75^{\circ} \mathrm{C}$. renders the corpuscles inactive. Either they are actually destroyed, or their virulence is attenuated to such a point that they can no longer be de- 
tected. In all instances where a complete destruction does not take place, the corpuscles manifest a recuperative power, the virulence being restored more or less readily by subsequent passages if the temperature to which they have been subjected is not higher than $72^{\circ} \mathrm{C}$.

\section{Staphylo-bacteriophage}

This race of the bacteriophage appears to be somewhat more sensitive than are many races, for after exposure to a temperature no higher than $60^{\circ} \mathrm{C}$. it would appear that some attenuation occurred. Subcultures from those tubes which failed to show a dissolution clearly demonstrate that the changed activity is referable to a simple attenuation, for even those suspensions inoculated with a bacteriophage previously heated for 30 minutes at $72^{\circ} \mathrm{C}$. gave plaques characteristic of an active bacteriophage. Here again, the original virulence may be restored to suspensions heated at $62,64,66$ and $68^{\circ} \mathrm{C}$. by two passages. With suspensions heated to 70 and $72^{\circ}$ six passages were required to bring back the virulence. A temperature of $75^{\circ} \mathrm{C}$. deprived the bacteriophage of all activity. From these experiments it might be concluded that all races of the bacteriophage react in the same manner to the influence of temperature. As a matter of fact with each individual race the attenuation begins at a given temperature, varying with the race, but as to the upper limit, all races are permanently destroyed, or at least rendered completely avirulent, at temperatures approximating $75^{\circ} \mathrm{C}$. (d'Herelle and Pozerski ${ }^{360}$ ).

It appears clearly from these experiments that attenuation through heat begins to occur at a temperature rather more than $10^{\circ}$ below the point where the activity becomes definitely lost. They show likewise that it is unsafe to conclude that the bacteriophage is destroyed simply because it ceases to provoke bacteriophagy. It is always necessary to prove the question of destruction by making a few passages at the expense of a susceptible bacterium, for if the attenuation has not been extreme the virulence may be enhanced again.

Otto, Munter and Winkler, ${ }^{496}$ as well as Hauduroy, ${ }^{288}$ have shown that the attenuating effects of temperature occur the more readily as the virulence is less strong. That such a relationship should obtain is obvious, for since the virulence becomes gradually and progressively attenuated as the temperature is raised it is clear that when the initial virulence is weak the race will reach the point of complete attenuation more quickly.

Otto, Munter and Winkler ${ }^{496}$ have shown also that with a single race 
of the bacteriophage the temperature of inactivation differs for each of the bacteria attacked, and may even be different for a single bacterium and a single bacteriophage from one experiment to another. Other investigators have obtained comparable results.

The following experiment shows that these variations are related to the actual intensity of the virulence for each bacterium attacked.

A race of the bacteriophage was isolated from the feces of a patient convalescent from benign infectious jaundice. During the febrile period in this case the intestinal $B$. coli had been almost completely replaced by $B$. enteritidis. This bacteriophage was first subjected to 12 passages at the expense of $B$. enteritidis (isolated from the stools of the patient at the onset of the disease). Following these 12 passages (each one being effected by inoculating $0.001 \mathrm{cc}$. of the suspension previously bacteriophaged into $10 \mathrm{cc}$. of fresh suspension) a selective isolation was made, in order to eliminate any other races of the bacteriophage which might possibly have been carried along from the fecal filtrate.

This pure bacteriophage showed virulences as follows:

B. enteritidis (strain from the patient) ................ ++++

B. coli (strain from the patient) .................... +++

B. dysenteriae Shiga .............................. ++++

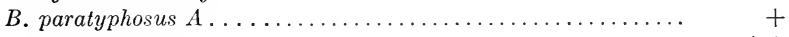

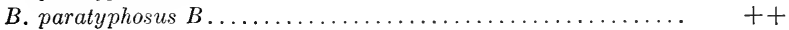

Sealed ampoules containing this bacteriophage were immersed for 30 minutes* in a water-bath regulated at the following temperatures: $60,63,65,68,70,73,75$, and $78^{\circ} \mathrm{C}$. A drop from each of these heated suspensions was added to a tube containing a suspension of susceptible bacteria, and the virulence was determined by the simplified procedure discussed in the section on "Evaluation of Virulence." (See table 27.)

It is not surprising then that those who have studied the resistance of different races of the bacteriophage to heat have obtained different figures for each race of bacteriophage, since everything depends upon the virulence.

Some authors (Weinberg and Aznar, ${ }^{624}$ Tchang Kouo Ngen and Wagemans ${ }^{595}$ ) have stated that the bacteriophage is destroyed only at a temperature of $80^{\circ} \mathrm{C}$. I am inclined to believe that their technic must have been defective for in accord with the majority of those who have published on the subject I have never yet found a bacteriophage,

* The ampoules were made of drawn out Pasteur pipettes, with thin walls, thus permitting the contents to quickly reach the temperature of the water-bath. This method is the only one which is free of criticism. 
even of maximum virulence, which was not rendered totally avirulent (or destroyed?) at a temperature in the neighborhood of $75^{\circ} \mathrm{C}$., nor have I found one which was rendered avirulent at temperatures below $65^{\circ} \mathrm{C}$.

Hauduroy ${ }^{288}$ also reported experiments showing that with all races of bacteriophage a true destruction takes place at the same temperature, although the temperature leading to a loss of virulence may be very different. He also noted that less heat is necessary to cause attenuation when the virulence is low. All of his experiments were performed with the same technic, hence his results are all comparable. And although the conclusion which he reaches is correct, the figures which he presents are certainly subject to revision. His method of testing consisted in placing the bacteriophage suspension in open tubes, and immersing them for 3 minutes in a water-bath regulated at the desired

TABLE 27

\begin{tabular}{c|c|c|c|c|c}
\hline \multirow{2}{*}{$\begin{array}{c}\text { BACTERIOPHAGE } \\
\text { SUSPENSION } \\
\text { HEATED AT }\end{array}$} & \multicolumn{5}{|c}{ VIRULENCE OF HEATED SUSPENSION FOR } \\
\cline { 2 - 6 } & B. enterititis & B. coli & B. shigae & $\begin{array}{c}\text { B. paratypho- } \\
\text { sus A. }\end{array}$ & $\begin{array}{c}\text { B. paratypho- } \\
\text { sus B. }\end{array}$ \\
\hline${ }^{\circ} \mathrm{C}$. & & & & & \\
60 & ++++ & +++ & ++++ & 0 & ++ \\
63 & +++ & ++ & ++++ & 0 & + \\
65 & +++ & ++ & +++ & 0 & + \\
68 & ++ & ++ & ++ & 0 & 0 \\
70 & ++ & 0 & ++ & 0 & 0 \\
73 & + & 0 & ++ & 0 & 0 \\
75 & 0 & 0 & + & 0 & 0 \\
78 & 0 & 0 & 0 & 0 & 0 \\
\hline
\end{tabular}

temperature. Certainly under such conditions the temperature reached by the liquid in the tube is not that of the water-bath. Nevertheless, by this method he found that all races of the bacteriophage were destroyed at $102^{\circ} \mathrm{C}$.

That the bacteriophage resists a temperature of $100^{\circ} \mathrm{C}$. has also been stated by Seiffert. ${ }^{582}$ He filtered a suspension of the bacteriophage through a Berkefeld candle, and he then suspended the candle in the neck of a flask containing boiling water. After 1 or 2 hours, during which time the candle remained in the steam, he filtered some sterile bouillon through the candle and determined whether bacteriophagy

* We may note that Tchang Kouo Ngen and Wagemans state that this bacteriophage, which resisted temperatures up to $80^{\circ} \mathrm{C}$. in one experiment, was inactivated at $72^{\circ} \mathrm{C}$. in a later test. This appears rather peculiar. 
took place when this filtrate was added to a suspension of susceptible bacteria. These experiments have been criticized by Gildemeister and Herzberg, ${ }^{235}$ by Prausnitz ${ }^{539}$ and by Flu. ${ }^{222}$ The last named author, in particular, has investigated this method, finding that if a culture of the staphylococcus is filtered through a very porous Berkefeld candle, and if this candle is then treated according to the technic of Seiffert, the temperature reached in the candle is insufficient to kill the staphylococci, although as is well-known the thermal death-point of this organism is about $60^{\circ} \mathrm{C}$. This renders it all the more evident why the bacteriophage appeared to resist such high temperatures.

One can only conclude from all of this that the bacteriophage corpuscle is destroyed at a temperature which it is impossible to accurately determine, but which is certainly about $75^{\circ} \mathrm{C}$. It is also obvious that the "apparent" destruction corresponds to a complete attenuation of the virulence, that this attenuation is progressive, and that it is proportionate to the temperature to which the corpuscles are exposed.

It is useless to cite figures for the different races, for whatever may be the bacterial species attacked, inactivation of the bacteriophage takes place, depending upon the virulence, at a temperature between 65 and $75^{\circ} \mathrm{C}$. Only the figures given by Gerretsen, Gryns, Sack, and Söhngen $^{228}$ for their bacteriophage races virulent for $B$. radicicola will be inserted here. This selection is made simply because their races are active for an organism which is but very distantly related to all of the other bacteria known to be susceptible up to the present time. They found that one race of this bacteriophage, virulent for B. radicicola, strain sarrandella, was inactivated at $65^{\circ} \mathrm{C}$, while the races virulent for $B$. radicicola, strains clover and lupine, resisted this temperature. Even with these newly discovered races, entirely unrelated to those previously known insofar as the bacterium involved is concerned, we find that the temperature of inactivation is essentially the same.

From the experiments of de Necker ${ }^{476}$ it appears that not all of the different corpuscles within a single suspension are inactivated at the same temperature. Some of the corpuscles appear to be very fragile, others more resistant. This is not at all surprising, for all bacterial cultures show the same thing. It has long been known that when a culture is exposed to heat a great many of the bacteria will die at a temperature far below that requisite to render the entire culture sterile. De Necker "titrated" the bacteriophage by the method of dilutions, a method which, in this case, is adequate, for here the differences are so considerable that the lack of precision does not invalidate the results. 
The suspension used was a Coli-bacteriophage, active in a dilution of $10^{-7}$.

After exposure for 30 minutes at $46^{\circ} \mathrm{C}$. it was still active at $10^{-7}$

After exposure for 30 minutes at 48 to $54^{\circ} \mathrm{C}$. it was still active at $10^{-6}$

After exposure for 30 minutes at 56 to $62^{\circ} \mathrm{C}$. it was still active at $10^{-5}$

After exposure for 30 minutes at $64^{\circ} \mathrm{C}$. it was still active at $10^{-4}$

After exposure for 30 minutes at $66^{\circ} \mathrm{C}$. it was still active at $10^{-3}$

After exposure for 30 minutes at $68^{\circ} \mathrm{C}$. it was inert

Bacteriophage corpuscles, without regard to what race they belong, are always more resistant than are the homologous susceptible bacteria. We have seen elsewhere that Bordet and Ciuca have taken advantage of this property to isolate the bacteriophage. By heating a secondary culture, or a mixed culture, at about $58^{\circ} \mathrm{C}$. the bacteria will be killed while the bacteriophage will not be entirely destroyed. But although they are not destroyed we now know that an attenuation in the virulence of the corpuscles begins at a temperature well below $58^{\circ} \mathrm{C}$., and from this it is evident that this method of isolating the bacteriophage should never be used when it is desired to carry out experimental work free of all criticism. Filtration through a candle or ultrafiltration through a membrane with large pores is always to be preferred.

Employment of this defective method of isolation opens the way for a number of errors. all too frequently appearing in some of the published work, which might readily have been avoided with a satisfactory technic. Gildemeister, a very skillful bacteriologist, has recently obtained questionable results because of such an error.

Some time ago I stated ${ }^{318}$ that experiment seems to show that corpuscles are more sensitive to heat immediately after bacteriophagy than they are several days later. Tomaselli ${ }^{599}$ has now confirmed this fact, and his experiments are here presented in summary.

A Shiga-bacteriophage was used, the tests being made immediately after dissolution was completed and again two days later. (See table 28.)

That the bacteriophage corpuscle appears to undergo a diminution of volume during the first few days after the bacteriophagic process is completed has been stated above. We now see that during this same period it appears to acquire a somewhat greater resistance to heat. This must be the result of a condensation of the corpuscular substance.

The only author who has measured the resistance of the bacteriophage when in the dry state is Hauduroy, who found that it was destroyed at a temperature of $135^{\circ} \mathrm{C}$. 
TABLE 28

\begin{tabular}{|c|c|c|c|c|c|c|c|c|c|c|c|c|}
\hline \multirow{3}{*}{$\begin{array}{c}\text { TEMPERA- } \\
\text { TURE }\end{array}$} & \multirow{3}{*}{$\begin{array}{l}\text { PERIOD OF } \\
\text { EXPOSURE } \\
\text { TO HEAT }\end{array}$} & \multicolumn{11}{|c|}{ ACTIVITY OF THE BACTERIOPHAGE AFTER HEATINO } \\
\hline & & \multicolumn{11}{|c|}{ Dilutions } \\
\hline & & $10^{-2}$ & $10^{-3}$ & $10^{-4}$ & $10^{-5}$ & $10^{-6}$ & $10^{-7}$ & $10^{-8}$ & $10^{-9}$ & $10^{-10}$ & $10^{-11}$ & $10^{-12}$ \\
\hline
\end{tabular}

I. Shiga-bacteriophage immediately after bacteriophagy

\begin{tabular}{|c|c|c|c|c|c|c|c|c|c|c|c|c|}
\hline \multirow[t]{2}{*}{${ }^{\circ} \mathrm{C}$. } & minutes & & & & & & & & & & & \\
\hline & 10 & $0^{*}$ & 0 & 0 & 0 & 0 & + & + & + & + & + & + \\
\hline \multirow[t]{3}{*}{40} & 20 & 0 & 0 & 0 & 0 & 0 & 0 & + & + & + & $t$ & + \\
\hline & 30 & 0 & 0 & 0 & 0 & 0 & 0 & + & + & + & + & + \\
\hline & 10 & 0 & 0 & 0 & 0 & + & + & + & + & + & + & + \\
\hline \multirow[t]{3}{*}{50} & 20 & 0 & 0 & 0 & 0 & \pm & + & + & + & + & + & + \\
\hline & 30 & 0 & 0 & 0 & 0 & \pm & + & + & + & + & + & + \\
\hline & 10 & 0 & 0 & + & + & + & + & + & + & + & + & + \\
\hline \multirow[t]{2}{*}{60} & 20 & 0 & + & + & + & + & + & + & + & + & + & + \\
\hline & 30 & 0 & + & + & + & + & + & + & + & + & + & + \\
\hline 70 & 10 & + & + & + & + & + & + & + & + & + & $t$ & + \\
\hline \multicolumn{2}{|c|}{ Unheated } & 0 & 0 & 0 & 0 & 0 & 0 & 0 & 0 & 0 & + & + \\
\hline
\end{tabular}

II. The same bacteriophage suspension two days after bacteriophagy

\begin{tabular}{|c|c|c|c|c|c|c|c|c|c|c|c|c|}
\hline \multirow{3}{*}{40} & 10 & 0 & 0 & 0 & 0 & 0 & 0 & 0 & + & + & + & + \\
\hline & 20 & 0 & 0 & 0 & 0 & 0 & 0 & + & + & + & + & + \\
\hline & 30 & 0 & 0 & 0 & 0 & 0 & 0 & + & + & + & + & + \\
\hline \multirow{3}{*}{50} & 10 & 0 & 0 & 0 & 0 & 0 & + & + & + & + & + & + \\
\hline & 20 & 0 & 0 & 0 & 0 & 0 & + & + & $t$ & + & + & + \\
\hline & 30 & 0 & 0 & 0 & 0 & 0 & + & + & + & + & + & + \\
\hline \multirow{3}{*}{60} & 10 & 0 & 0 & 0 & 0 & + & + & + & + & $t$ & + & + \\
\hline & 20 & 0 & 0 & 0 & + & + & + & + & + & + & + & + \\
\hline & 30 & 0 & 0 & + & + & + & + & + & + & + & + & + \\
\hline 70 & 10 & + & + & + & + & + & + & + & + & + & + & + \\
\hline \multicolumn{2}{|c|}{ Unheated } & 0 & 0 & 0 & 0 & 0 & 0 & 0 & 0 & 0 & \pm & + \\
\hline
\end{tabular}

$* 0=$ a dissolution of the bacterial suspension when the heated bacteriophage is added.

$+=$ the inoculated suspension remains turbid.

It would appear from the number of corpuscles present, as shown by the active dilution, that the bacteriophage employed by Tomaselli was of maximum virulence. It is certain that inactivation was not complete at $70^{\circ} \mathrm{C}$.; there was only an appreciable attenuation of the virulence, but this was sufficient to prevent a clearing of the medium. 
To turn to the other aspect of the subject, namely, the resistance of the bacteriophage corpuscle to cold, I am, insofar as I am aware, the only one who has studied the question. While at the University of Leiden I carried out at the Institute of Refrigeration a few experiments on the effect of cold upon the bacteriophage. The results are interesting.*

The suspensions were enclosed in thick glass tubes sealed in the flame. The tubes were then placed for 10 minutes in liquid air. In some instances exposure to the cold was repeated several times. The essential data obtained are given in table 29.

All of these races of the bacteriophage were of maximum virulence; control suspensions, inoculated with $0.001 \mathrm{cc}$. of an unfrozen filtrate, underwent bacteriophagy, dissolution being complete and permanent.

TABLE 29

\begin{tabular}{|c|c|c|c|}
\hline RACE OF BACTERIOPHAGE & $\begin{array}{c}\text { AGE (TIME ELAPSED } \\
\text { SINCE } \\
\text { BACTERIOPHAGY) }\end{array}$ & $\begin{array}{c}\text { NUMBER OF EXPOSURES } \\
\text { TO LIQUID AR, } \\
10 \text { MINUTES ON EACH } \\
\text { OCCASION }\end{array}$ & RESULT \\
\hline Shiga & 17 days & 1 & Resisted \\
\hline Shiga & 4 days & 1 & Resisted \\
\hline Shiga & 6 days & 3 & Resisted \\
\hline Shiga & 5 months & 1 & Destroyed \\
\hline Shiga & 5 months & 3 & Destroyed \\
\hline Staph. & 11 days & 1 & Resisted \\
\hline Staph. & 47 days & 1 & Destroyed \\
\hline Staph. & 3 months & 3 & Destroyed \\
\hline
\end{tabular}

These experiments show, insofar as they go, that the young corpuscles resist exposure to a temperature of about $-180^{\circ} \mathrm{C}$., while the older corpuscles are destroyed.

\section{ACTION OF INORGANIC CHEMICAL SUBSTANCES}

The reports of those who have determined the effect of inorganic chemical agents upon the bacteriophage will be merely summarized.

Acids and bases. Eliava and Pozerski ${ }^{192}$ found that a Shiga-bacteriophage was destroyed by standing for 24 hours in media with a $\mathrm{pH}$ of below 2.5 or above 8.4 . In these experiments the $\mathrm{pH}$ values were

* These experiments were made but a short time before my departure from Leiden, and I have not yet had an opportunity to repeat them. I present them, therefore, subject to confirmation. Causes of error are so easily overlooked that a single experiment of this type has but limited value. 
determined by Mouton by the electrometric method. Scheidegger ${ }^{568}$ reports that the corpuscles suspended in a medium with a $\mathrm{pH}$ of 4.5 remained alive for 2 hours; and according to Davison ${ }^{17 t}$ the bacteriophage is destroyed if $1 \mathrm{cc}$. of normal $\mathrm{NaOH}$ is added to 4 ce. of filtrate.

$\mathrm{Da}$ Costa $\mathrm{Cruz}^{15^{3}}$ noted that the corpuscles were flocculated in distilled water, but this finding does not agree with my observations provided the distilled water is neutral in reaction. Unquestionably flocculation takes place if the distilled water is acid, indeed, under such conditions the bacteria flocculate also but as a rule, it requires a somewhat higher acidity to cause bacterial flocculation.

Reichert ${ }^{550}$ has studied the action of acids and bases upon the bacteriophage very carefully, and according to him, the different virulences manifested by a race of the bacteriophage are differently affected, some resisting degrees of acidity or alkalinity which destroy others.

This can only mean that here the intensity of the virulence, just as is the case with heat, plays a determining rôle, within limits, upon the effects observed.

Mercuric chloride. Bacteriophage corpuscles suspended in physiological saline containing 0.5 per cent of this salt remain alive for 3 days, but they are destroyed after 4 days (d'Herelle ${ }^{321}$ ). By means of comparative determinations with the spores of $B$. anthracis Prausnitz and Firle ${ }^{539}$ have found that the corpuscles are slightly less resistant than the spores.

Sodium fluoride. Eliava and Pozerski ${ }^{192}$ have found that the bacteriophage remains alive for at least 24 hours in the presence of 2.5 per cent of this salt, while under these same conditions Prausnitz ${ }^{536}$ observed a considerable diminution in the number of corpuscles after 3 days. In a 1 per cent solution the corpuscles (Shiga-bacteriophage) are destroyed after 15 days (d'Herelle).

Potassium cyanide. That a very considerable reduction in the number of corpuscles occurred within 3 days in a medium containing 2.5 per cent of this salt has been reported by Prausnitz. ${ }^{536}$ In a 1 per cent solution, a Staphylo-bacteriophage was markedly attenuated after 15 days (d'Herelle), and destroyed after 1 month.

Sodium chloride. Marcuse ${ }^{438}$ states that whatever the concentration of the salt, no destruction of the corpuscles takes place. If a filtrate is mixed with a saturated solution of salt and if this is evaporated at a low temperature the virulent bacteriophage may still be found on the crystals.

Sodium sulfate (anhydrous). Flu found that he could impregnate 
anhydrous sodium sulfate with a bacteriophage filtrate, and triturate the mass so obtained, without causing a destruction of the corpuscles. Bacteria also resist this treatment.

Copper sulfate. A 1 per cent solution of this salt destroys the bacteriophage (Shiga) in 4 days. ${ }^{321}$

Oxygen. Arloing, Langeron and Sempé ${ }^{21}$ have reported that shaking in the open air has no destructive effect upon the bacteriophage.

\section{ACTION OF ORGANIC COMPOUNDS}

Alcohol. Kabeshima has stated ${ }^{388}$ that the bacteriophage resists the action of alcohol for several months, but I can not accept this statement for I have found, as has everyone else who has worked on the subject, that the corpuscles are destroyed in less than 48 hours by 90 per cent alcohol. Indeed, de Poorter and Maisin ${ }^{532}$ report that in 94 per cent alcohol, and even in 70 per cent, destruction occurs in less than 24 hours. Appelmans ${ }^{13}$ states that a bacteriophage, originally active in a dilution of $10^{-10}$, was active only to a dilution of $10^{-7}$ after 6 hours of contact with 50 per cent alcohol. He found further that this titre remained intact for at least 20 days.

Watanabe ${ }^{619}$ has observed a variation among different races as to their resistance to alcohol, for according to his experiments some races were already destroyed after 2 hours in 50 per cent alcohol, while other races, a majority, were destroyed after contact for 20 hours. All of the races with which he worked were destroyed by 75 per cent alcohol within a period of 30 minutes.

Once more it appears that there are differences in susceptibility, and these variations must be associated with differences in virulence.

Bronfenbrenner and Korb ${ }^{99}$ have found that the bacteriophage was rapidly destroyed by absolute alcohol at ordinary temperature, but that it resisted for 5 or 6 days if held at $0^{\circ} \mathrm{C}$.

Considering all of these findings it appears that the results obtained by the different workers are not strictly in accord, a fact unquestionably referable to differences in the technic employed. The fact, as I have shown, that a pure filtrate introduced into alcohol presents a much greater resistance than does the same filtrate previously diluted with water is suggestive. The reason for this difference is certainly the fact that in the first case the protein materials (derived from the culture medium and from the dissolved bacterial bodies) are energetically coagulated, the bacteriophage corpuscles become enclosed in a mass of material which protects them from the action of the alcohol. This 
protective action is still more marked with acetone, the coagulating action is still more energetic and the antiseptic action is correspondingly less.*

In this connection, I might remark that all authors state that by reagents of this type the bacteriophage is first precipitated and then destroyed. This conclusion is of significance not only in connection with this particular experiment but in the case of all substances which coagulate proteins. Inasmuch as the corpuscles are always found in suspension in liquids which contain protein materials (even in the case where the culture medium is synthetic, for the dissolved bacterial substance is present) we can not tell whether the corpuscles are precipitated directly as such or whether they are carried down mechanically enclosed by the precipitate which forms in the medium. That the corpuscles as such may be precipitated is possible, even probable, but as yet we can not prove it.

Acetone. If we add $0.25 \mathrm{cc}$. of an undiluted suspension of the bacteriophage to $5 \mathrm{cc}$. of acetone we find that a destruction of the corpuscles occurs,-after 15 days for a Staphylo-bacteriophage, after 25 days for a Shiga-bacteriophage.

If this experiment is repeat $\mathrm{d}$, except that the acetone is combined with the same bacteriophage when diluted 1:1000 with slightly alkaline distilled water it is found that the destruction of both races of the bacteriophage is complete after 3 days.

Chloroform. I have found that when a suspension of Shiga- or Staphylo-bacteriophage is kept over a layer of chloroform a very definite attenuation may be noted even after $3 \frac{1}{2}$ hours.

Essences. The corpuscles maintain their virulence unimpaired when held for 8 days in media saturated with essence of thyme, or essence of cloves (d'Herelle ${ }^{321}$ ).

Phenol. The bacteriophage (Shiga) is destroyed after contact for 7 days with 1 per cent phenol (d'Herelle $\left.{ }^{321}\right)$. In a 10 per cent solution of this antiseptic it is destroyed in less than 24 hours. Watanabe ${ }^{619}$ has observed that the rapidity of destruction by phenol depends, in some measure, upon the race of bacteriophage tested. Acting over a period of 24 hours, a 1 per cent solution destroyed some races, while with other races it required a 2.5 per cent solution, or even a 4 per cent solution, to exert the same effect.

* On several occasions I have observed a curious fact, namely, that bottles of acetone were contaminated by $B$. subtilis, whose spores appeared to resist the action of this substance for a long time. 
Lactic and oxalic acids. These acids, in 2 per cent solutions, destroy the corpuscles in less than 24 hours (de Poorter and Maisin ${ }^{532}$ ).

Formol. Prausnitz and Firle ${ }^{539}$ obtained the following results when $1 \mathrm{cc}$. of a bacteriophage suspension was added to $1 \mathrm{cc}$. of a 3 per cent solution of formalin (formic aldehyde concentration, 0.6 per cent).

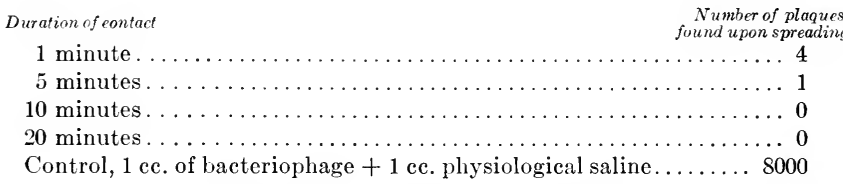

Antiformin. Destruction occurs within a few minutes in a 2 per cent solution (Seiffert).

Tetraline (hydrated derivative of naphthalene). The resistance of bacteriophage corpuscles to this antiseptic is essentially the same as that of anthrax spores (Prausnitz and Firle ${ }^{539}$ ).

TABLE 30

\begin{tabular}{c|c|c|c}
\hline $\begin{array}{c}\text { QUININE } \\
\text { HYDROCHLORIDE (10 PER } \\
\text { CENT SOLUTION) }\end{array}$ & PERIOD OF CONTACT & \multicolumn{2}{|c}{ RESULTS } \\
\cline { 2 - 4 } & & Plaques on agar & \multicolumn{1}{|c}{ In bouillon } \\
\hline$c c$. & hours & & \\
0 & 24 & $80-90$ & Dissolution \\
0.25 & 24 & $80-90$ & Dissolution \\
0.50 & 24 & $80-90$ & Dissolution \\
0.75 & 24 & $10-15$ & Dissolution \\
1.0 & 24 & None & No bacteriophagy \\
\hline
\end{tabular}

Lysol. Prausnitz and Firle ${ }^{539}$ have also tested this antiseptic, finding that the bacteriophage is a little more resistant than are the spores of B. anthracis.

Furfurol. Bacteriophage corpuscles are inert after exposure for 24 hours in a mixture of equal parts of corpuscle suspension and of 2 per cent furfurol.

Salts and derivatives of quinine. Eliava and Pozerski ${ }^{192}$ introduced 5 drops of a Shiga-bacteriophage suspension into $10 \mathrm{cc}$. of physiological saline and added to this dilution variable quantities of a neutral 10 per cent solution of quinine hydrochloride. Table 30 summarizes the results which they obtained.

They further state that the plaques obtained upon the agar spread from the control suspension and those derived from the first two dilu- 
tions were all of a diameter, about $2 \mathrm{~mm}$. The spreading corresponding to the $0.75 \mathrm{cc}$. dilution of the quinine salt gave, as indicated above, from 10 to 15 plaques, but here the plaques varied greatly in size, some having a diameter of about $2 \mathrm{~mm}$., others being far smaller.

But whatever may be the significance of this variability in plaque formation, it clearly appears that the bacteriophage is either destroyed or rendered avirulent after 24 hours in a 1 per cent solution of quinine hydrochloride.*

Just as for all antiseptic agents which have been tested, Watanabe ${ }^{19}$ found that different races of the bacteriophage show a variable degree of resistance for the salts of quinine. Destruction, or rather, as we have seen, avirulence is accomplished within 24 hours by the addition of from 5 to 50 per cent of a 10 per cent solution of quinine hydrochloride.

Wolff and Janzen ${ }^{628}$ have worked with a number of the quinine derivatives,--optochin, eucupine, vucine, and chinosol. To bacteriophage filtrates mixed with a suspension of a susceptible bacterium they added variable amounts of these antiseptics and found that at a certain concentration (with chinosol, 1:600) the mixture when spread on agar no longer gave plaques. At first sight it would seem that the corpuscles are here destroyed, but this is not the case. The corpuscles have simply become "latent." They have lost all activity for the bacterium, but they are not destroyed, for it is only necessary to add a drop of this mixture to a fresh suspension of the susceptible bacterium and to spread this over agar to obtain characteristic plaques. They performed this experiment with $B$. coli, B. typhosus, B. dysenteriae, and with the staphylococcus, using four races of the bacteriophage.

The result here is comparable to that which I observed with sodium fluoride, where also a quantity of antiseptic insufficient to kill the bacteria, even allowing their development, completely inhibited the bacteriophage corpuscles.

Malachite green, yatren, rivanol, and trypaflavine gave Wolff and Janzen similar results. And they further noted that in the presence of very minute quantities of the antiseptics (chinosol, $1: 4000$ ) the develop-

* Stimulated by these results reported by Eliava and Pozerski, BrocqRousseu, Urbain, and Forgeot have tested the activity of quinine salts for the streptococcus of equine strangles. They have seen that, just as with the bacteriophage, the streptococcus underwent an attenuation of virulence, passing to a complete lack of virulence, in the presence of quinine salts. The death of the streptococcus ultimately occurs, but only if the time of contact is prolonged beyond that necessary to cause a complete loss of virulence (Ann. Inst. Pasteur, $1923,37,322)$. 
ment of corpuscles appeared to be more active. This is not beyond reason, for we know, indeed, that very minute amounts of certain antiseptic substances exercise a stimulating action upon the growth of various species of bacteria.

Brutsaert ${ }^{104}$ reports that the corpuscles lose their virulence in 4 per cent solutions of quinine hydrochloride. Recovery of the lost virulence was slow; in his experiments it was not restored to its initial titre until after 20 to 22 passages.

Glycerol. The bacteriophage is destroyed by pure undiluted glycerol (d'Herelle and Bablet ${ }^{36,}{ }^{320}$ ). A mixture of 0.25 cc. of a bacteriophage (Shiga) and $5 \mathrm{cc}$. of anhydrous glycerol becomes completely inactive after 8 days if kept in sealed ampoules at a temperature of $37^{\circ} \mathrm{C}$.

Subsequent experiments have shown that the Staphylo-bacteriophage behaves just as do races active for the Shiga bacillus. The change brought about is a complete destruction, for it is impossible to restore the lost virulence by passages.

Proca ${ }^{542}$ suspended a mixed culture of bacteriophage- $B$. coli from agar in anhydrous glycerol and observed that after 24 hours the bacteriophage was destroyed. Transplants to bouillon gave normal cultures of $B$. coli. This would indicate that the bacteriophage is more sensitive to the action of glycerol than is the bacterium.*

Brutsaert $^{104}$ has also reported that the bacteriophage is destroyed by anhydrous glycerol.

In concluding this discussion of the effects of organic substances on the bacteriophage it may be added that Tomaselli ${ }^{599}$ states that filtrates of $B$. subtilis have no effect upon the bacteriophage corpuscles, and that Ionesco-Mihaiesti ${ }^{360}$ has shown that the corpuscles are unaffected by lecithin and cholesterol.

\section{VARIABILITY IN THE RESISTANCE OF THE BACTERIOPHAGE}

As first suggested by Otto, Munter and Winkler, ${ }^{496}$ and later confirmed by a number of authors (Bruynoghe and Brutsaert, ${ }^{118}$ Watanabe ${ }^{619}$ Reichert ${ }^{550}$ ), not only each race of the bacteriophage, but even each individual bacteriophage, behaves at different times in a different manner toward harmful agents, either physical or chemical. This individuality does not stop even there, for with a bacteriophage which is

* If this experiment of Proca is confirmed another method of purifying mixed cultures will be available. 
multivirulent the different virulences exhibited at the same time are not equally sensitive. Exposed to a given agent some of the virulences disappear, while others are retained (Reichert ${ }^{550}$ ), and recent investigations provide us with the reason for this diversity.

We can reveal the presence of the bacteriophage only through manifestations of its virulence. By the means now available the loss of virulence and destruction manifest themselves in the same manner, that is, by the lack of bacteriophagy. And yet, many experiments indicate that before an actual destruction occurs there is an attenuation of the bacteriophage or its virulences. When exposed to the action of many different physical or chemical agents, attenuation begins and progressively increases with the exposure so that with races having multiple virulences, the virulences disappear in the inverse order of their intensity, the weakest ones go first, the stronger ones next, and the chief and most intense one last. Up to the time when this chief one has disappeared interruption of the contact with the harmful agent leaves it still possible to restore the activity by the method of serial passages at the expense of susceptible bacteria. Working with Pozerski, I was the first to show this fact as it obtains in rejuvenation from the effects of temperature. But if the harmful action is prolonged too far, the primary virulence is lost and the loss is permanent. When this occurs is the bacteriophage dead? Does it still resist destruction for a time? It is impossible for us to tell.

\section{RÉSUMÉ}

The dimensions of the bacteriophage corpuscle are approximately those of the serum globulin micella $\left(\mathrm{d}^{\prime} \mathrm{Herelle}^{312}\right)$. Its diameter is about $20 \mu \mu$ (Prausnitz ${ }^{536}$ ). Its chemical reactions show that it is formed of protein substances (de Poorter and Maisin ${ }^{532}$ ).

The vitality of the bacteriophage corpuscle is very great (d'Herelle $^{321}$ ). Although the number of corpuscles diminish very considerably with preservation for several years, the viability is of much the same order as is that of bacterial spores.

The colloidal state is determined solely by physical properties, independent of the chemical constitution or of the living or inert state of a particle. These basic characteristic properties are insolubility and size. Inasmuch as the bacteriophage corpuscle possesses the properties which inevitably lead to the colloidal state, it naturally exists in this state. From the physical point of view the bacteriophage corpuscle is a colloidal micella. 
The bacteriophage corpuscle is flocculated by acids (da Costa Cruz ${ }^{156}$ ). It is adsorbed in an acid medium by kaolin and infusorial earth, but is not adsorbed by these substances in an alkaline medium (Gildemeister and Herzberg, ${ }^{235}$ Prausnitz and Firle $\left.{ }^{539}\right)$. It does not cause a reciprocal flocculation in the presence of electro-negative colloids; when admixed with such substances even bacteriophagy takes place normally (d'Herelle). All of its characters indicate that the bacteriophage corpuscle is the bearer of a negative electric charge (d'Herelle). It is an electronegative colloid, just as are the majority of bacterial species.

The bacteriophage corpuscle is rapidly destroyed by ultraviolet rays (Appelmans ${ }^{14}$ ). It is not destroyed by radium emanations (Brutsaert $\left.{ }^{105}\right)$. Here again it behaves like the bacteria.

Whatever may be the race of the bacteriophage, it becomes completely avirulent (or destroyed ?) at a temperature of approximately $75^{\circ} \mathrm{C}$. (d'Herelle and Pozerski ${ }^{360}$ ). The different corpuscles of a single suspension are inactivated by exposure to very different degrees of heating. With some an impairment in activity appears at a temperature as low as $45^{\circ} \mathrm{C}$. (De Necker ${ }^{476}$ ). The temperature of apparent inactivation is related to the virulence (Hauduroy ${ }^{288}$ ). With a single race of the bacteriophage having multiple virulences, these virulences disappear in the order of their intensity, the weakest first (d'Herelle).

When exposed to cold, the temperature of liquid air, experiment indicates that the resistance of young corpuscles is greater than is that of older ones (d'Herelle).

The resistance of bacteriophage corpuscles to the action of chemical agents is intermediate between that of vegetative forms and that of spores of $B$. subtilis (d'Herelle ${ }^{321}$ ). As for temperature, resistance is in direct proportion to the virulence, for with a single bacteriophage, the weak virulences disappear first (d'Herelle). 


\section{CHAPTER II}

\section{Hypotheses Concerning the Nature of the Bacteriophage}

\section{POSSIBLE HYPOTHESES}

We now know of what the phenomenon of bacteriophagy consists. We know that the agent which causes it exists in the state of invisible corpuscles, and that these corpuscles multiply during the course of their action, solely at the expense of living bacteria.

What can be the intimate nature of these corpuscles?

Let us first examine the general hypotheses which it is possible to formulate. There are three: namely,

I. Bacteriophagy may be caused by the presence of a foreign chemical principle, that is to say, a principle not derived from the bacterium which undergoes bacteriophagy.

II. Bacteriophagy may be effected through the action of a principle, either chemical or living, derived from the bacteria which undergo bacteriophagy.

III. Bacteriophagy may be caused by a living principle foreign to the bacterium.

These three major hypotheses cover all possibilities. Let us examine them in turn, and see which of them is acceptable, that is to say, which of them is not contradicted by the facts

\section{Hypothesis of a chemical principle foreign to the bacterium}

This hypothesis has been sponsored by Kabeshima. ${ }^{388}$ According to this author, there is within the intestinal tract of an animal, a catalyzer, derived without doubt from the leucocytes, which causes the dissolution of bacteria by activating some pro-ferment normally present in these bacteria.

We can not admit that a chemical principle, whether it be termed catalyzer or enzyme, derived from the tissues of an animal, is able to reproduce at the expense of bacteria, since this implies the power to transform "bacterium substance" into "catalyzer substance." To adopt this one must admit one of two things, either one must accept such a transformation and endow the catalyzer with the power of assimi- 
lation, that is, make it a living being, or one must assume the catalyzer to exist preformed in the bacterium. And if we accept this second interpretation we fall into the second of the possible hypotheses, which we will consider shortly. If on the other hand we can not admit either the one or the other of these two alternatives we are confronted by an absurdity, for such a chemical principle, incapable of reproduction, would quickly be eliminated in the course of successive passages. At that time when, because of dilution it would no longer be present as such, it would no longer be able to function, and serial activity would necessarily cease.

As a matter of fact, we can calculate at just what point in the successive passages every trace of the chemical principle introduced into the first suspension of the series would be eliminated. Obviously this is when there would remain less than the smallest possible particle of matter, that is, when the volume of the original material remaining is less than an electron, whose mass, as calculated by physicists, is equal to $10^{-27}$ grams.

To take a concrete example: Introduce into $10 \mathrm{cc}$, that is, 10 grams of a bacterial suspension, 0.001 gram of a fecal filtrate (or blood, or any other material containing the bacteriophage principle). The bacteria are dissolved. Then remove 0.001 gram of this dissolved suspension and add it to 10 grams of fresh suspension. Dissolution again takes place, and when finished again remove 0.001 gram and introduce it into a third 10 grams of bacterial suspension. Once more, a dissolution of the bacteria will take place. And in this way, as we already know, the passages can be continued indefinitely.

But, the 10 grams of the first suspension received $10^{-3}$ grams of the initial filtrate which contained the bacteriophage principle. A simple calculation shows that the second suspension inoculated can contain only $10^{-7}$ grams; the third only $10^{-11}$ grams, the fourth, $10^{-15}$ grams, and the seventh, $10^{-27}$ grams. This seventh suspension contains, then only $10^{-27}$ grams of the filtrate which introduced the bacteriophage principle into the first tube of the series. The quantity present is, at most, equal to a single electron. With the eighth passage, this quantity would fall to $10^{-31}$ grams, a quantity virtually representing less than nothing, since it is less than an electron. Nevertheless this eighth suspension is dissolved, just as will be the thousandth, or the two thousandth. How can this phenomenon continue to take place since the catalyzer or the enzyme,-chemical substances, introduced originally by the filtrate obtained from some substance from the body,-totally disappeared as far back in the series as the eighth passage? 
Those hypotheses, of whatever nature they may be, which attempt to attribute the phenomenon of bacteriophagy to the introduction into the first suspension of the series of some "chemical principle" foreign to the bacterium which undergoes lysis, whether this principle is designated by the name catalyzer, soluble ferment, or by any other name, is, from a mathematical point of view absurd, and consequently, can not be accepted.

\section{Hypothesis of a principle derived from the bacterium}

Expressed in this way, in general terms, this hypothesis admits of four solutions. Manifestly, in the first place, the principle may be an abnormal product elaborated by the bacterium, in which case it can only function as a pathological ferment, or it may be a normal physiological product present in all bacteria.

Upon further analysis each of these two concepts may be interpreted in two different ways. The principle elaborated by the bacterium, whether it be physiological or pathological, may be assumed to be either inert or living.

The general hypothesis, therefore, consisting of the concept that bacteriophagy is a phenomenon of dissolution caused by a principle elaborated by the bacterium itself, is open to any one of the four solutions:

A. The principle is abnormal and inert. If this is true it can only be an "autolysin" resulting from a vitiation of the bacterial metabolism.

B. The principle is abnormal and living. In this case it can only be a fragment of the bacterium freed at the moment of dissolution, and incidentally it must be a fragment which retains its eapacity to multiply.

C. The principle is normal and inert. It is then an autolytic enzyme elaborated by all bacteria.

D. The principle is normal and living. Here again it must still be a fragment of the bacterium, retaining the property of multiplication, and a normal constituent of all bacteria.

All of these hypotheses, with the exception of the second, have found supporters. The question of the bacteriophage has stirred up such diverse and singular conceptions that hardly anything can be said which has not had its day. But let us consider these four interpretations, and see how well they accord with the facts.

A. Hypothesis of an abnormal inert principle. The first to adopt this concept were Bordet and Ciuca..$^{85,86}$ Their theory comprised two ideas; first, under the action of some product elaborated by the leucocytes the bacteria undergo a nutritive vitiation leading to their dissolution, ${ }^{85}$ 
and second, the nutritive vitiation is hereditary, a conception rendered obligatory to explain the continuity of action in vitro, since obviously the original leucocytic product must disappear in the course of successive passages. Let us consider the rational basis of these two ideas, as well as the experimental data leading to their formulation.

1. Bordet and Ciuca gave guinea-pigs several intraperitoneal injections of a culture of $B$. coli. A few hours after the last injection they removed some of the peritoneal exudate and demonstrated that it contained a principle lysogenic for B. coli. Thus, they concluded that the primum movens in transmissible bacterial dissolution resided in the leucocytes. In their first communication, they announced the early publication of comparable experiments carried out with other bacterial species. This publication has never appeared, doubtless for good reasons.

Let us observe first that such an experiment does not prove the point. The bacteriophage principle may always be found in the intestine, and, we know that normally, the intestinal bacteria can gain entrance to the circulation. In the horse, for example, we know that during the digestive period the blood always contains bacteria, $B$. coli in particular. It may be, then, quite normal for bacteriophage corpuscles to penetrate the walls of the intestine and appear in the peritoneal cavity because of the irritation induced by the injection of bacteria (d'Herelle, ${ }^{336}$ ).

But this same experiment, the result of which is so inconclusive, does not constantly give the results described (d'Herelle ${ }^{328}$ ). I have attempted to repeat it, to satisfy myself as to the reasons why Bordet has not published further results on this phase of the subject. Upon several occasions, I have subjected guinea-pigs to repeated injections of $B$. coli, B. dysenteriae, or B. typhosus, but in no case have I been able to obtain an exudate containing the dissolving principle.

Furthermore, if the primum movens of the phenomenon of bacteriophagy is resident within the leucocytes, it should suffice to take the leucocytes of an experimentally hyperimmunized animal, to introduce them into a bacterial culture and thus provoke the phenomenon. Experiment shows that this is impossible. I have tried several times to isolate a bacteriophage principle from the leucocytic layer which rests on the clot after coagulation of the blood of horses which were producing anti-dysentery serum, that is, horses which had been receiving for several months, and in some instances for years, injections of dysentery bacilli. Without exception it has been impossible to detect in such 
material a principle causing the dissolution of dysentery bacilli $\left(\right.$ d'Herelle $\left.^{336}\right)$.

2. As for the theory offered by Bordet and Ciuca to explain serial action, it is built up entirely on the dictum that, the "nutritive vitiation" is "hereditary." The significance of such a statement is revealed by the following facts.

When a trace of a filtrate containing the bacteriophage principle is added to a suspension of susceptible bacteria the bacteria are dissolved. The limpid material thus formed is filtered through a porcelain candle impermeable to bacteria. A trace of the filtrate so obtained, is introduced into a second suspension containing only fresh normal bacteria, and their dissolution follows. This second suspension, now that it in turn has become limpid is filtered, and a trace of the filtrate is added to a third suspension, again containing only normal bacteria. This results in their dissolution. And this process can be continued in this way, serially, for an indefinite period.

Filtration through a porcelain candle intervening between each passage renders it certain that, with the trace of filtrate, none of the bacteria whatever of the suspension previously dissolved are passed on to the suspension into which this trace of filtrate goes, to later produce dissolution. Since none of the bacteria of the suspension which was apparently dissolved were transported into that which is next to undergo bacteriophagy where is the opportunity for the transmission of an acquired character? How can one invoke heredity when there are no descendents?

How, then, can Bordet and Ciuca expect us to believe that an hereditary character can be excreted in a soluble state, that this character can be passed through a porcelain filter, that it can maintain itself in a fluid, increasing and decreasing in potency, and that it can be communicated to a healthy bacterium by simple contact with this liquid? I think that it is needless to discuss such a theory which, although it has not been explicitly stated in these terms by Bordet and Ciuca, is the only one to accord with experimental facts and observations, if, in spite of everything, they would have us support the idea that the phenomenon of bacteriophagy is caused by an hereditary nutritive vitiation of the bacteria.*

It has seemed wise to some authors to vary this theory somewhat. Thus, Kuttner ${ }^{399}$ would derive the nutritive vitiation of the bacterium

* Bordet seems to have abandoned his hypothesis. Others, Ciuca for example, continue to support it, without, however, considering in any way the arguments, such as are here presented, which were published in $1923 .{ }^{347}$ 
from the action of some ferment originating in the intestinal tract or tissues of the animal. Lisbonne and Carrère ${ }^{420}$ would invoke a "bacterial antagonism."*

Seiffert ${ }^{578}$ is also the author of an hypothesis, according to which the phenomenon takes place through the action of some chemical substance outside of the bacterium. The facts show, he says, that bacteriophagy is effected through the action of a substance which is outside of the bacterium, hence it must necessarily be an "exogenous autolysis," due to an exogenous autolysin. This is all very simple. But words can not be accepted in the place of proof. It is desirable, at least, to explain in a plausible manner what this exogenous substance is and how it reproduces.

Doerr ${ }^{178}$ likens the bacteriophage principle to a toxin exercising its action upon the bacterial metabolism. This toxin should be regenerated by the diseased bacteria, standing thus, he says, in analogy with the phenomenon described by Peyton Rous as taking place in connection with chicken sarcoma. There is some logic in Doerr's point of view, but as a matter of fact it is not proved that the "cause" of the sarcomatous process is not a living being, an ultravirus which reproduces at the expense of the diseased cells. $t$ To establish an assumption of this kind it is first necessary to demonstrate that the sarcoma is caused by a toxin. However, Doerr states explicitly that the hypothesis of an autolysin is not plausible. Consequently, he admits that the something which he calls a toxin is a principle foreign to the bacterium, endowed with the power of multiplying at the expense of this bacterium, that is to say, he admits that the action continues in series because, in the course of each passage, "bacterial substance" is transformed into "lysogenic substance." If this "lysogenic substance" is living, we can comprehend very readily that it may be able to transform "bacterial substance" through a phenomenon of assimilation into the material of which it is itself composed. But Doerr assumes that it is an inert substance. Consequently it is essential to show that such a transformation is pos-

* I have shown ${ }^{337}$ that one of the two bacteria which, as they say, react on each other, must be in reality a culture admixed with the bacteriophage. Beckerich and Hauduroy, ${ }^{59,60}$ and later Bordet ${ }^{81}$ have observed the same thing. Lisbonne and Carrère seem, as a matter of fact, to have abandoned their hypothesis, for they have not replied to these contradictory observations.

Fabry and van Beneden, ${ }^{195}$ who had accepted the point of view of Lisbonne and Carrère, later recognized the fact that one of the two bacterial species involved in such an experiment was contaminated by the bacteriophage, and they have now adopted the hypothesis of the living nature of the bacteriophage.

$\dagger$ Leucocytes according to the work of Carrel. 
sible, and that this transformation can take place without the intervention of assimilative phenomena, for, assimilation being a physiological characteristic of life, a substance which assimilates is alive.

However, Doerr is consistent. He admits that the bacteriophage is an ultravirus to just the same degree as are the agents of rabies, of vaccinia, of encephalitis lethargica, and of sarcoma, but in his opinion all of these ultraviruses are simply products elaborated by the diseased cell. The demonstration of the contrary will be given in a later chapter.

Despite all these new terms, this is but a resurrection of the old theory of Stahl "all bodies brought to a state of putrefaction, readily transmit this state to another body as yet free of corruption." This theory of the multiplication of a principle by communicated motion was supported by Liebig in his famous discussion with Pasteur on the mechanism of fermentation. Pasteur demonstrated experimentally that such a conception was false, and it would have seemed that the facts were definitely accepted. Whether it be vital phenomena, or communicated motion, the discussion and the demonstrative experiments are based upon the same ideas; it is simply a case of descending a step or two in the scale as regards the magnitude of the heings involved.

Whatever may be the words used to clothe the hypothesis that bacteriophagy is caused by an abnormal inert substance derived from the bacterium, it will always be impossible to explain how it leads to a serial vitiation of the bacterial metabolism without the intervention of a foreign principle, functioning as a cause and perpetuating itself through the passages. Bordet clearly realized the necessity for such an explanation, and he provided the only one which can account for the serial vitiation in the bacterial metabolism without the intervention of a foreign factor. But his explanation does not accord with the facts, hence it can not be accepted.

In $1923^{347} \mathrm{I}$ published the arguments refuting the hypothesis, in the form in which it was developed, explaining bacteriophagy as being due to the action of an "abnormal autolysin." These arguments have since been repeated..$^{349,354}$ None of the adherents of this hypothesis have disposed of my objections, nor have they made any mention of them.

B. Hypothesis of a living abnormal principle, derived from the bacterium. As I stated above, an hypothesis of this nature has not yet been formulated. Despite its strangeness, it would not be, however, any more illogical than the preceding. Basically, it would be even less so.

If, at last, such an hypothesis is advanced, it obviously will be sub- 
ject to the same criticisms that have been directed toward the preceding hypothesis.

C. Hypothesis of a normal autolysin. This hypothesis together with that assuming the living nature of the bacteriophage, has shared in attracting the greatest number of supporters.*

The following have definitely declared themselves as being in favor of the idea of a normal autolysin: Wollstein, ${ }^{637}$ Gildemeister, ${ }^{22}$ da Costa Cruz, ${ }^{150}$ Davison, ${ }^{171}$ Otto, Munter, and Winkler, ${ }^{492}$ Bachmann and Aquino, ${ }^{39}$ Pico, ${ }^{518}$ Miessner and Baars, ${ }^{454}$ Weinberg and Aznar, ${ }^{623}$ Kraus and Marais, ${ }^{396}$ Ledingham, ${ }^{403}$ Ellis, ${ }^{193}$ Werthemann, ${ }^{626}$ Jötten, ${ }^{379}$ Proca, ${ }^{541}$ Borchardt, ${ }^{73}$ Putter and Vallen, ${ }^{54 t}$ Lepper, ${ }^{409}$ Meuli, ${ }^{452}$ Scheidegger, ${ }^{568}$ Eastwood, ${ }^{189}$ v. Angerer, ${ }^{9}$ Liebermann, ${ }^{417}$ Fejgin, ${ }^{198}$ Hadley, ${ }^{278}$ Arkwright, ${ }^{17}$ Marcuse, ${ }^{436}$ Schnabel, ${ }^{571}$ Yasaki, ${ }^{6.11}$ Petrovanu, ${ }^{502}$ Arnold and Weiss, ${ }^{25}$ and Ionesco-Mihaiesti. ${ }^{366}$

What are the arguments which have been advanced by these authors, particularly with reference to the fact that bacterial strains may be found containing the bacteriophage principle?

$A$ priori the idea of an autolytic process is not, indeed, absurd, for we know, as a matter of fact, that all cells contain within themselves ferments capable of dissolving the cellular body. It is well, however, to recall that under normal conditions of existence, it is rare to obtain a total autolysis, as a rule the autolytic process leads simply to a disaggregation involving but a limited number of cells. The dissolution is incomplete, and the process ends in the formation of an insoluble amorphous mass. Such is the case, and others might be mentioned, for autolysis of B. dysenteriae, B. coli, B. pestis, Staphylococcus albus and aureus, etc. Cultures or suspensions of these bacteria held in bouillon for even several months, contain amorphous particles, together with a great number of bacteria, dead but still intact. As a matter of fact, the culture is almost as turbid as it was at the beginning. The phenomenon of bacteriophagy is very different, for within a few hours all of the bacteria are completely dissolved, without residue of any kind, and the medium becomes as clear as uninoculated bouillon.

Furthermore, it is significant that a natural spontaneous autolysis is never observed with young bacteria, while bacteriophagy takes place

* It is interesting to see how those who support one or the other of these two hypotheses are distributed. In general, Anglo-Saxon students favor the autolytic hypothesis, while those using the Latin languages (with the exception of the South Americans) are, with but few exceptions, in favor of the hypothesis of the living nature of the bacteriophage. The Germans are divided, some favoring the first, some the second concept. 
with the greater vigor the younger the bacteria and when the conditions for life are at their optimum. And, as we know, the phenomenon takes place poorly, or may not occur at all, with suspensions of old bacteria.

The partisans of the autolytic theories are then, it seems to me, confronted by an obligation to explain the paradoxical fact presented by their conceptions, namely, that the phenomenon of bact eriophagy occurs with young bacteria which have no natural autolytic tendency, and does not take place with old bacteria which autolyse spontaneously.

But despite these contradictions, the fact that normal bacteria contain autolytic ferments makes it imperative that we analyse this hypothesis further. It is necessary to demonstrate, not that such an hypothesis is improbable, but that it is impossible. I am the more interested in this hypothesis, since when I was the only one interested in the phenomenon of bacteriophagy, and long before any of this discussion developed, I carefully considered this hypothesis as a possible explanation of the process which I was studying, ${ }^{315}$ only to reject it because it was contrary to the experimental facts. Although rejected at that time because it failed to accord with certain demonstrated experimental facts, the proof of its inadequacy is today on a distinctly different plane, for we are now able to present direct evidence, demonstrating that the lysogenic principle, the cause of bacteriophagy, is distinct from the autolysins and that it can not be present in a normal bacterium.

Let us consider first the validity of the argument upon which the hypothesis explaining bacteriophagy as being due to the action of a normal autolysin has been built.*

The argument advanced is correct, but it is open to several interpretations. Beyond question it is possible to isolate, from some bacterial strains, a principle which causes the phenomenon of bacteriophagy. Indeed I have reported that such an isolation is possible, and I was the first ${ }^{321}$ to show how such mixed bacterial strains could almost uniformly be obtained. It is only necessary to use strains of $B$. coli derived from cases of infection of the urinary tract. This observation has been since confirmed by several author's (among others, Tomaselli ${ }^{599}$ and Hauduroy. ${ }^{320}$

The question of natural mixed cultures has been discussed at length in

* The hypothesis of "splitter" of Bail can be refuted on the same grounds as that of an autolysin. 
Chapter V, where we found that even those who support the autolytic theory agree that bacterial strains from which it is possible to isolate a bacteriophage are the exception rather than the rule. The presence of the principle in the bacterium is, therefore, an abnormal occurrence, a point which causes the argument to lose much of its force, even reacting against the hypothesis which it pretends to support; for this fact suggests the idea that the "anomaly" may, indeed, be a principle foreign to the bacterium, that it is, in a word, a contamination of these rare "lysogenic" strains.

Is this hypothesis involving a possible contamination biologically absurd? By no means. For we find throughout nature many instances of the same type. We know, for example, that Paramecium bursaria, and Hydra viridis, are normally parasitized by a minute green alga, Zoöchlorella vulgaris. It is very rare to find paramecia which are free. It is even possible to carry the contaminated paramecia through successive generations and demonstrate, after a number of such passages, both the paramecia and the parasitizing Zoöchlorella in the cultures. If by chance some uncontaminated paramecia may be secured from some uncontaminated outside source it is only necessary to place them in a jar containing contaminated paramecia to have them all, in turn, become parasitized. And although this contamination becomes, in a sense, hereditary, it does not interfere with the normal reproductive capacity of the paramecia. Whitney* has succeeded in curing infected hydra by adding glycerol to the water to a concentration of 1 per cent. In this solution the hydras expelled the Zoöchlorella and acquired an immunity, for when re-transferred to some plain water containing the Zoöchlorella, they were not reinfected. Paramecia are, therefore, able to live without the Zoöchlorella, but the reciprocal of this is not true, for Zoöchlorella is an obligate parasite able to develop only within the host.

Another example may be mentioned. The Myxobacteria form a group of symbiotic beings, which may be cultivated serially, but after any number of cultivations whatever a Myxomycete and a bacterium will always be found in association. $\dagger$ The Myxomycete feeds upon

* Whitney, D. D.-Artificial removal of the green bodies of Hydra viridis. Biol. Bull., 1907, 13, 291: Further studies on the elimination of the green bodies from the endoderm cells of Hydra viridis. Ibid. 1908, 15, 241: See also

Caulery.-La symbiose chez les animaux. Bull. Inst. Pasteur, 1921, 19, $569 ; 617$.

† Pinoy, E.-Rôle des bactèries dans le dèveloppment de certains Myxomycètes. Ann. Inst. Pasteur, 1907, 21, 622; 686 . 
and develops at the expense of the bacterium, but this does not prevent an indefinite continuance of the symbiosis. By isolation procedures it is possible to obtain either pure bacteria, or a suspension containing only the spores of the Myxomycete, but while the bacteria can be cultivated in pure culture indefinitely, the spores of the Myxomycete will not germinate and will not give birth to vegetative forms unless the bacteria are present.

I emphasize these examples of symbiosis in which, despite the parasitism of the one of the two constituents, subcultures in series give symbiotic cultures indefinitely, for they show that, even if bacterial species normally contained the bacteriophage principle, it could not be interpreted as absolute proof that the principle is derived from the bacterium as such. It would still be necessary to exclude the chance that this was not simply another example of the symbiotic relationship, a symbiosis between bacteriophage and bacterium, both beings being alive.

The examples cited above offer a most perfect resemblance to the situation existing when a bacterial strain is contaminated by bacteriophage corpuscles. As stated above it is quite possible to interrupt the symbiosis between Zoöchlorella and Paramecium or between Myxomycete and Bacterium. And in the same way the symbiosis between bacteriophage and bacterium ean be interrupted, simply by applying the usual bacteriological procedure for the purification of bacterial cultures, that is, the method of colony isolation. After a series of two or three transfers by means of isolated colonies, often even after the first, the isolated colony will yield normal bacterial cultures no longer admixed with the bacteriophage principle.

Thus, we see, that, on the one hand, the implantation en bloc of a bacterial culture containing the lysogenic principle gives an indefinite series of cultures containing this principle, and that, on the other hand, a simple seeding by means of isolated colonies is adequate to break this symbiosis, for the re-seedings of isolated colonies give normal bacterial cultures. There is an absolute parallelism between symbiosis involving the Myxomycete and the Bacterium, and that involving the Bacteriophage and the Bacterium. The method of purification is the same in both cases, and in both cases also, the normal bacterium can be further cultivated alone in series, while the parasite, whether it be the Myxomycete or the Bacteriophage, can be cultivated only in the presence of the bacterium at whose expense it reproduces.

It would require volumes to exhaust this question of symbiosis in nature. The phenomenon is a general rather than an exceptional occur- 
rence. And its importance is very considerable, for symbiosis is in large measure responsible for evolution.* The term symbiosis is, however, rather poorly chosen, for it dates from an epoch when it was thought that the communal existence of the two beings necessarily involved a mutual benefit. We now know that symbiosis is always a struggle, which, as a rule, ends in the elimination of one of the two contestants.

But the history of science presents a very curious thing in the discussions which crop up each time that a new example of symbiosis is discovered. The last one, prior to the one now engaging our attention, related to the lichens. It is unwise to go into details which would carry us too far afield, although the alga-fungus symbiosis, constituting a lichen, is in all respects comparable to that between the bacterium and the bacteriophage It is sufficient to recall that when Schwendener had shown that the being which was thought to be an individual was in reality dual in nature, there were impassioned discussions, and Nylander, the most noted lichenologist of the day, refused up to his death to accept such a manifest heresy.

A mixed culture, comprising bacterium and bacteriophage, is in reality a culture of a microscopic lichen. A bacterium in symbiosis with a bacteriophage is a "microlichen." And it would not be strange (I say this advisedly, for, although as yet incontrovertible evidence is lacking, I have reason to suspect such a thing) if certain bacterial species may be recognized only when in the form of "microlichens," that is, as mixed cultures.

We have seen from our study of mixed cultures that it is experimentally easy to transform a normal culture into a "lysogenic" culture, the only essential being to transfer, en bloc, a secondary culture. Can such strains be purified? As we know it is only necessary to transfer them through individual isolated colonies and the majority become ultrapure.

Nevertheless, if we permit a secondary culture to age for several months, we find that the "bacteriophage-bacterium" symbiosis becomes more and more intimate. At the outset, if 20 colonies are isolated from such a secondary culture, one only (sometimes none) may be mixed, the other 19 being ultrapure. With an old mixed culture, the percentages are reversed, the mixed colonies greatly outnumber the ultrapure colonies.

For two years I have maintained two mixed cultures (derived from

* The study, as yet hardly touched upon, of the mutations which take place in the course of the Bacteriophage-Bacterium symbiosis, is of a nature to throw much light upon the phenomena of evolution. 
transplants of experimentally produced secondary cultures), one of bacteriophage-staphylococcus, the other of bacteriophage-coli, by transferring them en bloc on agar about once a month.

After two years, the cultures have an absolutely normal appearance, but filtrates from a transfer made in bouillon invariably cause bacteriophagy of a pure culture of the homologous susceptible bacteria. Butit has been impossible to obtain ultrapure colonies by agar isolations. With these old secondary cultures all colonies give cultures from which may be isolated a bacteriophage, showing thus that every bacterium in the mixed culture is contaminated. In order to derive an ultrapure bacterium from these it has been necessary to have recourse to the method described above, namely, effecting a series of cultures at a limiting temperature in a bouillon acidified to the growth limit. After this treatment an ordinary isolation on agar has yielded ultrapure colonies. The symbiosis becomes more and more intimate, therefore, as time passes, but a separation is always possible.

Could it not be assumed that the "bacteriophage-autolysin" exists in all bacteria even though in all of them it can not be demonstrated? The fact that bacterial strains can be purified already shows that this can not be true, but it is possible, in still another manner, to show that such an interpretation is merely a fabric of words. Subject a bacterial culture to the action of a virulent bacteriophage. Bacteriophagy takes place and this dissolution, say the adherents of the autolytic theory, is brought about through the action of an autolysin contained in the bacteria. If this explanation is correct, a normal culture of this same bacterium should contain it, for not to admit this is to imply that bacteriophagy is caused by something other than an autolysin. This question may be investigated by a method which leaves no question as to the significance of the results obtained.

Jaumain $^{377}$ has recently described a procedure which leads to the complete autolysis of several bacterial species, of the staphylococcus in particular. He prepares a bouillon suspension of bacteria removed from an agar slant and introduces this suspension into a test tube which is then sealed in the flame, making every effort to have as little air as possible remain in the sealed tube.

Several times I have attempted to perform experiments leading to a complete autolysis in order that I might compare a true autolysis with the autolysis induced by the bacteriophage, but my plans never succeeded, for the simple reason that, under natural conditions, autolysis does not take place with the species of bacteria for which I have up to 
the present time isolated active bacteriophage races. Consequently it was impossible for me to compare the phenomenon of bacteriophagy with an autolytic process which did not occur The new method of Jaumain, effecting autolysis in a limited amount of air, now renders such a comparison possible since it may be applied to the staphylococcus for which effective bacteriophages are available. It is only fair to add that Jaumain, a collaborator of Bordet, was the first to note that the liquid resulting from autolysis in confined air does not cause a dissolution of a fresh suspension of the homologous bacteria.

I have performed the experiment according to Jaumain's technic using strains of the staphylococcus for which I had a very active bacteriophage. This, naturally, anticipates any objection that the strains of the staphylococcus used were of a type which would not lend itself to bacteriophagy.

The experiment was performed with three different strains of the staphylococcus, and for each I demonstrated:

First, that suspensions in bouillon remained indefinitely turbid ( 6 months) and that such suspensions filtered serially did not cause the phenomenon of bacteriophagy; they were, therefore, not contaminated by a bacteriophage principle;

Second, that suspensions in bouillon were dissolved completely in less than 36 hours through the action of an added bacteriophage principle, the phenomenon being reproducible in series indefinitely, either with or without filtration between each passage. The dissolution was always complete, without any residue.

Pure suspensions of these staphylococcus strains in bouillon, without the addition of any substance whatever were sealed in tubes with a limited amount of air. Within a period of from 2 to 4 days at $37^{\circ} \mathrm{C}$. they were completely autolyzed.

Everyone who has wished to liken the phenomenon of bacteriophagy to autolytic processes has assumed, and, indeed, they could offer no other explanation, that the bacteria in being dissolved through the action of a filtrate, liberate into the medium the autolysin which they contained. Autolysis in confined air is induced necessarily by the action of an autolysin normally present in the bacterium and this autolysin must be liberated into the medium when the bacteria undergo dissolution.

This is, then, the autolytic process which has been advanced as an explanation of the phenomenon of bacteriophagy, and it is evident that if this hypothesis is correct, the limpid liquid which is obtained when 
autolysis is once completed ought to effect the phenomenon of bacteriophagy. But experiment shows that this liquid is absolutely inert. Bouillon seeded with the homologous staphylococcus yields, despite the addition of any quantity of the autolysis fluid, a normal culture, comparable in all respects to a control culture made by implanting the staphylococcus into plain bouillon. A suspension of the staphylococcus to which the autolysis fluid, filtered or unfiltered, is added in any quantity whatsoever, behaves just as does a normal control suspension. Spread over an agar surface it gives a normal culture, never showing any signs of the plaques characteristic of bacteriophagy.

These experiments show by a direct method that the bacteriophage principle is not an autolysin and that it is not present in the normal bacterium.

But, you may say, no doubt this is true, it may very well be that bacteriophagy is not a "normal autolysis," nevertheless bacteriophagy may occur as the result of some phenomenon in which autolysins intervene. Indeed! This is an entirely different affair, and is an hypothesis which we are quite willing to consider. But, however that may be, it is certain that the principle which "provokes" bacteriophagy is not a constituent of the normal bacterium; the experiments recorded above show this conclusively.

Very recently Gildemeister and Hermann thought that they had, at last, disclosed a fact supporting the idea that the bacteriophage is produced by the bacterium. They isolated a strain of $B$. coli which was "lysogenic;" whose filtrate caused an intense bacteriophagy of $B$. dysenteriae. But this took place only when they cultivated the colon bacillus at a temperature above $15^{\circ} \mathrm{C}$. In cultivations below this temperature they could not demonstrate the lytic principle. And so, they reasoned, if we make a series of cultures at $10^{\circ} \mathrm{C}$. we will lose the strain of bacteriophage and then if we subsequently cultivate it at $37^{\circ} \mathrm{C}$. the cultures should no longer be lysogenic. But, they found, on the contrary, that under these conditions the later cultures were perfectly lysogenic. From these olservations they concluded that the bacteriophage is a product of bacterial metabolism, produced only under restricted conditions, insofar as temperature is concerned, between $15^{\circ}$ and $37^{\circ} \mathrm{C}$.

The experiments of Gildemeister are always very well executed, but he should, nevertheless, employ methods beyond criticism. The method of isolating the bacteriophage which he employed is defective, and has frequently led to erroneous conclusions. To determine whether his 
cultures grown at $10^{\circ} \mathrm{C}$. were free of the bacteriophage he heated them at $58^{\circ} \mathrm{C}$., and as he was unable to reveal a bacteriophage after such treatment he concluded that it was not present. Such an assumption is not valid. In an earlier chapter some experiments have been detailed which show that the bacteriophage is attenuated at even lower temperatures than $58^{\circ} \mathrm{C}$. I would state once more that any method of isolation involving heating should never be used in important experiments if one wishes to avoid a very important source of error. Flu has informed me that he has repeated Gildemeister's experiments with the same strain of $B$. coli, (sent to him by Gildemeister) obtaining quite different results. His technic, however, differed for he used filtration in the place of heat, for isolating the bacteriophage. He found that after a series of ten successive cultivations at $10^{\circ} \mathrm{C}$. the filtrate prepared from the last culture contained a bacteriophage. Obviously this renders the conclusion of Gildemeister subject to revision. What happened in the original experiments was that by growth at $10^{\circ} \mathrm{C}$. an attenuation occurred, so that the bacteriophage became completely avirulent when heated to $58^{\circ} \mathrm{C}$.

The only thing disclosed by Gildemeister's experiments is that the bacteriophage, which as we know undergoes an attentuation when cultivated at high temperatures, is also attenuated when grown at a low temperature.

And finally, in order to finish with this question, it may be stated that some of the supporters of the autolysin hypotheses have, as a matter of fact, realized that they are inadequate to explain the facts, for bacteriophagy has no single character in common with autolysis. Thus, Otto and Munter ${ }^{496}$ have suggested that the normal autolysin may be enhanced by passage through filters of porcelain or by heating. This again is an explanation which does not conform to the facts, for with a bacteriophage of maximum virulence, bacteriophagy takes place without the subsequent development of secondary cultures, and with such a race it is possible to continue serial bacteriophagy indefinitely, inoculating each fresh bacterial suspension with a trace of the suspension previously bacteriophaged, without ever bringing the active principle in contact with a filter and without ever subjecting it to heat.

Experiment shows, therefore, that the principle responsible for bacteriophagy is not a constituent of normal bacilli; hence it can not be an autolysin. Does an autolysin play any part in the phenomenon? This is quite possible. But however that may be, the principle which is the essence of the phenomenon, which assures the continuity in 
series, is not itself an autolysin, since it is not present in the normal bacterium.

D. Hypothesis of a living principle, normally present in the bacteria. This is the hypothesis of Bail. I have been fortunate to find a résumé of this theory in a communication of this author recently published,,$^{50}$ for I confess that I would have been embarrassed to explain it. Here is the résumé.

Drawing an analogy with superior cells, there takes place in them two processes, especially in that which concerns the chromatin, the dissolution of the old substance and the reconstruction of that of the new cells. As these processes are always linked to changes in the chromatin, one has a right to attribute a causal relationship to the activated chromatin and to attribute to it the capacity of dissolution and of reconstruction. One might admit that these capacities may be separated in inferior cells. If, for some unknown reason, the capacity for reconstruction is lost, that of dissolution may remain active. In such a case the cellular elements would be dissolved, while their reproduction would be impossible. The chromatin continues but through this modification it has acquired new properties. It has become bacteriophage. In the presence of new cells in the process of multiplication, two phenomena occur, union with the bacteriophage followed by action of the bacteriophage. The new chromatin has two properties, dissolution and reconstruction, and now adding that property of the bacteriophage, dissolution, there will now be operative two dissolutions for each reconstruction. This last is not possible, hence the chromatin passes into the bacteriophage state. Consequently the latter increase in numbers.

As for chromatin it is necessary to consider the elements, the chromosomes, which are, in a general way, the elements of the hereditary substance. These elements are attacked individually by the bacteriophage. If, by this action, a chromosome, for example, is deprived of its chromatin two possibilities arise; either the cell can not live without it, in which case it dissolves, or, life is possible, in which case it becomes bacteriophage-resistant. This means that the bacteriophage no longer finds in this chromatin the element which it can attack; it is no longer itself, but something else. The more chromosomes, the more bacteriophages theoretically possible.

The more phrases, the more hypotheses, each one the more improbable than the others; they are cumulative. It is needless to enter here into a discussion of this hypothesis. The facts which have already been detailed in opposing the preceding hypothesis, and those which will be presented in the next chapter show that Bail's theory, as well as the other concepts which would derive the bacteriophage principle from the bacterium itself, "do not choose to be hampered by the facts."

Bail, and his pupils Nakamura, Watanabe, and Matsumoto (although these last have not ventured to clearly explain the above) are the only partisans of this strange theory. 


\section{The bacteriophage is a living being, foreign to the bacterium}

This is the hypothesis proposed in my first publication. I had, indeed, considered the hypothesis of a fermentative action, and I had discussed it even before any one else had advanced $i^{315}$ but since it did not conform to the facts it was rejected.

Let me first list the declared supporters, or those who have recognized that the theory which considers the bacteriophage as a living being, foreign to the bacterium is the one which best conforms to the facts. The list includes: Bablet, ${ }^{36}$ Gjørup, ${ }^{237}$ Eliava, ${ }^{359}$ Pozerski, ${ }^{191}$ Izar, ${ }^{367}$ Bruynoghe, ${ }^{113}$ Beckerich, ${ }^{57}$ Hauduroy, ${ }^{288}$ Janzen, ${ }^{374}$ Wolff, ${ }^{628}$ Polettini Bruno, ${ }^{529}$ Védrenne, ${ }^{610}$ Bastin, ${ }^{55}$ Prausnitz, ${ }^{536}$ Asheshov, ${ }^{27}$ Beijerinck, ${ }^{64}$ Kropveld; ${ }^{397}$ Tomaselli, ${ }^{599}$ Flu, ${ }^{216}$ Brutsaert, ${ }^{104}$ Caublot; ${ }^{140}$ Sardjito, ${ }^{566}$ Pondman, ${ }^{530}$ Philibert, ${ }^{509}$ Fabry and van Beneden, ${ }^{196}$ Reichert, ${ }^{550}$ Meissner, ${ }^{448}$ Kabelik, ${ }^{384}$ Schuurman, ${ }^{573}$ and Preisz. ${ }^{540}$ Maitland ${ }^{432}$ and Wollman, ${ }^{634}$ although not explicitly accepting this hypothesis, have brought forth evidence which convinces them that the bacteriophage is foreign to the bacterium.

As a matter of fact none of the authors who have adopted hypotheses other than that based on the living nature of the bacteriophage have expressly affirmed that any of the facts stand in contradiction to the hypothesis which I have formulated.

The reader who has given attention to all of the peculiarities of the phenomenon of bacteriophagy, as presented in the preceding pages, to the discussion of the resistance of bacteria to the action of the bacteriophage, and to the description of the characters of the principle, must have realized already that none of the facts stand in contradiction to the idea of the living nature of the principle which causes bacteriophagy.*

* I have already mentioned some of the odd arguments which have been advanced to oppose the living nature of the bacteriophage, such as the fact that it exists in the colloidal state, that it is subject to the laws of adsorption, of flocculation, etc., arguments which, as a matter of fact, are equivalent to saying that the fact that it partakes of the characters common to all living beings constitutes an objection to its living nature. But here is another argument, just as odd, but which, nevertheless, has been taken seriously by some of those who, on other grounds, support the hypothesis of its living nature. This argument is based on the fact that the first act of bacteriophagy consists in the fixation of the corpuscles to the bacteria. This is admitted, but it appears, indeed, that this is not "in favor" of the living nature of the principle.

If, however, the bacteriophage is a parasite of bacteria, it might be expected to behave as do all parasites, that is to say, that it might very well commence by 
All of the hypotheses designed to explain the phenomenon of bacteriophagy either as a result of the presence of a chemical principle, enzyme or catalyzer, foreign to the bacteria, or as the result of a vitiation of the bacterial metabolism under the influence of a chemical principle, are, by virtue of the fact of serial action, inadmissible simply because they are mathematically absurd.

On the other hand we have direct proof that the bacteriophage principle can not be an autolysin and that this principle can not be present in the normal bacterium which undergoes dissolution.

The result, then, is obvious, for, of all of the hypotheses advanced or which it is possible to formulate to explain the phenomenon of bacteriophagy, only one accords with the facts and with all of the facts. That one is the hypothesis which considers the phenomenon as being caused by a filtrable virus, by an ultramicrobe parasitizing the bacteria.

But even if all of this is true this conclusion can, logically, only be considered as a presumption. In order to tranform this presumption into a certainty it is essential to provide direct proof of the living nature of the bacteriophage principle. This is the purpose of the following chapter.

\section{RÉSUMÉ}

There are three possible hypotheses, a priori;

I. The bacteriophage principle is a chemical substance foreign to the bacterium.

II. The bacteriophage is a principle derived from the bacterium.

III. The bacteriophage is a living being foreign to the bacterium.

I. The first of these ends in mathematical absurdity.

II. The second hypothesis is open to four solutions:

A. It is an abnormal inert principle derived from the bacterium. This hypothesis is inadmissible, because it fails to consider the facts.

B. It is an abnormal living principle derived from the bacterium.

coming in contact with its prey. These authors would be the first to exclaim at the absurdity of the idea, if such an argument were advanced to disprove the living nature of the Hematozoön of malaria, or of a Piroplasma. What would happen if one were to affirm that these parasites can not be alive simply because they fix themselves electively to the red blood cells!

Unquestionably they would have a reply ready, "One may see the Hematozoön or the Piroplasm." Peculiar reasoning.

This argument is indeed typical of indirect argument, the sort which "fits in best" with the hypothesis of the author who has the most facile and flexible phraseology. 
This hypothesis has not yet been advanced, but like the preceding, it would receive no support from the facts.

C. It is an enzyme, normally elaborated by the bacterium. This enzyme can not be a normal autolysin. Among other objections, bacteriophagy, as is recognized even by those who support this hypothesis, has none of the appearances of an "autolysis," the characteristics of the two are even exactly opposed. This anomaly needs explanation if they would have us accept this view.

The principal argument advanced to support this hypothesis is the presence of a bacteriophage principle in cultures of some bacterial strains. This fact is in no way contradictory to the hypothesis of the living nature of the bacteriophage. Nature presents us with abundant evidence in the innumerable examples of such symbioses. Furthermore, the fact that it is possible to purify "lysogenic" strains shows that the bacteriophage behaves just as does any abnormal "contamination."

Finally it is possible to prove that the normal autolysin contained in a bacterium can not be the bacteriophage.

Since experiment shows that the bacteriophage principle is not a normal autolysin, the autolytic hypotheses of bacteriophagy are automatically excluded.

D. It is a living principle, existing normally in the bacterium. From the moment when experiment demonstrated that the bacteriophage principle is not contained in the normal bacterium this theory becomes as unfounded as the preceding. Furthermore, the experimental data presented in the next chapter will show the inadmissibility of this theory, as well as the deficiencies of the preceding.

III. The bacteriophage is a living being foreign to the bacterium. This is the sole hypothesis which is not in contradiction with any of the experimental facts of bacteriophagy. It is the only hypothesis which provides rational explanation of all of these facts, without necessitating recourse to any other hypothesis.

It is, therefore, the most probable hypothesis; the only one which takes into account all of the facts. 


\section{CHAPTER III}

\section{The Nature of the Bacteriophage}

\section{STATEMENT OF THE PROBLEM}

All of the arguments which have been advanced by the supporters of the different theories discussed in the preceding chapter are indirect arguments based upon circumstances of time or place. But it is not the place where a being is found, nor the circumstances which surround its discovery, that determine what must or must not be the intimate nature of the being.

Statements to the effect that under natural conditions the bacteriophage principle is found in some strains of bacteria; that the principle is encountered in the peritoneal cavity, in the blood, in the pus of abscesses, or in any tissue whatsoever, mean simply what they say. Can such things prove, beyond possible contradiction, whether the principle is a ferment or a living being?

If it can be definitely proved,-a proof which has not yet been adduced,- that the bacteriophage can act only upon a bacterium during the process of bacterial division, we will have additional information it is true, but will this exclude the possibility of the living nature of the principle? Would the fact that the moment of division is the critical moment for the cell exposed to the principle, and it seems that this is true for all cells exposed to ultraviruses, imply that the principle is inert?*

All of these things may be true, they say, nevertheless such and such a fact "fits in" better with this or that hypothesis. It is a question of evaluation, for one author the fact in question favors the hypothesis

* Naturally we need not discuss those strange arguments based on facts which, according to some authors, are incompatible with its living nature, such as the fact that it is flocculated by distilled water containing very small quantities of acid, or that it presents the characters of colloids. These authors have doubtless reflected that many bacteria are flocculated by acids, and that it is, therefore, simply a question of more or less. Doubtless they recall that the colloidal state is the one peculiar to all living matter. They have certainly determined whether the bacteriophage must be a colloid or a crystalloid, for there is no third state. Would they have needed to recognize in it the properties of crystalloids to enable them to believe that it might possibly be living? 
which he is supporting, for another it favors his. This is precisely the reason why indirect argument proves nothing. It neither supports nor condemns, for by a clever argument each one can "make it fit" with the hypothesis which he is defending.

An indirect argument lacks even a tentative value, as is shown by innumerable examples in the history of the sciences. In order to demonstrate the validity of any hypothesis, or rather to transform an hypothesis into an experimentally demonstrated certainty, it is necessary to support this hypothesis by direct arguments, that is to say, by indisputable facts which automatically and effectively exclude any and all other hypotheses.

In reality, it is all reduced to a question of logic. It is necessary first to give the question a logical expression, and then to ascertain whether in the present state of our knowledge we are able to provide the answer to this question.

Is there a property characteristic of life which will allow us to determine whether a being is living or dead, depending upon whether the being in question does or does not possess this property? If there is such a characteristic property, does or does not the bacteriophage property possess it? Briefly stated, this is the whole question.

If the bacteriophage principle does not possess this property, nothing can justify the statement that it is living. If it does possess it, discussion must end, for a being which possesses the characteristic by means of which we recognize life is necessarily living, and no amount of indirect argument can transform a living being into an inert one.

\section{THE CRITERIA OF LIFE}

What are the criteria of life? Can we consider as criteria such attributes as the faculty of motion, of increasing in volume, of exteriorizing a force, of exerting serial actions? Certainly not. For beyond question there are living beings which lack motility; an inert crystal may increase in volume; the wind gives expression to a physical force; tarnishing of tin transmits itself serially; and yet none of these things are caused by living beings.

What is it, then, that tells us that a being is living?

We do not know what life is, simply because as yet we are unable to identify that physico-chemical property which confers on matter the living state. But even if we do not yet know the essence of life we do know some of the resultants of it. We know that it is a composite of 
two fundamental properties,- the power of true assimilation, or assimilation of a heterologous medium, and the power of adaptation. We term all beings living which reveal to us that they are endowed with these properties; we call those inert in which these properties can not be detected. Moreover, these two powers are always found in association, hence they must both be expressions of the same physico-chemical property of matter.

Let us consider the two parts of this combination of properties. A crystal of copper sulfate, for example, immersed in a concentrated solution of copper sulfate increases in volume; it adds to itself molecules of the copper sulfate present in the solution. Is it living? No. For if one wished to explain this aggregation of molecules by terming it an assimilation, it would at once be obvious that the change is brought about in a homologous medium.

Assimilation in a heterologous medium implies the power of modifying the heterologous substances contained in this medium rendering them comparable to the distinctive substance of the being which brings about the transformation.

If the being, whose nature is under consideration, is visible macroscopically, it is, in general, not difficult to ascertain whether or not it possesses the power of assimilation in a heterologous medium. We observe a lamb and we see that it feeds on grass and increases in size; we see that it gives birth to other lambs, and we know that of necessity they must have been formed of the grass. We know that the lamb transforms "grass substance" into "sheep substance." It possesses the power of assimilation of a heterologous medium: it is living.

If the being whose nature we wish to determine is microscopic we say that it is living if it is able, just as was the case with the sheep, to assimilate a heterologous medium. We find that the bacterium multiplies in a man, for example, and we say that it transforms "man substance" into "bacterium substance." It possesses the power of assimilating a heterologous medium, and, therefore, by definition, it is living.

If we are dealing with a being of such minute size that it is impossible to see it even with the microseope, does this fact render the problem of its nature beyond solution? Is it impossible to ascertain whether or not it is capable of assimilating a heterologous medium? Not at all. For in no case do we see the actual assimilation; we observe only the effects of the process. The being increases in size or it multiplies in the heterologous medium, and that is sufficient to justify the conclusion that it possesses the power of assimilation. 
The power of adaptation may be defined as the faculty possessed by a being to respond by a specific opposing reaction to variations in the conditions of the medium.

This is the sum and substance of the question of the fundamental properties of living matter. Does this combination of properties constitute the "criterion" of life? We may reply in the affirmative, for all beings which man has classed in the category of beings which he terms "living" possess this combination of the two characters, while all of those which he allocates to the category termed "inert" lack it. When one of these beings which we term "living" is placed under conditions suited to the manifestation of these criteria and it no longer exercises these properties we feel warranted in refusing to consider it longer as being living; we say that it is "dead." For example, we heat a bacterial culture at a given temperature, and we implant a drop of the heated liquid into some fresh bouillon. If we find that the bacterium utilizes the nutritive substances of the bouillon for its development, if, in a word, it "assimilates," we say that it has not been killed. We affirm that it is still living. If, on the contrary, it does not "assimilate" we say that it is dead.*

If anyone refuses to admit that there is a "criterion" of life, and that this criterion is constituted of a combination of the powers of assimilation and of adaptation, all discussion as to the nature of any being whatsoever becomes vain. When such a point of view obtains the most that can be attained is a quarrel of words, devoid of real meaning. The matter ends in an impasse, and nothing remains but to close the discussion, frankly admitting that all knowledge of the nature of a being is impossible. This is said, however, without assuming that any student will arise to champion such an absurdity.

It remains to us, therefore, to attempt to determine whether the bacteriophage corpuscle does or does not possess this combination of properties which constitutes the criterion of life.

* "But" it may be said, "this is not quite true, we say that it is living because it multiplies." This is not correct. Unconsciously we reason differently. Immerse a crystal of copper sulfate in a saturated solution of this salt. The erystal multiplies. Do we infer that the crystal is living? No. Immerse a crystal of the same kind in a saturated solution of iron sulfate. There is no multiplication. Do we say that it is dead? Obviously not. It is then, not because the bacterium multiplies that we consider it a living being, but because it multiplies by using the heterologous sulsstances of the nutritive medium, transforming them into its own distinctive substance. We say it is living because it assimilates. 


\section{THE AUTONOMY OF THE BACTERIOPHAGE CORPUSCLE}

If the being, whose nature is in question, is macroscopically or microscopically visible, it is not, usually, difficult to ascertain if it possesses the power of assimilation of a heterologous medium. When a bacterium multiplies within the body of an animal or in bouillon, we conclude that it has transformed the animal substance or the nutritive substances of the artificial medium into "bacterium substance," and that, therefore, it possesses the power of assimilation in a heterologous medium.

If the being whose nature we desire to determine can not be seen because of its smallness, does the mere matter of size preclude the possibility of determining whether or not it has this power? Not at all. For in either case we do not see the assimilation. We deduce that it must occur because the being in question multiplies, using a heterologous substance in order to build up its own substance.

The bacteriophage corpuscle multiplies at the expense of the bacterium substance. Everyone admits this, and if the corpuscles were visible no one would question but what the process were one of assimilation. But in the case of the bacteriophage, and indeed, this is true for all ultraviruses, it may be objected that the substance of the corpuscle may be bacterial substance. In such a case the process would be a simple assimilation of a homologous medium, as in the case of the crystal which multiplies in a concentrated solution of a salt. Consequently, in order to demonstrate that the bacteriophage corpuscle assimilates when in a heterologous medium, it is necessary in the first place to show that "bacteriophage substance" differs from "bacterium substance," that is to say, it is essential to demonstrate that the bacteriophage corpuscle is an autonomous being, independent of the bacterium which undergoes its action.

Proofs of the autonomy of the bacteriophage corpuscle are both many and varied. Some of them will be presented in the next few pages.

I. Since the beginning of my study of the bacteriophage I have isolated more than 100 races of the Shiga-bacteriophage from the stools of normal men or from patients convalescent from dysentery and other liseases; from the excreta of a variety of animals,-- sheep, swine, cattle, logs, cats, rabbits, chickens, ducks, geese, etc.--from the water of rivers and wells, from tap-water, and from the sea, and I have yet to find two races of the bacteriophage which are strictly identical as to their powers of attack and as to the intensities of their action upon different species of the colon-typhoid-dysentery group of organisms..$^{312}$ This is true despite the fact that throughout these investigations I have always employed 
the same strain of B. dysenteriae Shiga-Kruse in effecting passages for the purpose of increasing the virulence of these races of the bacteriophage. It is evident that if the bacteriophage were a bacterial product all of these bacteriophage races ought to have been the same, presenting exactly the same properties, since in this case all were derived from the same bacterial substance. Inasmuch as this is not the case, since each bacteriophage possesses its own characters, it follows that each race is definitely individualistic, and, from this point of view, is quite independent of the bacterium at the expense of which it has multiplied. The bacteriophage is, therefore, an autonomous being.

II. Everyone admits that the bacterium can acquire a resistance to the bacteriophage. This is an actual immunity transmissible to the descendents throughout a certain number of generations. But in the absence of the bacteriophage this acquired immunity gradually diminishes and is finally lost. And when this has occurred the bacterium is again as susceptible as it was prior to the acquisition of the immunity. This fact can be understood only if the "substance" of the corpuscle differs from the "substance" of the bacterium, that is, it can not be interpreted unless the bacteriophage is an autonomous being, independent of the bacterium.

III. Under natural conditions "Iysogenic" strains of bacteria, from which it is possible to isolate a bacteriophage principle, may occasionally be encountered. Such strains can also be produced experimentally. We have seen above that it is possible to "purify" a bacterial strain by the ordinary bacteriological method of colony isolation. This suggests strongly that the bacteriophage corpuscle behaves like an "impurity" contaminating the bacterial culture. How would one expect by such a simple procedure as isolating individual colonies to purify a bacterial culture of some product which it itself produced? The bacteriophage corpuscle is therefore foreign to the bacterium, and being foreign, is autonomous.

IV. We have seen that B. typhosus is a heterologous species as regards the bacteriophage, that is, different strains of this bacillus are not all attacked by the same race of the bacteriophage. Each race of Typhobacteriophage has an affinity for some strains of B.typhosus and remains entirely inactive toward others. Here, we are dealing, not with an acquired resistance, but with a natural resistance, for this resistance is not lost by a series of successive cultivations in the absence of the bacteriophage. The majority of strains which are not attacked by one race of the bacteriophage are susceptible to another. 
Janzen and Wolff ${ }^{372,375}$ have taken advantage of this peculiarity to demonstrate the autonomy of the bacteriophage with reference to the bacterium, and their experiments are worthy of consideration.

They selected three races of the Typho-bacteriophage, attacking the different strains of B. typhosus, Re, Wi, and Sm. They maintained these three bacteriophage races with a single strain of $B$. typhosus (strain $\mathrm{Sm}$, isolated from the stools of the patient who furnished the bacteriophage $\mathrm{Sm}$ ). After a series of passages, all contacts being made with $B$. typhosus Sm, determinations of the activity of the three races for a number of typhoid strains showed the results in table 31 .*

It is very evident that if the bacteriophage were a bacterial product the three races of the bacteriophage should have become uniform, since all three developed during several passages at the expense of the same

TABLE 31

\begin{tabular}{|c|c|c|c|c|c|c|c|c|c|}
\hline \multirow{2}{*}{$\begin{array}{c}\text { STRAINS } \\
\text { OF B. } \\
\text { TYPHOSLS }\end{array}$} & \multicolumn{3}{|c|}{ BACTERIOPHAGE Re } & \multicolumn{3}{|c|}{ BACTERIOPHAGE Wi } & \multicolumn{3}{|c|}{ BACTERIOPHAGE Sm } \\
\hline & $\begin{array}{c}\text { Inhibi- } \\
\text { tion }\end{array}$ & $\begin{array}{l}\text { Dissolu- } \\
\text { tion }\end{array}$ & Plaques & $\begin{array}{l}\text { Inhibi- } \\
\text { tion }\end{array}$ & $\mid \begin{array}{c}\text { Dissolu- } \\
\text { tion }\end{array}$ & Plaques & $\underset{\text { Inhibi- }}{\text { tion }}$ & $\begin{array}{l}\text { Disso- } \\
\text { lution }\end{array}$ & Plaques \\
\hline $\mathrm{Wi}$ & 0 & 0 & 0 & $+t+t$ & $+t+t$ & $+t+t$ & 0 & 0 & 0 \\
\hline 1 & 0 & $+t+t$ & $+++t$ & $t+t+$ & $t+t+$ & $+t+t$ & 0 & $+t+$ & $+t+t$ \\
\hline 24 & 0 & + & $++t+$ & 0 & $+t+$ & $+t+t$ & 0 & 0 & 0 \\
\hline 27 & 0 & 0 & 0 & 0 & + & $+t+t$ & 0 & 0 & 0 \\
\hline 29 & $+t$ & $++t$ & $++t+1$ & ++ & $+t+t$ & $+++t$ & 0 & 0 & 0 \\
\hline
\end{tabular}

bacterium, B. typhosus Sm. But, as the experiment shows, this was not the case. Each bacterium retained its own characteristics and again it follows that the bacteriophage is an autonomous being, possessing its distinctive characters, independent of the bacterium at whose expense it has developed.

With regard to the characters of these races of the bacteriophage, Janzen and Wolff made an interesting incidental observation. They asked me to give them such races of the Typho-bacteriophage as I had in my collection. I sent them six tubes of the bacteriophage, marked $\tau v^{1}, \tau v^{2}, \tau v^{3}, \tau v^{4}, \tau v^{5}$, and $\tau v^{6} \cdot \dagger$ Some time later they asked me if I

* For the method of determining the degree of virulence of a bacteriophage see the section "Appreciation of Virulence." In the method employed by Janzen and Wolff three indices of activity were used: inhibitory power, dissolving power, and the formation of plaques on agar.

$\dagger$ For the sake of simplicity I have adopted the custom of designating the bacteriophages by the initial letter (Greek) of the bacterium for which the virulence was the strongest when the race was isolated. For example, $\delta$ indicates a Shiga-bacteriophage, $\sigma \varphi$ a Staphylo-bacteriophage, $\tau v$, a Typho-bacteriophage, $\chi$ a Coli-bacteriophage. To this is added the number of the race. 
were sure that I had sent them six different races. As I had been accustomed to give a different number to each race I replied to them in the affirmative, although I could not verify this statement from my experimental protocols which I was unable to consult since I was at the University of Leiden and my records of these races were in Paris.

They found, as a matter of fact, that after these six races of the bacteriophage had been subjected to serial passages with a single strain of B. typhosus, the virulences were as shown in table 32 .

From these results these races could clearly be divided into two groups one comprising races 1 and 2 , the other races $3,4,5$, and 6 . This indicated that there must have been some mistake on my part. And they had good reasons for such a suspicion. Some time later, being in Paris, I consulted my laboratory protocols, and I found that these races of the

TABLE 32

\begin{tabular}{c|c|c|c|c|c|c}
\hline \multirow{2}{*}{$\begin{array}{c}\text { STRAINS OF } \\
\text { B. TXYPHOSUS }\end{array}$} & \multicolumn{7}{|c}{ BACTERIOPHAGE RACES } \\
\cline { 2 - 6 } & 1 & 2 & 3 & 4 & 5 & 6 \\
\hline$M$ & 0 & 0 & + & + & + & + \\
3 & + & + & + & + & + & + \\
8 & + & + & 0 & 0 & 0 & 0 \\
16 & + & + & + & + & + & + \\
20 & 0 & 0 & + & + & + & + \\
\hline
\end{tabular}

bacteriophage had been isolated before I adopted the notation later used, and I had designated by the different numbers a bacteriophage of a single race which had been through passages with different strains. In reality, races 1 and 2 were derived from a convalescent from typhoid fever, while races $3,4,5$, and 6 were all obtained from another case.

The distinguishing characters of a race of the bacteriophage are so marked, even after repeated passages with different strains of a bacterium, or even after passages with bacteria of different species, that it is possible to distinguish them from all other races. This would not be the case if the bacteriophage were a product of the bacterium, for then it could not retain its individuality in this way.

$V$. Maitland performed an experiment which is essentially the reverse of that of Janzen and Wolff. He isolated a bacteriophage which was virulent for both $B$. paratyphosus $B$ and $B$. dysenteriae. This, he carried through a series of passages, on the one hand, with $B$. paratyphosus $B$, and on the other, with $B$. dysenteriae. He found that the bacteriophage remained the same, whether it was maintained at the expense of the one or the other of these bacteria. 
As Maitland very logically states, if the increase in the bacteriophage principle were the result of the metabolism of the bacterium, just as soon as one obtains an increase of a single principle at the expense of bacteria belonging to different species, the characters of the principle should differ to accord with the regeneration, depending upon whether it takes place with one bacterium or with another. But experiment shows that quite the opposite occurs, that the bacteriophage remains endowed with the same properties. Consequently Maitland has concluded that the bacteriophage is not a bacterial product, but that it is foreign to the bacterium.

VI. Asheshov ${ }^{30}$ isolated from the intestinal contents of a pig two different races of the bacteriophage, both active for $B$. dysenteriae Flexner. Upon agar, one formed very large plaques, 12 to $15 \mathrm{~mm}$. in diameter, the other produced only very minute plaques, from 1 to 2 $\mathrm{mm}$. He carried out two parallel series of passages at the expense of a single strain of the Flexner bacillus, the suspensions for each passage with the two races being prepared from the same agar culture. After a series of 15 passages, each of the bacteriophages had retained its own character, the one yielding large plaques, the other, minute ones. If the bacteriophage were a product of the bacterium, unquestionably the two should have become uniform. As this did not happen, Asheshov concluded that the bacteriophage is an autonomous entity, independent of the bacterium at the expense of which it multiplies.

VII. Gratia and de Namur ${ }^{274}$ have made use of bacteriophage races particularly well adapted to demonstrating the fixity of characters as well as the autonomy of the bacteriophage. For their experiment they selected the polyvirulent Staphylo-bacteriophage, race $h$, active for all strains of albus, aureus, and citreus, and a second staphylo-bacteriophage, race $v$, whose virulence is strictly limited to a single Stapnylococcus albus, strain $V$. They subjected the polyvirulent bacteriophage $h$ to a series of passages at the expense of staphylococcus $V$, and found that its properties were retained intact.

Inasmuch as this experiment seemed particularly well adapted to demonstrate the point, I have repeated it, using every precaution to forestall objections. ${ }^{350}$

Incidentally, it may be well to point out that for an experiment of this type to be beyond criticism, it is necessary to carry the race of bacteriophage (here, race $h$ ) through a sufficient number of passages with the given bacterium (here, strain $V^{r}$ ) so that by virtue of the successive dilutions it will be impossible that any of the original liquid containing the 
bacteriophage (that is, the material added to the first suspension to induce bacteriophagy) can remain. As we know, this degree of dilution can be calculated. Physicists have determined that the smallest particle of matter is the electron, whose mass is $1 \cdot 10^{-27}$ grams. When, as a result of successive dilutions, we introduce into one of the tubes of the series a quantity less than $1 \cdot 10^{-27}$ gram of the initial bacteriophage filtrate, we may be sure that we no longer have any of the initial filtrate, and consequently, none of the bacteriophage corpuscles (in the present experiment, bacteriophage $h$ ) inoculated in the first suspension of the series.

Still another precaution should be taken, namely, to heat, at $60^{\circ} \mathrm{C}$., the bacteriophage filtrate inoculated into the first suspension (here, Staphylococcus albus $V$ ). This eliminates the possible objection that this filtrate might have contained some filtrable form of the bacterium (Staphylococcus aureus) which, being inoculated in the first passage, might persist throughout the whole series, and which, indeed, may be the organism at whose expense the bacteriophage multiples and persists in its polyvirulence. ${ }^{*}$

Bearing these things in mind, this experiment was performed. In each of the successive passages $10 \mathrm{cc}$. of a staphylococcus $V$ suspension was inoculated with $0.001 \mathrm{cc}$. of a bacteriophage $h$ suspension, and the series was continued, the quantitative relationships being the same, the bacterial suspension being freshly prepared from the same strain, and the bacteriophage suspension being in every instance a heated filtrate of the last suspension dissolved. As we have shown elsewhere, in the seventh suspension of the series the degree of dilution of the initial suspension of bacteriophage is $1 \cdot 10^{-28}$, that is to say, in the present experiment $1 \mathrm{cc}$. of the seventh suspension can contain but $1 \cdot 10^{-28}$ grams of the original suspension of bacteriophage $h$. Such a quantity is imaginary and represents nothing real, since it is smaller than an electron. It is certain, therefore, that the eighth suspension of staphylococcus $V$ will not receive any of the original bacteriophage $h$ corpuscles. When the dissolution of the organisms of this eighth suspension is completed all of the corpuscles present will be those which must have reproduced at the expense of the Staphylococcus albus $V$. But, experiment shows that the bacteriophage corpuscles of this eighth suspension, when bacteriophaged, as also are those present in the fifteenth when the

* In repeating this experiment I have taken the precaution, not only to filter and to heat at $60^{\circ} \mathrm{C}$. the first suspension of bacteriophage $h$, but I have repeated both of these procedures with each of the 15 passages. 
passages are continued, show the distinctive characters of bacteriophage $h$, in particular, its polyvirulence.

This experiment, which offers no chance for misinterpretation, which yields a result so absolutely clear-cut, for it is impossible to confuse two characters as distinct as univirulence and multivirulence, demonstrates that the bacteriophage is an autonomous being, independent of the bacterium at the expense of which it multiplies.

As a matter of fact, bacteriophage $v$ attacks strain $V$ only. If it is a product derived from the metabolism of the bacterium it must be elaborated by the $V$ strain of Staphylococcus albus and its character of specificity depends upon the staphylococcus which produces it. If this conclusion is not admitted the alternative is to recognize that the properties of the bacteriophage are independent of the bacterium which undergoes its action, and this enforces acceptance of the autonomous nature of the bacteriophage. If, on the contrary, one considers the bacteriophage to be a product of bacterial metabolism and if this conception is valid, all of the bacteriophage corpuscles derived from the metabolism of staphylococcus $V$ should present the same characters, especially that of being univirulent. Bacteriophage $h$, because of the serial passages, should have lost its character of multivirulence. But the result is precisely the opposite. After any number whatever of such passages it remains multivirulent. From this, therefore, it is certain that the bacteriophage possesses its own characters; and but one deduction can be drawn, namely, that the bacteriophage is an autonomous being, independent of the bacterium at whose expense it multiplies.

VIII. I have repeated the preceding experiment in a somewhat different way. To begin with, bacteriophagy of a suspension of staphylococcus $V$ was induced by the inoculation of a suspension of bacteriophage $h$, previously heated to $60^{\circ} \mathrm{C}$. After a complete dissolution the limpid suspension was returned to the incubator at a temperature of $41^{\circ} \mathrm{C}$. After 3 days a secondary culture developed. For the past two years this mixed culture has been carried on by transfers made about once a month. After 24 subcultures a filtrate was made from a seeding of the mixed culture of the last transfer. This filtrate contained the bacteriophage, and this bacteriophage had all of the characters of bacteriophage $h$. It was multivirulent.

A mixed culture,-Bacteriophage $v$-Staphylococcus $T$,- -has been maintained for about four years, having undergone many transfers (from 30 to 40 ). It still contains a strictly monovirulent bacteriophage, attacking staphylococcus $V$ only. 
Like the preceding, this experiment leaves no doubt as to the independence of the characters of the bacteriophage from the bacterium at whose expense it has reproduced. The bacteriophage is, then, unquestionably autonomous.

IX. The autonomy of the bacteriophage is shown also by the experiments of Bordet and Ciuca. ${ }^{87}$ Indeed, these authors state that "Serological study indicates, therefore, that the lytic power is a really new character." This is, in terms adapted to their point of view, a recognition of autonomy.

We will see later, that as a result of the repeated injection of an animal with a bacteriophage suspension, an anti-bacteriophagic property appears in its serum.

Bordet and Ciuca treated some rabbits as follows:

Rabbit 1 ; received a series of injections of a culture of normal $B$. coli.

Rabbit 2 ; received a like number of injections of this same $B$. coli after it had been bacteriophaged.

Rabbit 3; received the same series of injections of a secondary culture derived from the same strain of $B$. coli.

They found that the sera of the last two animals neutralized the homologous bacteriophage, while the serum of the first had no neutralizing property whatever. The bacteriophage possesses, then, antigenic properties peculiar to itself and definitely different from those inherent in the bacteria at whose expense it develops. Thus, it is an autonomous being.

Wollman and Brutsaert ${ }^{+34}$ have recently repeated this experiment and have reached the same conclusion,- the autonomy of the bacteriophage.

$\mathrm{X}$. Proof of the autonomy is also derived from another experiment of the same authors. Bordet and Ciuca ${ }^{92}$ impregnated agar slants with the anti-bacteriophagic serum and then seeded them with a secondary culture of bacteriophage- $B$. coli. After incubation they found that the growth was a normal culture of $B$. coli, all sub-cultures were normal, and the bacteriophage had been eliminated,- that is, "cured" according to Bordet's expression.

If a bacterium infected by a bacteriophage can be "cured" by serum therapy it is evident that the bacteriophage must be a parasite, foreign to the bacterium.*

* Prausnitz ${ }^{536}$ saturated an anti-bacteriophagic serum (Shiga) with the humologous bacteria, and found that the antibacterial properties were removed while the anti-bacteriophagic properties persisted. He concluded from this that the bacteriophage is different from the bacterium, that is, an autonomous being. 
All of these experiments contribute in showing that the "substance" of the bacteriophage corpusele is different from the "substance" of the bacterium at whose expense it multiplies; that the characters of the corpuscle belong to it itself and are not determined by the characters of the bacteria which undergo its action. The bacteriophage corpuscle is, therefore, an autonomous being.

\section{THE POWER OF ASSIMILATION OF THE BACTERIOPHAGE CORPUSCLE}

The bacteriophage multiplies during the course of its action. We have seen that with highly potent races a single corpuscle inoculated into $10 \mathrm{cc}$. of a suspension of a susceptible bacterium will yield, when bacteriophagy is terminated, as many as 100,000 millions per cubic centimeter. The solitary one introduced into the $10 \mathrm{cc}$. of medium will have become a trillion; the suspension of bacterial cells will have become a suspension of bacteriophage corpuscles. The "mass" of bacteriophage substance has, then, increased from 1 to 1 trillion.

Proof has been adduced that the bacteriophage is not a product of bacterial metabolism; that the bacteriophage corpuscle is an autonomous being. And an autonomous being whose mass increases necessarily utilizes the substances found in its environment, transforming these heterologous substances into its own peculiar substance. Experiment shows that the bacteriophage multiplies at the expense of bacteria, that it must transform "bacterium substance" into "bacteriophage substance," and this can only mean that the bacteriophage corpuscle is endowed with the power of assimilating a heterologous medium.

\section{THE POWER OF ADAPTATION OF THE BACTERIOPHAGE CORPUSCLE}

I. We know that the vigor with which a bacteriophage corpuscle will attack a given bacterium is capable, according to the conditions of the experiment, of being enhanced or of being diminished. As a matter of fact, at the present time, this fact is universally accepted.

May one say, with Seiffert, ${ }^{578}$ that it is not really an exaltation or an attenuation in the virulence of the corpuscle for the bacterium, but

This experiment of Prausnitz is open to criticism, for one may object that if one saturates a serum which is both antitoxic and antibacterial with young bacteria the antibacterial properties will disappear while the antitoxic properties will remain, nevertheless, a toxin is certainly a bacterial product.

Prausnitz is correct in his deductions, but his experiment is not beyond criticism. Those of Bordet and Ciuca, on the contrary, are not open to attack. 
rather a variation in the bacterium, which "adapts itself" to produce variable amounts of the "lytic principle?" The obvious reply to this is, that proof being adduced that the bacteriophage corpuscle is a being independent of the bacterium, such an objection falls ipso facto. But there is no necessity to dispose of the question in this summary manner.

If a bacteriophage of low virulence is inoculated into a bacterial suspension, and if a series of cultures is made, transferring each time a trace of the preceding culture into some sterile bouillon, without either filtration or heating between each passage, will we obtain an increase in the virulence of the bacteriophage? Not at all. Everyone is quite agreed on this point, I believe. And yet, if the conception of Seiffert is correct, under these conditions the bacterium should accustom itself to produce a more and more potent "lytic" principle.

What is the procedure for obtaining an increase in the virulence of the bacteriophage corpuscle? Simply effecting serial passages, taking care to introduce in each succeeding suspension no bacteria from the preceding suspension which have been in contact with the bacteriophage. This we accomplish either by filtering the suspension where bacteriophagy has occurred, or by heating it at a temperature sufficiently high to destroy the bacteria. Under these conditions we find that with each new passage the virulence of the bacteriophage increases.

Wherein is the logic in speaking of an adaptation of the bacteria, when, with each passage, the bacteria of the preceding suspension which have been in contact with the bacteriophage are systematically discarded? Only the corpuscles suspended in the filtrate are carried along in the passages, only they may possibly become accustomed,-adapt themselves.

But why, indeed, are we obliged to filter (or heat, although this is far less satisfactory) between each passage? Because the bacterium also, like all living beings, possesses the power of adaptation. But far from adapting itself to elaborate a ferment directed toward its own destruction its adaptation consists in the acquisition of a resistance to the action of the bacteriophage.

An abundance of experimental data demonstrates this process of bacterial adaptation, and it shows with equal clearness that the result of the adaptation is exactly the opposite of the production of an "autolytic" principle. It is the development of a resistance to bacteriophagy. And meanwhile, the bacteriophage is adapting itself to an attack on the bacteria. 
As Beijerinck ${ }^{64}$ has very justly said, the single fact that the bacteriophage can undergo an exaltation or an attenuation of virulence is sufficient from the point of view of logic to prove that it is a living being.*

II. It has been demonstrated that the bacteriophage can accustom itself to the harmful action of glycerol. ${ }^{321}$ A mixture of 0.25 ce. of a suspension of Shiga-bacteriophage and of $0.75 \mathrm{cc}$. of neutral glycerol is made and the tube is sealed. $\dagger$ After a week the tube is opened and $1 \mathrm{cc}$. of anhydrous neutral glycerol is added. The tube is sealed and shaken. Each week this is repeated; the tube is opened, a cubic centimeter of glycerol is added, and the tube is again sealed. This process is continued up to the point where the total volume reaches $10 \mathrm{cc}$.

At this time another mixture is prepared; $0.25 \mathrm{cc}$. of the same suspension used above and $9.75 \mathrm{cc}$. of anhydrous neutral glycerol. The two mixtures are incubated at $37^{\circ} \mathrm{C}$. When examined after 10 days it is found that the bacteriophage of the second mixture, where the suspension was mixed at one time with the whole quantity of glycerol, is destroyed while in the first mixture, where the glycerol was added progressively, it is still present and virulent.

Brutsaert ${ }^{104}$ has, in effect, repeated this experiment. He mixed equal quantities of suspension and glycerol, and then evaporated the water. He found that the bacteriophage was destroyed when the evaporation was rapid, but that the virulence persisted when the evaporation was slow.

III. Prausnit $z^{53}$ has shown that the bacteriophage can accustom itself to an antibacteriophagic serum. We know that the serum of an animal prepared by injections of a suspension of the bacteriophage has the property of inhibiting the action of the bacteriophage. Prausnitz progressively increased the quantity of antiserum in a series of bacteriophage-bacterium mixtures and saw that an adaptation took place. The bacteriophage became resistant and caused bacteriophagy in the presence of quantities of serum which inhibited the action of a non-adapted bacteriophage.

*Perhaps the strangest feature in all of this discussion is the argument adduced by some authors, who, confronted by the evidence which $I$ have furnished, admit that the bacteriophage corpuscle possesses the characters of living beings, but affirm that this proves nothing more than that an inert substance can possess all of the characters of life! It is indeed interesting to see to what reaches of absurdity a preconceived idea can lead one, an idea which gradually becomes a fixed belief.

$\dagger$ In sealing, a long drawn-out neck should be provided, so that the tube can be repeatedly opened and sealed again in the flame. 
IV. I have found that the bacteriophage can develop a tolerance to the action of acids. ${ }^{332}$ If successive passages are made in media with increasing degrees of acidity a point is reached where the bacteriophage is perfectly active in media, which, with an unadapted bacteriophage of the same race, prevents multiplication.

Asheshov ${ }^{28}$ has performed the following experiments, which likewise demonstrate the acquisition of a tolerance to acidity. He chose a race

TABLE 33

\begin{tabular}{|c|c|c|c|c|c|c|}
\hline \multirow{3}{*}{ MEDIUM } & \multicolumn{6}{|c|}{$\begin{array}{l}\text { PROGRESSIVE PH CHANGES; TURBIDITY } \\
\text { OF THE MIXTURES }\end{array}$} \\
\hline & \multicolumn{2}{|c|}{ Immediately } & \multicolumn{2}{|c|}{ After $4 \frac{1}{2}$ hours } & \multicolumn{2}{|c|}{ After 8 hours } \\
\hline & $\mathrm{pH}$ & $\mid \begin{array}{c}\text { Turbid- } \\
\text { ity* }\end{array}$ & $\mathrm{pH}$ & Turbid- & $\mathrm{pH}$ & $\begin{array}{c}\text { Turbid- } \\
\text { ity }\end{array}$ \\
\hline Bouillon with 1 per cent glucose. & S.0 & 250 & 6.6 & 500 & $<6.6$ & $>500$ \\
\hline \multirow{2}{*}{ Ordinary bouillon . ............. } & 8.0 & 250 & 7.3 & 75 & 7.3 & 25 \\
\hline & 7.0 & 250 & 6.6 & 300 & 6.6 & 200 \\
\hline
\end{tabular}

* The degree of turbidity of the liquid, represented in millions of bacteria per cubic centimeter, based on comparison with titrated control tubes, obviously shows the degree of bacteriophagic action, or the inhibition of this action.

TABlE 34

\begin{tabular}{|c|c|c|c|c|c|c|c|c|}
\hline \multirow{3}{*}{ MEDIUM } & \multicolumn{8}{|c|}{ CHANGES IN $\mathrm{pH}$ AND IN TURBIDITY } \\
\hline & \multicolumn{2}{|c|}{ Immediately } & \multicolumn{2}{|c|}{$\begin{array}{c}\text { After } \\
3 \frac{1}{2} \text { hours }\end{array}$} & \multicolumn{2}{|c|}{ After 5 hours } & \multicolumn{2}{|c|}{ After 24 hours } \\
\hline & 党 & 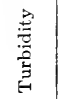 & 营 & 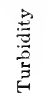 & 䓀 & 永 & 壳 & 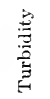 \\
\hline \multirow{2}{*}{1 per cent glucose bouillon.... } & S. 0 & 250 & 6.6 & 75 & 6.6 & 10 & $<6.6$ & 500 \\
\hline & 7.0 & 250 & 6.6 & 75 & $<6.6$ & 25 & $<6.6$ & 200 \\
\hline \multirow{2}{*}{ Ordinary bouillon. } & 8.0 & 250 & 7.7 & 450 & 7.7 & 400 & 7.6 & 50 \\
\hline & 6.6 & 250 & 6.6 & 350 & 6.6 & 350 & 6.6 & 50 \\
\hline
\end{tabular}

of bacteriophage (Flexner) which caused a weak clearing of a bacterial suspension in a neutral ( $\mathrm{pH}$ 7.0) medium, and which was completely inactive in acidified media (glucose). The effectiveness of the bacteriophage in these media is shown in table 33.

After seven passages in bouillon with an initial reaction of $\mathrm{pH} 7.0$ this bacteriophage reached the point where it would induce almost as complete a bacteriophagy as that occurring in alkaline bouillon. At the 
ninth passage it caused an almost complete dissolution of bacteria in glucose bouillon. The results of the twelfth passage are given in table 34 .

It is obvious [states this author] that after 12 passages the bacteriophage has become adapted to the harmful action of the medium and of the acid in statu nascendi. The final development (after 24 hours) of bacilli in the glucose bouillon must be attributed to the presence of bacteriophage-resistant bacteria, which have within this interval of time, so acidified the medium that even this race of acid-tolerant bacteriophage is unable to attack them.

It may be observed, however, that the activity of the bacteriophage weakens, although very slowly, during the course of passages through an acid medium. The number of bacteriophage corpuscles per cubic centimeter at the end of the experiment was comparatively smaller, just as the virulence was lower.

From this is appears that the bacteriophage although losing somewhat in virulence, may become accustomed to the harmful action of acids.

TABLE 35

\begin{tabular}{|c|c|c|c|}
\hline $\begin{array}{l}10 \mathrm{cC} \text {. OF } \\
\text { BOUILLON }\end{array}$ & $\begin{array}{l}\text { SUSCEPTI- } \\
\text { BLE } \\
\text { FLEXNER } \\
\text { CULTURE }\end{array}$ & BACTERIOPHAGE & $\begin{array}{c}\text { RESULT } \\
\text { AFTER } \\
5 \text { DAYS AT } \\
16^{\circ} \mathrm{C} .\end{array}$ \\
\hline$p H$ & & & \\
\hline 5.8 & 1 drop & 5 drops of the 47 th passage & Limpid \\
\hline 8.2 & 1 drop & 5 drops of the 47 th passage & Limpid \\
\hline 5.8 & 1 drop & None (control) & Turbid \\
\hline 5.8 & 1 drop & 5 drops of the unadapted bacteriophage & Turbid \\
\hline
\end{tabular}

Some of my experiments have shown the same thing, and that not only is the virulence of the bacteriophage attenuated by growth in an acid medium, but also by cultivation at a high temperature. And, as stated above, Gildemeister has shown that cultivation at a low temperature also results in a diminished virulence. The same thing occurs with pathogenic bacteria; they can be adapted to grow in an unfavorable medium, but their virulence suffers.

This is a fact worthy of note: Adaptation to a harmful action, instead of leading to a corresponding increase in virulence, is accompanied by an attenuation.

Schuurman ${ }^{573}$ attempted to adapt a Flexner-bacteriophage, which showed a maximum degree of virulence in a medium with a $\mathrm{pH}$ of 8.2 , to effect bacteriophagy in media of increasing degrees of acidity, the tubes in which the contacts were made being held at a temperature of $37^{\circ} \mathrm{C}$. At the twenty-second passage he obtained a complete dissolu- 
tion only in the media with a $\mathrm{pH}$ of 6.8 , but beyond this point, in higher concentrations of $\mathrm{H}$ ions, the reaction did not occur or was incomplete. Furthermore, he was unable to reduce this limiting concentration by continuing the contacts under these conditions. When, however, the passages were made at $16^{\circ} \mathrm{C}$. instead of at $37^{\circ}$, with the filtrations being made only after an incubation period of 48 hours, he obtained the results in table 35 at the forty-eighth passage.

The unadapted bacteriophage used in the above experiment had also undergone 47 passages, but in a medium with a $\mathrm{pH}$ of 8.2 .

Furthermore, in a bouillon with a reaction of 6.0 Schuurman obtained a complete bacteriophagy with the adapted bacteriophage at a temperature of $37^{\circ} \mathrm{C}$., while the same suspension inoculated with the bacteriophage, originally of the same race but which had passed through 48 passages in a medium at $\mathrm{pH}$ 8.2, failed to show any evidences of dissolution. These experiments show very clearly the effects of adaptation.

V. Wolff and Janzen ${ }^{628}$ have succeeded in rendering the bacteriophage tolerant toward different antiseptics, to chinosol in particular. When a quantity of chinosol sufficient to provide a final concentration of 1:600 is added to a bacterial suspension inoculated with a bacteriophage and, after 24 hours, a drop of this suspension is added to a fresh suspension, the latter, when spread on agar, yields no plaques. After contact for a week, when the bacteria are dead through the action of the antiseptic, a drop inoculated into a fresh suspension yields, after incubation, a very great many plaques. These experiments were performed with $B$. coli, $B$. typhosus, and B. dysenteriae, as well as with the staphylococcus.

Schuurman ${ }^{573}$ has also succeeded in demonstrating an adaptation of the bacteriophage to chinosol.

VI. Prausnitz and Firle, ${ }^{539}$ after confirming the earlier experiments of Prausnitz on adaptation to an antibacteriophagic serum (confirmed also by other authors,-Bruynoghe and Wagemans, ${ }^{127}$ d'Herelle $^{347}$ ) carried out some additional experiments demonstrating adaptation to different antiseptics. These are of sufficient interest to summarize here.

Experiments with phenol. For measuring the degree of adaptation they made comparative counts of the plaques derived from seeding a bacterial suspension inoculated with a bacteriophage adapted to the action of the antiseptic and of those obtained when a comparable suspension was inoculated with the non-accustomed bacteriophage, both specimens of the bacteriophage having been exposed to the action of the same concentration of the antiseptic. The results appear in tables 36 and 37. 
TABLE 36

Number of plaques (2 days at laboratory temperature)

\begin{tabular}{|c|c|c|c|}
\hline & \multicolumn{2}{|c|}{$\begin{array}{l}\text { EQUAL PARTS OF BACTERIO- } \\
\text { PHAGE AND }\end{array}$} & \multirow{2}{*}{ PER CENT LOSS } \\
\hline & $\begin{array}{l}1.5 \text { per cent } \\
\text { phenol }\end{array}$ & $\begin{array}{l}\text { Distilled } \\
\text { water }\end{array}$ & \\
\hline $\begin{array}{l}\text { Bacteriophage A (22 passages in phenol): } \\
\text { Plaque No. } 1 \ldots \ldots \ldots \ldots \ldots \ldots \ldots \ldots \ldots \\
\text { Plaque No. }\end{array}$ & 632 & 768 & 18 \\
\hline $\begin{array}{c}\text { Plaque No. } 2 \ldots \ldots \ldots \ldots \ldots \ldots \ldots \ldots \ldots \\
\text { Bacteriophage A (passages through } \\
\text { media without phenol) } \ldots \ldots \ldots \ldots \ldots\end{array}$ & $\$ 2$ & $\begin{array}{l}554 \\
578\end{array}$ & $\begin{array}{r}85 \\
100\end{array}$ \\
\hline
\end{tabular}

TABLE 37

Number of plaques after a contaet of from 1 to 4 days in a phenol medium, at laboratory temperature

\begin{tabular}{|c|c|c|c|c|c|c|}
\hline \multirow{2}{*}{$\begin{array}{l}\text { CONCENTRA- } \\
\text { TION } \\
\text { OF PHENOL }\end{array}$} & \multicolumn{3}{|c|}{$\begin{array}{l}\text { BACTERIOPHAGE A } \\
\text { (33 PASSAGES THROUGH A PHENOL MEDIUM) }\end{array}$} & \multicolumn{3}{|c|}{$\begin{array}{c}\text { BACTERIOPHAGE A } \\
\text { (PASSAGESTTHOUGH BOCILLON) }\end{array}$} \\
\hline & 1 day & 2 days & 4 days & 1 day & 2 days & 4 days \\
\hline per cent & & & & & & \\
\hline 1.0 & - & - & - & - & 3800 & \\
\hline 1.1 & - & - & - & - & 3500 & \\
\hline 1.2 & - & - & - & - & 748 & 630 \\
\hline 1.3 & - & - & - & - & 440 & 218 \\
\hline 1.4 & - & - & - & 32 & 2 & 2 \\
\hline 1.5 & 1240 & 2000 & 2000 & 3 & 0 & 0 \\
\hline 1.6 & 511 & 484 & 465 & - & & \\
\hline 1.7 & 232 & 121 & 114 & - & & \\
\hline 1.8 & 1 & 2 & 11 & - & & \\
\hline 1.9 & 0 & 0 & 0 & - & & \\
\hline 2.0 & 0 & 0 & 0 & & & \\
\hline
\end{tabular}

TABLE 38

Bacteriophage, having undergone 9 passages in media containing mercuric chloride:

A. Placed in a solution containing $1: 10,000$ of the salt . . . . . . . . . . . . . . . .

B. Placed in physiological saline.

About 2000

370

About 2000

410

Same bacteriophage, unadapted:

A. Placed in a solution containing 1:10,000 of

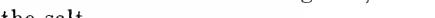

B. Placed in physiological saline . . . . . . .

NUMBER OF PLAQUES WHEN SPREAD OVER AGAR

\begin{tabular}{c|c}
\hline $\begin{array}{c}\text { After contact for } \\
\text { 1 day with } \\
\text { mercuric chloride }\end{array}$ & $\begin{array}{c}\text { After contact for } \\
\text { 2 days with } \\
\text { mercuric chloride }\end{array}$ \\
\cline { 1 - 2 } & \\
About 2000 & 370 \\
About 2000 & 410 \\
& \\
0 & 0 \\
454 & 174 \\
\hline
\end{tabular}


Experiments with mercuric chloride. Table 38 summarizes the results of an experiment involving the adaptation of the same bacteriophage (Shiga) to the action of corrosive sublimate.

Experiments with chloramine. The experiment dealing with adaptation to phenol has already shown that if the material is spread on agar during the adaptation the corpuscles of the different plaques, that is,

\section{TABLE 39}

\begin{tabular}{|c|c|c|c|}
\hline \multirow{2}{*}{$\begin{array}{l}23 \text { PASSAGES OF THE BACTERIOPHAGE } \\
\text { THROUGH CHLORAMINE }\end{array}$} & \multicolumn{2}{|c|}{ DILUTION, $1: 100,000$} & \multirow{2}{*}{$\begin{array}{c}\text { DILUTION, } \\
1: 1,000,000\end{array}$} \\
\hline & $\begin{array}{l}\text { Number of } \\
\text { plaques }\end{array}$ & $\begin{array}{l}\text { Per cent } \\
\text { of corpuseles } \\
\text { alive }\end{array}$ & \\
\hline \multicolumn{4}{|l|}{ Plaque No. $1+$} \\
\hline Chloramine $1: 1000 \ldots \ldots \ldots$ & 17 & 2.7 & 0 \\
\hline Chloramine $1: 1500 \ldots \ldots \ldots \ldots \ldots \ldots$ & 23 & 3.5 & 3 \\
\hline Distilled water.$\ldots \ldots \ldots \ldots \ldots \ldots$ & 660 & & 55 \\
\hline \multicolumn{4}{|l|}{ Plaque No. $2+$} \\
\hline Chloramine $1: 1000 \ldots \ldots \ldots \ldots \ldots$ & 11 & 1.5 & 0 \\
\hline Chloramine $1: 1500 \ldots \ldots \ldots \ldots \ldots$ & 22 & 3.0 & 3 \\
\hline Distilled water. . . . . . . . . . . & 725 & & 63 \\
\hline \multicolumn{4}{|l|}{ Plaque No. $3+$} \\
\hline Chloramine $1: 1000 \ldots \ldots \ldots \ldots \ldots$ & 35 & 4.6 & 2 \\
\hline Chloramine $1: 1500 \ldots \ldots \ldots \ldots \ldots$ & 47 & 6.2 & 4 \\
\hline Distilled water. . . . . . . . . . . & 760 & & 57 \\
\hline \multicolumn{4}{|l|}{ Plaque No. $4+$} \\
\hline Chloramine $1: 1000 \ldots \ldots \ldots \ldots \ldots$ & 3 & 1.6 & 0 \\
\hline Chloramine $1: 1500 \ldots \ldots \ldots \ldots \ldots$ & 7 & 3.7 & 1 \\
\hline 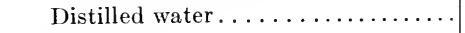 & 187 & & 20 \\
\hline \multicolumn{4}{|l|}{ Plaque No. $5+$} \\
\hline Chloramine $1: 1000 \ldots \ldots \ldots \ldots \ldots$ & 12 & 1.5 & 1 \\
\hline Chloramine $1: 1500 \ldots \ldots \ldots \ldots \ldots$ & 18 & 2.3 & 3 \\
\hline Distilled water.$\ldots \ldots \ldots \ldots \ldots \ldots$ & 790 & & 84 \\
\hline \multicolumn{4}{|l|}{$\begin{array}{l}\text { Same bacteriophage, unadapted; sus- } \\
\text { pended in: }\end{array}$} \\
\hline Chloramine $1: 1000 \ldots \ldots \ldots \ldots \ldots \ldots$ & 4 & 1.5 & 0 \\
\hline Chloramine $1: 1500 \ldots \ldots \ldots \ldots \ldots \ldots$ & 25 & 2.7 & 3 \\
\hline Distilled water $\ldots \ldots \ldots \ldots \ldots \ldots$ & 910 & & 85 \\
\hline
\end{tabular}

the different colonies of the bacteriophage, do not have an equal resistance, since with the corpuscles originating in one of the colonies (Plaque No. 1) of 100 corpuscles $\$ 2$ resisted the action of the phenol, and with those of the other (Plaque No. 2) only 15 resisted.

This inequality in the adaptation of the different corpuscles of a single suspension is well shown by the experiments on the action of chlor- 
amine (also performed by Prausnitz and Firle). They made 23 passages in media containing higher and higher concentrations of chloramine. They then spread the suspension on agar in order to secure isolated plaques, and separated these plaques for the following passage.

In the table covering their findings (table 39) the results show the outcome when suspensions of the bacteriophage derived from these different plaques were added to equal quantities of chloramine solution.

Adaptation to chloramine is acquired with greater difficulty than is that to phenol, and especially, that to mercuric chloride. The resistance of the corpuscles from Plaque No. 3 is, however, very appreciable, as the authors of this extremely interesting series of experiments have observed.

Considered as a whole, these experiments show, beyond doubt, that the bacteriophage corpuscle is capable of adapting itself to the conditions of the moment.

\section{THE FaCUlty of MUltiplication of the BaCteriophage CORPUSCLE}

Each of the two fundamental characteristics of living beings has a consequent phenomenon, which, always found in association with the basic character, forms to it a sort of corollary. For assimilation this corollary is the faculty of multiplication; for adaptation, the capacity for the characters of a single being to vary at different times, which in its turn necessarily involves a variability between beings belonging to the same species.

The power of assimilation has, then, for a corollary, the faculty of multiplication, a faculty which is exercised when the conditions are favorable, that is, when assimilation can take place freely. This is true, however, whether the assimilation takes place in a heterologous or in a homologous medium, for the crystal immersed in a concentrated solution of the homologous material multiplies, just as surely as does another being endowed with the power to assimilate in a heterologous medium providing it with assimilable substances.*

To consider more specifically the beings of the latter class, those capable of assimilating heterologous substances, we find that the elementary mass, when the being is immersed in a medium containing assimilable substances, tends to increase indefinitely, until the assimilable materials are exhausted, if we disregard, naturally, such limiting processes as the accumulation of waste materials. But in all beings endowed with this

* This clearly shows that multiplication is simply a corollary of assimilation. 
property the elementary particle is a colloidal polarized "micella," and by virtue of this fact its mass is limited by a purely physical phenomenon, as has been very clearly stated by Perrin. "If," he says, "one admits that the charges of the particles are due to the $\mathrm{H}^{+}$and the $\mathrm{OH}^{-}$ ions which are disseminated throughout the liquid and which become fixed to the particle, it is obvious that when the particle has attained a certain size, and provided the number of free ions is sufficiently great to afford more available for fixation, this increase in size will inherently be a cause of rupture, leading to a division into two parts." This physical phenomenon applies without discrimination to the cells of the body, to the bacterium, and to the infravisible virus.

The faculty of multiplication consequent upon assimilation in a homologous or heterologous medium is not, then, a characteristic of life. That all living beings possess it is true, but this is solely because they possess the power of assimilation. The bacteriophage corpuscle is endowed with this faculty, and from it all of the phenomena of bacteriophagy are derived.

\section{VARIABILITY OF THE BACTERIOPHAGE}

The power of adaptation, which is an attribute of all living beings, entails, naturally, a lack of fixity in the characters of a living being; it demands that they be dependent upon their environment. The same being placed under different conditions exhibits different characters.

But adaptation is accompanied by another phenomenon. Living matter, protoplasm, retains the impression of the reaction effected, an imprint whose mark in the substance is engraved the more deeply as the reaction is the more intense. Obviously, it is unnecessary to attach to the word "impression" any psychic meaning. As used here it implies simply that living matter which has once reacted to a given stimulus reacts again more quickly, and in general, more intensely when subjected to a second stimulus of the same nature as the first. That the reaction of living matter is specific, we have said. The "recollection" is likewise specific, for it modifies in no way the response to a different excitation.

This "reactional memory" is transmitted hereditarily, ${ }^{*}$ but if a stimu-

* Here, of course, we are not considering the case, still very obscure, of the transmission of characters to descendents through the intermediary of gametes. The only transmission of characters with which we are concerned here is that which takes place when a cell becomes two cells through division. Here, the transmission of acquired characters is beyond doubt. All immunity demonstrates this, as do the facts of all types of adaptation which can be experimentally effected with bacteria. 
lus of the same nature as the first fails to occur until after the lapse of a long time the "recollection" of the reaction effected is progressively lost.

The living being reacts continually. Since the conditions of the medium in which it lives are changing with each moment, and in very diverse ways, the specific reactions which it brings into play must adapt themselves to innumerable conditions. And the "recollection" of each of them imprints itself in the substance more or less strongly according to the energy expended in each of these reactions, and each "recollection," intense or mild, as the case may be, is transmitted to the descendents. As the conditions are different for each being of a single species, that is to say, for a single line, it results that each being presents distinctive characters and special aptitudes, either forced upon it through heredity or acquired through its own experience.

The less complex a being the greater is its faculty of adaptation. This fact can be experimentally demonstrated, for of the visible beings the bacteria have the most simple organization and we know that it is possible to induce them to develop under very diverse conditions, some extremely remote from their natural conditions of life. The greater the adaptability, the greater also is the variability. Indeed, when bacteriology was in its infancy, Pasteur showed that each bacterium within a single culture presented, together with characters belonging to the species, individual characters and aptitudes for adaptation.

Does the bacteriophage show this variability? The experimental data recorded on each page of this text have shown that there are no two races of the bacteriophage which are identical. Within a single suspension each corpuscle has its own individual virulence, and each presents an individual resistance to the action of physical or chemical agents. Furthermore, the experiments of Prausnitz and Firle have demonstrated that each corpuscle within a single suspension has a capacity for adaptation differing in degree from that of other corpuscles.

Some experiments of Wolff and Janzen $6^{30}$ dealing with variability are particularly interesting, being concerned with the variation occurring in the virulences when a given bacteriophage multiplies at the expense of bacteria of different species.*

* It may be mentioned that these experiments might have been cited to demonstrate the autonomy of the bacteriophage corpuscle as regards the bacterium. When different bacteriophages develop at the expense of a single bacterium, they should, if they are a product of bacterial metabolism, all tend to assume the same characters, in the present case to acquire the same virulences. But experiment shows the contrary. The aptitude to lose or to acquire a virulence does not depend on the bacterium "lysed"; this is a character appertaining to an autonomous entity. 
It is essential to state, that in order to forestall the possible objection that they worked with mixtures of bacteriophages, these authors purified their bacteriophage races by colony (plaque) isolations. Furthermore, all of their transfers were effected in the same manner, proceeding each time from a plaque.

Wolff and Janzen chose two races of Typho-bacteriophage (from my collection) and made serial passages with B. typhosus, with B. coli, and with B. dysenteriae Shiga. The virulences of these two races, as revealed after the passages, were compared with their original virulences. Table 40 presents their findings.

With bacteriophage No. 1 the "virulence" character did not change. Originally virulent for all three strains, it remained virulent for all three,

TABLE 40

\begin{tabular}{|c|c|c|c|c|}
\hline VIRULENT FOR & ORIGINALLY & \begin{tabular}{|c|} 
AFTER \\
PASSAGES \\
WITH \\
B. TYPHOSUB
\end{tabular} & $\begin{array}{c}\text { AFTER } \\
\text { PASSAGES } \\
\text { WITH } \\
\text { B. COLI }\end{array}$ & $\begin{array}{c}\text { AFTER } \\
\text { PASSAGES } \\
\text { WITH } \\
\text { B. DYSEN- } \\
\text { TERIAE }\end{array}$ \\
\hline
\end{tabular}

Bacteriophage No. 1

\begin{tabular}{|c|c|c|c|c|}
\hline B. typhosus.... & $t$ & + & + & + \\
\hline B. dysenteriae. & + & + & + & + \\
\hline
\end{tabular}

Bacteriophage No. 3

\begin{tabular}{|c|c|c|c|c|}
\hline 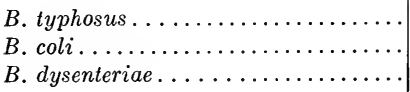 & $\begin{array}{l}+ \\
+ \\
+\end{array}$ & $\begin{array}{l}+ \\
0 \\
0\end{array}$ & $\begin{array}{l}+ \\
+ \\
+\end{array}$ & $\begin{array}{l}+ \\
+ \\
+\end{array}$ \\
\hline
\end{tabular}

the strain at whose expense it developed effecting no manifest modification. With bacteriophage No. 3, on the contrary, the same treatment resulted in a loss of virulence for $B$. coli and $B$. dysenteriae when it had developed at the expense of $B$. typhosus, but it retained its virulence intact, even that for B. typhosus, when it had multiplied at the expense of $B$. coli or of B. dysenteriae.

Wolff and Janzen next ascertained the virulences of the two races for different strains of B. typhosus after several passages with B. typhosus or $B$. coli. The results obtained with bacteriophage No. 3 are given in table 41 .

With the No. 1 race of the Typho-bacteriophage the virulences for these different strains of B. typhosus remained exactly the same, whether the bacteriophage had developed at the expense of B.typhosus, B. coli or the Shiga bacillus. 
We have seen in an earlier section that when a number of races of the bacteriophage are subjected to passages with a single strain of bacteria each race retains its own characters. Again this same fact appears, since each of the races, No. 1 and No. 3, retains its individuality following passages with the same strains of B. coli, B.dysenteriae, and B. typhosus. We now find that the passage of a single bacteriophage with bacteria of different strains does modify the characters. In this it behaves just as does a bacterium, as we must recognize if we but recall that Pasteur

TABLE 41

\begin{tabular}{|c|c|c|c|c|c|}
\hline & \multicolumn{5}{|c|}{$\begin{array}{l}\text { BACTERIOPHAGE NO. 3-ACTIVITY FOR STRAINS } \\
\text { OF B. TYPHOSUS }\end{array}$} \\
\hline & M & 3 & 8 & 9 & 20 \\
\hline \multicolumn{6}{|l|}{ Before the passages: } \\
\hline Inhibition* & 0 & ++ & + & $+t+$ & 0 \\
\hline Dissolution .......... & 0 & ++ & 0 & $+t$ & 0 \\
\hline Plaque formation... & $+t+t$ & $+t+t$ & 0 & $+t+t$ & $+t+t$ \\
\hline \multicolumn{6}{|l|}{$\begin{array}{l}\text { After passages with } B . \text { typhosus and } \\
\text { subsequent plaque isolation: }\end{array}$} \\
\hline Inhibition $\ldots \ldots \ldots \ldots \ldots \ldots$ & $+t$ & +++ & 0 & $+t+$ & $++t$ \\
\hline Dissolution. . . . . . . . . . . & + & ++ & 0 & $+t$ & + \\
\hline Plaque formation. .............. & $+t+t$ & $+t+t$ & 0 & ++++ & $+t+t$ \\
\hline \multicolumn{6}{|l|}{$\begin{array}{l}\text { After passages with } B . \text { coli and sub- } \\
\text { sequent plaque isolation: }\end{array}$} \\
\hline Inhibition $\ldots \ldots \ldots \ldots \ldots \ldots$ & 0 & 0 & 0 & 0 & 0 \\
\hline Dissolution. . . . . . . . . . . & 0 & 0 & 0 & 0 & 0 \\
\hline Plaque formation.............. & 0 & ++ & 0 & ++ & 0 \\
\hline
\end{tabular}

* See the section "Appreciation of Virulence" for the details of the method of titration adopted by Wolff and Janzen.

showed that by passage through pigeons the bacillus of swine fever became increasingly virulent, while it was attenuated by passages through rabbits. Today we know many instances of exactly comparable facts.

The above experiments show further that the capacity for variation involves not only the actually existing virulences of different races of the bacteriophage, but that it also extends to their tendency to preserve their initial virulences throughout a period of development at the expense of other differing bacterial species.

Even with the bacteriophage, variability is a characteristic phenome- 
non. Indeed, there is much reason to believe that the bacteriophage is the being which possesses this character to the highest degree. Furthermore, this character of variability is always proportionate to the more or less outspoken faculty of adaptation.

\section{THE BACTERIOPHAGE CORPUSCLE: A LIVING ULTRAVIRUS}

The first of all the scientific classifications is that which separates the beings known to man into two great categories; inert beings on the one hand, living beings on the other. This classification is based upon the fact that the living beings present certain properties which the others lack. Just as soon as it is determined that a being possesses, or does not possess, these properties, it is irrevocably placed in one or the other of these two categories.

If, perchance, it is found that a being, possessing the characters which classify it as "living," presents certain peculiarities that have up to that time not been detected in another being so classed, this in no way throws its nature into question. For just as soon as it possesses the "criteria" of life it is necessarily living, and the only choice is to recognize that the peculiarity which it presents is compatible with life. This sort of thing has occurred more than once before now in the history of science.

Experiment shows that the bacteriophage corpuscle, an autonomous being,* possesses the powers of assimilation in a heterologous medium and of adaptation, and thus, necessarily, the consequences of these two powers - the faculties of multiplication and of variability of characters.

This combination of characters constitutes the complete criterion of life. The bacteriophage corpuscle, conforming to this criterion in every respect, is a living being. This is no longer an hypothesis; it is a certainty.

It is this living being, this infravisible microbe, to which I have given

* The demonstration of the living nature of the bacteriophage under the form in which it is given in this chapter was published for the first time in $1923 .{ }^{347}$ It has been repeated, notably in the text published in the United States (Immunity in Natural Infectious Disease). Following its publication some of the discussion stopped. Nevertheless, there are still some writers who attempt to show that certain facts "fit in better" if one believes that the "lytic principle" is derived from the bacterium. None of them have discussed the proofs that I have adduced; none have even alluded to them.

Their attitude forces me to suggest that logically they might consider point by point the experimental facts which serve as the basis of my demonstration before they continue their study and their discussion of the nature of the bacteriophage. 
the name Protobios bacteriophagus (syn. Bacteriophagum intestinale d'Herelle) d'Herelle, 1918..$^{312,349}$ This infravisible microbe is the agent of an infectious disease occurring among bacteria, and this disease is bacteriophagy.*

* It should be remembered, however, that although up to the present time parasitism of bacteria has not been recognized we have for a long time observed and studied many parasites which incite infectious disease among the protozoa. Several examples will be found cited among the works of Metchnikoff. (Leçons sur la pathologie comparée de l'inflammation, Paris, 1892, Masson \& Cie. L'immunité dans les maladies infectieuses, Paris, 1901, Masson \& Cie.)

It may be well to mention a study of Dangeard entitled "Sur les parasites du noyau et du protoplasma" (Le Botaniste, 1894/95, 4, 199-248) for the facts disclosed by this investigator offer certain analogies to those presented in the preceding chapters. But there are these differences, namely, the parasite of Dangeard attacks a protozoan, and its dimensions are such that it can be readily observed microscopically and therefore classified.

The observations of Dangeard deal with an Oomycete, Nucleophaga amoeba Dangeard, which parasitizes the nucleus of Amoeba verrucosa Ehr. The Amoeba verrucosa has a large, doubly-contoured spherical nucleus, and also a nucleolus, likewise spherical, whose diameter is about two-thirds that of the nucleus. The substance of the nucleolus is very dense and stains with great intensity with various nuclear staining reagents. Between the nucleolus and the nuclear membrane is a space filled with the nuclear fluid.

The zoospore of Nucleophaga amoeba first penetrates the protoplasm of the amoeba but it never develops there; it passes into the nucleus through the membrane which it perforates, most certainly through the aid of a dissolving diastase. Dangeard has demonstrated the portal of entrance of the parasite as a minute circular opening, as though made by a punch, persisting after the entrance of the parasite. After its penetration into the nucleolus the parasite resembles a refractile corpuscle, increasing slowly in size in proportion as the nuclear substance disappears. When this nuclear material has been utilized completely the entire interior of the nucleus is filled and the membrane is distended. At this time the nucleus of the parasite, up to the present time single, actively divides and when sporulation is effected there are about one hundred regularly spaced nuclei. About each of these nuclei a zoospore organizes, and a sporangium is thus formed, containing distinct, rounded corpuscles, which contain nuclei at the time of sporulation.

Frequently a single amoeba is parasitized by two or perhaps several zoospores, and in such cases each develops separately and gives birth to a distinct sporangium. When the sporangium reaches maturity the protoplasm of the amoeba disintegrates, the sporangium ruptures, freeing the young zoospores, and these become distributed throughout the medium, ready to parasitize the healthy amoebae in their neighborhood.

It is evident that I have not made any comparison between Nucleophaga amoeba and Protobios bacteriophagus, and that these observations are mentioned simply because there is a certain resemblance between the two phenomena of destruction,- - that of the amoeba and that of the bacterium. 


\section{RÉSUMÉ}

All of the arguments which have been advanced by the adherents of the diverse hypotheses concerning the nature of the bacteriophage corpuscle are indirect arguments, that is to say, arguments which tend only to show that such and such a fact "fits in better," "explains better" such and such an hypothesis. Such arguments are valid only through interpretation, and each may be interpreted according to the taste of the individual; it is purely a question of dialectics.

Logically, a demonstration must be based on direct arguments, that is, on arguments which are not subject to interpretation, arguments which inherently exclude all hypotheses other than the one which they support. In this way only can an hypothesis be transformed into a certainty.

The question as to the nature of the bacteriophage corpuscle can be asked in but a single way: Is there a group of characters, constituting the "criterion of life" which permit us to place the being in question irrevocably either in the category of living beings or in the category of inert beings, depending upon whether it possesses or does not possess this combination of characters?

If this criterion exists, does the bacteriophage corpuscle conform to it, or does it not?

The criterion of life exists. It is formed of a combination of the powers of assimilation in a heterologous medium and of adaptation, with their corollaries, the faculty of multiplication and the capacity for variability of characters.

Experiment demonstrates that the bacteriophage is an autonomous being, independent of the bacterium at the expense of which it multiplies. By virtue of the fact that this autonomous being multiplies at the expense of the bacterium, heterologous with respect to itself, it must necessarily transform a heterologous substance into a homologous substance, a substance distinctively its own substance, and consequently it is endowed with the power of assimilation.

Various experiments show that the bacteriophage corpuscle is able to adapt itself to adverse conditions. The corpuscles therefore possess the faculties of reproduction and of variability.

The bacteriophage corpuscle possesses, therefore, precisely that combination of characters which permit us to recognize that a being is living, and anything which possesses the criteria of life is necessarily living.

The living nature of the bacteriophage being established, all facts 
pertaining to the bacteriophage must be interpreted to accord with the fundamental established idea. To do otherwise is to adopt a faulty system of logic.

It is to this being, infravisible, parasitic of bacteria, that I have given the name Protobios bacteriophagus (syn. Bacteriophagum intestinale) d'Herelle, 1918. 


\section{CHAPTER IV}

\section{The Unicity of the Bacteriophage Protobe}

\section{THE BACTERIOPHAGE PROTOBE}

We have now analyzed the experimental evidence which proves that the bacteriophage corpuscle possesses that composite of characters which allows us to distinguish the living nature of a being. There is, indeed, no antinomy between the behavior of the bacteriophage corpuscle, which is that of a parasite of bacteria, and its living nature, which is proved by its behavior.

If we consider fairly all of the known characteristics of the corpuscle we can find but a single peculiarity which differentiates it from all beings whose living nature has been accepted up to the present time. This one distinctive feature is its minute size. And since experiments demonstrate that its dimensions are approximately the same as those of the protein micella this excludes the idea of a cellular being.

The logic of the matter is simply this: The micella* is the smallest possible particle of matter in the colloidal state: The substance comprising all living beings is found in this state: $†$ Consequently the micella, the unit of colloidal matter is also the unit of living matter, and cells are constituted of a union of micellae. A cell being formed of a nucleus and a cytoplasm, by definition merely a single micella can not constitute a cell.

We are, then, confronted by the two following facts:

The bacteriophage corpuscle is formed of a single protein micella.

A bacteriophage corpuscle has the combination of characters which permit a substance to be classified in the category of living beings.

Two experimental facts can not be in contradiction with each other. If both facts are in agreement, and one, or both, contradict a theory,

* Or whatever may be the name by which we choose to designate the colloidal unit,-granule, particle, micella, or what not.

$\dagger$ This is universally admitted, and is, moreover, readily demonstrable. Living substance must be either a colloid or a crystalloid, for there are only the two choices.

$\ddagger$ We have shown, as will be recalled, that it presents the characters of protein substances,-among others, that of precipitation by saturation with the sulfates of sodium and ammonium. 
it is the theory which is defective. The theory, not the facts, must be modified. Such a state of affairs is not new; it has occurred many times in the history of science.

We must leave aside the virtually insoluble question of the first cause of life, but we may discover something about the source of this first cause, for there are only two possible hypotheses. It may result from a special organization, a cellular organization; or it may be derived from a particular state of protein matter. In the first case the smallest possible particle of living matter is the cell, in the second, it is the micella.

Up to the present, dominated by histologists whose conception stops precisely at the limit of visibility with the microscope, ${ }^{*}$ and whose reasoning is this- "all living beings that we see are cellular, consequently life results from a cellular organization,"- the first hypothesis has been generally adopted. Submission to this dictum of visibility is not, however, quite uniform, for certain independent minds whose opinion carries great weight, Beijerinck for example, have repudiated this concept absolutely.

The two experimental facts bearing upon the bacteriophage corpuscle are unqualifiedly in contradiction with this a priori hypothesis. But, as we have said, when hypothesis and experimental facts contradict one another it is the hypothesis which must yield to the facts. Just as soon as experiment shows that a corpuscle, constituted of a simple protein micella, is a living being, it follows that life does not require a cellular organization; that it results from a special physico-chemical state of matter, that is, the protein micella.

From the philosophical point of view, this fact is the most important one of the many which have been revealed through the study of the bacteriophage.

Up to the present time the infravisible agents of rabies, of variola, of vaccinia, of encephalitis lethargica, of the animal plagues, and of the mosaics of plants, have been considered by the majority of biologists as living beings, but this point of view has been, however, disputed. Although the proof of the living nature of the bacteriophage is only valid for itself alone, it is none the less true that the living nature of all

* Happily, physicists have succeeded in divorcing themselves from the idea of vision, which always leads to error, as the history of science abundantly proves. If they had studied the nature of matter with the mental concept of the histologists the electron would yet remain to be discovered. I hasten to add that many of the most eminent histologists are beginning to take exception to this point of view; they are abandoning their science for physical chemistry. 
of the beings which present the same characteristics is rendered the more probable by the facts disclosed in connection with the bacteriophage. These infravisible beings have been termed "invisible viruses," "ultraviruses," "ultramicrobes," etc. This does not give them a name, for these terms are simply qualifying terms, designating their state of invisibility.

In presenting the demonstration of the living nature of the bacteriophage, I have proposed to designate this group of living "micellar" beings by the name Protobes, ${ }^{347}$ a term which I have applied to the bacteriophage.*

\section{ASSIMILATION BY THE PROTOBES}

Here we step into the unknown. For the mere fact of knowing that a being assimilates reveals nothing as to the "mechanism" through which it assimilates. We know that there is a transformation of matter; that the bacteriophage protobe transforms "bacterium substance" into "bacteriophage substance." Let us see if by examination of the known facts we may gain some insight into the manner in which this transformation is effected.

In the first place, it seems to have been demonstrated that the metabolism of the parasitic protobes is limited to living matter; that this is the only material which they can utilize for their development.

The chemical transformation which the living matter of the prey undergoes, whether this be a bacterium in the case of the bacteriophage, a vegetable cell in the case of the mosaic protobe, the animal cell in the case of the protobe of variola, must be very extensive, for the antigenic properties are changed. It is for this reason that the injection of an animal with the "bacteriophage protobe substance" causes the appearance in the serum of this animal of an antibacteriophagic property, a property which is not manifested when the animal is injected with "bacterium substance."

On the other hand, can the assimilative process be as far-reaching in these rudimentary beings as it is in beings more highly organized and equipped with a digestive tract? To approach this point let us consider how assimilation is effected in those beings representing the next higher step in the scale,- the bacteria. It may be possible to apply the facts

* These beings, certainly the most simple that exist, are placed at the very bottom of the classification scheme as the "first living beings," and this is what

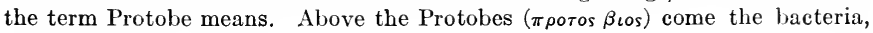
the Microbes ( $\mu \iota \chi \rho \circ \beta$ ○。s). 
here disclosed to the protobes, beings even more simple than the bacteria, and to ascertain whether these facts accord with their behavior.

It might be assumed, a priori, that there is "one" assimilation, that is to say, that the process always ends in the formation of a single substance and that this substance is identical with the substance of the being which assimilates. The facts are not in accord with this point of view.

When one supplies a metazoan provided with a specialized digestive tract any food material, from either the animal or the vegetablekingdom, which it is able to assimilate the assimilation is always complete, that is, the process ends in the formation of substances identical with those of which the metazoan is composed. Is the process the same in microörganisms, in particular, in the bacteria? Obviously not, for there is proof to the contrary. It is only necessary to provide a bacterium with different food materials to cause transformations in its antigenic properties, and this effectively shows that the constitution of the "substance" of this bacterium varies according to the nutritive materials which it assimilates.*

Here is the proof of his last statement, the experiments cited being those reported by Brocq-Rousseu, Urbain, and Forgeot. $\dagger$

For a long time we have known that a streptococcus, by passages through an animal, becomes what is termed a "passage streptococcus," but these authors should receive the credit for showing the nature of this transformation.

They utilized in their experiments a strangles antiserum (an antistreptococcus serum, specific for the streptococcus of equine strangles) which when titrated by the method of Calmette and Massol contained 1500 units of antibody. The antigen used consisted of a suspension of the streptococci killed by alcohol-ether, prepared in such a way that there was $1 \mathrm{cgm}$. of the bacterial bodies to $20 \mathrm{cc}$. of 0.9 per cent physiological saline.

Different animals were injected with different strains of the strangles streptococcus recently isolated from the lesions of infected horses. The strains were not passed through any other animal after isolation. For the injections they adopted the subcutaneous route with mice, the pleural route with rats, the intraperitoneal route with guinea pigs, and the intravenous or intraperitoneal routes with rabbits.

* This transformation in antigenic properties is a commonly observed fact. The following paragraphs, dealing with variations in the antigenic properties of microorganisms and of protobes, will present typical examples.

$\dagger$ Brocq-Rousseu, Urbain, and Forgeot.--Etudes sur le streptocoque gourmeaux. Ann. Inst. Pasteur, 1923, 37, 322. 
After the death of the injected animal, they withdrew aseptically a few drops of heart blood and seeded it immediately in serum-bouillon. The culture so obtained was tested against their strangles antiserum for its antigenic value. Table 42 summarizes their experiments, the figures indicating the results in units of antibody. There can be no question from these results but that the streptococcus acquires a new individuality within the body of each of the different animals.

The authors of these experiments conclude that the number of passages is a function of the resistance of the animal to infection; the longer death is delayed, they say, the more quickly the streptococcus

TABLE 42

\begin{tabular}{|c|c|c|c|c|c|c|}
\hline & \multicolumn{6}{|c|}{ STRAINS OF THE STRANGLES STREPTOCOCCUS } \\
\hline & 376 & A. 1109 & $\begin{array}{l}\text { Délici- } \\
\text { euse }\end{array}$ & BH542 & Carpano & File \\
\hline \multicolumn{7}{|c|}{ Passages through mice } \\
\hline First passage . . . . . . . & 500 & 500 & 500 & 500 & 500 & 500 \\
\hline Second passage.... & 300 & 150 & 300 & 300 & 300 & 75 \\
\hline Third passage.... & 50 & 30 & 50 & 50 & 50 & 10 \\
\hline Fourth passage... & 0 & 0 & 5 & 0 & 10 & 0 \\
\hline Fifth passage ........... & & & 0 & & 0 & \\
\hline
\end{tabular}

Passages through rats

\begin{tabular}{|c|c|c|c|c|c|c|}
\hline First passage . . . . . . . & 150 & 150 & 150 & 150 & 150 & 150 \\
\hline Second passage.... & 150 & 50 & 150 & 25 & 150 & 50 \\
\hline Third passage .... & 100 & 10 & 50 & 5 & 50 & 5 \\
\hline Fourth passage... . & 50 & 0 & 10 & 5 & 10 & 0 \\
\hline Fifth passage ........... & 10 & & 5 & 0 & 0 & \\
\hline Sixth passage . . . . . . . & 0 & & 0 & & & \\
\hline
\end{tabular}

Passages through rabbits*

\begin{tabular}{c|c|c}
\hline \multirow{2}{*}{ PASSAGE NuMber } & \multicolumn{2}{|c}{ Straivs } \\
\cline { 2 - 3 } & $\begin{array}{c}\text { Carpano (intravenously, } \\
\text { death in 2 days) }\end{array}$ & $\begin{array}{c}\text { Infutable (intraperitoneally, } \\
\text { death in 12 hours) }\end{array}$ \\
\hline 1 & 150 & 300 \\
2 & 50 & 300 \\
3 & 50 & 200 \\
4 & 0 & 75 \\
5 & & 20 \\
6 & & 15 \\
7 & & 15 \\
8 & & 15 \\
9 & & 0 \\
\hline
\end{tabular}


TABLE 42-Continued

\begin{tabular}{|c|c|c|}
\hline \multicolumn{3}{|c|}{ Passages through guinea pigs } \\
\hline \multirow{2}{*}{ PASSAGE NUMBER } & \multicolumn{2}{|c|}{ STRAINS } \\
\hline & $\begin{array}{l}\text { Carpano (intraperitoneally, } \\
\text { death in } 2 \text { to } 3 \text { days) }\end{array}$ & $\begin{array}{c}\text { Infutable (intravenously, } \\
\text { death in } 12 \text { hours) }\end{array}$ \\
\hline 1 & 1500 & 1200 \\
\hline 2 & 150 & 1000 \\
\hline 3 & 100 & 500 \\
\hline 4 & 50 & 500 \\
\hline 5 & 0 & 300 \\
\hline 6 & & 100 \\
\hline 7 & & 50 \\
\hline 8 & & 25 \\
\hline 9 & & 0 \\
\hline
\end{tabular}

* These experiments were carried out on the only two strains of the streptococcus which were sufficiently virulent to kill the rabbits. The same is true for the guinea-pig experiments.

adapts itself to the new organism which harbors it, and the more quickly it loses its equine character to become a streptococcus of the mouse, the rat, the rabbit, or the guinea pig.

It is certain, as these authors say, that the rapidity of the transformation is a function of resistance, but is it the resistance of the animal itself which is responsible, or may it be some other phenomenon accompanying resistance? The following experiments, made by these same investigators, suggest an answer.

A few years ago, in some experiments performed with Le Louet, I observed that the virulence of a bacterium for a given animal is exalted by cultivation in an artificial medium, a bouillon, prepared with the flesh of this animal.* The same thing has been found to occur with the strangles streptococcus for by cultivating this organism in Martin's bouillon to which the blood of some laboratory animal was added BrocqRousseu, Urbain and Forgeot observed that the virulence was increased for this animal. They further noted that this increase in virulence was associated with a transformation in antigenic properties, just as though the passages had been made through the animal itself. Table 43 summarizes their experiments.

These experiments solve the question. The explanation given by the authors, namely, that the transformation is a function of the resistance of the animal, can not be invoked. The alteration in properties here

* d'Herelle, F. and LeLouet, G.--Sur la vaccination antibarbonique par virus atténué. Ann. Inst. Pasteur, 1921, 35, 741. 
observed can only be a phenomenon linked with assimilation. When the antigenic properties of the streptococcus undergo a change it must mean that the substance of this streptococcus has changed, and that change varies with the nature of the foodstuff. The assimilation is not complete; the "substance" of a streptococcus which develops at the expense of "horse substance," although becoming "streptococcus sub-

TABLE 43

\begin{tabular}{r|r|r|r|r|r|r}
\hline \multirow{2}{*}{$\begin{array}{c}\text { PAssage } \\
\text { NUMBER }\end{array}$} & \multicolumn{6}{c}{ Strains of Streptococci } \\
\cline { 2 - 7 } & \multicolumn{1}{c}{376} & Ségala & Sauteur & Passe & A.1179 & Eclaireur* \\
\hline \multicolumn{7}{c}{ Medium: Bouillon, 4 cc. + guinea-pig blood, 1 cc. } \\
\hline 1 & 1400 & 1200 & 1200 & 1400 & 1200 & 1200 \\
5 & 500 & 500 & 500 & 1000 & 500 & 1000 \\
10 & 0 & 0 & 0 & 200 & 25 & 200 \\
15 & & & & 50 & 0 & 0 \\
17 & & & & 0 & & \\
\hline
\end{tabular}

Medium: Bouillon, 4 cc. + rabbit blood, 1 ec.

\begin{tabular}{r|r|r|r|r|r|r}
\hline 1 & 1400 & 1200 & 1200 & 1400 & 1200 & 1200 \\
5 & 700 & 300 & 300 & 500 & 1000 & 500 \\
10 & 100 & 50 & 50 & 0 & 200 & 50 \\
15 & 0 & 5 & 0 & & 25 & 50 \\
17 & & 0 & & & 0 & 5 \\
18 & & & & & & 0 \\
\hline
\end{tabular}

* Attention is called to the fact that the differences in the number of passages are due to the fact that it was necessary, in some cases, to make isolations from agar in order to separate the streptococci from associated organisms, which, despite the precautions taken, occasionally contaminated their cultures through blood stream invasions. In those instances in the table where the series is terminated the loss of antigenic power was complete at the tenth passage. The names applied to the strains of streptococci are the names of the horses from which they were isolated.

stance" retains, nevertheless, some of the characters of "horse substance," and the smaller the difference between the two substances, the greater is the virulence. The same thing takes place if the streptococcus is subjected to passage through another animal. $\dagger$

* This relation between the virulence and the quality of the substance of the bacterium is, without doubt, very simple. It may be associated with phagocytosis, which may be the more active as the substance of the bacterium differs from the substance of the animal invaded.

$\dagger$ It should be observed that these authors have noted that in order to induce a transformation in antigenic character by passages in artificial media, it is essential that the added animal product,- -blood,--be unmodified by heating. In effect, it is necessary that the animal substance be still "alive." 
In brief, then, these experiments, together with a number of facts previously known, show that assimilation in the bacteria is not complete. The substance of the bacterium varies according to the foodstuff utilized for its development, and this results in a variability of antigenic properties.

Is this also true for the protobes? This is a question which bears directly upon the subject of the unicity of the bacteriophage. We will consider this very shortly.

\section{THE CONCEPt OF SPECIES AMONG THE MICROBES AND THE PROTOBES}

Man has a tendency to generalize. Our knowledge of assimilation has been acquired by the study of superior animals, and it has been extended as such to all living beings. We have seen that this is certainly not correct in so far as bacteria are concerned. It is the same with regard to the concept of species, which, also, has been acquired in the first place by a study of superior animals and plants. To transfer this concept of species to rudimentary beings and to apply it in all of its details is again to follow a false trail.

Observation of nature shows that the facility with which adaptation takes place is directly proportional to simplicity of organization. With the superior animals adaptation takes place only within very narrow limits; as soon as a change, if pronounced and sudden, occurs in the conditions of the medium the being succumbs. And since variability is necessarily related to the facility of adaptation, the result is a high degree of homogeneity among the animals or the plants belonging to a single species. In so far as animals are concerned, and they have been more extensively studied from this point of view, the antigenic characters are identical in all of the individuals belonging to a single species. Indeed, a common antigenic character may extend beyond a single species, manifesting itself in neighboring species, a fact which has been utilized to disclose the bonds of relationship uniting them. This question of antigenic specificity in the animal and vegetable series offers a most interesting subject for study. But however great this specificity may be in other species, we have seen in an earlier section that with the bacteria the characters vary even within the individual according to the nature of the food. This variability in the substance of a single being involves as a consequence a variability in all of the other characters. What becomes, then, of the idea of species among these beings?

To take a concrete example. May we apply the idea of species in the 
same way to $B$. coli as to a superior plant, Melilotus altissima, for example? It is needless to enter here into comparisons such as anyone may make; to do so would but divert us from the more important subject.

We are forced to conclude that with these rudimentary beings, the bacteria, and unquestionably this is fully as true with the protobes, the concept of species can not have the same meaning as with beings higher in the scale of organization. As a matter of fact, if one wished to generalize, he would be forced to admit that a bacterium changes its species according to the conditions of the moment. A striking example is given us in the paper cited in the preceding section. Brocq-Rousseu, Urbain and Forgeot, after stating that "the strangles streptococcus is a species which can be differentiated from all of the other known streptococci," add, "by passages through animals, the strangles streptococcus loses its characters." Certainly, these two statements do not harmonize. It would be only too easy to multiply examples of contradictions of this kind.

\section{THE UNICITY OF THE SPECIES PROTOBIOS BACTERIOPHAGUS}

The fact that an "ultrapure" bacteriophage may show multiple virulences, that is to say, may parasitize bacteria belonging to different species, sometimes bacteria very remotely related, indicates that there is but a single species of the bacteriophage. Experiment shows further that this being is capable of adapting itself to the parasitism of bacteria which it did not previously invade. This realizes experimentally a process which is continually taking place in nature.

But if it is true that there is but a single species of bacteriophage, within this rudimentary being the phenomena of adaptation go on continually, the variability attained is extreme, and the point is reached where each race possesses its own characters (d'Herelle ${ }^{315}$ ).

This should not surprise us. The situation is exactly the same with the neighboring beings, the bacteria. We know very well that within any bacterial species there are no two strains which are, or can be, identical.

Objections to the concept of the unicity of the bacteriophage have been raised by a number of authors, but before discussing these objections I should first mention that none of them have explained just what they mean by "plurality of bacteriophages." If they mean that each strain, each race* of the bacteriophage possesses its own characters,

* Throughout this text the word "race" has been used intentionally in speaking of the bacteriophage, while the term "strain" has been reserved for bacteria. 
different from those of all other races their definition accords perfectly with what I have maintained consistently from the beginning of my studies, when I alone was occupied with the question. This interpretation I have adhered to throughout. Here is, in extenso, a passage from one of my papers published in 1919.315

I have stated above that I have not yet isolated two races of the bacteriophage which are absolutely identical. The differences consist in the extent of their action and in its intensity. When derived from the body some strains will be endowed with a bactericidal power for several of the intestinal bacilli; for example, they will attack, with different degrees of intensity, all of the dysentery bacilli, the typhoid bacillus, the paratyphoid strains, and $B$. coli; other strains will attack only a single bacterial species to the exclusion of all others, and a third race may attack two, the Flexner dysentery bacillus and paratyphoid $B$, for example. All possible combinations may occur. . . . And, since all possible combinations may be presented both as to extent and as to intensity, it may readily be understood that it is quite possible that millions of strains of the bacteriophage may be isolated before two are encountered which are identical.

I do not believe that I can express more clearly the idea that each strain-each race- possesses particular characters.

When these authors who support the idea of the "plurality of bacteriophages" take exception to this conception, that is, to the idea of a plurality of races which I was the first to observe, it can only mean that they affirm that there is a plurality, not of bacteriophages, but of species.

In order to avoid all confusion let me repeat that I affirm that the fact of variability, which can be induced experimentally within a single race, coupled with the fact of multiple virulences, shows that the differences between different races may be derived through adaptation, and that, in accord with the conditions of the moment, a "Shiga-bacteriophage" may be transformed into a "Staphylo-bacteriophage" or into a "Pestis-bacteriophage," in just the same way as by passages a "horse streptococcus" becomes a "rabbit streptococcus" or a "mouse streptococcus."

What, then, are the arguments advanced by these authors in support of their concept of plurality, that is, their belief that a Shiga-bacteriophage must remain a Shiga-bacteriophage always, that a Pestis-bacteriophage will never attack anything but $B$. pestis, and who affirm, in brief, to use the very expression of Bruynoghe,- the champion of the plurality

This plan has been followed simply for the sake of convenience and to avoid repetition and possible confusion. The implied meaning given to these terms, is, however, precisely that which ordinary usage accords them. 
of species idea,- that there is "not one bacteriophage but a great many bacteriophages, as different from each other as are the bacteria." 15

The arguments are four in number:

First. The antigenic variability of the bacteriophage races.

Second. The variability in resistance to different destructive agents.

Third. The fact that a bacterium which has developed a resistance to one bacteriophage may be attacked by another.

Fourth. Because different bacteriophage races may differ in the form of their colonies.

The reply to the first of these objections has already been given in the preceding paragraph. Just so surely as the antigenic properties of a bacterium like the streptococcus can be transformed by passages at the expense of different animals, it is but natural that the same fact should occur with the bacteriophage, and that the antigenic properties should differ in accord with the bacteria at the expense of which it has multiplied.

It is not without significance that, as we know, each strain of $B$. coli has its own individual antigenic properties, and that there are as many coli antigens as there are strains of $B$. coli. Has anyone yet ventured to advance the idea that each strain of $B$. coli represents an individual species? It is obvious that all strains of $B$. coli belong to a single species which is in a continual state of antigenic transformation. Upon what basis, then, can anyone advance the fact of the antigenic variability of the bacteriophage as an argument in favor of a plurality of species?

As for variability in resistance, clearly if the resistance of each race were a fixed property this argument would deserve consideration. But this is not what is observed. The studies of all authors who have considered the question, even including the collaborators of Bruynoghe, show that from one experiment to another a single bacteriophage presents considerable variations,- differences as great as are those observed between the different races.* This argument is therefore without value.

The argument based on the fact that one race of the bacteriophage may attack a bacterial strain which has acquired a resistance toward another race means nothing, for the same fact occurs with even a single race

* There is nothing strange in this, since with the bacteriophage as with pathogenic bacteria, virulence disappears prior to their destruction. We know that bacteria may become avirulent and yet remain alive. With the bacteriophage the same thing occurs. Absence of bacteriophagy does not necessarily imply total destruction of the being. With the bacteriophage an "apparent" destruction coincides with a loss in virulence, and this loss is effected through the action of a physical or chemical agent the more readily when the virulence is weak. 
I have performed with three different strains of B. typhosus the following experiment. By suitable contacts a strain of the typhoid bacillus is induced to acquire a resistance to a race of the bacteriophage which, at the same time was virulent for B. typhosus, B. coli, and B. dysenteriae. But, and this is the significant point, when tested later, this B. typhosus, although resistant to the bacteriophage developed through passages with $B$. typhosus, is bacteriophaged perfectly by the same race which in the meantime has undergone passages with either $B$. coli or with $B$. dysenteriae.

If we may find an inequality in the resistance acquired by one bacterium for a single bacteriophage developed at the expense of different bacteria, there is the more reason that it may be observed with different races. Certainly it can not weigh very heavily against the idea of the unicity of species of the bacteriophage.

As for the fourth objection, it disregards the fact, recorded by many authors, that the size of the plaque* is, with a given race of the bacteriophage, strictly related to its virulence. If the diameter of the plaque varies for a single race of the bacteriophage it can hardly cause astonishment if it varies for different races for the same reason.

The protobes, the bacteriophage in particular, like the microbes, the bacteria especially, are beings endowed with a very considerable power of adaptation. The variability which we find in antigenic properties shows further that assimilation is not brought about in the same manner in rudimentary beings as in beings more highly organized. According to the foodstuff utilized, even the substance of these beings shows variations. These two experimental facts demonstrate that as a consequence there must be an extreme variability in all characters in conformity to the conditions of the moment. There is but a single species of the bacteriophage, as is shown by the phenomenon of multiple virulences and by the possibility of experimental adaptation. But within this single species each race presents its own characters distinguishing it from other races, characters which are, nevertheless, variable from one time to another.

\section{THE MODE OF ACTION OF THE BACTERIOPHAGE}

Study of the phenomenon of bacteriophagy shows us that, most probably because of some chemotactic force, a bacteriophage protobe is

* Bruynoghe says "the form" of the colony. This term is incorrect, for plaques are always perfectly circular; only the diameter may vary. 
directed toward a bacterium and a contact is established. The protobe then penetrates the peripheral zone of the bacterium (the opening made remaining open) and multiplies within the bacterial protoplasm, forming a colony composed of a number of elements varying with the conditions of the moment. When the process of multiplication is completed the parasitized bacterium ruptures and undergoes a sudden dissolution. This liberates the young protobes, and each of the latter is then ready to repeat the process and parasitize the nearest bacterium. Thus the cycle begins anew, and continues until all of the bacteria present in the media have disappeared.*

How is this dissolution of the bacterial body brought about?

A priori, there are three possible hypotheses:

I. The bacteriophage protobe may itself act like an enzyme. In such a case it must be, in short, an enzyme of which each granule is capable of assimilation and of reproduction at the expense of the substances upon which it acts. This hypothesis is hardly probable, for the bacteriophage corpuscles, although minute, have a relatively large volume, $-20 \mu \mu-$ much larger than the granules of the known ferments.

II. The bacteriophage induces the bacterium to produce autolytic enzymes. The meaning of the first hypothesis was self-evident; this second one requires a little explanation.

Autolysis in a confined medium,- the method of Jaumain, ${ }^{377}$ - of which I have spoken above, shows us that under certain peculiar physical conditions, but without the intervention of any foreign substance, a non-spore-bearing bacterium may quickly produce autolysins capable of dissolving the bacterial body completely. The phenomenon is comparable to that which takes place in bacteriophagy, except that it is not transmissible in series. A permanent cause, persisting despite successive dilutions, is lacking.

For some time we have known that a great many spore-forming bacteria completely disappear after the formation of the spore. In this connection I have studied B. anthracis, an organism admirably suited and of particular interest from this point of view inasmuch as it is easy to obtain asporogenous varieties. It is found that, although with the sporulating races a dissolution of the vegetative forms in the medium may readily be observed after the formation of the spore, a natural spontaneous autolysis is never observed with the homologous asporogenous races. The autolysis is, therefore, predicated upon the presence of the spore within the interior of the bacterium.

* This is conditioned, naturally, upon the bacteriophage being sufficiently virulent. 
Considering the subject from a somewhat different point of view, there are many facts which show that the embryo, in animals as in plants, behaves like a parasite, and that the very distinctive reactions which it provokes may be brought about by a true parasite. As a striking example of this fact it is only necessary to recall that the considerable chemical activity mobilized by the plant at the moment of the formation of the seed results from the reaction of the flower to a stimulation of the embryo; but Bohn has shown that certain dipterathe Cecidomyia-deposit their eggs in plants belonging to the Vicia and Gallium genera, and that the presence of the egg leads to the formation of a false fruit, similar as to form, chemical composition and histological structure, to that of the true fruit.

There are, then, plants, in which the stimulation caused by the larva of a parasite produces the same effect as the stimulation caused by the embryo.

But to return to the bacteriophage; the bacteriophage corpuscle certainly penetrates the interior of the bacterium and multiplies there. It may be that the presence of the bacteriophage corpuscles within the interior of the bacterium causes reactions of the same type as those normally induced by the presence of the spore, that is to say, an autodissolution of the parasitized bacterium. The bacteriophage would then utilize for its metabolism the autolysed substances or at least, some of them.

This new conception of the mode of action of the protobe does not apply solely to the bacteriophage, but in general to all of the infravisible viruses which are invariably intracellular parasites.

Immunity in the cell attacked may be of one of two kinds, depending upon which of one of two processes becomes operative; a relative immunity depending on an adaptation to the stimulating action of the parasite, an adaptation which may progress even up to the point of a symbiosis, or a complete immunity, consisting in a digestion of the parasite.

III. The third possible hypothesis dealing with the mode of action of the bacteriophage assumes that the bacteriophage itself elaborates ferments which dissolve the substance of the bacteria, thus utilizing the substance of the bacteria for its development.

By analogy with the behavior of other living beings this hypothesis is the first to come to mind, but analogy most assuredly is not proof, and it may well be that in these rudimentary beings, occupying the very first step in the sequence of living creatures, behavior may be different. 
Bacteriophagy does not take place in physiological saline, despite the fact that the inoculation of $0.05 \mathrm{cc}$. of a bacteriophage having a maximum virulence into $10 \mathrm{cc}$. of a saline suspension is followed by a complete clearing of the medium. This experiment, performed several times, led me to believe that bacteriophagy could actually occur under such conditions. ${ }^{310,321}$ As a matter of fact this is not the case, for a multiplication of the bacteriophage corpuscles never occurs.* Several authors have made this same mistake. Obviously, this experiment might be interpreted as according with the hypothesis of the existence of an enzyme in the suspension.

There is one fact, however, which seems to favor this third possible hypothesis. By the addition of alcohol all of the protein substances in a suspension of the bacteriophage, the latter included, are precipitated. If the precipitate is allowed to remain in contact with the reagent for a period sufficiently long to kill the protobes, we find that the precipitate redissolved in saline exercises an inhibitory action upon the growth of a bacterium of the same species as that which had been bacteriophaged. This experiment has been questioned by a number of authors, and indeed, when I first recorded it, I presented it with some reservations. I have since repeated this experiment and I have again obtained an inhibitory effect. There is, nevertheless, an objection to the experiment which no one has as yet raised and which is still more serious. Even if the inhibiting action were extremely clear-cut, even if (as is not the case) the material caused a non-transmissible "lysis," one could not affirm that the "lytic substance" was a product of the bacteriophage rather than of the bacterium. I realize, then, that this observation is invalid as an argument.

Some other facts, not without interest may be recorded. From among my races of the Staphylo-bacteriophage I selected one which acts on some strains and not on others. When this race was added to a suspension containing a mixture of a susceptible staphylococcus and of a naturally resistant strain, the latter were to some extent dissolved. But here again, one might quite logically object that perhaps this dissolution of resistant organisms takes place through the action of an enzyme liberated by the susceptible staphylococci during their bacteriophagy.

* There is, however, some multiplication if the quantity of suspension inoculated is a little larger, a result most certainly to be ascribed to the fact that the bouillon present in the inoculated bacteriophage suspension serves as nutritive material. In fact, Maitland ${ }^{432}$ has shown that the addition of 1 per cent of bouillon to saline is sufficient to permit bacteriophagy to take place. 
While the following experiments can be very readily interpreted by the hypothesis of a lytic ferment secreted by the bacteriophage I hasten to add that they may also be interpreted in other ways, a fact which naturally deprives them of the character of proof.

Divide a suspension of staphylococci (125 million cocci per cubic centimeter) into two portions. Inoculate the first with 0.05 cc. of a Coli-bacteriophage, the second with 0.05 cc. of the same bacteriophage together with some susceptible $B$. coli. Both tubes are placed in a water-bath at $45^{\circ} \mathrm{C}$. After $3 \frac{1}{2}$ hours the opacity of the second suspension is about half of that of the first. Spreadings on agar show, after incubation, for the staphylococcus suspension a layer of growth extending over the entire surface of the agar, for the mixed staphylococcus-coli suspension, isolated colonies.

Results of the same nature have been secured in another experiment where a Coli-bacteriophage was combined, in one tube with Vibrio metchnikovii, and in another, with a mixture of the vibrio and B. coli.

The results are the same if a suspension of streptococci is used with the Coli-bacteriophage. Spreadings on agar of a drop of a suspension of streptococci inoculated with a Coli-bacteriophage give innumerable colonies; a spreading of a mixed streptococcus-coli suspension yields only a dozen colonies.

Here are some experiments of another type, carried out in physiological saline. A suspension of staphylococci, 250 million per cubic centimeter, is heated for a half hour at $70^{\circ} \mathrm{C}$., and tests show that the fluid is sterile. Each of three tubes receives this suspension. To the first of these add $1 \mathrm{cc}$. of a very potent Staphylo-bacteriophage, to the second $0.25 \mathrm{cc}$. , and to the third $0.1 \mathrm{cc}$. After 48 hours at laboratory temperature the first suspension is hardly cloudy (the turbidity is about equal to that of a suspension containing 40 million cocci per cubic centimeter), the second tube is about half as cloudy as at the beginning, while the third is unchanged, showing no evidence of clearing.

Obviously, these experiments are open to the criticism that they give no proof that the dissolving ferments are produced by the bacteriophage. This I realize perfectly, and the experiments are to be taken only at their face value.

As a matter of fact, up to the present time, no experiment has been performed which allows us to select between the three possible hypotheses of which I have spoken. The second, especially, is as probable as the third. 
The first essential toward gaining some light on this question is to study the nature of the products which result from the dissolution of bacteria through the action of the bacteriophage. The bacterium selected for this type of study should be $B$. coli, since this organism is readily bacteriophaged in a synthetic medium free of protein materials. Up to the present nothing has been done in this direction, or rather that which has beer done has no very great value if one may judge from the contradictory conclusions reached by the two authors who have attempted to ascertain the nature of the products resulting from the degradation of the bacteria. According to Otto and Sukkienikowa ${ }^{497}$ the bacteria are necessarily degraded into special products; according to Ionesco-Mihaiesti ${ }^{366}$ the bacteriophage does not attack the protein molecule of the bacterial substance at all, the process being simply a plasmolysis. It would seem that it would be well to have this question investigated by a chemist.

As for the fact that the injection of an animal with bacteriophage suspensions,- which as we know contain the dissolved bacterial substances in addition to the bacteriophage protobes themselves,- causes the appearance in the serum of both antibacteriophagic and antibacterial properties, this clearly shows that all of the bacterial substances are not modified by the bacteriophage. One portion remains chemically intact, since it retains its antigenic properties, while, on the contrary, another fraction necessarily undergoes a chemical transformation since it represents a new antigen. This last fraction corresponds to the bacterial substances which have been assimilated and transformed into bacteriophage substance.

\section{CONSEQUENCES RESULTING FROM THE LIVING NATURE OF THE} BACTERIOPHAGE*

Protobios bacteriophagus (syn. Bacteriophagum intestinale) forms a new species from the point of view of the systematist, but in what kingdom, animal or vegetable, does it belong?

In all probability to neither the one or the other. To explain: It is impossible for us to believe that life results in a spontaneous forma-

* I would refer the reader who may be interested in this aspect of the subject to Immunity in Natural Infectious Disease, Williams \& Wilkins, Baltimore, 1924. I have shown there the nature of the probable results of the existence of protobes as applied to the constitution of bacteria, beings which in reality are intermediate between the protobe and the cellular microbes. The bacteria must be aggregations of protobes, each micella being the less evolved bacterium, and capable of reproduction and of forming again the bacterial plasmodium. 
tion, as a result of a physical-chemical phenomenon associated with a particular constitution of matter, of a protozoan or a bacterium, for these organisms, although comparatively simple, are much too complex to be formed all at once.

A scientific conception of the origin of life should, on the contrary, be possible, just as soon as experiment demonstrates that there are beings of rudimentary organization, constituted of very simple assemblages of a few micellae, the most rudimentary of one only, which, nevertheless, possess all of the characteristics of living beings, even of those of a very complex organization, that is, the powers of assimilation and of adaptation, with their corollaries, the faculty of multiplication and the variability of characters. All these properties appear, indeed, to be bound up inseparably, resulting from a single cause.

We actually know a number of "micellar" beings. They are those which experiment up to the present time has shown to be able to pass through ultrafilters; - the agents of bacteriophagy, of the mosaics of plants, of the animal plagues, of variola, vaccinia, rabies, and of encephalitis lethargica. And the list is certainly not closed.*

These beings, manifesting what might be termed elementary life, most probably form a homogeneous group placed at the bottom of the two kingdoms, animal and vegetable, and from which are derived, by a simple phenomenon of aggregation, of synthesis, on the one hand the protophytes, and on the other, the protozoa. The name designating all of this group should be "Protobes."

We have up to now considered the question of the bacteriophage only in its relation to the phenomenon of bacterial dissolution. It has interests extending far beyond this.

In the last analysis, all of human science is actually directed toward the solution of two great problems; the nature of matter and the nature of life.

During the last few years physicists have materially advanced the knowledge of their problems. And the significant point is just this, it was not by studying matter in its tangible form that they succeeded.

* Among the diseases caused by the ultraviruses it is necessary to distinguish clearly those in which the agent is a protobe, from those whose agent is an infravisible form of a bacterium. To the causative agents of the second group I have applied the term ultrabacteria. In the last group we would certainly include scarlet fever, the agent of which must be an infravisible form of a streptococcus. And there must be many more diseases which come within this category. However, the difference between a protobe and the infravisible form of a bacterium is not great. (See Immunity in Natural Infectious Disease.) 
It was only when they attacked the infinitely small particles of chemical substances, when they studied the infravisible, that the day commenced to dawn. The meaning is clear. Surely it is not through studying visible organisms, complex ones, that biologists will reach the solution to their fundamental problems, for with such the investigator finds himself surrounded by a multiplicity of diverse factors, which by their very number and by their complexity effectively prevent advance into the unknown. The physicists have blazed the trail.

Here, as in the study of matter, it is necessary to approach the problem from the bottom. We do not know what life is, but we do know that life is certainly a physical property, a behavior of a colloidal micella of a particular constitution. In order to study this constitution, this behavior, we must necessarily turn to the smallest possible particle of autonomous living matter, where life presents itself in its most elementary form, where the complexity of the vital phenomena is least extensive. This infinitely small living being which it is necessary to study is, therefore, a protobe, and to be even more specific, it is that one which can most readily be observed, the bacteriophage.

\section{RÉSUMÉ}

The living state can only depend upon one of the two following conditions: either life results from an organization, or it results from a physico-chemical state of matter.

Up to the present time the first theory, the cellular theory, has been almost universally accepted.

The fact that the bacteriophage corpuscle has been demonstrated to be a simple protein micella, and that it is a living being, shows that the cellular concept of life is erroneous. Of necessity, then, the second alternative must be true. Life results from a particular physicochemical state of the protein micella.

With cellular beings, in particular with the metazoa provided with a digestive apparatus, assimilation is complete. That is, the substance of the food is transformed into substance identical with that of the being which assimilates. With the protobes, and also with the bacteria, experiment demonstrates that assimilation is incomplete. Although the substance of these beings is essentially different from that of the foodstuffs consumed, nevertheless, it varies with the nature of the food. as is proved by the variation in antigenic properties.

As a result of these variations, which are common to the protobes and to the microbes,- - to bacteria at least,- - there occurs a great variabil- 
ity among different representatives of a species. Each race of the bacteriophage presents special characters subject to variation from time to time.

The facts of multiple virulence and of an experimental adaptation to the parasitism of a species previously unattacked, show that all races of the bacteriophage belong to a single species, the special characters of each race being acquired by adaptation.

None of the arguments adduced by the partisans of the idea of a plurality of species of the bacteriophage are valid, for the facts upon which these arguments are based are observed just as clearly within a single race of the bacteriophage as among different races.

Only three hypotheses are possible as to the mode of action of the bacteriophage protobe:

It may act as an enzyme of which each granule is living;

It may act by causing the bacterium itself to elaborate autolytic enzymes;

It may itself elaborate bacteriolytic enzymes.

Upon the basis of our present knowledge it is impossible to choose from among these three hypotheses.

The fact that there is such a being as Protobios bacteriophagus permits us to approach, in a scientific way, the problem of the origin of life. 



\section{PAR'T III \\ THE BEHAVIOR OF' THE BACTERIOPHAGE PROTOBE}





\section{CHAPTER I}

\section{The Bacteriophage as an Antigen}

\section{INOCULATION OF THE BACTERIOPHAGE}

The normal habitat of the bacteriophage protobe is the digestive tract of animals, where it finds constantly the sole foodstuff at the expense of which it subsists, living bacteria. But this does not mean that the bacteriophage necessarily remains confined to the intestine. As a matter of fact it may be found in the urine $\left(d^{\prime} H e r e l l{ }^{310}\right)$, in the blood (d'Herelle ${ }^{313}$ ), and in other physiological and pathological products. Without entering into a discussion of this point at present, for we will later return to it, we may state that although the presence of the bacteriophage in the intestine is a constant occurrence, when it is found elsewhere its presence is incidental, and this in turn suggests that when it is found outside of its normal habitat it must have come from the intestine after passage into the circulation.

The presence of the bacteriophage protobe within the body does not lead to any disturbance and is not accompanied by any visible symptoms such as would inclicate its presence. I have inoculated rabbits with suspensions of the Shiga-bacteriophage ${ }^{311}$ without inducing in the animals the slightest detectable reaction. Similarly, no reactions at all appeared among the hundreds of fowl to which I administered, either by injection or by ingestion, bacteriophage races virulent for Eberthella sanguinaria (B. gallinarum). ${ }^{314}$ The hundreds of buffaloes which received the bacteriophage virulent for Pasteurella bovis also failed to show any reaction consequent to the injections. ${ }^{322}$

Bordet and Ciuca ${ }^{88}$ injected 2 cc. of a Coli-bacteriophage subcutaneously into a guinea pig. Seven hours later they bled the animal, observing aseptic precautions, and found that the blood contained a bacteriophage virulent for $B$. coli. They secured the same results with a rabbit which had received $20 \mathrm{cc}$. of the bacteriophage intravenously.

Appelmans ${ }^{12}$ found that after a subcutaneous injection the bacteriophage was eliminated rapidly from the different organs and tissues. It persisted in the spleen for several days after all of the other organs had become ultrasterile. Table 44 summarizes the results this 
author obtained with guinea pigs, when inoculated subcutaneously with 0.6 cc. of a suspension of the Coli-bacteriophage.

He failed to find a bacteriophage in the tissues or organs of normal uninoculated guinea pigs.

Other investigators have also attempted to determine the fate of the bacteriophage when inoculated, and their results do not agree, as is evident from the following résumé of the published findings. Werthemann ${ }^{626}$ reported that after the intravenous injection of guinea pigs, rabbits and frogs, the corpuscles disappear from the circulation slowly. In warm-blooded animals they could be found for 5 days; in the frog for 7 days. I have made comparable experiments with several different races of the bacteriophage, but I have never been able to detect the corpuscles in the blood 48 hours after the inoculation. According to Suzuki ${ }^{594}$ after an intravenous injection in the rabbit, the bacteriophage may be found in all of the organs, including the digestive tract, 1 hour later. After 3 hours they are to be found in the

TABLE 44

\begin{tabular}{|c|c|c|c|c|c|c|c|c|}
\hline \multirow{2}{*}{$\begin{array}{l}\text { INTERVAL } \\
\text { AFTER } \\
\text { INJECTION }\end{array}$} & \multicolumn{8}{|c|}{ BACTERIOPHAGE PRESENT OR ABSENT IN THE } \\
\hline & Liver & Kidney & Spleen & Heart & Blood & Urine & Testes & Lungs \\
\hline 24 hours & + & + & ++ & + & + & $+t$ & + & + \\
\hline 5 days & 0 & + & ++ & 0 & 0 & 0 & 0 & 0 \\
\hline 15 days & 0 & 0 & 0 & 0 & 0 & 0 & 0 & 0 \\
\hline
\end{tabular}

liver and spleen only, while after 24 hours they have completely disappeared from all of the organs of the body. After a subcutaneous injection the relative rates of disappearance were the same, although the elimination throughout was somewhat delayed. Thus, the bacteriophage was found in all of the organs after 3 hours, but it had completely disappeared after 24 hours. He also reports that after ingestion the corpuscles can be found in so short a time as 30 minutes in the lung and in the spleen. This would certainly seem to be erroneous.

The results of my own studies agree throughout with those of Appelmans. When ingested the bacteriophage does not appear to penetrate the intestinal wall under normal conditions, but on the contrary it does pass through readily when within the peritoneal cavity there is some agency causing irritation. In discussing the hypothesis of Bordet and Ciuca we have seen that these authors concluded that as a result of the intraperitoneal injection of bacterial cultures it was possible to isolate from the peritoneal exudate a bacteriophage virulent 
for the bacterium injected. I have shown ${ }^{328}$ that it is the exception for this experiment to give the result claimed for it by Bordet and Ciuca, but by modifying the conditions under which it is done only to the extent of having the animal ingest, a few hours before the intraperitoneal injection, a suspension of bacteriophage virulent for the bacterium injected, the experiment uniformly becomes successful. This proves that the bacteriophage isolated comes from the intestine, the passage into the peritoneum through the intestinal wall being induced by the irritation caused by the presence of the bacteria in the body cavity. Although lending but scant support to Bordet's hypothesis of the origin of the bacteriophage, this experiment does show one thing very definitely, namely, that a resorption of bacteriophage protobes through the intestinal wall can occur. The fact that the corpuscles can and do pass into the body has been confirmed by many investigators, whose contributions to the subject will be presented in the discussion to follow.

When bacteriophage protobes have penetrated into the tissues what is their fate?

The experiments of Appelmans ${ }^{12}$ already mentioned have shown us that bacteriophage protobes behave like bacterial spores; they become concentrated in the spleen where they persist for some time after they have disappeared from the other tissues. This strongly suggests that they must be carried there, or retained there, by the phagocytes.

Bruynoghe and Maisin ${ }^{123}$ have presented direct evidence that the bacteriophage protobes are phagocytized. They carefully mixed a trace of a bacteriophage suspension with $2 \mathrm{cc}$. of fresh sterile pus. The number of corpuscles present was determined immediately and again after incubation for 24 hours at $37^{\circ} \mathrm{C}$. Comparison of the two titrations showed that a very considerable diminution occurred, which, according to the experiment, represented a reduction by some hundreds or thousands of times.

It thus appears that the bacteriophage protobes behave in the body like saprophytic bacteria. But the substance of the protobes is protein in nature, and these proteins must function as antigens. That this is true is amply demonstrated by the data presented in the following section.

\section{ANTIBACTERIOPHAGIC SERA}

Bordet and Ciuca ${ }^{88}$ gave rabbits several injections of a suspension of Coli-bacteriophage. The animals were bled a few days after the 
last injection. Their first observation showed that the serum of the rabbit so treated had no effect upon the growth of $B$. coli. They then mixed a suspension of the homologous Coli-bacteriophage and the serum, and introducing 5 drops of this mixture into bouillon which they then seeded with a drop of a $B$. coli culture, they found that after incubation the colon bacilli had developed as abundantly as in a control tube which did not contain bacteriophage. From this they concluded that the serum of the animal, prepared by injections of the bacteriophage, possessed antibacteriophagic properties; that in the presence of the antiserum the bacteriophage failed to manifest its presence by the usual reaction.

They heated, according to their method, the culture which had received the mixture of antiserum and of bacteriophage at $58^{\circ} \mathrm{C}$., effected a few passages, and found that the bacteriophage was absent. Consequently, there has been, they said, a destruction through the action of the serum. We will ascertain in a moment if this last deduction is correct.

However that may be, whether the observed effect was due to an inhibition or a destruction, the fact remains that the serum of an animal, prepared by injections of a bacteriophage suspension, possesses an antibacteriophagic property.

The first serum obtained by Bordet and Ciuca neutralized the action of ten times its volume of a suspension of the homologous bacteriophage. They then found that the antiserum inhibited the process of bacteriophagy both on agar and in a liquid medium, for when they impregnated agar slants with the serum, and implanted them with a secondary culture of bacteriophage- $B$. coli, they obtained a normal growth, from which the subcultures remained normal indefinitely. The B. coli was "cured," as they expressed it. But if these bacteria have been cured, they remain resistant nevertheless. Bruynoghe $\mathrm{e}^{112}$ seeded a mixed culture of bacteriophage- $B$. coli into a mixture of equal parts of bouillon and antiserum. After a series of 6 subcultures he isolated colonies on agar and found that all of them were resistant.

Bordet and Ciuca also noted that the antibacteriophagic property of a serum could be established passively. When a normal rabbit is injected with some blood from a rabbit which has received repeated injections of the bacteriophage, within a few hours the blood of the normal rabbit has acquired the antibacteriophagic property.

And finally, they observed that after a series of injections of cultures of normal B. coli, - the same strain as that at whose expense the bac- 
teriophage had developed,-no antibacteriophagic property appeared in the serum, but if they injected a mixed culture the serum became antibacteriophagic. This might, indeed, have been predicted. I have shown that the injection of rabbits with very old mixed cultures (staphylococcus or Shiga) which had undergone several transplants, yields antibacteriophagic sera although they are weak.

The bacteriophage functions, therefore, as a specific antigen, an antigen not found in the normal bacterium. Such is the conclusion of these authors.

According to Prausnitz ${ }^{536}$ the antibacteriophagic property is thermostabile, resisting a temperature of $75^{\circ} \mathrm{C}$.

Otto, Munter and Winkler ${ }^{496}$ found that different races of the bacteriophage do not possess identical antigenic properties, and they also state that the higher the virulence of the bacteriophage, the stronger is the antigenic property. The protocols of their experiments do not seem to accord with this conclusion, as we will see later when we consider the question of the specificity of antibacteriophagic sera.

A very interesting observation made by these same authors, is that if the antibacteriophagic serum is combined with bacteria which have fixed the bacteriophage corpuscles, that is, when the serum is not added until an hour after the bacteriophage is introduced into the bacterial suspension, the corpuscles escape the action of the neutralizing principle, for when spread on agar, no growth appears after incubation. This is additional evidence that bacteriophagy can take place on a solid medium.*

These experiments of Otto, Munter and Winkler were confirmed by Wagemans, ${ }^{615}$ who also reported that different bacteriophages do not cause the appearance of "anti" properties having an equal neutralizing capacity. Certain of the antisera inhibited the action of the homologous bacteriophage when they were mixed with the suspension in a proportion of 1 to 1000, while others, under the same conditions, appeared to have no neutralizing property at all. $\dagger$ They proved that the nature of the animal inoculated contributed in no way to the differences in the activity of the antisera, but that the variable results

* This shows also that the bacteriophage corpuscles penetrate into the bacteria. If they were only adherent to the surface it would be difficult to understand why they should be protected against the action of the serum with which they are bathed.

† Comparable differences have been repeatedly observed with the bacteria and their antisera; never with the toxins. 
depended upon real differences in the antigenic property of the different races of the bacteriophage.

We have already mentioned that Prausnitz ${ }^{536}$ has shown that it is possible, by adaptation, to render a bacteriophage resistant to the action of its homologous antiserum. Bruynoghe and Wagemans ${ }^{127}$ confirmed this fact, but by a different procedure. They prepared three antisera of unequal neutralizing values for a single Coli-bacteriophage. Three successive passages were made with this bacteriophage, the first in media containing the least active serum, the second in media with the slightly more active serum, and the third in media containing the most active serum. After these three passages they found that the bacteriophage thus adapted caused a normal bacteri-

TABLE 45

\begin{tabular}{|c|c|c|c|c|c|c|}
\hline \multirow{2}{*}{$\begin{array}{c}\text { PROPORTION OF } \\
\text { BACTERIOPHAGE TO } \\
\text { ANTISERUM }\end{array}$} & \multicolumn{6}{|c|}{ COUNTS OF THE BACTERIOPHAGE CORPUSCLES MADE } \\
\hline & $\operatorname{Im}_{\text {mediately }}$ & $\begin{array}{c}\text { After } \\
1 \frac{1}{2} \text { hours }\end{array}$ & $\begin{array}{l}\text { After } \\
8 \text { hours }\end{array}$ & $\begin{array}{c}\text { After } \\
24 \text { hours }\end{array}$ & $\begin{array}{c}\text { After } \\
48 \text { hours }\end{array}$ & $\begin{array}{c}\text { Aitter } \\
96 \text { hours }\end{array}$ \\
\hline $1: 10$ & $1300^{*}$ & 28 & 1 & 2 & 0 & 0 \\
\hline $1: 20$ & $2000^{*}$ & 184 & 3 & 3 & 1 & 0 \\
\hline $1: 40$ & $2000^{*}$ & 560 & 6 & 3 & 1 & 0 \\
\hline $1: 80$ & $2000^{*}$ & 840 & 185 & 20 & 2 & 1 \\
\hline $1: 160$ & $3000^{*}$ & 960 & 260 & 47 & 1 & 4 \\
\hline $1: 320$ & $4000^{*}$ & $1800^{*}$ & 240 & 485 & 7 & 13 \\
\hline $1: 640$ & $4000^{*}$ & $2000^{*}$ & $1100^{*}$ & 710 & 750 & 16 \\
\hline $1: 1280$ & $4000^{*}$ & $3000^{*}$ & $1600^{*}$ & 905 & $1800^{*}$ & $1200^{*}$ \\
\hline $\begin{array}{l}\text { Bacteriophage }+ \\
\text { saline }\end{array}$ & $9000^{*}$ & $9000^{*}$ & $9000^{*}$ & $5000^{*}$ & $5000^{*}$ & $8000^{*}$ \\
\hline
\end{tabular}

* The figures marked with an asterisk were obtained by counting a segment of a plate, using a Wolfhügel apparatus.

ophagy in the presence of quantities of a homologous antiserum which completely inhibited the action of the same unadapted bacteriophage.

They found further that when the bacteriophage has become resistant, after having undergone passages in a normal medium sufficient in number to insure that all traces of the serum have been eliminated, it caused the development of "anti" properties when used as an antigen more active for the original unadapted bacteriophage than for the adapted bacteriophage- the corpuscles used in the immunization of the animals.

3. THE COURSE OF THE ACTION OF THE ANTIBACTERIOPHAGIC SERUM

The following experiment of Prausnitz $z^{536}$ shows something of the nature of the process of inactivation of bacteriophage corpuscles by an 
antiserum. Table 45, taken from Prausnitz, illustrates the effect of an antiserum for a Flexner-bacteriophage upon the homologous bacteriophage. The figures refer to the number of corpuscles in 0.001 cc., based upon counts made by plaque determinations.

In his discussion Prausnitz calls attention to the inequality in resistance of the different corpuscles present in a single suspension, a fact which is in agreement with what we know of the behavior of living beings. He also points out as worthy of comment the slowness with which the corpuscles are inactivated.

\section{VARIABILITY IN THE BEHAVIOR OF THE BACTERIOPHAGE}

When we undertake to consider this phase of the subject we are immediately confronted by experimental results which are absolutely

TABLE 46

\begin{tabular}{|c|c|c|c|c|c|c|c|c|}
\hline \multirow{3}{*}{$\begin{array}{l}\text { RACE OF BACTERIOPHAGE WITH } \\
\text { ANTI-KI SERUM }\end{array}$} & \multicolumn{8}{|c|}{ PLANTINGS MADE ON AGAR OF } \\
\hline & \multicolumn{4}{|c|}{$\begin{array}{l}\text { Bacteriophage anti-serum mix- } \\
\text { ture combined with }\end{array}$} & \multicolumn{4}{|c|}{$\begin{array}{c}\text { Bacteriophage only combined } \\
\text { with }\end{array}$} \\
\hline & $\begin{array}{c}B . \\
\text { dysen- } \\
\text { teriae } \\
\text { Shiga }\end{array}$ & $\mid \begin{array}{c}B . \\
\text { dysen- } \\
\text { teriae } \\
\text { Flexner }\end{array}$ & $\mid$\begin{tabular}{c|}
$B$. \\
dysen- \\
teriae \\
Hiss
\end{tabular} & $\begin{array}{c}\text { B. } \\
\text { typho- } \\
\text { sus }\end{array}$ & $\begin{array}{c}B . \\
\text { dysen- } \\
\text { teriae } \\
\text { Shiga }\end{array}$ & $\mid \begin{array}{c}B . \\
\text { dysen- } \\
\text { teriae } \\
\text { Flexner }\end{array}$ & $\begin{array}{c}B . \\
\text { dysen- } \\
\text { teriae } \\
\text { Hiss }\end{array}$ & $\underset{\substack{B \text { pho- } \\
\text { sus }}}{\text { B. }}$ \\
\hline K (homologous). & +3 & +2 & +2 & +4 & +4 & +4 & +4 & +4 \\
\hline$P \ldots \ldots$ & 0 & 0 & 0 & 0 & +4 & +4 & +4 & +4 \\
\hline S..... & +4 & +4 & +4 & +4 & +4 & +4 & +4 & +4 \\
\hline $\mathrm{T}$. & 0 & 0 & \pm & \pm & +4 & +4 & +4 & +4 \\
\hline $\mathrm{F}-2 \ldots \ldots$ & 0 & 0 & 0 & 0 & 0 & +4 & +4 & 0 \\
\hline $\mathrm{F}-23 \ldots \ldots$ & 0 & 0 & 0 & 0 & 0 & +4 & +4 & 0 \\
\hline$Y \ldots \ldots \ldots \ldots \ldots \ldots$ & 0 & 0 & 0 & 0 & 0 & +4 & +4 & 0 \\
\hline
\end{tabular}

contradictory, and which, superficially at least, leave the subject in the utmost confusion. Let us consider these results in the order of their publication.

Otto, Munter and Winkler ${ }^{496}$ report the following experiments.

A. A rabbit received 11 injections, at 8-day intervals, of a bacteriophage, race $\mathrm{K}$, whose virulences were

with $B$. dysenteriae Shiga bacteriophagy at $1 \cdot 10^{-9}$

with $B$. dysenteriae Flexner bacteriophagy at $1 \cdot 10^{-9}$

with $B$. dysenteriae Hiss bacteriophagy at $1 \cdot 10^{-9}$

with $B$. typhosus bacteriophagy at $1 \cdot 10^{-5}$

The serum of this animal was mixed in equal parts with suspensions of different races of the bacteriophage, and the bacteriophage-antiserum 
mixture was added to suspensions of the different bacteria for which the races of bacteriophage were virulent.*

Table 46 indicates the comparative results obtained with the bacteriophage-antiserum mixture and the bacteriophage alone.

B. A rabbit received 7 injections of bacteriophage F-2. Each injection was 2 cc.; the intervals between injections, 8 days. This race caused bacteriophagy:

\section{with $B$. dysenteriae Flexner at $1 \cdot 10^{-4}$ \\ with $B$. dysenteriae Hiss at $1 \cdot 10^{-6}$}

and was without action on $B$. dysenteriae Shiga and B. typhosus.

Determinations made as above (with the anti-K serum) gave the result shown in table 47 .

TABLE 47

\begin{tabular}{|c|c|c|c|c|c|c|c|c|}
\hline \multirow{3}{*}{$\begin{array}{l}\text { RACE OF BACTERIOPHAGE WITH } \\
\text { AN TI-F-2 SERUM }\end{array}$} & \multicolumn{8}{|c|}{ PLANTINGS MADE ON AGAR OF } \\
\hline & \multicolumn{4}{|c|}{$\begin{array}{l}\text { Bacteriophage anti-serum mix- } \\
\text { ture combined with }\end{array}$} & \multicolumn{4}{|c|}{$\begin{array}{c}\text { Bacteriophage only combined } \\
\text { with }\end{array}$} \\
\hline & $\begin{array}{c}\text { B. } \\
\text { dysen- } \\
\text { teriae } \\
\text { Shiga }\end{array}$ & $\mid \begin{array}{c}B . \\
\text { dysen- } \\
\text { teriae } \\
\text { Flexner }\end{array}$ & $\begin{array}{c}\text { B. } \\
\text { dysen- } \\
\text { teriae } \\
\text { Hiss }\end{array}$ & $\begin{array}{c}B . \\
\text { typho- } \\
\text { sus }\end{array}$ & $\begin{array}{c}\text { B. } \\
\text { dysen- } \\
\text { teriae } \\
\text { Shiga }\end{array}$ & $\mid$\begin{tabular}{c|}
$B$ \\
dysen- \\
teriae \\
Flexner
\end{tabular} & $\begin{array}{c}\text { B. } \\
\text { dysen- } \\
\text { teriae } \\
\text { Hiss }\end{array}$ & $\underset{\text { typho- }}{B \text { sus }}$ \\
\hline F-2 (homologous). & 0 & 0 & 0 & 0 & 0 & +4 & +4 & 0 \\
\hline K....... & +4 & +4 & +4 & +4 & +4 & +4 & +4 & +4 \\
\hline P. . & +3 & +3 & +2 & +2 & +4 & +3 & +3 & +4 \\
\hline T. & +4 & +3 & +3 & +4 & +4 & +4 & +4 & +4 \\
\hline $\mathrm{Y} \ldots \ldots$ & 0 & 0 & 0 & 0 & 0 & +4 & +4 & 0 \\
\hline
\end{tabular}

C. A rabbit received 15 injections, at intervals of 8 days of bacteriophage $\mathrm{Y}$. Two cubic centimeters were given at each injection and the rabbit was bled 10 days after the last injection. Table 48 gives the results of the tests of neutralizing action of the antiserum.

From these experiments it appears:

First, that in general, an antiserum for the Flexner-bacteriophage inactivates the virulence of the Flexner-bacteriophage for the Flexner bacillus (and for the Hiss bacillus, a very closely related, if not identical organism). This is true for a majority of the races of the bacteriophage.

* The results in the tables are expressed according to the scheme of notation of Otto; the various symbols meaning:

$0=$ absence of plaques when spread on agar.

$\pm=$ the agar medium shows a few scattered plaques.

+1 and $+2=$ the plaques are confluent, the figure indicating the relative number.

$+3=$ isolated bacterial colonies.

$+4=$ the agar medium shows no growth whatever. 
Second, that certain races of the bacteriophage are naturally refractory to the action of heterologous antiserum. Some of them are refractory even to a homologous antiserum.

Third, that the inactivating action of an antiserum for the different virulences of a single bacteriophage is extremely variable from one race to another. For example, in Experiment C, the last three races are multivirulent. Through the action of the antiserum all three races lose their virulences for the Flexner and Hiss bacilli, but at the same time, race $\mathrm{K}$ retains intact its virulence for Shiga and for B. typhosus, race $\mathrm{T}$ loses both, and race Ty loses much of its virulence for Shiga and retains that for $B$. typhosus intact.

TABLE 48

\begin{tabular}{|c|c|c|c|c|c|c|c|c|}
\hline \multirow{3}{*}{$\begin{array}{c}\text { RACE OF BACTERIOPHAGE:WITH } \\
\text { ANTI-Y SERUM }\end{array}$} & \multicolumn{8}{|c|}{ PLANTINGS MADE ON AGAR OF } \\
\hline & \multicolumn{4}{|c|}{$\begin{array}{l}\text { Bacteriophage anti-serum mix- } \\
\text { ture combined with }\end{array}$} & \multicolumn{4}{|c|}{$\begin{array}{l}\text { Bacteriophage only combined } \\
\text { with }\end{array}$} \\
\hline & $\begin{array}{c}B . \\
\text { dysen- } \\
\text { teriae } \\
\text { Shiga }\end{array}$ & $\mid \begin{array}{c}B . \\
\text { dysen- } \\
\text { teriae } \\
\text { Flexner }\end{array}$ & $\begin{array}{c}B . \\
\text { dysen- } \\
\text { teriae } \\
\text { Hiss }\end{array}$ & $\underset{\substack{\text { typho- } \\
\text { sus }}}{B .}$ & $\begin{array}{c}B . \\
\text { dysen- } \\
\text { teriae } \\
\text { Shiga }\end{array}$ & $\mid \begin{array}{c}R . \\
\text { dysen- } \\
\text { teriae } \\
\text { Flexner }\end{array}$ & $\begin{array}{c}B . \\
\text { dysen- } \\
\text { teriae } \\
\text { Hiss }\end{array}$ & $\begin{array}{c}B . \\
\text { typho- } \\
\text { sus }\end{array}$ \\
\hline 2. & 0 & 0 & 0 & 0 & 0 & +4 & +4 & 0 \\
\hline P. . & \pm & 0 & 0 & 0 & +4 & +3 & +3 & +4 \\
\hline$s \ldots \ldots \ldots \ldots$ & +4 & 0 & 0 & +4 & +4 & +4 & +4 & +4 \\
\hline $2-\mathrm{T} \ldots$ & +3 & 0 & 0 & +4 & +4 & +4 & +4 & +4 \\
\hline $\mathrm{F}-1 \ldots \ldots$ & 0 & +1 & 0 & 0 & 0 & +4 & +4 & 0 \\
\hline Sh....... & +4 & 0 & 0 & +4 & +4 & +1 & +1 & +4 \\
\hline K... & +4 & 0 & 0 & +4 & +4 & +4 & +4 & +4 \\
\hline Ту.......... & +1 & 0 & 0 & +4 & +4 & +3 & +3 & +4 \\
\hline $\mathrm{T} \ldots \ldots \ldots$ & 0 & 0 & 0 & 0 & +4 & +4 & +4 & +4 \\
\hline
\end{tabular}

Bruynoghe, and his collaborators Wagemans and Appelmans, ${ }^{116,615}$ also found that the antigenic value of different races of the bacteriophage is very variable, and although they agree with Otto on this point they differ entirely upon the question of antigenic specificity. According to Otto there is a specificity of virulence; according to Bruynoghe the specificity resides in the race. For the first (Otto), an animal prepared by injections of a bacteriophage developed at the expense of the Flexner bacillus neutralizes the virulence for Flexner bacilli (with few exceptions) of other races of the bacteriophage, leaving the other virulences unmodified. For Bruynoghe, the serum of an animal immunized by injections of one race of bacteriophage, whatever may be the bacterium at whose expense it has developed, neutralizes all of the virulences of this race, and solely of this race; remaining without neutralizing action for all other races of bacteriophage. 
I have quoted in detail the experimental results of Otto, since they show a great variability in the action of different antisera. It is unnecessary to reproduce those of Bruynoghe, for his results are absolute, bearing out his conclusion of race specificity.

The reason for these divergent results? It is, in part at least, due to the fact that Otto worked with races of the bacteriophage whose major virulence was for $B$. dysenteriae, while in Bruynoghe's experiments the primary virulence was for $B$. coli.

These results bring to mind the statement, so often made, that if one wishes to draw correct deductions and to generalize it is essential to work with different races of the bacteriophage, races of maximum virulence, and to select races active for bacterial species as distantly related as possible. When an experiment is repeated with three races of the bacteriophage, virulent respectively for B. dysenteriae Shiga, for $B$. typhosus, and for the staphylococcus, for example, and identical results are obtained, the chance for error in generalizing and in drawing conclusions from the experimental data is reduced materially. Unless results are confirmed by some such procedure erroneous conclusions are almost certain.

Wolff and Janzen ${ }^{630}$ report their results in using a bacteriophage virulent for both B. coli and B. typhosus. In the first place they started from isolated plaques on agar, thus insuring that their race was ultrapure. With this bacteriophage $\left(\tau v^{2}\right)$, virulent for both the typhoid and the colon bacillus, and developed at the expense of B.typhosus, they immunized a rabbit. They mixed 0.4 ce. of a suspension of this bacteriophage with $0.8 \mathrm{cc}$. of the antiserum, and allowed the mixture to stand at laboratory temperature for 24 hours. The mixture was prepared in cluplicate, and to one portion they added a suspension of B. typhosus, to the other, a suspension of B. coli. After the period of contact they spread these mixtures on agar. The results are shown in table 49 .

In a second experiment they produced two antisera, one (A), by immunizing an animal by injections of this bacteriophage, developed at the expense of $B$. coli, and a second (B), by injecting another rabbit with the same bacteriophage developed with B.typhosus. They found that the anti-A serum inactivated the virulence for $B$. typhosus and failed to neutralize the virulence for $B$. coli; and that the anti-B serum showed precisely the same reactions. Their results agree, therefore, with those of Otto, and are in conflict with those of Bruynoghe.

I have made a few experiments, using races that were certainly ultra- 
pure, obtained by the selective isolation method previously described. These are the conclusions to which I have arrived.

1. The antigenic value of different races of the bacteriophage is extremely variable. This agrees fully with all who have worked on the subject.

2. The neutralizing action of an antiserum is by no means limited to the race of the bacteriophage which served for the immunization of the animal. In this point my results agree with those of Otto, Munter and Winkler.

3. An antiserum shows a specificity of virulence and not of race. A serum derived from an animal immunized with suspensions of a bacteriophage developed at the expense of one bacterium inactivates the virulence for this bacterium, and leaves, in general but not always, the other virulences unimpaired. Otto, as well as Wolff, reached a similar conclusion.

TABLE 49

\begin{tabular}{|c|c|c|}
\hline \multirow{2}{*}{ MIXTURES } & \multicolumn{2}{|c|}{ PLAQUE COUNTS ON AGAR WITH } \\
\hline & B.typhosus & B. coli \\
\hline $\begin{array}{l}\text { Bacteriophage }+ \text { antiserum } \ldots \ldots \ldots \\
\text { Bacteriophage }+ \text { bouillon . . . . . . . }\end{array}$ & $\begin{array}{r}4 \\
450\end{array}$ & $\left.\begin{array}{l}\text { Infinity } \\
\text { Infinity }\end{array}\right\} \begin{array}{c}\text { In } 0.0001 \text { cc. of } \\
\text { the mixture }\end{array}$ \\
\hline
\end{tabular}

I have further observed that an antiserum for the Shiga, Flexner, or Hiss bacteriophage neutralizes the virulence for Shiga, Flexner, or Hiss bacilli of almost all other races of the bacteriophage. For the antibacteriophagic sera for the Coli-bacteriophage and the Typhoidbacteriophage the specificity of virulence is much less uniform, although an action upon other races may be observed.

\section{THE BACTERIOPHAGE ANTIGEN}

With Eliava I reported ${ }^{358}$ some experiments from which it appeared that all races of the bacteriophage must contain a common antigenic principle. As a matter of fact, any antibacteriophagic serum whatever-one prepared, for example, by injections of a shiga-bacteriophage - contains a sensitizer which causes the fixation of alexin in the presence of a suspension of bacteriophage of any race whatsoever, such as a Plague-bacteriophage.*

* Detailed protocols of these experiments have been presented in The Bacteriophage, Its Rôle in Immunity. It is unnecessary to repeat them here, for reasons which are obvious. 
Bruynoghe and Maisin ${ }^{122}$ confirmed these experiments, as did also Gratia and Jaumain. ${ }^{271}$ But while unquestionably an antiserum for the Shiga-bacteriophage contains a sensitizer which fixes alexin in the presence of a suspension of the Staphylo-bacteriophage, it also appears that this crossed fixation may be observed when, instead of using suspensions of the bacteriophage as antigens, bacterial autolysates of any species whatever, are employed. It is, then, the bacterium which contains the common antigen.

I must, and do, willingly, recognize experiments well performed, and I realize that had my data been correct and complete the conclusions which I drew would not have been presented.*

Does the inoculation of an animal with protobes of any kind, the agents of vaccinia and of rabies, for example, provoke the formation of sensitizers? This is a question which has been discussed freely, some investigators supporting their opinion by affirmative experiments, others confirming their point of view by negative results. Whatever may be the truth of the matter, it is obvious that the contradictory evidence proves, at least, that sensitizers for the protobes, if they exist at all, are certainly of but little potency .

\section{NATURE OF THE ANTIBACTERIOPHAGIC PROPERTY}

Immediately after Bordet and Ciuca published their paper ${ }^{88}$ announcing that the injection of an animal with suspensions of the bacteriophage caused the serum of this animal to manifest an antibacteriophagic property, and that by contact with such a serum the bacteriophage was destroyed, I carried out with Eliava ${ }^{359}$ some experiments in which we showed that, under the same conditions, the bacteriophage was not destroyed. The inactivation of bacteriophagic action was simply an inhibition.

We prepared a mixture of equal parts of an antiserum for the Shigabacteriophage and of a suspension of the Shiga-bacteriophage. This mixture was allowed to stand for 5 days under conditions which would favor a destruction of the corpuscles by the antiserum if such a destructive action was present. After contact between the two substances for the 5 days the mixture was tested to ascertain the condition of the bacteriophage. Three tubes, each containing 10 cc. of

* It may be remarked, however, that it is conceivable that the common antigen is some of the bacterial substances which have been transformed through bacteriophagy, even though the bacteriophage corpuscles themselves are not this antigen. 
bouillon, were implanted with a drop of a bouillon culture of $B$. dysenteriae Shiga. To the first of these tubes a drop of the antiserumbacteriophage mixture was added. The tube was thoroughly shaken, and a drop was transferred to the second tube. In the same way, a drop of the second tube was carried over to the third. In this way we procured a series of three tubes, all having essentially the same number of $B$. dysenteriae together with decreasing concentrations of the antiserum-bacteriophage mixture. The tubes were incubated at $37^{\circ} \mathrm{C}$. for 24 hours. Normal cultures of Shiga bacilli developed in the three bouillon tubes and plantings made from them on agar also yielded normal cultures. These results would indicate that the bacteriophage had been destroyed. But the experiment was continued further; the tubes were returned to the incubator, and after a second incubation for 24 hours it was found that in the first tube of the series a dissolution of the bacteria had commenced. Furthermore, transfers from this tube to agar remained sterile. Tubes 2 and 3 of the series still showed a normal culture of $B$. dysenteriae at this time, but after incubation for a further 24 hours dissolution had taken place in both of these. Agar cultures made from these tubes remained free of growth.

The conclusion that the effect observed here is that of an inhibition only has been questioned, the objection being based upon the observation that sometimes, with particularly active antisera and particularly sensitive races of the bacteriophage, bacteriophagy never takes place, even after several days, and that in such cases it is impossible, even by repeated passages, to reveal the presence of the bacteriophage. Hence, this proves that the bacteriophage is destroyed.

But even if such antisera exist, which may be possible, it does not alter the significance of the experiment reported above. For this experiment* shows unquestionably that after 24 hours there was a complete inhibition, for no plaques appeared on the agar transfers, even those made from the first tube where the number of corpuscles was very considerable. And it is just as certain that on the following day bacteriophagy was effected, and the spreading made on agar remained sterile. There was, therefore, an inhibition, with a later reactivation. In what way could this take place?

Prausnitz has demonstrated that in a bacteriophage suspension certain of the corpuscles are endowed with a very definite resistance, and that the number of these sero-resistant corpuscles is the greater when the quantity of antiserum added is small. This suggests the

* I have repeated this experiment since these results were first published. 
explanation for our results. It is probable that in our experiment a few of the corpuscles resisted the action of the antiserum - and in almost equal numbers in the three tubes, since although the number of corpuscles varied in the different tubes, the quantity of antiserum varied likewise in the same proportion. These sero-resistant corpuscles parasitized the nearest bacteria and reproduced, and thus were the origin of a sero-resistant line which ultimately provoked a complete bacteriophagy.

I do not believe that it is necessary to discuss further this question of destruction or inhibition. No one can longer have any real doubt on the point, for we have seen in an earlier paragraph that the experiments of a great many workers agree in showing that the inactivation brought about by an antiserum is very frequently limited to a single virulence, the other virulences remaining intact. Manifestly, under such circumstances, the bacteriophage protobe can not be destroyed; it can be but an inhibition.

The possible objection that the obvious inactivation of one virulence while another is retained may be due to the fact that the experiment was not performed with pure races of the bacteriophage, but rather with mixtures of different bacteriophages, each being then neutralized specifically by its antiserum, is valid for the experiments of Otto, but it can not hold for either those of Wolff or those which I have reported, for in both instances the races of the bacteriophage used were certainly ultrapure.

In what way does this inactivation of virulence occur? As a matter of fact, is the action of the antiserum on the protobe itself or on the bacterium? This it is impossible to answer definitely at the present time.

A number of authors, among whom Arnold and Weiss ${ }^{25}$ may be mentioned particularly, have attempted to show that the "neutralization" of a bacteriophage by an antiserum follows a course identical to that of the neutralization of a toxin by an antitoxin. These authors have adopted a method of reasoning which appears again and again throughout the study of the bacteriophage. They have selected a single, relatively unimportant fact, found that it has an analogy in some other phenomenon, and have generalized from this fact without taking into consideration all of the other facts which inherently contradict their deductions. Furthermore, in the case of the authors mentioned, their method of "titrating" the bacteriophage is so inadequate that it does not permit a satisfactory study of the phenomenon. 
Insofar as the inactivation of the bacteriophage by an anti-bacteriophagic serum is concerned there is nothing comparable with what takes place when a toxin is neutralized by an antitoxin. The following points bring out, I believe, the complete dissimilarity of the two phenomena.

1. Inactivation does not occur according to definite proportions; the great majority of the corpuscles are inactivated at the start, and then, in order to inactivate the few corpuscles remaining, those endowed with a natural sero-resistance, it is necessary to add greater and greater quantities of the antiserum.

2. Inactivation of the bacteriophage is an extremely slow process.

3. The antiserum "cures" secondary cultures. No one has ever advanced the concept of an antitoxic serum which should cause the bacterium to lose the property of elaborating an antitoxin.*

4. Different races of the bacteriophage, virulent for a single bacterium, possess markedly different antigenic properties; some apparently have none, for when inoculated into an animal they fail entirely to cause the appearance of an antibacteriophagic property. I do not believe that anyone can recall a comparable fact observed with the toxins.

5 . Certain races of bacteriophage inoculated into an animal do not lead to the formation of an homologous "anti" property only; the "anti" action is also directed against other races. We know of comparable facts in connection with the antigenic action of the bacteria, but never with the toxins.

6. An antibacteriophagic serum neutralizes a virulence of one bacteriophage and allows others to persist unimpaired. There is nothing comparable to this, quite naturally, with antitoxic sera.

7. In determining the power of inactivation of several anti-bacteriophagic sera (derived from animals prepared by inoculations of different races of the bacteriophage, but all virulent for a single bacterial species) we find that some sera exert their effect upon many races of the bacteriophage while with other sera the action is specific and limited to the homologous race of bacteriophage. Antitoxic sera show nothing comparable to this. $f$

* It is indeed evident, that when the inactivation of the bacteriophage by an antiserum is likened to the neutralization of a toxin by an antitoxin, it necessarily follows that the bacteriophage is likened to a toxin.

$\dagger$ That is, if we prepare three diphtheria antitoxins, for example, in three different animals immunized respectively with three different strains of Bact. diphtheriae, each of these three antitoxins may differ from the others quantitatively but not qualitatively. All three manifest an activity on any diphtheria toxin whatever. 
It is only necessary to consider the experimental data which have been detailed in this chapter to see the logic of these conclusions.

And finally, recognizing that an "inactivating principle" is present in an antibacteriophagic serum, the quite logical question is as to its nature. It can not be a sensitizer, for it brings about its effects without the intervention of alexin. Let us note in this connection that this is true for all antisera to the protobes. The anti-vaccinia sera, and anti-rabies sera, for example, inactivate the corresponding protobes in the same way without the intervention of alexin. This is, then, it appears, a general fact applying definitely to the protobes and to protobes exclusively.*

The "anti" principle which neutralizes the action of the protobes is, therefore, neither an antitoxin nor a sensitizer, and since it fails to conform to these recognized immunological principles I have proposed to term the active principle an antivirulin.

Study of the antibacteriophagic serum,--and such a study is in reality only beginning,-assumes a far greater importance in that the facts disclosed will not necessarily be limited solely to the bacteriophage, but may give us some information regarding the nature of the immunity to the pathogenic protobes.

In concluding the discussion of this subject I believe that it may be well to state that for many diseases there may be some question as to whether the causative agent is an ultravirus, that is, a protobe, or a microbe. Scarlet fever, for example, might be included in this class. But it would seem that the question may be settled readily. Does the heated serum $\dagger$ of a convalescent, by itself, neutralize the virus present in the blood of a patient? If the answer is in the affirmative, the agent is a protobe and not a microbe. $\ddagger$ With scarlet fever, for example, if the serum of a convalescent neutralizes the virus the primary agent is certainly not the streptococcus, at least, not the visible form of the streptococcus. If neutralization does not occur, a microbe is the primary agent,-in this case the streptococcus.

* This question of immunity against the ultraviruses, that is, against protobes, has been treated at length in the last two chapters of the text Immunity in Natural Infectious Disease, to which the reader who may be interested is referred.

$\dagger$ Prausnitz has shown that the antibacteriophagic substance resists a temperature of $75^{\circ} \mathrm{C}$.

$\ddagger$ One can not object that the neutralization may be exercised on a toxin, for a toxin is not a virus; it does not produce a serially transmissible disease. 


\section{THE COMPLEXITY OF AN ANTIBACTERIOPHAGIC SERUM}

The antibacteriophagic property which appears in the serum when an animal is injected with a suspension of the bacteriophage is necessarily produced because of a reaction by the animal to the bacteriophage protobe. But a bacteriophage suspension contains not only protobe substance, but other materials as well, such as:

1. The dissolved substances of the bacterial bodies.

2. The products resulting from bacteriophagy, no matter whether they are elaborated by the protobes or by the bacteria.*

3. Products elaborated in the physiology of the bacteria prior to their dissolution, such as the toxins.

The antibacteriophagic serum must, therefore, contain a complex of antibodies, and, as a matter of fact, various antibodies have been demonstrated. In their early work with the sera Bordet and Ciuca ${ }^{88}$ showed that such a serum had agglutinating properties for the bacterium bacteriophaged, as well as precipitating properties. With Eliava ${ }^{358}$ I found that it also contained a sensitizer for the bacterium concerned. Wollman and Goldenberg ${ }^{635}$ have shown that the antigenic value of the bacterial substances dissolved through the action of the bacteriophage is superior to the antigenic value of normal bacteria.

These facts make it evident, and this is a point of some significance from the point of view of the nature of the bacteriophagic process, that the greater part of the bacterial substance can not have undergone any great modification in the sense of a degradation during the

* That such products exist is proved by a number of the experiments described in Part I of this text, among others, by the experiment showing the absence of bacteriophagy in gelatin media because of the accumulation of these products at the surface of the medium. It may be recalled that this inhibition is due to the non-diffusibility of these substances into the gelatin, since on a layer of gelatin superimposed upon a substratum of agar, bacteriophagy takes place normally.

In the text The Bacteriophage, Its Rôle in Immunity ${ }^{321}$ I suggested that in bacterial suspensions which had undergone bacteriophagy there might be present both "lysins" elaborated by the bacteriophage and "antilysins" elaborated by the bacteria. I recognize, as I have already said, that such an inference can not be experimentally confirmed, and that at the present time, it is impossible to show that such products exist.

On the contrary, and here experiment is conclusive, in a bacteriophaged bacterial suspension there are abnormal substances which do not appear either in old cultures of bacteria or in autolysates. These substances appear only because of the presence of the bacteriophage, although there is no indication as to their origin. That is why I have designated these substances as "products resulting from bacteriophagy." 
process of bacteriophagy. The bacteriophage protobes utilize one fraction of the bacterium for their development, and this part is necessarily transformed since, as we have seen, bacteriophage substance differs from bacterium substance, but apparently the rest is not modified chemically, since its antigenic properties are the same after bacteriophagy as they were before. Otto and Winkler ${ }^{499}$ saturated an antibacteriophagic serum with the homologous bacterium and found that the antibacterial properties disappeared. Nevertheless, despite the fact that the bacterial antibodies were absorbed, the antibacteriophagic property persisted. From this they concluded that the bacteriophage possesses an antigenje function of its own.

Does the antibacteriophagic serum contain antibodies for the "products resulting from bacteriophagy"? It is impossible to say.

As for the presence of antibodies for the physiological products elaborated by the bacteria before they undergo dissolution, we will shortly see what happens.

\section{THE ANTI-ANTIBACTERIOPHAGIC SERUM}

In connection with the bacteriophage, as with so many other immunological studies, we have the question of anti-antibodies receiving attention. Fabry and van Beneden ${ }^{197}$ first prepared an antibacteriophagic serum in a rabbit by a series of injections of a bacteriophage suspension, the serum being collected when the antibacteriophagic property was sufficiently outspoken. They then immunized a dog with this antiserum, first injecting the serum subcutaneously, later intraperitoneally, in amounts of 1 to $2 \mathrm{cc}$. Two rabbits and three guinea pigs were also injected with the same antiserum. After 5 injections, given at 4 day intervals, they allowed the animals to remain without further treatment for a month, in order that the passively transferred immunity might disappear. The animals were then bled.

In the case of the rabbits and the guinea pigs they were unable to find the slightest evidence of an anti-antibacteriophagic property. With the dog serum, on the contrary, some interesting observations were made. A mixture of equal parts of the antibacteriophagic serum and of that of the dog immunized with this antiserum was incubated for an hour at $37^{\circ} \mathrm{C}$., and then allowed to stand over night at laboratory temperature. With this incubated mixture of the two sera the following tests were made.

Each of 12 tubes, containing $10 \mathrm{cc}$. of bouillon, was seeded with a drop of a culture of $B$. coli. To these implanted tubes, the various 
materials were added as indicated in table 50, which also records the results obtained.

From these experiments we find that the "antibacteriophage agent" contained in 4 drops of the antibacteriophage-coli serum is neutralized by 12 drops of the "anti-antiserum." This "anti-anti" property resisted heating at $56^{\circ} \mathrm{C}$. for 30 minutes.

Fabry and van Beneden concluded that the injection of an antibacteriophagic serum (rabbit) into a different and unrelated animal

TABLE 50

Each of the twelve tubes contains $10 \mathrm{cc}$. of bouillon implanted with 1 drop of B. coli culture.

\begin{tabular}{|c|c|c|c|c|}
\hline \multirow[b]{2}{*}{ TUBE } & \multicolumn{3}{|c|}{ ADDITIONAL MATERIALS ADDED } & \multirow[b]{2}{*}{ RESULT } \\
\hline & $\begin{array}{c}\text { Coli-bacterio- } \\
\text { phage }\end{array}$ & $\begin{array}{l}\text { Antibacterio- } \\
\text { phagic serum }\end{array}$ & $\left|\begin{array}{c}\text { Anti- } \\
\text { antibacteriophagic } \\
\text { serum }\end{array}\right|$ & \\
\hline 1 & & & & Growth \\
\hline 2 & 3 drops & & & No growth \\
\hline 3 & 8 drops & 4 drops & & Growth \\
\hline 4 & 3 drops & 3 drops & 3 drops & Growth \\
\hline 5 & 8 drops & 4 drops & 8 drops & Slight growth \\
\hline 6 & 8 drops & 4 drops & 12 drops & No growth \\
\hline 7 & 8 drops & 4 drops & 16 drops & No growth \\
\hline 8 & 8 drops & 4 drops & 20 drops & No growth \\
\hline 9 & & 3 drops & 3 drops & Growth \\
\hline 10 & 3 drops & & 3 drops & No growth \\
\hline 11 & 3 drops & \multicolumn{2}{|c|}{$\begin{array}{l}3 \text { drops of normal rabbit } \\
\text { serum }\end{array}$} & Growth + or $0^{*}$ \\
\hline 12 & \multicolumn{3}{|c|}{$\begin{array}{l}3 \text { drops }+6 \text { drops of normal serum }+6 \\
\text { drops of anti-anti serum }\end{array}$} & No growth \\
\hline
\end{tabular}

* In the course of a number of experiments they observed, in some instances, that the serum of a normal rabbit inhibited the action of the Coli-bacteriophage, while the sera of other rabbits were without inhibitory action.

species ( $\operatorname{dog}$ ) led to the formation within the dog of "anti-anti" properties capable of neutralizing in vitro the antibacteriophagic serum. In reality, this is not a true "anti-anti" action, and this is clearly shown by the fact that it is impossible to produce such a serum in animals of the same species or in related species. Certainly the effect observed is referable to a precipitation of the globulins. Dehne and Hamburger*

* Dehne R., and Hamburger, F.-Experimenteluntersuchungen über die Folgen parenteraler Einverleibung von Pferdserum. Wien. klin. Wchnschr., $1904,17,807$. 
many years ago thoroughly studied and explained this question of anti-antisera.

\section{THE ACTION OF ANTIBACTERIAL SERA}

Various authors (Bail, ${ }^{43}$ Machado and da Costa Cruz, ${ }^{153,427}$ and others) have attempted to show that a serum which is purely antibacterial can neutralize the action of the bacteriophage to the same degree and in the same way as does an antibacteriophagic serum.

Machado and da Costa Cruz ${ }^{153,427}$ provide experimental data according to which it would appear that the addition of a single drop of an anti-Shiga serum, with an agglutinating titre of $1: 5000$, to $10 \mathrm{cc}$. of bouillon inoculated with 16 drops of a suspension of the bacteriophage impairs bacteriophagic action to some extent. If 10 drops of the antibacterial serum is added to 16 drops of the bacteriophage suspension the action of the bacteriophage is completely prevented.

They further found that if an extract of Shiga bacilli was added to the bouillon the inhibitory action of the antibacterial serum was abolished; bacteriophagy took place.

They concluded, as had Bail in an earlier contribution, that the neutralizing action of an antibacterial serum and of an antibacteriophagic serum upon the bacteriophage is of the same nature.

But proof to the contrary can be obtained in a very simple manner. If a drop of a suspension of the bacteriophage is added to $10 \mathrm{cc}$. of the homologous antibacterial serum, that is, a serum agglutinating the bacterium at the expense of which the bacteriophage had developed, it will be found after any interval of time whatever, in my experiments varying from 1 day to 6 months, that the proportion of living and virulent corpuscles is exactly the same as in a control preparation made with physiological saline.

These experiments have been made with a Shiga-bacteriophage and an anti-Shiga serum, agglutinating at 1:6500, derived from a horse; with a Coli-bacteriophage and the serum of a rabbit which had received a series of injections with the same strain of $B$. coli as that used in making the passages of the bacteriophage; and with a Staphylo-bacteriophage and its corresponding antistaphylococcus serum. The results with these various antisera and with the diverse races of the bacteriophage warrant the conclusion that an antibacterial serum does not exercise the slightest destructive effect upon the bacteriophage itself.

In their determinations Machado and da Costa Cruz must have worked with a Shiga-bacteriophage of low virulence, for in repeating 
their experiments with a bacteriophage of maximum virulence, I found it necessary, in order to obtain an inhibition of bacteriophagy, to add to the bacterial suspension inoculated with the bacteriophage a quantity of antibacterial serum far greater than that used by these authors. I will cite one of these experiments illustrating the effects observed. All gave comparable results.

To tubes containing $10 \mathrm{cc}$. of bouillon ( $\mathrm{pH}$ 7.8) 16 drops of a Shigabacteriophage suspension were added, along with variable quantities of an anti-Shiga serum, agglutinating in a dilution of 1:6500. After incubation for 1 hour at $37^{\circ} \mathrm{C}$. the tubes were planted with 3 drops of

TABLE 51

\begin{tabular}{|c|c|c|c|c|}
\hline TUBE & $\begin{array}{l}\text { ANTI-SHIGA } \\
\text { SERUM }\end{array}$ & $\begin{array}{c}\text { SHIGA-BACTERIO- } \\
\text { PHAGE }\end{array}$ & SHIGA BACILLI & RESULT \\
\hline 1 & 10 drops & 16 drops & 3 drops & No growth \\
\hline 2 & 5 drops & 16 drops & 3 drops & No growth \\
\hline 3 & 1 drop & 16 drops & 3 drops & No growth \\
\hline 4 & 30 drops & 1 drop & 3 drops & Agglutinated \\
\hline 5 & 20 drops & 1 drop & 3 drops & Fine agglutinate \\
\hline 6 & 10 drops & 1 drop & 3 drops & No growth \\
\hline 7 & 10 drops & & 3 drops & $\begin{array}{l}\text { Heavy deposit; ag- } \\
\text { glutinated }\end{array}$ \\
\hline
\end{tabular}

TABLE 52

\begin{tabular}{|c|c|c|c|c|c|}
\hline TUBE & $\begin{array}{l}\text { ANTI-FLEXNER } \\
\text { SERUM }\end{array}$ & $\begin{array}{l}\text { FLEXNER } \\
\text { BACTERIO- } \\
\text { PHAGE }\end{array}$ & $\begin{array}{l}\text { FLEXNER } \\
\text { BACILLI }\end{array}$ & $\begin{array}{c}\text { EXTRACT } \\
\text { OF FLEXNER } \\
\text { BACILLI }\end{array}$ & $\begin{array}{l}\text { RESULTS AFTER } \\
24 \text { HOURS }\end{array}$ \\
\hline 1 & 5 drops & 2 drops & 3 drops & & Agglutinated \\
\hline 2 & 5 drops & 2 drops & 3 drops & 5 cc. & Clear \\
\hline 3 & 5 drops & 2 drops & 3 drops & 3 cc. & Clear \\
\hline 4 & 5 drops & 2 drops & & $1 \mathrm{cc}$. & Clear \\
\hline 5 & & & 3 drops & & Growth \\
\hline
\end{tabular}

a 24-hour bouillon culture of Shiga bacilli. The results were as shown in table 51 .

From a titration of this type it is obvious that the antibacterial serum has an inhibitory effect upon bacteriophagy, but it also shows, at least when working with a very virulent bacteriophage, that it is necessary that it be added to a high concentration. As a matter of fact, additional experiments have shown that the effect of an antibacterial serum becomes more outspoken as the bacteriophage used is of lower virulence.

As mentioned above, da Costa Cruz ${ }^{155}$ presents data showing the 
neutralizing action of bacterial extracts for the serum. Table 52 indicates the results obtained in one experiment of this type.

Hauduroy ${ }^{287}$ has also worked on the question of the inhibiting action of antibacterial sera and reached the conclusion that an inhibition does occur. But according to his protocols it requires far greater quantities of serum than is indicated by the experiments of da Costa Cruz. Hauduroy distributed a young culture of B. dysenteriae Shiga in tubes, $10 \mathrm{cc}$. to each, and inoculated each one with 1 drop of a suspension of the bacteriophage. He then added the anti-Shiga serum in quantities varying from 2 to 10 drops. Bacteriophagy took place normally. When the serum was added in quantities of between 12 and 34 drops the bacteriophagic process was retarded. It was only when the quantity of serum added was above 36 drops that inhibition occurred.

Studying the phenomenon further, he found that the inhibitory action was not due to some effect upon the bacteriophage itself, for if he filtered a suspension which remained turbid because of the large amount of added serum, he found that the bacteriophage was still alive and virulent in the filtrate. He concluded that the process of bacteriophagy was held in abeyance because some substance, secreted by the bacterium and essential to bacteriophagy, was flocculated by the serum.

If we consider the question as to the mechanism whereby an antibacterial serum may be inhibitory we immediately discover that there are two possible hypotheses which would explain the facts observed, and not a single explanation, as Hauduroy seems to believe.

The antibacterial serum exerts no direct action upon the bacteriophage protobe, as experiment shows. Consequently, the inhibiting action must be dependent upon some modification of the bacterium or upon some product elaborated by the bacterium.

We have seen in the section dealing with the nature of the bacteriophagic process that although the bacterium acted upon is in a medium containing quantities of an antiseptic which do not prevent its multiplication, the bacteriophage may, nevertheless, remain inert. The inhibiting action of the antibacterial serum can be explained perfectly upon the same basis, it making no difference whether the action is exerted by an antiseptic or by an antiserum.

On the other hand, we know that the first step in the phenomenon of bacteriophagy consists in the approach of the corpuscle to the susceptible bacterium. As the antibacterial serum most certainly modifies the surface tension of the bacterium, we have no reason to believe 
that this change may not prevent the fixation of the corpuscle to the bacterial cell.

To consider the phenomenon from another point of view, we know that in order for the fixation to take place it is essential that there be an approach of the corpuscles to the bacteria, and Kabelik ${ }^{384}$ seems to have shown that the primum movens for this coming together resides in a phenomenon of chemotaxis on the part of the bacteriophage corpuscle. This chemotaxis is necessarily determined by some bacterial product to be found in the liquid, in the same way, for example, as the chemotaxis of the fern antherozoid for the oosphere is determined by a secretion of malic acid by the latter. If the antibacterial substance precipitates the substance which determines the chemotaxis, the drawing together can not occur.*

Such are the hypotheses which may be formulated in an effort to explain the mechanism whereby an antibacterial serum modifies the process of bacteriophagy. Which hypothesis is true? I do not know. But in any case, one fact is certain, and that is that such a serum exerts no direct action upon the bacteriophage protobe itself.

\section{THE PHENOMENON OF ANTIPHYLAXIS}

When we immunize an animal by giving it a series of injections, using cultures of a toxic bacterium, such as B. dysenteriae Shiga, $\dagger$ we know that the serum of this animal acquires an antitoxic property.

A culture of Shiga bacilli, transformed through bacteriophagy to a suspension of bacteriophage protobes, still contains the bacillary toxins. To be convinced of this it is only necessary to inject rabbits, some with a quantity of culture sufficient to cause a posterior paralysis and death, and others with a suspension of the bacteriophage immediately after bacteriophagy. A comparison of the effects will show that the latter substance is as toxic as is a normal culture, indeed, it may be more so.

* To the observation that in order to fix itself to the bacterium the bacteriophage corpuscle may require that some product secreted by the bacterium be found in the liquid, Hauduroy draws the singular conclusion that the bacteriophage must be a "complex," formed of a living corpuscle and a soluble bacterial product. Man has an incessant need for oxygen, but it would be a poor definition of a man to say that he is a complex formed of a body and oxygen. Nor is the antherozoid of a fern formed of a complex with malic acid.

$\dagger$ Endo- or exo-toxins; what do we mean? As has been said by Nicolle the urine is an endo-urine or an exo-urine, depending upon whether it is in the bladder or in a test-tube. There is about the same difference between an endo- and an exo-toxin, 
When we immunize a rabbit with a suspenson of the bacteriophage, along with the protobes we inject of necessity the materials resulting from the dissolution of the bacterial bodies and also any toxins and like substances that may be present. We should, therefore, necessarily obtain a serum which at the same time will have antibacteriophagic, antibacterial, and antitoxic properties. We have seen that this is certainly true as regards the first two of these properties. In order to demonstrate whether antitoxic principles are also present, carry out the following experiment. ${ }^{321}$

Give a mouse, by subcutaneous injection, a lethal dose of a dysentery toxin prepared by the method of Rowland, as modified by Nicolle, ${ }^{*}$ and at the same time, $0.5 \mathrm{cc}$. of the antiserum for the Shiga-bacteriophage. Give a second mouse the same quantity of toxin and $0.5 \mathrm{cc}$. of an anti-Shiga serum. Give a third mouse a lethal dose of the toxin only. Subsequent events shows that the first mouse dies in about 30 hours after the injection; the second mouse lives indefinitely; and the third dies 4 days after the injection.

From this it is perfectly obvious that the antiserum for the Shigabacteriophage is not antitoxic, indeed, on the contrary it seems to be definitely sensitizing. Let us consider this singular phenomenon somewhat further.

Four mice receive subcutaneously a dose of dysentery toxin equal to one-tenth of the lethal dose. The first mouse is held as a control without further treatment. Two other mice receive in addition to the toxin $0.2 \mathrm{cc}$. of the antiserum for the Shiga-bacteriophage, and the last of the toxin-injected mice gets $0.1 \mathrm{cc}$. of the antiserum. The results: The first remains perfectly well indefinitely, the two which received the $0.2 \mathrm{cc}$. of dose of antiserum die after 40 hours, and the last mouse dies after 54 hours.

Here the results are even more clear-cut than in the previous experiment; the Shiga-bacteriophage antiserum sensitizes the animals to the action of the toxin. The results are the more striking when we consider that whatever the number of lethal doses of the Nicolle Shiga toxin injected into a mouse, death never occurs before the fourth day. Here, when the antibacteriophagic serum is injected with the toxin, even when added to a dose of toxin far below the normal minimal lethal dose, death takes place within 48 hours.

* The bacterial bodies are ground with anhydrous sodium sulfate, the powder so obtained is dried in the air, and dissolved in water at the time of injection. The turbid fluid thus obtained is centrifuged, and the clear supernatant portion is used for the injection. 
Let us take living dysentery bacilli, instead of toxin, and determine whether a similar sensitizing action is manifested here by the antiserum. Four mice receive subcutaneously a dose of dysentery bacilli equal to one-fifth of the lethal dose. The first mouse is held as a control. The second receives, also subcutaneously, $0.2 \mathrm{cc}$. of the antiserum, and the last two mice $0.1 \mathrm{cc}$. of the serum. The control animal lives, showing nothing abnormal. Those mice which received the antibacteriophagic serum in addition to the bacilli die in 7 to 9 days after the injection. Before death they show a paralysis of the posterior extremities, a symptom of intoxication which as a rule does not appear in mice following injections of $B$. dysenteriae, although a paralysis of this type is a common symptom in rabbits, more or less peculiar to the species. ${ }^{321}$

This experiment leaves no room for doubt; the antibacteriophagic serum possesses, not an antitoxic property, but a property which is precisely the opposite of antitoxic. It sensitizes the animal to the toxin. For this state of hypersensitivity I have coined the term "antiphylaxis." 349

One other experiment may be cited, since it shows another aspect of the phenomenon of antiphylaxis. Give a group of mice 5 successive injections at weekly intervals, using an old suspension of the Shigabacteriophage in doses of $0.01,0.02,0.05,0.1$, and $0.15 \mathrm{cc}$. When tested by the injection of Shiga toxin 15 days after the last injection it is found that these mice are killed by quantities of toxin equal to a fifth of the lethal dose for control mice. ${ }^{349}$

That the phenomenon of antiphylaxis occurs has been confirmed by a number of authors, whose contributions to this phase of bacteriophagy are of sufficient theoretical interest, as well as of practical importance, to warrant discussion here.

In the course of experiments on the immunization of rabbits with suspensions of the Staphylo-bacteriophage Gratia ${ }^{257}$ observed that when the sera had acquired a strong antibacteriophagic power the animals were hypersensitive to the injection of cultures of the staphylococcus. With minimal injections of the coccus they died in less than 4 days, one with a miliary infection of the spleen, another with a similar infection involving the kidneys. The normal control rabbits died only after a period of 8 days, and even then only when they had been injected with a much larger quantity of the culture. This shows a diminished resistance to the staphylococcus, comparable to that which I had demonstrated in mice with $B$. dysenteriae.

Perhaps even more conclusive are the following experiments of 
Hauduroy. ${ }^{296}$ He immunized a number of young rabbits, weighing from 1300 to 1700 grams, giving them a series of 7 injections of a suspension of the Staphylo-bacteriophage. All injections were made subcutaneously, at 5- to 6-day intervals, and $2 \mathrm{cc}$. of the suspension were given on each occasion. The bacteriophage used was of maximum virulence. The suspensions were filtered, and were held for at least 15 days to prove their sterility before they were used.

Other rabbits, which were to serve as controls, received a like number of injections of sterile bouillon.

Considering the effects as they developed during and after this treatment, the results may be divided into two groups:

First group: A number of the rabbits became emaciated very quickly, and died, either before or immediately after the series of 7 injections was completed. At autopsy Hauduroy never found staphylococcus lesions of the organs in these animals, but aluays multiple subcutaneous abscesses due to staphylococei. When these abscesses ulcerated the lesion did not appear, in any case, to show any tendency toward a spontaneous cicatrization.

Second group: The other animals increased in weight in an approximately normal fashion throughout the entire course of treatment. Abscesses never appeared. But if, a few days after the last injection of the bacteriophage, these apparently healthy "immunized" rabbits were given intravenously a dose of the staphylococcus which was never fatal for the controls, they died within 24 hours, and the staphylococcus injected could be recovered from the heart blood.

The control rabbits, that is, those which had received the injections of bouillon, increased in weight normally, showed no abscess formation, and resisted intravenous injections of the staphylococci.

This experiment of Hauduroy is very interesting, for it shows that antiphylaxis, developed by a series of injections of a suspension of the bacteriophage, may be so potent that the animal may lose its natural immunity to a bacterium.

Confirming this significant finding, I also have observed upon several occasions the marked emaciation and spontaneous death of rabbits as a result of a series of injections of suspensions of the Staphylobacteriophage. At autopsy these animals presented most often, as in the experiments of Hauduroy, subcutaneous abscesses. On two occasions I have found miliary abscesses of the liver.

Not all races of the bacteriophage possess the property of causing antiphylaxis, and, curiously enough, the capacity to incite this reaction 
is related to the extent of the virulences. With a polyvirulent race I have found that it is sufficient to give repeated injections of the bacteriophage, to see, after a variable length of time, the animals begin to emaciate and finally die spontaneously. With some animals death occurred after only 5 injections; with others a far greater number was necessary - in one case 17 . On the contrary, in another experiment using a bacteriophage whose virulence was strictly limited to one strain of Staphylococcus albus six rabbits withstood a series of 30 injections without showing emaciation or any other obvious disturbance. After the completion of this long "immunizing" course, four of the rabbits were inoculated with variable quantities of a culture of Staphylococcus aureus. Two succumbed like the controls which had received a like dose. There was, then, no antiphylaxis toward this strain of the staphylococcus which was refractory to the action of the univirulent bacteriophage used in the preparatory injections. The remaining two of the original 6 "immunized" rabbits received weekly injections of a suspension of the multivirulent Staphylo-bacteriophage. After the fifth and sixth injections a developing emaciation became obvious, and without any further treatment the rabbits died, one on the twelfth, the other on the sixteenth day after the last injection.

One might conclude that as regards the staphylococcus, antiphylaxis is a function of the virulence range of the race of bacteriophage injected.

This phenomenon of antiphylaxis is of very great interest from the immunological point of view, for it is entirely possible that under certain circumstances it may occur naturally. It represents the first example of a contra-immunity, in the true sense of the word, either active or passive.

As a matter of fact it is very difficult to formulate an explanatory hypothesis as to the nature of this singular phenomenon, for almost any idea advanced leads to rather strange deductions. If it were only a bacterial antiphylaxis, that is, a sensitization to the bacterium, the many facts which will appear in the following pages would permit us to understand it. But it is a toxin antiphylaxis. How is this to be explained?

If we should find that the serum of an animal showed an anti-antitoxic property it would unquestionably be because we had injected an antitoxin. Following out this line of reasoning it must be that a suspension of the Shiga-bacteriophage contains an antitoxin for the Shiga bacillus. Whence is this antitoxin derived? If it exists in the 
Shiga-bacteriophage suspension, when, as we know it did not exist in the Shiga culture, it must be that its presence is associated with the bacteriophage protobe, suggesting that the latter in some way possesses the power of transforming the toxin of the Shiga bacillus into a substance possessing at least some of the properties of an antitoxin.

The reader will do well not to hastily assert the utter impossibility of this before he has read what is to be said with reference to bacillary dysentery in the remainder of this text. I may say here that the following facts seem to favor this hypothesis.

I have found ${ }^{321}$ that a suspension of the Shiga-bacteriophage is, immediately after bacteriophagy, as toxic (sometimes even more toxic) as a normal culture of Shiga bacilli of the same strain as that which was dissolved. As the bacteriophage suspension ages, this toxicity diminishes, and after about a month with some strains it is almost zero, with others very greatly reduced. On the other hand we know that the dysentery toxin is very resistant. If the toxin is destroyed in the presence of the bacteriophage it can only be through the action of the latter. Furthermore, under certain circumstances, suspensions which have become atoxic protect the rabbit against a fatal dose of toxin.

I have observed that antibacteriophagic sera show a higher antiphylactic value when the animals have been "immunized" with old bacteriophage suspensions, - those which have become atoxic. The serum of a rabbit which has received injections of fresh, toxic, suspensions possesses a less marked antiphylactic property than does that of another rabbit, prepared by the same number of injections of the same suspension when it is 20 to 30 days old.

All of this seems to indicate that there must be in the bacteriophage suspensions antitoxic principles elaborated through the action of the bacteriophage protobes.

\section{THE OPSONIC ACTION OF BACTERIOPHAGE SUSPENSIONS}

In concluding this discussion of the antigenic nature of the bacteriophage I will present the results of the experiments performed with Eliava on the question of the opsonic action manifested by suspensions of the bacteriophage. ${ }^{321}$

In the following experiments the opsonic power has been determined by the method of Wright and Douglas, making a mixture of one part of the fluid of which the opsonic action is to be measured, one part of a suspension of leucocytes, and one part of a suspension of the 
bacteria against which the opsonic effect is to be determined. The mixture is aspirated in a capillary pipette which is sealed and placed in the water-bath at $38^{\circ} \mathrm{C}$. for fifteen minutes. The contents of the pipette are then spread on a slide, stained, and examined.

As reagents we have taken: Guinea pig leucocytes, a Shiga-bacteriophage suspension, and a suspension of Shiga bacilli.

1. Control.

Leucocytes, bacilli, ordinary bouillon

100 leucocytes phagocytize 36 bacilli

$$
\text { Opsonic index }=1
$$

2. Leucocytes, bacilli, bacteriophage suspension two years old" 100 leucocytes phagocytize 692 bacilli

Opsonic index $=19.2$

3. The same mixture, except the bacteriophage suspension is diluted $1: 250$

100 leucocytes phagocytize 156 bacilli

Opsonic index $=4.3$

4. Leucocytes, bacilli, bacteriophage suspension six days old 100 leucocytes phagocytize 1510 bacilli

Opsonic index $=41.9$

5. The same mixture, except the bacteriophage suspension is diluted $1: 250$

100 leucocytes phagocytize 146 bacilli

Opsonic index $=4.1$

6. The same mixture, except the bacteriophage suspension is heated at $60^{\circ} \mathrm{C}$. for 30 minutes

100 leucocytes phagocytize 728 bacilli

Opsonic index $=20.2$

7. The same mixture, except the bacteriophage suspension is heated and diluted to $1: 250$

100 leucocytes phagocytize 101 bacilli

Opsonic index $=2.7$

In the mixtures 2,4 , and 6 , the indices recorded represent a minimum. Many leucocytes contain so many phagocytized bacilli that counting is impossible. Since in the above counts only those cells which did not contain masses of bacteria have been included, the actual index is therefore somewhat higher.*

* It will be recognized that the opsonic indices obtained with sera are far below these secured with the bacteriophage suspensions. With the former an index of 2 is exceptional. 
The opsonic action of a bacteriophage suspension manifests itself with such rapidity that it is improbable that the opsonic power can be exercised directly by the protobes. We have seen, in fact, that the bacteria are parasitized only after an appreciable lapse of time,ten to twenty minutes.

An experiment conducted with the following reagents indicates the course of the reaction. Leucocyte suspension, Shiga suspension, and Shiga-bacteriophage suspension are mixed in equal parts. After various periods of incubation drops of the mixture are examined showing results as given in table 53 .

After ten minutes some of the leucocytes are so completely filled with bacilli that counting is impossible. The figure given is a minimum based only on leucocytes in which masses of bacteria were not present to interfere with enumeration.

TABLE 53

\begin{tabular}{|c|c|c|}
\hline TIME INTERVALS & $\begin{array}{c}\text { NUMBER OF } \\
\text { BACILLI IN } 100 \\
\text { LEUCOCYTES }\end{array}$ & INDEX \\
\hline Immediately after mixing $\ldots \ldots \ldots \ldots \ldots \ldots$ & 197 & 5.4 \\
\hline After $2 \frac{\mathrm{I}}{2}$ minutes . . . . . . . . . & 362 & 10.0 \\
\hline After 5 minutes.... & 372 & 10.3 \\
\hline 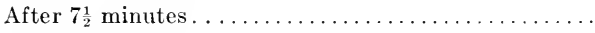 & 440 & 12.2 \\
\hline After 10 minutes . . . . . . . . . . . . . . . . & 824 & 23.0 \\
\hline
\end{tabular}

The opsonic power must be exerted, not by the bacteriophage protobes themselves, but by some principle which originated during the course of the bacteriophagic process. With the facts now available it is impossible to speak with any degree of assurance as to what the nature of this principle may be.*

It will be recalled that in an earlier chapter it was stated that by the addition of alcohol to a bacteriophage suspension a precipitate formed, which, in one way or another, involved the bacteriophage protobes. It is of interest to ascertain whether the material thrown down in such a precipitated suspension exerts any opsonic action. The following experiment indicates that the active principle appears, in part at least, in this precipitate.

Two milligrams of the alcoholic precipitate of a Shiga-bacteriophage

* I first advanced the hypothesis ${ }^{321}$ that this principle must be the "lysin" secreted by the bacteriophage protobe. This is indeed possible, but as yet, as I have already said, this can not be proved. 
suspension, still moist, are dissolved in 10 cc. of physiological saline. A mixture is made containing this solution, a suspension of Shiga bacilli, and a leucocytic suspension in equal parts. The mixture is held at a temperature of $38^{\circ} \mathrm{C}$. Microscopic examination of stained preparations, made after 15 minutes, shows that 100 leucocytes have taken up 536 bacilli (this count is a minimum, since some of the leucocytes had taken up so many bacilli that they could not be counted). The index is 14.9.

It is interesting to determine whether the active principle, causing such a marked stimulation in opsonic action, is also operative upon bacteria which have developed an acquired resistance to bacteriophagy. The following experiments bear on this point.

Mix equal parts of a suspension of Shiga bacilli resistant to the action of the bacteriophage, a Shiga-bacteriophage suspension two years old, and a suspension of leucocytes. After fifteen minutes, 100 leucocytes have ingested 8 bacteria. The index is thus 0.22 , or 90 times less than with normal bacilli.

Prepare a similar mixture, but with a bacteriophage suspension six days old. Here, 100 leucocytes have phagocytized 13 bacilli. The index is 0.38 , or 108 times less than with normal bacilli.

Another mixture is made, using the solution of the alcoholic precipitate. 100 leucocytes have phagocytized 19 bacilli. The index is 0.53 , or, 28 times less than with normal bacilli.

From this it is clear that bacteria which resist the bacteriophage also resist phagocytosis.

The same experiment has been performed with a race of the Barbonebacteriophage and the bacterium of barbone. The results were comparable, as follows:

(A) 1. Control. Mixture of equal parts of leucocyte suspension, bouillon, and suspension of the bacterium of barbone.

After fifteen minutes at $38^{\circ} \mathrm{C}$. there are no bacteria in 100 leucocytes.

2. Mixture of equal parts of leucocyte suspension, the suspension of the bacterium of barbone, and a Barbone-bacteriophage, $\$$ months old.

After fifteen minutes 100 leucocytes have ingested 109 bacteria.

3 . The same mixture, except that the bacteriophage suspension is diluted 1:250.

One hundred leucocytes have phagocytized 52 bacteria.

4. Mixture of one-third leucocyte suspension, one-third bacterial suspension, and one-third solution of the alcohol precipitate of a recent suspension of the Barbone-bacteriophage ( $2 \mathrm{mgm}$. of precipitate in 10 cc.of saline).

One hundred leucocytes have phagocytized 239 bacteria. 
(B) A mixture is made of equal parts of leucocyte suspension, culture of the bacterium of barbone, and a fresh (four days old) suspension of the Barbone-bacteriophage. During incubation at $38^{\circ} \mathrm{C}$. drops taken for examination show:

Immediately, in 100 leucocytes there are 30 bacteria.

After two and one-half minutes in 100 leucocytes there are 139 bacteria.

After five minutes, in 100 leucocytes there are 201 bacteria.

After seven and one-half minutes, in 100 leucocytes there are 271 bacteria.

After ten minutes, in 100 leucocytes there are 269 bacteria.

In the control mixture, made with bouillon, no bacteria were phagocytized.

Here it is impossible to calculate the opsonic indices, since no phagocytosis occurred in the control mixture. The indices are infinity.

As was the case with the dysentery bacillus, the bacterium of barbone which resists the action of the bacteriophage is also resistant to phagocytosis.

Prepare a mixture of one-third leucocytic suspension, one-third of the same suspension of Barbone-bacteriophage as that used in the preceding experiment, and one-third of a suspension of the bacterium of barbone resistant to dissolution. After fifteen minutes at $37^{\circ} \mathrm{C} .100$ leucocytes have ingested 3 bacteria, that is to say, 90 times less than with normal bacteria.

The race of Shiga-bacteriophage employed in the experiments previously described manifests a definite, although feeble, virulence for B. typhosus. The following experiments show that it also exerts a definite opsonic action on this bacillus.

1. Mix equal parts of bouillon, leucocyte suspension, and B. typhosus suspension.

After fifteen minutes at $38^{\circ} \mathrm{C} .100$ leucocytes have phagocytized 68 bacilli. The opsonic index is 1 .

2. Mix equal parts of Shiga-bacteriophage suspension, leucocyte suspension, and typhoid suspension.

After fifteen minutes 100 leucocytes have ingested 203 bacilli. Opsonic index $=3$.

3. Mix equal parts of leucocyte suspension, typhoid suspension and the solution of the alcoholic precipitate (the same one as that employed in the experiments with the dysentery bacillus). 
After fifteen minutes 100 leucocytes have ingested 109 bacilli. Opsonic index $=1.6$.

Considering the data presented in the above experiments, there can be no question but what some factor present in a bacteriophage suspension exerts a very definite effect upon opsonization with the homologous, bacteriophage-susceptible, bacteria.

But these principles, formed during the process of bacteriophagy, possess still another property. For when used as antibody in the complement fixation reaction it functions as an amboceptor. The experiment cited below is taken from among many others which gave identical results.

Antigen: This is prepared according to the method of Maurice Nicolle. One loopful of an agar culture of $B$. dysenteriae Shiga is suspended in $4 \mathrm{cc}$. of saline. This suspension, heated at $100^{\circ} \mathrm{C}$. for five minutes, then cooled, serves as antigen.

TABLE 54

\begin{tabular}{|c|c|c|c|c|c|c|c|}
\hline TUBE & $\begin{array}{l}\text { ANTI- } \\
\text { GEN }\end{array}$ & $\begin{array}{l}\text { ANTI- } \\
\text { BODY }\end{array}$ & $\begin{array}{l}\text { COMPLE- } \\
\text { MENT }\end{array}$ & SALINE & & \begin{tabular}{|l} 
HEMO- \\
LYTIC \\
SYSTEM
\end{tabular} & RESULT \\
\hline & $c c$. & $c c$. & $c c$. & $c c$. & & cc. & \\
\hline 1 & 0.5 & 0.2 & 0.2 & 1.6 & $\stackrel{\Xi}{=}$ & 1 & ++ \\
\hline 2 & 0.5 & 0.4 & 0.2 & 1.4 & +0 & 1 & $+t$ \\
\hline 3 & 0.5 & 0.5 & 0.2 & 1.3 & $\Xi \stackrel{\oplus}{\oplus} \dot{0}$ & 1 & $+t+$ \\
\hline 4 & 0.5 & 0.6 & 0.2 & 1.2 & 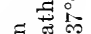 & 1 & +++ \\
\hline 5 & 0.5 & - & 0.2 & 1.8 & .00 & 1 & Complete hemolysis \\
\hline 6 & - & 0.6 & 0.2 & 1.7 & 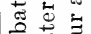 & 1 & Complete hemolysis, rapid \\
\hline 7 & - & - & 0.2 & 2.3 & 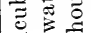 & 1 & Complete hemolysis \\
\hline 8 & - & - & - & 2.5 & $\Xi$ & 1 & $+t+t$ \\
\hline
\end{tabular}

Antibody: An alcohol precipitate of a Shiga-bacteriophage suspension is taken into solution in a quantity of saline equal to the original volume of the suspension. This serves as antibody.

The complement is fresh guinea pig serum, titrated.

The hemolytic system is the usual anti-sheep system. (See table 54.)

That there is something in the bacteriophage suspension which modifies the complement fixation reaction is obvious. Certainly the principles present in the solution used as antibody do not of themselves fix complement, as is evident from the results obtained with tube 6 , where it is clear that hemolysis is stimulated.

With the very fragmentary knowledge which we now have, it is hardly possible to draw any conclusions from this experiment. 
But however that may be, insofar as opsonic activity is concerned, whatever may be the agent which is operative, it is certain that during the course of bacteriophagy some product is formed which possesses a very considerable opsonic action.

\section{RÉSUMÉ}

Although the normal habitat of the bacteriophage is the intestinal tract, it is able to pass into the circulation, and thence into the tissues (d'Herelle ${ }^{310,313}$ ).

The presence of the bacteriophage within the tissues does not cause any detectable disturbance (d'Herelle ${ }^{311}$ ).

Bacteriophage corpuscles introduced into the body by a parenteral route behave like the spores of saprophytic bacteria. They are eliminated quickly from the circulation, and are to be found last in the spleen (Appelmans ${ }^{12}$ ).

The repeated injection of an animal with suspensions of the bacteriophage causes the appearance in the serum of an antibacteriophagic property (Bordet and Ciuca ${ }^{88}$ ).

The intensity of the antigenic properties of different races of the bacteriophage is highly variable (Otto, Munter and Winkler ${ }^{496}$ ).

Within a single bacteriophage suspension the sensitivity to the inhibiting action of an antibacteriophagic serum varies greatly from one corpuscle to another (Prausnitz ${ }^{536}$ ).

Different races of the bacteriophage behave very differently toward an antibacteriophagic serum (Otto, Munter and Winkler ${ }^{496}$ ).

An antibacteriophagic serum does not destroy the bacteriophage corpuscles; the effect is an inhibition of their action (d'Herelle and Eliava $\left.^{359}\right)$.

The inhibition of bacteriophagic action by an antiserum has no points of resemblance to a neutralization of a toxin by the homologous antitoxin. The inhibitory effect is not that of a sensitizer (d'Herelle $\left.{ }^{349}\right)$.

The nature of the reaction of an antibacteriophagic serum upon the bacteriophage is not distinctive; this type of reaction is common to all anti-protobian sera. The injection of a protobe (ultravirus) of any kind into an animal leads to the appearance of a specific "antivirulin" in the serum (d'Herelle ${ }^{349}$ ).

Antibacterial sera exert an inhibitory action upon bacteriophagy (Bail ${ }^{43}$ ). This inhibitory action is entirely different from the inhibitory action of an antibacteriophagic serum on the protobe itself. The antibacterial serum does not modify the bacteriophage (Hauduroy ${ }^{287}$ ). 
The repeated injection of an animal with a bacteriophage suspension, instead of immunizing the animal, may sensitize it, inducing the antiphylactic state (d'Herelle ${ }^{321}$ ).

This state of antiphylaxis may be sufficiently pronounced to cause the animal to lose its naturally refractory condition for a given bacterium (Hauduroy ${ }^{296}$ ).

Suspensions of the bacteriophage possess a powerful opsonic action (d'Herelle and Eliava ${ }^{321}$ ). 


\section{CHAPTER II}

\section{The Ubiquity of the Bacteriophage}

\section{THE BACTERIOPHAGE IN THE INTESTINAL TRACT}

Throughout the remainder of this text the subjects under discussion will involve, directly or indirectly, the question of the multiple virulences which a race of the bacteriophage may show in the intestine. But before presenting this material I must first reply to an objection which has been raised by several authors, wherein they question whether the activity which a filtrate of the stool manifests against different bacterial species is due to the presence of one bacteriophage endowed wicn multpie virulences, or whether it is a natural result of the simultaneous presence of several races of the bacteriophage.

Anticipating somewhat the facts which are to be presented, we may say at once that different races of the bacteriophage exist, and are found, frequently, in any material which may have been, at any time, contaminated by the excreta of any animal whatever. For example, drinking water, among other things, commonly contains the bacteriophage. This being the case, the ingestion of different races of the bacteriophage must be a constant occurrence, both by man and by animals. One would think, a priori, that a specimen of excreta should, therefore, contain bacteriophages belonging to different races. But as a matter of fact, the experiments to be detailed in the following chapters show that this is not the case, and thatitis rare to find in the filtrate of a stool from a given individual, not many races, but even two.

How can we explain this finding which seems to be in conflict with the fact that we daily ingest different races?

Experiment shows that within the intestinal tract of each individual there is, as a rule, one race of the bacteriophage living in a symbiotic relationship with the $B$. coli present in the intestine of this individual. Thus, of two possibilities, one must occur; either the different races ingested are not able to adapt themselves to the bacteriophagy of the $B$. coli strain there present, with the result that they are quickly eliminated, or, the behavior of the bacteriophage is not the same in vivo as in vitro, and that in the intestine the different races quickly lose their particular distinctive characters and become uniform. It is difficult to 
choose between these two hypotheses, for we have no direct proof of either one, but the following experiment seems to favor the first of them, although, as will appear, it by no means eliminates the second.

I have a race of the bacteriophage, $\mathrm{K}$ (isolated from the feces of a cholera convalescent), which is very virulent for strain $\mathrm{C}$ of $B$. coli (isolated from a case of cystitis) and for the dysentery organisms, Shiga, Flexner, and Hiss. During the course of some studies made on a perfectly normal individual I found that the stools contained a bacteriophage virulent for strain $\mathrm{V}$ of $B$. coli (an old stock culture strain). I had this individual ingest 2 cc. of a suspension of bacteriophage $\mathrm{K}$, and 36 hours later I found that both races of the bacteriophage were present in his stools, the race belonging to the individual himself, and race $K$. Specimens collected 2, 3, and 4 days after the ingestion showed the same thing. Those of the 5th, 6th, and 7th days showed only the race which was present in the intestine before the ingestion. Race $\mathrm{K}$ had disappeared.

Similarly, we will see that when there exists in the intestine a culture of a bacterium susceptible to the race of bacteriophage which is ingested, the latter develops at the expense of these bacteria, but that it disappears very soon after the bacteriophagy in vivo is completed.

Whether the ingested bacteriophage corpuscles quickly lose their characters in vivo, a change which does not occur in in vitro experiments and which the observations made on the in vivo process renders improbable, or whether they are very quickly eliminated, the fact remains that all of the bacteriophage corpuscles to be found in a filtrate from the stool, either normal or pathological, belong to but a single race.

The following fact, clearly demonstrated by Tomaselli59s is of interest in this connection. He showed that within the intestine of a given individual a special race, always the same, persists. From an individual who has had typhoid fever, even though the disease occurred several years previously, he found that he could repeatedly recover from the feces a race of the bacteriophage showing a virulence for $B$. typhosus, a virulence which is but rarely demonstrated in the bacteriophage of those who do not have the disease. This indicates a persistence, throughout several years, of a single race, and this is hardly compatible with the hypothesis that in vivo the races of the bacteriophage daily ingested become uniform.

The facts suggest, therefore, that in the intestine of each living being there is a race of the bacteriophage possessing special characters, a race which is in a sense peculiar to the individual inasmuch as it possesses its own different and variable virulences. 
One of the most important points to be considered in the discussion of the material which is to make up the remainder of this text, is that we must differentiate between the degree which the diverse virulences may attain by adaptation in vitro, and the degree of virulence possessed by a bacteriophage in the intestine at the time of observation. For our present purposes the latter is the more significant and in what follows, when we refer to a bacteriophage as having a given degree of virulence this will refer to the degree of virulence which the bacteriophage has, or had, at the time of isolation from the stool, or other product.

The notation adopted for recording the degree of virulence is the simplified scheme which has been described in the section dealing with "The Appreciation of Virulence." It will be remembered that, according to this scheme,

$$
\begin{aligned}
0 & =\text { no virulence } \\
+ & =\text { weak virulence } \\
++ & =\text { moderate virulence } \\
+++ & =\text { strong virulence } \\
++++ & =\text { maximum virulence }
\end{aligned}
$$

When the virulence is spoken of as "weak," "moderate," etc., the meaning is that which has been given to these terms in the section referred to.

\section{THE BACTERIOPHAGE IN HEALTHY MAN}

In order to demonstrate that the intestinal tract of the normal healthy man contains a bacteriophage I have made systematic examinations on a person in a perfect state of health. ${ }^{316}$ Throughout a year I collected a specimen of stool every two weeks, and the virulence of the bacteriophage found upon each occasion was tested against strains of the following bacterial species: $B$. coli, $B$. dysenteriae Shiga, B. dysenteriae Flexner, B. dysenteriae Hiss, B. typhosus, B. paratyphosus $A$, and $B$. paratyphosus $B$. Later, where indicated, the tests were extended to other bacterial species of particular interest.

With the first examinations a weak activity $(+)$, especially for B. coli, was not detected or remained doubtful, but when the tests were repeated after several months, with the same filtrates which had been held during the interval in sealed ampoules, using a more satisfactory technic, the bacteriophage was clearly demonstrated. As will be seen upon examining the table where the results are recorded, some of the examinations remained negative; the bacteriophage appeared to be absent. Would it have been the same if it had been possible to test the filtrate against 
all of the bacteria which may be found in the intestine? An answer to this question was sought. A specimen taken on July 1st was inactive toward the eight species of bacteria routinely employed, and it was tested against a different series of bacteria, selected at random. The filtrate showed a high activity for an organism of the Salmonella (hog cholera)

TABLE 55

\begin{tabular}{|c|c|c|c|c|c|c|c|c|}
\hline \multirow{3}{*}{ DATE } & \multicolumn{8}{|c|}{ VIRULENCE OF THE INTESTINAL BACTERIOPHAGE FOR } \\
\hline & \multirow[b]{2}{*}{ B. coli } & \multicolumn{3}{|c|}{$B$, dysenteriae } & \multicolumn{3}{|c|}{ Bacillus } & \multirow[b]{2}{*}{ Other organisms } \\
\hline & & $\stackrel{.00}{\Xi}$ & 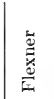 & $\stackrel{\substack{n \\
\stackrel{D}{\Xi}}}{\stackrel{D}{\Xi}}$ & $\frac{\sqrt[9]{3}}{\stackrel{3}{a}}$ & 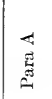 & $\stackrel{n}{\vec{w}}$ & \\
\hline January $15 \ldots$. & 0 & 0 & 0 & 0 & 0 & 0 & 0 & \\
\hline February $1 \ldots$ & + & 0 & 0 & 0 & 0 & 0 & 0 & \\
\hline February $15 \ldots \ldots$ & 0 & 0 & 0 & 0 & 0 & 0 & 0 & \\
\hline March $1 \ldots \ldots \ldots$ & 0 & 0 & 0 & 0 & 0 & 0 & $++t$ & \\
\hline March $15 \ldots \ldots \ldots$ & + & 0 & 0 & 0 & 0 & 0 & 0 & \\
\hline April $1 \ldots \ldots \ldots$ & + & 0 & 0 & 0 & 0 & 0 & 0 & \\
\hline April $15 \ldots \ldots \ldots$ & +++ & 0 & 0 & 0 & 0 & 0 & 0 & \\
\hline May $1 \ldots \ldots \ldots$ & + & 0 & 0 & 0 & 0 & 0 & ++ & \\
\hline May $15 \ldots \ldots \ldots$ & + & 0 & 0 & 0 & 0 & 0 & 0 & \\
\hline June $1 \ldots \ldots \ldots$ & 0 & 0 & 0 & 0 & 0 & 0 & 0 & \\
\hline June $15 \ldots \ldots \ldots$ & + & 0 & ++ & +++ & 0 & 0 & 0 & \\
\hline July $1 \ldots \ldots \ldots$ & 0 & 0 & 0 & 0 & 0 & 0 & 0 & Salmonella +++ \\
\hline July $15 \ldots \ldots \ldots$ & $++t$ & 0 & 0 & 0 & 0 & 0 & 0 & \\
\hline August $1 \ldots \ldots$ & + & + & 0 & 0 & 0 & 0 & 0 & \\
\hline August $15 \ldots \ldots$ & $++t$ & +++ & 0 & 0 & 0 & 0 & 0 & \\
\hline September $1 \ldots \ldots$ & +++ & 0 & 0 & 0 & 0 & 0 & 0 & \\
\hline September $15 \ldots$ & + & +++ & 0 & 0 & 0 & 0 & 0 & \\
\hline October $1 \ldots \ldots$ & + & 0 & 0 & 0 & 0 & 0 & +++ & \\
\hline October $15 \ldots \ldots$ & + & +++ & ++ & 0 & 0 & 0 & 0 & \\
\hline November $1 \ldots .$. & + & 0 & 0 & 0 & 0 & 0 & 0 & \\
\hline November $15 \ldots$. . & + & 0 & 0 & 0 & 0 & ++ & 0 & \\
\hline December $1 \ldots \ldots$ & 0 & 0 & 0 & 0 & 0 & 0 & 0 & B. enteritidis ++ \\
\hline December $15 \ldots$ & + & 0 & 0 & 0 & 0 & 0 & 0 & \\
\hline
\end{tabular}

group. When the same experimental tests were repeated on December 1 st this filtrate was active for $B$. enteritidis.

The results of all of these tests are given in table 55 .

From these determinations I reached the conclusion that in those cases where the bacteriophage can not be demonstrated the failure is due solely to the fact that the method employed is defective. This defect in the method is due primarily to the fact that $B$. coli is a heterogeneous species, hence the inactivity of a filtrate for a strain of this 
organism means rather that the strain is not susceptible, and not that the bacteriophage is absent. And from this and other studies ${ }^{313,316,321}$ I have concluded that the bacteriophage is constantly present in the intestinal tract of all normal men, where it grows at the expense of $B$. coli and perhaps of other banal bacteria found there.

These conclusions have been subjected to attack by several authors, and even by some of those who have found the bacteriophage in normal men, their argument being that the condition is not habitual.

Recently I have carried out some more of these studies, following a a different and more rational method. In its essentials this method involved the following procedures, all carried out with the same specimen of fecal material.

First, a filtrate is prepared, according to the usual technic, which has been described.

Second, the bacteria of the stool are isolated on Endo medium. After incubation, it is found that the reddish colonies of $B$. coli assume three different forms,

(a) those perfectly circular, with a rounded surface;

(b) those perfectly circular, umbilicated;

(c) those which are irregular and flat. These last are, in general, mixed colonies.

Twelve of the circular colonies are removed and transferred to tubes of bouillon. At the same time, if any colorless colonies are present on the plates, three or four of these are also transferred to bouillon tubes. To each of these implanted tubes 3 drops of filtrate are added, and the tubes are incubated at 28 to $30^{\circ} \mathrm{C}$.

After incubation for 24 hours each tube is examined for the presence of the bacteriophage, by spreading the culture on agar, and, if necessary, by making two or three passages, always at the expense of the $B$. coli derived from the colony which was used to seed the first tube.

During a period of three months, at irregular intervals, I secured 17 specimens of the feces of a normal person.*

In each of the 17 specimens the bacteriophage protobe was present. But the proportion of susceptible colonies of $B$. coli found on the plates varied from one examination to another. In this respect, the results were:

In 1 specimen, 5 of the 12 colonies were susceptible;

* The same person who had provided the specimens which gave the results incorporated in table 55. As a matter of fact, he is the author of these lines. When these specimens were examined he was living at Alexandria, as were the 6 individuals mentioned in a later paragraph. 
In 3 specimens, 4 of the 12 colonies from each were susceptible; In 7 specimens, 3 of the 12 colonies from each were susceptible; In 4 specimens, 2 of the 12 colonies from each were susceptible;

In 2 specimens, none of the 12 colonies in either one was susceptible.

In the last two cases, - those where all of the organisms proved to be resistant,- I subjected the 12 colonies in each case to a series of transfers in an acid medium, the acidity being at the limit for growth of the organism at $41^{\circ} \mathrm{C}$., and between each two passages I isolated colonies on a 1 per cent glucose agar, according to the method of Roux, described above. The sequence of cultures was, therefore, alternately, growth in an acid medium, isolation on agar, growth in an acid medium, isolation on agar, etc. At the 5th, 10th, 15th, 20th, and 25th isolations I determined whether any of the colonies had lost their resistance, whether they had become susceptible to the bacteriophage contained in the filtrate made from the same specimen from which the 12 original colonies had been isolated. With the two filtrates the results were:

Filtrate 1.

At the 5th passage, none of the colonies had become susceptible;

At the 10th passage, 1 of the colonies had become susceptible;

At the 15th passage, 1 of the colonies was still susceptible;

At the 20th passage, 4 of the colonies had become susceptible;

At the 25 th passage, 5 of the colonies had become susceptible.

Filtrate 2 .

At the 5th passage, 1 of the colonies had become susceptible;

At the 10th passage, 1 colony was still susceptible;

At the 15th passage, 1 colony was still susceptible;

At the 20th passage, 6 of the colonies had become susceptible;

At the 25th passage, 7 of the colonies had become susceptible.

It is very probable that had the passages been continued other colonies would have become susceptible, but this is of no consequence for the matter with which we are at present concerned.

In the course of these 17 examinations I also isolated certain colonies which were colorless on Endo,-14 in all, which upon microscopic examination proved to be bacilli. Of the 14,6 proved to be $B$. fecalis alkaligenes, for they failed to ferment glucose. The identity of the others was not determined. Of the 14 colonies 13 were susceptible to the action of the bacteriophage present in the filtrate of the specimen which yielded the colony.

I have procured specimens from 6 other normal persons, and the examinations were carried out in the same manner. Of the 12 colonies isolated in each case from the specimen which furnished the filtrate; 
In 2 cases, 4 of the 12 colonies were susceptible;

In 1 case, 2 of the 12 colonies were susceptible;

In 2 cases, 1 of the 12 colonies was susceptible;

In 1 case none of the colonies was susceptible.

In this last case I carried out a series of purifications, as in the two negative instances reported above, and at the 10th passage 1 of the 12 colonies was found to be susceptible.*

My first experiments upon the subject had shown that "the bacteriophage exists in the intestine, where it lives normally at the expense of the B. coli." "311 The experiments which I have made since have verified this observation and permit me to affirm that the bacteriophage protobe is always, in every individual, a normal inhabitant of the intestinal tract, where it persists because of a symbiosis with the colon bacillus. But in order to demonstrate its constant presence it is not sufficient to combine any stool filtrate whatever with any strain of $B$. coli whatever. It is essential to adopt an adequate method, such as that which has been described.

Many authors have confirmed this observation that the bacteriophage is present in the intestinal contents of normal man. Dumas ${ }^{188}$ found the bacteriophage present in 5 of 8 healthy persons which he examined. Debré and Haguenau ${ }^{175}$ also found it in some individuals who had no intestimal disturbance, as did Beckerich and Hauduroy ${ }^{57}$ and da Costa Cruz. ${ }^{153}$ This last author examined the same healthy person at 5 different times and on 3 occasions he found a bacteriophage.

These different authors did not demonstrate the bacteriophage solely because of its virulence for $B$. coli, but because at the time of examination the virulence of the bacteriophage in the individual examined extended to a dysentery or a paratyphoid bacillus. This occurs very frequently, as I reported in some of my earlier studies. ${ }^{311,}{ }^{316}$

Tomaselli ${ }^{599}$ procured specimens of the stools of 10 normal persons, and from his determinations, he reported the following virulences:

In 3 persons:

B. dysenteriae Shiga +++

B. typhosus (2 of 4 strains tested) +

B. paratyphosus $B$ ( 4 of 4 strains tested) +

In 1 person:

B. dysenteriae Shiga +

B. typhosus (1 of 4 strains tested) +

B. paratyphosus $B$ (3 of 4 strains tested) +++

* The purifications were not continued, since what I wished to show had been demonstrated at this point. 
In 1 person:

B. dyscnteriae Shiga ++

B. typhosus (3 of 4 strains tested) ++

In 1 person:

B. dysenteriae Shiga +

B. paratyphosus $A$ (4 of 4 strains tested) +

In 4 persons:

B. dysenteriae Shiga ++

It is needless to introduce more data bearing on this point. That the bacteriophage may be present in the normal intestinal tract is virtually accepted by everyone, but a great many seem to think that its presence there is accidental. Such an opinion can be based only upon investigations carried out by an inadequate technic. I repeat: in every intestine there is a race of the bacteriophage protobe maintaining itself there because of a symbiosis with $B$. coli.*

One question comes immediately to mind; When does this intestinal bacteriophage arrive? Does it exist in the fetus, or is it implanted after birth? And if the latter is true, when?

We know that the intestinal contents of the fetus are sterile, the normal meconium is free of organisms at the time of birth. This phase of sterility is promptly followed by a phase of increasing contamination, which begins, within the first few hours after birth, as a result of the first deglutitive movements which carry a varied flora into the digestive tract of the infant. At first there is a predominance of Staphylococcus albus and of $B$. coli, with some of the anaerobes, and the body reacts by a desquamation of the epithelium of the digestive tube.

At about the fourth day for the breast-fed infant, somewhat later and in an incomplete manner for those fed artificially, the flora is transformed. The anaerobes, B. bifidus in particular, replace the earlier flora. With the breast-fed infant the bacterial content is almost entirely made up of $B$. bifidus.

In connection with his thesis, Védrenne ${ }^{610}$ made a study of the bacteriophage in infancy. He was unable to demonstrate the presence of a bacteriophage virulent for B. coli, B. dysenteriae Hiss, Staphylococcus

* Upon several occasions I have remarked that the symbiosis is always due to a parasitism of the one or the other of the two antagonists. All symbioses which occur naturally are comparable to that which exists between a leper and the bacillus of Hansen. The old concept of an association between two organisms for the purpose of a common benefit is false and is never observed in nature, as has been shown by Noël Bernard. 
albus, or B. proteus, either in the meconium or in the stools of infants up to the 4 th day.

Surányi and Kramár ${ }^{592}$ have made some systematic studies of the same nature. They uniformly failed to find the bacteriophage in the meconium, and in no case could one be demonstrated in the stools of the infant before the 4 th day. It may be added that in the course of my studies I have had occasion to examine 4 infants, aged, respectively, $7,9,10$, and 12 days. In each of these cases I found a bacteriophageB. coli symbiosis.

One might conclude from these studies that the bacteriophage makes its appearance in the intestine of man between the 4 th and the 7 th days after birth. It is probable that its appearance coincides with the ingestion of a colon bacillus infected with a bacteriophage protobe.

\section{THE BACTERIOPHAGE IN ANIMALS}

It is much easier to prove that the bacteriophage is normally present in the intestinal tract of animals than in man, for in domestic animals certainly, the virulence is rarely limited to $B$. coli, but extends to the dysentery strains and to the paratyphoid bacilli (d'Herelle, ${ }^{314,316}$ ).

This difference in the nature of the virulences of the intestinal bacteriophage in man and in the animals can hardly be explained except in a single way,- because of their mode of life the animals are much more exposed to the frequent ingestion of different bacilli. In animals the bacteriophage is found constantly, therefore, in the presence of varied species of bacteria, and against these it builds up its faculty of virulence.

This hypothesis receives support from the fact that although the intestinal bacteriophage of animals which live an unconfined life almost always manifests multiple virulences, with laboratory animals, restricted to their own cages and under conditions more closely approaching those of human beings, the virulence is often restricted to the intestinal colon strain.

We will see later that in a group of persons exposed to the possible ingestion of a pathogenic bacillus, we find that in all of them the intestinal bacteriophage manifests a virulence for the bacillus in question.

Confirming this observation, the following tables will show us that in animals which find themselves in an environment contaminated by a bacterium, even though the organism is not pathogenic for the animal in question, we find that the intestinal bacteriophage acquires a virulence for this bacterium, while in all of the animals of the same species 
living in regions where this bacterium is lacking, a corresponding virulence of the intestinal bacteriophage can never be detected.

All of these facts show that if the intestinal bacteriophage of domestic animals manifests, with very few exceptions, a virulence for the dysentery bacilli and for the paratyphoid organisms, it is simply because these bacteria must be extremely abundant in the environment and that

TABLE 56

\begin{tabular}{|c|c|c|c|c|c|c|c|c|}
\hline \multirow{2}{*}{ HORSE } & \multirow{2}{*}{ B. COLI } & \multicolumn{3}{|c|}{ B. DYSENTERIAE } & \multicolumn{3}{|c|}{ BACILLUS } & \multirow{2}{*}{$\begin{array}{l}\text { B. GALLI- } \\
\text { NARUM }\end{array}$} \\
\hline & & Shiga & Flexner & Hiss & Typhosus & Para A & Para B & \\
\hline 1 & $+t$ & $+t+t$ & $+t$ & $+t$ & 0 & 0 & 0 & - \\
\hline 2 & + & + & + & 0 & 0 & 0 & 0 & - \\
\hline 3 & + & $+t$ & 0 & 0 & $+t$ & + & + & - \\
\hline 4 & $+t$ & $+t$ & + & + & 0 & + & $+t$ & 0 \\
\hline 5 & ++ & +++ & $+t+$ & $+t+$ & 0 & 0 & 0 & 0 \\
\hline 6 & + & 0 & $+t+$ & $+t+$ & 0 & 0 & 0 & 0 \\
\hline 7 & ++ & $++t+$ & $+t+t$ & $t+$ & 0 & 0 & 0 & - \\
\hline$\delta$ & ++ & + & $+++t$ & $++t+$ & 0 & 0 & 0 & - \\
\hline 9 & $+t$ & ++++ & $+t$ & $+t$ & 0 & 0 & 0 & - \\
\hline 10 & $+t$ & 0 & ++ & ++ & 0 & 0 & + & - \\
\hline 11 & + & 0 & + & ++ & 0 & 0 & 0 & 0 \\
\hline 12 & $+t$ & $++t$ & + & ++ & 0 & 0 & + & 0 \\
\hline 13 & $+t$ & $+t$ & $+t$ & + & + & 0 & 0 & 0 \\
\hline 14 & ++ & $+t+t$ & ++ & ++ & 0 & 0 & 0 & 0 \\
\hline 15 & 0 & $++t+$ & $+t$ & +++ & 0 & 0 & 0 & 0 \\
\hline 16 & $+t$ & ++++ & $+t+t$ & $+t+t$ & $+t$ & $+t+$ & $+t$ & ++ \\
\hline 17 & ++ & $+t+$ & $+t$ & ++ & + & $+t$ & 0 & ++ \\
\hline 18 & +++ & $+t$ & $+t+$ & +++ & $++t$ & $+t+$ & ++ & +++ \\
\hline 19 & $+t$ & ++++ & + & + & 0 & 0 & ++ & 0 \\
\hline 20 & 0 & $+t$ & 0 & 0 & 0 & 0 & 0 & 0 \\
\hline 21 & + & + & + & 0 & 0 & + & ++ & 0 \\
\hline 22 & $+t$ & 0 & 0 & 0 & 0 & 0 & $+t$ & 0 \\
\hline 23 & $+t$ & $++t$ & $+t$ & $+t$ & 0 & 0 & ++ & 0 \\
\hline 24 & + & $+++t$ & $+t+$ & ++ & ++ & 0 & + & 0 \\
\hline 25 & + & ++ & 0 & 0 & 0 & 0 & 0 & 0 \\
\hline 26 & +++ & $++t$ & $+t$ & ++ & 0 & 0 & $+t+$ & 0 \\
\hline
\end{tabular}

their ingestion by the animals is not an exceptional occurrence $\left(d^{\prime} H_{e r e l l e}^{316}\right)$.

The results of some of the studies which I have made upon the distribution of the bacteriophage among different animal species ${ }^{314,316,321}$ and the virulences which these races of the bacteriophage possess are presented in the following sections. 


\section{THE BACTERIOPHAGE IN THE HORSE}

Sixty-two specimens of manure derived from horses living both in cities and in the country, in France and in Indo-China, have been examined and all contained an active bacteriophage. A list of the animals is given, and the results of the examination are recorded in table 56 .

The following notes, descriptive of the animals examined, show somewhat the diverse conditions under which these animals lived.

No. 1. Horse No. 21 of the Pasteur Institute. This horse was used in the production of anti-dysentery serum. The examination was made three days after the injection of Shiga toxin.

No. 2. Horse No. 21 (above), tested ten days later.

No. 3. Horse No. 21 (above), tested four months later, the examination being made 48 hours after a toxin injection.

No. 4. Horse No. 114. Used in the production of Shiga antidysentery serum.

No. 5. Horse No. 18. Used in the production of Shiga anti-dysentery serum.

No. 6. Horse No. 18 (above), tested four months later. The specimen was collected 48 hours after the injection of Shiga toxin.

No. 7. Horse No. 64. This horse had received injections of atoxic dysentery bacilli-Flexner and Hiss - for two years.

No. 8. Horse No. 65. This horse had received injections of atoxic dysentery bacilli-Flexner and Hiss - for two years.

No. 9. Horse No. 68. This horse had received injections of atoxic dysentery bacilli-Flexner and Hiss-for two years.

No. 10. This horse was receiving injections of B. anthracis.

No. 11. This horse was receiving injections of B. anthracis.

No. 12. A carriage horse in Paris.

No. 13. A carriage horse in Paris.

No. 14. A carriage horse in Paris.

No. 15. The same animal as No. 14 (above) but tested four days later.

No. 16. A farm horse on a farm where avian typhosis was present.

No. 17. A farm horse on a farm where avian typhosis was present.

No. 18. A farm horse on a farm where avian typhosis was present.

No. 19. A race horse at Chantilly.

No. 20. A race horse at Chantilly.

No. 21. The same animal as No. 19 (above) but tested eight days later. 
No. 22. The same animal as No. 20 (above) but tested eight days later.

No. 23. A carriage horse at Saïgon.

No. 24. A carriage horse at Saïgon.

No. 25. A saddle-horse at Nha-Trang (Annam).

No. 26. A saddle-horse at Phantiet (Annam).

There is no point in adding to this list; the thirty-six other specimens gave entirely comparable results.

Incidentally, horses No. 19 and No. 20 were examined to see if the bacteriophage presented a virulence for various other bacteria, including the following organisms:

1. A cocco-bacillus (?) isolated from the nasal mucus of a horse, in the same stable, which showed the evening before an elevation of temperature:

Horse No. $19(++)$, horse No. $20(++)$.

2. A cocco-bacillus isolated by Césari from the blood of a horse slaughtered in the abattoir of Vaugirard:

Horse No. $19(+)$, horse No. $20(++)$.

3. Salmonella (hog cholera):

Horse No. $19(++)$, horse No. $20(++)$.

4. B. enteritidis: Horse No. 19 (0), horse No. $20(+)$.

These results show that at a single time the bacteriophage may show a virulence for a large number of bacteria. It is significant that only in horses Nos. 16, 17, and 18, which lived in an environment contaminated by $B$. gallinarium, did the intestinal bacteriophage show a definite virulence for this bacterium.

Examination was made of twenty-three specimens of serum, of clot remaining after the decantation of the serum, and of the leucocytic layer on top of this clot, taken from horses harboring in their intestines a bacteriophage active for $B$. dysenteriae. In no case was a bacteriophage found. In all instances the specimens of blood were collected about two weeks after the last injection of toxin or of bacilli. All the specimens of blood examined came from horses furnishing anti-dysentery serum. It was therefore not determined whether the bacteriophage may not pass into the circulation immediately after the injection, especially when living bacteria are used. As a matter of fact the passage of the intestinal bacteriophage into the circulation has been observed in the rat, and in the fowl in cases of septicemia. In all cases the demonstration of the presence, it might be said constant presence, in the excreta of the horse of a bacteriophage active for the Shiga bacillus, and the absence 
of this bacteriophage in the blood, show in an unquestionable manner that the intestine is the only locality where the bacteriophage normally grows.

These results have received confirmation by all who have made a study of this type; everyone has demonstrated the habitual presence of a race of the bacteriophage virulent for $B$. dysenteriae in the intestinal tract of the horse. There is no particular advantage to be gained in citing all of the results reported on this subject, but those of Tomaselli ${ }^{599}$ are worthy of particular mention. He followed over a period of time, making repeated examinations, the variations in the virulences of the intestinal bacteriophage in two horses, which, during the period of study were subjected to varied conditions of activity and of food. Briefly, his findings are as follows:

When resting in the stable; fed upon dry hay:

The intestinal bacteriophage showed virulences for:

$$
\begin{aligned}
& \text { B. dysenteriae Shiga }++ \\
& \text { B. dysenteriae Flexner }++
\end{aligned}
$$

After a period of excessive labor; fed upon dry hay:

The intestinal bacteriophage showed virulences for:
B. dysenteriae Shiga +++
B. dysenteriae Flexner +++
B. dysenteriae Hiss +

When resting in the stable; fed on green food:

The intestinal bacteriophage showed virulences for:
B. dysenteriae Shiga ++++
B. dysenteriae Flexner ++++
B. dysenteriae Hiss ++
B. of Gaertner +
B. coli ++

After heavy labor; fed on green food:

The intestinal bacteriophage showed virulences for:
B. dysenteriae Shiga ++++
B. dysenteriae Flexner ++++
B. dysenteriae Hiss +++
B. of Gaertner +
B. coli $+++^{*}$

* These virulences for $B$. coli refer to the activity for a laboratory strain of this bacillus. Tomaselli did not test the races isolated upon the strains present in the intestinal tracts of the horses themselves. 


\section{THE BACTERIOPHAGE IN THE CHICKEN AND IN THE GOOSE}

During the course of my experiments on the application of the bacteriophage to specific purposes I have made rather extensive studies of the activity of the intestinal bacteriophage to be found in chickens. The chickens examined were in different countries and were living under very unlike conditions as to nourishment and other environmental factors. The examinations made upon geese were carried out on these animals as found in farm-yards infected with avian typhosis, a disease to which the goose is refractory.

TABLE 57

\begin{tabular}{|c|c|c|c|c|c|c|c|c|c|}
\hline \multirow{2}{*}{ NUMBER } & \multirow{2}{*}{ B. COLI } & \multicolumn{3}{|c|}{ B DYSENTERIAE } & \multicolumn{3}{|c|}{ BACILLUS } & \multirow{2}{*}{$\begin{array}{l}\text { B. GALLI- } \\
\text { NARUM }\end{array}$} & \multirow{2}{*}{$\begin{array}{l}\text { BACT. } \\
\text { BAR- } \\
\text { BONE }\end{array}$} \\
\hline & & Shiga & Flexner & Hiss & $\underset{\text { supho- }}{\text { Typho }}$ & Para A & Para B & & \\
\hline 1 & 0 & 0 & + & $+t+$ & 0 & 0 & 0 & 0 & - \\
\hline 2 & ++ & ++ & 0 & 0 & + & 0 & + & 0 & - \\
\hline 3 & + & $+t+$ & $+t+$ & $+t+$ & + & + & $+t$ & ++ & - \\
\hline 4 & ++ & + & + & ++ & ++ & 0 & + & + & - \\
\hline 5 & +++ & $++t+$ & $+t+$ & $++t$ & ++ & $+t$ & $+t$ & $+t+t$ & - \\
\hline 6 & ++ & $++t$ & $++t$ & +++ & 0 & + & $+t+$ & $+t+$ & - \\
\hline 7 & + & ++ & 0 & 0 & 0 & 0 & $+t$ & 0 & - \\
\hline 8 & 0 & 0 & ++ & + & 0 & 0 & + & 0 & - \\
\hline 9 & ++ & $+t+$ & + & ++ & 0 & 0 & 0 & 0 & 0 \\
\hline 10 & + & + & 0 & $++t$ & 0 & 0 & ++ & 0 & 0 \\
\hline 11 & ++ & +++ & ++ & ++ & 0 & 0 & ++ & 0 & + \\
\hline 12 & 0 & + & 0 & + & ++ & + & $+t$ & 0 & ++ \\
\hline 13 & +++ & $+1++$ & +++ & $+t+$ & + & ++ & ++ & ++ & - \\
\hline 14 & ++ & $+t$ & ++ & ++++ & 0 & 0 & $+t+$ & ++ & - \\
\hline
\end{tabular}

I have made seventy examinations of the excreta of fowls, and have tested the bacteriophage for virulence against the eight bacterial strains selected. It is needless to give all the results since they were all of the same nature. As examples, the results of only one or two tests in each lot will be given. Table 57 clearly shows the range of virulences presented by the intestinal bacteriophage in these animals. The following supplementary notes are self-explanatory.

Nos. 1 and 2 represent chickens living in France in regions free of avian typhosis ( 12 specimens examined).

Nos. 3 and 4 represent healthy fowls living in regions where avian typhosis was present (19 other examinations carried out on the eight test bacteria gave comparable results, particularly as regards $B$. gallinarum). 
Nos. 5 and 6 represent chickens which had recovered from avian typhosis (4 tests made).

Nos. 7 and 8 represent chickens which died of avian typhosis (8 other tests gave similar results). The bacteriophage was present but was not virulent for the pathogenic bacillus.

Nos. 9 and 10 were chickens living in Cochin-China in regions free of both avian typhosis and barbone.

Nos. 11 and 12 represent chickens living in Cochin-China in areas where barbone was present but free of avian typhosis (11 tests, all essentially the same).

Nos. 13 and 14 were chickens in France living in regions where avian typhosis was present.*

\section{THE BACTERIOPHAGE AS FOUND IN A NUMBER OF DIFFERENT SPECIES}

Throughout the course of the many experiments performed there has been an opportunity to study the nature of the intestinal bacteriophage as found in a considerable number of different animal species. That such a bacteriophage is to be found within the intestinal tract becomes obvious when we consider the very diverse species in which it has already been disclosed. The results obtained in examining the excreta of some of these animals are presented in table 58. Explanation of the numbers appearing in the first column follows.

No. 1. A monkey, confined in a eage in Paris.

Nos. 2 and 3. Cats in Paris.

Nos. 4 and 5. Cattle living on a farm where avian typhosis was present.

Nos. 6 and 7. Cattle in France, in a region free of epizootic diseases.

Nos. 8 and 9. Steers in Cochin-China, living in regions free of epizootics (42 other comparable tests).

Nos. 10 and 11. Buffaloes living in regions free of barbone (14 other comparable tests).

Nos. 12 and 13. Healthy buffaloes living in regions where barbone was present ( 24 other comparable tests).

Nos. 14 and 15. (for comparison) Buffaloes sick (14) or dead (15)

* I would recommend that bacteriologists desiring to procure strains of the bacteriophage investigate principally the excreta of horses and chickens, particularly at the beginning of their work. It is from these animals that is most easy to isolate strains of the bacteriophage having a high activity when taken from the body. These excreta are, moreover, more readily procured than the feces of convalescents. 
of barbone. Eight other tests have been made; five were comparable to those cited. In three others a bacteriophage was not found; if it was present it was inactive for the eight test organisms.

Nos. 16 and 17. Swine in Cochin-China, in a barbone area.

Nos. 18 and 19. Swine in Paris.

Nos. 20 and 21. Swine in France, on a farm infected with avian typhosis ( 4 other comparable results).

TABLE 58

\begin{tabular}{|c|c|c|c|c|c|c|c|c|c|}
\hline \multirow{2}{*}{ NUMBER } & \multirow{2}{*}{ B. COLI } & \multicolumn{3}{|c|}{ B. DYSENTERTAE } & \multicolumn{3}{|c|}{ BACILlús } & \multirow{2}{*}{$\begin{array}{c}\text { BAC- } \\
\text { TERIUAI } \\
\text { OF } \\
\text { BARBONE }\end{array}$} & \multirow{2}{*}{$\begin{array}{c}\text { B. } \\
\text { GALLI- } \\
\text { NAREM }\end{array}$} \\
\hline & & Shiga & Flexner & Hiss & $\begin{array}{c}\text { Typho- } \\
\text { sus }\end{array}$ & Para A & Para B & & \\
\hline 1 & + & $+t$ & 0 & 0 & 0 & 0 & 0 & - & - \\
\hline 2 & 0 & + & ++ & + & 0 & 0 & 0 & - & - \\
\hline 3 & + & 0 & + & + & 0 & 0 & 0 & - & - \\
\hline 4 & 0 & 0 & 0 & $+t+$ & 0 & 0 & 0 & - & + \\
\hline 5 & ++ & ++ & + & + & 0 & 0 & 0 & - & $+t$ \\
\hline 6 & + & $+t$ & ++ & 0 & 0 & 0 & 0 & - & 0 \\
\hline 7 & 0 & $+t$ & 0 & 0 & 0 & 0 & 0 & - & 0 \\
\hline 8 & ++ & +++ & ++ & + & 0 & 0 & $+t$ & - & 0 \\
\hline 9 & + & 0 & + & 0 & 0 & 0 & 0 & - & 0 \\
\hline 10 & + & $+++t$ & ++ & 0 & 0 & 0 & 0 & 0 & - \\
\hline 11 & ++ & 0 & $+t+$ & 0 & 0 & 0 & 0 & 0 & - \\
\hline 12 & $+t+$ & + & 0 & 0 & 0 & 0 & 0 & $+t$ & - \\
\hline 13 & $+t+$ & ++ & $+t$ & 0 & 0 & 0 & 0 & $+t+$ & - \\
\hline 14 & ++ & $+t+$ & ++ & 0 & 0 & 0 & + & 0 & - \\
\hline 15 & $++t$ & + & + & 0 & 0 & 0 & 0 & 0 & - \\
\hline 16 & + & +++ & 0 & + & 0 & 0 & + & + & 0 \\
\hline 17 & ++ & + & 0 & 0 & 0 & 0 & 0 & + & 0 \\
\hline 18 & + & 0 & 0 & 0 & + & 0 & $t+$ & - & 0 \\
\hline 19 & + & ++ & 0 & + & 0 & 0 & 0 & - & 0 \\
\hline 20 & ++ & $+t$ & $+t+$ & $+t+$ & 0 & 0 & $+t+$ & - & ++ \\
\hline 21 & +++ & + & 0 & + & + & + & ++ & - & + \\
\hline 22 & + & ++ & + & $+t+$ & 0 & 0 & 0 & - & - \\
\hline 23 & 0 & + & 0 & 0 & 0 & 0 & 0 & - & - \\
\hline 24 & ++ & 0 & + & 0 & 0 & 0 & 0 & - & - \\
\hline 25 & ++ & 0 & 0 & 0 & 0 & 0 & 0 & - & - \\
\hline
\end{tabular}

Nos. 22 and 23. Rabbits living in cages at the Pasteur Institute (4 other comparable findings).

Nos. 24 and 25. Goats in Paris. ${ }^{316,321}$

7. THE VIRULENCE OF THE BACTERIOPHAGE IN THE NORMAL ANIMAL

I have attempted to discover whether the activity manifested for different bacteria by a filtrate of the excreta of an animal is due to the 
presence in this filtrate of a single race of the bacteriophage or to the presence of several different races.

All of the experiments made to this end have been conducted in the same manner. First, the activity of the filtrate on different bacterial species was determined, and then a series of 10 to 12 passages with the bacterium for which the activity proved to be the strongest was made. This procedure for increasing the virulence was followed by spreading the bacterium-bacteriophage mixture on agar. From these cultures three plaques were selected and inoculated into three bacterial suspensions, always of the same bacterium at the expense of which the 10 or 12 passages had been made. Finally, selective isolations in bouillon, by the inoculation of single corpuscles were made.

Being thus certain that pure races had been obtained, I ascertained the different virulences of each of the three plaques.

Naturally, for these tests I selected filtrates active for different species of bacteria. The results follow.

\section{Bacteriophage from the horse (Phantiet)}

This filtrate had been held in a sealed ampoule for two years. When tested for its virulences it showed:
B. coli $^{*}++$
B. dysenteriae Shiga +++
B. dysenteriae Flexner +++
B. dysenteriae Hiss ++
B. paratyphosus $B+$

After the virulence had been increased by means of passages with B. dysenteriae Shiga, and the purification procedure had been effected, determinations made with the three plaques isolated gave:

\begin{tabular}{lccc} 
& \multicolumn{3}{c}{ Plaques } \\
B. coli & 1 & 2 & 3 \\
B. dysenteriae Shiga & ++++ & ++++ & ++++ \\
B. dysenteriae Flexner & ++++ & ++++ & ++++ \\
B. dysenteriae Hiss & ++ & + & +++ \\
B. paratyphosus B & ++ & ++ & +
\end{tabular}

From this we can only conclude that the activity of the original filtrate was due to the multiple virulences of but a single race of the bacteriophage.

* The strain of $B$. coli used in this study, and in those which follow was a very susceptible old stock strain. 
2. Bacteriophage from the horse (Alexandria)

The original filtrate showed virulences as follows:
B. dysenteriae Shiga
$+++$
B. dysenteriae Flexner
$++t$
$B$. dysenteriae Hiss
0
B. paratyphosus $B$
$+++$
B. of Gaertner
$++$

After a series of passages with $B$. dysenteriae Flexner, followed by purification, the virulences of the three plaques isolated were:

\section{Plaques}

1

B. dysenteriae Shiga

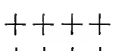

B. dysenteriae Flexner $+++$

B. dysenteriae Hiss

0

2

3

$B$. paratyphosus $B$

$+++$

$+++$

B. of Gaertner

B. $\operatorname{coli}$

$+$

$+$

$+++$

$+++$

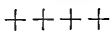

0

0

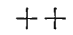

$++$

$++$

$+$

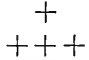

Here again, the activity of the original filtrate was due to the multiple virulences of a single race of bacteriophage protobes.

\section{Bacteriophage from a chicken (Alexandria)}

The intestinal bacteriophage of the original filtrate showed the following virulences:

B. dysenteriae Shiga ++++

B. dysenteriae Flexner +++

B. dysenteriae Hiss ++++

B. paratyphosus $\mathrm{B}++++$

B. coli ++

With this bacteriophage passages were made with $B$. paratyphosus $B$, and following the purification, the virulences of the three plaques proved to be:
B. dysenteriae Shiga
1
B. dysenteriae Flexner
$+$
0
B. dysenteriae Hiss
$+++$
$B$. paratyphosus $B$
B. coli$$
+++
$$
0
Plaques
2
0
0
$++$
$+++$
0

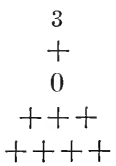
0

In this case there seem to have been two races in the original filtrate. In order to be certain of this I returned to the original filtrate, which 
had been preserved in a sealed ampoule during the period of the above manipulation, and I made a series of 12 passages at the expense of B. dysenteriae Shiga. Three plaques were selected and the race was purified by isolations from the limiting dilution. Virulence determinations on these three plaques showed:

\section{Plaques}

1

B. dysenteriae Shiga

B. dysenteriae Flexner

B. dysenteriae Hiss

B. paratyphosus $B$

B. coli

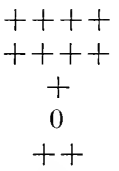

2

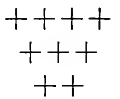

0

$+$
3

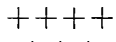

$++$

$+$

0

$+$

This proves that there were, indeed, two races of the bacteriophage, the one active for $B$. dysenteriae Shiga, the Hiss strain, and $B$. paratyphosus $B$, the other virulent for the three dysentery strains and for B. coli.

\section{Bacteriophage from a dog (Alexandria)}

The original filtrate showed virulences for:

B. dysenteriae Shiga +++

B. dysenteriae Flexner ++

B. dysenteriae Hiss 0

B. paratyphosus $B \quad 0$

B. suipestifer ++++

B. coli +++

Passages were made with $B$. suipestifer; purifications were made, and the three plaques isolated showed virulences as follows:

\begin{tabular}{lccc} 
& \multicolumn{3}{c}{ Plaques } \\
B. dysenteriae Shiga & 1 & 2 & 3 \\
B. dysenteriae Flexner & + & + & + \\
B. dysenteriae Hiss & 0 & 0 & 0 \\
B. paratyphosus B & 0 & 0 & 0 \\
B. suipestifer & ++++ & +++ & ++++ \\
B. coli & ++ & ++ & +
\end{tabular}

Obviously, this filtrate contained but a single race.

It may be concluded from these experiments that as a rule but a single race of the bacteriophage can be found within the digestive tract of an animal at a given time. This is the more strange in view of the 
fact that animals are unquestionably ingesting all of the time a wide variety of races. The most logical explanation for this apparently illogical situation is that most probably only that race which forms a symbiosis with the intestinal $B$. coli persists.

\section{THE BACTERIOPHAGE IN THE EXTERNAL ENVIRONMENT}

From the data which have been presented in previous chapters it is obvious that the bacteriophage protobe possesses a high degree of vitality. Its resistance to the different destructive agencies to which it is exposed in nature, - a resistance almost equal to that of the bacterial spores,-permits it to survive the majority of influences to which natural conditions subject it. Because of its minute size it is certainly carried by seepage water through soils which are impermeable to bacteria. It should, because of these facts be found in all materials which, at any time, have been exposed to pollution by excreta. And this is, in fact, what the studies of many investigators show to be the case.

Without discussing the presence of the bacteriophage in food products, and it has been found there (sausage; van der Hoeden ${ }^{364}$ ) because of some accidental contamination occurring in the abattoir or in the manipulation of the product; without considering its presence, a perfectly natural thing, in products derived from the intestinal mucosa, such as enterokinase (Pico, ${ }^{510}$ Kuttner, ${ }^{399}$ Borchardt ${ }^{75}$ ), ${ }^{*}$ it is of significance to know that the bacteriophage may be found in the external world, particularly in drinking water.

* The presence of the bacteriophage being constant in the intestine as is readily demonstrated, particularly in animals because of the multiple virulences which it shows, it is perfectly natural to find it in products derived from the intestine. The three authors mentioned have, however, constructed theories as to the nature of the bacteriophage on the basis of their findings. According to their method of reasoning, inasmuch as the bacteriophage may be found in products derived from the intestine it must be an intestinal enzyme. As it is easy to demonstrate that $B$. coli is present in commercial preparations of enterokinase, and even of pancreatin, one might just as logically conclude that $B$. coli is derived from the intestinal enzymes. As a matter of fact, Combiesco ${ }^{148}$ has shown that when the bacteriophage is present in preparations of trypsin and of enterokinase, it represents an impurity, for it resists a temperature which destroys the enzyme. Following out the same idea, I have purified contaminated samples of enterokinase by treating them with anhydrous glycerol or with alcohol. The bacteriophage is destroyed by either the glycerol or alcohol, while the enzymes are left unmodified, at least for a period of time adequate for the destruction of the bacteriophage. $\mathrm{Flu}^{213}$ has shown that the pancreatic juice of the cat, if collected without admixture with other substances, never contains a bacteriophage. 
Dumas $^{188}$ was the first to find a bacteriophage, virulent for either B. coli or the Shiga organism, in the drinking water of Paris. He also found it in garden soil, which was not peculiar since such soil is heavily charged with manure.

Beckerich and Hauduroy ${ }^{58}$ isolated races of the bacteriophage from the water of the river Ill, as well as from that of the Rhine, which, after filtration through sand, serves as drinking water for Strasbourg.

I have isolated a bacteriophage virulent for $B$. coli from a specimen of sea water collected in the estuary of Mekong; a race virulent for $B$. dysenteriae from the water off Alexandria; and a similar race from a specimen of water collected outside of Marseilles. On the contrary, I failed to find it in a specimen of water collected from the Indian Ocean, at approximately $60^{\circ}$ longitude, and $10^{\circ}$ north latitude.

Various authors, Arloing, Sempé and Chavanne, among others, report having found virulent races in the water of different rivers and wells. These races have shown virulences for such organisms as $B$. typhosus, B. coli, B. dysenteriae, and the paratyphoids.

\section{RÉSUMÉ}

Within the intestinal tract of every man, and of every animal, there is a race of the bacteriophage living in symbiosis with $B$. coli and the other bacteria of the normal flora (d'Herelle ${ }^{316}$ ).

The bacteriophage is not present in the intestinal tract of the fetus, nor is it found in the new-born infant (Védrenne ${ }^{610}$ ). In the infant it appears, as a rule, some time between the 4 th and 7 th days after birth (d'Herelle).

In the normal man the intestinal bacteriophage usually shows a virulence only for the strain of $B$. coli with which it is maintaining a symbiotic relationship. But to this there are many exceptions, cases where the virulence extends to different bacterial species (d'Herelle ${ }^{316}$ ).

In the normal animal the intestinal bacteriophage can be demonstrated somewhat more readily than in man. This is due to the fact that in the animal the virulences of the race present almost always extend to the dysentery and paratyphoid bacilli (d'Herelle).

As a rule, the intestinal tract of man, and of animals, contains but a single race of the bacteriophage (d'Herelle).

Everything which, under natural conditions, has once been exposed to pollution by excreta, may contain bacteriophage protobes. It is always present in the water of some rivers $\left(\right.$ Dumas $\left.^{188}\right)$; in cultivated soils (Dumas ${ }^{188}$ ), and often in sea water near the coast (d'Herelle ${ }^{321}$ ). 


\section{CHAPTER III}

\section{The Behavior of the Bacteriophage in Disease}

\section{VARIATIONS IN THE VIRULENCE OF THE BACTERIOPHAGE}

There is, then, in the intestinal tract of each man, indeed, in that of every animal, vertebrate or invertebrate, a race of the bacteriophage. Unquestionably we can consider this point as established. Experiments in vitro have shown us that this bacteriophage has the power to adapt itself to the parasitism of different species of bacteria. Under certain circumstances it can probably adapt itself to any and all species. These two facts being true, the logical question is: What happens when a pathogenic bacterium gains access to the digestive tract of a being which already has within its intestine a bacteriophage protobe to parasitize this bacterium?

We know from common experience that a normal man is sometimes attacked by slight transitory intestinal disturbances; upsets which quickly pass off, without the person becoming really sick. Let us first see what happens within the intestinal tract in cases of this type.

The following observations were made on the same normal man who was examined periodically throughout a year, providing the data reported in the preceding chapter on the uniform presence of a race of the bacteriophage in the intestinal tract.

During the course of this same year, this individual showed at two different times, July 3 , and September 26 , slight intestinal disturbances lasting some hours. The first time there was no obvious cause; the second attack followed a suspected meal taken in a village tavern. On each occasion specimens of the stools were examined on the following days. The results are recorded in table 59 .

The results are here so clear-cut that no comment is necessary.

Additional information is provided by the results of the stool examinations of three adult persons, a mother and two daughters, who lived together. Within a period of 15 days, during which time daily examinations were being made, one of them, I, without being otherwise disturbed, had a few stools of somewhat less than a normal consistency. These occurred on the 17 th and 18 th of July.*

* A few cases of dysentery had occurred during the preceding days in the village where the persons lived. This was, as a matter of fact, the reason for undertaking the systematic examinations. 
The results of these examinations appear in table 60 , the designations, I, II, and III, referring to the three persons mentioned above.

The marked change in the virulences of the intestinal bacteriophage,

\begin{tabular}{|c|c|c|c|c|c|c|c|}
\hline \multicolumn{8}{|c|}{3 LE 59} \\
\hline \multirow{2}{*}{ DATE } & \multirow{2}{*}{ B. COLI } & \multicolumn{3}{|c|}{ B. DYSENTERIAE } & \multicolumn{3}{|c|}{ BACILLUS } \\
\hline & & Shiga & $\begin{array}{c}\text { Flex- } \\
\text { ner }\end{array}$ & Hiss & $\underset{\text { sus }}{\text { Typho- }}$ & Para A & Para B \\
\hline July $4 \ldots$ & +++ & 0 & $+t+$ & ++ & 0 & 0 & 0 \\
\hline July 5. & ++ & + & +++ & + & 0 & 0 & 0 \\
\hline July $6 \ldots$ & +++ & + & $+t$ & + & 0 & 0 & 0 \\
\hline July $7 \ldots$ & ++ & 0 & $+t$ & 0 & 0 & 0 & 0 \\
\hline July $8 \ldots \ldots$ & + & 0 & 0 & 0 & 0 & 0 & 0 \\
\hline September 27 . & +++ & ++ & + & + & + & 0 & $+t+t$ \\
\hline September 28 . & +++ & + & 0 & 0 & 0 & 0 & $+++t$ \\
\hline September 29. & +++ & 0 & 0 & 0 & 0 & 0 & ++ \\
\hline September $30 \ldots$ & ++ & 0 & 0 & 0 & 0 & 0 & $+t+$ \\
\hline October $1 \ldots$. & + & 0 & 0 & 0 & 0 & 0 & $+t+$ \\
\hline October $2 \ldots$. & + & 0 & 0 & 0 & 0 & 0 & + \\
\hline October $3 \ldots$ & + & 0 & 0 & 0 & 0 & 0 & 0 \\
\hline
\end{tabular}

TABLE 60

\begin{tabular}{|c|c|c|c|}
\hline DATE & I & I! & III \\
\hline July $15 \ldots \ldots \ldots \ldots \ldots$ & $\mathrm{C}+$ & $\mathrm{C}+++$ & 0 \\
\hline July $16 \ldots \ldots \ldots \ldots \ldots$ & $\mathrm{C}+$ & $\mathrm{C}+++$ & $\mathrm{C}+++$ \\
\hline July $17 \ldots \ldots \ldots$ & 0 & $\mathrm{C}+$ & 0 \\
\hline July $18 \ldots \ldots \ldots \ldots \ldots$ & $\mathrm{C}+++\mathrm{H}+++$ & 0 & 0 \\
\hline July $19 \ldots \ldots \ldots \ldots \ldots$ & $\mathrm{C}+++\mathrm{H}++$ & 0 & $\mathrm{C}++\mathrm{B}+$ \\
\hline July $20 \ldots \ldots \ldots \ldots$ & $\mathrm{C}++\mathrm{H}+++$ & 0 & $\mathrm{C}+\mathrm{B}+$ \\
\hline July $21 \ldots \ldots \ldots \ldots \ldots$ & $\mathrm{C}++\mathrm{H}+$ & $\mathrm{C}+$ & 0 \\
\hline July $22 \ldots \ldots \ldots \ldots$ & 0 & 0 & 0 \\
\hline July $23 \ldots \ldots \ldots \ldots$ & 0 & 0 & $\mathrm{C}+$ \\
\hline July $24 \ldots \ldots \ldots$ & $\mathrm{C}+++$ & 0 & 0 \\
\hline July $25 \ldots \ldots \ldots \ldots \ldots$ & $\mathrm{C}++$ & 0 & 0 \\
\hline July $26 \ldots \ldots \ldots \ldots$ & 0 & 0 & 0 \\
\hline July $27 \ldots \ldots \ldots \ldots$ & $\mathrm{C}++\mathrm{Sh} .+++$ & $\mathrm{C}++$ & $\mathrm{C}+++$ \\
\hline July $28 \ldots \ldots \ldots \ldots \ldots$ & $\mathrm{C}+\mathrm{Sh} .+$ & 0 & 0 \\
\hline July $29 \ldots \ldots \ldots$ & 0 & $\mathrm{C}+$ & 0 \\
\hline July $30 \ldots \ldots \ldots \ldots \ldots$ & $\mathrm{C}+$ & $\mathrm{C}+$ & 0 \\
\hline
\end{tabular}

$\mathbf{C}=B$. coli: $\mathrm{H}=B$. dysenteriae Hiss; Sh. = B. dysenteriae Shiga; $\mathrm{B}=B$. paratyphosus $\mathrm{B}$.

as observed here, can not fail of being significant, for we find here, that upon the occurrence of the slightest intestinal disturbance the virulence 
of the bacteriophage protobe is increased, both for $B$. coli and for the pathogenic organism.

These findings immediately suggest the idea that there may have been in these three cases the beginning of an infection by these pathogenic bacteria for which the virulence of the bacteriophage became manifest, but that the infections were aborted because of the rapid adaptation on the part of the intestinal bacteriophage, thus resulting in a bacteriophagy in vivo (d'Herelle ${ }^{316,321}$ ).

Study of the behavior of the intestinal bacteriophage in outspoken disease shows us that this hypothesis is correct.

\section{THE BACTERIOPHAGE IN BACILLARY DYSENTERY}

The charts presented in this section show, much better than any explanation, the relations which exist between the condition of the patient and the virulence of the intestinal bacteriophage for the pathogenic bacterium. The upper tracing gives the number of stools in 24 hours; the single line indicating stools without blood, the double line those containing blood and mucus. On the lower portion of the chart is indicated (1) by the dotted line, the virulence of the bacteriophage for the colon bacillus; (2) by the broken line, the virulence of the bacteriophage for a stock strain of the Shiga bacillus which had been maintained for a long time under laboratory cultivation; and (3) by the solid line, the virulence for the Shiga strain recovered from the patient himself.

The five cases given as examples were treated at the Pasteur Hospital. It was thus possible to follow them with all necessary attention and to obtain material for examination as often as the investigation demanded; at least once, often several times, during the course of each day.

For these examples, cases of different severity have been selected. In all of them $B$. dysenteriae Shiga was isolated from the stools at the beginning of the disease.

Case 1. Germaine Mel. . . . . (sixteen years, chart 1). This was a mild case of dysentery. The patient was an inmate in an institution where there were about thirty young girls. During the period from the 12 th to the 22 nd of July about twenty of these girls presented intestinal disturbances of sudden onset, accompanied by a profuse diarrhea, followed by a rapid amelioration of symptoms. Within one or two days after the onset all had again become normal. In only one or two cases did the stools contain traces of blood. In order to establish a diagnosis the directrix was asked to send a patient to the Hospital during the earliest symptoms. 
Germaine Mel. . . . . entered the Hospital on the 18th of July. From the first stool passed after her arrival a bacillus presenting the biochemical characteristics of the Shiga bacillus was isolated after considerable difficulty. It was inagglutinable, and it was only after three transfers on agar that agglutination was secured $(1: 500)$.

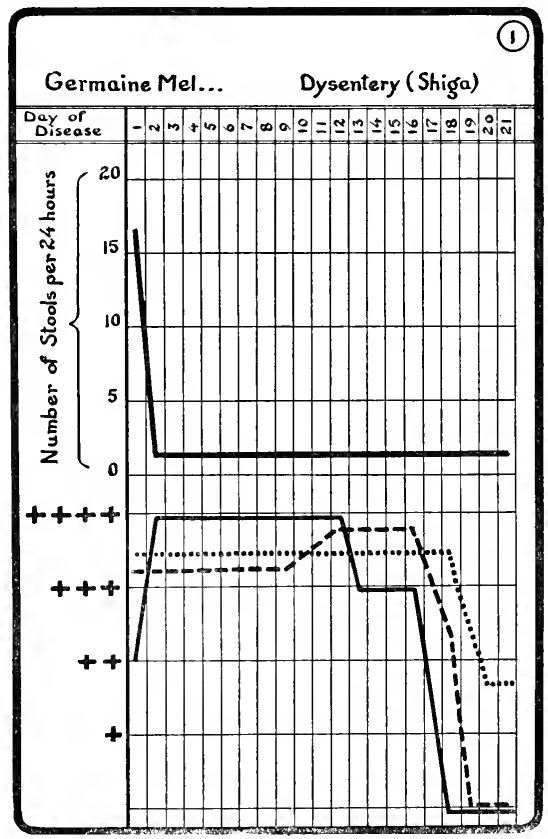

Chart. 1. Germaine Mel. . . . . Dysentery (Shiga)
Virulence for $\left\{\begin{array}{l}\text { B. dysenteriae from the patient- } \\ \text { B. dysenteriae, stock strain----... } \\ \text { B. coli....... }\end{array}\right.$

As can be seen from the tracings, the number of fluid stools, seventeen on the first day, fell quickly during the second day to two, without medication.

The intestinal bacteriophage, isolated from the fifth stool of the first day, was endowed with an extreme virulence for the bacillus causing 
the infection, and with a somewhat lower grade of virulence for the stock Shiga strain and for B. coli.

The stools of eleven of the inmates of this institution were examined. Among the number were nine who had shown intestinal disturbances

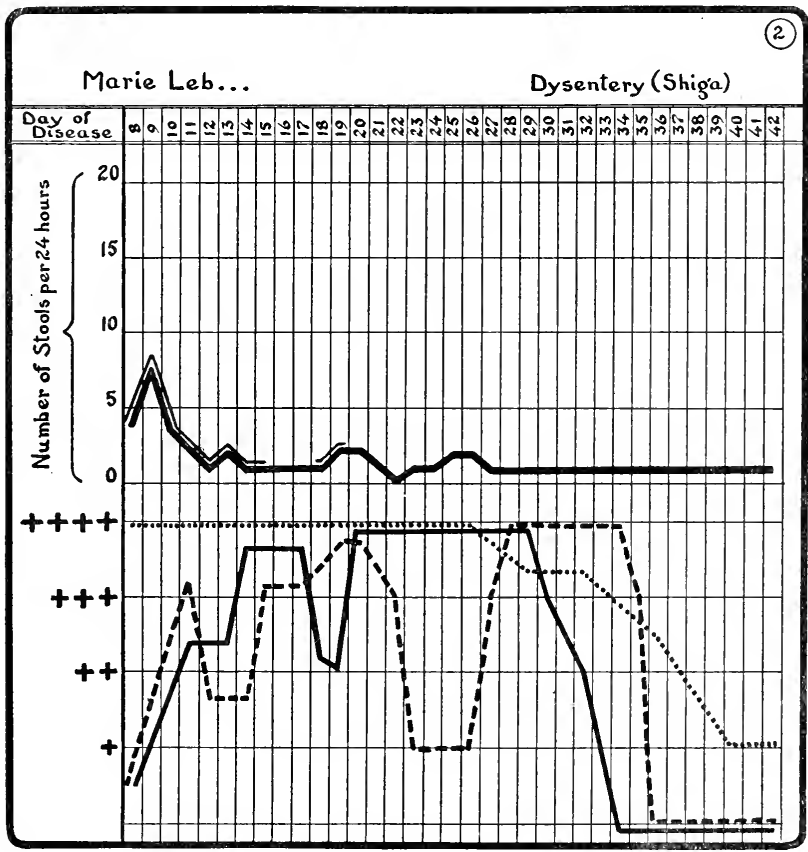

Chart 2. Marie Leb. . . . . (26 years) Dysentery (Shiga)

Virulence for $\left\{\begin{array}{l}\text { B. dysenteriae from the patient-- } \\ \text { B. dysenteriae, stock strain------- } \\ \text { B. coli....... }\end{array}\right.$

Stools contained blood $=$

uwo or three days previously. Two had shown no morbid symptoms. All of those examined contained a bacteriophage with a high or maximum virulence for the Shiga strain isolated from the stool of Germaine Mel. . . . . as well as for the stock strain of Shiga and for B. coli. 
Therefore, with Germaine Mel. . . . . there was a bacteriophage of maximum activity, even from the beginning of the disease. Recovery took place within twenty-four hours.

Case 2. Marie Leb. . . . . (twenty-six years, chart 2). This

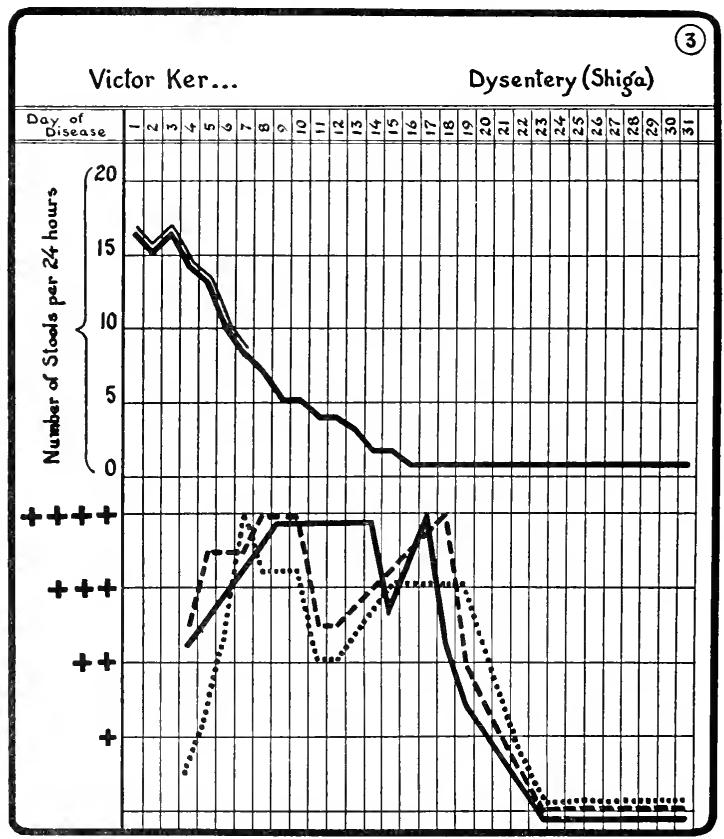

Chart 3. Victor Ker. . . . . (6 years) Dysentery (Shiga)
Virulence for
B. dysenteriae from the patient-
$B$. dysenteriae, stock strain-
B. $\operatorname{coli} \ldots . . .$. .
Stools contained blood=

case was one with a mild dysentery, due to B. dysenteriae Shiga. The stools were typical, containing blood and mucus. She was admitted to the Hospital on the eighth day of the disease. The first stool containing blood had been passed the day before.

Upon entrance to the Hospital the feces contained a bacteriophage 
active for the Shiga organism ( + ), extremely active for $B$. coli $(++++)$, and but very slightly active for the dysentery bacillus found in the patient $(+)$. Against this last bacillus the virulence increased during the course of the three following days, reached its maxi-

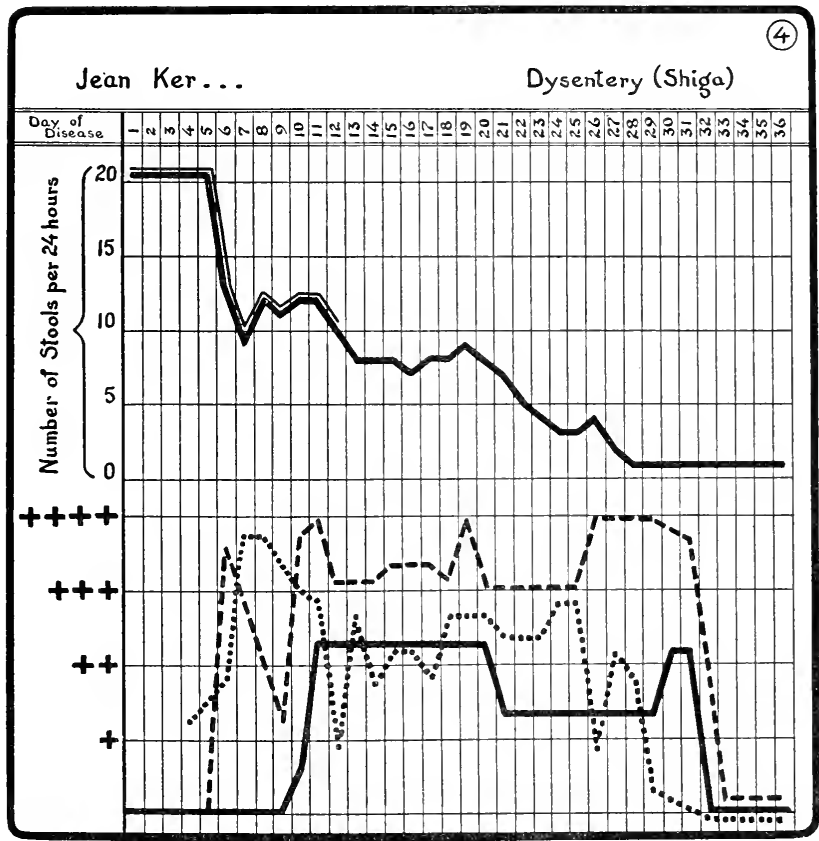

Chart 4. Jean Ker. . . . . (6 years) Dysentery (Shiga)
Virulence for $\left\{\begin{array}{l}B . \text { dysenteriae from the patient-- } \\ B . \text { dysenteriae, stock strain --.-- }\end{array}\right.$
B. $\operatorname{coli} \ldots . .$. ...
Stools contained blood

mum activity $(++++)$, fell away somewhat $(++)$, and then definitely regained its full virulence $(++++)$. These fluctuations in virulence were reflected in the condition of the patient. At the end of convalescence there remained only a slight activity $(+)$ of the bacteriophage and that was directed against B. coli. 
Case 3. Victor Ker. . . . . (five years, chart 3). The dysentery was caused by the Shiga bacillus, was of moderate severity, and was contracted by contact with the patient next discussed. When admitted to the Hospital, on the third day of the disease, the intestinal bacteriophage already manifested an average virulence $(++)$ for the stock Shiga strain as well as for the strain isolated from the patient. This virulence increased rapidly and maintained a high value up to the time of complete convalescence $(+++)$. It then abruptly disappeared.

Case 4. Jean Ker. . . . . (six years, chart 4). This patient was a brother of the foregoing. The general condition was poor when admitted to the Hospital on the third day of the disease. There were from twenty to thirty bloody stools a day; a severe dysentery due to the

TABLE 61

\begin{tabular}{|c|c|c|c|c|}
\hline \multirow[b]{2}{*}{ DAY OF DISEASE } & \multirow[b]{2}{*}{ NUMBER OF BLOODY STOOLS } & \multicolumn{3}{|c|}{ VIRCLENCE OF THE BACTERIOPHAGE FOR } \\
\hline & & $\begin{array}{l}\text { B. dysenteriae } \\
\text { (patient) }\end{array}$ & $\begin{array}{l}\text { B. dysenteriae } \\
\text { (stock) }\end{array}$ & B. coli \\
\hline 5 th & 23 & 0 & + & + \\
\hline 6 th & 13 & 0 & ++++ & ++ \\
\hline 7 th & 9 & 0 & +++ & $+++t$ \\
\hline Sth & 12 & 0 & ++ & $++t+$ \\
\hline 9 th & 11 & 0 & + & ++++ \\
\hline 10 th & 12 & + & ++++ & +++ \\
\hline 11th & 12 & $+t+$ & $+++t$ & $++t$ \\
\hline 12 th & $\begin{array}{c}\text { (4 of } 6 \text { stools without } \\
\text { blood) }\end{array}$ & +++ & ++++ & + \\
\hline
\end{tabular}

Shiga bacillus. On the fourth day of the disease there were twentyfour bloody stools. The bacteriophage was feebly active (+) for $B$. coli and was inactive for the Shiga bacillus. The record is shown in table 61 .

From this time on improvement became more and more marked. The activity of the bacteriophage did not disappear after convalescence had been established.

In the first three of the cases reported above the dysentery was mild. The bacteriophage was active at the onset, the bacterium did not acquire a resistance, and its growth was quickly suppressed. In the last case there was a struggle and the bacillus acquired a resistance which was finally overcome. The condition of this patient was much more serious.

Case 5. Lans. . . . . (seventy years, chart 5). This case illustrates an extremely severe dysentery due to the Shiga bacillus. The patient entered the Hospital on the second day of the disease. 


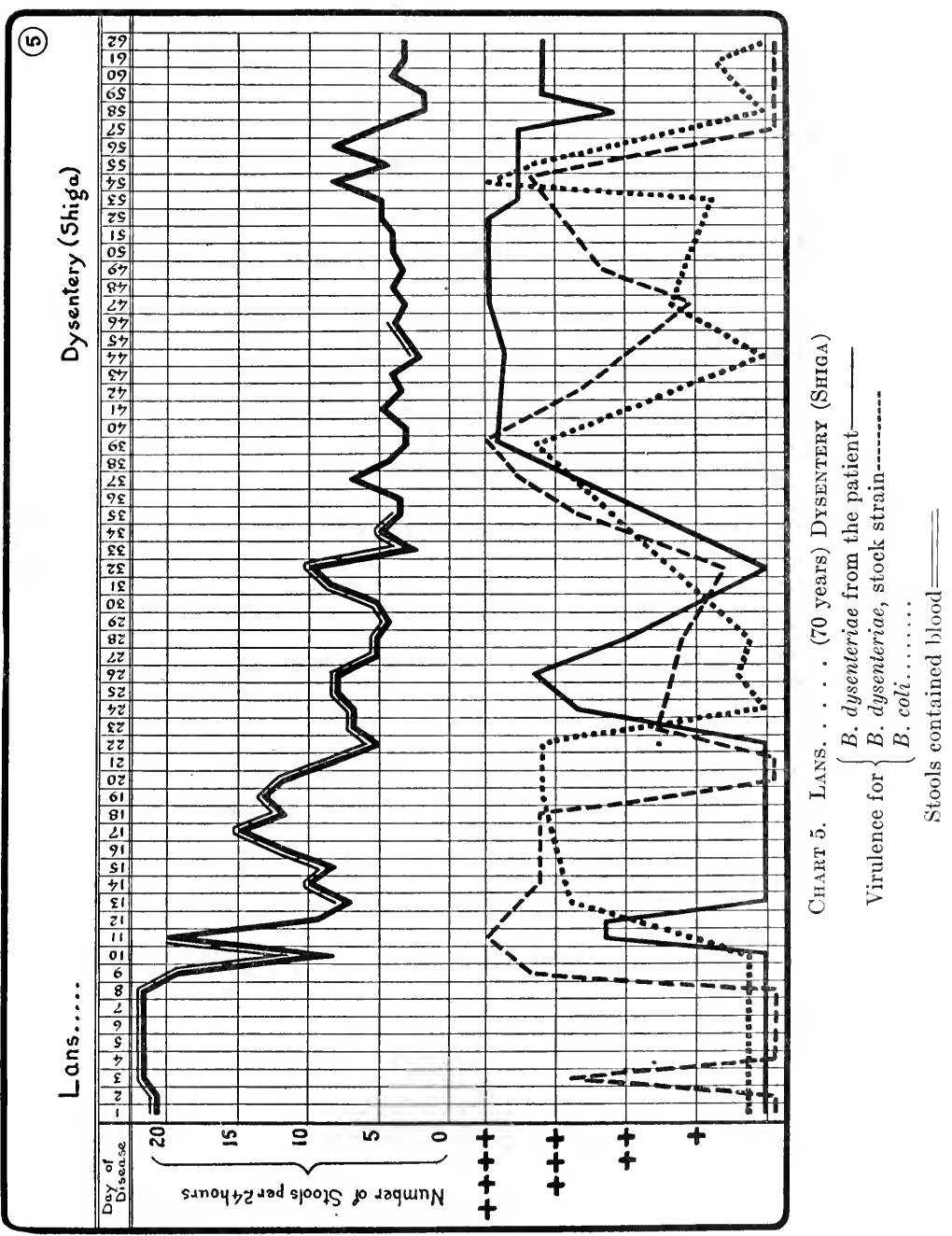


In this case the struggle was prolonged, with fluctuations due to the mixed cultures formed in the intestine. The condition of the patient registered faithfully the changes in the struggle. It may be noted particularly that the bacteriophage manifests a transitory activity on the eleventh day of the disease and the stools temporarily lose their bloody character. But the bacillus increases its resistance and this permits it to develop, and blood reappears in the stools. The disease is only definitely overcome at a time when the virulence of the bacteriophage is sufficiently high to dominate the resistance of the bacterium.

In addition to the five cases which have just been presented as illustrating the manner in which the bacteriophage behaves, a number of other cases have been followed, both in France and in Indo-China. Of these other cases, 17 which varied materially in the severity of the infection, were examined daily throughout the period of the disease, and 29 more were examined less frequently.

In all of the cases the intestinal bacteriophage showed changes in its virulence comparable to those recorded above. These changes may be summarized as follows:

1. In case of recovery, the virulence of the bacteriophage commences to manifest itself in a marked manner toward B. coli.

2. The virulence next extends to the type strain of the Shiga bacillus, that is to say, toward a strain which has been for a long time under artificial cultivation and which, for this reason, has been deprived of much of its resistance.

3. It manifests itself next, more or less quickly, toward the Shiga bacillus isolated from the patient himself at the onset of the disease.*

4. In all cases the fluctuations in the virulence, as well as the fluctuations in the resistance of the bacteria, parallel the state of the patient, and the onset of improvement coincides with the moment when the virulence of the bacteriophage dominates clearly the resistance of the bacterium. We thus see reproduced in vivo the same phenomena as we have observed in vitro; permanent and complete dissolution, mixed cultures with negative transfers, mixed cultures with alternations in the dominating force.

In Indo-China an opportunity was afforded to follow four fatal

* Obviously it is necessary to preserve this strain without replanting. The isolated colonies obtained on the original plates are planted on several agar tubes and a portion is taken from these tubes for the tests conducted during the course of the disease. It is well-known that resistance is attenuated by successive transplantations. 
cases of bacillary dysentery in natives. At no time during the course of the infection did the intestinal bacteriophage show a trace of activity for the Shiga bacillus, either for the stock strain or for those isolated from the stools of the patients.

A last case, of particular interest, may be presented, for it shows that bacteria may become resistant, acquiring a refractory state, in vivo, as well as in vitro. While undoubtedly such a change in the bacterium may occur within the body, such cases must be very rare, even exceptional.

Alix Desp . . . . (fifty-six years). The patient entered the Pasteur Hospital on September 26, 1919. At the time of admission there was a profuse mucous diarrhea with thirty to forty stools a day. Examination of the intestinal contents gave an almost pure culture of a dysentery bacillus presenting atypical characters, as follows:

Non-motile bacillus. Gram negative. Indol positive. No blackening of lead acetate agar. No change in neutral red media. Litmus sugar agar media not fermented with any of the sugars. In Barsiekow's medium, maltose and lactose are unchanged, glucose and mannite are turned red. After six transfers on agar it agglutinated to the titre (1:6000) with a Hiss agglutinating serum, to $1: 400$ with an anti-Flexner serum of which the titre was 1:6000, and was not agglutinated at $1: 20$ by an anti-Shiga serum.

In spite of these atypical characters it was, then, a Hiss strain possessing weak fermentative properties.

When secured from the body this bacillus was not affected by a bacteriophage very virulent for a normal Hiss bacillus, but it became susceptible to dissolution after about a dozen transplantations. It was, then, a bacillus which was refractory to the bacteriophage when recovered from the body.

At the same time a strain of bacteriophage was isolated from the stools of the patient. This presented the following virulences: Shiga 0 , Flexner ++ ; stock culture strain of Hiss ++++ ; B. coli + ; the Hiss strain from the patient + . After twelve subcultures of the Hiss strain from the patient the virulence of the filtrate was again tested. Perfect dissolution was secured, showing that the bacillus had lost its resistance by transfers on agar.

The bacteriophage of the patient was active to a maximum degree against a stock strain of the Hiss bacillus but it was only slightly active for the individual strain causing the infection, with which it formed in vitro, mixed cultures indefinitely cultivable. There was likewise in 
the intestine of the patient a mixed culture of the bacteriophage and the refractory Hiss strain.

In spite of every care and repeated injections of anti-dysentery serum the patient became weaker and weaker; the temperature oscillated between $38^{\circ}$ in the morning and $40^{\circ} \mathrm{C}$. in the evening; the number of stools gradually increased and became uncountable on about the thirtieth day; and at about this time the patient fell into a marasmic condition, the temperature stayed at about $38^{\circ} \mathrm{C}$. and death occurred on the thirty-fifth day.

Bacteriologically, the stools, tested each day, showed an almost constant bacterial flora. The pathogenic bacillus was always abundant, often in almost pure culture, and presented the characteristics described. The virulence of the bacteriophage increased continuously until the fifteenth day when it became fixed, showing: Shiga ++++ ; Flexner ++++ ; Hiss ++++ , B. typhosus $+++;$ B. paratyphosus $A+++$; $B$. paratyphosus $B+++; B$. coli ++++ ; bacillus of the patient 0 (completely refractory) when freshly isolated, +++ after fifteen transplants.

At autopsy* there was isolated from the contents of the colon, from a fragment of mucous ulceration, from the liver, from the spleen, and from the heart blood, a Hiss dysentery bacillus, presenting the same characteristics as that which had been isolated at the beginning of the disease. From all the organs a bacteriophage was isolated presenting the same characters as that which had been isolated from the stools and whose virulence has been indicated.

This case, altogether exceptional (I believe that it is the first case reported of a $B$. dysenteriae Hiss septicemia) is very interesting for it shows in an unquestioned manner the rôle that the bacteriophage plays in the defense of the organism. In all of the cases examined heretofore we have seen, either recovery starting from the time when the bacteriophage had acquired sufficient virulence to dominate the pathogenic bacillus, or death in the case of the lack of such an adaptation. In this last case, the bacteria developed a refractory condition, the bacteriophage was overcome and remained without action whatever its virulence may have been. The barrier thus being lacking, the bacteria developed freely and invaded the entire organism. The patient succumbed to a septicemia with the Hiss bacillus.

This unusual case provides us with new information. A bac-

* Performed by L. Géry, whom I thank for the specimens he was kind enough to send me. 
terium is pathogenic for a given organism if it secretes substances toxic for the cells of this organism. It is the more virulent the more capable it is of development at the expense of this organism. The dysentery bacilli are pathogens because of this secretion of toxic substances, for they do not invade the organism, as a rule, but remain localized in the intestine and in the intestinal mucosa. Nevertheless, in the case of the woman Desp. . . . . the Hiss strain was accidentally endowed with an extreme virulence, and this solely because the bacteriophage had been overcome. This suggests an idea which we will have occasion to confirm in the following chapters,- that the virulence of a bacterium at any given moment is the greater if its resistance to the bacteriophage is at this time high.

The case Desp. . . . . . is exceptional. As a general rule death occurs in dysentery, not because of the acquisition by the bacterium of a refractory condition, but by a failure of the bacteriophage to adapt itself to bacteriophagy of the pathogenic organism. In the four cases mentioned above which were fatal, a bacteriophage active for the Shiga bacillus could not be isolated at any period of the disease.

We may summarize the situation, insofar as bacillary dysentery is concerned, in the following way. The pathogenesis and the pathology of bacillary dysentery are dominated by two factors, operating in different directions; the dysentery bacillus as the pathogenic agent and the bacteriophage as the agent of immunity. The history of a case of dysentery is only the story of the struggle, occurring within the body, between these two factors, and the condition of the patient faithfully reflects the vicissitudes of the struggle.

In case of a rapid enhancement in the virulence of the intestinal bacteriophage toward a pathogenic bacillus, the latter is unable to develop a resistance and is destroyed in the struggle, so that the disease aborts before the appearance of any symptoms or manifests itself by only a transitory disturbance.

The increase in the virulence of the bacteriophage for the invading bacterium may be retarded for one of two reasons:-First, as a result of unfavorable intestinal conditions. (We have seen the considerable importance, in vitro, of very slight variations in the reaction of the medium on the development of the bacteriophage.) In accordance with the chemical and physical state of the intestinal contents, one bacterium is favored at the expense of another; the intestinal fermentations, and as a result, the reaction of the medium will vary according to the predominating flora. The development of the bacteriophage is 
then doubly influenced. First, by a change in the state of the medium itself, and second, by changes in the flora which increase or decrease, according to circumstances, the bacterial species at the expense of which it normally develops. This of course necessitates variations in virulence in response to the variation in the bacterial species. Moreover, it has been known for a long time that catarrhal diarrhea affects (provoked by the ingestion of undigestible foodstuffs, of green fruits in particular, or by the "froid au ventre" so common in tropical countries) the incidence of certain intestinal diseases-dysentery and cholera among others.

Second, as a result of a more or less marked degree of resistance of the invading bacillus to the bacteriophage. We have seen that in the course of the disease the pathogenic agent defends itself. Such a bacillus in a state of resistance, ingested by a healthy person will develop in spite of the presence of a bacteriophage, particularly if the latter is but slightly active, whereas a non-resistant bacillus is destroyed without any very great difficulty.

In cases of bacillary dysentery, even very severe, but in which the patient improves rapidly, the bacteriophage shows its virulence in a very active manner at the outset, not only for laboratory strains of the bacillus, but for the strain secured from the patient himself, and this takes place at the moment when the symptoms begin to improve. There may be a rapid increase in the virulence of the bacteriophage without a corresponding resistance in the bacterium.

In cases where the disease is prolonged, one of two situations may develop.

1. The bacteriophage shows no, or but slight, activity as long as the condition of the patient remains stationary. The improvement occurs when the activity of the bacteriophage manifests itself in an energetic manner, not only for the stock cultures of the bacillus but also for the strain derived from the patient. There has been a delay in the adaptation, then the sudden acquisition of a high virulence. Recovery takes place promptly, for the pathogenic bacterium is not able to develop a resistance.

2. At a given moment of the disease the virulence of the bacteriophage manifests a more or less energetic action on the stock bacilli, but on the contrary, the virulence is inappreciable or but very weak for the strain taken from the patient. Here there has been a delay in the adaptation, since the bacteriophage has gradually acquired virulence for the pathogenic bacillus, but this delay has allowed sufficient time for the creation 
of a resistant race of the latter. As a result there is a struggle, and the condition of the patient reveals the fluctuations of the struggle.

This conflict is particularly to be noted in cases of long duration with a relapse. During the latter, especially, the virulence of the bacteriophage shows daily fluctuations. At certain times it may be extreme for the stock culture, although uniformly very weak for the strain causing the infection. Recovery begins to take place at the moment when the bacteriophage shows an activity as intense for one strain as for the other.

Under two circumstances the disease may have a fatal issue:

1. When the bacteriophage exerts no protective action through a lack of adaptation to the pathogenic bacillus. Here there is no struggle at all, and the bacterium develops freely. In the great majority of such cases non-adaptation is the cause of death, which then occurs quickly.

2. In certain exceptional cases the pathogenic bacterium acquires an almost absolute resistance,- - a refractory state. And the bacteriophage, whatever the degree of virulence it acquires, remains ineffective. From this moment, when the bacterium becomes equal to the bacteriophage, the entire body is invaded and death ensues after a greater or less length of time.

\section{THE BACTERIOPHAGE IN TYPHOID FEVER}

Several cases of typhoid fever of varied severity have been studied by the same method as that employed in bacillary dysentery. Fourteen of these were in the Pasteur Hospital for treatment, and of these the stools were examined at least once a day throughout the course of the disease and during convalescence. Fourteen more, under treatment in other hospitals, were followed with somewhat fewer examinations. In all of the charts which follow, the following data are presented; in the upper portion is the curve showing the temperature: in the lower portion there are three tracings, (1) in dotted line, showing the curve of the virulence of the bacteriophage for B. coli, (2) in broken line, showing the virulence of the bacteriophage for an old laboratory strain of $B$. typhosus, a strain which has undergone a great many transfers on laboratory media (this same strain was used in all the cases studied), and (3) in solid line, indicating the curve of virulence of the bacteriophage for the strain of $B$. typhosus from the patient himself, isolated either by stool culture or by blood culture.

In order to use bacilli as comparable as possible with those found in the body of the patient the strains were transplanted as infrequently 
as possible. In each case an agar tube was inoculated with a colony taken from the primary culture, and each time that a fresh culture was needed for the preparation of suspensions against which the filtrates containing the bacteriophage from the patient were to be tested, it was

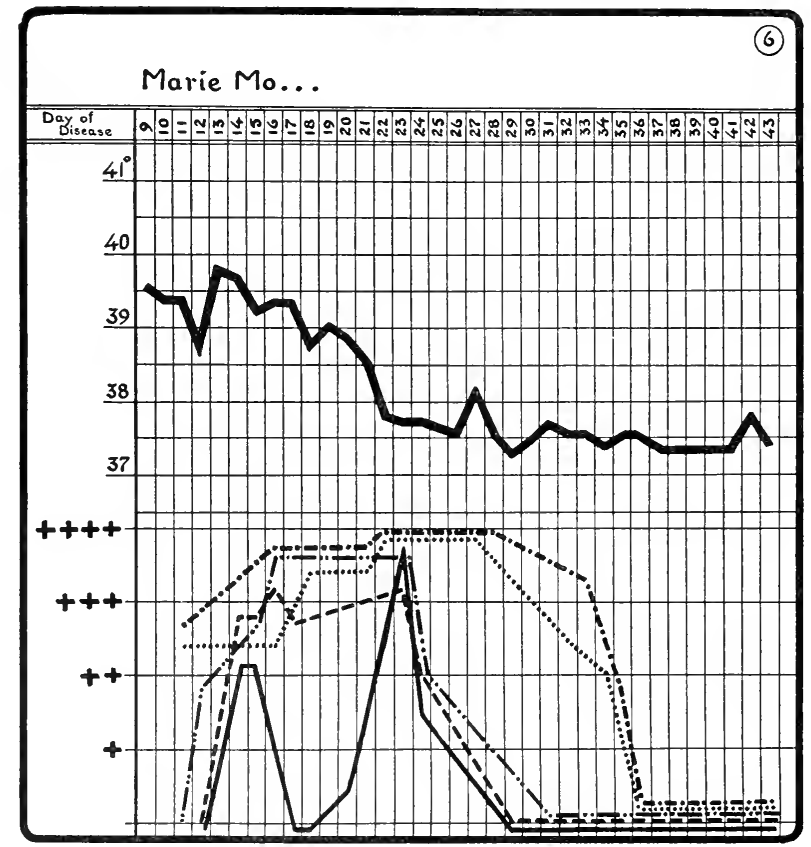

Chart 6. Marie Mo. . . . . (55 years) Clinically, Typhoid Fever

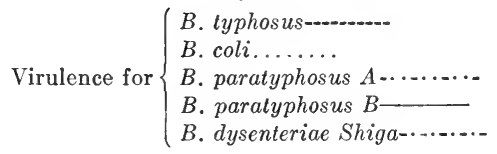

always taken from this tube. In this way, the bacteriophage throughout the course of the disease was tested against a culture as nearly constant as possible, uniform especially from the point of view of the resistance of the bacterium. 
For the first three curves only (charts 6,7 , and 8) the organism of the patients had not been isolated (they had fevers which appeared benign) and the curves of the virulence of the bacteriophage against the bacillus of the patient is, of course, lacking. For these three cases

Louis $\mathrm{Pi} . .$.

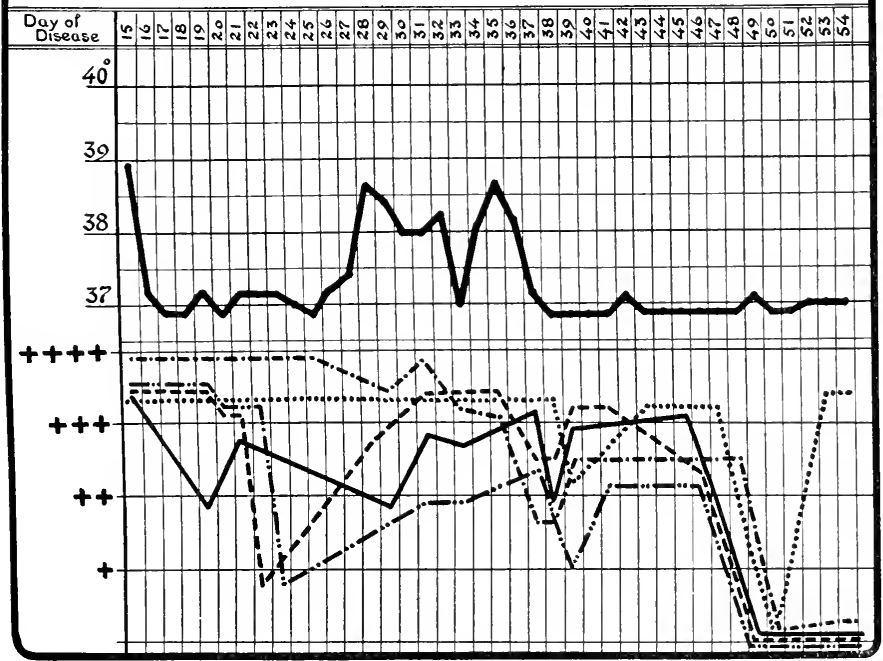

Chart 7. Louis Pi. . . . (17 years) Clinically, Typhoid Fever

\begin{tabular}{|c|c|}
\hline \multirow{3}{*}{ Virulence for } & B. typhosus--..- \\
\hline & B. paratyphosus $A$ - \\
\hline & $\begin{array}{l}\text { B. pola....... } \\
\text { B. paratyphosus B- } \\
\text { B. dysenteriae Shigo }\end{array}$ \\
\hline
\end{tabular}

the virulence of the bacteriophage against a Shiga dysentery strain, and against the paratyphoids $\mathrm{A}$ and $\mathrm{B}$ are given.

We will select as examples cases of different severity.

\section{Mild infections}

These were cases of typhoid fever or paratyphoid fever running a mild course. Clinically they were typhoid fever but the blood and stool 
cultures were negative. The curves for these three cases are given herewith.

1. Marie Mo. . . . . (fifty-five years, chart 6).

2. Louis Pi. . . . (seventeen years, chart 7).

3. François Jod. . . . (thirty-four years, chart 8).

François Jod...

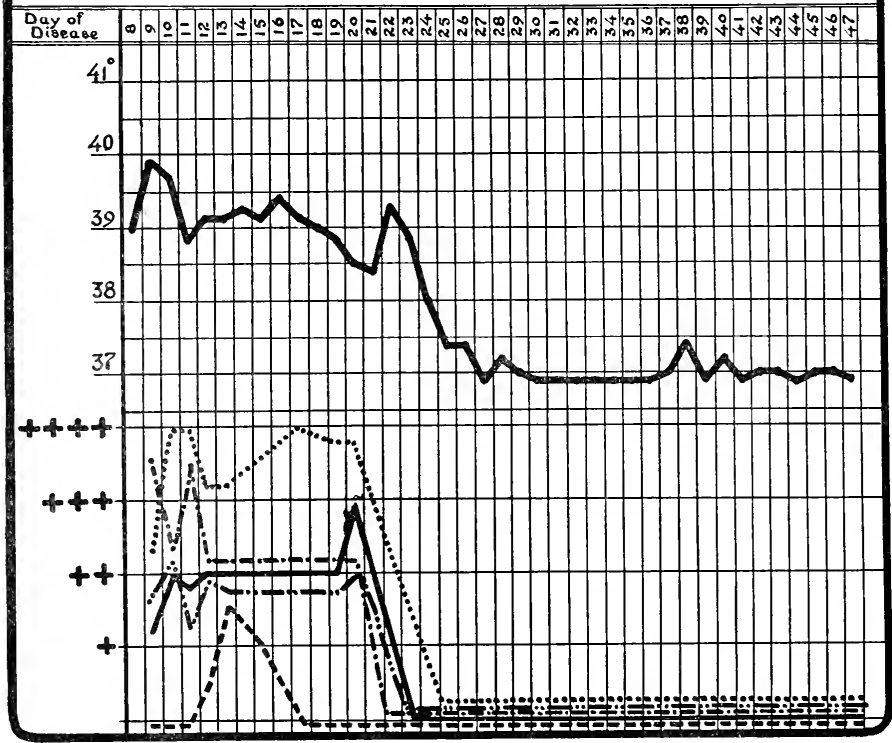

Chart 8. Frangois Jod. . . . (34 years) Clinically, Typhoid Fever

Virulence for $\left\{\begin{array}{l}\text { B. typhosus-.-...- } \\ \text { B. coli....... } \\ \text { B. paratyphosus A-........ } \\ \text { B. paratyphosus B-. } \\ \text { B. dysenteriae Shiga-....... }\end{array}\right.$

In these cases the virulence of the intestinal bacteriophage was determined for B. coli, B. typhosus, B. paratyphosus $A$ and $B$, and $B$. dysenteriae Shiga. It is needless to comment on these observations, 

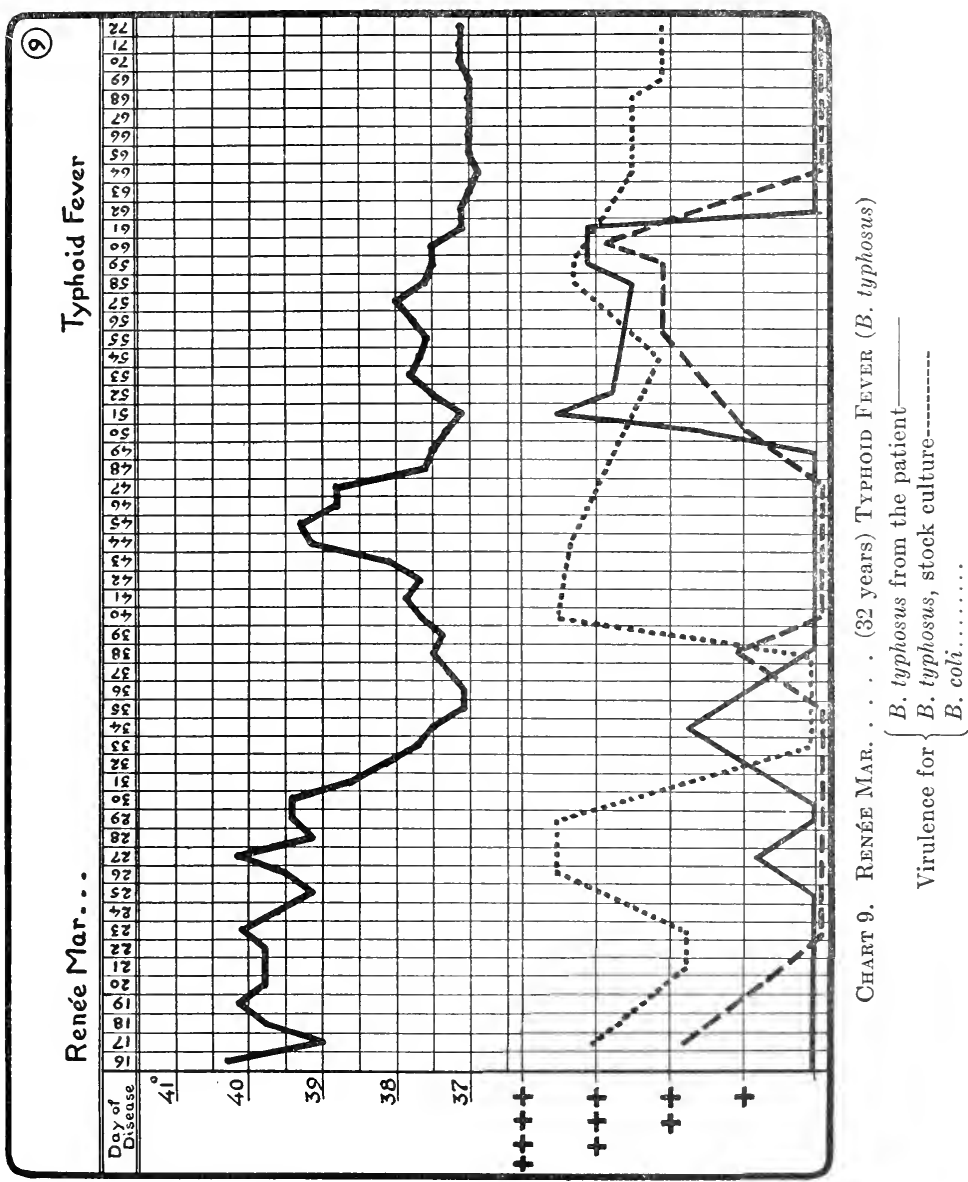
since examination of the curves is more instructive than would be an explanation.

What is the causative bacillus in each of these three cases? It is indeed difficult to make a diagnosis by means of the bacteriophage, which as we have seen, but rarely develops a single virulence.

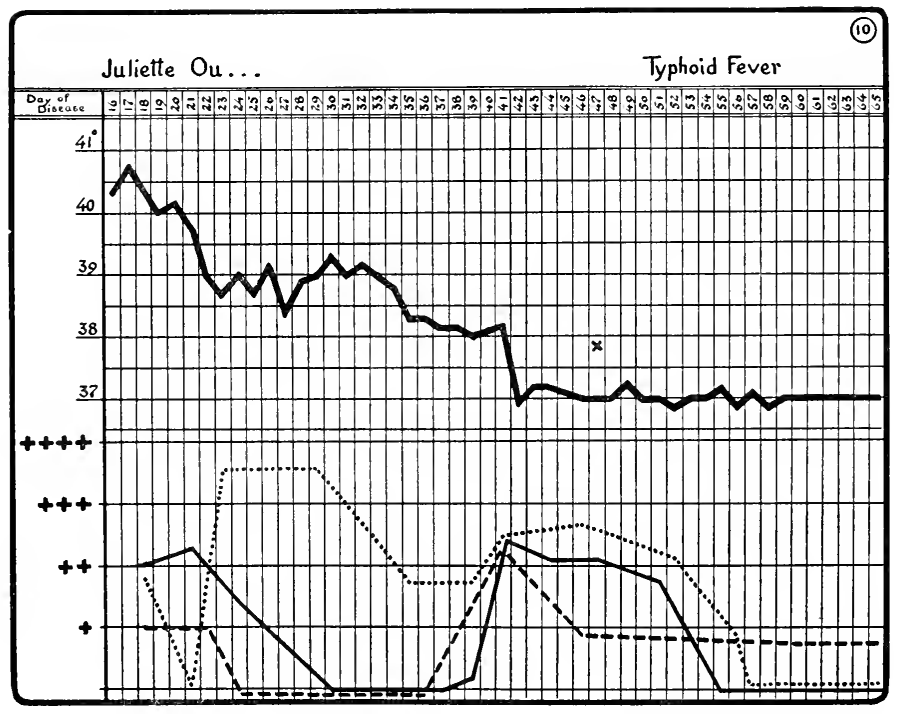

Chart 10. Juliette Ou. . . . . (36 years) Typhoid Fever (B. typhosus)

Virulence for $\left\{\begin{array}{l}\text { B. typhosus from the patient------ } \\ \text { B.typhosus, stock culture----- } \\ \text { B. coli........ }\end{array}\right.$
"x" Convalescence

This virulence extends to other bacteria of the same group to a more or less marked degree, and this fact is particularly in evidence when working with the representatives of the colon-typhoid-paratyphoiddysentery group. It appears, however, in the case of Louis Pi. . . . . that the causative bacillus must have been the typhoid bacillus, with Marie Mo. . . . . B. paratyphosus A, and in François Jod. . . . . $B$. paratyphosus $B$.

It should be noted that in these three cases in which improvement 


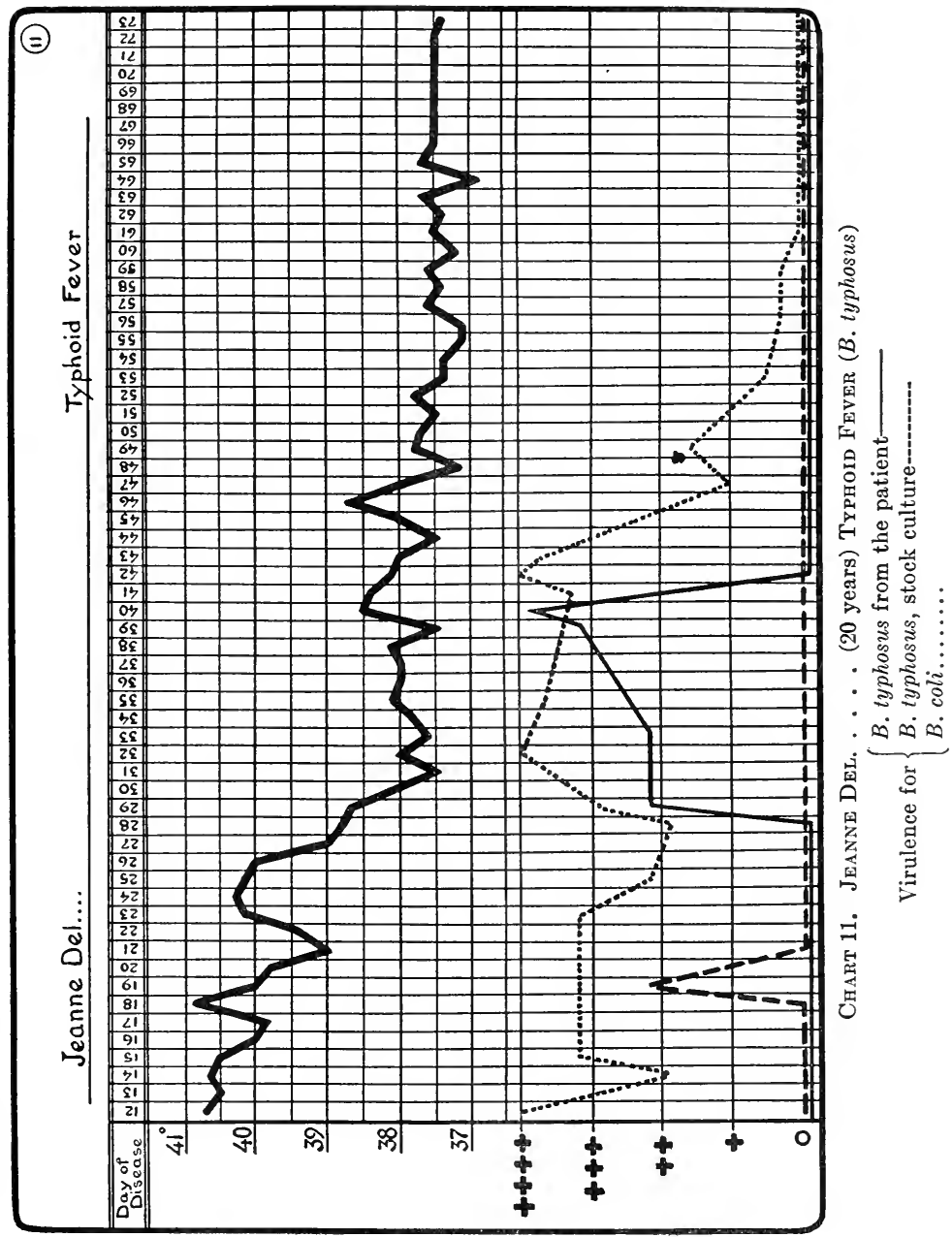


was rapid, the curves representing the virulence of the bacteriophage are comparable. It is also to be noted that in all, the accessory virulences for B. dysenteriae Shiga and for B. coli are very high, and that the acquisition of virulence for $B$. typhosus and $B$. paratyphosus $A$ and $B$ is early and is maintained up to the beginning of convalescence. In the case of Louis Pi. . . . . the abrupt deflection in virulence on the

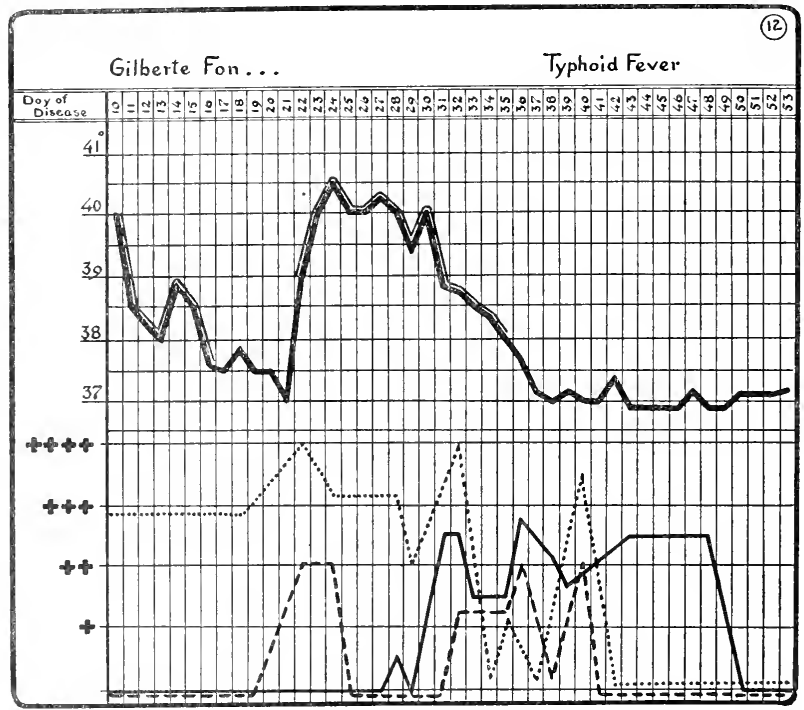

Chart 12. Gilberte Fon. . . . (4 years) Typhoid Fever (B. typhosus)

Virulence for $\left\{\begin{array}{l}\text { B. typhosus from the patient--..- } \\ \text { B.typhosus, stock strain---- } \\ \text { B. coli........ }\end{array}\right.$
Condition serious

twenty-second day preceded a slight relapse which occurred on the twenty-fifth to the thirty-second day. This complication did not prove serious since the virulence of the bacteriophage increased gradually from the twenty-third day.

\section{Severe infections}

The three cases to be cited here were serious, with both stool and blood cultures positive. All were infected with B. typhosus. 
1. Renée Mar. . . . . (thirty-two years, chart 9).

The bacteriophage was from the beginning virulent for $B$. coli and remained so during the course of the disease, throughout convalescence and up to the time when the patient was discharged from the hospital completely cured. It may be noted that the acquisition of virulence by the bacteriophage for the bacillus of the infection coincided with the first defervescence. Then this virulence became reduced and the temperature again went up. The infection was definitely overcome at the time when this virulence was again established.

2. Juliette Ou. . . . . (thirty-six years, chart 10).

3. Jeanne Del. . . . . (twenty years, chart 11).

The curves for these cases are self-explanatory.

\section{Typhoid fever with relapse}

1. Gilberte Fon. . . . . (four years, chart 12).

On the sixteenth day the disease appeared ended. However, the virulence of the bacteriophage toward the bacillus of the patient disappeared before the end of the crisis and the destruction of the pathogenic bacteria was not complete. Whereupon there was a relapse, very severe, which did not show improvement until the bacteriophage recuperated with a virulence sufficient to control the resistance of the bacterium.

\section{Typhoid fever of extreme severity}

1. Andrée Dess. . . . (thirty years, chart 13).

2. Jeanne Cot. . . . . (twenty-four years, chart 14).

In these two cases strains of $B$. typhosus were isolated at different times during the course of the disease. These bacilli presented a marked resistance to the action of a very active strain of Typhoid-bacteriophage and lost this resistance only after about ten transfers on agar. It is to be noted that at their isolation from the body these bacilli were inagglutinable (this fact has frequently been observed) and that they did not become agglutinable until after a series of subcultures. This transitory inagglutinability is, as we have seen, associated with resistance to the action of the bacteriophage.

Examination of the curves shows clearly the struggle which was carried on within the organism between the bacterium and the bacteriophage and the repercussions of this campaign upon the state of the patient. 


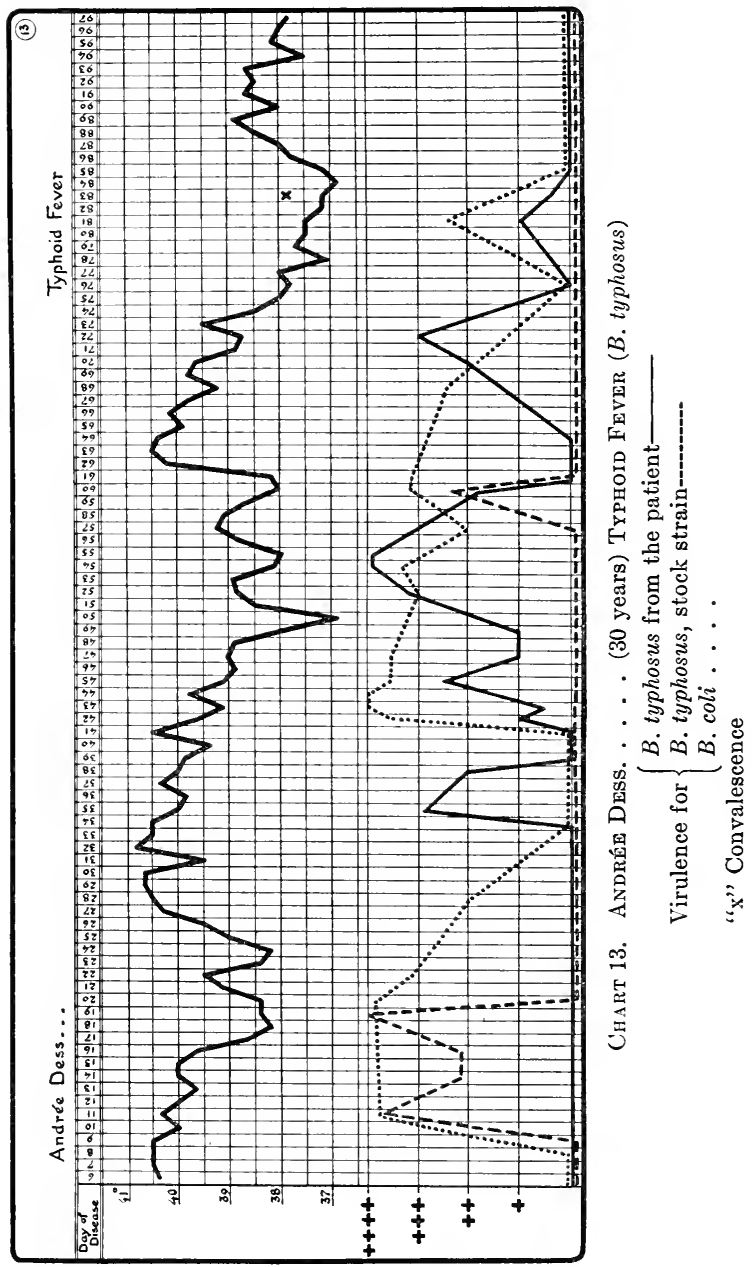




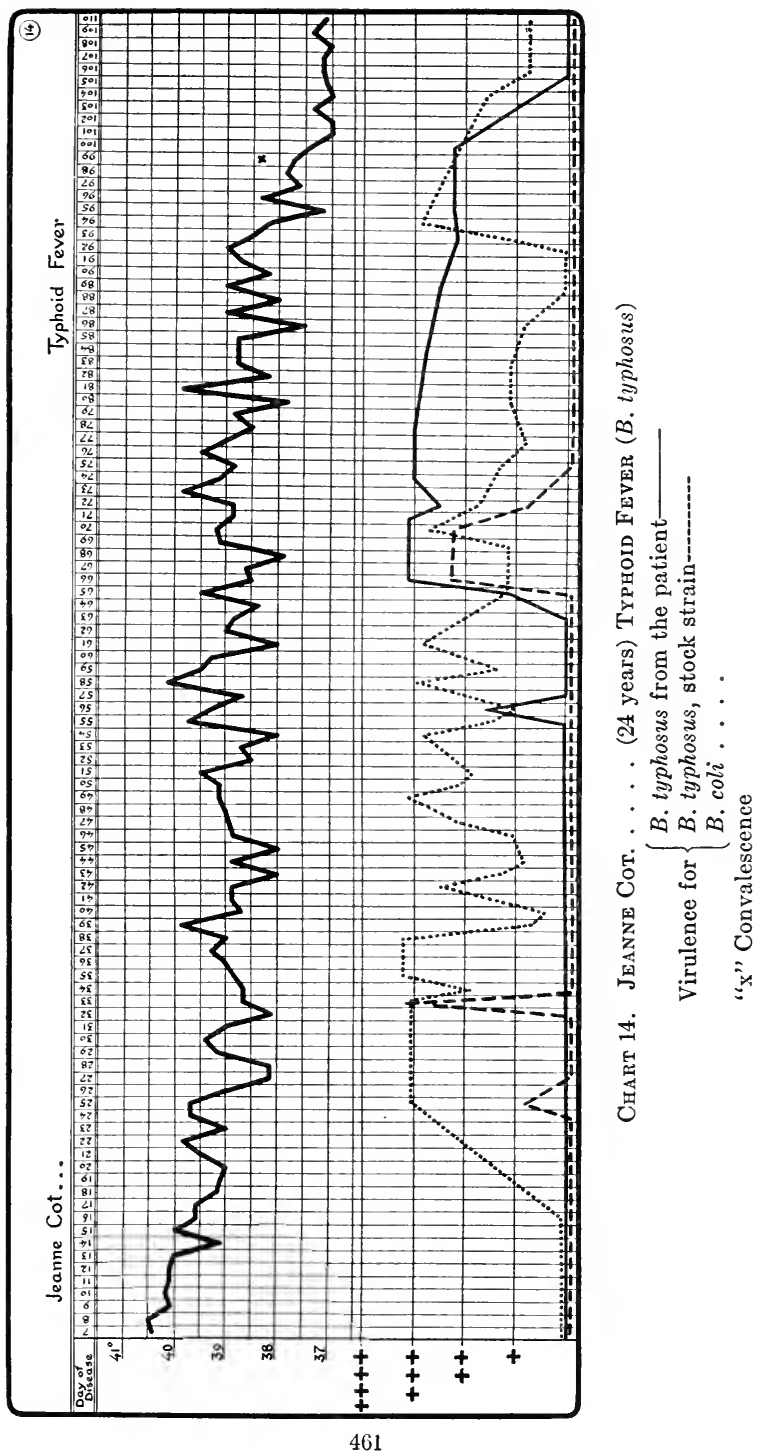


We find then, in typhoid fever,-an intestinal infection complicated by a septicemia - the same facts as seen in bacillary dysentery.

The virulence of the bacteriophage isolated from the stools of the typhoid patient is not limited, in general, to a single pathogenic bacillus; at one and the same time it extends, in some degree, to some or all of the bacilli of the colon-typhoid-dysentery group. This fact is particularly noted in mild cases or those of average severity. In the severe cases the bactericidal action is more specific and is often limited to the specific pathogenic organism and to $B$. coli, the latter always being attacked. In certain very severe cases the specificity becomes such that up to the beginning of actual improvement only the bacillus isolated from the patient is attacked, whether it has been secured by stool or by blood culture, to the exclusion of other bacilli, taken either from old laboratory cultures or from strains recently isolated from other patients. It seems, then, that in the course of their struggle each of the two organisms present,-bacteriophage and bacterium-acquires an individual personality, which differentiates them from other organisms of the same species rendered banal as a result of cultivation.

In addition to 28 non-fatal cases which were studied in order to determine the influence of the bacteriophage on the course of the disease, three fatal cases were observed. In these three cases, at no period of the disease could the presence of a bacteriophage be demonstrated active for B. typhosus, either for a stock strain or for the bacillus from the patient. Furthermore, examination of the strains from the intestinal contents from five individuals who had died of typhoid fever failed to show any activity for the typhoid bacillus. But the bacteriophage was not entirely absent, since in six of these eight cases a bacteriophage of moderate activity for the colon bacillus was found. This bacteriophage did not, however, show any activity for the pathogenic organisms. Death in typhoid fever results, usually, because of a failure of the bacteriophage to adapt itself to the bacteriophagy of the invading bacillus.

May death occur because of the acquisition of a resistant condition by the typhoid bacillus, which protects it from the action of the bacteriophage, as we have seen in the case of dysentery? There has been no opportunity to establish this up to the present but it is the more probable, since, in vitro as in vivo, the tendency toward resistance is certainly more marked in the typhoid bacillus than for B. dysenteriae. In any case, this cause of death is certainly the exception, even in typhoid. It must necessarily accompany a septicemia when it occurs.

In typhoid, as in dysentery, the investigation of the virulence of the 
bacteriophage is of prognostic significance. It is sufficient to test simultaneously the virulence of the intestinal bacteriophage of the patient for $B$. coli, for the pathogenic bacillus taken from the patient, and for a stock culture of $B$. typhosus. A comparison of these three results furnishes the information desired. The detection of resistance in the pathogenic bacterium would indicate a poor prognosis, and that in proportion as the resistance is the more pronounced. The establishment of a refractory state in the bacterium, resulting in the formation of a mixed culture in the intestine accompanying a septicemia, implies a fatal outcome and suggests that death will not be long delayed.

If we consider all of the data which have been presented with reference to the relation of the bacteriophage to typhoid fever, we may summarize the results somewhat as follows:

In all of the cases of typhoid fever studied, whatever may have been their severity, the appearance in the intestinal bacteriophage of a virulence for the pathogenic bacillus has been preceded by an increase in virulence for $B$. coli. This has always begun in the course of the second week and has rapidly attained great intensity. This activity was maintained during the entire course of the infection and appreciably decreased only during convalescence, sometimes even later. On the contrary, the development of a virulence for the pathogenic bacillus has varied according to the severity of the disease. In cases that were mild or of average severity the activity of the bacteriophage for this bacillus appeared before the end of the second week and disappeared toward the end of convalescence. The activity for B. coli and for $B$. typhosus is, in these cases, parallel. In the severe cases an activity for the typhoid bacillus only began to manifest itself in an energetic manner towards the beginning of definite improvement. It persisted for a greater or less length of time, in some cases up to the middle of the period of convalescence.

In the forms with relapse and recrudescence the struggle was complicated by the fact of the acquisition of a resistance by the bacteria, and it was only toward the decline of this relapse that the virulence of the bacteriophage was sufficient to definitely control the resistance of the bacterium. Here, the activity of the bacteriophage was maintained up to complete recovery, that is to say, up to the moment when, because of a total destruction of the pathogenic bacteria, the bacteriophage was no longer able to develop at their expense.

In all cases, the condition of the patient faithfully registered the vicissitudes of the struggle taking place within the body between the bacteriophage and the invading bacterium. 
A number of authors, basing their opinions on their own studies, have taken exception to these conclusions. They have filtered a specimen of stool taken from a person at any time during the convalescent period, they have combined this filtrate with any typhoid bacillus that happened to be available, and they have found that the filtrate was inert. From such a study they concluded that their observations did not support my conclusions. Most assuredly their results did not conform to mine, nor did their procedure resemble the method which I employed. I have, upon several occasions, insisted upon the mode of procedure to be followed by those who wish to correctly disclose the facts, and if $I$ have insisted on this, it is simply because I am convinced that to follow any less exacting method is to invite precisely those errors which have been committed, together with the faulty deductions consequent to them. It is only necessary to consult the curves presented above to see that, in the majority of cases, the bacteriophage does not retain its virulence for the pathogenic bacterium. Immediately after the in vivo bacteriophagy is completed the virulence disappears. Furthermore, we know very well that $B$. typhosus is a heterogeneous species as regards the bacteriophage, and to take any strain of this bacillus at all in order to determine whether the race of bacteriophage present in a patient is virulent for $B$. typhosus is simply to confess that the nature of the phenomenon of bacteriophagy is not too thoroughly appreciated.

There is but a single method which will allow one to determine the behavior of the bacteriophage in the course of disease in general and in typhoid fever in particular, and that method consists in the daily collection of specimens of the stools of the patient, from the beginning of the disease up to the end of convalescence, and in combining filtrates of the specimens so obtained with cultures of the pathogenic bacterium isolated from the patient himself at the beginning of the disease.

To work in any other way, and then declare that the results do not agree with mine, is to beg the question.

One author only has applied this rational method to his studies, and that is Hauduroy. ${ }^{294}$

As this question of bacteriophagy in vivo is of fundamental importance in its bearing on the new conception of immunity, I am inserting here, in extenso, some of the statements made in Hauduroy's paper. $\mathrm{He}$ states:

It is not possible to reproduce here all of the curves and all of the observations which I have made. I shall content myself with recording a few selected cases taken from among those which seem to me to be the most typical. 


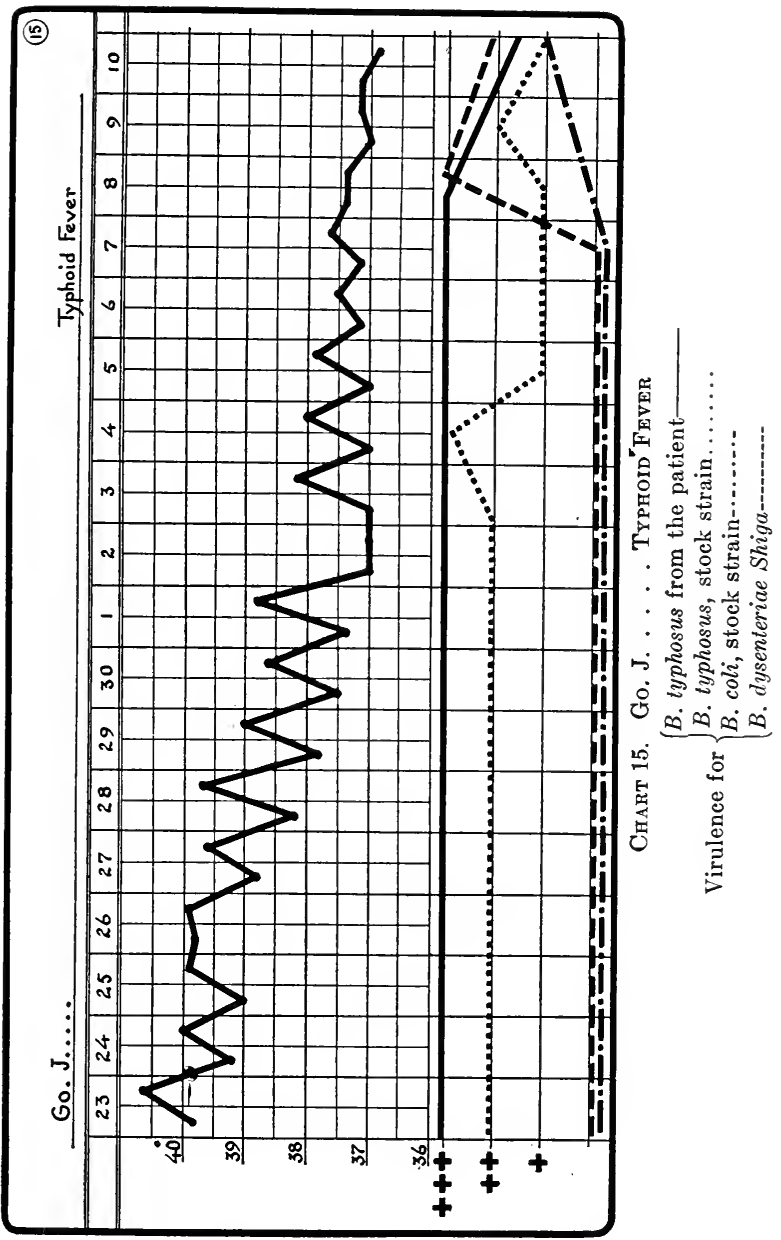




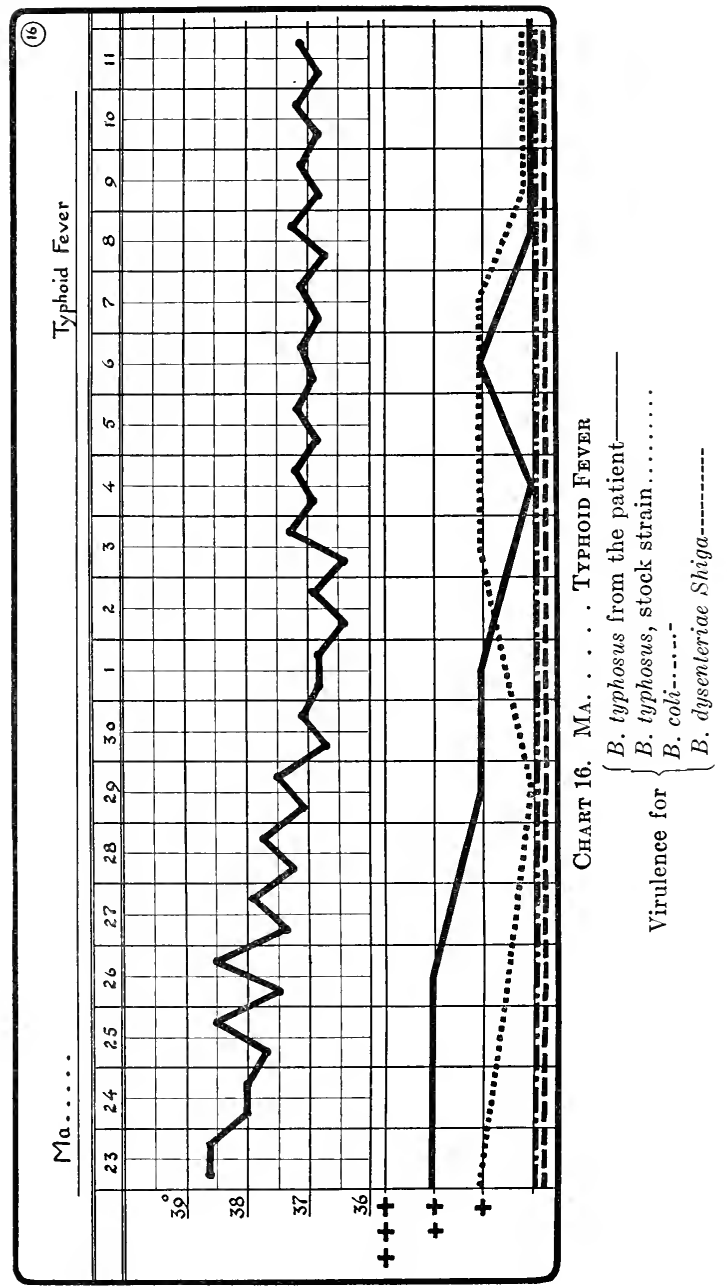


Considering the cases from the point of view of the time when the bacteriophage appeared in the stools, and on the manner in which it remained there, the observations may be divided into two groups.

Group One:

Case Go. J. . . . . Mrs. Go. J. (chart 15), aged 33 years, entered the hospital on April 21. The disease began 8 days before admission, the symptoms being fever, anorexia, insomnia, and head-ache. Upon admission the abdomen was but slightly distended, and not painful. The tongue was coated. The heart sounds were normal; the pulse of good quality. A blood culture yielded $B$. typhosus. The curve which is reproduced here shows in parallel the temperature, and the virulences of the intestinal bacteriophage for the typhoid strain from the patient, for a laboratory strain of $B$. typhosus, for a colon bacillus, and for a strain of B. dysenteriae Shiga.

In brief, the case was one of typhoid fever of a moderate severity, in which it was possible to demonstrate a lytic principle active for the infecting bacillus throughout the entire course of the defervescence.

Case Ma . . . Mr. Ma . . . ., aged 34 years (chart 16), entered the hospital on April 23. For the past two weeks he had complained of head-ache, pains in the lumbar region, a general fatigue, and for the past 8 days he had had some fever. Upon admission to the hospital the abdomen was neither tender nor distended. The symptoms of typhoid fever were not severe. Vomiting was frequent. Rose spots were present. The pulse was good.

The case was one of typhoid fever of moderate severity, in which it was possible to demonstrate a lytic principle active for the bacillus causing the infection. This lytic principle was present throughout the entire period of the defervescence.

Other illustrative cases of this type might be included, but they would simply duplicate the two cases which have been presented.

A consideration of this first group permits the following conclusions:

In a certain proportion of typhoid fever cases, at the same time that the disease shows regression, when the temperature falls, one may find in the stools of the patient a lytic principle (the bacteriophage) which in vitro destroys the infecting bacillus. This lytic principle is of maximum activity, in general, at the time when the period of defervescence begins. Often, when the temperature has reached normal, the lytic principle disappears. In our observations we have not found any definite relationship between the lytic power of the bacteriophage for the infecting bacillus and its lytic power for the stock strains of the bacteria which were tested.

Group Two:

Case Fe . . . (chart 17) Mrs. Fe. entered the hospital on April 21. She had been sick for about 9 days. Rose spots were present. The pulse was good. The blood culture yielded B. typhosus. Chart 17 shows the observations made.

To summarize this case: A patient having a moderately severe typhoid fever, in which, at one time there appeared in the stools a bacteriophage very active for the bacillus causing the disease. This bacteriophage disappeared almost immediately, and it was impossible to discover it again. The beginning of convalescence coincided with the appearance of the bacteriophage.

There is no point in presenting any further cases of this same type, for they add no new points of interest. 


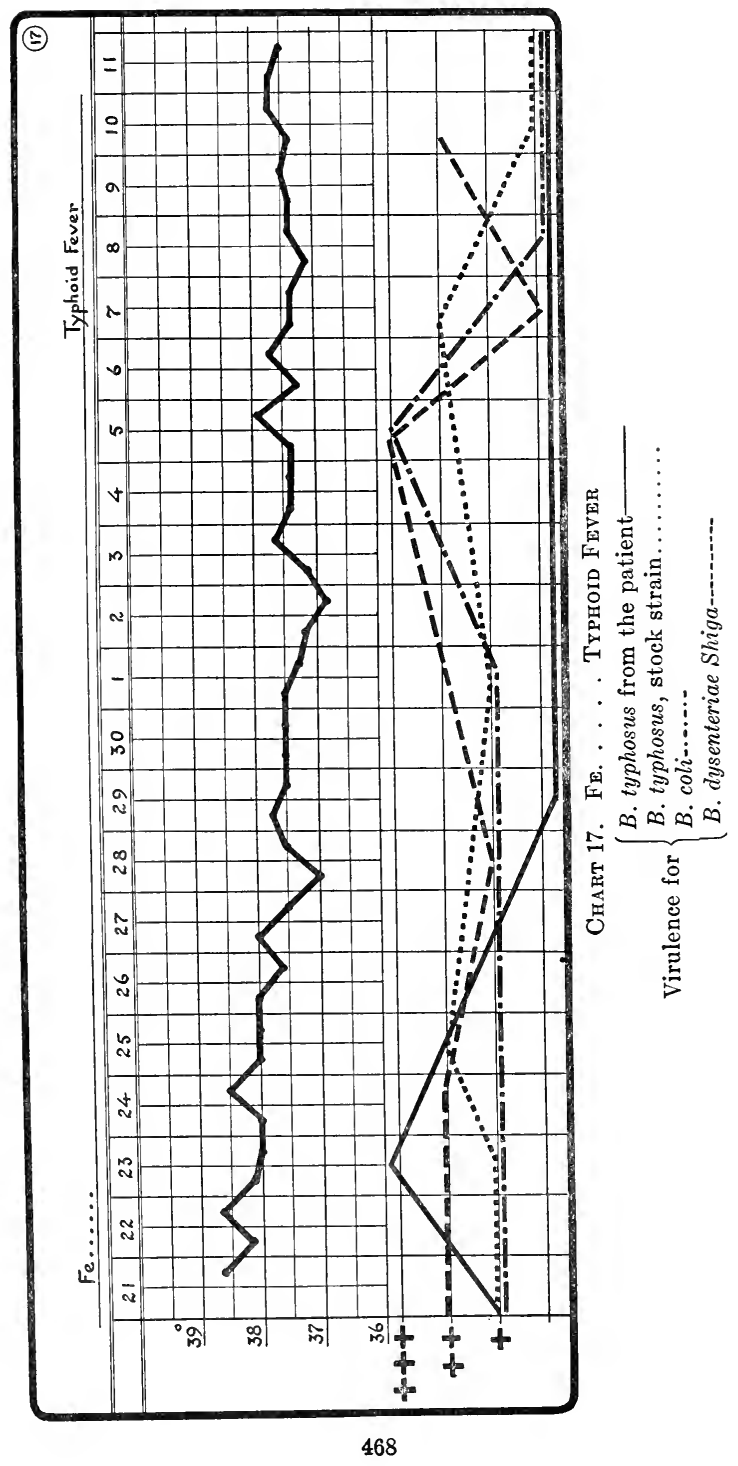




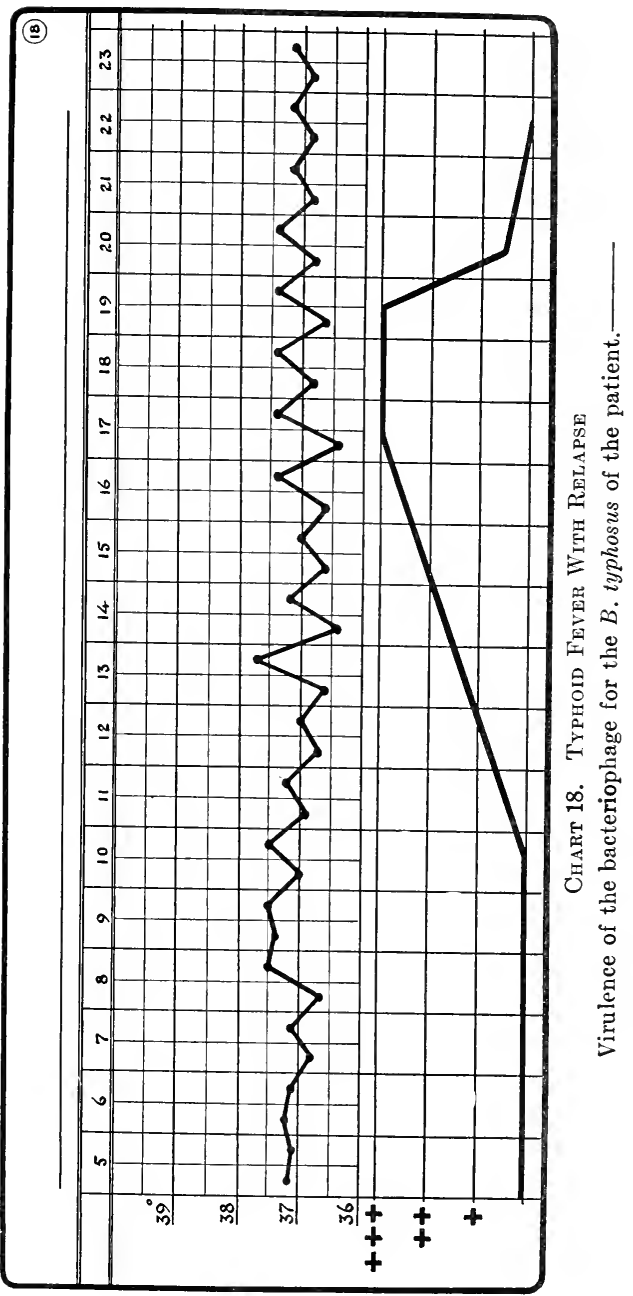


All of the cases discussed up to this time have ended in a complete recovery without complicating incidents. A number of the other cases have showed relapses, and the charts of all of these resemble the one which follows. In some of the cases a relapse has only been threatened; cases in which the temperature, once become normal, has showed a tendency throughout a few days to rise again. But despite this event, recovery took place without any serious complications. Chart 18 is illustrative of a case of this type.

These observations of Hauduroy agree in all respects with those which I have reported.

Typhoid fever is not a purely intestinal infection as is dysentery. In the latter it can be understood how, when all of the pathogenic bacteria of the intestine or of the mucosa, that is, those in proximity to the bacteriophage corpuscles, have been destroyed the disease ends ipso facto. In typhoid fever there is in addition a septicemia and even though the destruction of the bacilli contained in the intestinal contents is sufficient to delay the appearance of the disease or to restrain it from the beginning, it may not be adequate to overcome the infection once the pathogenic bacilli have invaded the organism.

In the last chapter we learned that the "products resulting from bacteriophagy" are possessed of an extremely potent opsonizing power. For example, we may precipitate a suspension of the Typhoid-bacteriophage by the addition of four volumes of 96 per cent alcohol. We allow this precipitate to remain in contact with the alcohol for 48 hours, a period adequate to ensure the complete destruction of all of the bacteriophage protobes. One centigram of this moist precipitate is dissolved in $10 \mathrm{cc}$. of saline. If, with this, we determine the opsonic index, we find that the leucocytes become so loaded with typhoid bacilli that it is quite impossible to count the number of organisms ingested. The opsonic index found is certainly higher than 50. It is possible that these "products of bacteriophagy," occurring in the intestine as the bacteriophage acquires a virulence adequate to dissolve the typhoid bacilli, may be resorbed and pass into the circulation, thus assuring a destruction of the bacilli through a phagocytic process.

On the other hand, as we know, the bacteriophage does not remain strictly localized in the intestinal tract; at times it passes into the circulation.

Inasmuch as I have not had an opportunity to make studies on the blood of patients, I have been forced to study the experimental disease induced in rats by the ingestion of particularly virulent strains of $B$. typhi murium. In these examinations I have found that every time 
one of the rats survived the infection consequent to the ingestion,- and survival is relatively frequent,-- $\mathrm{I}$ could demonstrate in the blood, removed by heart puncture on the 4th to 6th day after the infecting meal, a bacteriophage virulent for the pathogenic bacterium. ${ }^{313}, 321$

From these experimental studies I concluded that it is quite probable that a similar event occurs in human typhoid fever.

Beckerich and Hauduroy ${ }^{57}$ were the first to demonstrate the presence of a bacteriophage virulent for B. typhosus in blood cultures which failed to give a growth of the typhoid bacillus. Following this observation, Hauduroy ${ }^{294}$ systematically studied the question of the passage of the bacteriophage into the circulation during the course of typhoid fever. His observations were made on cases with positive, as well as those with negative blood cultures. I am inserting some of his conclusions in extenso.

Cases with Negative Blood Cultures.

The table given below summarizes the results obtained in a scries of cases of this type.

\begin{tabular}{|c|c|c|c|c|}
\hline \multirow{2}{*}{ PATIENT } & \multirow{2}{*}{$\begin{array}{c}\text { NUMBER } \\
\text { OF PASSAGES } \\
\text { MADE WITH } \\
\text { THE FILTRATE } \\
\text { OF THE BLOOD }\end{array}$} & \multicolumn{3}{|c|}{ BACTERIA SUBJECTED TO TEST } \\
\hline & & B. typhosus & B. coli & $\begin{array}{l}\text { B. dysenteriae } \\
\text { Shiga* }\end{array}$ \\
\hline Ler............ & 5 & Lysis & No lysis & No lysis \\
\hline Fo $\ldots \ldots \ldots \ldots \ldots$ & 5 & Lysis & No lysis & No lysis \\
\hline $\operatorname{Tr}$. & 6 & Lysis & No lysis & No lysis \\
\hline Go..... & 4 & Lysis & No lysis & No lysis \\
\hline Ro.... & 2 & Lysis & No lysis & No lysis \\
\hline $\operatorname{Ri} \ldots \ldots \ldots \ldots \ldots \ldots$ & 2 & Lysis & No lysis & No lysis \\
\hline
\end{tabular}

*All of the bacterial strains used in this study were laboratory strains known to be susceptible.

Here are the curves showing the temperature reactions of two of these cases. The time at which the blood was collected which later showed the presence of a bacteriophage active for B. typhosus is indicated.

Case Tr. . . . The blood culture was negative. The bacteriophage was found in the blood at the moment when the patient showed a true crisis; at a time when the oscillations in the temperature were very considerable. Upon the day following that when the blood yielded a bacteriophage convalescence began.

Case Ri. . . . This patient had a typhoid fever of the abortive type. Admission to the hospital occurred on the 3rd day of the disease, and on the 7th day defervescence was complete. A blood culture taken on the 11th day was negative, but the blood at this time contained a bacteriophage very active for the typhoid bacillus. We will have occasion to mention the bacteriologic findings on this patient again, for they are of very great interest. 
Case Ler. . . . ., whose chart is not given, also gave a negative blood culture. Here, again, the blood contained a bacteriophage at the time when the patient's temperature began to fall. The fall in temperature was gradual, and continuous, and convalescence occurred without incident.

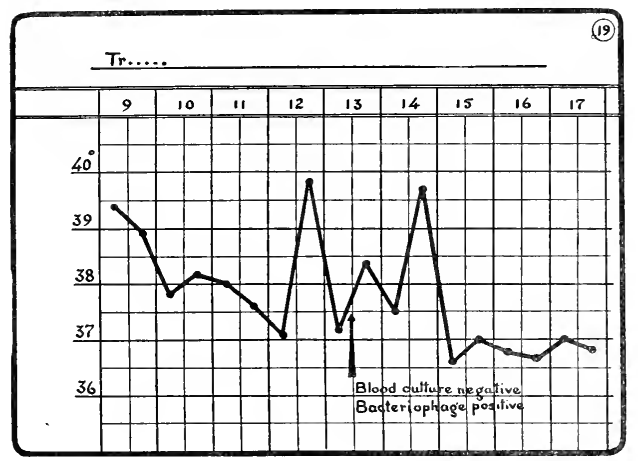

Chart 19. Case Tr. . . . Clinically, Typhoid Fever The blood culture was negative; the bacieriophige was present

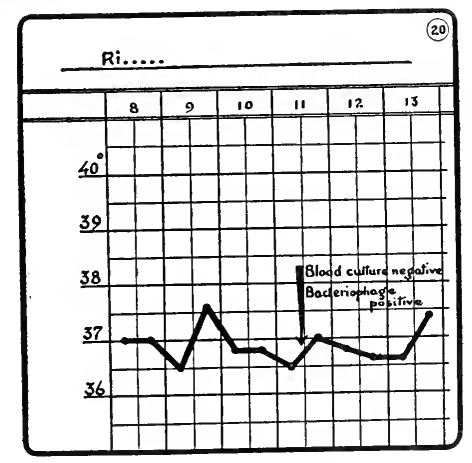

Chart 20. Case Ri. . . . Clinically, Typhoid Fever

The blood culture was negative; the bacteriophage was present

We may summarize the findings on this group of cases by saying that very of ten the blood cultures in typhoid fever are negative at the time when defervescence begins; and that when a bacteriophage is found in the blood stream of these cases 
with a negative blood culture, the temperature always falls during the following days.

Why is it that the filtrate from a blood which contains the bacteriophage fails to cause a dissolution of the typhoid bacilli without further treatment? As a matter of fact, experience shows that it may be necessary to make 2,4 , or even 6 passages before a complete dissolution of the typhoid bacillus will take place. But, in these cases with a negative blood culture, the bacillus under test is the organism of a stock laboratory strain, and it is not particularly astonishing that the virulence of the bacteriophage may have to be enhanced before a macroscopic effect can be observed.

It is also pertinent to observe that the lysis which occurs is specific. That is, it takes place only with $B$. typhosus and not with the related organisms B.coli and B. dysenteriae Shiga.

In brief, then, it is possible to find in negative blood cultures taken during the later stages of typhoid fever a bacteriophage dissolving in a specific fashion the typhoid bacilli of a laboratory stock culture. The lysis is not obtained by a simple contact of the filtrate and the bacterium, but only after a few passages with intervening filtrations.

Cases with Positive Blood Cultures.

In the table below are listed some of the cases which gave positive blood cultures. The manner of testing the filtrates of the blood, and the organisms against which they were tested, are the same as in the preceding group.

\begin{tabular}{|c|c|c|c|c|}
\hline \multirow{2}{*}{ PATIENT } & \multirow{2}{*}{$\begin{array}{l}\text { NUMBER } \\
\text { OF PASSAGES } \\
\text { MADE WITH } \\
\text { THE BLOOD } \\
\text { FILTRATE }\end{array}$} & \multicolumn{3}{|c|}{ BACTERIA SUBJECTED TO TEST } \\
\hline & & B.typhosus & B. coli & $\begin{array}{l}\text { B. dysenteriae } \\
\text { Shiga }\end{array}$ \\
\hline $\mathrm{Se} \ldots \ldots \ldots$ & 7 & Lysis & No lysis & No lysis \\
\hline Be. L . . . . . . . . . & 8 & Lysis & No lysis & No lysis \\
\hline Du. G... & 8 & Lysis & No lysis & No lysis \\
\hline Be. A......... & 6 & Lysis & No lysis & No lysis \\
\hline Go. J . . . . . & 6 & Lysis & No lysis & No lysis \\
\hline Go. A....... & 6 & Lysis & No lysis & No lysis \\
\hline Th.......... & 6 & Lysis & No lysis & No lysis \\
\hline Ka........... & 6 & Lysis & No lysis & No lysis \\
\hline $\mathrm{Ba} \ldots \ldots \ldots \ldots$ & 6 & Lysis & No lysis & No lysis \\
\hline
\end{tabular}

Considering the data afforded by this group of cases it is obvious that in cases yielding a positive blood culture, near the end of the septicemia a bacteriophage may always be found which is able to dissolve the typhoid bacillus.

At first sight, a result of this type would seem to be paradoxical. But let us consider the facts in somewhat greater detail. The correlation of the available data may permit us to understand and interpret such a situation. In the first place, is there any relationship between the temperature of the patient, the positive blood culture, and the presence of the bacteriophage?

Let us select from among our cases but one as an example, that of patient Go. A. . . . . and examine it from this point of view. 
The temperature chart of this patient is here given.

From this chart we see that the blood culture taken on the 25th, when the temperature was still above $38^{\circ} \mathrm{C}$., was positive, and that within 2 or 3 days convalescence became established. This has been a common observation in all of our cases; they have all showed the same thing. Every time that the blood has yielded a typhoid bacillus and at the same time a bacteriophage active for the typhoid bacillus (another strain) we have observed a very definite fall in the temperature within a few days after the blood culture was taken.

We know from common experience that blood cultures are sometimes positive, sometimes negative, at the end of the second week, and especially during the third week. Correlating the results obtained in the two groups of cases reported above, those with, and those without positive blood cultures, we find that invariably before, or during, the fall in temperature a bacteriophage can be encountered in the blood stream. Unfortunately, we have not been able to procure

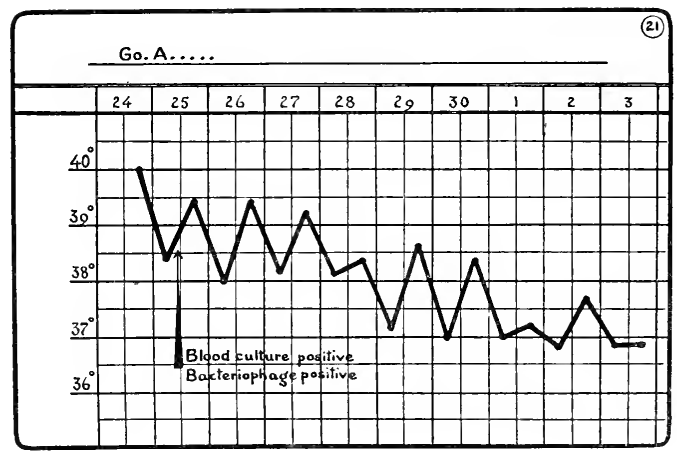

Chart 21. Case Go. A. . . . Typhold Fever

The blood culture yielded both a bacteriophage and B. typhosus

specimens of blood at the onset or during the incubation period of the disease, because of circumstances beyond our control, chiefly the fact that patients do not enter the hospital until late in the disease.

As stated above such results appear paradoxical. It is quite logical to find a bacteriophage in a negative blood culture, but it seems illogical to find it in the positive blood culture cases as well. As a matter of fact, this contradiction is more apparent than real, and the following observation, we believe, opens the way to an explanation.

In the case of the patient Ri. . . . , of whom we have spoken above, we find that the patient entered the hospital on the 3rd day of the disease, that on the 7 th day defervescence began, and that on the 11th day the blood was taken for examination. This blood culture was incubated, and daily examinations were made by removing sterilely a drop of the culture, spreading it on a slide and staining it. 
The stained smear prepared on the 2nd day of incubation showed microscopically a few bacilli having the morphology and the staining reactions of the typhoid bacillus. On the third day they were also found, in slightly increased numbers. The number found in the successive smears continued to increase up to the 6th day, and from that time on it progressively diminished. By the 10th day it was impossible to find a single organism in several smears. Meantime, at no period was it possible to obtain a growth of the organisms seen microseopically upon either solid or liquid media. When this culture, which we would term negative, was filtered the filtrate readily yielded a race of the baeteriophage which was very active for $B$. typhosus.

What happened here? Apparently, in taking this blood culture we withdrew from the blood stream of the patient both the bacterium of the disease and a bacteriophage capable of destroying this bacterium. The process occurring in vitro which we have witnessed on the slides probably takes place in vivo also, and undoubtedly with greater rapidity. This would explain the temporary occurrence of bacteria, their disappearance, and the impossibility of securing a positive blood culture at all times.

Is it not possible to consider this blood culture as being intermediate between a positive culture and a negative culture? Had it been taken a few days earlier it would have been positive. But in the interim, the struggle which has taken place between the bacterium and the bacteriophage has turned the tide to the advantage of the latter. A few days earlier this had not occurred, either because the bacteriophage at that time was not sufficiently active, or because the bacterium had then an adequate resistance. As a matter of fact, in a series of his communications on the subject of bacteriophagy, Gratia has shown that a pure culture of a bacterium may be dissoeiated from the point of view of resistance to the action of the bacteriophage. Mutations, reversions in particular, are possible. Thus, it is easy to conceive that a resistant bacterium may give birth to susceptible organisms, and it is probable that such reversions take place with particular facility within the body.

Had the culture been taken a little later,- - a few days or even a few hours,--it is possible that an in vitro destruetion the bacterium might not have occurred ${ }^{*}$ for a bacteriophage might have by then disappeared from the eirculation.

The paradoxical situation to which we ealled attention above, is, therefore, only an apparent eontradiction. In all blood cultures made prior to the fall in the temperature the bacteriophage may be found. The culture is positive, or negative, according to whether the specimen is removed before or after the destruction of the bacterium by the lytic principle.

With these facts at hand, there are still two points which are of very definite interest from the point of view of the study of bacteriophagy. In the first place, we should be quite sure that there is not a baeteriophage present in the blood of the normal healthy individual as well as in the blood of the diseased. And in the second place, we are interested in determining whether this lytic principle is, in fact, identical with the bacteriophage of d'Herelle.

* It should be remembered that we are here speaking of the culture strain in an artificial mixture of filtrate and bacteria. 
In order to gain information on these two points we have procured blood by venepuncture from a group of 11 apparently healthy individuals. In none of these did we ever succeed in demonstrating a lytic principle.

We have also examined the blood of various domestic animals, - sheep, chickens, and horses,-and in healthy animals of all of these species a lytic principle has never been found.

And finally, in order to afford an additional check on the results reported above, we made examinations of the blood of persons suffering from diseases other than typhoid fever (2 cases of tuberculous meningitis, 2 cases of pulmonary tuberculosis, and a number of cases of various infectious diseases). In no case could a lytic principle active for $B$. typhosus be disclosed.

With reference to the other point at issue, it should be stated that the filtrates which gave the d'Herelle phenomenon were subjected to the following tests:

First, serial transmissibility of the action:

Second, susceptibility to heat inactivation, with the possible reactivation.

The lytic principles which we recovered from the blood stream of our patients showed differing susceptibilities to heating, the sensitivity being related to the virulence of the principle. Most of them were inactivated at $70^{\circ} \mathrm{C}$., a few at temperatures between 75 and $80^{\circ} \mathrm{C} .^{*}$ Reactivation was always possible.

Third, the formation of clear plaques on agar slants.

The fact that the principles found by us in these cases conformed in all of these respects to the reactions induced by the bacteriophage clearly proves that they were, indeed, races of the bacteriophage with which we were working and not some bacteriolytic property of the serum as we have been accustomed to conceive the latter.

I may add that I have never found in the blood the bacteria-dissolving principle, not bacteriophagous in nature, which has been reported to be present in stools. $\dagger$

Such are the conclusions of Hauduroy, and he ends his discussion in these words: "We can not summarize our observations better than to borrow from d'Herelle one of his conclusions, namely, that in every case (of typhoid fever) the condition of the patient registers faithfully the fluctuations in the struggle taking place within the body between the bacteriophage and the invading bacterium."

I may contribute something to one point brought out in the studies of this author. I have had an opportunity to examine four blood

* Heating in an open tube for 5 minutes.

$\dagger I$ have noted in the stools of cholera convalescents, and even in those of persons who have received injections of heat-killed cholera vibrios ${ }^{341}$ a principle, of ferment nature, capable of dissolving the cholera vibrio. Hauduroy has found this same fermenting principle in the intestinal tract in patients convalescent from typhoid fever. Most certainly this principle is derived from the body of the patient, and has nothing in common with the bacteriophage (it is effective in proportion to its concentration; its action is not transmitted in series). Surely it plays some rôle in immunity, and offers a problem worthy of study. 
cultures taken during the first week of typhoid fever. All four were positive for B. typhosus, but in none of them could I detect a bacteriophage. In making the tests I combined the filtrates of the blood with 6 different laboratory strains of B.typhosus and with the strains isolated from the stools of each of the patients. In each instance 12 consecutive passages were made. Examinations made of the intestinal bacteriophage in these four cases showed that in no instance did it possess any virulence for B. typhosus, either for the stock culture strains or for the strains isolated from the blood or from the stools.

Of 5 cases in which positive blood cultures were secured during the second week of the disease, four contained no detectable bacteriophage, but from the fifth case, on the contrary, I isolated a bacteriophage virulent for the strain of $B$. typhosus procured from the stools four days previously. In the first four cases I was unable to show that the intestinal bacteriophage had any virulence for B. typhosus of any strain whatever. In the fifth, the race of bacteriophage present in the intestine had a moderate virulence for the strain isolated 4 days previously from the stools.

It seems, then, that the presence of the bacteriophage in the blood may be correlative to its acquisition of virulence in the intestine.

In another communication on the same subject ${ }^{306}$ Hauduroy presents the following observations, which throw some light on the fact, apparently paradoxical, of the possible presence of the bacteriophage in a positive blood culture. In the course of his investigations he secured, quite by chance, blood cultures in three cases at a time which, although the temperature was then between 39 and $40^{\circ} \mathrm{C}$., happened to be almost immediately before defervescence began. In these three cases the fall in temperature was abrupt and the patients recovered without incident. The blood cultures were placed in the incubator and were examined daily for several days. After 24 to 48 hours, he found that all three contained organisms,-motile, Gram-negative, morphologically to all appearances B. typhosus. Two or three days later these bacilli had disappeared. It was impossible for him to find a single bacterium,* and search for the bacteriophage yielded a race extremely active for $B$. typhosus. As Hauduroy remarks, the blood cultures in these three cases were temporarily positive.

In brief, we may say that in a blood culture containing both $B$. typhosus and the bacteriophage, the result of the culture depends upon

* The observation of Eliava, mentioned in the Introduction to this text, relative to the disappearance of the cholera vibrios from cultures made of the water of the Koura river, may be recalled in this connection. 
the virulence of the last and upon the capacity of the first to develop a resistance. If virulence is the factor which prevails, the bacteria are bacteriophaged, as in the three cultures mentioned above; if the resistance dominates, a mixed culture is formed.

Another fact of equal importance may be deduced from the results reported above, and that is, that the adaptation of the bacteriophage appears to be accomplished more readily and more rapidly in vivo than in vitro. This situation is readily comprehended. In vitro, the bacterium has to struggle against the bacteriophage only; in vivo, this struggle is superimposed upon the attack which the body itself makes, and certainly the body is not passive. Phagocytic processes, in particular, must enter into the struggle, and we have already emphasized the potency of the "products resulting from bacteriophagy" as regards opsonic action.

The conclusion of all of these studies is that recovery in typhoid fever, as well as in the paratyphoid fevers, is caused by an adaptation of the intestinal bacteriophage to parasitism of the pathogenic bacterium and by the in vivo bacteriophagy which results. In typhoid fever, as in dysentery, the reactions of the body itself seem to have but a secondary influence in the processes of recovery, an influence which is limited to favoring the action of the bacteriophage.

As for the deaths which occur from typhoid fever (excluding the complications, such as intestinal hemorrhage) we have seen that these result, either because of an inertia of the bacteriophage or because the bacterium has a chance, and a capacity, to acquire a refractory state. This refractory state results in the formation of a mixed culture in the body, and recent studies indicate that in such a case there may be a formation of "ultrabacteria" of $B$. typhosus, with an invasion of all of the organs by these filtrable forms.

Friedberger and Meissner* inoculated some fragments of the organs of patients who had died of typhoid fever into the peritoneal cavity of guinea pigs. They then made a series of passages by inoculating into the peritoneum of each new guinea pig a fragment of the brain (or of any organ) of the preceding pig. From the second passage on it was impossible for them to disclose any bacteria of any kind in the organs of the sick guinea pigs, nevertheless, these organs were infected, since the disease was transmitted serially. Cultures of these infected organs gave no growth on the usual media.

* Friedberger, E., and Meissner, G.-Zur Pathogenese der experimentellen Typhusinfektion, Klin. Wehnschr., 1923, 19, 403. 
Fejgin ${ }^{204,205}$ has recently reported some experiments showing that the "invisible virus" of Friedberger and Meissner is nothing other than the filtrable form which $B$. typhosus assumes when in symbiosis with the bacteriophage.

The action of the bacteriophage in disease is very complex, and we will be the more convinced of this as we study other diseases, but whatever may be its mode, or modes, of action, the whole pathogenesis of the disease and its issue are determined by the behavior of the bacteriophage.

\section{THE BACTERIOPHAGE IN AVIAN TYPHOSIS}

Avian typhosis is a disease affecting principally the Gallinaceae. Despite its frequency it for a long time remained undetected, confounded with chicken cholera. This last disease is, in reality, very rare. In 1919, in investigating epizootics for the purpose of testing on domestic animals, which allow of experimentation, the conclusions reached as to the rôle of the bacteriophage in human dysentery and typhoid, an extended focus of "chicken cholera" was found in the Department of the Aube. In the first examinations the error which had been made became apparent; it was the disease known in the United States as "fowl typhoid," whose existence in France had up to that time been unrecognized. Shortly after this numerous foci throughout the surrounding territory were discovered.

Fowl typhoid, which will here be called fowl typhosis, is a very interesting disease. Its study is complicated by the existence of several "paratyphoses" which resemble still more the human typhoid. The pathogenic agent, B. gallinarum Klein, studied by Moore under the name of $B$. sanguinarium, presents, with the exception of motility, all of the characteristics of the bacillus of Eberth (B. typhosus). It is even agglutinated to titre by an antityphoid serum. Aside from this type bacillus there are often found, in the same foci, bacilli presenting different agglutinative and biochemical reactions. The clinical type of the infection which they provoke does not differ from that caused by the typhoid type. These differing species of bacteria have up to the present been studied only by American workers; Ph. Hadley among others, who describes $B$. pullorum $A, B$. pullorum $B, B$. jeffersonii, B. rettgeri, and $B$. pfaffi. A discussion of the distinctive characters of these different bacilli will not be presented here since it would not be germane to 
the study with which we are concerned.* It is sufficient to know that in France in the epizootic of 1919 the most frequent pathogenic agent was of the B. gallinarum type (found in 57 of 73 examinations). Along with $B$. gallinarum other forms have been found: $B$. pullorum $A$ (once), B. pullorum $B$ (6 times), B. jeffersonii (4 times), and B. pfaffi (4 times). In a single focus, of which the centre was found in the village of Trainel (Aube), a paratyphosis infection occurred due solely to $B$. pfaffi without admixture with bacilli of the true typhosis type.

The clinical picture hardly varies whatever may be the causative bacillus. A typical observation follows.

On the evening of May 24 the chicken appeared perfectly well. On the morning of May 25 it remained apathetically on the ground of the poultry-yard and took no measures for its defense. The next day, toward noon, it appeared somnolent, the plumage rough, the eyes halfclosed, the crest slightly violet colored. It did not eat or drink, and remained humped up "in a ball." The inspirations were deep, twentyfive per minute. There was a greenish yellow diarrhea with portions definitely yellow. The condition became worse in the afternoon. It fell on its side at about 8 o'clock and died a few minutes later. The necropsy showed the crest to be violet in color, with spots of the same nature over the skin. The liver was voluminous, congested, and presented foci of degeneration. There was a pericarditis.

By direct microscopic examination the blood at first appeared negative, but a very careful search revealed three bacilli in a whole smear. The blood and tissues when cultured gave a pure growth of $B$. gallinarum, and this organism was also found, very abundantly, in the intestinal contents.

Sometimes death occurs more rapidly still, in certain cases in a striking manner. Epizootics of avian typhosis have a high mortality. In 1919 foci existed throughout the extent of France. In general, the epizootic begins quickly; within the space of three or four weeks a half, three-quarters, sometimes more, of the fowls on a farm succumb. Then the disease assumes a sporadic character, only an occasional animal dying during the course of a year. The disease may disappear for a few months and then reappear. The annual mortality amounts to forty to seventy per cent of the population of the infected poultry-

* Readers who are interested in the subject will find much useful information in the contribution of $\mathrm{Ph}$. Hadley, The Colon-Typhoid Intermediates as Causative Agents of Diseases in Birds. Bulletin No. 174, Rhode Island Agric. Exper. Sta., 1918. 
yards. Young adults are the most susceptible, then the old animals; the chicks are in general spared.

Epizootics of typhosis extend rapidly over large areas; certain Departments were contaminated throughout in 1919. The establishment of a new focus begins by the importation of the organism from an infected region, either through the agency of a flock of sheep or herd of cattle, or by horsemen (this last mode of dissemination was particularly frequent during the war; this explains the extension of the disease during the years 1917 and 1918). The disease rages for a few days on a farm, passes to a neighboring farm, and then extends rapidly into the surrounding villages.

The pathogenic bacillus remains alive and virulent during several months in the regions where the infection has been epidemic. In several tests it has been shown that an isolated infected chicken-yard, cleaned and left unoccupied for six to eight months, still contains virulent bacilli, for, when repopulated with chickens from a region free of the disease, the infection breaks out again within a few days among the new occupants.

Avian typhosis being a disease in general but little known, I have thought it useful to consider it in some detail, since it will allow us the better to understand the facts now to be presented.

Because of the exceptional severity of the infection in avian typhosis it has been possible to follow only four cases which recovered. In all, the picture has been identical. In the morning the infected chicken remains on the ground, "balled up," the feathers roughened, and with the characteristic diarrhea. The appearance is the same as in the fowls which succumb. At this stage of the infection examination of the feces gives results such as:

B. gallinarum, present in abundance.

Intestinal bacteriophage, virulent for $B . c o l i+$ (in 2 cases) or ++ (in 2 cases); for B. gallinarum 0 (in the four cases). The blood culture was positive in the two cases in which it was done; the blood for culture being taken aseptically by puncture of the crest.

During the course of the day the condition remains the same as that shown by animals which die. This state is prolonged and the next morning the chicken still appears the same. Examination of the feces at this time shows:

B. gallinarum present in three cases, absent in one.

Intestinal bacteriophage virulent for $B$. coli +++ (4 cases); for B. gallinarum + (in 3 cases) +++ (in 1 case). Towards noon, in 
one case, in the course of the afternoon in the three others, blood cultures were negative. In three cases a bacteriophage active for B. gallinarum was found in the blood. The blood which was ultrasterile was that of the chicken whose condition was the best at this time and which had shown no pathogenic bacilli in the intestinal tract in the morning. The presence of the bacteriophage in the blood is extremely transitory.

On the morning of the third day the animals appeared normal, they drank a great deal, ate some grain, and the diarrhea was less profuse. Examination of the feces showed:

$B$. gallinarum absent in the four cases.

Intestinal bacteriophage active for $B$. coli +++ (4 cases), for $B$. gallinarum $+++(3$ cases $)++++(1$ case $)$. Blood cultures were negative: no bacilli, no bacteriophage.

On the fourth day the animals were practically normal.

In the four chickens which recovered the intestinal bacteriophage remained active for $B$. gallinarum for a very long time. After three months it showed the same degree of activity as at the time of recovery. In one of them, in which it was possible to make an examination after five months, it was still as active as at first. We will see, from experimental observations that this persistence of virulence depends solely upon the fact that the pathogenic bacillus, distributed in profusion in the exterior environment, is frequently ingested by the animal and this maintains the virulence of the intestinal bacteriophage since it is able to grow at its expense.

The feces of about one hundred chickens which had died of avian typhosis were examined. In no case was there a bacteriophage active for B. gallinarum or for any of the bacillary agents of the paratyphoses. Nevertheless the bacteriophage had been present for it could be disclosed (91 times in 97 examinations) because of the activity shown for one or several species of the colon-typhoid-dysentery group. One sees clearly, then, that the lack of defense is not due to the absence of the bacteriophage, but solely to the fact that the intestinal bacteriophage remained passive because it failed to acquire a virulence for the pathogenic bacillus.

To summarize: as in dysentery and in typhoid fever in human beings, the acquisition of virulence by the intestinal bacteriophage for the pathogenic bacterium is the sine qua non of recovery.

Kramer ${ }^{39 t}$ also, has found that the bacteriophage plays an important rôle in immunity to avian typhosis. We will consider his contribution to the subject in more detail a little later. 


\section{THE BACTERIOPHAGE IN STAPHYLOCOCCUS AND STREPTOCOCCUS} INFECTIONS

But very little is known of how the bacteriophage behaves in infectious processes due to these Gram-positive cocci, but that the bacteriophage is found in association with such infections is beyond question.

I have reported ${ }^{321}$ the isolation of a bacteriophage virulent for the staphylococcus from an abscess after a bite on the finger made by a guinea pig. Gratia ${ }^{258}$ isolated a bacteriophage from an abscess on the face. Callow ${ }^{135}$ recovered a race from the pus in a case of furunculosis, and Kropveld ${ }^{397}$ invariably found it in furunculosis and in the pus of anthrax cases.

The behavior of the bacteriophage in staphylococcus infections offers a very interesting field for study.

In 2 cases where $I$ isolated races from the sanguineous pus of anthrax lesions, opened at the beginning of the fluctuation, the races found showed, in addition to a virulence for Staphylococcus aureus, a very definite virulence for some colonies of $B$. coli isolated from the stools of the same individuals.

In studying some cases of streptococcus angina, Piorkowski ${ }^{526}$ demonstrated a bacteriophage in scrapings from the tonsils. From his studies he concluded that when the virulence of the races for the streptococcus was high it should be considered as a favorable sign.

Eichhoff has carried out a series of investigations, in Küttner's clinic in Berlin and at the Institute of Hygiene at Breslau, in which he isolated races of the bacteriophage virulent for the staphylococcus and for the streptococcus from the pus of various types of lesion, as well as from the stools of patients recovering from infections due to these organisms. Incidentally, he showed that certain races of the bacteriophage virulent for B. dysenteriae Shiga exert a destructive action upon the staphylococcus and the streptococcus.

\section{THE BACTERIOPHAGE IN COLON BACILLUS INFECTIONS}

Sometimes the colon bacillus may become pathogenic and may be encountered as the etiological agent in diverse localized infections, or even in septicemias. It at first appears strange that so common an organism, a normal inhabitant of the intestine, should at a particular time develop pathogenicity. There must be "a something" which differentiates the pathogenic $B$. coli from the banal $B$. coli. It is this which I_have tried to determine. 
Five specimens of infected urine secured from individuals with pyelonephritis have been examined. In all of these cases not only was the colon bacillus present, but there was a mixed culture of $B$. coli and the bacteriophage, as shown by inoculation of the urine on agar. In one of the cases simple plating of the urine on agar gave a colon culture studded with plaques, in the other four, agar cultures made after a bouillon growth gave the same appearance. The colon bacillus possessed a high resistance, although it was not entirely refractory. Thus the struggle continued in the organism. The ordinary $B$. coli is not pathogenic. The resistant $B$. coli becomes so because of its resistance to the action of the bacteriophage.

Tomaselli examined 5 urines derived from patients with pyelonephritis, and in the 5 cases he found, as had $I$ in other cases of this type, a mixed culture of bacteriophage- $B$. coli. On the contrary, in cases of simple cystitis without renal lesions he observed that the bladder contained only an ultrapure culture of $B$. coli, that is, the bacteriophage was not present. As an incidental point this author suggests that this fact may perhaps be of diagnostic significance in detecting the presence of a renal lesion.

My own studies permit me to confirm the statement that the bacteriophage is absent from the urines of cases with a simple cystitis, although the number of cases which I have examined (eight) does not warrant a final conclusion. Aside from the 5 cases of pyelonephritis mentioned above, I have since seen 7 other cases of $B$. coli infection of the urinary tract in which the cystitis was accompanied by renal lesions, and in all of these a mixed culture was found.

This introduces a new idea as to the behavior of the bacteriophage,to be added to those which we have acquired in the study of the diseases previously discussed. B. coli certainly does not possess a highly developed pathogenic tendency. We know, for example, that in certain animals (horses) this bacillus normally passes into the blood during the digestive period, and that in spite of this, it never causes any lesions, a proof that it is quickly eliminated,--probably by phagocytosis. For what reason, then, does this bacillus sometimes become implanted in a lesion?

The observations recorded above offer the answer to this question. The infection occurs,-and persists,--because the bacillus is introduced as a mixed culture, and consequently is a bacterium resistant to bacteriophagy. Against such a bacterium the body is powerless. We have already shown by in vitro experiments that a bacterium resistant to the bacteriophage is likewise resistant to phagocytosis. 


\section{THE BACTERIOPHAGE IN TYPHUS EXANTHEMATICUS}

Having learned from the above brief discussion of the behavior of the bacteriophage in colon bacillus infections that under certain circumstances the bacteriophage may assume a new and distinctive rôle in the origin of an infectious process, let us now turn to an entirely different disease, typhus fever, where we will discover still another aspect of the complex question of the bacteriophage and bacteriophagy.

For our knowledge of the behavior of the bacteriophage in this disease Fejgin ${ }^{201,202}$ is largely responsible. She began by isolating a bacteriophage from an old strain of $B$. proteus $X_{19}$. The virulence of this race she enhanced by repeated passages. In studying the mutant strains of $B$. proteus which form in secondary cultures* she found that filtrates of these cultures often became turbid because of the development of bacteria identical with the mutant forms previously studied.

Continuing with the problem, she injected guinea pigs with the clear filtrates and found that animals so inoculated presented the same thermic reactions as did guinea pigs inoculated, either with the blood of patients with typhus exanthematicus, or with virulent materials derived from passage guinea pigs. Such a finding is of extreme interest, but her next observation is still more strange, for she found that guinea pigs injected with these filtrates were thereafter immunized against injections of virulent materials, whether the latter were derived directly from a patient with typhus fever or from passage guinea pigs. Reciprocally, a guinea pig which had reacted to the typhus material was refractory to the filtrates of the $B$. proteus $X_{19}$ bacteriophage.

And, as though to add further interest to these results, she found that from guinea pigs bled on the $3 \mathrm{rd}$ or 4 th days of the febrile period which followed the injection of a filtrate she could isolate from the blood and from the organs (using the egg medium of Besredka under anaerobic conditions) the same mutant forms as she had previously recovered, either from the cultures which developed in the filtrates or from the organs of guinea pigs inoculated with material derived from patients having typhus fever.

As I stated above, if these studies are confirmed,-and it seems, indeed, that this work of Fejgin was carefully done,- they will explain both the etiology of typhus exanthematicus and the relation which exists between the agent of this disease and $B$. proteus $X_{19}$.

* We have considered the studies of Fejgin on the mutant forms in an earlier chapter. 
Fejgin has not, however, drawn any very definite conclusions from her studies, certainly because of a false conception of bacteriophagy. She simply states that there must exist some relation between typhus exanthematicus, $B$. proteus $X_{19}$, and the bacteriophage.

Having recourse to the facts which have been presented in the first part of this text, the etiology of typhus fever could be explained as follows: The $B$. proteus $X_{19}$, developing as a mixed culture with the bacteriophage, becomes transformed into its micellar ultrabacterial infravisible form, ${ }^{*}$ and these ultrabacteria, capable of reproducing in this form, would really be the agents of typhus exanthematicus.

The bacteriophage protobe is not a providential agent found in the intestine, in order to assure the "defense" of the individual who harbors it against the bacteria which try continually to invade it. The bacteriophage protobe is simply a parasite of bacteria, occurring by preference in the intestinal tract because it there finds the most favorable conditions for its development, and in this it behaves like all living beings which multiply in that place where they are best able to exercise their faculty of assimilation. The bacteriophage does not exist for an "end," and if, generally, it is a cause of recovery from disease because it is able to exercise its faculty of assimilation of pathogenic bacteria, it is equally able in other cases to be an indirect cause of disease.

For an analogy we have only to turn to the white blood cell. The phagocyte is a "salutary agent" when it engulfs a microbe which it can digest; it is an "agent of destruction,"- even of death,-when, en-

* We have seen in Part I that this is a common phenomenon which may be observed with all bacteria, and that the micellar form which is produced occurs as a reaction of the bacterium to the presence of the bacteriophage.

In speaking of these ultrabacteria I suggested a hypothesis on the subject of the agent of scarlet fever. Opportunities to apply this hypothesis are abundant. For example, it would be strange if the Leptospira discovered by Noguchi is really the agent of yellow fever (a disease with which I am familiar from having studied it in Yucatan). I have spent weary days with my eye to the microscope examining slides of the organs and tissues of individuals dead of yellow fever, and never yet did I see a single Leptospira. All of this would be explained if the true parasite is a filtrable form of the Leptospira of Noguchi. As a matter of fact, the spirochetes are undifferentiated microbes, which must be, like the bacteria, micellar plasmodia. ${ }^{349}$

Insofar as typhus exanthematicus is concerned, there is an opportunity to attempt treatment by the injection of a mixture of different races of the bacteriophage, virulent for $B$. proteus $X_{19}$, if among the races available there are any virulent for the proteus strain found in the patient. 
countering on a mucous surface a bacterium which it can not digest, it phagocytizes it and carries it into the body.

The bacteriophage protobe is a "salutary agent" when it causes bacteriophagy in vivo; it is harmful to the individual who harbors it when it forms a symbiotic relationship with a microbe which has an acquired resistance to bacteriophagy. Such a symbiosis causes the bacterium to suddenly acquire a resistance to phagocytosis, and it also confers upon the bacterium the property of yielding "ultrabacterial" filtrable forms, as appears to be the case in typhus fever, in typhoid fever, and doubtless in other diseases.

Within the body, there are not "reactions of defense;" there are simply reactions, salutary or harmful according to the conditions under which they take place.

\section{THE BACTERIOPHAGE IN PLANTS}

We know that on the roots of the Leguminosae we find nodules which are due to the reaction of the plant to the presence of nitrifying bacteria, $B$. radicicola. Here there is a symbiosis between the plant and the bacterium, but it is a symbiosis in the true sense of the word, that is to say, it is a mitigated parasitism. B. radicicola attempts to invade the tissues of the plant but there is some "cause" which prevents this invasion.

Many authors have sought for this cause. They have observed that in certain cases an alteration of the bacteria takes place; the plant, they have said, imposes a teratological form on the bacterium, and sometimes succeeds in eliminating it.

Zinzer* inoculated cultures of $B$. radicicola into the stems of the Leguminosae and found that the bacteria were destroyed without becoming implanted in the tissues. On the other hand, Giornini, while studying the tumor of the olive tree, saw that the "tissues" in the region of the tumor possessed agglutinating properties, and even destructive properties, for the pathogenic bacterium.

In this destruction, the tissues of the plant did not effect the phenomenon without some agency, and the nature of this has been shown by the work of the Dutch authors, Gerretsen, Sack, Gryns, and Söhngen. ${ }^{228}$

They worked with the nodules found on the roots of clover, sarrandella and lupin. They washed these nodules in a 1 per cent mercuric chloride

* Zinzer.-Ueber das verhalten von Bakterien, insbesondere von IInöllschenbakterien in lebenden pflanzlichen Geweben. Jahrb. f. Wiss. Bot., 1897, 30, 423. 
solution, in alcohol, and finally in distilled water. Fragments of the nodules were placed on agar seeded with cultures of $B$. radicicola, and they noted that after incubation each fragment occupied the centre of a circle within which no growth had taken place. The action was specific; a fragment of a given nodule had an action only upon the strain of $B$. radicicola derived from a plant of the same species.

They subjected these nodules to extraction and made filtrates. These filtrates contained races of the bacteriophage of high virulence, each race being in general virulent for only those strains of $B$. radicicola isolated from plants of the same species. But this specificity was not quite absolute, for a bacteriophage isolated from the nodules of clover, as also another race isolated from lupin, had a weak, but definite, virulence for a strain of $B$. radicicola obtained from the nodules of the bean. They were able to isolate races of the bacteriophage virulent for $B$. radicicola from field soil, but did not find them in the earth of uncultivated areas or forests.

Like all other races of the bacteriophage, the corpuscles passed through collodion membranes sufficiently permeable to allow the micellae of serum globulin to pass. This explains the diffusion of the bacteriophage throughout the different parts of the plant, for these authors have noted its presence in the stems, but not in the leaves. They concluded that the fact that $B$. radicicola does not invade the entire plant is due to the presence in the plant of a virulent race of the bacteriophage.

With plants, as with animals, antibacterial immunity is "exogenous;" it is the bacteriophage protobe. This is a general fact, throughout nature.

\section{RÉSUMÉ}

The observations made in bacillary dysentery, in the typhoid and paratyphoid fevers, and in avian typhosis, show that the behavior of the intestinal bacteriophage toward a pathogenic bacterium which becomes implanted in the intestinal tract varies; being determined, (a) by the conditions found in the intestine, and (b) by the hereditary characters of the bacteriophage and of the bacterium.

The behavior of the bacteriophage may be summarized as follows:

All of the conditions of medium and of characters working to the advantage of the bacteriophage, its virulence immediately increases for the invading pathogenic bacterium; the result being the immediate elimination of the bacterium before it can develop within the individual and cause any disturbance. 
The conditions being less favorable for the bacteriophage, its virulence increases less rapidly, bacteriophagy takes place after a longer latent period; the result being that after a few mild symptoms, the disease is aborted.

When the virulence of the bacteriophage is built up only after a considerable delay, the disease follows its course up to that time when the acquired virulence permits a complete bacteriophagy; the result being that at this time the symptoms of the disease regress, disappear, and the individual enters upon convalescence.

When the virulence of the bacteriophage is enhanced very slowly and the delay in increased activity permits the bacterium to acquire a resistance, or when the bacterium at the time of invasion already has a resistance acquired outside of the body now invaded, a struggle takes place, the contending forces being the virulence of the bacteriophage on one hand and the resistance of the bacterium on the other, and the condition of the patient reflects the ups and downs of this campaign. The result here may vary; if the conditions of environment and heredity favor the bacteriophage, the virulence permanently overcomes the resistance, complete bacteriophagy takes place; convalescence is established. If, on the contrary, the conditions favor the bacterium, it acquires a refractory state, it is able to develop without hindrance; the individual dies.

When, as a last possibility, the intestinal bacteriophage remains inert, the bacterium develops freely without any necessity for acquiring a resistance; the result being the death of the individual

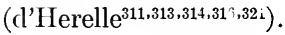

The bacteriophage does not remain confined to the intestine; it passes into the circulation, from there into the tissues, and thence to the lesions where bacteria are to be found upon which it can exert its faculty of assimilation; the result being an in vivo bacteriophagy with an elimination of the invading microbe. This is what takes place in experimental typhoid infection of the rat (d'Herelle ${ }^{313}$ ); in avian typhosis (d'Herelle $^{321}$ ); in bubonic plague (d'Herelle ${ }^{321}$ ); in typhoid fever (Beckerich and Hauduroy ${ }^{57}$ ); in staphylococcus infections (d'Herelle ${ }^{321}$ ); and in streptococcus infections (Eichhoff).

The process of in vivo bacteriophagy is not restricted to animals. It also occurs in plants, as in the case of the Leguminosae infected with B. radicicola (Gerretsen, Sack, Gryns, and Söhngen ${ }^{228}$ ). 


\section{CHAPTER IV}

\section{The Behavior of the Bacteriophage in Epidemics}

\section{AVIAN TYPHOSIS}

In the preceding chapter we have dealt with the behavior of the hacteriophage protobe within the intestinal tract of animals having the disease avian typhosis. Let us now see what happens in those animals which live in regions where this disease is prevalent but which resist infection.

Let us consider first a fact bearing on the territory involved in the epizootic. During the period involved in this study eighty-one examinations were made upon the feces of barn-yard animals, not only in France but also in Indo-China, in regions where avian typhosis had not occurred in epidemic form among the fowls for several years. In each of these examinations a bacteriophage active for one or several of the bacilli of the colon-typhoid-dysentery group was isolated, but in no instance has the bacteriophage shown any detectable activity for $B$. gallinarum.

In contaminated regions the situation is quite different. As an example, observations made on a farm located at Pougy-sur-Aube may be cited, where the disease was followed very closely. The disease appeared in 1917 in July. Within the period of a month fifty-one of the ninety-eight fowls died; then the epizootic disappeared. In May, 1918, it reappeared in less violent form. Twenty-five of one hundred and four fowls died in the period from May to September, and it again disappeared. In 1919 it broke out again early in April. On the 21st of May, twenty-one of eighty had died. At this time I beganmy observations.

On May 21, specimens of the excrement of thirty of the fifty-nine survivors were taken. Examination made later in the laboratory, showed in twenty-six a bacteriophage of weak or moderate activity for B. gallinarum ( 23 were,+ 3 were ++ ), in four it was absent. On May 22 , two chickens contracted the disease. The specimens taken the day before were numbered and examination showed that an active bacteriophage had not been found in these two animals. On May 23 one of the two chickens affected the day before died. On May 24, a third chicken, sick in the morning, died in the following night. Its excrement, col- 
lected on May 22, did not contain a bacteriophage active for B. gallinarum. On the morning of May 24 the chicken which had been taken sick on May 22 and which had resisted showed in its intestinal contents a bacteriophage of extreme activity $(++++)$ toward the pathogenic bacillus. On May 26 the fourth chicken, one of those whose feces had not showed an active bacteriophage when examined on May 22 , was affected. It resisted, and on May 28 its symptoms had disappeared. The disease disappeared suddenly and during the next three months no new cases developed.

On May 30 the feces of thirty chickens were examined and the following results were obtained:

Virulence for B. gallinarum; in five ++++ , in twenty-one +++ , in four ++ .

We see, then, on May 22, four animals among thirty in which the intestinal bacteriophage lacked activity for the pathogenic bacillus. These four animals contracted the disease during the four following days. In the twenty-six specimens collected on May 22 and showing positive results, the bacteriophage showed a relatively weak virulence. Nine days later this activity was very much greater, that is, at the time when the epizootic ceased. What, then, took place in this interval? The bird which became sick on May 22 and which resisted showed in its feces, when examined on May 24, a bacteriophage endowed with a considerable activity for the pathogenic agent.

Here is a second example of the same general nature, giving the results secured on farm M. . . . . at Véricourt (Aube). The epizootic first appeared among the flock of twenty-five chickens in May, 1919. The first animal died on May 18. On the next day twelve specimens of excreta were collected at random. Three only contained a bacteriophage, and that of feeble activity, for B. gallinarum. From May 19 to 26 twelve birds contracted the disease and of these eleven died. One, which became sick on May 23, showed on May 25 a strongly active bacteriophage $(+++$ for $B$. gallinarum $)$ and recovered. The epidemic stopped abruptly. On May 27 twelve specimens were taken at random. In all a bacteriophage active for B. gallinarum was found (in $1++++$, in $9+++$, in $2++$ ).

A third example may be mentioned, in which the infection was a paratyphosis.* On October 15, strains of $B$. pfaffi were isolated from two specimens of blood, taken from animals which had died in a chicken-

* These experiments were carried ont with the assistance of M. Micheau, D. V. M. at Trainel (Aube). 
yard where for about a month there had been an infection presenting the characters of typhosis. From specimens of the feces taken from two healthy animals living in the same yard two races of bacteriophage were isolated, one showing a low virulence $(+)$ for $B$. pfaffi, the other showing no activity for this bacillus. Towards the end of the month three chickens became sick, recovered after an interval of two or three days, and then the epizootic ceased. Six specimens of feces examined at this time all showed a bacteriophage of high virulence $(+++)$ for $B$. pfaffi. Against B. gallinarum four were inactive and two showed a weak virulence $(+)$.

B. pfaffi was therefore the cause, for when the epizootic broke out three months later the eighty chickens which had survived received a subcutaneous injection of 0.5 cc. of a suspension of the Pfaffi-bacteriophage and the epidemic stopped abruptly and permanently from the time of the injection. We will see later that this abrupt cessation is the rule following immunization by means of a suspension of the bacteriophage.

These facts can be explained in only one way. A weak or moderate activity of the intestinal bacteriophage for the pathogenic bacterium is sufficient to render the animal resistant to infection. The pathogenic bacteria which are able to penetrate into the intestine are destroyed before they can multiply. But it is not the same once the disease has appeared and the organism is invaded. The animal recovers-and recovery is very rare in typhosis - villy because of a rapid adaptation of the bacteriophage and the acquisition of a high virulence which leads to an intensive destruction. This bacteriophage with exalted virulence is distributed broadcast with the excreta of the recovered or convalescent animals, and persists, indeed, during several months after recovery. This bacteriophage is necessarily ingested by the other animals of the barn-yard which become, in fact, "infected" by an extremely active bacteriophage and by this means acquire a complete protection against the disease, in spite of the presence of the pathogenic organism in the environment, and in spite of its frequent ingestion, an ingestion which serves to maintain the virulence of the bacteriophage.

These hypotheses are not simply idle speculation, for the interpretation given to these observed facts is confirmed by experiments which provide, in a controlled manner the natural conditions of the epizootic. Furthermore, it will be seen that the rôle of defense assigned to the bacteriophage is confirmed by the immunization of several thousand animals by the administration of suspensions of an active bacteriophage. 
Before discussing these control experiments I ought to mention that, thanks to the kindness of the veterinarians of different regions invaded by typhosis, I have been able to procure numerous specimens of blood and excreta taken from sick chickens, from chickens which had died or from those which had recovered, derived from eleven different foci scattered throughout all France. This allows me to generalize from the facts that I have personally observed.

\section{Control experiments}

The control experiments have been conducted in Paris, that is to say, entirely outside of the epizootic area.

Six chickens, procured from a region free of infection, were placed under observation. Their excreta were examined daily for ten days for the purpose of establishing the complete absence of a bacteriophage active for $B$. gallinarum.

Chicken No. 1 then received, per os, 1 cc. of a suspension of a race of bacteriophage very active for B. gallinarum $(++++)$.

Chicken No 2 received 0.5 cc. of the same suspension by subcutaneous injection.

The next day examination of the feces of these two animals showed the presence of a bacteriophage strongly virulent for B. gallinarum. Therefore, the bacteriophage passed into the intestine, whether ingested or injected. This same fact has since been verified with man and with different animals.

Chicken No. 1 next received per os daily for twenty-five days, 2 cc. of a bouillon culture of $B$. gallinarum. The active bacteriophage persisted in the intestine with its primary virulence $(++++)$ and maintained itself up to nine days after the last dose of the pathogenic organism.

Chicken No. 2, which had received nothing after the inoculation of the active bacteriophage ceased to show a race active for $B$. gallinarum within three days after the injection. In other words, chicken No. 1, subjected to repeated reinfections, retained an intestinal bacteriophage active for B. gallinarum for thirty-four days, while chicken No. 2, not infected, for only three days.

It follows that the intestinal bacteriophage remains active only if it is able to develop in the intestine at the expense of this bacterium, but in such a case it remains active just so long as this condition is fulfilled. Inversely, the presence in the intestine of a bacteriophage possessing virulence for a given bacterium indicates that this bacterium was a short time previously in the intestine. 
In the course of the preceding experiment chickens Nos. 3 and 4 were placed in contact with chicken No. 1 . They all ate and drank from the same containers, the more so since they were changed about in the pens in such a manner as to simulate conditions of life analogous to those of the chicken-yard. Two days after the first contact, in the case of chicken No. 3, three days after with chicken No. 4, their excreta contained a bacteriophage very virulent for $B$. gallinarum $(++++)$. From this time on they each received each day for twenty-one days, 2 cc. of a bouillon culture of $B$. gallinarum. At no time did they appear sick. The intestinal bacteriophage remained active for the bacillus throughout the entire period of the administration of the pathogenic bacillus, and even longer - seven days in No. 4 and ten days in No. 3 . The intestinal bacteriophage did not then disappear, for as in the case of chickens Nos. 1 and 2, it remained active for one or several members of the colon-typhoid-dysentery group. But the virulence for B. gallinarum did not persist when the ingestion of cultures of this last bacillus was stopped. The experiment with chickens Nos. 3 and 4 shows clearly that the bacteriophage protobe is infectious in exactly the same sense as is the pathogenic bacillus itself, since these birds were "contaminated" by contact with chicken No. 1 .

Chickens Nos. 5 and 6 , which had not been in contact with the other chickens, and which on repeated examinations were shown to be free of a bacteriophage active for B. gallinarum, each received per os, on some bread, a single dose of 2 cc. of a bouillon culture of $B$. gallinarum. Three days after the infecting meal diarrhea appeared and they died two and three days later, after having shown all of the symptoms of the natural disease. Necropsy showed the presence of the same lesions. Cultures of the blood gave pure cultures of the pathognic bacillus, which was likewise found in abundance in the intestinal contents.

Chickens Nos. 1, 3, and 4, which had resisted repeated ingestions of $B$. gallinarum culture without showing the least inconvenience, were therefore immunized; the first as a result of the ingestion of a bacteriophage active for the pathogenic bacterium, the two others by simple association with the first.

About one month after the virulence of the bacteriophage for $B$. gallinarum had disappeared in chickens Nos. 1, 2, 3, and 4 each of them was given on each of the three days 2 ec. of a culture of the bacillus. In all the intestinal bacteriophage showed a new virulence for the pathogenic organism. None of them showed the slightest trouble. 
In all of these experiments the infections have been made with bouillon cultures of B.gallinarum prepared directly from the blood of chickens dead of spontaneous natural infection. This is essential because of the loss in virulence of this organism which takes place under artificial cultivation.

With chickens Nos. 5 and 6 the ingestion of the pathogenic bacillus caused a fatal attack of typhosis. The intestinal bacteriophage at no time manifested an activity for the causative organism. In chickens Nos. 1, 2, 3, and 4, on the contrary, the ingestion of the same culture caused no disturbance and their intestinal bacteriophage which for about a month had showed no activity for the bacillus, rapidly recuperated its first activity. It has, therefore, not disappeared from the intestine, although its activity was no longer evident, but when it found itself again in contact in the intestine with the pathogenic organism it rapidly regained its potency.

This "latent virulence" may be maintained for a very long time. In this connection I may recall the fact cited of a strain of bacteriophage still possessing after three years and more than 1000 passages in vitro, always with the Shiga bacillus, the power to attack B. coli and B. typhosus. It showed a weak power, but was capable of rapid augmentation by transfers at the expense of these organisms. This is exactly what this experiment shows us to take place in vivo in the chicken.

Can a chicken contract typhosis in spite of the presence of an active bacteriophage in the intestine? It certainly can. As we have seen in many experiments the bacterium may develop a resistance to the action of the bacteriophage and this resistance is one of the factors comprising the virulence of the bacterium. We have then, on the one hand, the bacterium, which when introduced into the organism may acquire a resistance to the action of the bacteriophage ranging from zero to absolute resistance, and on the other hand, the bacteriophage, which at the same time may possess a virulence running from zero to extreme activity. Infection occurs, or does not occur, according to whether the algebraic sum of virulence + resistance is in favor of the one or the other of the two organisms present. Once the disease has manifested itself, the virulence of the one and the resistance of the other become increased or attenuated according to the conditions of the moment and the aptitudes previously acquired which favor the one or the other of the two agents. The sequence in which the events of this struggle occur determine the issue.

The observations made in natural discase and the experiments which 
confirm the deductions which these observations suggest, show that the bacteriophage protobe is always present in the intestine of the chicken, whether it is healthy or sick, whether it lives in a locality free of infection or in an epizootic zone.

Against a definite bacterium, B. gallinarum in so far as avian typhosis is concerned, the intestinal bacteriophage may be virulent or avirulent, and in the first case its virulence may be exercised according to a scale which passes from the smallest degree capable of detection to one of extreme activity.

Virulence of the bacteriophage protobe for B. gallinarum is only observed in an infected locality. The absence of such a virulence is equally the rule with animals which are about to die and with those which have died.

In a contaminated area animals which harbor in their intestine a bacteriophage endowed with sufficient virulence for the pathogenic bacterium are by this very fact protected against the disease, and they remain so, provided the actual or latent virulence of the bacteriophage is maintained at a level sufficiently high to effect a rapid destruction of the pathogenic bacilii ingested.

The ingestion of pathogenic bacilli at sufficiently frequent intervals constitutes the principal factor in maintaining the virulence for the given bacterium. Among the factors which contribute to diminishing the virulence or causing the virulence of the bacteriophage for a pathogenic bacterium to disappear, I would place as most significant the introduction into the organism of bacteria endowed with a resistance to the action of the bacteriophage. We have clearly seen this fact in the course of the experimental study of the phenomenon of the resistance of bacteria. Another possible factor, influencing the activity of the bacteriophage is the reaction of the medium in the intestine, which may vary according to the accidental conditions of the moment, the type of food, etc. The importance of the reaction of the medium for the dissolution of bacteria in vitro has already been demonstrated.

A bacteriophage which has lost its virulence for the pathogenic bacterium lacks the power to exercise it because of the absence of this bacterium, but it possesses, nevertheless, a latent virulence. When placed again after a greater or less length of time in the presence of this bacterium it regains its original virulence.

The fact of the habitual virulence of the intestinal bacteriophage for $B$. gallinarum in the infected regions indicates the frequency of the ingestion of these bacilli, and consequently the excessive contamination of the environment by the pathogenic organism. 
In contaminated regions the animal in which the intestinal bacteriophage does not enjoy any activity for B. gallinarum quickly contracts the disease. It may resist and recover, but this is the exception, occurring only when the intestinal bacteriophage quickly acquires a virulence for the infecting bacillus. In the contrary, and usual, case the animal succumbs.

In a chicken which recover's, the intestinal bacteriophage acquires a considerable virulence against the pathogenic bacterium and maintains this for a very long time; in fact, as long as the exterior environment remains infected. This persistence of virulence is maintained by the frequent ingestion of pathogenic organisms, which allow the bacteriophage to multiply at the expense of the particular organism. The resistant animal disseminates in its excreta the bacteriophage of enhanced virulence; the animals which associate with it become "contaminated" and by this fact they enter the same class of resistant animals as those which have recovered. Recovery of one animal in a barnyard often marks the end of an epizootic, or its arrest for a few months.

The study of an epidemic of a vian typhosis shows, in a word, that the history of the contagion reflects, in the last analysis, the story of the struggle between the two agents - the pathogenic bacterium and the bacteriophage protobe-and since this last is transmissible from individual to individual the immunity is contagious in the same sense as the disease itself. The beginning of an epizootic is marked by a diffusion of the bacteria, the end by a diffusion of bacteriophage virulent for these bacteria. We will encounter the same facts in another disease; in hemorrhagic septicemia in the buffalo (d'Herelle ${ }^{321}$ ).

\section{2. hemorRhagic SEPTicemia of the BUfFalo (BARbone)}

\section{Barbone, the disease}

Unlike avian typhosis barbone does not present intestinal symptoms; it is of the hemorrhagic septicemia type. The pathogenic organism is a Pasteurella. Cultures of the organism in beef bouillon maintain their virulence for a considerable time at least eighteen months. The inoculation of a buffalo or of a cow with $0.0002 \mathrm{cc}$. of a virulent culture kills the animal in between thirty-six and forty hours with all the symptoms of the spontaneously acquired disease. At necropsy identical lesions are found and the pathogenic bacterium swarms in the blood and in the organs.*

* In two different attempts I have proved that diluted blood or macerations of organs (liver and lung) taken from animals dead of spontaneous infection, filtered through a Chamberland filter $\left(\mathrm{L}_{2}\right)$ and inoculated in large amounts into the buffalo or into cattle do not cause the slightest disease symptoms. 
The buffalo is par excellence the beast of burden in the cultivation of rice-fields; it replaces the ox in all southern Asia and in the islands of the Sunda Straits. It is utilized in certain regions of Italy, in Egypt, in Hungary, and in the Balkans. Wherever the buffalo lives there also will be found barbone, the most terrible, without doubt, of all the contagious diseases. The reports indicate a mortality of from 70 to 95 per cent. I was present during an epizootic which raged in June, 1920, in the Province of Bac Lieu (Cochin-China) where among the thirty thousand buffaloes of the region ten thousand died, and I did not have an opportunity to observe a single animal which recovered. Recovery may occur, but it is certainly rare, and the mortality in Cochin-China is certainly above 99 per cent of the animals affected.

The average duration of the evolution of the disease is but eighteen to twenty-four hours; rarely thirty-six. Death sometimes takes place without precursory symptoms. An animal yoked to a plow stops, remains motionless for a few moments with a haggard aspect and then falls as though struck by lightning. In typical cases, which can be reproduced in a perfect manner in experimental infection, the animal appears dejected, the eyes fixed, the head lowered. The temperature rapidly mounts to 41.5 to $42.5^{\circ} \mathrm{C}$., the respiration, at first accelerated, becomes slowed and then dyspneic, the inspirations less and less frequent. The animal shows meteorism; it lies flat on the ground in complete lateral decubitus usually a short time before death which is preceded by cramps and at times convulsions.

Often tumefaction is to be observed, appearing usually in the region of the throat and extending back to the shoulder. The engorgement is produced by a gelatinous exudate of a yellow color within the connective tissue. At times the tumefaction appears in another part of the body, or it may be entirely lacking. This tumefaction, as shown in experimental infection, marks the portal of entrance of the pathogenic bacteria. Infection usually occurs by way of the digestive tract and the virus most frequently penetrates the tissues through some portion of the nasopharynx. A tumefaction on another part of the body-thigh, abdomen, rump - indicates a reinfection by the penetration of the virus through an excoriation. Examination of cadavers shows that the absence of tumefaction indicates an infection by way of the stomach and intestine.

Bovines and the buffalo are equally susceptible, as was noted a long time ago by Piot in Egypt. The statistics of Indo-China indicate, it is true, that the mortality from barbone is but slight for cattle, but this 
is solely due to the fact that these animals are present in but small numbers in the regions where barbone rages; regions which are extremely humid and admirably adapted to the buffalo, a semi-aquatic animal. The rare cattle found sometimes in such regions contract the disease and die like the buffalo, after having presented identical symptoms.

The effect of low places and swamps on the contagion has been from time immemorial recognized by the natives. When it is possible, as soon as a case of barbone is detected in a neighborhood, they hasten to collect their animals and remove them to a more elevated region. It is known, moreover, that the organisms of the Pasteurella group remain virulent for a very long time in the mud of the marshes and in the slime of the streams.

\section{Rôle of the bacteriophage in the disease}

In Cochin-China barbone is always present in sporadic form causing each year numerous small epizooties which remain localized in individual villages. A localized epidemic observed in Long Huu in the Province of Gocong may serve as an example.

From May 5 to 13, 1920, seventeen buffaloes died: on May 5, one; May 7, three; May 8, two; May 9, one; May 10, two; May 11, four; May 12, three; and May 13, one. Then the epizootic stopped and not a single case was detected during the next six months.

Specimens of the feces of four of these animals were collected, either before death or from the cadaver. None contained a bacteriophage active for the bacterium of barbone. On May 13 specimens of feces were collected from healthy animals, as follows:

First. From a buffalo in a stable where two animals had died, one on May 12, the other on May 13.

Second. From three buffaloes in a stable where one had died on May 5.

Third. From two buffaloes in a stable where two had died, one on May 8, the other on May 11.

Fourth. From four buffaloes in a stable which had not been invaded.

Fifth. From one buffalo, living alone in a stable located at a distance of about five kilometers from the village of Long Huu.

Sixth. From eight buffaloes in the surrounding villages, from eleven to nineteen kilometers distant.

Of all the specimens, those in the first, second, third and fourth groups gave a bacteriophage of weak or average activity $(+$ or ++$)$ for the bacterium of barbone. An active bacteriophage was not found in the specimens from groups 5 and 6 . 
Again on May 19 specimens were collected in Long Huu, as follows:

First. From the buffalo which had furnished specimen No. 1 on May 13.

Second. From two buffaloes living in a stable where three had died from May 7 to May 12. These specimens all gave a bacteriophage moderately virulent $(++)$ for the bacterium of barbone.

The animals which resisted, therefore, showed in their intestine a bacteriophage virulent for the pathogenic bacterium.

The epizootic does not always remain localized in a village. At times it spreads rapidly from village to village and within a few days will extend over a very considerable territory. It is rarely possible to determine the primary focus, so great is the speed with which it spreads. The mortality then becomes considerable, the losses often amounting to tens of thousands of animals, as has been observed many times in China, in British India, and in the Dutch East Indies. Sometimes even, as actually happened in Java, the buffalo, as a race, is practically eliminated.

In the first two weeks of June, 1920, the epizootic became general in the Province of Bac Lieu and in certain parts of the adjacent provinces (western Cochin-China). It was possible to examine the blood of eleven animals which died in widely scattered parts of the area invaded, and in all the bacterium of barbone was found in considerable quantity.* The epizootic died out during the first fortnight of July. It had persisted for a month, killing a third of the animals in the district.

The region of Thoi Binh was particularly affected, the loss amounting to more than fifty per cent of the buffaloes in the locality. From July 8 to 13 , at the time when the epizootic was disappearing (the last animal to be affected died on July 12), twenty specimens of feces were collected. These were taken from buffaloes which had resisted the infection and which at no time showed any evidence of the typical symptoms of the disease. All of the animals examined lived on the farms of the village of Thoi Binh or in the neighboring hamlets within a radius of fifteen kilometers.

Tests for the virulence of the intestinal bacteriophage against the bacterium of barbone gave the results shown in table 62 .

* Bacteriological diagnosis is easy, even if the only available material is some blood or a fragment of an organ taken without any special precautions in the field, as is usual in such eountries. Even if the specimen is some days old it is only necessary to smear it over the shaved skin of a rabbit. If the bacterium of barbone is present the animal will die within 24 hours, and the organism will be found in pure culture in the blood, from which it may be readily isolated. This is also the best method for detecting the bacterium in soil or in fecal material. 
From this it appears that the intestinal bacteriophage is endowed with virulence for the bacterium of barbone in all the buffaloes which the disease had spared.

In the course of different trips across Indo-China, I collected fortyone specimens of feces from buffaloes, each specimen collected in a different village in which no buffaloes had died of barbone for at least two years. In only three of these specimens could a bacteriophage active for the bacterium of barbone be demonstrated, and in these cases it was

TABLE 62

\begin{tabular}{|c|c|c|c|c|}
\hline FARM & MORTALITY & $\begin{array}{c}\text { THE LAST } \\
\text { [ANIMAL DIED } \\
\text { ON }\end{array}$ & $\begin{array}{c}\text { NUMBER } \\
\text { OF ANIMALS } \\
\text { WHICH } \\
\text { RESISTED }\end{array}$ & $\begin{array}{l}\text { VIRULENCE } \\
\text { OF THE } \\
\text { BACTERIO- } \\
\text { PHAGE }\end{array}$ \\
\hline 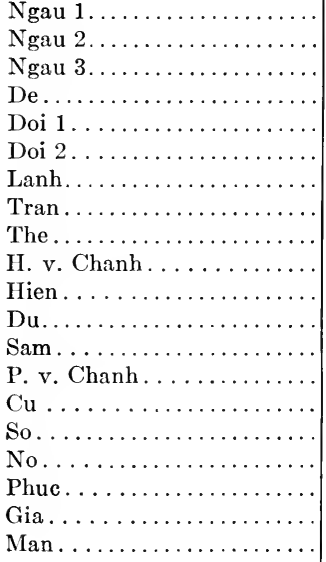 & $\begin{array}{l}2 \\
5 \\
9 \\
1 \\
3 \\
2 \\
0 \\
0 \\
2 \\
6 \\
1 \\
3 \\
5 \\
8 \\
8 \\
1\end{array}$ & $\begin{array}{l}\text { July } 7 \\
\text { June } 10 \\
\text { June } 28 \\
\text { July } 2 \\
\text { July } 4 \\
\text { July } 11 \\
\text { July } 2\end{array}$ & $\begin{array}{r}1 \\
4 \\
\\
4 \\
2 \\
1 \\
2 \\
4 \\
6 \\
8 \\
8 \\
5 \\
5 \\
10 \\
4 \\
3 \\
3\end{array}$ & $\begin{array}{l}++ \\
+ \\
+++ \\
+ \\
++++ \\
+++ \\
++ \\
++++ \\
++++ \\
++++ \\
++++ \\
+ \\
++ \\
++++ \\
+++ \\
++ \\
+++ \\
+++ \\
+++ \\
++\end{array}$ \\
\hline
\end{tabular}

weak $(+)$. Nevertheless, the intestinal bacteriophage was present in all; but although it was active for one or another of the intestinal organisms, its virulence was weak or lacking for the bacterium of barbone.

We will see later, on the contrary, that in a contaminated area at the time when the epizootic dies out, the intestinal bacteriophage of all of the buffaloes which escaped the disease is virulent for the bacterium, the causative agent of the epizootic. Ne find here, then, the same facts as were observed in the study of avian typhosis; that the protection of the body in the case of barbone, a septicemic disease, is assured by the bacteriophage. 
In the buffaloes of a region ravaged by the disease the bacteriophage preserves for a very long time its virulence for the pathogenic bacterium. This, the following example shows.

In November, 1919, a localized epizootic of barbone occurred among the buffaloes of the village of Phuoc Thien (Province of Bien Hoa). On a farm having twenty-one buffaloes seven died-two adult animals and five aged from one to two years. The disease died out, or to speak more correctly, after this, two animals recovered one after another. On the 12th of the following April, that is to say, five months later, specimens of the feces of eight of the surviving animals were collected. All contained a bacteriophage active for the bacterium of barbone (six were ++ , two were + ).

Two specimens of the mud of a water-hole where the animals were accustomed to remain immersed up to the neck during the hottest hours of the day were also examined. In both a bacteriophage virulent $(++)$ for the bacterium of barbone was found. The destruction of the pathogenic bacterium in the external medium must often be effected by the bacteriophage, for it is certain that if the bacterium of barbone has once been introduced into a water-hole by a sick animal the bacteriophage present there must destroy it. Furthermore, this fact shows one of the modes of "contagion" of the active bacteriophage. A single buffalo, in the intestine of which the bacteriophage has acquired a virulence for the pathogenic bacterium, is sufficient to "contaminate" all the herd which frequent the water-hole. Localized epizootics are of short duration, but in spite of this we find that the pathogenic bacterium persists for several months in the external world and that the ingestion of the bacteria by buffaloes is frequent, since the virulence of the bacteriophage maintains itself against this bacterium. The repeated ingestion of a bacterium is, as we have seen, essential for the permanence of the virulence of the bacteriophage toward this bacterium. The epizootic dies out, not because of an absence of pathogenic bacteria but because of the presence of a virulent bacteriophage in the intestine of all exposed animals.

All of the observations are therefore comparable, whether they deal with avian typhosis or with barbone in the buffalo. These epizootics of very different nature were investigated intentionally, that the general nature of the rôle of the bacteriophage in disease and in immunity might be the better established.

One may at first be quite astonished that the intestinal bacteriophage, whose rôle can easily be conceived in infections with intestinal manifes- 
tations, constitutes a defense of the organism in septicemias. In reality, whatever may be the infection, the pathogenic bacterium always gets into the intestine. Let us take a localized disease, cerebrospinal meningitis, for example. We know that the initial symptom is a rhinopharyngitis and that even healthy subjects who have been in contact with a patient often carry the specific organism in the nasopharynx. There can be no doubt but that a fair number of the meningococci present in the rhino-pharynx are swallowed and pass into the intestine. It is needless to insist on this, that, aside from a few rare exceptions to which we will later return, whatever may be the disease under consideration, the portal of entrance of the virus is either the buccal route or by way of the respiratory tract. In either case the ingestion of organisms is, it might be said, obligatory. The pathogenic bacterium is always at some time in contact with the intestinal bacteriophage, this organism therefore is thus able to adapt itself to the bacteriophagy of the bacterium and to acquire a virulence.

In the particular case of barbone the pathogenic bacterium is found freely disseminated through the exterior world in contaminated regions. In an epizootic zone I have been able, in two different trials, to isolate it from the mud of a marsh where the buffaloes were accustomed to bury themselves. This is but natural since the bacterium of barbone is found in the intestinal tract of sick animals or of those which have succumbed. The ingestion of the pathogenic bacterium by the animals which remain immersed for whole hours in a mire containing these organisms is necessarily frequent. If the animal which ingests them has an erosion at any point in the digestive tract it is susceptible to infection. Otherwise the bacteria reach the intestine and come within the range of the intestinal bacteriophage which can then acquire a virulence for the virus. If this takes place the animal is thenceforth protected from the infection and becomes a carrier of the virulent bacteriophage. A diseased animal propagates his disease; an animal in a resistant condition propagates his immunity (d'Herelle and Le Louet ${ }^{321}$ ).

\section{BUBONIC PLAGUE}

Through a lack of favorable circumstances it has not been possible to follow the evolution of the intestinal bacteriophage in man affected with plague. The few cases that have been examined have all been fatal, and at no time could the intestinal bacteriophage be shown to have the least virulence for $B$. pestis. The activity in these cases remained restricted to $B$. coli. However, the stools of two convalescent 
individuals have been secured and examined. According to the physicians treating the cases the material was collected on the sixth and the eleventh days after the beginning of convalescence. Examination showed, in the first case, bacteriophage of average virulence $(++)$ and in the second case, one of fecble virulence $(+)$ for $B$. pestis. The virulence of the first of these strains has been enhanced in vitro and the bacteriophage has been maintained in culture.

An attempt was made to find a bacteriophage active against this bacillus in the feces of twenty-two natives living in regions free of plague, but in no case could a strain be isolated. However, in view of the particular mode of infection in bubonic plague the study of its propagation in man offers only a matter of secondary interest, at least from the epidemiological point of view. We know that an epidemic of plague in man is always consequent to an epizootic among rats. That which it is interesting to study is, therefore, the epizootic, the primary cause of the epidemic. In order to attain a correct interpretation of results it is essential to follow the natural order of things. From the point of view of man the epidemic is obviously the important fact; from the point of view of nature this is but a secondary incident, for if we were able to suppress the epizootic the epidemic would cease spontaneously.

From what we actually know about the epidemiology of plague, it results that all of the rats living in a city where there has been a case of plague in man are the animals which have resisted the contagion, either because they were infected and recovered, or because they remained unaffected. I have then, investigated the virulence of the intestinal bacteriophage of the rat toward B. pestis.

First. Twenty-one specimens of the excrement of rats taken from towns in Indo-China free of plague were examined. The intestinal bacteriophage was found, active against one or another of the intestinal bacteria, but it never showed any virulence whatever for $B$. pestis.

Second. A small epidemic of plague (eleven fatal cases) occurred in the village of Bac Lieu, in the eastern part of Indo-China, during July, 1920. On the following 6th of November I procured in this town four specimens of the excreta of rats, each specimen composed of some dozens of particles, and certainly derived from several individuals. The tests for virulence against $B$. pestis gave the following results:

Specimen derived from a granary ++

Specimen derived from the embarkment quay +++

Specimen derived from a decorticating mill +++

Specimen procured in the house of a native ++++ 
Those rats which have survived an epizootic, therefore, harbor in their intestine a bacteriophage possessed of a high virulence for $B$. pestis.

Plague has existed in the sporadic form in the region of Phantiet, in southern Annam, for about twenty years. I obtained specimens of the excrement of rats in the infected villages, each specimen being composed of the feces derived from several animals. The results of the tests for the virulence of the intestinal bacteriophage in these specimens were:

Village Virulence
Thieu Duc ++
Hung Long +
Duc Hang +++
Duc Thang ++
Tri Long ++
Phu Tay +++
Cu Long +

The results are thus identical with those secured at Bac Lieu, although the virulence seems to be somewhat lower, but this can only be of relative importance since in the present case each specimen was composed of the excreta taken from several rats; the results then, indicate only an average.

Is the bacteriophage present in all of the rats of an infected region or only in a certain number? At Phantiet I collected the excrement of six young rats, according to their weight, aged from three to four weeks. Examination showed that four of the specimens contained a bacteriophage active for $B$. pestis ( + ) while two did not. These last two animals were therefore susceptible to plague.

From the results given above one may conclude that, as for avian typhosis and for barbone, the cause of the resistance against $B$. pestis is the presence in the organism of a bacteriophage possessing a virulence for this bacillus.

How is the adaptation effected in the case of B. pestis? At different times it has been noted that the bacillus has been found in the intestinal contents of victims of plague. Thus, it is possible for them to be disseminated by the feces throughout the external world where they may again be ingested. The bodies of dead rats constitute another mode of dissemination. These bodies are often devoured by the surviving rats and this extends the infection. In those animals which resist and which are infected the intestinal bacteriophage is maintained virulent for the 
pathogenic bacillus. But observation and direct experimentation have shown us that a bacteriophage is only possessed of a virulence for a bacterium when the ingestions of this bacterium are frequent. The permanence of the virulence of the intestinal bacteriophage of the rat against the plague bacillus indicates the persistence of this bacillus in the external world, at least for several months after the last human case has taken place. Moreover, the revival of the epidemic each year in certain localities, Bac Lieu for example, shows that it can not be otherwise* (d'Herelle, ${ }^{321}$ ).

\section{BACILLARY DYSENTERY}

During the course of the epidemic of dysentery which occurred in the region of Paris during the early autumn of 1918, an opportunity was given to observe twenty-nine cases of benign diarrhea. In all of these cases a bacteriophage of very high or extreme activity for the Shiga bacillus was isolated from stools taken the day after the malaise. This bacillus was the cause of all the severe cases studied at this same time.

Living at this time in a locality (Meulan) where several severe cases of dysentery were noted together with a large number of cases of transitory diarrhea, I examined the stools of nine persons who were healthy, but who lived in contact with individuals who had had dysentery. From these nine individuals a bacteriophage of average or high activity for the Shiga bacillus was isolated. We have noted above that the same fact was observed in the institution where Germaine Mel. . . . . had contracted dysentery. Individuals who are exposed to infection and who resist show therefore in their intestine a bacteriophage virulent for the causative pathogenic bacillus, exactly like the affected individuals who recover.

This would make it appear that in an epidemic period the simple cases of diarrhea must in reality be cases of aborted bacillary dysentery,

* Demonstration of the presence of a bacteriophage active for $B$. pestis in the rats of a locality would in certain cases be very useful, for it would indicate the presence of the bacillus in the exterior world and the possibility of a renewal of the epidemic. Such a demonstration might also be useful in establishing a retrospective or doubtful diagnosis. Suppose a few suspicious deaths have occurred in a group some time previously. The presence in the rats of the neighborhood of a bacteriophage showing a virulence for $B$. pestis would eliminate all doubt; the deaths were due to plague. Or, the question of the nature of a epizootic among the rats may be in question. Was the mortality due to plague? The demonstration of a bacteriophage active for $B$. pestis either in the dead rats or in those that have survived provides the answer. 
thanks to the rapidity with which the intestinal bacteriophage adapts itself to bacteriophagy against pathogenic bacteria. And healthy individuals, living in contact with affected people, are only spared by virtue of a still more rapid adaptation occurring before morbid symptoms appear (d'Herelle ${ }^{321}$ ).

\section{FLACHERIE OF THE SILK-WORMI}

A few experiments have been made on this disease, but only for the purpose of determining if defense against infection in invertebrates is also assured by the bacteriophage.

In a breeding-place in Cochin-China a certain number of silk worms died of a disease presenting all of the characteristics of flacherie. Examination of the excreta of the sick worms, as well as of the cadavers, showed the presence of a cocco-bacillus, Gram-negative, which was not present in the dejections of healthy worms. The ingestion, on mulberry leaves, of some of the culture of this cocco-bacillus reproduced the disease; eleven out of twelve worms dying in from six to eleven days after the infecting feeding.

Three filtrates were prepared from the excreta of healthy worms living in the baskets where the affected worms were found. These three filtrates contained a bacteriophage of moderate or high virulence $(++,+++,+++)$ for the cocco-bacillus. On the other hand, two filtrates were prepared, the one with the intestinal contents of a sick worm, the other with the intestinal contents of a worm which had died of the infection. Neither contained a bacteriophage active for the coccobacillus.

These experiments have not been carried further, since the desired end had been attained. They were adequate to show that the facts observed in infectious disease in mammals were reproduced in an infectious disease of an invertebrate. From this it seems logical to conclude that the defense of the organism by the bacteriophage must constitute a general fact throughout all animals (d'Herelle ${ }^{321}$ ).

\section{RÉSUMÉ}

Whether it be a disease purely intestinal in nature, as bacillary dysentery; a disease both intestinal and septicemic, as avian typhosis; a pure septicemic disease, as barbone in the buffalo; or a septicemia with glandular localizations, as bubonic plague; the behavior of the bacteriophage protobe during an epidemic is the same.

During an epidemic we find, reproduced on a large scale among a 
community of individuals, the struggle which occurs within the single infected individual between the bacterium and the protobe.

The bacteriophage protobe is transmissible from one individual to another just as is the bacterium itself. The history of an epidemic is, in the last analysis, the story of an infection with two microorganisms. The epidemic ceases at the moment when all susceptible individuals harbor a bacteriophage active for the causative organism of the epidemic. Either the bacteriophage has acquired virulence in the body of the individual who harbors it, or this individual has been "contaminated" by a bacteriophage which has acquired a virulence in another individual for the specific bacterium involved (d'Herelle ${ }^{321}$ ). 


\section{CHAPTER V}

\section{Immunization with BaCteriophage Suspensions}

\section{THE PROBLEM OF PROPHYLACTIC IMMUNIZATION}

The fact that following the administration of bacteriophage suspensions by injection an effective and durable immunity is obtained has been stated in a number of my publications. ${ }^{311,321,322}$ As we will see, this conclusion based on my own experiments, has been confirmed by a number of different authors. Other authors, on the contrary, report that their attempts at immunization against experimental infections with bacteriophage suspensions resulted in very definite failures. Among this last group may be mentioned Meissner and Baars, ${ }^{456}$ Kramer ${ }^{394}$ and Levy, ${ }^{414}$ all of whom selected as a test disease the infection of mice caused by $B$. typhi murium. I may add that I, also, have failed up to the present time to demonstrate an acquired immunity in experimental plague of the guinea pig, and Flu has informed me that he has been equally unsuccessful.

To what is this lack of success in experimental disease due, when such an efficient immunity is obtained in several natural infectious diseases? Undoubtedly the reasons are multiple.

In the first place, it is obviously foolish to assume that one can take any suspension of the bacteriophage whatever and inject any quantity whatever into any kind of an animal and thus obtain an immunity. We will see, for example, that although the question of the size of the dose injected does not appear to be of any great importance in the case of avian typhosis, it is, on the contrary, of the utmost importance in the case of hemorrhagic septicemia in the buffalo.

In the second place, the question of the experimental animal is another factor of consequence. This is shown very clearly by the fact that, stimulated in some way by an injection of a suspension of the bacteriophage, buffalo and cattle acquire a solid and clurable immunity against hemorrhagic septicemia, while, on the contrary, it has been quite impossible, in spite of many attempts, to obtain the slightest immunity in rabbits. Cattle, animals naturally susceptible, are, therefore, capable of being immunized; the rabbit, an animal which does not contract barbone spontaneously, can not be immunized. 
But while many of these questions are of the greatest theoretical interest, I must add that I am absolutely indifferent toward the study of such fictitious immunities, developed for fictitious experimental diseases. The only thing that interests me in this connection is the immunity obtained against the natural disease. Upon several occasions I have expressed my ideas on this subject. ${ }^{321,349}$

\section{IMMUNIZATION AGAINST AVIAN TYPHOSIS}

Immunization experiments made in a region where the epizootic was present, as was true in the case of avian typhosis, presented an especial difficulty, or rather, a complication. It has been mentioned that aside from the typical typhosis, due to B. gallinarum, there are several varieties of paratyphoses, each caused by a particular species of bacterium. The differences which these bacterial species present from the biochemical and agglutinative points of view and which serve to differentiate them are of no particular significance from the point of view of this study. In so far as action of the bacteriophage is concerned for each of these, a race of bacteriophage having an extreme virulence $(++++)$ for $B$. gallinarum possesses the same activity for all the French and American strains as well as for B. jeffersonii. The activity is less pronounced for $B$. pullorum $A$, still less for $B$. pullorum $B$, and is lacking for $B$. pfaffi and for $B$. rettgeri. With such a race of bacteriophage the reactions are: $B$. gallinarum,$++++ B$. jeffersonii ++++ , B. pullorum $A++, B$. pullorum $B+, B$. pfaffi 0 , and $B$. rettgeri 0 . Inversely, a race of bacteriophage secured from fowls resistant to paratyphosis due to $B . p f a f f$ (focus at Trainel, Aube) had the following virulences: $B$. gallinarum $0, B$. jeffersonii $0, B$. pullorum $A$ 0, B. pullorum $B+, B$. pfaffi ++++ , and B. rettgeri 0 .

The immunization experiments thus become singularly complicated, particularly since both typhosis and the paratyphoses may be found in the same areas, as is also true for human enteric infections. In routine practise the solution is simple; it is sufficient to immunize the poultry with a mixture of different races of the bacteriophage active against the diverse pathogens, the causes of typhosis and the paratyphoses. In the preliminary investigations this was not possible, for the differences between the different diseases had not been recognized when I first undertook this study. The different bacilli, the agents of the paratyphoses, had been studied in the United States but their simultaneous presence in foci of typhosis had not been noted. And in so far as B. pfaffi is concerned, discovered by Pfaff in an epi- 
zootic in Vienna, it had not then been incriminated as capable of producing disease in the Gallinaceae. These facts have only been disclosed gradually in the course of these investigations.

The suspensions of bacteriophage used in the immunization experiments were prepared in the following manner:

A culture of B. gallinarum, in Martin bouillon, aged nine or ten hours, that is, very young but showing a definite turbidity, is inoculated with a bacteriophage isolated from the excreta of a recovered chicken and possessing a high virulence for the pathogenic bacillus. After about 12 hours the bacterial dissolution is completely finished and the bouillon is perfectly limpid. This material is filtered through a bougie and distributed into ampoules which are sealed.

The dose employed for immunization has been in all cases 0.5 cc., given subcutaneously. The point of injection is of no importance for the slightest local or general reaction has never been observed.

Experiment $I$. The following experiments were conducted in 1919 and 1920 in the neighborhood of Agen with the assistance of M. Lambert, D.V.M.

Barnyard 1. The epizootic began in August, 1919. By October 2, 110 of 160 fowls had died. The 50 survivors, of which 5 were already affected were inoculated with the bacteriophage. The 5 sick chickens recovered and the epizootic stopped abruptly and definitely on the same day as the immunization.

Barnyard 2. The epizootic began about August 20. By October 6, 120 of 200 fowls had died. The 80 survivors, of which 7 were sick, received an injection of the Gallinarum-bacteriophage. The 7 recovered; the epizootic immediately and permanently disappeared.

Barnyard 3. The epizootic began October 10. By the 15th, 21 fowls had died. The 130 that were alive, of which 8 were already sick, were inoculated. The 8 recovered and the epizootic disappeared from the day of the inoculation.

Barnyard 4. The epizootic began about November 15. By December 1, 26 of 51 fowls were dead. The 25 survivors, among which were 4 which were infected, were inoculated. One of the sick animals died, the other 3 recovered. The mortality stopped from the date of the inoculation.

Barnyard 5. The epizootic began about November 25. By December 1, 7 of 60 chickens had succumbed. The 53 survivors were inoculated. Of these 4 were sick. The sick animals recovered and no new cases appeared. 
Barnyard 6. The epizootic began on December 16 . On the 28th, 40 of 142 fowls had died. The 102 survivors, of which 3 were infected, were inoculated. The sick recovered and the disease abruptly stopped.

Barnyard 7. The epizootic began on January 2. By January 14, 15 of 50 animals had died. The 35 survivors were inoculated. No new cases developed from this time on.

Barnyard 8. The epizootic began about January 15 with a daily mortality of 4 to 6 fowls. On January 21 the 121 survivors, including 5 which were sick, were inoculated. The sick recovered and the epizootic stopped at once.

Barnyard 9. The epizootic began about February 10. By February 20, 14 chickens had died from among the original 84 . The 70 survivors were inoculated and the disease disappeared at once.

Barnyard 10. The epizootic began about February 25. By March 1, 20 chickens had died. The 120 survivors, of which 5 were sick, were inoculated. The 5 recovered and the epizootic stopped.

Barnyard 11. The epizootic began on February 4. From February 4 to 10,10 chickens died. On February 10 the 48 living fowls were inoculated in the wing with 0.5 cc. of the Gallinarum-bacteriophage, as had been all the chickens in the ten preceding experiments. The epizootic continued its course and 5 chickens died from February 10 to 17 . On February 17 the 43 fowls which remained were inoculated with 0.5 cc. of a mixture of four races of the bacteriophage: active against B. gallinarum, B. pullorum, A, B. pullorum B, and B. pfaffi. The epizootic stopped immediately after this second inoculation.

Barnyard 12. This barnyard was adjacent to the preceding and here the same facts were observed. A first inoculation made on February 9 on 80 chickens with a suspension of the Gallinarum-bacteriophage was without effect. The epizootic stopped abruptly after an inoculation of bacteriophage active for the bacillary agents of the paratyphoses, made on February 17.

Examination of the blood of fowls dead in Barnyard No. 12 resulted in the isolation of a $B$. pfaffi type of bacillus. This organism, then, was responsible for the epizootics in groups 11 and 12 . In this connection I will only mention the instance of the epizootic of paratyphosis at Trainel mentioned elsewhere. This outbreak was likewise due to B. pfaff $\mathrm{f}$ and was controlled by the inoculation of a Pfaffi-bacteriophage.

Experiment $I I$. This was performed at Poully en Auxois with the assistance of MM. Voillot and Bouhier, D.V.M.

Barnyard 1. On January 5, 20 chickens were taken at random from 
a poultry-yard containing about 100 fowls where typhosis had appeared. These 20 were immunized with a suspension of Gallinarum-bacteriophage. On February 7 the immunized birds were all alive and in perfect condition, while the epizootic had continued to spread among the non-immunized animals, of which only about 20 remained.

Barnyard 2. On February 23 the surviving chickens of a poultryyard containing at that time 102 animals were immunized. The epizootic which began about 10 days previously, and which had resulted in a daily mortality of 4 or 5 chickens, stopped quickly and permanently from the time of the immunization. The epizootic continued, on the contrary, to ravage with the same intensity as formerly in all the neighboring poultry-yards which served as controls.

Experiment III. This experiment was conducted at Provins, with the aid of M. Sorriau, D.V.M., in an important poultry-yard where typhosis was present in endemic form.

For several months the daily mortality had been 2 or 3 fowls. On January 25 the 225 survivors were immunized. The epizootic immediately and permanently disappeared from the date of the immunization.

Experiment $I V$. Performed at Rouillac, Charente, with the assistance of M. Chollet, D.V.M.

On December 15, 100 fowls were immunized in a poultry-yard where typhosis had appeared about ten days previously. The daily mortality had been from 4 to 6 animals. With the immunization there was an immediate and permanent cessation of the epizootic. Typhosis continued to prevail on all the neighboring farms. Among the 100 chickens inoculated, about 12 were already affected. Of these only 2 died, 2 and 3 hours after the injection.

Experiment $V$. This test was conducted with the assistance of Dr. Ormières at Carcassonne.

The epizootic began during the month of August. By October 1, 80 chickens had succumbed. The 120 survivors were immunized. The epizootic stopped immediately and no further cases appeared after the clate of the immunization.

Experiment VI. This experiment was conducted with the assistance of M. Mesnard, Departmental Veterinarian at Angoulême. In these experiments the chickens were immunized by the ingestion, on bread, of about 1 cc. of a Gallinarum-bacteriophage.

$A$. On July 2 the 50 chickens surviving in a poultry-yard where typhosis had been prevalent for six weeks, with a daily mortality of 
2 or 3 fowls, each ingested about $1 \mathrm{cc}$. of the bacteriophage suspension. Seven months later no new case had developed since the time of the ingestion.

$B$. The same test was performed on October 15 on about 100 chickens on a neighboring farm where typhosis had been present for several months. The epizootic was immediately and completely checked.

In both of these cases the disease continued to spread throughout the neighboring poultry-yards that were held as controls.

It appears needless to multiply such examples. In all cases the picture has been the same. The epizootic disappeared from the time that the bacteriophage virulent for the pathogenic bacterium, the cause of the epizootic, had been introduced into the organism of the susceptible animal, whether this introduction was by injection or ingestion. We will see later that this last mode of administration is somewhat less efficient than injection.

On the contrary, injections of a suspension of a bacteriophage active for B. gallinarum, the specific cause of typhosis, had in general, no effect when the epizootic was a paratyphosis, particularly in the case of infections due to $B$. pfaffi. In practise, it is only necessary to inject a mixture of different races of bacteriophage active for the various pathogenic bacteria that may produce the epizootic. This mixture should also include a race active for chicken cholera. It will be very easy to accomplish this, for the dose of $0.5 \mathrm{cc}$. which $\mathrm{I}$ have arbitrarily adopted is indeed much larger than necessary, as we will see. Even in mixing five or six different strains of bacteriophage, the quantity necessary to effect immunization is not more than a fraction of a cubic centimeter.

In the course of the experiments cited there has been no selection. All of the animals of the poultry-yard, even though they were moribund, received the immunizing injection. About 100 sick chickens have therefore been injected, and the mortality among these has been 5 per cent. This is an appreciable reduction since the mortality among affected animals varies from 100 per cent at the beginning of the epizootic to 95 per cent, when, after some weeks, the disease appears only in sporadic cases.

A suspension of the bacteriophage, as we have shown in several ways, is composed of bacteriophage corpuscles suspended in a medium containing the dissolved bacterial substance, the bacteria which have been destroyed by the action of the protobes. What, among these different principles, is the one which plays the active rôle in the protec- 
tion of the healthy animal or in the one already sick, under the conditions of the experiment, that is, in a contaminated area? Unquestionably it is the bacteriophage corpuscles themselves. The immediate protection assured by the injection or even by the ingestion of the bacteriophage suspension suffices to demonstrate this. An organic immunity necessarily requires a certain time for its development. Other phenomena of immunity, organic in nature, are produced only after an incubation period, as the experiments on barbone will show.

For the moment, let us conclude only that with sensitive animals immunized by the injection of a suspension of the bacteriophage active for the causative pathogenic bacterium, in a contaminated area, that is to say, in an area where frequent reinfections may take place as a result of the dissemination of the pathogenic bacteria in the external environment, the principal rôle of protection is played by the bacteriophage itself. The other phenomena of immunity which may later develop, stimulated by the other substances contained in the material injected, play no rôle under such conditions unless it be a very secondary one. We will see that this proposition becomes reversed when similar experiments are carried out in a non-contaminated area. ${ }^{314,321}$

At the Institute of Parasitic and Infectious Diseases at Utrecht, Kramer ${ }^{394}$ has carried out a series of experiments under conditions comparable to those under which my work was done. And as this question of the immunity conferred by the bacteriophage is of the greatest importance from the point of view of our knowledge of "exogenous" immunity, I venture to insert in this text summaries of the different experiments made by this author, since it may be that this confirmation of my findings may prompt other investigators to follow this method.

In the first place, Kramer calls attention to the fact that, contrary to what is reported by other authors, de Blieck was unable to obtain an immunity against natural avian typhosis by injections, single or repeated, of vaccines made of heated bacterial bodies, or even by employing "auto-vaccines." Incidentally, the experiments of Kramer also show that a passive immunity is not conferred by the so-called antibacterial sera.

Without further discussion, let us consider Kramer's experiments and the results which he obtained. The data presented below is extracted from his protocols.

Observation 1. Epizootic typhosis.

Fifty of 100 pullets had died within a period of 8 days. Cultural 
diagnosis established $B$. gallinarum as the causative agent. The 50 survivors, of which half were already affected, received one intramuscular injection of $2 \mathrm{cc}$. of a suspension of bacteriophage virulent for the pathogenic bacterium. During the three following weeks there were 8 deaths, and then the epizootic again broke out with its original vigor. The 22 survivors received a second injection. The disease stopped permanently.

Observation 2. Enzootic due to B. gallinarum.

There had been from 1 to 2 deaths per day. The 160 survivors received an injection of $1 \mathrm{cc}$. of the bacteriophage suspension. The mortality was arrested, only to later break out again. Four weeks after the first, a second injection was given. The enzootic then stopped permanently.*

Observation 3. Enzootic due to B. gallinarum.

Within the past year there had been 250 deaths. The 150 survivors were divided into three lots, the animals comprising the different groups receiving $2 \mathrm{cc} ., 1 \mathrm{cc}$, or $0.5 \mathrm{cc}$. of the bacteriophage suspension. The enzootic was suddenly arrested from the day of the injection. None of the animals died during the 6 months following.

Observation 4. Enzootic due to B. gallinarum.

This occurred in a brood of chicks. It had lasted for 4 weeks, with from 1 to 4 deaths per day. Each of the 80 survivors (out of an original 150) received an injection of $1 \mathrm{cc}$. of the bacteriophage suspension. There were 4 deaths on the day of the injection, 2 on the next day, and then the enzootic permanently disappeared.

Observation 5. Enzootic due to B. gallinarum.

The pullets had received a previous injection of an anti-gallinarum serum, but no effect upon the mortality had been observed. The 300 survivors received an injection of $1 \mathrm{cc}$. of bacteriophage suspension. The enzootic stopped at once and did not recur.

Observation 6. Epizootic due to B. gallinarum. There had been 3 to 4 deaths per day. One cubic centimeter of a bacteriophage suspension was injected into each of the 150 survivors. The disease disappeared immediately and permanently.

Observation 7. Epizootic due to B. gallinarum.

Of 110 pullets 30 had died within the past 2 weeks. Among the 80 survivors, 40 received an injection of an anti-gallinarum serum,

* "Arrest" of the epizootic or enzootic means that from the time indicated none of the animals died. This is not a relative measure of effect, but an absolute value. 
and the remaining 40 received an injection of bacteriophage suspension. The disease suddenly stopped in the lot which had received the bacteriophage, while the mortality continued without diminution in the group which had received the serum. After a few days, the survivors of the serum-treated lot were inoculated with 1 ec. of bacteriophage suspension. Thereupon the mortality in this group suddenly stopped.

Observation 8 . Epizootic due to $B$. gallinarum.

There had been 3 to 4 deaths per day. An injection of 1 cc. of bacteriophage suspension was given to each of the 100 survivors. The epizootic was immediately and permanently arrested.

Observation 9. An enzootic involving a group of farms.

On one of the farms 98 out of a total of 150 pullets had died within the past 6 months. The mortality was very irregular; some weeks none of the animals died, in others as many as 12 succumbed. Among the survivors, 25 were inoculated with $0.5 \mathrm{cc}$. of the bacteriophage suspension, the other 27 with $1 \mathrm{cc}$. The disease disappeared from this barn-yard from the day of the inoculation, although it continued as before to destroy the animals of the neighboring farms where the pullets had not been immunized.

Observation 10. Epizootic due to B. gallinarum.

Out of a total of 140 chickens, 15 had died within 4 days. Each of the survivor's received an injection of $0.5 \mathrm{cc}$. of a bacteriophage suspension. Three chickens which were very sick at the time of inoculation died; no further deaths occurred.

Observation 11. Epizootic due to B. gallinarum.

The record showed 150 deaths within the past 2 months, out of a total number of 400 . The 250 survivors received an injection of $0.5 \mathrm{ec}$. of a bacteriophage suspension. In the 2 months which followed a few of the pullets died from time to time. A second injection of $0.1 \mathrm{cc}$. of bacteriophage suspension was given. Thereupon the enzootic stopped permanently.

Here, as a result of the first injection, the epizootic assumed the character of an enzootic, and a second injection was necessary to arrest the enzootic.

Observation 12. Epizootic due to B. gallinarum.

This outbreak took place on a farm which had three separate chicken yards. The epizootic began in yard No. 1, containing 225 chickens. The mortality here was 7 to 8 per day. The disease next extended to yard No. 2; and at the time when the experiment began it had not yet reached yard No. 3 . 
Yard No. 1. The 100 survivors received an injection of 0.5 cc. of bacteriophage suspension. Five chickens died immediately after the injection, and then there were no further deaths.

Yard No. 2. Here there were 3 deaths per day. The 24 survivors, despite the fact that the majority of them were already infected and showed the symptoms of the disease, received an injection of 0.5 cc. of bacteriophage suspension. The animals that were very sick died, then the epizootic was arrested.

Yard No. 3. The disease had not yet invaded this yard, in which there were 100 chickens. All of them received as a prophylactic measure an injection of $4 \mathrm{cc}$. of an anti-gallinarum serum. Four days after this injection the epizootic began, 4 or 5 animals dying each day. Five days later, when 80 chickens still remained alive, each one received 0.5 cc. of bacteriophage suspension. The epizootic stopped at once.

Observation 13. Epizootic due to B. gallinarum.

The race of bacteriophage used in this experiment had but a weak virulence for a strain of $B$. gallinarum isolated from the blood of one of the chickens dying in the henyard. The 100 survivors received an inoculation of $0.5 \mathrm{cc}$. of the suspension of bacteriophage of low virulence. There was no effect. Two weeks later a second injection was given. The mortality diminished, but the disease did not disappear. This experiment is extremely interesting for it shows two things:

First: That immunization does not take place if the bacteriophage inoculated does not possess a virulence sufficient to overcome the pathogenic organisms;

Second: That it is clearly the bacteriophage which is effective, and effective as a parasite of the pathogenic bacteria. If the active principle were some soluble substance of the bacteria, the virulence of the bacteriophage, with the associated possibility of secondary cultures, would be of little consequence.

Observation 14. Enzootic due to B. gallinarum.

The infection here had lasted throughout several months. An injection of $0.5 \mathrm{cc}$. of a bacteriophage suspension was given to each of the 200 survivors. The enzootic stopped abruptly and finally.

Observation 15. Epizootic due to an atypical B. gallinarum.

In this epizootic there had been 3 or 4 deaths per day. Isolation of the pathogenic bacterium showed that the causative agent was an atypical strain of $B$. gallinarum, but the race of bacteriophage available possessed a very high virulence for this bacillus. The 317 survivors 
received an injection of $0.1 \mathrm{cc}$. of the bacteriophage. A large number of these animals were already showing symptoms at the time of the inoculation. All of the sick chickens recovered and the epizootic permanently disappeared. This experiment confirms that recorded above, in that it shows that the effective principle is the bacteriophage protobe itself, functioning because of its virulence. The dissolved bacterial substances do not contribute in any way to the processes of immunity which immediately follow the inoculation.

Observation 16. Epizootic due to B. gallinarum.

Within the past month there had been 23 deaths. The 71 survivors received an injection of $0.1 \mathrm{cc}$. of a bacteriophage suspension. The epizootic stopped immediately and completely.

Observation 17. Epizootic due to B. pullorum $A$.

This started in a brood of chicks when they were 4 days old. Between the 5th and 10th days there were 80 deaths out of a total of 137 . On the 10th day the chicks ingested with the drinking water a suspension of bacteriophage which was virulent for both B. gallinarum and $B$. pullorum. Three more chicks died and then the disease was arrested.

Observation 18. Epizootic due to B. pullorum $A$.

This epizootic was also among young chicks. The Gallinarumbacteriophage showed a definite virulence for the B. pullorum isolated from the intestine of one of the chicks of this brood which had died. Of the 500 chicks, 150 had died within the first two weeks after hatching. The survivors ingested a suspension of bacteriophage mixed in the drinking water. The epizootic was transformed into an enzootic, that is, the disease persisted but with a lessened and very irregular mortality.

Observation 19. Epizootic due to B. pullorum.

This developed among chicks 6 days old. Here again the bacteriophage was administered by ingestion in the drinking water. The race used was virulent for $B$. gallinarum. Out of a total of 500 chicks, an average of 16 had died daily prior to the ingestion. An in vitro test showed that the Gallinarum-bacteriophage possessed also a strong virulence for a strain of $B$. pullorum isolated from a chick of this hatching which had died. This bacteriophage was mixed with the drinking water. The mortality diminished considerably, although during the few days following the ingestion 30 chicks died. The suspension was again added to the water and a second ingestion was accomplished. From this time on the disease disappeared completely.

Observation 20. Epizootic in 14 day old chicks. 
There remained but 93 out of a total of 200 . The race of the bacteriophage used proved to be very virulent for the pathogenic bacillus isolated from one of the dead chicks. The survivors received an injection of $0.5 \mathrm{cc}$. of the bacteriophage suspension. Two died after the injection and then the epizootic ceased.

Kramer concluded from these experiments that the bacteriophage certainly plays a rôle in immunity.

To this modest conclusion I may add that the experiments of this author show definitely that the immunity conferred experimentally is proportionate to the virulence which the bacteriophage administered has for the strain of the pathogenic bacterium which is causing the epizootic.

A favorable result, either in prophylaxis or in therapy, can hardly be expected unless a race of the bacteriophage is used which has a maximum virulence for the pathogenic agent against which the development of an immunity is desired. To attempt to work with races of low virulence is simply to invite certain failure.

\section{IMMUNIZATION AGAINST BARBONE}

Together with Le Louet, one-time Chief of the Veterinary Service of Cochin-China, I have made some studies on immunity in barbone, or hemorrhagic septicemia of the buffalo.

Thanks to the liberality of the Government of Cochin-China, which placed at our disposal all the animals, steers and buffaloes, which we needed, we have been able to study in detail certain of the conditions underlying immunization by means of the bacteriophage. Barbone is, indeed, an ideal disease for a study of this type. The blood taken from an animal about to die of the disease can be preserved in sealed ampoules for at least six months without any loss in the virulence of the bacteria present. Bouillon inoculated with a drop of this blood yields a culture which regularly kills the steer or the buffalo in a dose of 0.0002 ec. With half this dose, 0.0001 ec., usually one out of two animals will be killed. Experimental infection reproduces the spontaneous disease in the most minute details; the same temperature curve, the same symptoms, the characteristic edema at the point of entrance of the virus. Like the natural infection, the disease is fatal; all animals succumb and death occurs in the same length of time in the two cases, within twelve to eighteen hours from the appearance of the first symptoms. The lesions to be found at autopsy are identical. Immunization experiments conducted with such a disease provide, then, absolute results. 
I may state here, once for all, that each time that the immunity of one animal has been tested by the inoculation of a culture of the bacterium of barbone this test has been controlled by the injection of an equal dose into a control animal of the same weight, and never has the control resisted. Furthermore, although there can be no possible doubt concerning the cause of death, confirmation has always been made by microscopic examination, by blood culture, and by the demonstration of the lesions at autopsy. The temperature of the experimental animals was taken regularly, morning and evening, and the slightest reaction in the immunized animals could not have passed unobserved.

The race of bacteriophage employed for the preparation of the suspensions destined for use in the immunization experiments had been isolated from the feces of a buffalo which had passed unaffected through the epizootic mentioned in the preceding chapter. This bacteriophage possessed, when derived from the organism, a strong virulence $(+++)$ for the bacterium of barbone. After about ten passages in vitro the virulence became extreme $(++++)$, and at this time it was used.

A fairly turbid bouillon culture of the bacterium of barbone about 12 hours old received one drop of the previously described active $(++++)$ Barbone-bacteriophage. After about 12 hours the medium became perfectly limpid. This culture was filtered through a Chamberland filter $\left(\mathrm{L}_{3}\right)$ and distributed into ampoules, which were sealed. I would call attention to the necessity of employing only suspensions in which the dissolution of the bacteria has been completc. Such suspensions ought, moreover, to be filtered because of the fact that a sccondary culture may develop in some of the tubes.

The suspensions of Barbone-bacteriophage have been used after a variable length of time,--from twenty days to five months after their preparation. No difference has ever been observed in their mode of action, whatever the time elapsed between the date of preparation and the time of use.

All of the experiments, except those dealing with the effect of the age of the animal upon the development of immunity, have been effected on steers of the indigenous race, in a perfect state of health, aged from twelve to eighteen months, and of an average weight of $100 \mathrm{kgms}$. , * and on buffaloes aged from one to twelve years. The bovine race and the buffalo are equally susceptible to barbone. In

* The race in Indo-China is of small size. 
Egypt, Piot has been herds of cattle decimated to the same extent as the herds of buffalo. According to our observations the buffalo may be rather easier to immunize than cattle.

Let us consider first the experiments conducted for the purpose of determining what conditions control the development of the immunity resulting from the injection of a suspension of the bacteriophage. The size of the dose and the age of the animals are the two principal factors whose variation has the greatest influence on the result. To facilitate discussion, we may consider the effects of smaller and smaller doses, although in reality the chronological order of the experiments was somewhat different, since the tests were first made with the injection of a dose arbitrarily fixed at $5 \mathrm{cc}$. In this experiment the animals all died, when the test injection was given twenty days later. Thinking that the immunizing dose was inadequate it was increased in the next test to $20 \mathrm{cc}$. Here again, the results were the same. It was only somewhat later, when smaller doses were employed, that the treatment proved to be efficacious. We have seen already that immunization by means of bacteriophage cultures presents individual peculiarities.

\section{Determination of the immunizing dose}

I. Eight steers received $20 \mathrm{cc}$. of the bacteriophage suspension subcutaneously. Six of these were tested after a lapse of time varying from fifteen to forty days by the inoculation of a quantity of barbone culture representing certainly 50 fatal doses. All died in the same length of time as the control animals. The remaining 2 were tested also with 50 fatal doses, sixty days after the immunizing injection. They showed no obvious disturbance. The two controls died in nineteen and twenty-two hours after the inoculation of virulent material.

II. Four steers received, subcutaneously, 5 cc. of the bacteriophage suspension. Three were tested after thirteen, fifteen and twentyeight days by the inoculation of 50 lethal doses of virulent bacilli. All died in the same length of time as the controls. The fourth was tested on the fortieth day. It showed no reaction. The control died in twenty-two hours.

III. Forty-one animals; 25 steers, 4 buffaloes aged from one to two years, and 12 adult buffaloes, received an injection of $0.25 \mathrm{cc}$. of the bacteriophage suspension.

$A$. Eight steers were tested between the third and twelfth days 
following the injection by the inoculation of virulent culture, representing, according to the weight of the animal, from 5 to 1000 sureiy fatal doses. All died.

$B$. Twelve steers and one buffalo were tested between the 13 th and 20th days, all by the inoculation of 1000 surely fatal doses of barbone

TABLE 63

\begin{tabular}{l|c|l}
\hline \multicolumn{1}{c|}{ ANIMAL } & $\begin{array}{c}\text { TEST INJECTION } \\
\text { 1000 FATAL } \\
\text { DOSES GIVEN } \\
\text { AFTER }\end{array}$ & \\
\hline days & 13 & Resisted without showing any reaction \\
Buffalo & 15 & Died 26 hours after the inoculation \\
Steer No. 50 & 15 & Died 20 hours after the inoculation \\
Steer No. 52 & 15 & Died 23 hours after the inoculation \\
Steer No. 53 & 15 & Died 26 hours after the inoculation \\
Steer No. 55 & 15 & Died 25 hours after the inoculation \\
Steer No. 56 & 15 & Resisted, without showing any symptoms \\
Steer No. 38 & 16 & Died 68 hours after the inoculation \\
Steer No. 27 & 16 & Resisted, without showing any symptoms \\
Steer No. 20 & 17 & Resisted, without showing any symptoms \\
Steer No. 28 & 17 & Resisted, without showing any symptoms \\
Steer No. 30 & 17 & Died 34 hours after the inoculation \\
Steer No. 89 & 17 & Died 32 hours after the inoculation \\
Steer No. 90 &
\end{tabular}

TABLE 64

\begin{tabular}{c|c|c}
\hline \multicolumn{1}{c|}{ ANimal } & TESTEd AFTER & \multicolumn{1}{c}{ REsUlt } \\
\cline { 2 - 3 } Steer No. 107 & 1 & \\
Steer No. 103 & 1 & Resisted, no reaction whatever \\
Steer No. 106 & 2 & Resisted, no reaction \\
Steer No. 101 & 3 & Died 36 hours after the inoculation \\
Steer No. 83 & 4 & Died 28 hours after the inoculation \\
Steer No. 84 & 4 & Resisted, no reaction \\
Steer No. 104 & 5 & Resisted, no reaction \\
\hline
\end{tabular}

culture. Five resisted, the others succumbed. The experiment is given in detail in table 63 .

Two control steers died in 22 and 26 hours after the inoculation, and one buffalo, as control, died in 19 hours.

$C$. Twenty animals; 5 steers, 3 young buffaloes, and 12 adult buffaloes were tested during the period from the twenty-first to the sixtieth day after the immunizing injection. All received 1000 surely 
fatal doses of culture. All resisted without showing any reaction. Five control animals died, all between 16 and 23 hours after the inoculation of culture.

IV. Eight steers received 0.04 cc. of bacteriophage suspension. They were tested after a variable number of days by the inoculation of 5 surely fatal doses of virulent culture. The results are shown in table 64 . The last steer, No. 102, was tested 60 days after the injection of the immunizing dose by the inoculation of 50 surely fatal doses of culture. It resisted without showing any disturbance.

V. A last experiment, as a control, was performed with a view to testing the practical application of immunization of buffaloes against barbone by M. Le Louet after my departure from Saigon. Twelve steers received by subcutaneous injection $0.25 \mathrm{cc}$. of bacteriophage suspension. They were tested 25 days later by the inoculation of 2000 surely fatal doses of barbone culture. They resisted without showing the slightest reaction. The controls died in from 18 to 22 hours after the inoculation.

The injection of the bacteriophage did not produce in any of the animals, even in $20 \mathrm{cc}$. doses, the slightest reaction, either local or general. The temperature curve following the immunizing injection could be superimposed throughout on the curves of normal untreated animals. From this it is clear that, contrary to general belief, an immunity bordering on the refractory state may be acquired without the manifestation of the slightest reaction.

During the course of these experiments aphthous fever made its appearance at Saigon. The animals in the course of immunization contracted it but this complication in no instance exerted any influence upon the development of immunity to barbone.

From these different experiments it may be deduced that with a large dose of bacteriophage suspension the immunity is slow in being established; about forty to sixty days with a dose of 20 cc., more than twenty-eight days with $5 \mathrm{cc}$. With $0.25 \mathrm{cc}$. it is not effective for all animals until about the twentieth day. It then permits them to resist without apparent discomfort two thousand surely fatal doses of the culture of barbone, that is to say, the immunity conferred borders on the refractory state. With the minimal dose of 0.04 cc., or less than a normal drop, a solid immunity is acquired by the fourth day. We are not concerned for the moment with the steers which have resisted after twenty-four hours; the immunity which they enjoy is of a different order, as we will see later. 
We have seen in the discussion of the phenomenon of antiphylaxis that the serum of rabbits which have received several injections of bacteriophage suspension possesses the property of sensitizing the animals against the bacteria for which the bacteriophage injected was active. The delay in the establishment of the immunity as a result of the injection of large doses of Barbone-bacteriophage ought to induce this same phenomenon. The injection produces in the animals two phenomena of different orders: an immunity and a sensitization which varies in intensity according to the dose inoculated. With a small dose the first surpasses the second which disappears quickly; with a large dose, on the contrary, the inhibitive action persists for a very long time -about sixty days for an injection of $20 \mathrm{cc}$.

The experiments further show that the immunity conferred by the injection of suspensions of the bacteriophage is absolute when once established, and is negative during the period of incubation. There is no intermediary state. The animals, young or old, which receive the test inoculation during the period of incubation die, with very few exceptions, in the same time as the controls, even if this inoculation is made at a time very close to that where all the immunized animals resist. On the other hand, all those which are tested after the incubation period resist without presenting any apparent malaise, whatever the test dose may be. It seems indeed, as a result of these findings, that after an incubation time, more or less protracted according to the amount of bacteriophage injected, a period during which the animal remains as sensitive as a normal animal, the immunity increases very rapidly once its manifestation has commenced. In a word, the release of immunity is abrupt.

\section{Effect of the age of the animals on the acquisition of immunity}

We have seen that thirty-two animals, steers, young buffaloes, or adult buffaloes of less than twelve years, have all acquired an immunity that approaches the refractory condition within twenty days following the injection of $0.25 \mathrm{cc}$. of the bacteriophage suspension. We wished to see how this would compare with the results obtained in old animals.

Three buffaloes between fourteen and sixteen years and five very old animals no longer working and certainly more than twenty years old*

* The buffalo usually lives about twenty-five or thirty years. The Annamite never kills a buffalo; old and no longer able to work, it is fed and cared for as well as are the younger animals. The attachment of the natives for these buffa- 
received $0.25 \mathrm{ec}$. of the suspension of bacteriophage. All eight were tested forty-three days later by the inoculation of 1000 surely fatal doses of bacterium barbone culture at the same time as a normal control animal. This last died in seventeen hours. One of the three youngest buffaloes showed no reaction other than a transitory edema at the site of the inoculation, the other two showed a voluminous edema and were obviously sick, but all three recovered and could be considered normal six days after the test inoculation. The five very old buffaloes succumbed after 48,53, 54, 60, and 142 hours; that is, after a time considerably longer than the control. Fifteen young animals immunized and tested at the same time failed to show any reaction to the test injection.

It is evident that although the test dose was enormous, that did not alter the fact that in the old animals the acquisition of immunity was much more difficult, somewhat in proportion to the age. The relative immunity against an extremely severe experimental test is observed only in these old animals; with the young or with adults in the prime of life, the immunity, as we have seen, is absent during the incubation period and complete once it has appeared at all.

\section{The duration of the immunity}

After my departure from Indo-China, my collaborator M. Le Louet, continued the experiments with a view to ascertaining the duration of the immunity produced by the inoculation of a suspension of the bacteriophage. In January, 1921, he injected 15 steers, aged about one year, with a cubic centimeter of a suspension of the bacteriophage that was about onemonth old, that is, a bouillon culture of the bacterium of barbone which had been dissolved by the bacteriophage one month before use. In March, 1922, all of the animals were tested, along with nine controls, by the inoculation of $0.1 \mathrm{cc}$. of a virulent culture of the bacterium of barbone. The virulence of this culture was such that in amounts of $0.002 \mathrm{cc}$. it regularly killed steers in less than thirty-six hours. Of the animals thus infected all of the controls died in from seventeen to twenty-three hours after the injection, while of the vaccinated animals ten resisted without any evident reaction and five died in from two to five days after inoculation.

loes is such that it is difficult to find a person who will scll one of these animals. Those which served in the experiments were procured, some through the agency of the Governor of Cochin-China, M. le Gallen; others by M. Privé, Director of the plantations of An Loc and Suzannah, without considering the possible loss. I offer them my sincere thanks. 
This experiment shows that fourteen months after the immunizing injection two-thirds of the animals possessed an immunity sufficiently strong to enable them to withstand a massive dose of the pathogenic bacterium.

\section{The immunizing principle}

Under the conditions of the experiment, that is to say, in a non. contaminated area, what, in the bacteriophage suspension, is the principle which brings about the immunization?

A preparation of the bacteriophage contains, as we know:

1. The bacteriophage protobes and

2. The soluble substances contained in the culture medium. These are the soluble substances derived from the bacterial bodies at the expense of which the bacteriophage has developed, the "products resulting from bacteriophagy," and the metabolic products elaborated by the bacteria prior to their dissolution.

The course of the phenomenon alone, has shown us already that the immunizing principle must be different according as the immunity is developed in a contaminated area, as was the case in the experiments made on typhosis, or in a non-contaminated area, as in those on barbone. In the first, the immunity is acquired immediately; in the second, it becomes effective only after an incubation period. However, direct experiment allows us to confirm this idea.

1. If one injects steers, by the subcutaneous route, with from 5 to $20 \mathrm{cc}$. of Barbone-bacteriophage it is possible to isolate the active protobes from the blood throughout the first twenty-four hours after the injection. After this period they have disappeared. Experimentfurther shows that the protobes pass quickly into the intestine. They can be isolated from the intestine within about twelve hours after the injection and they persist there for a somewhat longer time than in the circulation: for two or three days (up to six days in a single case). In all instances they have disappeared long before the immunity is established. Let us repeat that this applies only to the case where the introduction of the bacteriophage into the organism takes place in a territory free from the infection. We have seen, for example, that five months after the termination of an epizootic of barbone it is still possible to isolate a bacteriophage active for the pathogenic bacterium from the excreta of buffaloes which have resisted. On the other hand experimentation in the chicken has shown us that the activity of the bacteriophage for the pathogenic bacillus is maintained just as long as the experimental animal continues to ingest these bacteria. 
2. Bablet has shown that the bacteriophage corpuscles are destroyed by preservation for a week in glycerine. We know that this substance exerts no destructive influence on either the diastases or the toxins. It may be assumed, therefore, that in a mixture of bacteriophage suspension and glycerine the protobes alone will be destroyed while the immunizing substances contained in the medium will remain intact. Starting from this hypothesis, we mixed $0.5 \mathrm{cc}$. of a suspension of the Barbone-bacteriophage with $9.5 \mathrm{cc}$. of glycerine. After holding the mixture at incubator temperature $\left(37^{\circ} \mathrm{C}\right.$.) for ten days, and after we were assured that the corpuscles were effectively destroyed, we inoculated two steers with this liquid, diluted in $500 \mathrm{cc}$. of saline. Each steer thus received $0.25 \mathrm{cc}$. of the original suspension. Tests after forty-five days, respectively with 5 and 50 fatal doses of a culture of the bacterium of barbone, showed that these two animals resisted. They had acquired an immunity in spite of the destruction of the bacteriophage protobes.

In the case of experimental barbone the tests were made in a barbonefree region, and the principle which is responsible for the development of the immunity is most probably constituted of the substance of the bacterial cells. The rôle which the bacteriophage plays here is to dissolve the bacteria, in which condition the bacterial substance is in a state particularly adapted to stimulating the cells of the body which enter into the production of organic immunity. The substance of the bacterial body dissolves in the medium under the influence of the dissolving principle elaborated by the protobes, but it is not present in the same condition as in the body of the living bacterium, for the bacteriophage does not simply produce a disintegration. This is shown by the fact that the culture medium becomes perfectly limpid, whereas the medium remains cloudy when a simple disintegration takes place. As we have seen in several tests, the destruction of the bacterium by the bacteriophage is a process of solution. Indeed, it is rather the substances composing the bacterial body which are dissolved. This process is of necessity accompanied by a change in state. It is, then, not proper to speak of the bacterial substance as the principle which provokes the acquisition of immunity; it is in reality the products resulting from the degradation of the substances composing the bacterial cells which are effective.

It is obvious that this is yet only an hypothesis, experiment showing only that the principle which provokes the appearance of immunity is not, under the conditions of the experiment, the bacteriophage con- 
sidered as a living being. Aside from the dissolved bacterial substance do the other substances present in the culture play any part in the production of immunity? In the present state of these investigations it is impossible to affirm or deny this.

We have tested the action of temperature on the immunizing element contained in the bacteriophage suspension. To this end, we have repeated the experiment of the bacteriophage treated with glycerine, with the difference that the suspension was previously subjected to a temperature of $56^{\circ} \mathrm{C}$. maintained for a half hour. Two steers have each received a dose of this preparation heated and glycerinized, corresponding to $0.25 \mathrm{cc}$. of the original suspension. After forty-five days they were tested, the one with five, the other with fifty, fatal doses of barbone culture. The first resisted, the second died. The immunizing principle contained in the bacteriophage suspension is not destroyed but is sensibly weakened by heating for a half hour at $56^{\circ} \mathrm{C}$.

Although it is not yet possible to know with certainty the nature of the process which controls the development of organic immunity, we are at least able to recognize the result and to note the property which distinguishes the animal immunized by an injection of the bacteriophage from a normal animal.

In the case of the immunity acquired as a result of an attack of a contagious disease the blood possesses preventive properties. The blood of immunized animals enjoys the same property, as the following experiments show.

I. Steer No. 54 received on November $5,0.25$ cc. of a Barbonebacteriophage. Fourteen days later $500 \mathrm{cc}$. of blood was taken into a flask containing $25 \mathrm{cc}$. of a 10 per cent solution of sodium citrate. The blood was immediately injected into the jugular vein of steer No. 43. This last animal was tested twenty-three hours later by the injection of 1000 fatal doses of the bacterium of barbone culture. It failed to show the least evidence of infection. A control died in twentythree hours. Steer No. 54 likewise resisted the inoculation of 1000 fatal doses, given on December 1st.

II. The experiment given above was repeated. Steer No. 112 received into the jugular vein $500 \mathrm{cc}$. of blood from steer No. 95. Both of them resisted the test injections.

III. Steer No. 104 received on December 29 a subcutaneous injection of $0.04 \mathrm{cc}$. of a suspension of the bacteriophage. Four days later $500 \mathrm{cc}$. of blood were taken as before and this was transfused into steer No. 108. The next day the two steers resisted the inoculation of five fatal doses, which killed the control animal in thirty-two hours. 
IV. The above experiment (III) was repeated. The steer which received the blood of the immunized animal was not tested by the inoculation of 50 fatal doses until forty-five days after the transfusion. It resisted, without showing any apparent disturbance, as did also the steer which was immunized directly.

This last experiment does not, however, prove anything with regard to the duration of passive immunity conferred by the blood of an immunized animal, for it was performed with homologous blood, and we know that an immunity thus produced is of much longer duration than that produced with heterologous blood. In any case, the immunity thus conferred is extremely powerful and these experiments open the way for further investigations on the production of therapeutic sera in animals immunized by a single injection of an active bacteriophage, not only for barbone, but for other diseases as well.

One might conceive that the "principle" which is contained in the blood of the immunized animal and which confers the passive immunity is nothing more than the suspension of the bacteriophage persisting in the circulation. But this is impossible, for if the blood is taken at a time sufficiently close to the immunizing injection of the bacteriophage, it is in no way effective. That is, blood taken during the incubation period confers no immunity to the transfused animal.

Steers Nos. 89 and 90 received on December 19, 0.25 ec. of the bacteriophage suspension subcutaneously. Sixteen days later 500 ec. of blood were withdrawn from each animal and transfused into steers Nos. 92 and 93. The four animals, tested the next day, died with no greater delay than the controls. Steer No. 46 received on November 5, $20 \mathrm{cc}$. of the bacteriophage. On November 19, $500 \mathrm{ec}$. of blood were taken and transfused into steer No. 42. These two animals died in the same time as the control after a test injection.

As is to be seen, the incubation period of immunity in animals which receive the immunizing injection of bacteriophage suspension parallels the appearance of the protective power in their blood. Immunity develops abruptly; in the same way the protective power of theblood manifests itself suddenly, and at the same moment.

What then, is the immunizing principle which makes its sudden appearance in the blood at the moment when immunity is established, even in animals which have received only the minimal dose of a single drop of the bacteriophage? Can it be an amboceptor? By no means, for the complement fixation reaction shows that the sera of animals immunized with suspensions of the bacteriophage do not contain a 
specific amboceptor in detectable quantity. In conducting the reaction of Bordet with even 0.5 cc. one obtains an exactly comparable hemolysis, of the same intensity and in the same time, as that which occurs in a control tube containing the same quantity of normal serum.

Examination of the opsonic power of two of these sera gave indices of 0.3 and 0.4 ; indices which are essentially negative.

The serum containing the protective principle does not contain destructive substances or even substances delaying the growth of the bacterium of barbone. Bouillon, mixed with such a serum, in any proportion (from $0.05 \mathrm{cc}$. to $3 \mathrm{cc}$. per $10 \mathrm{cc}$. of bouillon), with or without the addition of fresh guinea pig serum, furnishes a medium which, when inoculated, gives luxuriant cultures of the bacterium of barbone.

Finally, the serum contains no traces of agglutinins.

Organic immunity, then, is not due to the presence of an amboceptor, nor to the presence of an opsonin in the blood of the vaccinated subjects. The blood contains neither agglutinins nor inhibiting substances. The immunity is most probably antitoxic.

We have seen in the experiments performed on avian typhosis, that, in an infected area, the protection of the animal is immediate and that this protection is assured only by the presence of bacteriophage corpuscles virulent for the pathogenic bacterium. We have again found this immediate immunity in the case of barbone. It is that which protected steers Nos. 103 and 107 against the inoculation of five fatal doses of culture when given only twenty-four hours after the injection of the bacteriophage.

In typhosis, this heterologous immunity has been permanent, for the daily reinfections which occur in the infected area allow the bacteriophage to multiply at the expense of the pathogenic bacteria ingested and thus to maintain its virulence for this bacterium. In barbone, this same thing takes place in an infected area, since we have seen that the bacteriophage virulent for the bacterium of barbone was present in the intestine of buffaloes five months after the complete disappearance of the epizootic.

In a non-infected region, and this was the case in the experiments performed on barbone, the mechanism is not same. In the absence of reinfection the bacteriophage active for the bacterium is eliminated very rapidly, since it is not able to multiply at the expense of this bacterium. The heterologous immunity disappears with it, - that is to say, after one or two days,- - and the animal then becomes susceptible. It remains in this condition throughout the entire duration of the in- 
cubation of the organic immunity, which develops under the influence of the soluble products contained in the culture of the bacteriophage. Once this organic immunity is established the animal is refractory.

We will see in connection with dysentery, that when a suspension of the bacteriophage is injected the organism responds by the production of an antitoxin. It is probable that the same thing takes place in barbone and that the protective principle present in the blood, since it is neither an amboceptor nor an opsonin, is likewise an antitoxin; the response of the organism to the injection of the modified substance of the dissolved bacterial cells contained in the suspension of the bacteriophage.

To summarize: the injection of the buffalo or of cattle, with a suspension of the bacteriophage active for the bacterium of barbone confers:

1. An heterologous immunity, solely due to the presence in the body of bacteriophage protobes virulent for the bacterium of barbone, which assures the destruction of the bacteria upon their introduction into the organism. This immunity terminates just as soon as the protobes are eliminated from the body. In the absence of frequent reinfections this elimination is very rapid, since the continued growth and the maintenance of virulence can not persist.

2. An homologous, or organic and powerful immunity, induced by a reaction of the tissues of the animal to the soluble principles contained in the bacteriophage suspension injected. This organic immunity is characterized principally by the appearance in the blood of an extremely potent immunizing substance-probably an antitoxin. The organic immunity establishes itself abruptly after an incubation period, which varies with the dose injected, being longer as the amount of injected culture is increased.

A single injection of 0.04 cc., or less than a normal sized drop, into a steer of $100 \mathrm{kgm}$. weight places the animal within 4 days in a condition where it can withstand a test inoculation of five fatal doses. Sixty days later the animal resists a test inoculation representing fifty surely fatal doses.

The blood of an immunized animal injected into a normal animal confers on the latter a passive immunity as solid as that enjoyed by the actively immunized one itself, even if this last one has received but a single injection of $0.04 \mathrm{cc}$. of bacteriophage suspension. And this passive immunity, under experimental conditions at least, is still intact forty-five days after the injection of the blood. . $^{3 * 1}$

As stated above, after my departure from Indo-China Le Louet 
continued the experiments on the duration of the immunity to barbone, showing that even 14 months after an immunizing injection of the Barbone-bacteriophage a very considerable immunity remained.

As a result of these experiments, Le Louet, who in the meantime had become Inspector-General of the Veterinary Service of Indo-China, applied this process of immunization on a large scale. The bacteriophage suspensions virulent for Pasteurella bovis were prepared at the Pasteur Institute at Saïgon. By September, 1923, 12,000 buffaloes had been immunized, and the first results on this group were reported by Le Louet at the Congress of the Tropical Medical Association of the Extreme Orient (Singapore, September, 1923).

He stated that up to that time, none of the 12,000 buffaloes which had been immunized had contracted the disease, despite the fact that it was prevalent in Indo-China.

Since then, the vaccinations have been continued. The mortality from barbone among the buffaloes has been reduced to actually nothing, and according to Le Louet ${ }^{405}$ it appears as though the foci of the disease have died out.

Comment on these results is unnecessary.

\section{IMMUNIZATION AGAINST BACILLARY DYSENTERY}

"The cultures of Shiga lysed by the invisible microbe, which are in reality cultures of the anti-microbe, possess the property of immunizing the rabbit against a dose of Shiga bacilli which will kill the controls in five days." This statement is taken from my first communication on the bacteriophage. The experimental data upon which this affirmation was based are given in the following protocols.

The rabbit, although naturally refractory to bacillary dysentery is, on the contrary, susceptible to the inoculation of dysentery toxin. This animal could, then, be utilized for the preliminary antitoxic immunization experiments. The following experiments showed at once that the Shiga-bacteriophage suspension, a short time after dissolution, is toxic, although to a less degree than is a normal culture of Shiga bacilli.

Rabbit No. 1. One cubic centimeter of a normal culture of Shiga bacilli was injected intravenously on August 10. The animal died on August 16.

Rabbit No. 2. Two cubic centimeters of a normal culture of Shiga bacilli were injected subcutaneously on August 10. The animal died on August 16.

Rabbit No. 3. One cubic centimeter of a Shiga bacillus culture which 
had been subjected to bacteriophagy for six hours was injected intravenously. (The amount of bacillary substance here was the same as in the preceding.) The rabbit lived.

Rabbit No. 4. Two cubic centimeters of a Shiga culture which had been undergoing bacteriophagy for six hours were injected subcutaneously. The animal died on August 16. This rabbit had also been injected on August 10.

Six days after the completion of the dissolution the toxicity of the culture was markedly diminished, as the following tests show:

Rabbit No. 5. Two cubic centimeters of a Shiga bacillus culture which had been dissolved 6 days previously were injected intravenously on August 10. The animal lived.

Rabbit No. 6. Three cubic centimeters of the Shiga culture which had been dissolved for 6 days were injected subcutaneously on August 10. This rabbit also lived.

Rabbit No. 7. Five cubic centimeters of the Shiga culture which had been dissolved for 6 days were injected intravenously on August 10. The rabbit died on August 21.

When the tests were done with a Shiga culture which had been dissolved for a month the toxicity had disappeared, as is shown by the following.

Rabbit No. 8. Fifteen cubic centimeters of Shiga culture which had been dissolved for one month were injected subcutaneously. The rabbit lived. The injection was given on August 10 .

Rabbit No. 9. Ten cubic centimeters of this same preparation were injected intravenously on August 10. This rabbit lived.

The following protocol illustrates an immunization experiment.

On August 23, eight rabbits received a subcutaneous injection of 0.25 cc. of a suspension of the Shiga-bacteriophage, two months after the dissolution was completed. These animals were tested by the injection of 3 cc. of a twenty-four hour bouillon culture of Shiga bacilli. For the strain employed this represented two surely fatal doses. The strain of Shiga used in the test differed from that used to prepare the suspension dissolved by the bacteriophage.

Rabbit No. 10; tested after twenty-eight hours. Died six days later.

Rabbit No. 11; tested after four days. Died five days later.

Rabbit No. 12; tested after six days. Lived.

Rabbit No. 13; tested after eight days. Lived.

Rabbit No. 14; tested after ten days. Lived.

Rabbit No. 15; tested after one month. Lived. 
Rabbit No. 16; tested after two months. Lived.

Rabbit No. 17; tested after three months. Lived.

All the control rabbits inoculated with half the dose, that is, with 1.5 cc. of the Shiga culture, died in from four to seven days.

The rabbit is, therefore, immunized against two surely fatal doses of B. dysenteriae Shiga culture by the injection of a quarter of a cubic centimeter of a suspension of the Shiga-bacteriophage. The antitoxic immunity is established six days after the injection and persists for at least three months.

In an experiment of this kind there can be no question of the nature of the process. The bacteriophage as a living being can not be the cause of the immunity. The responsible agent must be the soluble principles contained in the culture medium. ${ }^{311,321 *}$

Kabeshima ${ }^{386}$ was the first to confirm these experiments. He injected into the ear-vein of each of 10 rabbits, weighing about $2 \mathrm{kgm}$., 1 cc. of a suspension of the Shiga-bacteriophage. The suspensions inoculated were of different ages, some of the rabbits receiving suspensions which had been prepared for 3 weeks, others receiving suspensions which were from 3 months to $2 \frac{1}{2}$ years old, some of the latter being suspensions which I had turned over to him for his experiments made in my laboratory.

Eleven days after the injections, the 10 immunized rabbits, as well as 10 controls, received an inoculation of 3 fatal doses of a culture of the Shiga bacillus. The 10 controls died within 12 hours. All of the immunized animals resisted, and, although they became somewhat emaciated they regained their original weight within periods of from 2 to 17 days.

Kabeshima then attempted to determine at what time the immunity appeared. (Naturally, in this case it is an antitoxic immunity.) Each of 10 rabbits received an intravenous injection of 1 cc. of a Shigabacteriophage suspension. They were then inoculated, one by one, after variable lengths of time, with a surely fatal dose of the Shiga bacil-

* Several immunizing experiments with the bacteriophage for B. typhosus and for the paratyphoid organisms have been performed upon laboratory animals, both rabbits and guinea pigs. In all cases these showed a perfect immunization; -provided it is permissible to employ the word immunization when the process is carried out in refractory animals.

Not attributing any value to experiments of this type I have not included them in the monograph. In all cases the bacteriophage administered, either when given by subcutaneous injection or by the buccal route, has been isolated a few hours later from the intestinal tract. 
lus. He found that, even after 24 hours, there was some increased resistance, and that the immunity was fully acquired by the 4th day.

In a third series of experiments he tested the subcutaneous route as a mode of administration of the bacteriophage suspension. A single injection of $2 \mathrm{cc}$. by this route gave him the same results as had 1 cc. by the intravenous route, provided the suspension was not more than 3 months old. Two injections of a suspension $2 \frac{1}{2}$ years old perfectly immunized the animals.

Under the direction of Flu, Sardjito ${ }^{566}$ made a study of this question the subject of his thesis for the doctorate. He prepared his suspensions of the bacteriophage by causing a very virulent race of the bacteriophage to act upon a suspension containing 300 million bacilli per cubic centimeter, the Shiga strain being very toxic. When, after bacteriophagy, the fluid was perfectly limpid, he added a new quantity of a concentrated suspension of bacilli, in such a way as to restore the count to 300 million per cubic centimeter. Again after bacteriophagy he restored the bacterial suspension a third time, in the same way. When bacteriophagy was finished, the suspension then containing the dissolved bacterial substance of 900 million bacteria per cc., he filtered through a candle and used the fresh filtrate for his injections.*

Six rabbits inoculated by the subcutaneous route with $0.25 \mathrm{cc}$. of this bacteriophage suspension were tested after a variable length of time by the injection of 4 surely fatal doses of a culture of Shiga bacilli. The results in this series were:

Two rabbits, tested after 45 days, resisted without loss of weight. Three rabbits tested after 37 days, 2 resisted without loss in weight, 1 died.

One rabbit tested after 31 days died.

Therefore, after the injection of 0.25 cc. of a suspension of the bacteriophage 4 out of 6 rabbits had an antitoxic immunity sufficient to enable them to resist the injection of 4 lethal doses of Shiga bacillus culture.

In a second series, 7 rabbits received a subcutaneous injection of 0.12 cc. of the bacteriophage suspension. They were tested by the inoculation of 4 fatal doses, as follows:

* This was a mistake. Sardjito thus lost 9 animals because of the toxicity of the fresh filtrate. He mentions, however, that this course was taken because of the urgency of eireumstances. As I have shown elsewhere, the toxicity diminishes considerably as the suspension ages. When the time comes for applying this method of immunization against bacillary dysentery to man, it will be advisable to age the suspensions for a least a month before use. 
Three were injected after 24 days. All 3 resisted, 2 without loss of weight, 1 lost 200 grams.

Two were tested after 17 days. One resisted, the other died.

Two were tested after 10 days. Both resisted, one without loss in weight, the other lost 50 grams.

Thus, of the 7 rabbits inoculated with but $0.12 \mathrm{cc}$. of bacteriophage suspension, 6 resisted the inoculation of 4 fatal doses of culture. A single one did not have an immunity sufficient to overcome this strong intoxication.

Sardjito then attempted to ascertain whether the bacteriophage exerts an opsonic action in vivo comparable to that which I had shown to occur in vitro. His results indicated that the subcutaneous inoculation of a suspension of the bacteriophage increased the phagocytic power of the rabbit from 100 to 200 per cent.

These experiments upon rabbits revealed another fact which is indeed difficult to understand, namely, the injection of the bacteriophage suspensions neutralized in vivo the dysentery toxin

When rabbits which had received by subcutaneous injection a surely fatal dose of Shiga bacilli were given 6 hours later, also by the subcutaneous route, $1 \mathrm{cc}$. of bacteriophage suspension (one month old) 8 of the 11 animals survived. All 6 of the control rabbits died. In a second experiment the bacteriophage was not injected until 16 hours after the injection of Shiga bacilli. Here, 2 of the 6 treated animals lived. All 4 of the controls died.

On the other hand, upon many occasions I have injected simultaneously the bacteriophage and the bacilli into different regions of the body and I have yet to protect an animal from death in this way. Apparently unless the bacteriophage is injected either before or after the infecting injection it is without neutralizing effect. For this fact I can offer no explanation.

This distinct antitoxic action of suspensions of the bacteriophage which have developed at the expense of $B$. dysenteriae is in some way related to the phenomenon of antiphylaxis. As was stated when we were considering this phenomenon it seems logical to believe that when a series of injections of bacteriophage cause within the animal the development of a toxic hypersensitivity it must mean that the suspensions contain an antitoxic substance or property. There are other experiments which suggest the same thing.

Such are the experiments made up to the present time upon the immunity conferred by the inoculation of suspensions of the bacteriophage. It is to be hoped that experiments will be made on man, partic- 
ularly in connection with protection against bacillary dysentery, and it seems that such a course is the more logical since, as we shall see, the administration of bacteriophage suspensions is being applied on a large scale in the specific therapy of this disease.

\section{RÉSUMÉ}

Experiments have been carried out in three very different infectious diseases:

a. One an infectious disease in which the portal of entry of the causative agent is the intestinal tract (avian typhosis);

b. One a septicemic disease (barbone of the buffalo);

c. One a toxic disease (injection of the rabbit with Shiga bacilli).

The experiments have been carried out under different environmental conditions:

a. In an environment contaminated by the infecting agent (avian typhosis);

$b$. In an environment free of contamination (barbone).

The results obtained with these differing diseases, under these varied circumstances permit the following conclusions:

The experiments made upon barbone of the buffalo in an uncontaminated environment show that after the injection of a bacteriophage suspension there is an initial, and very short, period of immunity, -24 to 48 hours, - followed by a period in which the animal has returned to its normal susceptibility, the length of which is increased as the dose of suspension given was the greater. After a variable time, -20 to 30 days after doses of 1 to $2 \mathrm{cc}$. in the buffalo,-an immunity is established which borders on the refractory state (d'Herelle and Le Louet $\left.{ }^{321}\right)$. This second period of immunity persists for at least 14 months (Le Louet ${ }^{405}$ ).

The first period of immunity is "exogenous" in nature. It is due to the presence in the body of bacteriophage protobes virulent for the pathogenic bacterium. In a contaminated environment this period of exogenous immunity persists as long as re-infections occur; re-infections which permit the protobes to multiply and maintain themselves. In an uncontaminated enviromment the bacteriophage is unable to multiply because of a lack of susceptible bacteria. Elimination from the body is rapid, and the exogenous immunity ceases immediately after this elimination is complete.

The second period of immunity, is certainly endogenous.* It is the

* As I use the terms, "exogenous immunity" is that which is due to the presence of the foreign principle in the body, - as the bacteriophage corpuscle itself,"endogenous immunity" is that which results from a reaction of the body itself ${ }^{311,321,349}$ 
result of some reaction on the part of the body of the animal, the stimulus being provided by the products of bacterial dissolution as contained in the bacteriophage suspension. These bacterial products, produced through bacteriophagy, appear to be in a distinctive and peculiar physical state, one particularly suited to causing a reaction of immunity by the body of the animal (d'Herelle ${ }^{321,349}$ ). The extensive immunization experiments made under natural conditions of exposure to infection have confirmed those made in the laboratory (Le Louet ${ }^{405}$ ).

In an epidemic environment, the experiments made on natural typhosis show that the immunity conferred by the administration of bacteriophage suspensions is acquired immediately. It begins at the moment of the inoculation, and is lasting. Experiment shows clearly that the immunizing agent is the bacteriophage,- as a living parasite of bacteria. Under the conditions of an infected environment the bacteriophage maintains itself because of the frequently occurring reinfections which permit the corpuscles to multiply and perpetuate themselves within the body of the animal. After an incubation period this lasting exogenous immunity is followed by an endogenous immunity, resulting, as in the previous case, from a reaction of the body to the bacterial substances present in the suspension of bacteriophage protobes inoculated (d'Herelle ${ }^{321}$ ).

In brief, the mechanism is always the same; the only difference being that immediately acquired exogenous immunity is transitory in an uncontaminated environment; permanent in a contaminated environment.

The antitoxic immunity which develops after the injection of bacteriophage suspensions is also endogenous in origin, due to the inoculation of the bacterial products present in the suspension. These products seem here to be found in a physical state particularly adapted to exciting an antitoxic reaction response on the part of the animal body (d'Herelle ${ }^{321}$ ). 


\section{CHAPTER VI}

\section{Specific Therapy with Bacteriophage Suspensions}

\section{THE SPECIFIC THERAPY OF BACILLARY DYSENTERY}

Before undertaking experiments on man I had to assure myself that the administration of suspensions of the Shiga-bacteriophage caused no reaction. First, I ingested increasing quantities of such suspensions, aged from six days to a month, from one to thirty cubic centimeters, without detecting the slightest malaise. Three persons in my family next ingested variable quantities several times without showing the least disturbance. I then injected myself subcutaneously with one cubic centimeter of a forty-day old suspension. There was neither a local nor a general reaction. In all the cases, twenty-four hours after the ingestion or after the injection, I was able to isolate from the stools a bacteriophage possessing for the Shiga bacillus an activity equal to that of the race administered. More recently G. Eliava has received by subcutaneous injection $5 \mathrm{cc}$. of a suspension of Shiga-bacteriophage aged thirty days. No reaction, local or general, followed.

It is known that the subcutaneous injection of Shiga bacilli, killed by any procedure whatsoever, can not be performed because of the extremely violent reactions produced, which are due to the toxicity of the bacillus. This is precisely the reason that vaccine prophylaxis is not applied to dysentery as it is in the case of typhoid. The absolute innocuity of injections of the Shiga-bacteriophage suspensions, which contain the substance of the bacterial bodies in a dissolved state, shows indeed that these substances undergo profound modifications under the influence of the dissolving agents of the bacteriophage protobe. Nevertheless, these new substances possess a specific immunizing power much more potent than the original substance. The experiments on rabbits, and in particular the results secured in immunization against barbone, demonstrate this beyond possible doubt.

Prophylactic vaccination against bacillary dysentery by means of suspensions of the anti-dysentery bacteriophage is therefore applicable to man. In practice, quite naturally, the prophylactic injections should be performed with a mixture of bacteriophage races-antiShiga, anti-Flexner, and anti-Hiss. Such a mixture would constitute a polyvalent dysentery vaccine. 
The Shiga bacillus is one of the most toxic organisms known, and it may be assumed that the harmlessness of injections of such a bacteriophaged culture indicates a general law, whatever may be the bacterium against which the bacteriophage is prepared. In order to test this hypothesis, I injected myself, subcutaneously, with half a cubic centimeter of Plague-bacteriophage. No reaction, either general or local, followed. Stool examination made twenty-four hours after the injection showed that a bacteriophage, equal in virulence to that injected, was present. The inoculation experiment was repeated with Typhoidbacteriophage. G. Eliava repeated it with the Staphylococcusbacteriophage, and the same results were secured in both cases. These observations are confirmed in part by another fact, observed in several tests, that following the administration of the bacteriophage, either by injection or by ingestion, the bacteriophage passes in a short time into the intestine. It is eliminated rapidly if it fails to encounter the bacterium against which it has a virulence, that is to say, in an uninfected individual. On the contrary, it grows and maintains its virulence if it is in contact with this bacterium, a condition which, as we have seen in several instances, is produced in an infected environment among animals which remained healthy, or which had been infected and were recovered.

After being assured that no harmful effects attended the ingestion of the Shiga-bacteriophage, this treatment was applied for therapeutic purposes to patients affected with bacillary dysentery.* As in the experimental work, so also here in the clinical tests, the therapy has been limited to those cases in which the etiology of the infection was proved by the isolation of the pathogenic organism, and where, in addition, the virulence of the intestinal bacteriophage was negative

* These experiments were made with the assistance of M. Nadal, on the service of Dr. Hutinel, at the Hôpital des Enfants Malades.

Tests have also been made in cases of toxic diarrhea of infants, but they will not be discussed here since a conclusion regarding them has not yet been reached. In those cases there is an especial difficulty, for the pathogenic organism is still unknown. It was at first thought that this might be determined through the ability to isolate and cultivate an active race of bacteriophage which might be used for curative purposes. It is indeed probable that there is, not one, but several diarrheas of infants caused by different bacterial types, as the experiments of Nobécourt made during the past few years would also indicate. The solution of the prolstem is not impossible but it would be necessary to administer to the affected infants a mixture of diverse races of the bacteriophage, active against the diverse bacterial types capable of inciting the diarrhea. It can readily be conceived that under such eircumstances the investigation must be protracted. 
toward the different dysentery bacilli at the time of the administration of the bacteriophage suspension. It is evident that in routine practice it would not be necessary to investigate all these points, especially since the administration of the bacteriophage is always inoffensive.

In each of the following cases the only treatment instituted has been the ingestion of the bacteriophage.

Robert K. . . . (eleven years). This was a case of bacillary dysentery of moderate severity with from 5 to 7 bloody stools a day.

August 1. The stool examination showed: B. dysenteriae Shiga present.

The intestinal bacteriophage with virulences as follows: $B . \operatorname{coli}++$, Shiga 0 , Flexner 0 , Hiss 0 .

August 2. At 10 o'clock in the morning the patient ingested 2 cc. of a Shiga-bacteriophage. This filtrate had been held for thirty-five days. During the afternoon of this day there were 3 bloody stools, in the evening there was one stool and that was free of blood.

August 3. During this day there was only the one formed stool. Examination showed: B. dysenteriae Shiga absent.

The intestinal bacteriophage with virulences as follows: $B$. coli ++++ , shiga $t+++$, Flexner +++ , Hiss $t++$.

August 8 . The intestinal bacteriophage was active as follows: $B$. coli +++ , Shiga + , Flexner 0, Hiss + .

August 9. The patient was discharged from the hospital.

André B. . . . . (ten years). A case of bacillary dysentery of moderate severity. During the period from August 25 to 29 inclusive there were 9 to 11 bloody stools a day.

August 28. Stool examination showed: B. dysenteriae Shiga present.

Intestinal bacteriophage active as follows: $B$. coli + , Shiga 0, Flexner 0 , Hiss 0 .

August 29. At 4 p.m. the patient ingested 2 cc. of a Shiga-bacteriophage suspension which had been prepared for 2 months.

August 30. There was one bloody stool in the morning and during the afternoon and the night there were 5 stools, none of which showed any blood.

August 31. There were 3 fluid, but not bloody, stools.

Examination showed: Shiga bacilli not present.

The intestinal bacteriophage active as follows: B. coli ++++ , Flexner ++ , Hiss +++ , Shiga ++++ . 
September 1. There was one fluid stool, without blood.

September 2. There was one fluid stool, without blood.

September 3. There was one formed stool. Examination of the intestinal bacteriophage showed: $B$. coli +++ , Shiga ++++ , Flexner +++ , Hiss + .

September 8. Reactions with the intestinal bacteriophage were: B. coli ++ , Shiga 0, Flexner 0, Hiss + .

September 9 . The patient was discharged from the hospital.

Robert D. . . . . (twelve years). This patient had a very severe dysentery, with vomiting, cold sweats, chilling of the extremities, and involuntary and uncountable stools.

September 8. The stools could not be counted. They were fetid, purulent, and streaked with blood. Examination showed: B. dysenteriae Shiga present; about 1 out of every 10 colonies on the plates was the dysentery bacillus.

The intestinal bacteriophage showed no virulence for $B$. coli, or for the Shiga, Flexner, or Hiss organisms.

September 9. Two cubic centimeters of a suspension of Shiga-bacteriophage were ingested at 11 o'clock. This suspension was three and one-half months old. During the afternoon and the night the stools became less numerous but continued bloody.

September 10. There were 6 fluid stools, without blood. Examination showed: B. dysenteriae Shiga, not present.

Intestinal bacteriophage active as follows: $B$. coli ++++ , Shiga ++++ , Flexner ++++ , Hiss +++ .

September 11. There were 2 normal, formed stools.

September 20. The patient was discharged from the hospital.

Julien D. . . . . (three and one-half years). This was a case of very severe dysentery. The general condition of the patient was very bad. A sister of the patient had died at home of dysentery on September 8 .

From the 11th to the 13th of September the number of stools, all of which were bloody, could not be counted.

September 13. The patient entered the hospital. Examination showed: B. dysenteriae Shiga present, the dysentery bacilli constituting about 4 of every 5 colonies on the plates.

The intestinal bacteriophage was without activity for either $B$. coli or the dysentery organisms. 
September 13 . The patient ingested $2 \mathrm{cc}$. of Shiga-bacteriophage at 5 o'clock. This filtrate had been prepared for fifteen days.

September 14. There were 6 bloody stools. The intestinal bacteriophage showed virulences as follows: $B$. coli +++ , Shiga +++ , Flexner ++ , Hiss + .

September 15. During the day there was one bloody stool. There were also 5 stools without blood. Examination showed: $B$. dysenteriae Shiga absent.

The intestinal bacteriophage with activities as follows: $B$. coli ++++ , Shiga ++++ , Flexner +++ , Hiss $t++$.

September 16. There were 4 stools, all without blood. The intestinal bacteriophage showed: B. coli +++ , Shiga ++++ , Flexner ++ , Hiss +++ .

September 17. During the day there were one fluid stool and 2 formed stools. The intestinal bacteriophage showed: B. coli ++++ , Shiga ++++ , Flexner ++ , Hiss + .

September 18. There were 2 formed stools. The intestinal bacteriophage was virulent as follows: $B$. coli ++++ , Shiga ++++ , Flexner + , Hiss ++ .

September 26. The patient was discharged from the hospital. On this date the virulence of the intestinal bacteriophage was: $B$. coli +++ , Shiga 0, Flexner 0, Hiss + .

Emile D. . . . . (seven and one-half years). This patient was a brother of the preceding case, and showed a very severe dysentery. On the 11th and 12 th of September there were 20 to 25 fetid stools, fluid but not bloody.

September 12. Examination showed that the Shiga bacilli were very abundant. The intestinal bacteriophage was inactive for $B$. coli or for the dysentery organisms.

September 13 . There were 25 bloody stools. At 5 o'clock the patient ingested $2 \mathrm{cc}$. of bacteriophage suspension which had been prepared $6 \frac{1}{2}$ months previously.

September 14 . There were 4 bloody stools in the morning and 2 stools without blood in the afternoon. Examination of one of the latter showed no Shiga bacilli. The virulences of the intestinal bacteriophage were: B. coli ++++ , Shiga ++++ , Flexner ++ , Hiss +++ .

September 15 . There were 5 stools, all free of blood.

September 16,17 , and 18 . During these days there were 3 or 4 stools a day. None of these contained blood. 
September 19. There were 2 formed stools on this day.

September 28. The patient was discharged from the hospital.

In all of these cases the general condition of the patient always paralleled the severity of the intestinal symptoms.

Two other cases of dysentery due to the Shiga bacillus, treated in the same manner, but outside of the hospital, gave comparable results. In these there was a cessation of the bloody stools with improvement in the general condition in the twenty-four hours immediately following the administration of Shiga-bacteriophage suspensions.

Since the publication of these results ${ }^{321}$ this method of treating bacillary dysentery has been applied extensively, and the deduction from my experiments, which to me appeared so logical, that the bacteriophage should constitute a specific treatment of dysentery ${ }^{321}$ has been absolutely confirmed.

At the Institute Oswaldo Cruz at Rio de Janiero a test of the method, involving an initial group of 24 patients was undertaken by da Costa Cruz. ${ }^{157}$ To all of these cases the suspension of the bacteriophage was administered per os in a quantity of $2 \mathrm{cc}$. In 22 of the patients the stools lost their bloody character within 24 hours after the ingestion, and in the majority an improvement in the condition was evident within 4 to 6 hours. In the other 2 cases, after a temporary improvement, blood reappeared in the stools a few hours later. In these, a second dose was followed within the succeeding 24 hours, by improvement leading to a permanent convalescence.

Confronted by such results the Institute Oswaldo Cruz undertook the preparation of bacteriophage suspensions virulent for $B$. dysenteriae on a large scale. The results of da Costa Cruz, published in June, $1924,{ }^{165}$ are given here, quoting directly from his report.

Up to the present, we have prepared about 10,000 ampoules of the bacteriophage which have been distributed to several hospitals and to a number of physicians in the different States of Brazil.

As a preliminary test of the method, the bacteriophage was first sent to Dr. Nicolau da Costa (Para), to Dr. Cassio Miranda (Director of the Rural Prophylactic Service of Maranhao), to Dr. Cavalcanti (Director of the Rural Prophylactic Service of Pernambuco), to Dr. Travassos (Assistant at the Institute of Angra dos Reis, Rio), and to Dr. A. L. Barros Barreto (Director of Rural Prophylaxis of Parana).

The results obtained by these colleagues fully confirmed those which we had observed.

As a result, we have been obliged to send material to a great many doctors, 
distributed throughout all of the different States, who have requested preparations of the bacteriophage.

The Director of Public Health of the State of Bahia has kept us informed of the results obtained in a series of patients, and at Sao Paolo a very large number of cases have been observed by various physicians who have applied the treatment in their individual clinics. All have reported good results. Here in Rio de Janiero, the method has been thoroughly tried out, and is today the routine method of treatment.

Nevertheless, we still advise the use of serum in cases infected with the Shiga bacillus, since the toxin is not neutralized by the bacteriophage.

It is possible to find only a few rare cases in which the administration of the bacteriophage has not been followed by benefit to the patient. Of these cases, the first was a patient infected with the Hiss strain of the bacillus, perfectly susceptible in vitro to the bacteriophage, but in whom the bacteriophage failed, for reasons not yet discovered, to exert its action in the intestinal tract. The second was an infant who died of a very severe infection caused by the Shiga bacillus, in spite of the fact that both serum and bacteriophage treatment were used. These are the only two completely unsuccessful cases which have up to the present come to our attention, but to judge from the very wide use which has been made of the method it would seem that there must be other cases of failure of which we have not yet heard.

The therapeutic effect,--the sudden change in the condition of the patient,consequent to the administration of the bacteriophage has astonished all of the physicians who have, worked with it. A few hours after the administration of the first dose improvement has been noted, and the rapidity of the recovery has been a cause of amazement. In examining the collected data pertaining to the patients treated it appears that the majority of them had first received all known treatments, and had been given the bacteriophage as a last resort. We are, therefore, absolutely convinced that it has saved the lives of a great many patients.

The dysentery bacteriophage is by far the best therapeutic agent in the bacillary dysenteries known up to the present time.

Its action incomparably surpasses that of all other agents, including serum, for in the majority of cases the symptoms materially diminish in intensity within 4 to $S$ hours after treatment, and the patient enters upon convalescence within 24 to 48 hours.

The only criticism that I would make of the conclusions of this scientist at the Institute Oswaldo Cruz bears on the use of anti-dysentery serum. The results observed in the 7 cases of severe dysentery which have been cited above, as well as many comparable observations which I have made since those results were first reported, all dealing with cases of dysentery caused by the Shiga bacillus, have shown that the administration of the bacteriophage alone results in a prompt and complete cure, without requiring the administration of serum. I might say further that I have gained the impression from two cases where I have been obliged (because of the demands of the parents) to give an 
injection of serum at the same time that I administered the bacteriophage, that convalescence was retarded, probably as a result of serum reaction complications.

Serum may perhaps be indicated in those cases where the toxic symptoms are manifest at the time when the bacteriophage treatment is given; in all others serum causes more bother than benefit. It seems, indeed, that everything may not have been considered, for in a recent publication, Pereira ${ }^{500 *}$ reports on 23 cases of bacillary dysentery treated at the Hospital Sao Pedro at Porto Allegre. All of these were treated solely by the ingestion of bacteriophage suspensions, despite the fact that the Shiga bacillus was isolated from the stools of a majority of the patients. The conclusions of Pereira, presented below, confirm my first observations, and those of da Costa Cruz as well.

The anti-dysentery bacteriophage constitutes a specific treatment for the bacillary dysenteries, whether they be caused by the Shiga bacillus or by the paradysentery organisms.

Without causing general reactions, the ingestion of this principle modifies completely, within a very short space of time, the evolution of the dysenteric syndrome. Recovery, in a clinical and in a bacteriological sense, is complete.

By the use of the bacteriophage the development of carriers is avoided, for dysentery bacilli are never found in the excreta of patients who have been treated by the ingestion of the bacteriophage.

Pereira ends his discussion by suggesting that a milk diet is contraindicated during treatment with the bacteriophage, and this is indeed quite logical, for it is obviously necessary to carefully avoid everything which may change the reaction of the contents of the intestinal tract toward the acid side.

A final observation of Pereira is noteworthy, for he is of the opinion that since the systematic application of the method of treating the dysenteries by means of the bacteriophage was instituted, the mortality from enteric diseases in general has considerably diminished at the hospital. Serum treatment, he adds, comes far from giving as conclusive results. $\dagger$

But to obtain such results I can not emphasize too strongly the point that it is absolutely indispensable that the bacteriophage be of maximum virulence for the bacterium upon which it is to act. If it is impossible to

* The Oswaldo Cruz Prize, having been awarded to Pereira for his work, it would seem that the Institute itself must sanction his conclusions, and that they must agree with those obtained at the Institute itself.

$\dagger$ Recently Spence and McKinley ${ }^{580}$ have again confirmed the value of bacteriophagic therapy in bacillary dysentery. 
obtain a maximal virulence as I have defined it (complete and permanent dissolution of normal suspensions of 250 million bacilli per cubic centimeter) races of the bacteriophage with a very high virulence may be used, but those less active should never be employed. To use, either for prophylactic or for therapeutic purposes, races of the bacteriophage of moderate or even strong virulence, is to decide in advance that success or failure makes no particular difference.

I should also add that it is indispensable, even though the race of bacteriophage used is of maximum virulence and does not permit secondary cultures to develop, to filter the suspensions to be used for therapeutic purposes through a candle of known efficiency.

In addition, and on this point of general application it is impossible to place too much emphasis, it is imperative to administer the suspension just as early in the course of the disease as is possible. Early in the disease the bacteriophage has but one thing to do-to cause bacteriophagy,-and this is all that is required. Naturally, the bacteriophage is unable to cause even the slightest modification in organic lesions which have already developed. And, inasmuch as the administration of the bacteriophage is always harmless, even if the diagnosis is later proved to have been erroneous, it should be used at once without awaiting a confirmatory laboratory diagnosis. ${ }^{321}$

As regards dysentery, Otto and Munter, ${ }^{492}$ and later Davison, ${ }^{173}$ reported that their attempts at treating bacillary dysentery were complete failures. It is only fair to say that these authors made their attempts early in their study of bacteriophagy, without apparently having a sufficient knowledge of the distinctive characteristics of the bacteriophage, and, indeed, since in both of these reports the conclusion is reached that the active principle is a ferment derived from the bacterium, any idea of virulence could hardly have been within their conception. They most certainly used bacteriophage races of weak virulence, and the results could not be other than those which they obtained. This hypothesis seems the more probable, in that it so happens that da Costa Cruz passed through the same experience before he obtained the very beautiful results which we have just noted Two years prior to the publication of the report from which we have just quoted, that is, at the beginning of his work on the subject of bacteriophagy, he attempted the treatment of dysentery with suspensions of the bacteriophage, and he concluded that the therapeutic value was precisely zero, "quite the opposite to what d'Herelle assumed."”*

* In his paper ${ }^{157}$ he states that the results obtained in this first series of experiments were completely negative, "despite the great activity of the bacteriophage 
After having followed for two years his studies on the subject of bacteriophagy, now understanding the mechanism of the phenomenon more completely, he used bacteriophage races whose virulence was in fact very high. The results obtained are those reported above.*

\section{BACTERIOPHAGE THERAPY IN DIFFERENT INTESTINAL DISTURBANCES}

In the paper of da Costa Cruz from which we have quoted the following comments appear.

In addition, we have been surprised at the results obtained in cases of chronic colitis of undetermined nature, of several years duration,- - up to 15, - and which have recovered following bacteriophage therapy.

We have also observed one case of alimentary intolerance which recovered quickly and permanently after administration of the bacteriophage. This offers additional evidence that these conditions are of bacterial origin, associated with typical dysentery bacilli or with the very closely related $B$. coli forms. The bacteriophage has always been administered per os, twice a day, morning and evening, in doses of $2 \mathrm{cc}$. diluted in a glass of water.

I have myself applied treatment by the bacteriophage to about 100 cases of intestinal disturbances,- patients with a colitis, or an enteritis without a definite cause, and I have obtained a permanent recovery in almost all of the cases. I have always used mixtures of different races of the bacteriophage, including races virulent for the dysentery and paradysentery bacilli, for the paratyphoids $\mathrm{A}$ and $\mathrm{B}$, for $B$. enteritidis, and for B. suipestifer. As da Costa Cruz implies, in these eases it is often necessary to continue the ingestions throughout several days.

\section{BACTERIOPHAGE THERAPY IN TYPHOID AND THE PARATYPHOID FEVERS}

In the experiments which were made on preventive inoculation against avian typhosis, among the fowls vaccinated there were about 100 which were already sick at the time when the suspension of the bacteriophage

for the Shiga bacillus," and he adds in parenthesis, that the bacteriophage was active in vitro up to a dilution of $1: 100$. This very brief record shows that, contrary to what he then believed, the bacteriophage used in his therapeutic tests had but a low virulence, and not a high virulence, for had this been the case it would have been active, not at $1: 100$, but in a dilution at least a million times greater.

* I further commentary on these early adverse reports is provided by the fact that Munter, as a result of some observations made with Boenheim, ${ }^{467}$ renouncing his first conclusions, has affirmed that it is possible to obtain some very interesting results in the bacillary dysenteries of children. 
was injected. The mortality in the epizootics which I observed was nearly 100 per cent. In the chickens which received the bacteriophage suspension the mortality was about 5 per cent.

As the typhoid and paratyphoid bacilli are closely related to $B$. gallinarum, and since in many respects typhosis closely resembles human typhoid fever, I thought that a specific bacteriophage therapy of this last disease could be readily accomplished. This was a mistake.

Beckerich and Hauduroy ${ }^{58}$ treated 12 cases of typhoid and paratyphoid fevers with the bacteriophage. They report as follows:

In 2 cases of the ataxoadynamic form in the adult, as well as in 2 cases in children, all very severe cases, the administration of the bacteriophage proved of no benefit.

In 2 cases of the ordinary form, treated between the 9 th and 18 th days of the disease, the patients were given by ingestion $2 \mathrm{cc}$. of a suspension of the bacteriophage virulent for B. typhosus. Two hours after the treatment a crisis occurred, with sweating, which was foi'towed by a permanent fall in temperature in 48 hours.

In 2 very severe cases of infection by $B$. paratyphosus $B$ in infants, the bacteriophage was adminstered, on the 9 th day in one, on the $23 \mathrm{rd}$ day in the other, by the subcutaneous injection of $1 \mathrm{cc}$. and the simultaneous ingestion of $2 \mathrm{cc}$. In the first case the temperature dropped to normal within 24 hours; within 48 hours in the second case. As in the preceding cases successfully treated a sudoral crisis occurred about 2 hours after the administration of the bacteriophage.

In 2 cases of severe typhoid fever in adults, on the 10th and on the 14th days respectively, the bacteriophage was administered by mouth (5 cc.) and by subcutaneous injection $(1$ cc.). Permanent defervescence followed within 48 hours.

In all of the cases which have been described, the pathogenic bacillus was isolated by blood culture shortly before the administration of the bacteriophage, indicating that the disease was in its full development.

Hauduroy and Arsimoles ${ }^{307}$ report a $B$. typhosus infection, assuming the form of a dysentery,- -severe abdominal pain, colic, vomiting, mucous and bloody stools, and fever. The patient was given per os a bacteriophage virulent for both $B$. typhosus and B. dysenteriae. The blood quickly disappeared from the stools and convalescence began within 48 hours.

Alessandrini and Doria ${ }^{2}$ treated 18 cases of typhoid fever with the bacteriophage. In 9 the therapeutic result was very clear-cut, $-\mathrm{a}$ rapid improvement in the general condition with a complete loss of 
fever within a week. They noted that the higher the virulence of the bacteriophage for the bacterium causing the infection the sharper were the results and the more rapid the recovery.

Smith $^{586}$ has employed the bacteriophage therapeutically in 7 cases. In 5 he obtained a defervescence which began immediately after the administration. In the other 2 no benefit resulted, in spite of the fact that the bacilli from the blood, from the urine, and from the feces were susceptible to the bacteriophage used.

Richet, Azerad and Delarne ${ }^{554}$ have published notes on a case of a patient affected with a very severe typhoid of the ataxoadynamic type, which they treated by injections of bacteriophage. They state: "Improvement was apparent within 24 hours. Within 3 days the condition was radically transformed. In our opinion no other therapy would have caused as rapid an improvement. It appears to us to have acted as a specific therapy should act."

Philibert and Hauduroy ${ }^{509}$ have applied this treatment to a number of cases of typhoid fever with very variable results; the effect was very definite in some cases, entirely negative in others.

Herdershee and Wolff ${ }^{309}$ have treated about 100 cases without beneficial results.

It is worthy of note, however, that the conclusions of all of these authors is, in one respect, very definite. Either improvement with the tall in temperature occurs promptly after the administration of the bacteriophage, or no improvement at all results. There is no interinediate. The action is either complete or it is entirely lacking.

We thus have available the following facts:

The studies made on dysentery show that the condition of the patient is strictly related to the virulence of the intestinal bacteriophage for the pathogenic bacterium. Furthermore, the administration for therapeutic purposes of a suspension of the bacteriophage virulent for the pathogenic organism acts in all cases in a specific manner and causes a prompt cure.

In typhoid and the paratyphoid fevers, the situation is the same as regards the condition of the patient, this is always directly related to the virulence of the intestinal bacteriophage for the pathogenic bacillus. But in these infections, the situation does not parallel what is observed in dysentery, at least in the majority of cases the specific therapeutic effect is lacking. When such an effect does occur, it takes place just as in dysentery, but as a rule beneficial action does not attend the treatment. 
For this difference in behavior and in effect there must be a cause.

Marcuse $^{436}$ attributes the failures in typhoid fever to the assumption that a contact between the bacteriophage and the bacteria does not occur. It is necessary to have recourse, therefore, in typhoid fever to intravenous injections given in conjunction with ingestion. It is possible that this may account for the failure in some cases, but I do not think that it will explain all of them.

The thought naturally occurs that while the dysentery bacilli are a homogeneous species, the typhoid and paratyphoid bacilli are a heterogeneous species, and that this might explain the difference in the behavior of the bacteriophage suspensions when introduced into the body of the patient. Here again, certain failures may be explained upon such a basis, but I do not think that this can be the principal cause of lack of success.

Very recently Hauduroy has discovered a fact which may better explain the failures. ${ }^{304}$ He has found that in vitro the addition of bile to the medium inhibits bacteriophagy. The bacteriophage protobe is not destroyed, but its action is completely inhibited. In vivo the bacilli which are found in the gall bladder are thus completely protected against bacteriophagy, and consequently when the infection involves the bladder the administration of bacteriophage can have no effect upon the course of the disease.

An earlier finding of Hauduroy is in accord with this conclusion. He observed in a case where the administration of the bacteriophage had no effect upon the course of the disease that there was, nevertheless, a diminution and even a disappearance, of the typhoid bacilli from the feces. But this disappearance was only transitory, for a short time afterward they were again present as abundantly as before the administration of the bacteriophage.

The inhibitory action which bile exerts on bacteriophagy will certainly explain some of the failures, but it is probable that even this is not the key to the situation.

Is the typhoid bacillus which we know, which we see under the microscope, always the agent of the typhoid and paratyphoid fevers? In the forms of the disease of average severity, and more probably in the benign forms, it is probable; in the severe forms, it is doubtful. What, then, is the true agent in these?

We have discussed this question in an earlier chapter, and recent experimental studies show, that in reality certain forms of typhoid fever, the inost severe ones, must unquestionably be double infections 
in a sense, the two agents being the known visible form of B.typhosus and the infravisible form.* Insofar as the disease caused by the infravisible form of $B$. typhosus is concerned, the bacteriophage may have no action.

\section{BACTERIOPHAGE THERAPY OF COLON BACILLUS INFECTIONS}

Let us first state that since $B$. coli is, of all bacterial species the most heterogeneous, up to such time as some one succeeds in isolating a polyvirulent race of the bacteriophage - it will always be necessary to utilize in the treatment of each particular case what is improperly termed an "autobacteriophage." Treatment can be effected only by testing those races of the Coli-bacteriophage available in the laboratory and using that race which proves to be most virulent for the bacillus causing the infection. This has been the procedure of those who have attempted the bacteriophage treatment of $B$. coli infections.

Beckerich and Hauduroy ${ }^{58}$ were the first to publish results in this field, their report comprising 3 cases of puerperal pyelo-cystitis treated by a single subcutaneous injection of $1 \mathrm{cc}$. of a Coli-bacteriophage suspension. In the two cases where the $B$. coli isolated from the urine was susceptible, a sudoral crisis occurred 2 hours after the injection, the temperature quickly fell to normal, and cure was definite after 48 hours. In the third case the urinary colon bacillus was not susceptible, and no benefit followed the treatment.

These same authors ${ }^{62}$ then published the results obtained in 11 cases of urinary tract infection; 9 were recoveries, 2 were failures. Haudu${ }^{\prime} \mathrm{y}^{302}$ then applied the method in a large number of cases. Here are four of his observations, taken as examples. All are alike, however, in that the bacteriophage caused recovery. Again we find the same situation, namely, if recovery takes place at all, it occurs quickly. The effect seems to be "all or nothing.'

Case 1. Mrs. H. . . . . 35 years old. The patient had a chronic cystitis of 3 years duration, consequent to a puerperal infection. At the time when she appeared for treatment, urine secretion was abundant, and urination was frequent, with pain and burning. There was no

* There must also be forms of typhoid fever caused by the infravisible form alone, for in certain morljid conditions presenting clinically all of the symptoms of a severe typhoid, a most careful study will not permit the isolation of any organism capable of being considered as the cause of the disease.

$\dagger$ We have seen that Gratia has succeeded in isolating a polyvirulent race active for staphylococei. This offers hope that such a one may be found for B. coli. 
fever. The general condition was poor; the patient was emaciated. Up to the present time the disease had run a cyclic course, acute attacks following periods of remission.

The present attack had persisted for several weeks. Treatment of various kinds, - application of heat to the pelvis, urotropin,--had been instituted, but no improvement resulted. Bacteriological examination showed the urine to be virtually a culture of $B$. coli, and this $B$. coli was susceptible to a race of the bacteriophage maintained in the laboratory. Two cubic centimeters of this bacteriophage suspension were injected subcutaneously in the thigh. The next day there was a slight redness and swelling and a little pain. A second injection of $2 \mathrm{cc}$. was given on the following day. A local and not painful reaction followed.

The day following the second injection the pain associated with urination had disappeared, and urination was less frequent. During the following days the need for urination became less and less frequent. On the 5th day, bacteriological examination showed that the urine was sterile. Two months later the patient had gained 11 pounds, the general condition was excellent, urination was normal and recovery was complete and permanent.

In brief, this was a case of chronic cystitis due to $B$. coli. Medical treatment had failed. Clinical and bacteriological cure occurred, at the expense of a very slight local reaction, within 7 days after 2 injections of the bacteriophage.

Case 2. Mrs. F. A primipara in the 5th month of pregnancy. An acute case of pyelonephritis on the right side. Eight days after the onset of the attack the temperature had fallen somewhat, but the kidney remained large, very sensitive, and the urine was cloudy. Bacteriological examination showed $B$. coli to be present; the B. coli was susceptible.

The patient was given a subcutaneous injection of 1 cc. of Colibacteriophage and it was instilled into the bladder twice. Improvement was very prompt. The patient got up a few days after the injection and at this time the urine was clear and culturally sterile.

Case 3. A female child, 1 year old. The general condition was poor. There was a $B$. coli pyelitis. The treatment was:

On April 19, a subcutaneous injection of $0.75 \mathrm{cc}$.

On April 22, a second injection of the same bacteriophage.

This same day the urine was clear. Bacteriological examination on April 25 showed the urine to be sterile. Recovery was complete.

Case 4. An infant with a recent acute pyelitis. The urine yielded an alundance of $B$. coli; the strain was susceptible. Two injections of 0.5 ce. of a Coli-bacteriophage were given, with a 48-hour interval. 
Recovery, clinically and bacteriologically, was complete 4 days after the first injection.

Along with the many cases where recovery was obtained in a manner as abrupt as in the cases cited above, Hauduroy encountered some absolute failures.*

Philibert and his associates $\left({ }^{507}\right)$, at the Hôpitaux de Paris, have treated many cases of $B$. coli urinary infections. Here are their conclusions:

Colon bacillus infections, particularly septicemia and pyelonephritis, are improved or cured with great facility by the anti-coli bacteriophage. Recovery, occurring in all of the cases which we have observed within 24 to 48 hours, is evidenced by a clearing of the urine, a cessation of the pains, a disappearance of the pollakiuria, a regression of the renal swelling, a fall in the temperature, and in a general improvement. The fall in temperature may take place either abruptly, or by lysis, and is accompanied by a diaphoretic crisis.

After remarking that in certain cases, recovery does not take place, a result which, they say, is primarily due to the fact that the bacillus of the patient is not susceptible to the bacteriophage used, they add:

Every case of colibacilluria is subject to this treatment, whether it is a case of pyelonephritis of pregnancy, of calculus, of intestinal or of septicemic origin. We of ten encountered coli bacillurias associated with, or secondary to, intestinal disturbances in tabetics, in patients who were constipated or had an enteritis, and it seemed as though the intestinal disturbance as well was improved by the bacteriophage treatment.

Arloing, Dufour, Bouvier and Sempé ${ }^{20}$ have also obtained extremely rapid cures in cases of pyelonephritis, $\dagger$ and Nunter and Boenheim ${ }^{467}$ report the successful treatment of pyelitis in infants, while Pereira ${ }^{500}$ has treated urinary infections in adults.

* The method employed by Hauduroy for the preparation of the suspensions of Coli-bacteriophage was as follows: After having isolated a large number of races of the bacteriophage virulent for $B$. coli he maintained by successive passages two races which were distinctive because of their wide range of virulences. The $B$. coli under test is planted in bouillon. When the turbidity is slight, but quite evident, a mixture of these two races of the bacteriophage is added, and bacteriophagy is allowed to take place at $22^{\circ} \mathrm{C}$. When the material becomes clear a concentrated suspension of the $B$. coli strain is added and bacteriophagy takes place a second time at $22^{\circ} \mathrm{C}$. The medium again being clear, it is filtered through a candle and distributed in ampoules which are kept in the ice-box until used.

$\dagger$ Marcuse ${ }^{436}$ has succeeded in producing an experimental colon bacillus eystitis in guinea pigs. They were cured by the bacteriophage. 
I have treated a dozen cases of simple cystitis or pyelonephritis, in which I had previously shown that the pathogenic B. coli was susceptible to one of my races of bacteriophage. In the treatment of all of these cases the suspension of the bacteriophage which I used was prepared by the complete bacteriophagy of a suspension of $B$. coli containing 250 million bacilli per cubic centimeter. In some of the cases the bacteriophage used was developed at the expense of the colon strain isolated from the patient, in others, it was developed at the expense of a laboratory strain. I was unable to detect any difference in the therapeutic effects; the single condition essential to a therapeutic success being that the bacteriophage inoculated be active for the pathogenic bacillus of the patient.

I have always applied two injections of a maximum of 2 cc., at a 24 hour, or at a maximum, a 48 hour interval, and I have given at the same time bladder instillations with a 1:10 dilution in saline of the bacteriophage suspension.

We have seen that repeated injections of the bacteriophage may lead to the development of the state of antiphylaxis. Hence, the injections should not be repeated indefinitely. Furthermore, experiment shows that two are sufficient, for if beneficial results are not obtained with them, it means that the race employed is devoid of action. As for the instillations, they may be repeated as many times as seem advisable, for they do not lead to antiphylaxis. Ingestions are also harmless in this respect as I have shown. To test this last point, I ingested daily throughout a period of a month, $10 \mathrm{cc}$. of bacteriophage suspensions virulent for $B$. coli, B. dysenteriae, and for B. paratyphosus $A$ and $B$. No harmful effects followed.

But to return to the treatment of $B$. coli infections of the urinary tract. In the 12 cases which I have treated, being assured always in advance that the bacterium was susceptible to the bacteriophage, clinical and bacteriological cure was obtained within 5 days of the last injection, with the exception of the first case treated. Here is the history of this patient.

A woman, aged 30 years, had suffered from a cystitis for 7 years. The patient had been under the care of several specialists who had applied varicus forms of treatment, including auto-vaccines at two different times, some 30 injections being given in each series. In neither instance could any beneficial effect be observed.

The $B$. coli isolated from the urine proved to be very susceptible and I prepared material for use in treatment by the bacteriophagy of a 
suspension, 250 million bacilli per cubic centimeter, of the strain derived from the patient. Clearing was perfect in 8 hours. I gave, with a 48 hour interval, two injections of $2 \mathrm{cc}$. of this filtered suspension. Fifteen days after the last injection there had been no improvement, for the urine was as heavily loaded with $B$. coli as before, in fact, it was simply a culture of the bacillus.

I then gave the patient a bladder instillation, using $20 \mathrm{cc}$. of the same bacteriophage diluted with physiological saline. Five days later the urine was sterile. Cure was permanent, for 6 months later examination showed that the urine was still sterile.

Since this experience I have always combined subcutaneous injection with bladder lavage, a procedure which offers no inconvenience.

I have had occasion to treat one case of angiocholecystitis. The patient, a woman aged 32 years, had been in the hospital for 6 months. During this time the temperature had varied between 37.8 and $39^{\circ} \mathrm{C}$. The infection had resisted all of the usual types of treatment, including vaccine therapy. The patient was extremely weakened and emaciated. Two hours after the injection of $2 \mathrm{cc}$. of a suspension of the bacteriophage (a race isolated 2 years previously from the stools of a cholera convalescent, and developed at the expense of a stock strain of $B$. coli) the temperature rose and quickly reached $40.5^{\circ} \mathrm{C}$. It stayed there from the 7 th to the 11th hour after the injection. Then there occurred a rapid fall, without general disturbances but with a profuse sweating, and 22 hours after the injection the temperature was $37.2^{\circ} \mathrm{C}$. and the patient entered upon convalescence. She left the hospital in a perfect state of health 12 days later. For the 2 years following her discharge from the hospital she has shown no morbid symptoms.

What is the principle which causes the recovery in these $B$. coli infections? Is it the dissolved bacterial substance, or is it the bacteriophage itself acting as a parasite of the bacteria?

In order to reach an answer to this question it is only necessary to recall that in all cases, whether cure resulted or whether the diseased state persisted without change, the dissolved bacterial substance was injected. But recovery followed only when the bacteriophage inoculated was virulent for the pathogenic bacterium. It is, then, the "virulence" which is effective. This means that recovery occurs because the bacteriophage protobes parasitize and destroy the pathogenic bacteria.

The treatment of infections due to $B$. coli by the bacteriophage is specific. When someone succeeds in isolating a race of the bacterio- 
phage possessing a virulence for all strains of $B$. coli, the treatment can be applied as a routine measure, without the necessity of first determining whether the bacillus in each patient is susceptible to some race of the bacteriophage which is available. This lack of a polyvirulent race is a complication which considerably limits the general application of the treatment to infectious processes whose etiological agent is the colon bacillus.

\section{BACTERIOPHAGE THERAPY OF STAPHYLOCOCCUS INFECTIONS}

Bruynoghe and Maisin ${ }^{121}$ were the first to use the Staphylo-bacteriophage therapeutically. They applied it in the treatment of 6 patients with anthrax or furunculosis, injecting as closely as possible to the lesions a single dose, varying from 0.5 to $2 \mathrm{cc}$. Their bacteriophage had been heated at $56^{\circ} \mathrm{C}$. They observed a rapid diminution in the induration and often its complete disappearance within 24 to 48 hours. Infections which had already suppurated opened and dried up quickly. They noted that in some individuals there occurred a rise in temperature following the inoculation, while in others the temperature was not changed. They gained the impression that a febrile reaction occurred especially in those patients having extensive lesions where the rapid dissolution of the cocci involved the resorption of large quantities of the bacterial products. As for the local reaction, they noted that the point of injection was painful and slightly edematous for a period of 24 hours.

These authors were the first to publish their results on the treatment of staphylococcus infections, but certainly Gratia, ${ }^{258}$ making his experiments at the same time, should receive the credit for having clearly shown the advantages of the bacteriophage in the treatment of these infections.

We know that the staphylococcus is a heterogeneous species as regards bacteriophagy, but Gratia isolated a race of the bacteriophage which is polyvirulent. By repeated determinations he demonstrated that all strains of the staphylococcus,-albus, aureus, and citreus,which he tested were susceptible to this race. I have also found that none of the strains, about 100 in number, which I have isolated from a series of patients are resistant to this race. Micrococcus tetragenus is also susceptible to it. Without this discovery of Gratia we would have been obliged, as in the case of $B$. coli infections, to determine for each patient the virulence of the race for the causative staphylococcus, and in case of a resistance, to effect passages to bring about an adaptation, a tiresome procedure and one which would materially restrict the application of the treatment. Having a polyvirulent race, it is possible to 
employ it as a "stock bacteriophage," as is the case in the treatment of the dysenteries.

Gratia, ${ }^{257}$ at the hospital at Bruxelles, has treated some 50 cases of different staphylococcus infections. At the beginning he injected the suspension directly into the lesion, a procedure, in general, attended with much pain. But from the first case of folliculitis treated, he saw that this mode of procedure offered no advantages, and that the injection could be made in any portion of the body and give identical results.

In cases of staphylococcus septicemia he used the intravenous route.

The doses used varied from 0.5 to $3 \mathrm{cc}$., usually but one injection being given. In certain cases two injections,-- the maximum number,were given with a 24 hour interval.

He found that in certain cases the general reaction following the injection passed unperceived, while in others, there was a transitory elevation of temperature. A strong general reaction, as a rule, followed intravenous administration.

The local reaction consists in an erythematous induration at the point of injection, accompanied with pain and pruritus. The inflamed area may be as large as the palm of the hand, and the reaction may persist for 24 to 36 hours. At the locus of the lesions a congestive reaction is observed. "In this connection he mentions two very interesting cases.

A patient had a folliculitis of the left fore-arm, and also showed on physical examination, the scar of an appendectomy done 4 months previously. Immediately after the injection of $1.5 \mathrm{cc}$. of bacteriophage suspension subcutaneously in the arm the patient showed, not only a local reaction at the point of inoculation and in the foci of the folliculitis, but also an intense congestion in the operative cicatrix, which became red, painful, and which 2 days later, eliminated two infected silk threads.

The other case is that of a man who had suffered from a chronic furunculosis for 15 years, and at the time of treatment had many small furuncles upon different parts of the body. Immediately after the injection of $1.5 \mathrm{cc}$. of the Staphylo-bacteriophage, given subcutaneously in the left arm, he showed first the usual local reaction, and later, on the next day, although in the meantime all of the furuncles had aborted, an urticarial crisis, accompanied by a rise in temperature to $40^{\circ} \mathrm{C}$. The attack persisted throughout the night, and reappeared in an identical manner 4 days later, without any other disagreeable results.

The conclusions of Gratia follow:

. . . we think that we may conclude that injections such as we have given are inoffensive. They influence the evolution of folliculitis, furunculosis, an- 
thrax, and subcutaneous abscesses, giving unquestioned favorable effects. As the local congestive reaction, which persists for 24 hours, disappears, there is a very marked reduction of the local tenderness and a quick disappearance of the erythema, which, before vanishing, assumes a bluish tinge. There is an early softening of the induration and the edema disappears. Very often the cores, the purulent masses, undergo a rapid liquefaction, and they may be evacuated through a very small opening, or they may even be gradually absorbed without leaving a scar.

It may be added that of the 50 cases treated within a period of three months only one had a recurrence.

The authors mentioned above have used suspensions of the bacteriophage which had been heated at 56 to $58^{\circ} \mathrm{C}$. Following my suggestion, Bastin $^{55}$ worked with suspensions which were simply filtered. In contrast to the findings of the preceding authors he did not find that the inoculations caused any appreciable general reaction, and that the local reactions, although of variable intensity, were but slight. From his experience he concluded that:

Contrary to that which has been observed following the injection of heated suspensions, the inoculation of a fresh suspension does not cause any significant general reaction, and the local reaction is minimal. We think that the reactional phenomena reported by Bruynoghe and Maisin, by Gratia and Jaumain, must be due to the injection of albuminous substances which have been modified by the heating.

Whatever may be the reason for these differences, I can fully confirm the fact that the inoculation of suspensions of a virulent Staphylobacteriophage, simply filtered through a candle, does not cause any material general reaction, while the local reaction is essentially the same as that which follows the injection of heated suspensions of staphylococci (vaccines).

Nelson Barbosa ${ }^{478}$ has applied bacteriophagic treatment to cases with staphylococcus cystitis, with recovery, both clinical and bacteriological, within 2 or 3 days following the injection of 0.5 to 1 cc. of a bacteriophage suspension.

Hauduroy ${ }^{302}$ also, has successfully used bacteriophage treatment in staphylococcus infections.

Bazy $^{56}$ reports on the use of bacteriophagic therapy in all types of localized pyogenic surgical infections. In some cases he brought the bacteriophage directly into contact with the lesion (anthrax, localized abscesses) by encircling the lesion with a series of injections. In other cases he injected the suspension subcutaneously in some part more 
remote from the lesion. The results were essentially the same in both cases.

One might say, he states, that they have been excellent, and that the procedure has invariably been well tolerated by the patients. The therapeutic effects may be summarized thus:-In all cases where the pathological condition involved only purely reactional phenomena a rapid regression of the lesions and the restitutio ad integrum was witnessed. Wherever there was a tendency toward suppuration and necrosis, this developed rapidly, the abscesses matured better and more quickly, and the necrotic material was eliminated more rapidly. In short, recovery was accomplished after a very short delay.*

* It is interesting that Bazy attributes the curative power, not to the bacteriophage itself, but to the dissolved bacterial substances. Some of the reasons which he advances for this view are rather peculiar. "In vitro, he says, we observe the formation of secondary cultures." Surely, and we know that secondary cultures with races of maximum virulence occur, or do not occur, according to the conditions of the experiment,- the reaction of the medium, the temperature, etc. But the fact that under certain conditions they do develop in vitro does not prove that they must also occur in vivo.

Ile affirms, in support of his thesis, that Gratia has obtained the same results with filtrates of cultures which have autolyzed spontaneously, and with suspensions of the bacteriophage which have been heated at $70^{\circ} \mathrm{C}$. Let us state at once that heating at $70^{\circ} \mathrm{C}$. does not necessarily destroy the bacteriophage, but this is of little moment.

Bazy refers to two of Gratia's publications but he alters the scnse strangely. In the first, Gratia ${ }^{257}$ states that he wished to determine whether the local and general reactions following the injections were due to the presence of the bacteriophage or of the bacterial substances. He concluded that the latter are the cause, because suspensions heated at $70^{\circ} \mathrm{C}$. cause the same reactions. But he does not say a word about the therapeutic effects obtained with such heated suspensions.

In the second paper, criticizing the experiments of Besredka on local immunity, Gratia $^{262}$ injects into the skin of rabbits, either suspensions of the bacteriophage, or autolysates, or pure sterile bouillon. After a certain interval he injects these animals, in the region of the first injection, with a staphylococcus culture. And, while in the unprepared control animals there occurs a large local lesion, in those which have previously been inoculated, even with pure bouillon, nolesion develops.

Gratia associates this experiment with the old experiment of Metchnikoff, which showed that if some pure sterile boullon was inoculated into the peritoneal cavity of guinea pigs a few hours before the injection of a culture of vibrios, the guinea pigs were protected.

This experiment of Gratia has no relation to the therapeutic treatment of staphylococcus infections. In case it is desired to bring out such a relationship, I would simply advise Bazy to attempt the treatment of cholera or of furunculosis, by a single injection of some sterile bouillon.

Gratia's experiment, and the conclusions which he has drawn with reference to the theories of Besredka are perfectly logical. Bazy has attempted to generalize from them, and has derived some very absurd conclusions. 
Gougerot and Peyre ${ }^{244}$ have applied the bacteriophage treatment to sycosis, a disease up to the present time incurable, and whose etiology even, is subject to discussion. The experiments of these authors disclose a therapeutic procedure, and show at the same time that the infectious agent must be a staphylococcus. ${ }^{*}$

They first applied the bacteriophage by the subcutaneous route, as in other staphylococcus infections, but the result was clearly negative. To quote from their paper:

Local treatment is the only method to yield results. It is necessary to patiently open each pustular element with a pipette containing the suspension of bacteriophage in such a way as to inoculate each pustule. All of the elements must be attacked, even though they be minute. A compress moistened with the bacteriophage suspension is placed over the area involved. . . . . Frequently on the next day a disagreeable reaction is observed, and it is necessary to protect the patient because of the annoyance of the lesions. As a matter of fact, each pustule increases in size, and it becomes surrounded by an inflammatory zone, indurated and red. One gains the impression that a struggle between the staphylococcus and the bacteriophage is going on. And then, at the end of 48 hours, the injected lesions have dried up and disappeared. Every other day it is necessary to repeat this procedure, certain pustules having been overlooked or having appeared after the first treatment. Lack of success is due solely to neglect on the part of the patient to have all of the cutaneous elements treated. At the end of 8 to 10 applications improvement is manifest.

In two cases it has been possible for us to state that cure has resulted.

The first case was a physician having a pustular sycosis of the superficial variety with erythemato-squamous dermo-epidermitis.

The second case was that of a tailor having a sycosis with large hypodermic nodules and a diffuse dermo-epidermitis, involving the moustache, the beard, and the pubis.

In two other cases now undergoing treatment,--pustular sycosis with amianthoid squamous epidermitis; and a pustular sycosis with an oozing ulcerous dermo-epidermitis,-a very great improvement has already been obtained, in spite of the fact that the classic forms of treatment were unable to prevent the spread of the lesions.

These attempts, some 100 or more, at the treatment of diverse infectious processes due to the staphylococcus permit us to recommend the following mode of procedure.

In the first place utilize a race of the bacteriophage which is polyvir-

* It is impossible, as a matter of fact, to definitely affirm this, for it may quite logically be objected that, inasmuch as the bacteriophage possesses the power of adaptation, especially in the direction of the acquisition of virulences, it may acquire in the lesion the power of attacking the microbe " $\mathrm{X}$," the true causative agent of the disease. 
ulent (race $h$ of Gratia, for example) with a maximum virulence. After the complete bacteriophagy of a suspension containing about 250 million cocci per cubic centimeter, filter the suspension; never heat it.

For furunculosis and folliculitis inject $1 \mathrm{cc}$. of the suspension subcutaneously, at any point whatever, but preferably under the skin of the abdomen. The injection should be once repeated, after an interval not greater than 48 hours.

Local abscesses and anthrax should be treated by an inoculation of 1 to 2 cc. into the immediate vicinity of the lesion, by one, or better several, injections surrounding the focus. When it is possible, it is still better to inject directly into the lesion itself, using a very fine needle. In this case 0.25 to $0.5 \mathrm{cc}$. of the bacteriophage suspension is adequate.

For septicemias, a single intravenous injection of 2 to $3 \mathrm{cc}$. should be given.

For sycosis, the technic of Cougerot and Peyre should be adopted.

For urinary tract infections, give two subcutaneous injections of $1 \mathrm{cc}$, with a 48 hour interval. At the same time, morning and evening instill into the bladder $10 \mathrm{cc}$. of the bacteriophage suspension diluted in 40 cc. of physiological saline. The instillations may be repeated over as long a period as is desired, without any harmful results. Generally, however, treatment is completed in 2 days, that is, with two injections and four instillations. These usually effect a cure.

What element present in the bacteriophage suspension causes the recovery? It is certain that the dissolved bacterial substances do not remain inert, but the following facts show, it seems to me, that the principal rôle is played by the bacteriophage protobes themselves as living beings parasitic of the staphylococci.

Everyone who has worked with vaccine therapy knows that in the great majority of cases a very great many injections of autogenous vaccines are necessary in order to obtain a demonstrable therapeutic effect, and even then such an effect is not always observed. All of those who have worked with bacteriophagic therapy mention the fact that they have successfully treated cases which had resisted a series of injections of autogenous vaccines. And yet a single injection of a bacteriophage suspension usually caused an immediate cure.

We have already considered the question of the autonomy of the bacteriophage as regards the bacterium; we may recall the experiment of Gratia carried out with the two races of the bacteriophage, one univirulent, solely active for one strain of Staphylococcus albus, the other polyvirulent. In this connection the following experiment is of interest, since it forms a corollary to Gratia's. 
I prepared two suspensions, both derived through the bacteriophagy of a single strain of Staphylococcus albus. One suspension was obtained by means of a univirulent race of the bacteriophage active for this one strain only, while the other suspension was the result of the action of the polyvirulent race of Gratia.

Two patients having a recurrent furunculosis received two injections, with a 48 hour interval, of the suspension made with the univirulent race. No improvement resulted in either case. Three weeks later in one case, 40 days in the other, a single injection of the suspension made with the polyvirulent race led to recovery. Nevertheless, in the two suspensions there was the dissolved substances of the same staphylococcus, the strain of Staphylococcus albus which was susceptible to the univirulent race.

And finally, a last fact may be presented. Perhaps this is the most clear-cut of all, for it shows how fundamental is the difference between a "vaccine" and a suspension of the bacteriophage. The repeated injection of laboratory animals with the first, as we know, causes an immunity,-entirely artificial it is true,-but which is, at least, entirely different from the state of antiphylaxis resulting from the repeated injection of a bacteriophage suspension. As a matter of fact, this single difference, all by itself, shows that the treatment by the bacteriophage,-"phagotherapy," as it might be termed,--bears no relationship to vaccine therapy.

\section{BACTERIOPHAGE THERAPY OF INFECTED WOUNDS}

In the work of McKinley ${ }^{460}$ we discover a new field for the application of bacteriophagic therapy,- the treatment of infected wounds.

The suspensions of the bacteriophage which he used were prepared in the following manner. After isolating a race of the bacteriophage and increasing its virulence to such a point that a bacteriophaged suspension spread on agar gave no colonies, he added to 30 to $50 \mathrm{cc}$. of the suspension $1 \mathrm{cc}$. of a 24 hour culture of the susceptible bacterium. After bacteriophagy he added a further $2 \mathrm{cc}$. of a fresh 24 hour culture, and when bacteriophagy was again ended he made a third addition of 3 cc. of culture, then, after dissolution, a fourth addition of $4 \mathrm{cc}$., and finally, a fifth of $5 \mathrm{cc}$. When, after this final addition of cocci, bacteriophagy was completed, he filtered the suspension through a candle. This filtrate was used in his therapeutic work.*

* The method of preparation as previously described,-complete bacteriophagy of a bacterial suspension containing about 250 million organisms per cubic 
McKinley reports the treatment of 4 cases of old wounds, heavily infected, chiefly by the staphylococcus. In all of the cases the results were essentially the same; the suppuration stopped shortly after the application of the bacteriophage and the processes of healing began promptly. His observations recorded as to one of these cases are summarized here, this particular case being selected because of the marked general reaction which was caused by the third injection, given 6 days after the first.

J. N. C., a sheet metal worker, aged 57 , entered the hospital on October 11 , as the result of a fall from a height of 30 feet. He landed on both feet, suffering a compound fracture of the right tibia and fibula and a crushing fracture of the os calcis, with a dislocation of the astragalus of the left foot. The bones of the right leg were set, and both the right leg and the left foot were placed in casts.

On March 26, a piece of bone about 3 inches $(7.62 \mathrm{~cm}$.) long was removed from the right leg, and the cast reapplied. The wound was badly infected with Staphylococcus aureus.

April 10: Two cubic centimeters of staphylococcus bacteriophage were injected into the discharging wound and subcutaneously around the wound.

April 11: The discharge was markedly decreased.

April 12: Two cubic centimeters of bacteriophage were again injected into the wound and subcutaneously around the wound. One cubic centimeter was given subcutaneously into the right arm.

April 13: There was practically no discharge.

April 14: The wound appeared much improved. There was practically no discharge.

April 15: The condition was about the same.

April 16: Two cubic centimeters of bacteriophage were again injected into the wound and subcutaneously around it. Treatment was given at 8 o'clock in the morning. At noon the temperature was $103^{\circ} \mathrm{F}$. The patient began to have cramping pains all over the body and marked emesis. Chills were marked and lasted until 5 o'clock that afternoon.

April 17: The patient's condition was a little improved. The wound was healing well. There was no discharge.

April 18: His general condition was much better. His temperature was normal. He had no chills. He had little nausea but felt weak. The wound was healing well.

April 23: The patient's condition was good. The wound was healing. There was no discharge. The wound, formerly about 1 inch $(2.54 \mathrm{~cm}$.) deep, continued to heal, and on May 18 healing was nearly complete, except for a slight depression which contained a small amount of blood clot.

centimeter,-is more simple and much quicker, and is certainly to be preferred. Furthermore, it conforms to the procedure which I have employed throughout for all of the suspensions which have been used so successfully for therapeutic purposes. 
May 18: The blood clot was cleaned out, and bacteriophage was placed on the dressing and laid over the wound. The patient had a marked reaction, beginning about 8 o'clock that night, simulating the one described above, only more marked. He was nauseated and had chills and fever for a week, together with marked swelling of the right leg and foot.

June 2: The wound was healed. Roentgen-ray examination showed union of the fractured bones.

From my first publications ${ }^{321}$ on the application of the bacteriophage to therapeutic purposes I emphasized the fact that it is not wise to multiply injections. All of the observations which have been made since justify me in repeating this recommendation. It is never necessary to give more than 3 injections, and it is particularly important that they should be given within an interval of time not greater than 48 hours between the first and the last injection. In the great majority of cases a single injection is adequate to lead to recovery, and nothing is gained by repeating the treatment.

This warning applies only to treatments given by a parenteral route. Administration by mouth, by rectum, by bladder instillation, or by ntroduction into the vagina, can be repeated without danger as many times as seems best, and the intervals at which they are given are of no moment, from the point of view of inciting harmful effects.

It may be added that the bacteriophage treatment of infected wounds is actually being used in a number of hospitals, but in view of the publications already made on the subject, those who are applying this form of therapy have not yet thought it necessary to publish their observations. They feel, however, that the method has become an established procedure. It so happens that in the surgical service of one of the Paris hospitals they have recently shown me a number of patients who had been treated by the bacteriophage. Among them was one who was admitted, three weeks previously, with a large suppurating wound of the thigh, with diffuse phlegmon. The general condition of the patient was very poor; temperature above $40^{\circ} \mathrm{C}$., adynamic, and the condition of the leg was such that the surgeon believed amputation necessary. But the general condition of the patient was so poor that he deferred the operation until the next day. As a sort of last chance, he injected $2 \mathrm{cc}$. of a Staphylo-bacteriophage suspension into the muscular tissues surrounding the wound. By the next morning the temperature had fallen, the general condition was improved, and suppuration was markedly diminished. Conditions as regards the infection were so far improved that it was determined to postpone the operation. During 
the following days improvement continued. At the time when I saw the patient the general condition was perfect, and the healing of the wound was far advanced.

Treatment by the bacteriophage is so simple and the action is so rapid, that it should be applied routinely in all cases of suppurating wounds.

\section{BACTERIOPHAGE THERAPY OF STREPTOCOCCUS INFECTIONS}

In connection with his work on staphyloccoccus infections McKinley ${ }^{460}$ has reported one case of a streptococcus infection treated with the bacteriophage.

F. N., aged 39. The patient was first seen on March 9, the present illness being of 3 weeks duration. The essential points in the examination were; many streptococci in smears of the fluid from the chest, and the presence of an abscess of the lower part of the right lung, as revealed by Roentgen-ray examination.

March 13: A rib resected and drainage instituted.

March 14: There was considerable drainage with a foul odor.

March 15: The condition was the same.

March 17: The condition was the same. Thirty cubic centimeters of a streptococcus bacteriophage were injected into the cavity.

March 18: There was a large amount of drainage. The odor was not so bad.

March 19: There was a small amount of drainage and hardly any odor.

March 20: Thirty cubic centimeters of bacteriophage were injected into the cavity. There was little discharge and no odor.

March 21: The condition remained the same.

March 22: There was practically no discharge and no odor.

March 23: There was no discharge. Twenty-five cubic centimeters of bacteriophage were injected into the cavity.

March 25: The patient was discharged from the hospital. He said that he "felt fine."

May 15: The patient returned and said that he had felt perfectly well since leaving the hospital.

Needless to say, the conditions under which the bacteriophage may be used in streptococcus infections, and the limitations as regards repeated administration, are the same as in the case of staphylococcus infections.

\section{BACTERIOPHAGE THERAPY IN BUBONIC PLAGUE}

In 1920, while in Indo-China, I isolated several races of the bacteriophage virulent for $B$. pestis. One race, in particular derived from a 
specimen of rat excreta collected at Bac-Lieu after an epidemic of plague, possessed from the beginning a high virulence $(+++)$, which became very high indeed $(++++)$ at the second passage with a strain of $B$. pestis.

In order to determine the toxicity of the Pestis-bacteriophage a guinea pig was injected subcutaneously with $1 \mathrm{cc}$. of the filtered suspension. There was no apparent disturbance. I then injected $1 \mathrm{cc}$. subcutaneously into my own arm. The general reaction was practically negative,--perfectly normal sleep during the following night, a slight headache in the morning which disappeared immediately after taking 1 gr. of aspirin. The local reaction, which lasted for 48 hours, consisted of a slight edema, and the point of injection was somewhat tender. In all respects, the reaction to the filtrate was definitely less marked than that following an injection of heat-killed cultures of $B$. pestis.

As a result of this test $I$ felt warranted in attempting to use the Pestis-bacteriophage in the treatment of plague in human beings. Unfortunately, I had succeeded in isolating races of the bacteriophage highly virulent for $B$. pestis only a very short time before my departure from Indo-China, so that it was impossible for me to make the desired tests. I gave Dr. Galinier, Colonial Physician at Phantiet, where plague is endemic, a dozen ampoules of the bacteriophage, asking him to inject, subcutaneously, 1 cc. of the suspension into some patients with bubonic plague. Shortly after my return to Paris I received a cablegram, requesting that, in view of the excellent results secured, I send him more of the suspension. Having learned in the meantime that he had received instructions to inject anti-plague serum conjointly with the bacteriophage I did not think it wise to continue the tests under these conditions.

As though to provide the material needful to reaching a conclusion on this question of bacteriophagic treatment in plague, there have appeared, just as I am writing this present chapter, three cases of plague on board a steamship here in the port of Alexandria. These three patients were removed to the hospital of the Quarantine Station, where I have been able to treat them. A few days later a fourth case was discovered and removed from another ship.

The suspensions used for the treatment were prepared in the following manner: To $10 \mathrm{ce}$. of a culture of B. pestis in bouillon, 24 hours old ( $\mathrm{pH}$ of the medium, 7.6 ; incubation at $32^{\circ} \mathrm{C}$.) were added $3 \mathrm{cc}$. of fresh bouillon, and this was then inoculated with $0.02 \mathrm{cc}$. of a bacteriophage sus- 
pension having a very high virulence $(++++)$ for $B$. pestis.* This race of the bacteriophage is the one which $\mathrm{I}$ isolated from the excreta of the rat at Bac-Lieu in Indo-China, and since its isolation it had undergone about 100 passages. After 20 hours incubation at $37^{\circ} \mathrm{C}$. bacteriophagy was complete. The clear liquid was filtered in the morning and the patients were inoculated a few hours later.

In each of the four cases the sole method of treatment was the injection of the bacteriophage, given directly into the bubo. The results are presented here in some detail.

Case 1. Georges Cap. . . . . 18 years old. On July 10, 1925, he complained of fever, lassitude, vertigo, headache, and examination showed a bilateral tonsillitis. On the following day his condition became worse. During the night of the 11 th and 12 th the crural gland on each side swelled. He was brought to the hospital on July 12th.

Some of the material from the bubo on the left side was removed, and direct examination of smears showed cocco-bacilli with bipolar staining. The cultures which later developed from this material yielded perfectly characteristic $B$. pestis, which were agglutinated by a known anti-plague serum. An inoculated guinea pig died after 68 hours, showing the lesions characteristic of experimental plague.

On July 13th, at 2 p.m., the patient had a temperature of $40.3^{\circ} \mathrm{C}$., the pulse was 130 , the face was congested, the eyes were injected and drooping, and prostration was extreme. The two buboes were of the size of nuts, and painful when pressed. I gave an injection of $0.5 \mathrm{cc}$. of Pestis-bacteriophage into each of the two buboes, the needle being introduced to about the center of the bubo. Apparently the injections caused no pain, since the patient showed no reactions of defense.

At 8 p.m., of this same day there was some sweating of the axillae and the forearms.

On the morning of the 14 th the condition of the patient was completely changed. He stated that he felt weak, but that he was not in pain and felt well. The buboes were somewhat painful and had slightly increased in volume.

On the 15th he sat up in bed; and on the 16th he begged for food.

On the 16th the buboes had increased in size to that of a small hen's egg, but they were not painful. Puncture of the left bubo with a syringe permitted the withdrawal of a few drops of bloody fluid. Direct

* B. pestis, as has been stated, is a very homogeneous species as regards the bacteriophage. All of the strains which I have tested, laboratory cultures as well as strains freshly isolated from buboes, have showed practically the same susceptibility. 
examination showed nothing in the way of organisms, and the culture made from it failed to yield a growth. Some of it was inoculated into a guinea pig, and the guinea pig is still alive as this is written (10 days later). The aspirated material, examined for bacteriophage, showed that it was present, with a very high virulence for $B$. pestis $(++++)$.

On the 25th both of the buboes were incised very freely. Both contained some purulent material. By August 8th healing was complete, a fact of some interest in that, as we know, usually in such cases the healing process is very slow, sometimes requiring a month.

The curve showing the temperature of this patient is here given (chart 22), and it may be said, that here, as in the other three cases, the temperature changes closely parallel the general condition of the patient.

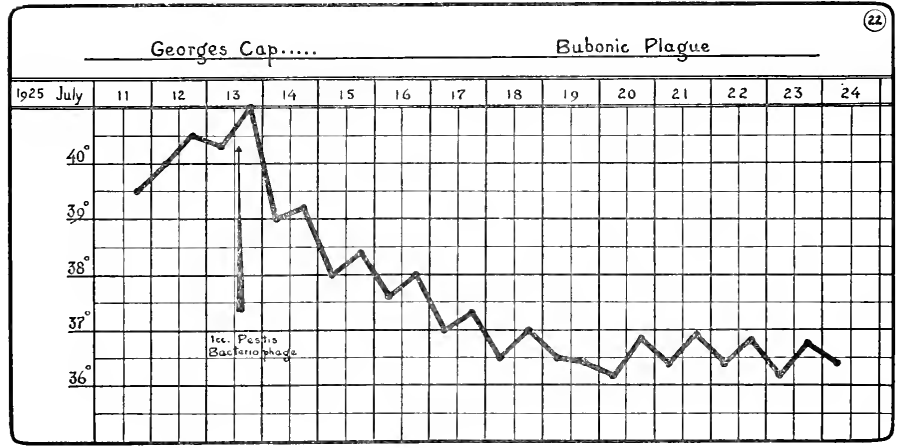

Chart 22. Georges Cap. . . . Bubonic Plague

Treatment consisted in a single injection of Pestis-bacteriophage

Case 2. Nicolas Sw. . . . . , 40 years old. Second Officer.

The crew and passengers of the steamship, having been disembarked at the Quarantine Station, four sailors, under the command of this officer were left on board. During the night of July 12th and 13th, he was taken with fever, chills, and had a frontal headache. On the morning of the 13th he was transferred to the hospital. At 2 p.m. of this same day he had a temperature of $40.2^{\circ} \mathrm{C}$., a pulse of 124 , irregular with skipped-beats, the conjunctivae were injected, and he was markedly prostrated. The right crural gland was swollen, painful, and of the size of a nut. Some of the material from the gland was removed for bacteriological examination. In view of the clear-cut symptoms, associated with the presence of a previous case of plague, 1 cc. of bac- 
teriophage suspension was injected directly into the center of the bubo without awaiting the result of the laboratory findings.

The results of the bacteriological examination, subsequently reported, were that the material from the bubo examined in direct smear revealed cocco-bacilli with bipolar staining; that cultures gave organisms having the characteristics of $B$. pestis, agglutinable by an anti-plague serum, and that a guinea pig inoculated with some of the material died 78 hours after the injection, presenting the lesions of experimental plague.

The injection of the bacteriophage did not cause any reaction. The patient passed a good night. On the next morning his condition was completely changed. Like his companion who had been inoculated at

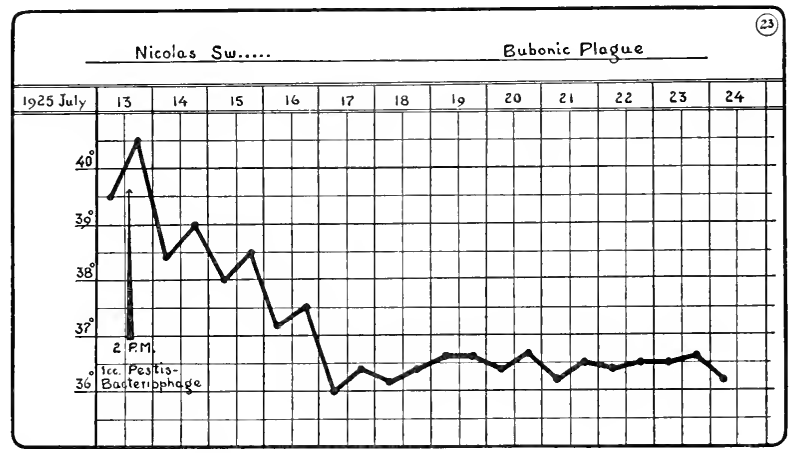

Chart 23. Nicholas Sw. . . . Bubonic Plague

Treatment consisted in a single injection of Pestis-bacteriophage

the same time, he declared that he felt well, but rather weak. His temperature was $38.4^{\circ} \mathrm{C}$, his pulse 82 , but still irregular. The gland was slightly increased in size, but was hardly painful at all, it requiring a strong pressure to cause the patient to complain.

Improvement was still more marked on the next day, but the pulse had not regained its regularity.

On the 16th, the patient, a vigorous man, demanded vehemently that he be given a substantial meal.

The curve showing the course of the temperature is here given (chart 23).

On the 16th some of the material from the bubo was aspirated, and, as in the preceding case, the bloody fluid showed nothing upon direct 
examination except some red blood cells and some polymorphonuclear cells, about 1 to every 40 red cells. Plantings made upon media remained sterile. Guinea pigs inoculated have lived for 10 days-up to the present time. To the bouillon implanted with the material, which had remained sterile, I added a suspension of $B$. pestis (the strain isolated from the patient 3 days previously) and bacteriophagy was complete in 20 hours.

After two passages, the race of bacteriophage derived from the bubo caused a complete dissolution in 6 hours. In this case also, the bubo was opened on the 25th. Only a few drons of bloody fluid were found: there was no pus. The incisions had healed completely on August 5th.

Case 3. Theodoro Cass. . . . . cabin-boy, 16 years old.

On the 12th of July he presented the symptoms of a febrile gastric disturbance, and on this same day he was isolated in the hospital. His condition grew worse on the evening of the 14th.

On the morning of the 15 th the pulse was irregular at 126 ; the temperature was $39.4^{\circ} \mathrm{C}$; the conjunctivae were injected; and there was a marked prostration. During the night a swelling of the right submaxillary gland developed, it becoming the size of a hazelnut, and was painful when pressed.

Material aspirated from the gland revealed cocco-bacilli in direct smears. Cultures from the material gave typical B. pestis, and a guinea pig inoculated with it died 56 hours later with all of the lesions characteristic of experimental plague in this animal.

On the 15 th, at 3 p.m., I injected 1 cc. of the bacteriophage suspension directly into the center of the swollen gland.

On the morning of the 16th, all of the symptoms, with the exception of the bubo, had disappeared. The patient was lively, and was sitting up in bed when I made my visit. His temperature was $37.2^{\circ} \mathrm{C}$; the pulse 70. He stated that he had felt no ill effects as a result of the injection and that he had slept well. I had requested the attendants to watch him closely throughout the night and they confirmed his statement - they had observed no evidence of a reaction, no sweating and no restlessness. A few hours after the injection the patient had told them that he felt better and that he was going to sleep. When he woke up he told them that he was well.

Considering the nature of the disease, and the serious condition of the three patients, I had feared that there might be a marked general reaction as a result of the in vivo bacteriophagy of the plague bacilli. But absolutely nothing of the kind occurred. There was not even a local 
reaction, for 16 hours after the injection, in all cases, the bubo was less painful.

On the morning of the 18th, as with the preceding cases, 60 hours after the inoculation, I withdrew some of the material from the gland. Direct examination, culture, and guinea pig inoculation, all showed that the contents of the bubo were sterile. A suspension of plague bacilli, added to the bouillon which had shown no growth, failed to develop and were completely bacteriophaged in 7 hours.

The bubo absorbed slowly. On the 8 th of August it was still as large as a pea.

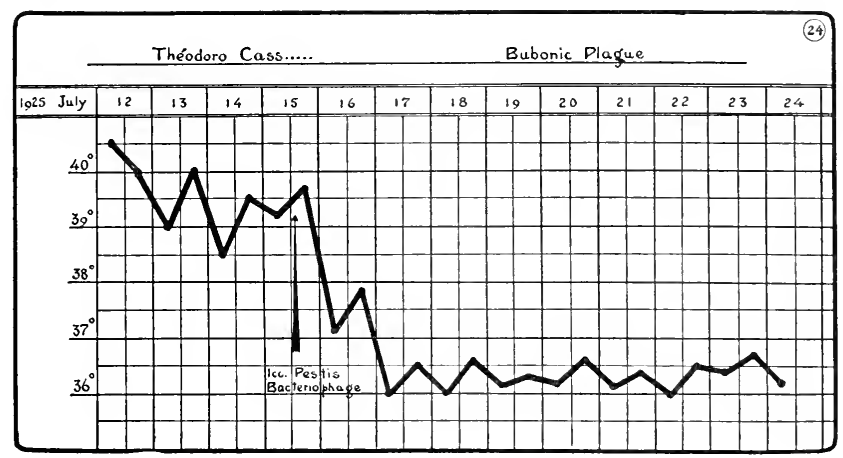

Chart 24. Théodoro Cass. . . . Bubonic Plague

Treatment consisted in a single injection of Pestis-bacteriophage

The temperature curve is given (chart 24).

Case 4. A. Stell. . . . . 32 years old. Steward.

During the morning of July 25 , while still at sea, the patient was taken with fever, chills, headache, and had a swelling in the region of the groin. On the forenoon of July 27 th the ship arrived at Alexandria, and at the time of the Quarantine Service inspection the patient had a temperature of $40^{\circ} \mathrm{C}$., the eyes were injected, and the tongue was dry and brownish. The prostration was marked. The patient could speak only with difficulty. The pulse was 120 and irregular. In the right groin there was a bubo about the size of a nut and very painful.

At 4 o'clock in the afternoon I removed some of the contents of the gland, and bacteriological examination later confirmed the diagnosis of bubonic plague. Immediately after the removal of the material from 
the gland I injected into the bubo $1 \mathrm{cc}$. of a suspension of bacteriophage virulent for $B$. pestis. This treatment was given, therefore, about 60 hours after the onset of the disease.

On the 28th the bubo was much less painful, and there was a slight fall in the temperature. The general condition of the patient appeared somewhat better, but from the manifest disturbances in the heart action there seemed to be a severe toxemia. Camphorated oil was injected and sparteine was administered.

On the 29th the temperature again rose and the patient became delirious. The tongue was dry and brownish. The condition adynamic. The pulse was weak, 110 beats per minute, with a pause between every three or four beats. At 4 o'clock in the afternoon I gave a second injection into the bubo, $1.5 \mathrm{cc}$. of the bacteriophage suspension being given.

On the morning of the 30th the condition of the patient was much better. While on the previous evening the case had seemed hopeless, in the morning the patient was stronger, the temperature had fallen, the pulse was 92 , and the irregularities occurred about every 15 beats. By evening the improvement was still more marked. The pulse was 76 and the pauses in the beats occurred only about once in 25 beats.

On the 31st the patient was bright and the general condition was good. Pulse 80, of good quality and regular.

Throughout the following days the improvement continued.

On August 2nd, at about 5 p.m., the patient complained of a pain in the left groin. On the 3 rd the left crural gland was swollen to the size of a small nut. The gland was aspirated and a few drops of pus were obtained. Direct examination showed only polymorphonuclear leucocytes with a few coccobacilli with bipolar staining. Seedings of this material in broth and on agar remained sterile. A guinea pig inoculated with the material showed no evidence of infection up to 8 days later. The bouillon which had been implanted and which had remained without growth was seeded after 48 hours with a $B$. pestis culture. Bacteriophagy was complete in 8 hours. It is of interest to note that the two inoculations of bacteriophage had been made into the right bubo, and that nothing had been injected into the one on the left side.

On the 8th of August this bubo had in large part been absorbed. Chart 25 gives the curve showing the fluctuations in the temperature of this patient.

In 1921 I published the observations on 5 patients affected with bacillary dysentery which I had treated by the ingestion of the bacterio- 
phage. I concluded that 5 cases were obviously inadequate to permit me to state that phagotherapy represented a specific treatment for this infection, but that what had been observed warranted me in thinking that it might be so. Four years have elapsed since then; the treatment of some tens of thousands of cases has shown that my beliefs were wellfounded.

These four cases of plague which I have just had an opportunity to treat justify me, I believe, in repeating what I said in the case of bacillary dysentery, namely, that bacteriophage treatment may be a specific therapy for the disease.

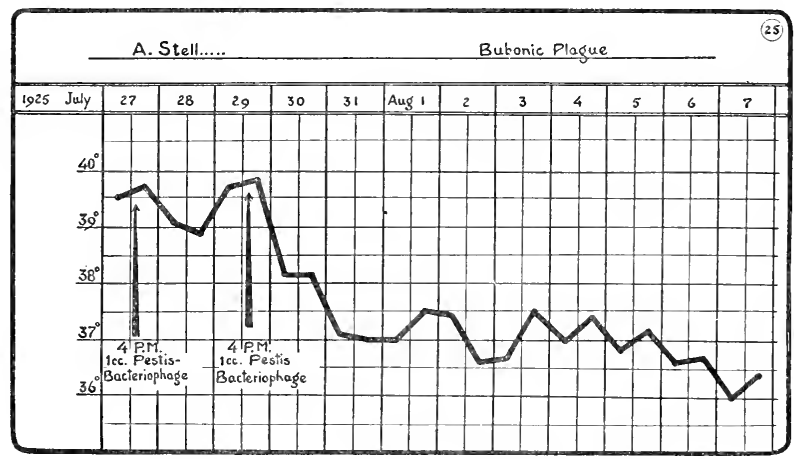

Chart 25. A. Stell. . . . Bubonic Plague

Treatment: Two injections of Pestis-bacteriophage

Since $B$. pestis, like $B$. dysenteriae, is a very homogenous species as regards bacteriophagy, it will be possible to use, as I did in the four cases reported above, a "stock-bacteriophage," without the necessity of preliminary laboratory tests. On the basis of the results reported above, admittedly few, I would recommend that injections be made directly into the bubo; that a dose of $2 \mathrm{cc}$. be given; and that the treatment be given as promptly as possible. Inasmuch as the injection of bacteriophage virulent for $B$. pestis is absolutely harmless even if it is later discovered that the diagnosis was wrong, it is unnecessary to await a report from the laboratory. When a case of suspected plague is encountered the proper procedure is first to remove some material from the gland for later examination (wherever this is possible) and then 
immediately after this removal to inject the suspension of bacteriophage. Needless to say, the bacteriophage injected should be of maximum, or at least very high $(++++)$ virulence.

If the temperature shows a progressive fall after this initial injection I would advise that no further treatment be given. But if the temperature still remains high on the day following the first treatment, a second injection should be given, and, if necessary, a third on the next day. If, as was the situation in Case 4 reported above, after the temperature shows an initial fall it again rises, make a second injection.

If buboes are not present, as in the case of septicemic or pneumonic plague, intravenous injection of the bacteriophage should be tried. From the nature of the effects observed in the few patients which I have treated it would seem that, aside from the bacteriophagy which occurs in vivo due to the inoculated bacteriophage, the injection of the suspension exerts an antitoxic effect. I have already called attention to this same fact in connection with the injection of suspensions developed at the expense of $B$. dysenteriae Shiga.

\section{APHTHOUS FEVER}

It may not be out of place to mention here the studies of Meier ${ }^{445}$ on aphthous fever, although it has not been demonstrated that the bacteriophage is definitely responsible for the results.

Impressed by a number of resemblances between what I have described in connection with immunity in barbone of the buffalo and what he had observed in aphthous fever, Meier undertook the studies indicated below. He procured some of the excreta from a cow which had had aphthous fever and which appeared to be recovering and from this he prepared a filtrate. A few cubic centimeters of this filtrate were injected into cows which were already infected and definitely sick and into other animals which presented suspicious symptoms of the disease. The results were decisive-the sick animals recovered quickly, the suspected ones failed to develop the disease.

Furthermore, the curative effect showed the features of a serial action. Thus, the sick cows inoculated with the initial filtrate derived from the naturally convalescent animal recovered promptly (within a few hours (4) in some cases). Twelve hours after the inoculation he collected feces from these treated cows (incidentally, during this period the character of the feces had changed, both in consistency and in color) and prepared fresh filtrates. These also had a curative effect, causing a very prompt improvement in condition when injected into sick animals. 
Meier found that the filtrates were effective only when injected early in the disease; when given after the animal had been sick for some days the injections were without benefit, indeed, at times it appeared as though they aggravated the condition.

The principle which causes recovery does not remain long in the intestine; after two weeks it had completely disappeared.

Among the experiments of Meier we may mention the following. In a single barn there were 9 cows, one of which showed the symptoms of aphthous fever. All 9 were injected with filtrate. The sick cow recovered promptly, the other 8 remained free from the disease. A comparable experiment made in a second barn gave the same results. In all, Meier's experiments involve some 650 animals, including both cattle and goats.

Such are the facts as recorded by Meier. Aphthous fever is a common disease with which experimental work can readily be performed, and it is to be hoped that these investigations will be continued and confirmed.

\section{RÉSUMÉ}

The experimental studies upon the possible utilization of the bacteriophage protobe in the treatment of infectious disease, comprising already a very considerable number of cases, show that the use of the bacteriophage-phagotherapy-represents a specific therapy for the bacillary dysenteries and for staphylococcus infections.

The results obtained in a few cases of bubonic plague allow us to hope that it is the same for this disease.

With respect to the other infectious diseases, the results obtained are encouraging, but as yet a perfected method of treatment has not been attained.

\section{CONCLUSION}

In Part Three of this text we have considered what is now known on the subject of exogenous immunity. The era of discussion is not yet passed, indeed the discussion is far from closed, but in the meantime therapy by means of the bacteriophage-phagotherapy - is being applied more and more extensively. Those authors, who, two or three years ago, declared that their experience was in direct contradiction to mine, that they obtained no beneficial results from the use of the bacteriophage, one after another reconsider their first conclusions.

For certain diseases-bacillary dysentery, staphylococcus infections, colon bacillus infections-phagotherapy is becoming an established 
procedure, and many authors consider it specific. I hope that this will presently be true for plague.*

In order to properly close this text, perhaps I should draw some general broad conclusions. Possibly I should discuss the very material changes in our concepts brought about by our knowledge of the bacteriophage protobe and bacteriophagy, particularly in that which concerns pure Biology and in that which deals with Immunity. I deem it best, however, to refrain from discussing these phases of the subject that this text may not be unreasonably lengthened, for if we were to enter into this field a second volume would be necessary. It so happens, indeed, that these are precisely the general considerations which have been made the subject of a recently published book,- "Immunity in Natural Infectious Disease," - to which I would refer the interested reader.

* As was remarked some two years ago by an English medical journal, in the prophylactic and therapeutic use of the bacteriophage there is a vast field for commercial exploitation. This has already begun. I can not witness it without apprehension, and it is very desirable that commercial products be very rigidly controlled. It would be far better if scientific Institutes, following the example of the Institute Oswaldo Cruz, would prepare the suspensions of the bacteriophage destined for use as a therapeutic agent.

Too often, commercial firms mislead both physicians and the public by clever quotations (clever in the sense that they avoid conflict with the law) tending to make it appear that such and such a scientist supervises their products, or even controls them. I now declare that I am, and always will remain, a stranger to all commercial enterprises. I may go even further in this direction and state that every time that I have treated a patient it has been done solely from a scientific motive. 


\section{BIBLIOGRAPHY}

1. Alessandrini, A., And Doria R.: Bacteriophagum antityphicum polyvalens. Med. Klin., 1924, 20, 1447.

2. —— Il batteriofago nella terapia del tifo addominale. Il Policlinico (sez. pract.), 1924, $81,109$.

3. Algalia: El bacteriofago. Siglo Medico, 1921, 68, 984.

4. Alphonsi: Guérison rapide de deux cas de pyélo-néphrite gravidique traités par le bactériophage de d'Herelle. Bull. de la Soc. d'obstét. et de gyn. de Paris, 1924, 13, 573.

5. Amedille, P.: Le bactériophage de d'Herelle. Ann. de méd., 1921, 9, 197.

6. Andervont, H., ANd Simon, C. E.: On the origin of the so-called pellucid areas which develop on agar cultures of certain spore-bearing bacteria. Am. J. Hyg., 1924, 4, 386.

7. von Angerer, K.: Über das d'Herellesche Phänomen. Klin. Wchnschr., $1922,1,654$.

8. — : [Discussion to] Weitere Untersuchungen über das d'Herellesche Phänomen [by Gildemeister et al.]. Centralbl. f. Bakt., I, Orig., 1922/23, $89, * 204$.

9. — Ứber Bakteriophagie. Kilin. Wchnschr., 1923, 2, 810.

10. — : Über das d'Herellesche Phänomen. Klin. Wchnschr., 1923, 2, 1194.

11. — : Beiträge zum Bakteriophagenproblem. Arch. f. Hyg., 1924, 92, 312 .

12. Appelmans, R.: Le bactériophage dans l'organisme. Compt. rend. Soc. de biol., 1921, 85, 722 .

13. - Le dosage du bactériophage. Compt. rend. Soc. de biol., 1921, 85, 1098.

14. - : Quelques applications de la méthode de dosage du bactériophage. Compt. rend. Soc. de biol., 1922, $86,508$.

15. - : Au sujet de la valeur thérapeutique du bactériophage. Arch. internat. de pharmacod., 1922, 27,85 .

16. - and Wagemans, J.: Bactériophages de diverses provenances. Compt. rend. Soc. de biol., 1922, 86,738 .

17. Arkwright, J. A.: The source and characteristics of certain cultures sensitive to bacteriophage. Brit. J. Exp. Path., 1924, 5, 23.

18. Arloing, F., and Chavanne: Propriétés empêchantes des eaux de l'Isère à l'égard de diverses cultures microbiennes. Compt. rend. Soc. de biol., 1925, 92, 257.

19. —— De l'influence sur le bactériophage des électrolytes et de la concentration en ions $\mathrm{H}$ de milieu. Compt. rend. Soc. de biol., 1925, 93, 531.

20. - D Drour, Bouvier, and Sempé: Traitement de la pyélonéphrite par le bactériophage de d'Herelle. Compt. rend. Soc. méd. des Hôp. de Lyon, 1924, May 13. 
21. Arloing, F., Langeron, L., and Sempé: Recherehes comparatives sur l'action de certains agents physiques sur le bactériophage. Une diastase et le complément. Compt. rend. Soc. de biol., 1925, 92, 260.

22. — and Sempé: Propriétés empêchantes des eaux du Rhône et de la Saône sur la développement de certaines bactéries. Compt. rend. Soc. de biol., $1924,91,667$.

23. - - - and Chavanne: Propriétés antimicrobiennes de diverses eaux fluviales ou marines. Pouvoir bactériophagique. Bull. Acad. de Méd., 1925, 3 s., 93, 184.

24. Arnold, L.: Baeteriophage phenomena. J. Lab. and Clin. Med., 1923, 8, $720 ; 813$.

25. - AND WEIss, E.: A study of bacteriophage with antibacteriophage serum. J. Infect. Diseases, 1924, 35, 505.

26. —— The Twort-d'Herelle phenomenon; the resemblance of bacteriophage to toxins and ferments. J. Infect. Diseases, 1924, 85, 603.

27. Asheshov, I. N.: Sur les particularités de quelques souches de bactériophage. Compt. rend. Soc. de biol., 1922, 87, 1341.

28. - L L'aceoutumance du baetériophage. Compt. rend. Soc. de biol., $1922,87,1343$.

29. - L L'attenuation du bactériophage par vieillissement. Compt. rend. Soc. de biol., 1923, $89,118$.

30. - : Quelques reeherches sur la nature des plages de bactériophage. Compt. rend. Soe. de biol., 1923, 89, 120.

31. - : The bacteriophage. Discovery, Lond., 1924, 5, 182.

32. - Experimental studies on the bacteriophage. J. Infect. Diseases, $1924,34,536$.

33. - - Le pouvoir antigène des lysats ultrastériles. Compt. rend. Soc. de biol., 1925, 93, 643.

34. - - Immunisation des bactéries contre le bactériophage. Compt. rend. Soc. de biol., 1925, 93, 644 .

35. Azerad, E.: Le bactériophage de d'Herelle. Gaz. de Hôp., Par., 1924, 97, 629 .

36. Bablet, J.: Sur le prineipe bactériophage de d'Herelle. Compt. rend. Soc. de biol., 1920, 83, 1322.

37. Bachmann, A.: El bacteriofago de d'Herelle. Comprobaction di su existencia entre nososbios. Sa preseneia en los pozos ciegos. Prensa méd. Argentina, 1922, Mar. 20.

38. - AND AqUino, L. I.: Sobre autolisis baeteriana transmisible en serie. Semana méd., Buenos Aires, 1922, 29, 432.

39. — - Sobre el baeteriofago. Bol. Asoc. méd. Argent., Buenos Aires, 1922, 35, Sect. Soc. de biol., 18.

40. ——: Sur le bactériophage. Compt. rend. Soc. de biol., 1922, 86, 1108.

41. - AND DE LA BARRERA, J.: Quelques variations sérologiques du Bacille paratyphique A. Compt. rend. Soc. de biol., 1923, 89, 756 .

42. BaIL, O.: Das Bakteriophage Virus von d'Herelle. Wien. klin. Wchnschr., 1921, 34, 237.

43. - : Bakteriophage Wirkungen gegen Flexner- und Koli-Bakterien. Wien. klin. Wehnschr., 1921, 34, 448. 
44. BaIl, O.: Ueber Shiga-Bakteriophagen. Wien. klin. Wehnschr., 1921, 34,555 .

45. — : Elementarbakteriophagen des Shigabazillus. Wien. klin. Wehnschr., 1922, 35, 722; 743; 765 .

46. —— B B B - Kabhtungen an Koli-bakteriophagen. Med. Klin., 1923, 19, 144.

47. — Ueber das Lysozym A. Flemings. Wien. klin. Wchnschr., 1923, 36, 107.

45. — : Versuche über die Vielheit von Bakteriophagen. Zeitschr. f. Immunitätsforsch., 1923, 38, 57.

49. - - Untersuchungen über die M-Konzentration von Bakterien $u$. Bakteriophagen. Arch. f. Hyg., 1924, 94, 54.

50. - Theorie der Bakteriophagen Wirkungen. Bull. techn. d. Sc. méd., Genève, 1925, 1, 23.

51. - AND Matsumoto, T.: Die Anhäufungsmöglichkeit von Bakteriophagen und Bakterien. Med. Klin., 1923, 19, 1579.

52. - AND OKUDA, S.: Der Abbau lebender Bakterien durch Bakteriophagen. Arch. f. Hyg., 1923, 92, 251.

53. - and Watanabe, T.: Ueber Mischbakteriophagen. Wien. klin. Wehnschr., 1922, 85, 169.

54. —— : Versuche über spezifische Bakteriophagenwirkung. Wien. klin. Wchnschr., 1922, 85,362 .

55. Bastin, A. H. C. P.: Le phénomène de d'Herelle considéré dans ses rapports avec la biologie générale, la pathogénie, la prophylaxie, et la thérapeutique des infections. Thèse. Lille. Leblanc and Durant, 1922, 112 pp.

56. BAZY, L.: Traitement des infections chirurgicales à staphylocoques par le bactériophage anti-staphylococcique. Compt. rend. Soc. de biol., 1925, $92,485$.

57. Beckerich, A., and Hauduroy, P.: Au sujet du titrage du bactériophage. Compt. rend. Soc. de biol., 1922, 86, 165.

58. — - Le bactériophage dans le traitement de la fièvre typhoïde. Compt. rend. Soc. de biol., 1922, $86,168$.

59. —_ - Au sujet de l'obtention de bactériophage par antagonisme microbien. Compt. rend. Soc. de biol., 1922, 86,881 .

60. - Sur l'obtention de bactériophage par antagonisme microbien. Réponse à MM. Lisbonne et Carrère. Compt. rend. Soc. de biol., 1922, $87,1124$.

61. __ L L bactériophage de d'Herelle, son rôle dans l'immunité, ses applications thérapeutiques. Le Méd. d'Alsace et Loraine, 1922, July.

62. — Le traitement des infections urinaires à colibacilles par le bactériophage de d'Herelle. Bull. méd., Par., 1923, 87, 273.

63. —— Le bactériophage de d'Herelle: ses applications thérapeutiques. J. Bact., 1923, 8, 163.

64. Beijerinck: Pasteur en de Ultramicrobiologie. Chemisch. Weekblad, Pasteur Number, 1922, Nov. 25.

65. Bergstrand, H.: Sur la lyse mierobienne transmissible. Compt. rend. Soc. de biol., 1922, 86, 489 . 
66. Bergstrand, H.: Sur la variation des bactéries. Compt. rend. Soc. de biol., 1922, 86,492 .

67. —— : [Le bacteriophage.] Svensk. Läk.-Sällsk. Handl., Stockholm, 1922, $48,24$.

68. Bertarelli, E.: Il batteriofago nella biologia e nella medicina. Med. ital., Milano, 1922, 3, 250.

69. Bremond, A. G.: Einige Bakteriophagenuntersuchungen. Zeitschr. f. Hyg. u. Infektionskrankh., 1924, 103, 681.

70. Blatr, J. E.: A lytic principle (bacteriophage) for Corynebacterium diphtheriae. J. Infect. Diseases, 1924, 35, 401.

71. Blanc, J.: Transformation de Bacilles pyocyaniques en bacilles sans pigments. Compt. rend. Soc. de biol., 1923, 88, 52 .

72. Bogendörfer, L.: Hemmungsstoffe aus Bakterien und ihren Kultursubstraten. Zeitschr. f. d. ges. exper. Med., 1924, 41, 620.

73. Borchardt, W.: Biologische Untersuchungen über die Natur des d'Herelleschen Phänomens. Klin. Wchnschr., 1923, 2, 295.

74. ——: Weitere biologische Beiträge zum d'Herelleschen Phänomen. Klin. Wchnschr., 1923, 2, 791.

75. — : Biologische Beiträge zum d'Herelleschen Phänomen. Zeitschr. f. Immunitätsforsch., 1923, $87,1$.

76. — : Ist das lytische Agens des d'Herelleschen Phänomens flüchtig? Klin. Wehnschr., 1924, 3, 278.

77. Bordet, J.: Hemolytic exudates and transmissible bacterial autolysis. Johns Hopkins Hosp. Bull., 1921, 32, 302.

78. - Concerning the theories of the so-called "bacteriophage." Brit. M. J., 1922, ii, 296.

79. —- La bactériophagie ou l'autophagie microbienne transmissible. In Soc. roy. d. sc. méd. et nat. de Brux., vol. jubil., Brux., 1922, 353.

80. - Obtention de principes de faible puissance dans l'autolyse microbienne transmissible. Compt. rend. Soc. de biol., 1922, 87, 987.

81. - - La théorie de l'antagonisme microbien dans la genèse de la lyse transmissible. Compt. rend. Soc. de biol., 1923, $88,1211$.

82. - - L Les théories de la lyse microbienne transmissible (à propos d'une note de la Costa Cruz). Compt. rend. Soc. de biol., 1923, 89, 963.

83. —- : Microbic transmissible autolysis. Brit. M. J., 1923, i, 175.

84. - - A Apparition spontanée du pouvoir lysogène dans les cultures pures. Compt. rend. Soc. de biol., 1924, 90, 96.

85. — AND CidCA, M.: Exsudats leucocytaires et autolyse microbienne transmissible. Compt. rend. Soc. de biol., 1920, 83, 1293.

86. —— - Le bactériophage de d'Herelle; sa production et son interprétation. Compt. rend. Soc. de biol., 1920, 8s, 1296.

87. — - Déterminisme de l'autolyse microbienne transmissible. Compt. rend. Soc. de biol., 1921, 84, 276.

88. ——: Spécificité de l'autolyse microbienne transmissible. Compt. rend. Soc. de biol., 1921, 84, 278.

89. - - Autolyse microbienne et sérum antilytique. Compt. rend. Soc. de biol., 1921, 84, 280. 
90. Bordet, J., And Ciuca, M.: Remarques sur l'historique des recherches concernant la lyse microbienne transmissible. Compt. rend. Soc. de biol., 1921, 84, 746.

91. —_ Evolution des cultures de coli lysogène. Compt. rend. Soc. de biol., 1921, 84, 747 .

92. —— Guérison et retour a l'état primitif par le sérum antilytique du coli lysogène. Compt. rend. Soc. biol., 1921, 84, 748.

93. —— : Sur la régénération du principe actif dans l'autolyse microbienne. Compt. rend. Soc. de biol., 1921, 85, 1095.

94. et les conditions de régénération du principe actif. Compt. rend. Soc. de biol., 1922, 86, 295.

95. ㄴ. : Variations d'énergie du principe actif dans l'autolyse microbienne transmissible. Compt. rend. Soc. de biol., 1922, 87, 366.

96. Botez, A.: La bactériolyse en série par le violet de méthyle. Compt. rend. Soc. de biol., 1921, 85, 585 .

97. Boulet, P.: Le bactériophage de d'Herelle existe-t-il? Thèse. Montpellier. L'Abeille, 1924.

98. Bronfenbrenner, J.: Further studies on so-called bacteriophage. Proc. Soc. Exp. Biol. and Med., 1924, 22, 81.

99. - AND Korb, C.: Is the bacteriophage of d'Herelle volatile? Proc. Soc. Exp. Biol. and Med., 1924, 21, 175.

100. ㄴ. : Effect of alcohol on the so-called bacteriophage of d'Herelle. Proc. Soc. Exp. Biol. and Med., 1924, 21, 177.

101. - On the factors influencing the appearance of placques of bacterial lysis. Proc. Soc. Exp. Biol. and Med., 1924, 21, 315.

102. — : Studies on the bacteriophage of d'Herelle. I. Is the lytic principle volatile? J. Exp. Med., 1925, 41, 73.

103. - Studies on the bacteriophage of d'Herelle. II. Effect of alcohol on the bacteriophage of d'Herelle. J. Exp. Med., 1925, 42, 419 .

104. Brutsaert, P.: La virulence des bactériophages. Compt. rend. Soc. de biol., 1923, $89,87$.

105. —— Le bactériophage et le radium. Compt. rend. Soc. de biol., 1923, 89 , 90.

106. — : Les bactériophages dans les milieux fluorés. Compt. rend. Soc. de biol., 1923, 89, 1173.

107. —_- Influence des électrolytes sur le phénomène de d'Herelle. Compt. rend. Soc. de biol., 1923, $89,1175$.

108. - L L'agglutination des microbes résistants. Compt. rend. Soc. de biol., 1924, 90, 645 .

109. —— Les bactériophages et les microbes dans le bouillon hypersalé. Compt. rend. Soc. de biol., 1924, 90, 646.

110. - L Le bactériophage dans les milieux gélatinés. Compt. rend. Soc. de biol., 1924, 90, 1292.

111. - L Les bactériophages et les microbes dans le bouillon hypersalé. Bull. Soc. belge de biol., 1924, 1, 646. 
112. Bruynoghe, R.: Au sujet de la guérison des germes devenus résistants au principe bactériophage. Compt. rend. Soc. de biol., 1921, 85, 20.

113. - - Au sujet de la nature du principe bactériophage. Compt. rend. Soc. de biol., 1921, 85, 258.

114. - C C Contribution à l'étude de la nature des bactériophages. Bull. Acad. roy. de méd. de Belg., Brux., 1923, 5.s., 3, 360.

115. - L Les bactériophages. Arch. internat. de méd. expér., Liége, 1924, i, 17 .

115. ㄴ N Notre contribution à l'étude des bactériophages. Bull. techn. d. sc. méd., Genève, 1925, 1, 1.

116. — - AND Appelmans, R.: La neutralisation des bactériophages de provenance différente. Compt. rend. Soc. de biol., 1922, 87, 96.

117. — : La neutralisation des bactériophages. Arch. internat. de pharmacod., 1922/23, 2\%, 81 .

118. — and Brutsaert, P.: La résistance des bactériophages a l'action de certaines substances chimiques. Compt. rend. Soc. de biol., 1923, 88, 966.

119. - AND MaIsin, J.: Au sujet des microbes devenus résistants au principe bactériophage. Compt. rend. Soc. de biol., 1921, 84, 847 .

120. ——— Le principe bactériophage du staphylocoque. Compt. rend. Soc. de biol., 1921, 85, 1118.

121. - Essais de thérapeutique au moyen du bactériophage du staphylocoque. Compt. rend. Soc. de biol., 1921, 85, 1120.

122. —— Au sujet de l'unité du principe bactériophage. Compt. rend. Soc. de biol., 1921, 85, 1122 .

123. — - La phagocytose du bactériophage. Compt. rend. Soc. de biol., 1922, 86, 292.

124. - - Au sujet de la réaction consécutive à l'injection du bactériophage. Compt. rend. Soc. de biol., 1922, 86, 294.

125. — : Réponse a la note de MM. Gratia et Jaumain relative aux réactions produites par l'injection de bactériophages. Compt. rend. Soc. de biol., 1922, 86,739 .

126. — and Mund, W.: Les microbes irradiés et le bactériophage. Compt. rend. Soc. de biol., 1925, 92, 464.

127. - AND Wagemans, J.: La résistance des bactériophages au sérum neutralisant. Compt. rend. Soc. de biol., 1923, 88, 968.

128. —- Sur la complexité de certaines bactériophages. Compt. rend. Soc. de biol., 1923, 89,85 .

129. Bull, C. G.: Bacteriophage. Physiol. Rev., 1923, 5, 95.

130. Bürgers: Bedingt die Entdeckung des bakteriophagen Virus eine Änderung unserer Anschauungen über Infektion und Immunität? Klin. Wchnschr., 1923, 2, 567.

131. — and Bachmann, W.: Bakteriophagenstudien. Zeitschr. f. Hyg. u. Infektionskrankh., 1924, 101, 350.

132. Burnet, F. M.: The nature of the acquired resistance to bacteriophage action. J. Path. and Bact., 1925, 28, 407.

133. - The conditions governing the appearance of tâches vièrges in bacteriophage activity. J. Path. and Bact., 1925, 28, 419. 
134. Busson, B., and Ogata, N.: Untersuchungen über sekundäre und bakteriophagenresistente Dysenteriestämme und ihre Beziehung zu den sogenannten Schmitzstämmen. Wien. klin. Wehnschr., 1924, 37, 665.

135. Callow, B. R.: Bacteriophage phenomena with Staphylococcus aureus. J. Infect. Diseases, 1922, 30, 643.

136. Čančik, J.: [Bacteriophagy in Bacillus pyocyaneus eultures.] Časop. lék. česk., Praha, 1923, 62, 25.-Abs. in J. Am. Med. Assn., 1923, 80, 970.

137. - : [The Twort-d'Herelle Phenomenon.] Časop. lék. česk., Praha, 1923, 62, 183.-Abs. in Wien. klin. Wehnschr., 1923, 36, 125.

138. Caplazi, A.: Die Destillation der übertragbaren Lysine (Bakteriophagen). Zeitschr. f. Hyg. u. Infektionskrankh., 1924, 102, 438.

139. Carrère, J. Étude du phénomène de bactériolyse transmissible. Montpel. méd., 1924, 46, 161; 185; 209.

140. Caublot, P.: Technique des manipulations et contribution à l'ćtude du bactériophage de d'Herelle. Thèse. Paris, A. Legrand. 1923, 75 pp.

141. - : Le bactériophage du Pneumobacille de Friedländer. Compt. rend. Soc. de biol., 1924, 90, 622 .

142. Chaubaud, J.: Le bactériophage de d'Herelle. Arch. de méd. et pharm. nav., Par., 1922, 112, 203.

143. Ciaccio, C.: Contriluuto allo studio della lise batterica transmissibile. I. Ricerche sul meccanismo col quale si estrinseca la lise batterica. Ann. d'ig., Roma, 1924, 34, 477.

144. Cirvca, M. : Présence de principe lytique pour le Bacille de Shiga et le Colibacille dans les selles des cholériques. Compt. rend. Soc. de biol., 1923, $88,143$.

145. - L Lyse transmissible en absence d'électrolytes libres. Compt. rend. Soc. de biol., 1924, 90, 521.

146. - and Manoliu, E.: Action inhibitrice du filtrat de cultures et lyse transmissible au cours de la fièvre typhoïde. Compt. rend. Soc. de biol., 1924, 91, 1225.

147. - and Petrovand, E.: Sur quelques particularités du "principe lytique" d'origine intestinale. Compt. rend. Soc. de biol., 1923, 88, 922.

148. Сombiesco, D.: Sur le phénomène de d'Herelle. Compt. rend. Soc. de biol., $1922,87,17$.

149. - and Magherd, A.: Sur la lyse du Bacille pyocyanique. Compt. rend. Soc. de biol., 1923, $88,912$.

150. DA Costa CrUz, J.: Sobre a autolyse microbiana transmissivel; bacteriophago de d'Herelle. Brazil-med., Rio de Jan., 1922, 36, 45.

151. - : Sobre a lyse microbiana transmissivel (bacteriophago de d'Herelle). Brazil-med., Rio de Jan., 1922, 36, 96.

152. - - Sobre a lyse microbiana transmissivel (bacteriophago de d'Herelle). Brazil-med., Rio de Jan., 1922, 36, 131.

153. — : Sur la lyse microbienne transmissible (Bactériophage de d'Herelle). Mem. Inst. Oswaldo Cruz, 1922, 14, 81.

154. — - Sobre a lyse microbiana transmissivel (bacteriophago de d'Herelle). Mem. Inst. Oswaldo Cruz, 1922, 16, 104.

155. — : Sobre a lyse microbiana transmissivel (bacteriophago de d'Herelle). Brazil-med., Rio de Jan., 1923, 37, 44. 
156. DA Costa Cruz, J.: A respecto de natureza do bacteriophago. A questão dos virus filtraveis e dos fermentos infecciosos. Brazil-med., Rio de Jan., 1923, 37, 201.

157. ——: O bacteriophago en therapeutica. Brazil-med., Rio de Jan., 1923, 37, 298.

158. - A influencia dos electrolytos sobre a lyse pelo bacteriophago. Brazil-med., Rio de Jan., 1923, $37,341$.

159. - : Sur la nature du bactériophage. Influence des électrolytes. Compt. rend. Soc. de biol., 1923, 89, 759 .

160. —_: A influencia do $\mathrm{pH}$ sobre o bacteriophago de d'Herelle. Brazilmed., Rio de Jan., 1924, $38, \$ 50$.

161. - - Sur l'influence des électrolytes dans la lyse par le bactériophage. Compt. rend. Soc. de biol., 1924, 90, 236.

162. - : Sur la nature du bactériophage. À propos d'une note de F. d'Herelle. Compt. rend. Soc. de biol., 1924, 90, 694 .

163. —_ L L'influence du pH sur la lyse par le bactériophage. Compt. rend. Soc. de biol., 1924, 90, 878.

164. - : Sur le mécanisme de l'action anti-lytique du sérum anti-bactérien dans la lyse par le bactériophage. Compt. rend. Soc. de biol., 1924, 91, 840.

165. - L Le traitement des dysentéries bacillaires par le bactériophage. Compt. rend. Soc. de biol., 1924, 91, 845 .

166. —_ Influence de la concentration des bactéries sur la production du bactériophage. Compt. rend. Soc. de biol., 1925, 92, 310.

167. - L L'influence du ClNa sur le bactériophage. Compt. rend. Soc. de biol., 1925, 93, 37.

168. Courcoux, Philibert, and Corday: Un cas de pyélonéphrite gravidique traitée par le bactériophage de d'Herelle. Bull. et mém. Soc. méd. d. Hôp. de Par., 1922, 3. s., 46, 1151.

169. Cutrer, R. K.: Bacillary dysentery: Report of a case, with a study of the intestinal bacteriophage. J. Am. Med. Assn., 1923, 80, 1674.

170. Damade, E. M. J. R.: Êtude d'une épidémie de dysentérie bacillaire. Thèse. Bordeaux, 1919, $108 \mathrm{pp}$.

171. Davison, W. C.: Nature and therapeutic application of bacteriolysants (d'Herelle's phenomenon). Abstracts Bact., 1921, 6, 27.

172. - : Filtrable "substance" antagonistic to dysentery and other organisms (d'Herelle's phenomenon, bacteriophage, bacteriolytic agent, bacteriolysant, etc.). Abstracts Bact., 1922, 6, 159.

173. - The bacteriolysant therapy of bacillary dysentery in children. Therapeutic application of bacteriolysants; d'Herelle's phenomenon. Am. J. Dis. Children, 1922, 23, 531.

174. - Observations on the properties of bacteriolysants (d'Herelle's phenomenon, bacteriophage, bacteriolytic agent, etc.). J. Bact., 1922, 7,$475 ; 491$.

175. Debré, R., and Hagdenad, J.: Quelques particularités du "phénomène de d'Herelle." Compt. rend. Soc. de biol., 1920, 83, 1348.

176. —_ : Quelques particularités du "phénomène de d'Herelle." Compt. rend. Soe. de biol., 1920, 83, 1368. 
177. Delezenne, C., and Ledebt, S.: Sur la transmission en série du pouvoir protéolytique initialement conféré au suc pancréatique inactif par l'entérokinase. Compt. rend. Acad. sci., 1922, 175, 779.

178. Doerr, R.: Die Bakteriophagen (Phänomen von Twort und d'Herelle). Klin. Wchnschr., 1922, 1, 1489; 1537.

179. - Die invisiblen Ansteckungsstoffe und ihre Beziehungen zu Problemen der allgemeinen Biologie. Klin. Wchnschr., 1923, 2, 909.

180. - - Ueber Bakteriophagen. Schweiz. med. Wchnschr., 1923, 5s, 1009.

181. - AND Berger, W.: Studien zum Bakteriophagenproblem. III. Mitt. Die antagonistische Wirkung von Gelatine und Agar auf den Ablauf der Bakteriophagenreaktion. Zeitschr. f. Hyg. u. Infektionskrankh., 1922, 97,422 .

182. - AND GRÜninger, W.: Experimentelle Untersuchungen über die Beziehungen von Bakterien und Bakteriophagen zur Galle. Schweiz. med. Wchnschr., 1922, 52, 761.

183. ﹎. _ - Studien zu Bakteriophagenproblem. I Mitt. Zeitliche und quantitative Beziehungen zwischen Bakterienvermehrung und Zunahme des lytische Agens. Zeitschr. f. Hyg. u. Infektionskrankh., 1922, 97, 209.

184. — - And Rose, G.: Die Thermoresistenz der übertragbaren Lysine (Bakteriophagen). Schweiz. med. Wchnschr., 1924, 54, 10.

185. — AND ZDANskr, E.: Studien zum Bakteriophagenproblem. V. Quantitativen und qualitativen Nachweis der Lysine. Ihr Dispersitätsgrad und die Aufteilbarkeit ihrer Lösungen. Zeitschr. f. Hyg. u. Infektionskrankh., 1923, 100, 79.

186. Doria, R.: Contributo allo studio sulla natura del batteriofago. Ann. d'ig., Roma, 1924, 34, 65 .

187. Dujarric de la Rivière, R.: Flocculation et bactériophage de d'Herelle. Compt. rend. Soc. de biol., 1925, 93, 498.

188. Dumas, J.: Sur la présence du bactériophage dans l'intestin sain, dans la terre, et dans l'eau. Compt. rend. Soc. de biol., 1920, 83, 1314.

189. EAstwood, A.: Bacterial variation and transmissible autolysis; The relation of bacterial enzymes to bacterial structure. Gt. Brit. Ministry of Health. Repts. on Pub. Health and Medical Subjects, 1923, 18, 14.

190. Eichnoff: Ist das d'Herellesche Phänomen von Bedeutung für die Chirurgie? Deutsche med. Wehnschr., 1922, 48, 756.

191. Eliava, G., And Pozerski, E.: Sur les caractères nouveaux présentés par le bacille de Shiga ayant résisté a l'action du bactériophage de d'Herelle. Compt. rend. Soc. de biol., 1921, 84, 708.

192. - - De l'action destructive des sels de quinine sur le bactériophage de d'Herelle. Compt. rend. Soc. de biol., 1921, 85, 139.

193. Ellis, A. W. M.: Discussion on the bacteriophage (bacteriolysin). Brit. M. J., 1922, ii, 298.

194. Emsmann: Werner von Siemens und das "Bakteriophage Virus." Zeitschr. f. ärztl. Fortbild., Jena, 1922, 19, 124.

195. Fabry, P.: Autolyse microbienne transmissible obtenue par antagonisme microbien. Compt. rend. Soc. de biol., 1922, 87, 369. 
196. Fabry, P., and van Beneden, J.: À propos de l'obtention de l'autolyse transmissible par antagonisme. Compt. rend. Soc. de biol., 1924, 90, 109.

197. - S Sérum antilytique et antisérum anti-antilytique. Compt. rend. Soc. de biol., 1924, 90, 111.

198. FeJGin, B.: Contribution à l'etude des races résistantes du Bacille de ShigaKruse. Compt. rend. Soc. de biol., 1923, 89, 1381.

199. — : Examen de quelques microbes isolés des matières fécales. Compt. rend. Soc. de biol., 1923, 89, 1383.

200. - - Sur les variations brusques du Proteus $\mathrm{HX}_{19}$ survenues sous l'influence de l'agent lytique anti- $\mathrm{HX}_{19}$ et leur rapport avec les souches isolées des cobayes infectés avec le virus de passage du typhus exanthématique. Compt. rend. Soc. de biol., 1924, 90, 1106.

201. - - Recherches sur le typhus exanthématique expérimental. Compt. rend. Soc. de biol., 1924, 90, 1200.

202. - Examen de quelques souches isolées des cobayes inoculés avec le bactériophage anti-HX ${ }_{19}$. Compt. rend. Soc. de biol., 1924, 90, 1202.

203. - : O Bakteriofagach. Medy. Doswiadezalnej, Varsovie, 1924, 2, No. 34 .

204. - - : Sur la forme filtrant de Bacille d'Eberth. Compt. rend. Soc. de biol., 1925, 92, 1528.

205. ——: Sur les cultures secondaires du Bacille typhique isolé des organes des cobayes infectés avec le virus de la fièvre typhoïde. Compt. rend. Soc. de biol., 1925, $92,1530$.

206. ——: Sur le principe lytique antidiphterique. Compt. rend. Soc. de biol., 1925, 93, 365 .

207. - and Supniewski, J.: Sur la nature du phénomène de d'Herelle. Compt. rend. Soc. de biol., 1923, 89, 1385.

208. Fleming, A.: On a remarkable bacteriolytic element found in tissues and secretions. Proc. Roy. Soc., Lond., 1922, 93B, 306.

209. - Further observations on a bacteriolytic element found in tissues and secretions. Proc. Roy. Soc., Lond., 1922/23, 94B, 142.

210. - AND Allison, V. D.: Observations on a bacteriolytic substance ("Lysozyme") found in secretions and tissues. Brit. J. Exp. Path., 1922, 3,252 .

211. FLV, P. C.: Das Verhalten eines inagglutinablen Flexner-Bakterium gegenüber anti-Flexner-Bakteriophagen. Centralbl. f. Bakt., I, Orig., 1923, 90,374 .

212. - Die Natur des Bakteriophagen und die Bildung von Bakteriophagen in alten Bouillonkulturen pathogenen Mikroorganismen. Centralbl. f. Bakt., I, Orig., 1923, 90, 362 .

213. - - Sur la prétendue action déchaînante de la pancréatine sur le principe bactériophage. Compt. rend. Soc. de biol., 1923, 89, 970.

214. - De forming van Bacteriophaagen in oude bouilloneultures van pathogene darmbacterien en de natur van de Bacteriophaag. Nederl. Tijdschr. v. Geneesk., Haarlem, 1923, 67, 226.

215. - On the bacteriophage and the self-purification of water. Verhandel. v. k. Akad. v. Wetensch., Amsterdam, 1923, 26, 116. 
216. FLU, P. C.: Het gedrag van een inaglutinabele Bacterium Flexner tegenover Bacteriophagen anti-Flexner. Tijdschr. v. Vergelijk. Geneesk., 1923, 8 , No. 4 .

217. - - De Bacteriophaag en de selfreiniging van oppervlaktewater. Tijdschr. v. Vergelijk. Geneesk., 1923, 8, No. 4.

218. - - Een method voor het onderzoek van reinkulturen op besmetting met een bacteriophaag. Tijdschr. v. Vergelijk. Geneesk., 1924, 10, 183.

219. — : Tryptische fermenten en de formeng van Bacteriophagen. Tijdschr. v. Vergelijk. Geneesk., 1924, 10, 189.

220. — : Over Cholerabacteriophagen. Tijdschr. v. Vergelijk. Geneesk., 1924, 10, 196.

221. —- Kunnen bacteriophagen door een $3 \%$ agargel diffundeeren? Tijdschr. v. Vergelijk. Geneesk., 1924, 10, No. 4.

222. - - Over de thermoresistentie van bacteriophagen en de vraag of reactiveering van tot $100^{\circ}$ vahitte bacteriophagen door filtratie mogelijk is. Tijdschr. v. Vergelijk. Geneesk., 1924, 10, No. 4.

223. Françon, F., and Marquézy, R.: Le phénomène de d'Herelle; les faits, les interprétations; les applications. Bull. méd., Par., 1922, $36,33$.

224. Friedberger, E., ANd Vallen, J.: Über die Wirkung eines Typhusbakteriophagen in Gegenwart von roten Blutkörperchen. Klin. Wchnschr., 1923, 2, 1649 .

225. Friedmann, U.: Über das d'Herellephänomen. Naturwissenschaften, 1921, $9,1010$.

226. Friel, A. R.: The d'Herelle phenomenon. Lancet, 1922, i, 251.

227. Gercke, A.: Untersuchungen über die Frage nach der Flüchtigkeit der Bakteriophagen-Lysine. Centralbl. f. Bakt., I. Orig., 1925, 94, 387.

228. Gerretsen, F. C., Gryns, A., Sack, J., and Söhngen, N.: Das Vorkommen eines Bakteriophagen in den Wurzelknöllchen der Leguminosen. Centralbl. f. Bakt., II. Orig., 1923/24, 60, 311.

229. Gildemeister, E.: Ueber das d'Herelleschen Phänomen. Berl. klin. Wchnschr., 1921, $58,1355$.

230. ——: Weitere Untersuchungen über das d'Herellesche Phänomen. Centralbl. f. Bakt., I. Orig., 1922, 89 , *181.

231. —- Weitere Untersuchungen über das d'Herellesche Phänomen. Klin. Wchnschr., 1922, 1, 1859.

232. — and Herzberg, K.: Ueber das d'Herellesche Phänomen. III. Mitt. Centralbl. f. Bakt., I. Orig., 1924, 91, 12.

233. — - U U U U C Der das d'Herellesche Phänomen. IV. Mitt. Centralbl. f. Bakt., I. Orig., 1924, 91, 228.

234. —— Zur Theorie der Bakteriophagen (d'Herelle-Lysine). VI. Mitt. Über das d'Herellesche Phänomen. Centralbl. f. Bakt., I. Orig., 1924, 93, 402 .

235. —— Zur Frage der Destillierbarkeit und Flüchtigkeit der d'Herelle-Lysine. V. Mitt. Ueber das d'Herellesche Phänomen. Klin. Wchnschr., 1924, 3, 186.

236. —— : Erwiderung [to] Zur Frage der Destillierbarkeit und Flüchtigkeit der d'Herelle-Lysine, von O. Olsen und Y. Yasaki. Klin. Wchnschr., 1924, $8,278$. 
237. GJ $\varnothing \mathrm{R} \cup \mathrm{P}$, E.: [Bacteriophagum intestinale.] Hospitalstid., 1921, 64, 214; 234. 238. - - Sur la présence de bactériophages (d'Herelle) chez les typhiques et les paratyphiques. Acta. med. scandin., Kristiania, 1924, Suppl. 7, 365 .

239. Goglia, G.: Il batteriofago nella terapia del tifo addominale. Folia med., Napoli, 1924, 10, 109.

240. Gomez, J. M.: Recherches sur la bactériophagie; action sur les erythrocytes, les toxines, les sucres. Brazil-med., Rio de Jan., 1922, 36, pt. 2, 412.

241. Gorter, E.: Filtreerbaar virus. Nederl. Vereen. v. Kindergeneesk., 1924, May 24.

242. Gory, M.: Transformation muqueuse du Bacillus coli. Compt. rend. Soc. de biol., 1923, 88,49 .

243. Govgerot, and Peyre, E.: Essai de traitement par le bactériophage dans les infections cutanées. Bull. Soc. franc. de dermat. et syph., 1924, 31, 420 .

244. - L Le bactériophage dans le traitement des affections cutanées. Compt. rend. Soc. de biol., 1924, 91, 452.

245. Gbzony, L., and Surányi, L.: Reduktionsversuche mit Bakteriophagen. Centralbl. f. Bakt. I. Orig., 1925, 95, 353.

246. Gratia, A.: Studies on the d'Herelle Phenomenon. J. Exp. Med., 1921, 34, 115.

247. - Studies on the lytic agent of Bordet and Ciuca. Proc. Soc. Exp. Biol. and Med., 1921, 18, 192.

248. - - Preliminary report on a staphylococcus bacteriophage. Proc. Soc. Exp. Biol. and Med., 1921, 18, 217.

249. - Influence de la reaction du milieu sur l'autolyse microbienne transmissible. Compt. rend. Soc. de biol., 1921, 84, 275.

250. — : De l'adaptation héreditaire du colibacille a l'autolyse microbienne transmissible. Compt. rend. Soc. de biol., 1921, 84, 750.

251. - Dissociation d'une souche de colihacille en deux types d'individus de propriétés et de virulence differéntes. Compt. rend. Soc. de biol., $1921,84,751$.

252. ——: De la signification des "colonies de bactériophage" de d'Herelle. Compt. rend. Soc. de biol., 1921, 84, 753.

253. - : Sur la spécificité du principe lytique. Compt. rend. Soc. de biol., $1921,84,755$.

254. - - L L'autolyse transmissible du staphylocoque et l'action coagulante des cultures lysées. Compt. rend. Soc. de biol., 1921, 85, 25.

255. - : Autolyse transmissible et variations microbiennes. Compt. rend. Soc. de biol., 1921, 85, 251.

256. - Discussion on the bacteriophage (bacteriolysin). IV. Brit. M. J., 1922, ii, 296.

257. - _ La lyse transmissible du staphylocoque et ses applications thérapeutiques. Bull. Acad. Roy. de méd. de Belg., Brux., 1922, 5. s., 2, 72.

258. - L La lyse transmissible du staphylocoque. Sa produetion, ses applications thérapeutiques. Compt. rend. Soc. de biol., 1922, 86, 276.

259. — : The Twort-d'Herelle phenomenon. II. Lysis and microbic variation. J. Exp. Med., 1922, 35, 287. 
260. Gratia, A.: Hétérogénéité du principe lytique du colibacille. Compt. rend. Soc. de biol., 1923, 89, 821 .

261. - - Relations entre la variabilité du colibacille et l'hétérogénéité du principe lytique correspondant. Compt. rend. Soc. de biol., 1923, 89, 824 .

262. — : Phagocytose et immunité locale. Compt. rend. Soc. de biol., 1923, 89,826 .

263. ——: Le problème des variations microbiennes. Ann. et Bull. Soc. Roy. d. sc. méd. et nat. de Brux., 1924, 79, 62.

264. - and Dath, S.: Propriétés bacteriolytiques de certaines moisissures. Compt. rend. Soc. de biol., 1924, 91, 1442.

265. - - Moisissures et microbes bactériophages. Compt. rend. Soc. de biol., 1925, 92, 461.

266. - - A A propos de l'action bactériolytique du Streptothrix. Compt. rend. Soc. de biol., 1925, 93, 451.

267. - and Doyle, D.: Les effets des injections de bactériophage dans la septicémie colibacillaire expérimentale. Compt. rend. Soc. de biol., $1925,93,452$.

268. - and Jaumain, D.: Identité du phénomène de Twort et du phénomène de d'Herelle. Compt. rend. Soc. de biol., 1921, 85, 880 .

269. ——: Dualité du principe lytique du colibacille et du staphylocoque. Compt. rend. Soc. de biol., 1921, 85, 882 .

270. ——. Au sujet des réactions consécutives aux injections de principe lytique staphylococeirque. Compt. rend. Soc. de biol., 1922, $86,519$.

271. —— - Réaction de fixation de l'alexine et specificité antigènique des principes lytiques. Compt. rend. Soc. de biol., 1922, 87, 99.

272. — and de Kiruif, L.: Au sujet de la titration du bactériophage. Compt. rend. Soc. de biol., 1923, 88,30 s.

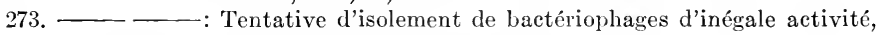
à partir d'un principe lytique coli manifestant des variations d'énergie. Compt. rend. Soc. de biol., 1923, $88,629$.

274. —- AND DE NAMUR, M.: Individualité des principes lytiques staphylococciques de provenances différentes. Compt. rend. Soc. de biol., 1922, 87, 364 .

275. - and Rhodes, B.: Action du principe lytique sur les émulsions de staphylocoques vivants et de staphylocoques tués. Compt. rend. Soc. de biol., 1923, $89,1171$.

276. ——_ De l'action lytique des staphylocoques vivants sur les staphylocoques tués. Compt. rend. Soc. de biol., 1924, 90, 640 .

277. v. Gruber: [Discussion to] Weitere Untersuchungen über das d'Herellesche Phänomen [by Gildemeister et al.]. Centralbl. f. Bakt., I. Orig., 1922 ‘23, $89, * 205$.

278. Hadley, P.: Transmissible lysis of Bacillus pyocyaneus. Papers Mich. Acad. sc. arts \& lett., 1923, N. York, 1924, 3, 316.

279. — : The variation in size of lytic areas and its significance. J. Bact., 1924, 9, 397.

280. J. Bact., 1924, 9, 405. 
281. Hadley, P.: Transmissible lysis of Bacillus pyocyaneus. J. Infect. Diseases, $1924,34,260$.

282. Hajós, K.: Beiträge zur Frage der wachstumshemmenden Wirkung von Bouillonkulturen. Centralbl. f. Bakt., I. Orig., 1922, 88, 583.

283. —_ Z Zur Natur des d'Herelleschen Phänomens. Bemerkungen zur Mitteilung von Putter und Vallen. Klin. Wehnschr., 1923, 2, 931.

284. - - Untersuchungen über die Natur der bakteriolytischen Substanz. Zeitschr. f. Immunitätsforsch., 1923, 37, 147.

285. Hankin, E.: L'action bactéricide des eaux de la Jumna et du Gange. Ann. Inst. Pasteur, 1896, 10, 511.

286. Hauduroy, P.: Sur les lysins du bactériophage de d'Herelle. Compt. rend. Soc. de biol., 1922, 87,964 .

287. — - De l'action du sérum antidysénterique sur la lyse du Bacille de Shiga par le bactériophage de d'Herelle. Compt. rend. Soc. de biol., 1922, 87, 966.

288. — Influence du chauffage sur le bactériophage de d'Herelle. Compt. rend. Soc. de biol., 1922, 87, 1089.

289. - : Sur la constitution du bactériophage de d'Herelle et sur le mécanisme de la lyse. Arch. internat. de pharmacod., 1923/24, 28, 1.

290. - - Sur la constitution du bactériophage de d'Herelle. Compt. rend. Soc. de biol., 1923, 88,59 .

291. — - Recherches du bactériophage de d'Herelle dans différents milieux. Compt. rend. Soc. de biol., 1923, 88, 1084.

292. — - Le rôle du bactériophage dans la fièvre typhoïde; sa présence dans les selles. Compt. rend. Soc. de biol., 1923, 89, 791.

293. — - Le rôle du bactériophage dans la fièvre typhoïde; sa présence dans le sang. Compt. rend. Soc. de biol., 1923, 89, 875 .

294. - - E Etude sur une épidémie de fièvre typhoïde. Le rôle du bactériophage de d'Herelle. J. physiol. path. gén., 1924, 22, 904.

295. - - Les applications du bactériophage de d'Herelle en thérapeutique et en hygiéne. Ann. de méd., Par., 1924, 16, 340.

296. - - Sensibilisation d'animaux à certaines infections par une vaccination anti-bactériophage. Compt. rend. Soc. de biol., 1924, 90, 290.

297. —— : Action de la gélatine sur le phénomène de d'Herelle. Compt. rend. Soc. de biol., 1924, 90, 1463.

298. — - Les cultures secondaires, après filtration, dans le phénomène de d'Herelle. Compt. rend. Soc. de biol., 1924, 91, 1209.

299. - - Les cultures secondaires, après filtration, dans le phénomène de d'Herelle. Compt. rend. Soc. de biol., 1924, 91, 1325.

300. - - Le bactériophage dans le fièvre typhö̈de. Sa présence constante dans les selles et dans le sang au moment de la convalescence. Rev. path. comparée, 1924, Feb. 20, 148.

301. — - Le bactériophage de d'Herelle. Rev. prat. d. Mal. des pays chauds, 1924, 3, No. 6.

302. ——: Le bactériophage de d'Herelle. Paris, Le François, 1925, 212 pp.

303. - - Le rôle du bactériophage dans l'immunité. Bull. techn. d. sc. méd., Genève, 1925, 1, 49 .

304. - - : Action de la bile sur le bactériophage et importance de cette action. Compt. rend. soc. de biol., 1925, 92, 1442. 
305. Hauduroy, P.: Le rôle du bactériophage dans la fièvre typhoïde. Compt. rend. Soc. de biol., 1925, 93, 100.

306. — - Le rôle du bactériophage de d'Herelle dans la guérison de la fièvre typhoïde. Presse méd., 1925, 38, 525.

307. - AND ARsimoles: Syndrome dysentériforme produit par le Bacille typhique. Guérison par le bactériophage de d'Herelle. Progrès méd., 1923, 3. s., 36,61 .

308. - - And Peyre, E.: Le bactériophage du Bacille pyocyanique. Compt. rend. Soc. de biol., 1923, 88, 688 .

309. Herderschee, D., and Wolff, L. K.: Geneeskrachtige waarde van den Typhusbacteriophaag end. Nederl. Tijdschr. v. Geneesk., 1924, 68, pt. 1, 2706.

310. D'Herelle, F.: Sur un microbe invisible antagoniste des bacilles dysentériques. Compt. rend. Acad. sci., 1917, 165, 373.

311. - Sur le rôle du microbe filtrant bactériophage dans la dysentérie bacillaire. Compt. rend. Acad. sci., 1918, 167, 970 .

312. - - Technique de la recherche du microbe filtrant bactériophage (Bacteriophagum intestinale). Compt. rend. Soc. de biol., 1918, 81, 1160.

313. — - Du rôle du microbe filtrant bactériophage dans la fièvre typhoïde. Compt. rend. Acad. sci., 1919, 168, 631.

314. —— : Sur le rôle du microbe bactériophage dans la typhose aviaire. Compt. rend. Acad. sci., 1919, 169, 932.

315. — : Sur le mierobe bactériophage. Compt. rend. Soc. de biol., 1919, 82, 1237.

316. —— : Le processus de défense contre les bacilles intestinaux et l'étiologie des maladies d'origine intestinale. Compt. rend. Acad. sci., 1920, 170, 72 .

317. — : Sur la résistance des bactéries a l'action du microbe bactériophage. Compt. rend. Soc. de biol., 1920, 83, 97.

318. — - Sur le microbe bactériophage. Compt. rend. Soc. de biol., 1920, 8s, 247.

319. — : Sur le microbe bactériophage. Compt. rend. Soc. de biol., 1920, $83,1318$.

320. — : Sur la nature du principe bactériophage. Compt. rend. Soc. de biol., 1920, 88, 1320.

321. - L L bactériophage: son rôle dans l'immunité. Paris, Masson et Cie., 1921, 277 pp. Monographe de l'Inst. Pasteur.

322. —- Le microbe bactériophage, agent d'immunité dans la peste et le barbone. Compt. rend. Acad. sci., 1921, 172, 99.

323. - - : Sur la nature du bactériophage (Bacteriophagum intestinale de d'Herelle, 1918). Compt. rend. Soc. de biol., 1921, 84, 339.

324. —— Phénomènes coïncidant avec l'acquisition de la résistance des bactéries à l'action du bactériophage. Compt. rend. Soc. de biol., 1921, 84,384 .

325. —— : Rôle du bactériophage dans l'immunité. Compt. rend. Soc. de biol., 1921, 84, 538 .

326. - - Sur l'historique du bactériophage. Compt. rend. Soc. de biol., $1921,84,863$, 
327. D'Herelle, F.: Sur la nature du bactériophage. Compt. rend. Soc. de biol., 1921, 84, 908 .

328. — : L'ultramicrobe bactériophage. Compt. rend. Soc. de biol., 1921, 85,767 .

329. - : Le bactériophage. La Nature, 1921, Oct. 1, 219.

330. - Le bactériophage. Son rôle dans l'immunité. Presse méd., 1921, 29,463 .

331. - - The bacteriophage; Its rôle in immunity. Authorized translation by George H. Smith. Baltimore, Williams and Wilkins Co., 1922, 287 pp.

332. — - Der Bakteriophage und seine Bedeutung für die Immunität. Nach einem erweiterten und verbesserten Text des Autors übersetzt von $\mathbf{R}$. Pfreimbter, W. Sell \& L. Pistorius. Braunschwieg, F. Vieweg \& Sohn., $1922, x i v+214 \mathrm{pp}$.

333. —- De Bacteriophaag. Traduction, Storm van Leeuwen.

334. - The nature of the bacteriophage. Brit. M. J., 1922, ii, 289.

335. ——: Sur les anti-lysines d'origine bactérienne. Compt. rend. Soc. de biol., $1922,86,360$.

336. ——: Sur la présence du bactériophage dans les leucocytes. Compt. rend. Soc. de biol., 1922, $86,477$.

337. — : Sur la prétendue production d'un principe lytique sous l'influence d'un antagonisme microbien. Compt. rend. Soc. de biol., 1922, 86, 663.

338. — : Sur une cause d'erreur pouvant intervenir dans l'étude du bactériophage. Compt. rend. Soc. de biol., 1922, 87, 665.

339. — : Les défenses de l'organisme. Paris, E. Flammarion, 1923, 229 pp. (Bibliothéque philosophie scientifique).

340. - : Action du fluorure de sodium sur le bactériophage. Compt. rend. Soc. de biol., 1923, 88, 407.

341. — : Sur un "principe bactériolysant" non bactériophage, existant dans l'intestin des cholériques. Compt. rend. Soc. de biol., 1923, 88, 723.

342. - Observations au sujet des expériences concernant le phénomène de bactériophage. Compt. rend. Soc. de biol., 1923, 89, 231.

343. — : Sur la nature du bactériophage. Compt. rend. Soc. de biol., 1923, 89,914 .

344. —— La nature du bactériophage. Nederl. Maandschr. v. Geneesk., Leiden, 1923, 11, 737.

345. — : La nature du bactériophage. Nederl. Tijdschr. v. Geneesk., 1923, $67,2969$.

346. - : Autolysis and bacteriophagis. J. State Med., Lond., 1923, 31, 461.

347. - : Le bactériophage. Rev. path. comparée., 1923, 23, No. 238, 11.

348. - C Culture du bactériophage sans intervention de bactéries vivantes. Verhandl. v. k. Akad. v. Wetensch., Amsterdam, 1923, 26, 486.

349. - - Immunity in natural infectious disease. Authorized English edition by George H. Smith. Baltimore, Williams \& Wilkins Co., 1924, 399 pp.

350. - : Sur l'autonomie du bactériophage. Compt. rend. Soc. de biol., 1924, 90, 25.

351. - : Sur l'état physique du bactériophage. Compt. rend. Soc. de biol., 1924, 90, 27. 
352. D'Herelle, F.: Sur la constance des propriétés du bactériophage. Compt. rend. Soc. de biol., 1924, 90, 481.

353. — : De bacteriophaag. Nederl. Tijdschr. v. Geneesk., 1924, Jan., 1598.

354. — : La nature du bactériophage. Tijdschr. v. Vergelijk. Geneesk., 1924, 10, Mar.

355. —_ L La nature du bactériophage. Bull. techn. d. sc. méd., Genève, 1925, $1,11$.

356. — Sur la nature du bactériophage. Compt. rend. Soc. de biol., 1925, 93, 509 .

357. —_: De ultravirus. Nederl. Maandschr. v. Geneesk., Leiden, 1925, 13, 69.

358. —— and Eliava, G.: Unicité du bactériophage; Sur la lysine du bactériophage. Compt. rend. Soc. de biol., 1921, 85, 701.

359. - Sur le sérum anti-bactériophage. Compt. rend. Soc. de biol., 1921, 84, 719 .

360. - and Pozersini, E.: Action de la témperature sur le bactériophage. Compt. rend. Soc. de biol., 1921, 85, 1011.

361. Herzberg, K.: [Discussion to] Neuartige Lysine bei Mycoidesbakterien [by Sartorius]. Centralbl. f. Bakt., I. Orig., 1924, 93, 165.

362. Hoder, F.: Vergleichende Untersuchungen von Coli-Dysenterie- und Paratyphusbakteriophagen. Centralbl. f. Bakt., I. Orig., 1924, 93, 424.

363. —— Ueber Zusammenhänge zwischen Bakteriophagen und Bakterienmutation. Zeitschr. f. Immunitätsforsch., 1925, 42, 197.

364. van der Hoeden: [Bactériophage isolé des saucisses.] Nederl. betr. d. Volkgezondheit., 1923, July, 535.

365. Ікома, T.: Studien über Bakteriophagenwirkung. Centralbl. f. Bakt., I. Orig., 1924, 91,554 .

366. Ionesco-Minaiesti, C.: Studies on the Twort-d'Herelle phenomenon. J. Exp. Med., 1924, 40, 317.

367. Izar, G.: Sui considetti batteriofagi. Ac. Gioemia d. sci. nat. in Catania, 1921, May 7.

368. - - Sui cosi detti batteriofagi. Folia med., Napoli, 1922, 8, 1.

369. JacobsthaL, E.: [Discussion to] Untersuchungen über den d'Herelleschen Bakteriophagen. Centralbl. f. Bakt., 1922/23, I. Orig., 89, *203.

370. Janzen, J. W., and Wolff, L. K.: Over de reiniging der Pokkenlymphe. Nederl. Tijdschr. v. Geneesk., 1922, i, 2252.

371. ——: Ueber die Reinigung der Pockenlymphe. Ueber den Typhusbakteriophagen. Centralbl. f. Bakt., 1923, 90, 41.

372. — - Ueber den Typhusbakteriophag. Centralbl. f. Bakt., I. Orig., 1923, 90, 6 .

373. - - Bakteriophag studies. Nederl. Tijdschr. v. Geneesk., 1923, 67 , pt. 1, 2107; pt. 2, 147.

374. ——: Studies about d'Herelle's bacteriophagus. Verhandel. v. k. Akad. v. Wetensch., Amsterdam, 1923, 25, 31.

375. - : Studies on the bacteriophagus of d'Herelle. II. Verhandel. v. k. Akad. v. Wetensch., Amsterdam, 1923, 25, 87.

376. ——: Studies on the bacteriophagus of d'Herelle. Verhandel. v. k. Akad. v. Wetensch., Amsterdam, 1923, 25, 171. 
377. Jaumain, D.: Autolyse microbienne en tubes scellés. Compt. rend. Soc. de biol., 1922, 87, 790 .

378. - and Meuleman, M.: Absorption du principe lytique par les microbes tués. Compt. rend. Soc. de biol., 1922, 87, 362.

379. Jötten, K. W.: Ueber das sogennannte d'Herellesche Phänomen. Klin. Wchnschr., 1922, 1, 2181.

380. - : [Discussion to] Weitere Uutersuchungen über das d'Herellesche Phänomen [by Gildemeister]. Centralbl. f. Bakt., I. Orig., 1922/23, $89, * 202$.

381. — : Ưber das sogenannte d'Herellesche Phänomen. Klin. Wchnschr., 1923, 2, 103.

382. KabELIK, J.: L'influence de l'indicateur "universel" sur le bactériophage. Compt. rend. Soc. de biol., 1925, 92, 1055.

383. - : La symbiose bactériophagienne. Compt. rend. Soc. de biol., 1925, 92, 1057.

384. - and Kukula, K.: La chimiotaxie du bactériophage. Compt. rend. Soc. de biol., 1925, 92, 1058.

385. — , ToMÁsĚK, V., AND BođCEK, J.: [Bacteriophagum intestinale.] Biologické Listy, 1922, Nos. 3/4; Abst.-in Centralbl. f. Bakt. I. Ref., $1922 / 23,74,328$.

386. Kabeshima, T.: Recherches expérimentales sur la vaccination préventive contre Bacille dysentérique de Shiga. Compt. rend. Acad. sci., 1919, $169,1061$.

387. - - Thérapie expérimentale des porteurs de germes. Compt. rend. Acad. sci., 1920, 170, 71.

388. - : Sur un ferment d'immunité bacteriolysant, du mécanisme d'immunité infectieuse intestinale de la nature du dit "microbe filtrant bactériophage" de d'Herelle. Compt. rend. Soc. de biol., 1920, 83, 219.

389. — : Sur le ferment d'immunité bacteriolysant. Compt. rend. Soc. de biol., 1920, 83, 471 .

390. Keller, W.: Über Lysin und Trypsin. (Ein beitrag zur Biologie des Twortd'Herelleschen Phänomens.) Zeitschr. f. Hyg. u. Infektionskrankh., $1924,103,177$.

391. Kimura, S.: Ueber Schleimbildung bei Bakterien unter dem Einfluss von Bakteriophagen. Zeitschr. f. Immunitätsforsch., 1925, 42, 507.

392. King, D. S.: The Twort-d'Herelle phenomenon (bacteriophagy); Its possible relation to therapeutics. Med. Clin. N. Am., Phila., 1923, 7, 883.

393. Kister, J.: Gegenwärtiger Stand der "Bakteriophagenfrage." Zusammenfassende Besprechung der bisherigen Veröffentlichungen. Centralbl. f. d. ges. Hyg., 1924, 8,$1 ; 81$.

394. Kramer, I. M.: Bijdrage tot de kennis van de rol van den bacteriophaag (d'Herelle) bij infectie en immuniteit. Thèse. Utrecht, Schotanus \& Jens, 1925, $120 \mathrm{pp}$.

395. Kraus, R., and Gomez, J. M.: Experiencias sobre bacteriophagia. Brazilmed., Rio de Jan., 1922, 36, 267.

396. - and Marrey, P.: Experiencias sobre bacteriophagia. Brazil-med., Rio de Jan., 1922, 36, 227.

397. Kropveld, S. M.: Studies over den bacteriophaag tegen staphylokokken. Nederl. Tijdschr. v. Geneesk., 1923, 67, i, 1228。 
398. Kuttner, A. G.: Preliminary report on a typhoid bacteriophage. Proc. Soc. Exp. Biol. and Med., 1921, 18, 158.

399. - On the influence of tissue enzymes on the bacteriophage principle. Proc. Soc. Exp. Biol. and Med., 1921, 18, 222.

400. - B Bacteriophage phenomena. J. Bact., 1923, 8, 49.

401. Le Blaye, R.: Le bactériophage et ses applications thérapeutiques. Arch. méd.-chir. de Provence, Tours, 1923, 13, 177.

402. — : Traitement spécifique de la fièvre typhoïde. Bull. Soc. de méd. de la Vienne, Poitiers, 1924, p. 87.

403. Ledingham, J. C. G.: Discussion on the bacteriophage (bacteriolysin). Brit. M. J., 1922, ii, 297.

404. Lehndorff, H.: Therapeutische Anwendung des bakteriophagen Lysins (Twort-d'Herelle) bei Kinderkrankheiten. Wien. med. Wchnschr., 1924, $74,1051$.

405. Le Louet: Immunisation contre le barbone au moyen du bactériophage. Arch. d. Inst. Pasteur d'Indo-Chine, 1925, No. 1, 91.

406. Lemos Monteiro, J.: Sobre o phenomeno Twort-d'Herelle; aç̧ão bacteriolytica e acção bacteriophagica. Brazil-med., Rio de Jan., 1922, 36, 72 .

407. - S Sobre o phenomeno de d'Herelle; presença do principio lytico nas culturas, em meio solido, do bacillo anthracis, B. pestis e B. dys. Shiga Kruse. Brazil-med., Rio de Jan., 1922, 36, 297.

408. Lepper, E. H.: On the mode of production of bacteriophage. Brit. J. Exp. Path., 1923, 4, 53.

409. - - The rate and progress of bacteriophage action. Brit. J. Exp. Path., 1923, 4, 204.

410. - - The reproduction of bacteriophage when the sensitive organism is grown in a synthetic medium. Brit. J. Exp. Path., 1924, 5, 40.

411. Lesbre, P.: Bactériophage et anatoxine dans la vaccination anti-dysentérique par voie buccale. Compt. rend. Soc. de biol., 1925, 93, 614 .

412. Levaditi, C., and Nicolad, S.: Propriétés physiques des ultravirus neurotropes. Compt. rend. Soc. de biol., 1923, 88, 66.

413. Levinson, L.: Contribution a l'étude du phénomène du bactériophage. Thèse. Paris. A. Legrand, 1922, $38 \mathrm{pp}$.

414. Levy, M. M.: Essai d'immunisation contre la typhose des souris à l'aide du bactériophage. Compt. rend. Soc. de biol., 1925, 93, 82 .

415. - : Essai de traitement de la typhose murine par le bactériophage. Compt. rend. Soc. de biol., 1925, 93, 395.

416. - Essai de protection de la souris contre la typhose murine par l'ingestion de bactériophage. Compt. rend. Soc. de biol., 1925, 93, 396.

417. von Liebermann, L.: Die Erklärung des d'Herelleschen Phänomens mittels Modellversuches. Klin. Wchnschr., 1923, 2, 1240.

418. Lisbonne, Boulet, and Carrère, L.: Sur l'obtention du principe bactériophagique au moyen d'exsudats leucocytaires in vitro. Bull. Soc. de sc. méd. et biol. Montpellier, 1921/22, 3, 229.

419. - — - - - - Sur l'obtention du principe bactériophagique au moyen d'exsudats leucocytaires in vitro. Compt. rend. Soc. de biol., $1922,86,340$. 
420. Lisbonne and Carrère, L.: Antagonisme microbien et lyse transmissible du Bacille de Shiga. Compt. rend. Soc. de biol., 1922, 86, 569.

421. - : Sur l'obtention du principe bactériophagique par antagonisme microbien. Compt. rend. Soc. de biol., 1922, 8\%, 1011.

422. — : Obtention du principe lytique pour le Bacille de Shiga par culture filtrée de B. coli. Compt. rend. Soc. de biol., 1923, 88, 724.

423. - - - Influence des électrolytes sur la lyse microbienne transmissible. Compt. rend. Soc. de biol., 1923, 89, 865 .

424. — - Sur l'apparition spontanée du pouvoir lysogéne dans les cultures pures. Compt. rend. Soc. de biol., 1924, 90, 265.

425. Lisch, H.: Ueber die soganannten Pyocyaneus-Bakteriophagen. Centralbl. f. Bakt., I. Orig., 1924, 93, 421.

426. Lode, A.: Hemmung- und Bakteriophagenwirkung beim Bacillus pyocyaneus. Arch. f. Hyg., 1923, 93, 267.

427. Machado, A., And da Costa Cruz, J.: Sobre a autolyse microbiana transmissivel; bacteriophago de d'Herelle. Brazil-med., Rio de Jan., 1921, $35,347$.

428. Maisin, J.: Au sujet de la nature du principe bactériophage. Compt. rend. Soc. de biol., 1921, 84, 467.

429. - : Adaptation du bactériophage. Compt. rend. Soc. de biol., 1921, 84, 468 .

430. - Au sujet du principe bactériophage et des anticorps. Compt. rend. Soc. de biol., 1921, 84, 755 .

431. - : Les bactériophages. Arch. internat. de pharmacod., 1922, 26, 215.

432. Maitland, H. B.: Experiments employing a quantitative method in a study of the d'Herelle phenomenon. Brit. J. Exp. Path., 1922, 3, 173.

433. Mallmann, W. L., and Hemstreet, C.: Isolation of an inhibitory substance from plants. J. Agric. Research., 1924/25, 28, 599.

434. Manolid, E.: Sur la présence d'un principe lytique anti-Shiga dans un exsudat pathologique dû au Bacille typhique. Compt. rend. Soc. de biol., 1925, 92, 724 .

435. Marcuse, K.: Demonstration zu Methodik des d'Herelleschen Phänomens. Klin. Wehnschr., 1922, 1, 2452.

436. - - Grundlagen und Aufgaben der Lysintherapie (d'Herelle's Bakteriophagen). Deutsche. med. Wchnschr., 1924, 50, 334.

437. - Untersuchungen über das d'Herellesche Phänomen. II. Untersuchungen ïber die Bedeutung der Leukocyten fur des d'Herellesche Phänomen. Zeitschr. f. Hyg. u. Infektionskrankh., 1924, 102, 206.

438. - - Untersuchungen über das d'Herellesche Phänomen. I. Zur Methodik der Konservierung des Lysins. Zeitschr. f. Hyg. u. Infektionskrankh., 1923/24, 101, 375.

439. Marshall, M. S.: The quantitative estimation of bacteriophage. Papers Mich. Acad. sc., arts, \& lett., 1923, N. York, 1924, 3, 327.

440. - Observations on d'Herelle's bacteriophage. J. Infect. Diseases, $1925,3 \%, 126$.

441. Matsumoto, T.: Ueber die Vielheit von Bakteriophagen. Wien. klin. Wchnschr., 1923, 36, 759.

442. — : Versuche ïber die Vermehrung von Bakteriophagen. Centralbl. f. Bakt., I. Orig., 1924, 91, 413. 
443. Matsumoto, T.: Ueber das Verhalten konzentrierter Bakteriophagen. Zeitschr. f. Immunitätsforsch., 1924, 40, 214.

444. ——: Bestimmungsversuche von Bakteriophagen. Zeitschr. f. Immunitätsforsch., 1924, 41, 1 .

445. Meier, J. J.: Mond- en klauwzeerbackteriophaag. Tijdschr. v. Diergeneesk., 1925, 52, No. 7.

446. - - Un bactériophage de la fièvre aphteuse. Recueil méd. vet., 1925, Mar. $15,135$.

447. - Un bactériophage de la fièvre aphteuse. Berl. terärztl. Wchnschr., 1924, Feb. 20.

448. Meissner, G.: Ueber Bakteriophagen gegen Choleravibrionen. Centralbl. f. Bakt., I. Orig., 1924, 91, 149.

449. - Versuche über die Flüchtigkeit und Kochbeständigkeit des d'Herelleschen Bakteriophagen. Centralbl. f. Bakt., I. Orig., 1924, 92, 424.

450. - Die Bindungsverhaltnisse zwisehen Bakteriophagen und Bakterien. Centralbl. f. Bakt., I. Orig., 1924, 93, 489.

451. Metalnikoff, S.: B. dysentérique et bactériophage de d'Herelle chez les chenilles de Galleria mellonella. Compt. rend. Soc. de biol., 1920, 83, 667.

452. Meuli, H.: Studien zum Bakteriophagenproblem. II. Mitt. Die Konzentration des lytischen Prinzips und ihre Beziehungen zum Ablauf der Bakteriophagenreaktion. Zeitschr. f. Hyg. u. Infektionskrankh., 1923, $99,46$.

453. Meyn, A.: Über das d'Herellesche Phänomen und Versuche zu seinem Nachweis an Streptococcus equi und Bacterium bipolare avisepticum. Deutsche tierärztl. Wchnschr., 1925, No. 16, 259.

454. Miessner, H., And BaArs, G.: Bakteriolysate und das Phänomen von d'Herelle. Deutsche. tierärztl. Wchnschr., 1922, 30, 207.

455. — - Paratyphusbakteriophagen. Deutsche.tierärztl. Wehnschr., $1922,30,211$.

456. — : Das d'Herellesche Phänomen an Rotlaufbakterien. Centralbl. f. Bakt., I. Orig., 1924, 93, 131.

457. Miller, E. C. L.: Bacteriophage. Virginia Med. Monthly, 1923, 50, 180.

458. Mchinley, E. B.: Notes on d'Herelle's phenomenon. Adaptation of bacteriophage antagonistic to Bacillus dysenteriae and other bacilli to various cocci. Development of a polyvalent bacteriolysant. (Preliminary report.) J. Lab. \& Clin. Med., 1922/23, 8, 311.

459. - Further notes on d'Herelle's phenomenon. J. Lab. \& Clin. Med. $1923,9,185$.

460. - The bacteriophage in the treatment of infections. Arch. Int. Med., 1923, 32, 899 .

461. - - The relation of digestive enzymes and ferments to the phenomenon of d'Herelle. J. Bact., 1923, 8, 543.

462. M'Leod, J. W.: Discussion on the bacteriophage (bacteriolysin). Brit. M. J., 1922, ii, 298.

463. - AND GoRdon, J.: On the production of peroxides by pneumococci and other bacteria. J. Path. and Bact., 1922, 25, 139.

464. - AND Grovenlock, P.: The production of bactericidins by microorganisms. Lancet, 1921, i, 900. 
465. Morales Villazon, N.: Bactériophage efficace contre le Bacille de la peste. Compt. rend. Soc. de biol., 1923, 89, 754.

466. - : Estudio de un bacteriofago eficaz para el bacilo de la peste. Rev. Assn. Med. Argentina, 1923, 36, 453.

467. Munter, H., and Boenheim, C.: Über therapeutische Versuche mit bakteriophagem Lysin bei Kindern und Saüglingen. Zeitschr. f. Kinderheilk., 1925, 39, 388.

468. Nakamura, O.: Die Hemmung der Bakteriophagenwirkung durch Gelatine. Arch. f. Hyg., 1923, 92, 61.

469. —— Der Verhalten von Bakteriophagen in Gelatine. Wien. klin. Wchnschr., 1923, 36, 86 .

470. - - Ueber Lysozymewirkung. Wien. klin. Wchnschr., 1923, 36, 322.

471. - : Ueber Lysozymwirkungen. Zeitschr. f. Immunitätsforsch., 1923/24, 38,425 .

472. Nakashima, T.: Beitrag zum Vorkommen und Verhalten des bakteriophagen Lysins in Abwässern. Centralbl. f. Bakt., I. Orig., 1925, 94, 303.

473. NAL, D.: Il batteriofago di d'Herelle. Biochem. e terap. sper., Milano, $1922,9,54$.

474. NAst, A. G.: The Twort-d'Herelle phenomenon. J. Am. Inst. Homeop., $1922 / 23,15,979$.

475. DE NeCKer, J.: Au sujet de l'action inhibitive du principe bactériophage sur le développement des microbes receptifs. Compt. rend. Soc. de biol., $1921,85,742$.

476. ——: De l'influence de la chaleur sur le principe bactériophage. Compt. rend. Soc. de biol., 1922, 86, 736 .

477. — : De l'adsorption du principe bactériophage par les colloïdes. Compt. rend. Soc. de biol., 1922, 87, 1247.

478. Nelson Barbosa: Acçáo curativa do bacteriofago estaphylococcico. Brazil-med., Rio de Jan., 1923, 37, 297.

479. Оенцer, R.: Symbiose und Kommende Zelltheorie. Centralbl. f. Bakt., I. Orig., 1924, 93, 216.

480. OGata, N.: Zur Entstehung des Bakteriophagen in alten Kulturen. Centralbl. f. Bakt., I. Orig., 1924, 93, 329.

481. Окамото, T.: Untersuchungen über die Beziehungen des d'Herelleschen Phänomens zum N-Stoffwechsel der Bakterien. Zeitschr. f. Immunitätsforsch., 1925, 42, 161.

482. OKuda, S.: Pyocyaneusbakteriophagen. Arch. f. Hyg., 1923, $92,109$.

483. - : Ueber Pyocyaneusbakteriophagen. Wien. klin. Wchnschr., 1923, $36,125$.

484. - Weitere Untersuchungen an Pyocyaneusbakteriophagen. Wien. klin. Wchnschr., 1923, 36,638 .

485. Olden, O., and Yasaki, Y.: Die Flüchtigkeit des d'Herelleschen übertragbaren lytischen Agens. Klin. Wchnschr., 1923, 2, 1879.

486. ——_ Z Zur Frage der Destillierbarkeit und Flüchtigkeit der d'Herelle-Lysine. Kilin. Wchnschr., 1924, 3, 278.

487. —— : Erwiderung [to] "Ist das lytisch Agens des d'Herelleschen Phänomens flüchtig" [by] Borchardt. KKlin. Wchnschr., 1924, 3, 279.

488. - - D Das Verhalten des lytischen Agens d'Herelles bei der Destillation und in Durchlüftungsversuchen. (Untersuchungen über Bakteriophagen II.) Zeitschr. f. Hyg. u. Infektionskrankh., 1924, 102, 540. 
489. Osumi, S. Serologische Studien mit einem Bakteriophagen. Zeitschr. f. Immunitätsforsch., 1924, 40, 261.

490. Отто, R.: [Discussion to] Weitere Untersuchungen über das d'Herelleschen Phänomen. [By Gildemeister et al.] Centralbl. f. Bakt., I. Orig., $1922 / 23,89, * 204$.

491. - : Das sogenannten d'Herelleschen Phänomen. Zeitschr. f. ärztl. Fortbild., Jena, 1923, 20, 253.

492. - and Munter: Zum d'Herelleschen Phänomen. Deutsche med. Wchnschr., 1921, 47, 1579.

493. — - Das bakteriophage Lysin; seine Beziehungen zum Bakterium und zu dem Antilysin. Zeitschr. f. Hyg. u. Infektionskrankh., 1922, 98, 302 .

494. —— : Bakteriophagie. Ergebn. d. Hyg., Bakteriol., Immunitätsforsch., u. exp. Therapie, 1923, $6,1$.

495. —— Weitere Untersuchungen zum d'Herelleschen Phänomen. Zeitschr. f. Hyg. u. Infektionskrankh., 1923, 100, 402.

496. — - - AND Winkler, W. F.: Beiträge zum d'Herelleschen Phänomen. Zeitschr. f. Hyg. u. Infektionskrankh., 1922, 96, 118.

497. - and Sukiennikowa, N.: Bakteriophagen Lysin und Paragglutination. Zeitschr. f. Hyg. u. Infektionskrankh., 1923, 101, 119.

498. - and Winkler, W. F.: Über die Natur des d'Herelleschen Bakteriophagen. Deutsche med. Wchnschr., 1922, 48, 383.

499. Pauron: L'ultramicrobe bactériophage; son rôle dans la prophylaxie et la thérapeutique des infections. Arch. de méd. et pharm. mil., Par., $1923,78,195$.

500. Pereira, O.: O phenomeno de d'Herelle e as infeccoes pelos bacilos coli e dysentericos. Thèse. Porto Alegre, Lib. do Globo., 1924.

501. Pesch, K. L.: Milzbrand-Pseudobakteriophagen. Centralbl. f. Bakt., I. Orig., 1924, 93, 525 .

502. Petrovanu, G.: Sur la présence du principe lytique dans l'exsudat amygdalien de diverses angines. Compt. rend. Soc. de biol., 1924, 91, 502.

503. - - Recherches sur l'existence du principe lytique dans la péritonite cholérique expérimentale. Compt. rend. Soc. de biol., 1924, 91, 735.

504. — : Recherches sur la présence du principe lytique vis-à-vis du Vibrion cholérique dans la paroi de l'intestin grêle. Compt. rend. Soc. de biol., $1924,91,754$.

505. Peyre, E.: Le bactériophage. Progrès méd., 1923, 3. s., 98, 53.

506. Pfreimbter, R., Sell, W., and Pistorius, L.: Eine neue Methodik zum Nachweis des d'Herelleschen Virus. München. med. Wchnschr., 1922, $69,495$.

507. Philibert, A.: Les applications thérapeutiques du bactériophage. Clinique et Lab., Par., 1924, Jan. 20.

508. ——: Le principe bactériophage (bactériophage de d'Herelle), immunité; vaccination. Médecine, Par., 1923/24, 5, 191.

509. — and Hauduroy, P.: Le rôle du bactériophage de d'Herelle dans l'immunité. Rev. path. comparée, 1923, No. 240, Dec. 5.

510. PIco, C. E.: Sur la nature du principe bactériophage de Twort-d'Herelle. Compt. rend. Soc. de biol., 1922, 86, 1106. 
511. Prco, C. E.: Précédents historiques sur la lyse microbienne transmissible. Compt. rend. Soc. de biol., 1922, 87, 685 .

512. - Le principe lytique est-il contenu dans les bactéries? Compt. rend. Soc. de biol., 1922, 87, 687 .

513. - : À propos de la note de Combiesco sur le phénomène de d'Herelle. Compt. rend. Soc. de biol., 1922, 87, 826.

514. - Autolyse transmissible du Bacillus anthracis sans intervention de l'hypothétique virus bactériophage. Compt. rend. Soc. de biol., 1922, 87,836 .

515. - Observaciones a la nota de Combiesco sobre el fenomeno de d'Herelle. Rev. Asoc. Med. Argentina, Buenos Aires, 1922, 35, Sect. Soc. de biol., 220.

516. - : Autolysis transmissible del Bacillus antracis sin intervencion del supuesto virus bacteriofago. Rev. Asoc. Med. Argentina, Buenos Aires, 1922, 35, Sect. Soc. de biol., 268.

517. - Bacteriophagie sans le virus bacteriophage. Rev. Asoc. Med. Argentina, Buenos Aires, 1922, 35, 860 .

518. _ - Sobre la naturaleza del principio bacteriofago de Twort-d'Herelle. Semana med., 1922, 29, 415.

519. — : [Transmissible autolysis of anthrax bacilli without the intervention of the supposed bacteriophage virus.] Semana med., 1922, 29, 448.

520. - Existe el virus bacteriofago de d'Herelle? Semana med., 1922, 29, 675.

521. - Action déchainante de la pancréatine sur l'autolyse microbienne transmissible. Compt. rend. Soc. de biol., 1923, 89, 753.

522. — - Sobre la action desencadenante de la pancreatina sobre la autolisis microbiana transmissivel. Rev. Asoc. Med. Argentina, Buenos Aires, 1923, 36, Sect. Soc. de biol., 154.

523. - Action déchaînante de la pancréatine sur l'autolyse microbienne transmissible. Compt. rend. Soc. de biol., 1924, $91,31$.

524. - Sobre la accion desencadenante de la pancreatina en la autolisis microbiana transmissivel. Rev. Asoc. Med. Argentina, Buenos Aires, 1924, 37, Sect. Soc. de biol., 68 .

525. Pierret, R., and Bilouet, V.: Le bactériophage de d'Herelle chez le nouveau-né. Compt. rend. Soc. de biol., 1925, 93, 635.

526. Pionkowski, G.: Beitrag zur Streptokokkenfrage. Anwendung des d'Herelleschen Phänomens auf Streptokokken. Med. Klin., 1922, 18, 474.

527. Plicque, A. F.: Le bactériophage intestinal. J. de méd. et chir., Par., 1923, 94, 108.

528. Pock-Steen, P. H.: [Le bacteriophage.] Ugesk. f. Laeger., Københ., 1922, 84,319 .

529. Polettini Bruno: Sulla pretesa transmissibilita in serie della batteriolisi provocata dal violetto di metile. Pathologica, 1922, 14, 157.

530. Pondman, A.: Proeven tot het verkrijgen van het verschijnsel van d'Herelle uit reinculturen. Thèse. Leiden, C. de Boer jr., 1923, 80 pp.

531. Pons, R.: Bactériophage du pyocyanique. Compt. rend. Soc. de biol., 1923, 89, 77.

532. De Poorter, P., ANd Maisin, J.: Contribution à I'étude de la nature du principe bactériophage. Arch. internat. pharmacod., 1921, 25, 473. 
533. Ротоскі and Fisch: Remarques sur le mode d'emploi des filtrates microbiens en applications locales. Bull. Soc. d'Obstet. et de Gynécol., 1924, 13, 483.

534. — - Remarques sur le mode d'emploi des filtrats microbiens en applications locales. Gynécologie, Par., 1924, 23, 541.

535. Prausnitz, C.: Untersuchungen über den d'Herelleschen Bakteriophagen. I. Mitt. Die Natur des Bakteriophagen. Centralbl. f. Bakt., I. Orig., $1922,89,{ }^{*} 187$.

536. —— Über die Natur des d'Herelleschen Phänomens. K'lin. Wchnschr., 1922, 1, 1639.

537. —— Das Twort-d'Herellesche Phänomen. Klin. Wchnschr., 1923, 2, 2007.

538. — - Das Twort-d'Herellesche Phänomen. Med. Klin., 1923, 19, 1316.

539. - ANd Firle, E.: Neuere Untersuchungen über das Wesen des Bakteriophagen. Centralbl. f. Bakt., I. Orig., 1924, 93, 148.

540. Preisz, H.: Die Bakteriophagie. Jena. G. Fischer, 1925, 110 pp.

541. Proca, G.: Sur la lysine des bactéries lysogènes. Compt. rend. Soc. de biol., 1923, 88, 909 .

542. —_ : Particularités de la lysine du B. coli. Compt. rend. Soc. de biol., 1923, 89, 274.

543. —_ : Sur les extraits de cultures lysogènes. Compt. rend. Soc. de biol., $1923,89,631$.

544. Puntoni, V.: Il batteriofago. Ann. d'ig., Roma, 1920, 30, 643.

545. — : I recenti studi sulla natura dei batteriofago. Ann. d'ig., Roma, $1921,31,250$.

546. Putter, E., and Vallen, S.: Zur Natur des d'Herelleschen Phänomens. Klin. Wchnschr., 1923, 2, 339.

547. — : Zur Natur des d'Herelleschen Phänomens: Berichtigung. Klin. Wchnschr., 1923, 2, 1072.

548. Quiroga, R.: Bactériophage de Bacille pyocyanique. Compt. rend. Soc. de biol., 1923, 88, 363 .

549. Racchiusa, S.: Il batteriofago in rapporto all'autosterilizzazione del terreno. Ann. d'ig., Roma, 1923, 33, 396.

550. Reichert, F.: Untersuchungen über das d'Herellesche Phänomen. Centralbl. f. Bakt., I. Orig., 1924, 91, 235.

551. Remot1, E.: Contributo allo studio della lisi batterica transmissible. II. Azione conparativa del batteriofago e dei filtrati batterici sulle sostanze proteiche. Ann. d'ig., Roma, 1924, 34, 480.

552. Rhodes, B.: The phenomenon of bacteriophage. J. Lab. and Clin. Med., $1922,7,288$.

553. Richaud, A.: La bactériophagie de d'Herelle. J. de pharm. et chim.,Par., 1922, 7. s., 25, 429.

554. Richet, C., Azerad and Delarne: Sur un cas de fièvre typhoïde ataxoadynamique très rapidement gueri par un stock-bactériophage antiEberth. Bull. et mém. Soc. Méd. d. Hôp. de Par., 1924, 40, 838.

555. Richet, C., and Hauduroy, P.: Essais d'immunisations contre la typhose des souris avec le bactériophage de d'Herelle. Compt. rend. Soc. de biol., 1925, 93, 222 . 
556. Rigobello, G.: Il batteriofago. Pavia, 1923, 257 pp.

557. Rimpad, W.: Das d'Herellesche Phänomen. München. med. Wchnschr., 1921, 68, 1649.

558. Romagnolo, C.: Il batteriofago de d'Herelle. Minerva med., Torino, $1922,2,482$.

559. Rosenthal, L.: La lyse des Bacilles diphteriques effectuée par un Streptothrix. Compt. rend. Soc. de biol., 1925, 93, 77.

560. Rossi, L.: Sul virus batteriofago d'Herelle. N. Ercolani, Torino, 1921, 26, $381 ; 416 ; 441$.

561. Rouslacroix, A.: Le phénomène de la lyse bactérienne transmissible (bactériophage de d'Herelle). Prat. méd. franç., Par., 1921/22, 1, 1029.

562. Saldanha, A.: Phénomène de d'Herelle. Compt. rend. Soc. de biol., 1922, $86,623$.

563. Salimbeni, A.: Sur la nature de bactériophage de d'Herelle. Compt. rend. Acad. sci., 1920, 171, 1240.

564. — : Sur le bactériophage de d'Herelle. Compt. rend. Soc. de biol., $1920,83,1545$.

565. SAnderson, E. S.: A note on the bacteriophage with respect to complement fixation tests. J. Immunol., 1925, 10, 625 .

566. SARDJITo, M.: Immunisatie tegen bacillaire dysenterie door middel van den bacteriophaag anti-dysenteriae Shiga-Kruse. These. Leiden, E. Ijido, $1923,67 \mathrm{pp}$.

567. SArtorius: Neuartige Lysine bei Mycoidesbakterien. Centralbl. f. Bakt., I. Orig., 1924, 93, 162.

568. Scheidegger, E.: Studien zum Bakteriophagenproblem. IV. Mitt. Der Einfluss der Wasserstoffionenkonzentration auf das lytische Agens und den Bakteriolyse. Zeitschr. f. Hyg. u. Infektionskrankh., 1923, 99, 403.

569. Schlossberger, H.: Das d'Herellesche Phänomen. Zentralbl. f. Haut- u. Geschlechtskr., 1922, 4, 401.

570. Schnabel, A.: [Discussion to] Weitere Untersuchungen über das d'Herellesche Phänomen [by Gildemeister et al.]. Centralbl. f. Bakt., I. Orig., 1922/23, 89, *204.

571. — : Die Übertragung allergischer Zustände bei Bakterien. Klin. Wchnschr., 1924, 3, 566 .

572. Schudrman, C. J.: Der Bakteriophage, ein lebender Organismus. Centralbl. f. Bakt., I. Orig., 1925, 95, 97.

573. — : De bakteriophaag; een Ultramicrobe. Thèse. Leiden, Van Duesburg, 1925, $155 \mathrm{pp}$.

574. Schwarz, G.: Bakterizidie und Temperatur. Deutsche. med. Wchnschr., $1924,50,754$.

575. Seiffert, W.: Ein wirksames Filtrat durch Passage mit verscheidenen Bakterien polyvalent. Kklin. Wchnschr., 1922, 1, 2547.

576. —_: Das d'Herellesche Phänomen. Med. Klin., 1922, 18, 997; 1093; 1121.

577. — : Über das d'Herelleschen Phänomen. Soziale Hyg. u. Mikrobiol., 1922, Sept. 21.

578. — : Das d'Herellesche Phänomen als "exogene Autolyse der Bakterien." Zeitschr. f. Hyg. u. Infektionskrankh., 1922, 98, 482.

579. ——: Ein Beitrag zur Variation der Bakterien und zum d'Herelleschen Phänomen. Centralbl. f. Bakt., I. Orig., 1922/23, 89, *195. 
580. Selffert, W.: Der Charakter des d'Herelleschen Phänomens. Klin. Wchnschr., 1923, 2, 1049.

581. — - Zum d'Herelleschen Phänomen. K'lin. Wchnschr., 1923, 2, 1479.

582. — : Neue Untersuchungen über den Charakter des d'Herelleschen Phänomens. Med. Klin., 1923, 19, 833.

583. — - Der Charakter des d'Herelleschen Phänomens. Zeitschr. f. Immunitätsforsch., 1923/24, 38, 292.

584. Seiser, A.: Untersuchungen über das Phänomen von d'Herelle. Arch. f. Hyg., 1923, 92, 189.

585. Simon, C. E.: Filterable viruses. Physiol. Reviews, 1923, 3, 508.

586. Sмiтн, J.: The bacteriophage in the treatment of typhoid fever. Brit. M. J., 1924, ii, 47.

587. Sonnenschein, C.: Zur Kenntnis bakteriophagenähnlicher Erscheinungen.

Centralbl. f. Bakt., I. Orig., 1925, 95, 257.

588. Sчёт, W.: Die Flüchtigkeit des d'Herelleschen flüchtigen Agens. Med. Ḱlin., 1924, 20, 184.

589. Spence, R. C., And Mchinley, E. B.: The therapeutic value of bacteriophage in the treatment of bacillary dysentery. Southern M. J., 1924, 17,563 .

590. Sumryosнi, Y.: Bauchhöhlenexsudat und Bakteriophage. Zeitschr. f. Immunitätsforsch., 1924, 39, 377.

591. Surányi, L., and Kramár, E.: Ueber das Vorkommen des d'Herelleschen Bakteriophagen in Säuglingsstühlen. Monatschr. f. Kinderheilk., 1923, 26,392 .

592. — : Ueber das Vorkommen des d'Herelleschen Bakteriophagen in Stühlen von Neugebornen. Monatschr. f. Kinderheilk., 1924, 28, 330.

593. Suzuki, T.: De verspreiding van bakteriophagus in dysentery-faeces van kinderen. J. Oriental Med., 1923, 1, 137.

594. — : Albumin-free bacteriophage, personally produced. J. Oriental Med., 1924, 2, 125.

595. Tchang Kouo NGen, and Wagemans, J.: Résistance des bactériophages a la chaleur. Compt. rend. Soc. de biol., 1922, 87, 1253.

596. - - Résistance des bactériophages a la chaleur. Compt. rend. Soc. de biol., 1923, 88, 303.

597. Thévenot: Le bacille bactériophage de d'Herelle. J. de méd. de Lyon, 1921, Mar. 5.

598. Thiroux: Le bactériophage d'Herelle. Ann. de méd. et de pharm. colon., Par., 1921, 19, 209.

599. Tomaselli, C.: Contributo allo studio del batteriofago. Milano, A. Cordiani, $1923,53 \mathrm{pp}$.

600. Turro, R.: Extraction des ferments cellulaires. Compt. rend. Soc. de biol., 1921, 84, 60 .

601. - : Extraction des ferments cellulaires. Compt. rend. Soc. de biol., $1921,84,290$.

602. - - Extraction des ferments cellulaires. Compt. rend. Soc. de biol., $1921,84,375$.

603. - : Extraction des ferments cellulaires. Compt. rend. Soc. de biol., $1921,84,435$. 
604. Twort, F. W.: An investigation on the nature of ultramicroscopic viruses. Lancet, 1915, ii, 124.

605. — : Researches on dysentery. Brit. J. Exp. Path., 1920, 1, 237.

606. — : The bacteriophage; the breaking down of bacteria by associated filter-passing lysins. Brit. M. J., 1922, ii, 293.

607. - : The ultramicroscopic viruses. J. State Med., Lond., 1923, 31, 351. 608. Uhlenhuth: [Discussion to] Weitere Untersuchungen über das d'Herellesche Phänomen [by Gildemeister et al.]. Centralbl. f. Bakt., I. Orig., 1922/23, 89, *204.

609. Vallen, I.: Ueber Schädigung der Leukozyten beim d'Herelleschen Phänomen. Centralbl. f. Bakt., I. Orig., 1924, 91, 424.

610. Védrenne, R.: Le bactériophage chez le nourisson. Thèse. Paris, Jouve et Cie., 1922, $38 \mathrm{pp}$.

611. Violato, A.: Considerazione sul comportamento del batteriofago nelle occlusioni intestinali. Cultura med. mod., Palermo, 1924, 3, 449.

612. Wagemans, J.: Au sujet de la constitution du bactériophage. Compt. rend. Soc. de biol., 1922, 8\%, 1244.

613. - La recherche des bactériophages dans la nature. Arch. internat. de pharmacod., 1923/24, 28, 159.

614. - Sur la constitution des bactériophages et leur neutralisation. Arch. internat. de pharmacod., 1923/24, 28, 181.

615. - L La neutralisation des bactériophages. Compt. rend. Soc. de biol., $1923,88,304$.

616. VAN DER WALLE, N.: Nieuwere ondersachingen over den bacteriophaag. Nederl. Tijdschr. v. Geneesk., 1923, 67, 589.

617. Wassermann, A., And Ficker, M.: Über die Rolle von Aktivatoren dei der Bildung von giften Spaltprodukten im Darminhalt. Klin. Wchnschr., $1922,1,1159$.

618. Watanabe, T.: Desinfektionsversuche mit Bakteriophagen. Wien. klin. Wehnsehr., 1921, 34, 522 .

619. - D D Dinfektionsversuche mit Bakteriophagen. Arch. f. Hyg., 1923, $92,1$.

620. - U Ueber die Natur des bakteriophagen Virus. Wien. klin. Wchnschr., $1922,35,53$.

621. - Über die Wirkung von Staphylokokkenbakteriophagen. Wien. klin. Wchnschr., 1922, 35, 603.

622. - : Serologisehe Untersuchungen an Shigabakteriophagen. Zeitschr. f. Immunitätsforsch., 1923, 37, 106.

623. Weinberg, M., and Aznar, P.: Autobactériolysines et le phénomène de d'Herelle. Compt. rend. Soc. de biol., 1922, 86, 833 .

624. - Ouelques faits nouveaux sur les autobactériolysines. Compt. rend. Soc. de biol., $1922,8 \%, 136$.

625. Weiss, E., AND ARNOLD, L.: A study of antigenic properties of bacteriophage. J. Infect. Diseases, 1924, 34, 317.

626. Werthemann, A.: Das Verhalten der übertragbaren Lysine (Bakteriophagen) in der Zirkulation von Kalt- und Warmblütern. Arch. f. Hyg., $1922,91,255$. 
627. WolfF, L. K.: [État actuel de la question du bactériophage.] Nederl. Tijdschr. v. Geneesk., 1922, 66, 479.

628. - - and Janzen, J. W.: Action de divers antiseptiques sur le bactériophage de d'Herelle. Compt. rend. Soc. de biol., 1922, 87, 1087.

629. - Over den typhusbacteriophag. Nederl. Tijdschr. v, Geneesk., 1922, No. 17, 1818.

630. —— Sur la virulence multiple du bactériophage. Ann. Inst. Pasteur, 1923, 37, 1064.

631. Wollman, E.: À propos de la note de MM. Bordet et Ciuca (Phénomène de d'Herelle, autolyse microbienne transmissible de J. Bordet et M. Ciuca, et hypothèse de la pangénèse de Darwin). Compt. rend. Soc. de biol., $1920,83,1478$.

632. — : Sur le phénomène de d'Herelle. Compt. rend. Soc. de biol., 1921, $84,3$.

633. — : Recherches sur le phénomène de d'Herelle. Action de la trypsine sur le bactériophage du Bacille de Shiga. Compt. rend. Soc. de biol., 1924, 90, 59.

634. - - and Brutsaert, P.: L'autonomie antigène des bactériophages. Compt. rend. Soc. de biol., 1925, 92, 1284.

635. - and Goldenberg, L.: Le phénomène de d'Herelle et la réaction de fixation. Compt. rend. Soc. de biol., 1921, 85, 772.

636. ——and Wollman, E.: Recherches sur la phénomène de d'Herelle. Pluralité et autonomie des bactériophages. Compt. rend. Soc. de biol., $1925,92,552$.

637. Wollstein, M.: Studies on the phenomenon of d'Herelle with Bacillus dysenteriae. J. Exp. Med., 1921, 34, 467.

638. Wоорсоск, H. M.: An introduction to the study of haematophagy. I. Haematophagy as a normal occurrence. II. Haematophagy as a pathological occurrence. J. Roy. Army Med. Corps, 1921, 37, 321; 418.

639. - Are the active principles of filter-passing and ultramicroscopic viruses living organisms or enzymes? J. Roy. Army Med. Corps, 1922, $39,243$.

640. YASAKI, Y.: Wirkung und Neubildung des d'Herelleschen Agens in ihren Beziehungen zu vitalen Vorgängen in der Bakterienzelle. (Untersuchungen über Bakteriophagen. I.). Zeitschr. f. Hyg. u. Infektionskrankh., 1924, 102, 526 .

641. —_ : Die Abhängigkeit der Eigenschaften des lytisches Agens d'Herelles von der Verdünnung und vom Medium. Untersuchungen über Bakteriophagen. III. Zeitschr. f. Hyg. u. Infektionskrankh., 1924, 102, 554.

642. ZDANSKY, E.: Gewinnung spezifischer Bakteriophagen und über bakteriophagentherapeutische Versuche. Med. Kilin., 1924, 20, 1485.

643. — : Kritische und experimentelle Beiträge zur Frage der Wirkungsmöglichkeit der Bakteriophagen im Warmblüterorganismus und in der freien Natur. Zeitschr. f. Hyg. u. Infektionskrankh., 1924, 103, 164.

644. —— : Ueber die Bedeutung der Salze für die übertragbaren Lysine (Bakteriophagen). Wien. klin. Wehnschr., 1924, 37, 141.

645. Zingher, A.: The bacteriophage reaction of d'Herelle. Proc. N. York Path. Soc., 1921, 21, 2. 
646. Zoeller, C.: Action des rayons ultraviolets sur une souche de bactériophage. Compt. rend. Soc. de biol., 1923, 89, 860.

647. - ANd Manoussakis: Kérato-conjunctivite expérimentale a Bacille pyocyanique. De l'action d'un bactériophage antipyocyanique. Compt. rend. Soc. de biol., 1924, 91, 548 .

The author, and the translator as well, would consider it a favor if those who publish upon the subject of the bacteriophage in any of its many phases will send them reprints of their work. This will be of material assistance in the preparation of future editions of the text. 


\section{INDEX OF AUTHORS CITED}

Alessandrini (with Doria); 550

Allison (see Fleming)

v. Angerer; $134,275,316$

Appelmans; 142, 291, 302, 381, 383, 389; (with Wagemans); 191

Aquino (see Bachmann)

Arkwright; 212, 316

Arloing (with Dufour, Bouvier and Sempé); 555; (with Sempé and Chavanne); 436; (with Langeron and Sempé); 290, 302

Arnold (with Weiss); 316, 394

Arsimoles (see Hauduroy)

Asheshov; 70, 166, 287, 326, 337, 344

Azerad (see Richet)

Aznar (see Weinberg)

Baars (see Miessner)

Bablet; 71, 326, 528; (with d'Herelle); 306

Bachmann (with Aquino); 316; (with de la Barrera); 252; (see also Bürgers)

Baerthleins; 253

Bail; 90, 158, 171, 193, 232, 317, 325, 400; (with Matsumoto); 129

de la Barrera (see Bachmann)

Bastin; 326, 560

Bazy; 560, 561

Bechhold; 25

Beckerich; 326; (with Hauduroy); 145, 146, 233, 243, 251, 266, 314, $422,436,471,550,553$

Beguet; 1, 2

Beijerinck; 326, 343

van Beneden (see Fabry)

Berger (see Doerr)

Bernard; 211, 222

Blair ; 258

de Blieck; 515

Boenheim (see Munter)

Bohn; 371
Borchardt; 280, 316, 435

Bordet; 199, 201, 222, 314; (with Ciuca); 3S, 49, 161, 168, 198, 201, 203, 207, 208, 209, 218, 221, 223, 256, 29s, $311,312,313,340,381,383,384$, 392,397

Borrel; 25

Botez; 259

Bouin (see Velu)

Bouvier (see Arloing)

Brocq-Rousseu (with Urbain and Forgeot); 305,361

Bronfenbrenner (with Korb) ; 280, 302

Brutsaert; 64, 69, 72, 201, 209, 292, 306, 326, 343; (see also Bruynoghe; see also Wollman)

Bruynoghe; 203, 207, 236, 326, 367, 369, 389, 390; (with Brutsaert); 306; (with Maisin); 207, 383, 392, 558; (with Wagemans); 346, 386

Bürgers (with Bachmann); 236, 258

Callow; 483

Carré (see Valeé)

Carrère (see Lisbonne)

Caublot; 256, 326

Caulery; 318

Chavanne (see Arloing)

Cheyssial; 2

Ciuca; 69 ; (see also Bordet)

Combiesco; 435; (with Magheru); 267

Conradi (with Kurpjuweit); 7

da Costa Cruz; 45, 68, 108, 114, 178, 287, 301, 316, 401, 422, 545, 548, 549 ; (see also Machado)

Crendiropoulo; 264

Dangeard; 19,355

Davison; 43, 219, 301, 316, 548

Debré (with Haguenau); 422

Dehne (with Hamburger); 399

Delarne (see Richet) 
Doerr; 63, 109, 117, 134, 143, 273, 314, 315; (with Berger); 63, 90; (with Grüninger); 59, 121, 122, 123, 124

Doria (see Alessandrini)

Duclaux; 31

Dufour (see Arloing)

Dumas; 37, 243, 422, 436

Eastwood; 316

Eichhoff; 483

Eichhorn; 266

Eijkman; 6, 85

Einstein; 83

Eliava; 12, 161, 178, 263, 326, 477; (with d'Herelle); 391, 392, 397, 408; (with Pozerski); 203, 300, 301, 304

Ellis; 58, 316

Emmerich (with Löw); 6, 9

Fabry (with van Beneden); 314, 326, 398,399

Faltin; 7

Fejgin; 257, 258, 316, 479, 485, 486

Firle (see Prausnitz)

Fleming (with Allison); 17

Flu; 8, 10, 195, 211, 223, 226, 234, 235, 238, 262, 263, 264, 277, 297, 301, $324,326,435,509$

Forgeot (see Brocq-Rousseu)

Friedberger (with Meissner); 478

Gamaleia; 6, 267

Gerretsen (with Gryns, Sack and Söhngen); 259, 286, 291, 297, 487

Gildemeister; 11, 209, 232, 233, 234, 291, 298, 316, 324, 345; (with Hermann); 323; (with Herzberg); $280,289,297$

Giornini; 487

Gృørup; 326

Gohs; 58

Goldenberg (see Wollman)

Gomez (see Kraus)

Gory; 210

Gougerot (with Peyre); 562

Gratia; 16, 45, 46, 169, 187, 191, 198, $202,209,218,219,221,222,223,256$,
265, 267, 405, 483, 558, 559, 561; (with Jaumain); 210, 392; (with deKruif); 86, 144, 198, 199; (with de Namur); 337; (with Rhodes); 49 Grüninger (see Doerr)

Gryns (see Gerretsen)

Hadley; 197, 267, 316, 479

Haffkine; 11

Haguenau (see Debré)

Hajós; 7

Hamburger (see Dehne)

Hankin; 5, 7

Hauduroy; 64, 114, 138, 228, 229, 230, 251, 291, 294, 296, 298, 317, 326, 402, 403, 406, 464, 470, 471, 476, 477, 552, 553, 555, 560; (with Arsimoles); 550; (see also Beckerich; see also Philibert)

Herdershee (with Wolff); 551

Hermann (see Gildemeister)

Herzberg (see Gildemeister)

Ionesco-Mihaiesti; 306, 316, 374

Izar; 163, 226, 326

Janzen; 326; (with Wolff); 107, 108, 146, 163, 169, 176, 248, 335; (see also Wolff)

Jaumain; 321, 370; (with Meuleman); 108; (see also Gratia)

Jeantet; 113

Jötten; 232, 261, 277, 316

Kabelik; 43, 110, 326, 403

Kabeshima; 71, 282, 288, 292, 302, 308, 535

Korb (see Bronfenbrenner)

Kramár (see Surányi)

Kramer; 482, 509, 515, 520

Kraus (with Gomez); 266; (with Marais); 316

Kropveld; 326, 483

deKruif (see Gratia)

Kruse (with Pansini); 6

Kuttner; 49, 59, 207, 208, 209, 292, 313, 435 
Langeron (see Arloing)

Ledingham; 316

LeLouet; 524, 526, 533; (with d'Herelle); 363,520

Lemos Monteiro; 266

Lepper; 316

Levaditi; 38; (with Nicolau); 275, 281, 287, 291

Levy; 509

Liebermann; 316

Lisbonne (with Carrère); 314

Lisch; 269

Löw (see Emmerich)

Machado (with da Costa Cruz); 400

Magheru (see Combiesco)

Maisin; 287; (see also Bruynoghe)

Maitland; 43, 49, 119, 127, 148, 326, 336

Malfitano; 6, 25, 27; (with Strada); 6

Marais (see Kraus)

Marcuse; 301, 316, 552, 555

Martin; 23, 24

Matsumoto; 325 ; (see also Bail)

McKinley; 178, 266, 564, 567; (see also Spence)

Meier ; 576

Meissner; 261, 262, 280, 326; (see also Friedberger)

Metchnikoff; 9,355

Meuleman (see Jaumain)

Meuli; 125, 316

Miessner (with Baars); 316, 509

Munter (with Boenheim); 549, 555; (see also Otto)

\section{Nageotte; 11}

Nakamura; 63, \$9, 90, 325

de Namur (see Gratia)

de Necker; 290, 291, 297

Nelson Barbosa; 560

Nicolau (see Levaditi)

Nylander; 320

Olsen (with Yasaki); 277

Otto; 141, 143, 176, 233, 389, 390, 391, 394; (with Munter); 6, 37, 67, 147, 232, 233, 268, 290, 324, 548; (with Munter and Winkler); 257, 294,
306, 316, 385, 387, 391; (with Sukiennikowa); 374 ; (with Winkler); 398

Pansini (see Kruse)

Pereira; 547, 555

Pesch; 267

Petrovanu; 316

Pfreimbter (with Sell and Pistorius); 147

Philibert; 326, 555; (with Hauduroy); 551

Pico; 7, 266, 316, 435

Pinoy; 12, 103, 318

Piorkowski; 266, 483

Pistorius (see Pfreimbter)

Polettini Bruno; 259, 326

Pondman; 114, 234, 326

dePoorter (with Maisin); 282, 287, $290,302,304$

Pozerski; 326; (with d'Herelle); 165, 292, 293; (see also Eliava)

Prausnitz; 123, 134, 237, 275, 289, $297,301,326,340,341,343,385$, 386, 387, 393, 396; (with Firle); $277,290,301,304,346,349,351$

Preisz; 326

Proca; 306, 316

Putter (with Vallen); 316

Quiroga; 267

Rahn; 7

Reichert; 235, 243, 251, 301, 306, 307, 326

Rhodes (see Gratia)

Richet (with Azerad and Delarne); 551

Roux (with Salimbeni); 24

Sack (see Gerretsen)

Salimbeni (see Roux)

Sardjito; 326, 536, 537

Scheidegger; 45, 188, 301, 316

Schnabel; 316

Schuurman ; $326,345,346$

Schwendener; 320

Seiffert; 164, 187, 232, 2S6, 289, 296, 304,341 
Seiser; 70

Sell (see Pfriembter)

Sempé (see Arloing)

Smith; 551

Söhngen (see Gerretsen)

Spät; 280

Spence (with McKinley); 547

Strade (see Malfitano)

Sukiennikowa (see Otto)

Surányi (with Kiramár); 424

Suzuki; 382

Tchang Kouo Ngen (with Wagemans); 295,296

Tomaselli; 38, 72, 226, 227, 228, 233, $259,298,306,317,326,417,422,428$

Twort; 13, 264, 267

Urbain (see Brocq-Rousseu)

Valeé (with Carré); 291

Vallen (see Putter)

van der Hoeden; 435
Védrenne; 326, 423

Velu; 2; (with Bouin); 2

Wagemans; 171,385 , 389; (see also Appelmans); (see also Bruynoghe); (see also Tchang Kouo Ngen)

Watanabe; 302, 303, 305, 306, 325

Weinberg; 233; (with Aznar); 232, 295, 316

Weiss (see Arnold)

Werthemann; 122, 145, 316, 382

Whitney; 318

Winkler (see Otto)

Wolff; 326, 391, 394; (with Janzen); 305, 346, 351, 352, 390; (see also Janzen); (see also Herdershee)

Wollman; 102, 202, 275, 2S2, 283, 326; (with Brutsaert); 340 ; (with Goldenberg); 397

Wollstein; 63, 202, 316

Yasaki; 316; (see also Olsen)

Zinzer; 487

Zoeller; 291 


\section{INDEX}

Abscesses, bacteriophage in; 483

Acetone, effect on bacteriophage; 288, 303

Acid, adaptation of bacteriophage to; 344

- - effect on bacteriophage; 304

Acquired resistance and secondary cultures; 191

-_ of bacteria; 217

- - specificity of ; 192

Acquisition of resistance by bacteria; 194

— of virulence by bacteriophage; 175

Adaptation of bacteriophage; 175,341 , 386

Adsorption of bacteriophage; 24, 288

— of viruses; 291

Agar, diffusibility of bacteriophage in; 277

-, effect on bacteriophagy; 63

-

Age, effect on bacteriophage; 276,285 , 300

- of bacteria, effect on bacteriophagy; 46,88

Agglutinability of cholera vibrios; 264

_- of resistant bacteria; 218

Agglutinated cultures, bacteriophagy with; 213

—— , growth of; 209

Agglutinating property of antibacteriophagic sera; 397

Agglutination in bacteriophagy; 73

— of bacterial mutants; 252,258

Agglutinin absorption; 109

Alcohol, effect on bacteriophage; 2SS, 302

Animal charcoal, adsorption of bacteriophage by; 290

Animals, kacteriophage in; 424

Anthrax, bacteriophage therapy of; 558

Anti-antibacteriophagic sera; 398
Antibacterial sera, effect on bacteriophage; 400

Antibacteriophagic property, nature of ; 392

- sera, adaptation of bacteriophage to; 343

—— complement fixation with; 391

—— complexity of; 397

—— developed resistance to; 386

—_ inhibition by; 203, 340

——, mode of action of; 386

—

—— - specificity of action of ; 388

Antibodies of antibacteriophagic sera; 397

Antibody fixation, relation to bacteriophage fixation; 109

Antiformin, effect on bacteriophage; 304

Antigen of the bacteriophage; 391

Antigenic function of bacteriophage; 381

- value of bacteriophage and virulence; 389

Antiphylaxis, nature of; 403

Antiseptics, adaptation to; 346

_- , attenuation of virulence by; 168

- _. effect on bacteriophagy; 71

Antitoxic action of dysentery-bacteriophage; 537

Aphthous fever, relation of bacteriophage to; 576

Appelmans' method of virulence determination; 142

Arrest of bacteriophage multiplication; 127

Ascitic fluid, effect on bacteriophagy; 71

Assimilation by bacteriophage; 341

— by protobes; 360

_ by strangles streptococci; 361

Attenuation of bacterioohage; 292 
Attenuation of bacteriophage virulence; 165,197

Autolysin hypothesis of bacteriophage; 314

Autolysis, bacterial; 321

— , relation to bacteriophagy; 6

Autonomy of bacteriophage; 333

Avian typhosis, bacteriophage in; 479 , 490

—— immunization against; 510 481

—— - symptomatology of ; 480

Bacillary dysentery, bacteriophage in; 439,506

- bacteriophage therapy of; 540

—— immunization against; 533

$B$. aerogenes form of $B$. coli; 210

$B$. anthracis dissolution; 6,266

B. of barbone, distribution of; 502

$B$. coli, colony types of $; 420$

- , heterogeneity of ; 168,255 infections, bacteriophage in; 483 553

-

- , mutations of ; 221,255

—-, number dissolved by bacteriophage; 54

B. diphtheriae, heterogeneity of ; 258

B. dysenteriae Hiss septicemia; 448

B. dysenteriae, homogeneity of ; 168, 242

- mutations of; 223

— number dissolved by bacterio phage; 54

- , toxicity of ; 403

B. fluorescens, relation to Myxomycete; 103

B. of Friedländer, heterogeneity of ; 256

B. gallinarum in avian typhosis; 480

- number dissolved by bacteriophage; 55

B. jeffersonii, homogeneity of; 245

- in avian typhosis; 480

$B$. paratyphosus, heterogeneity of ; 251,254
B. paralyphosus, mutations of ; 252

- number dissolved by bacteriophage; 54

B. pestis, bacteriophage races for; 247

- homogeneity of ; 168,247

- number dissolved by bacteriophage; 55 "suicide"; 12

B. pfaff, homogeneity of; 245

- in avian typhosis; 480

$B$. proteus cultures, bacteriophage from, 485

-

- mutants; 257

B. pullorum, homogeneity of; 245

- in avian typhosis; 480

B. pyocyaneus dissolution; 9,267

B. radicicola, bacteriophagy of; 487

- , heterogeneity of; 259

B. rettgeri, homogeneity of; 245

B. sanguinaria, homogeneity of; 245

B. suipestifer, heterogeneity of; 254

B. typhi murium, heterogeneity of ; $\mathbf{2 5 4}$

—_ infection in mice; 509

B. lyphosus, heterogeneity of : 168, 248

- number dissolved by bacteriophage; 54

—, ultrabacterial form of ; 478

Bacteria, acquired immunity in; 169

195

_- fixation of bacteriophage to; 104

—_, natural immunity in; 169

— of secondary cultures: 206

- , penetration by bacteriophage; 112

_- , relation to plaque formation; $\$ 7$

164

Bacterial autolysis; 321

- cultures, bacteriophage in; 10 , $11,37,232,257,261,485$

- - ultrapure; 207

- destruction and bacteriophage multiplication; 121

dissolution, mechanism of; 113

- - microscopic control of; 130

$\ldots$ _ 
Bacterial dissolution, relation to bacteriophage multiplication; 125

- growth stimulation by bacteriophage; 73

_ immunity and secondary cultures; 191

- metabolic products, effect on bacteriophagy; 128

- morphology, effect of bacteriophage on; 112, 130

- numbers, effect on bacteriophagy; 50

- products, bacteriophagy with; 102

- resistance, acquired; 193, 217

- and bacteriophage multiplication; 210

—— - and mutation; 219

—_ - effect of temperature on; 162

—— effect on agglutinability; 218

_— - effect on biochemistry; 218

- - natural; 193, 217

—— - significance of; 182

rupture during bacteriophagy; 113

species, concept of; 365

-, heterogeneous; 168

- homogeneous; 168

state, effect on bacteriophage multiplication; 124

— symbiosis with bacteriophage; 211

- toxicity, effect of bacteriophagy on; 403

Bacterioclysis; i3

- of B. pyocyaneus; 267

— of staphylococci; 264

Bacteriolysis, definition of; 5

_- relation to inhibition; 51

Bacteriophage, adaptation of; 175,341

sera; 343,386

— , adaptation to acid; 344

—, adaptation to antiseptics; 346

-, adaptation to glycerol; 343

—, adsorption of; 24,288

—, antigen of; 391

_-, antigenic function of; 381

- antiphylactic property of; 403

—, antisera to ; 203, 340,383, 391, 397

- - appearance in infants; 423
Bacteriophage, assimilation by; 341 , 360

- attenuation of; 165, 197, 292

__ autonomy of; 333

-, behavior in epidemics; 490

- behavior in secondary cultures; 196

- carriers; 10, 11, 37, 194, 232, 257, 261,485

—, catalyzer nature of ; 309

— 257

- - centrifugation of ; 280

—- chemotaxis of; 109

—, colloidal state of; 281

— complement fixation with; 391

- conservation of; 283

corpuscles, colloidal state of; 281

- , enumeration of; 96

_ granules; 132

——, size of; 134,275

, corpuscular nature of; 82, 273

- , definition of ; 18

- , desiccation of ; 287

- destruction by resistant bacteria; 195,211

— , determination of virulence of; 141

- , diffusibility of ; 277

- , digestion of; 282

—-, discovery of ; 2

- distillation of; 278

—, distribution of; 37

—, effect of acetone on; 288, 303 .

- , effect of acid on; 304

_- , effect of age on; 276, 285

-, effect of alcohol on; 288, 302

- , effect of antibacterial sera on; 400

_- effect of antiformin on; 304

- , effect of antiseptics on; 71,168 , 346

-, effect of bile on; 71, 552

—-, effect of chloroform on; 303

—-, effect of cold on; 300

- - effect of colloids on; 290

-, effect of concentration of; 55

—, effect of copper sulfate on; 302

-, effect of diet on; 428 
Bacteriophage, effect of dyes on; 67, 305

- , effect of essences on; 303

- , effect of enzymes on; 282

-, effect of formol on; 304

-, effect of furfurol on; 304

—-, effect of glycerol on; 286, 306

300

—

-, effect of lysol on; 304

- effect of mercuric chloride on; 301

_- effect of organic compounds on; 302

-, effect of oxygen on; 302

- - effect of papaine on; 282

- - effect of $\mathrm{pH}$ on; $43,69,88,126$, 184, 187, 287, 300

_-, effect of phenol on; 303

- , effect of potassium cyanide on; 301

- effect of quinine on; 304

- , effect of radium on; 292

_- effect of sodium chloride on; 301

- - effect of sodium fluoride on; 301

_-, effect of sodium sulfate on; 301

--, effect of temperature on; 38, 59, 91, 123, 161, 183, 292, 409

_- effect of tetraline on; 304

- effect of trypsin on; 282

-

-

_- effect on electrical potential; 135

-, effect on phagocytosis; 408

-, elective fixation of; 105

-

- evaporation of ; 286, 301

- expression of degrees of virulence of ; 141

—, extraction by sodium sulfate; 195

—_, fate of ingested; 416

—, ferment secretion by; 373

—, filtration of ; 21,274

- f. fixation to bacterium; 104

- fixation, relation to antibody fixation; 109

- flocculation of; 287, 301
Bacteriophage, hypotheses concerning; 309

- immunization against avian typhosis; 511

—— against barbone; 520

—— against dysentery; 533

- - against experimental infections; 509

— in abscesses; 483

_ in animals; 424

in avian typhosis; 479,490

in B. coli infections; 483

in bacterial cultures; $10,11,37$, $232,257,261,485$

— in barbone; 497

— in blood; $37,427,470$

— in buffaloes; 431,497

— in cats; 431

in cattle; 431

in cystitis; 484

in dogs; 434

in dysentery; $439,449,506$

in enterokinase; 435

in external environment; 435

in flacherie; 507

in food products; 435

in fowls; 429,433

in furunculosis; 483

in goats; 431

in horses; 425, 432

in $\operatorname{man} ; 418,437$

in monkeys; 430

in plants; 487

in plague; 503

in pus; $37,248,483$

in pyelonephritis; 484

in rabbits; 431

in sausage; 435

in soil; $37,436,488$

in staphylococcus infections; 483

in streptococcus infections; 483

in swine; 431

in typhoid fever; 451

in typhus fever; 485

in urine; 37,484

in vaccinia virus; 16

in water; $12,37,243,251,436$

ingestion of; 416 
Bacteriophage, irradiation of ; 291

- , isolation of ; 21,38

- , isolation of pure races; 156

- , isolation from mixed cultures; 234,237

- leucocytic origin of ; 311

—

- metabolic products, inhibition by; 216

- , mode of action of; 369

-, multiple races of; 416

_ multiple virulences of ; 432

multiplication, arrest of; 127

—— factors influencing; 121,349 - with resistant bacteria; 210

- , nature of ; 309,329

_, nature of substance of; 282

_

— 274

—

_

—, physical state of ; 273

_- precipitation of ; 287

- , preservation of ; 285

__, production of antisera to; 383

- , pure races of; 155

__, qualitative variations in; 139

— races in barbone; 246

- in dysentery; 242

__ in flacherie; 256

—_ in fowl typhosis; 242,246

— in paratyphoid fever; 252

— in plague; 247

—— in plant nodules; 259

—

in typhoid fever; 248

$\ldots$ - individuality of; 169

—— of intestinal tract; 416

$\longrightarrow$ - pure; 155

- $\ldots$, separation of; 156

__

__ - variation in virulence of ; 152

- , rate of multiplication of $; 115$

$\ldots$, refractile index of; 134

_ relation to aphthous fever; 576

__ , relation to Flattenformen; 11

_- relation to Hankin's principle; 7
Bacteriophage, relation to origin of life; 375

_- relation to principle of Emmerich and Löw; 10

—_, relation to viruses; 358

—, sedimentation of; 280

—

- , serial action with; 40

145

—, solubility of ; 282

_- species, concept of ; 365

—_, symbiosis with bacteria; 211

- therapy of avian typhosis; 490

— of $B$. coli infections; 553

— of bubonic plague; 567

— in chronic colitis; 549

- of dysentery; 540

— of paratyphoid fever; 550

— of staphylococcus infections;

558

—— of streptococcus infections;

567

—— of typhoid fever; 549

- thermal death point of; 292

- ubiquity of ; 416

- , ultrafiltration of ; 274

—, unicity of; 358

-, variability of; 350

__, variations in virulence of; 137 , 159,437

virulence, acquisition of; 175

__ and colony type; 209

_ - , attenuation of $; 165$

- _ Appelmans method of determining; 142

—_, Beckerich and Hauduroy method of determining; 145

$\ldots \_$, d'Herelle method of determining; 150

$\ldots$, virulences in normal animals ; 431 virulence, increase in; 160

___ Janzen and Wolff method of determining; 146

—_ Maitland method of determining; 148

__ _ - multiple; 170 
Bacteriophage virulence, Otto and Munter method of determining; 147

-

——, Pfreimbter, Sell and Pistorius method of determining; 147

_-

—_

_of determining; 142

—— Werthemann expression of; 145

- vitality of ; 283

- volatility of ; 8,277

Bacteriophagum intestinale, living virus; 355

Bacteriophagy, agglutination in; 73

— and ferment action; 371

- antiserum inhibition of ; 340

- cause of mutations; 220

— , culture media for $; 42$

—, dark-field observation of ; 113,131

—, effect of agar on; 63

_- effect of age of bacteria on; 46

—, effect of antiseptics on; 71

- effect of ascitic fluid on; 71

- effect of bacterial metabolic products on; 128

- - effect of bacterial numbers on; 50

_- effect of bile on; 71,552

—, effect of calcium chloride on; 70

- , effect of chloroform on; 71

- , effect of colloids on; 65

-, effect of dyestuffs on; 67

- effect of electrolytes on; 68

-, effect of gelatine on; 63,216

- , effect of gums on; 63

_-, effect of magnesium sulfate on; 71

- , effect of metabolic products on; 48

- effect of $\mathrm{pH}$ on; 43, 69, 184, 187

—, effect of phosphates on; 71

—, effect of potassium chloride on; 71

-, effect of pressure on; 63

—, effect of quinine on; 71

-, effect of salts on; 70

—, effect of serum on; 71
Bacteriophagy, effect of sodium fluoride on; 71

- , effect of sugars on; 70

—

—, effect of urine on; 71

_ , effect of vaccinated medium on; 127

_- effect of viscosity on; 63

_-, effect on bacterial toxicity; 403

—, granule formation in; 130

— in absence of bacterial cells; 102 in avian typhosis; 479,490

— in bacillary dysentery; 439,506

- in B. coli infections; 483

— in barbone; 497

— in flacherie; 507

— in mixed cultures; 178,208

_- in plague; 503

in plants; 487

in staphylococcus infections; 483

in streptococcus infections; 483

in synthetic media; 46

in typhoid fever; 451

in typhus fever; 485

, inlibition by antibacterial sera; 400

—, mechanism of ; 101

_, microscopic aspects of; 130

_

— of agglutinated cultures; 213

— on solid media; 76

—-, phenomena simulating; 266

_, plaque formation in; 76

- products of ; 374

_- relation to autolysis; 6,321

—-, relation to bacteriolysis; 5,13

- , relation to Twort phenomenon; 13

- - rupture of bacteria in; 113

—-, stained preparations in; 114

—, staining of bacteria during; 130

—-, stimulation of bacterial growth by ; 73

—, ultrabacteria formed in; 226

__ with dead bacteria; 47

Bail's hypothesis of bacteriophage; 325 Barbone, bacteriophage from; 247, 497

— immunity, duration of; $\mathbf{5 2 6}$ 
Barbone, immunization against; 520

- of buffaloes; 497

Barbone-bacteriophage, filtrability of; 274

—, immunization with; 520

— immunizing principle in; 527

— in buffaloes; 499

— in cattle; 431

— in fowl; 429

_- opsonic action of ; 411

-_, sources of ; 247

- - , spontaneous attenuation of; 167

Beckerich and Hauduroy method of determining virulence; 145

Bile, effect on bacteriophagy; 71,552

Biochemistry of mixed cultures; 214

—_ of resistant bacteria; 218

Blood, bacteriophage in; 37, 251, 255, $256,427,470$

- cultures, bacteriophage in: 470

Body fluids, effect on bacteriophagy ; 71

Bordet's hypothesis of bacteriophage; 311

Bubonic plague, bacteriophage in; 503

- - - bacteriophage therapy of; 567

Buffaloes, bacteriophage in; 247,431 , 497

- - hemorrhagic septicemia of ; 497

- - immunization with bacteriophage; 520

Calcium chloride, effect on bacteriophagy ; 70

Carriers of bacteriophage; 194

Cats, intestinal bacteriophage of ; 431

Cattle, intestinal bacteriophage of ; 431

Centrifugation of bacteriophage; 104, 280

Chemotaxis of bacteriophage; 109

Chickens, avian typhosis of; 479

—, bacteriophage immunization of ; 511

—, bacteriophage in; 246, 429, 433, 490

- - epidemic avian typhosis in; 490

Chloramine, adaptation of bacteriophage to; 348
Chloroform, effect on bacteriophagy; 71,303

Cholera, bacteriophage races from; 261 Cholera-bacteriophage in stock cultures; 10

—, sources of ; 261

- - spontaneous attenuation of ; 167

Chronic colitis, bacteriophage therapy of ; 549

Coccobacillus acridiorum infection of locusts; 1

Cold, effect on bacteriophage; 300

Coli-bacteriophage, antisera to; 384

- - , attenuation by resistant bacteria; 198

—_, corpuscular nature of ; 85

- effect of antibacterial sera on; 400

—-, effect of antiseptics on; 72

—, effect of $\mathrm{pH}$ on; 45,187

- - effect of radium on; 292

—_, effect of temperature on; 293

—, effect of viscosity on; 63

_- elimination of injected; 381

—_, filtrability of; 274

—, fixation of ; 108

- , formation of ultrabacteria with; 226

__ in avian typhosis; 481

in buffaloes; 431

in cats; 431

in cattle; 431

- in dogs; 434

— in dysentery ; 440,542

in fowl; 429,433

in goats; 431

in horses; 425, 432

in man; 438

in monkeys; 431

in rabbits; 431

in stock cultures; 11

in swine; 431

in typhoid fever; 452

in urinary infections; 484

in water; 436

—, normal presence of; 418

- -, rate of multiplication of ; 121

— , sources of ; 255

_- specificity of; 168 
Coli-bacteriophage, spontaneous attenuation of; 167

—-, temperature limits of; 59

- - therapy of B. coli infections; 553 , virulence attenuation of; 165

_- virulence determination of; 145

—, virulence increase of; 202

-, volatility of; 280

Collodion filters, preparation of; 25 membranes, passage of bacteriophage; 274

-_ sacs, bacteriophagy with; 102

Colloidal state of bacteriophage; 281

Colloids, effect on bacteriophagy; 65 , 290

Colon bacillus infections, bacteriophage in; 483

of ; 553 , bacteriophage therapy

Colony form of mixed cultures; 210

- types of B. coli; 420

Complement fixation, effect of bacteriophage on; 413

_-_ _ with bacteriophage; 391

Conservation of bacteriophage; 283

Copper sulfate, effect on bacteriophage; 302

Corpuscular nature of bacteriophage; 82,273

Corpuscles, enumeration of ; 96

- , liberation of ; 105

- , rate of multiplication of; 115

Criteria of life; 330

Culture media for bacteriophagy; 42

Cystitis, bacteriophage in; 484

- bacteriophage therapy of; 553 , 560

_- mixed cultures in; 232

Dark-field observation of bacteriophagy; 113,131

Dead bacteria, bacteriophagy with; 47

Denitrification of ultrafilters; 30

Desiccation of bacteriophage; 286

Destruction of bacteriophage by resistant bacteria; 195, 211

Determination of bacteriophage virulence; 141
Dictyostelium mucoroides, multiplication of; 103

Diet, effect on bacteriophage; 428

Diffusibility of bacteriophage; 277

Diffusion and plaque formation; 89

Digestion of bacteriophage by ferments; 282

Diphtheria-bacteriophage, sources of; 258

Discovery of bacteriophage; 2

Disease, behavior of bacteriophage in; 437

- - experimental and natural; 509

Dissolution of bacteria by bacteriophage; 113

- - of bacteria, products of; 374

Distillation of bacteriophage; 278

Distribution of bacteriophage; 37

Doerr's hypothesis of bacteriophage; 314

Dogs, bacteriophage of ; 434

Dyestuffs, effect on bacteriophagy; 67 , 305

Dysentery, bacteriophage in; 3,242 , 439, 506

__ bacteriophage therapy of; 540

_-, immunization against; 533

--., Subtilis-bacteriophage from; 261

Dysentery-bacteriophage, acquired resistance to; 193

- - action of antibacteriophagic sera on; 387

- - , action on dead bacteria; 48

—, adaptation of ; 178

-_, adaptation to acid; 344

__ , agglutination by; 73

_- , antiphylaxis with; 404

_- , antitoxic action of ; 537

—, attenuation by resistant bacteria; 197

- -, behavior in mixed cultures; 179

- -, corpuscular nature of; 82

-_, discovery of; 3

- - , effect of acetone on; 303

-

-, effect of antisera on; 388,392

-

—, effect of bacterial numbers on; 52 
Dysentery-bacteriophage, effect of chloroform on; 71, 303

- - effect of colloidal silver on ; 66

- - effect of copper sulfate on; 302

- - effect of glycerol on; 306

-

- , effect of phenol on; 303

-

- - , effect of quinine on; 304

-

__, effect of sodium fluoride on; 71 , 301

-—, effect of sugars on; 70

- - effect of temperature on; 123 , 184, 292

_- enumeration by plaque counts; 96

-

_. , fixation to bacteria; 104

—

_—, formation of ultrabacteria with; 226

—, growth in bacterial products; 102

_- immunization with; 533

—— in bacillary dysentery; 439, 506, 542

—— in buffaloes; 431

in cats; 431

- in cattle; 431

in dogs; 434

- in fowl; 429

- in goats; 431

in horses; 425

- in man; 438

- in monkeys; 431

in rabbits; 431

- in swine; 431

in typhoid fever; 452

- in water; 436

—, isolation of ; 40,137

_- limiting concentration of ; 56

—_, maximum titre of; 127

—, microscopic aspects of; 130

-

__ , origin of secondary cultures with; 189

- , penetration of bacteria by $; 112$

_- , persistence of virulences of; 171

_- plaque formation by; 77,90
Dysentery-bacteriophage, qualitative variations in; 139

- , rate of fixation of $; 120$

- , rate of multiplication of; 116, 124

- - , sedimentation of ; 280

_- , size of plaques of 92

- , source of plaques with; 87

-

_- , spontaneous attenuation of; 166

-

__ therapy of dysentery; 542

-_, variation in; 333

-

—

-_, virulence range of ; 242

-

-

Elective fixation of bacteriophage; 105

Electrolytes, effect on bacteriophagy; 68

Enteritidis-bacteriophage, sources of; 254

Enterococcus-bacteriophage, sources of ; 266

Enterokinase, bacteriophage in $; 435$

Enumeration of bacteriophage corpuscles; 96

Enzyme digestion of bacteriophage; 282

Epidemics, behavior of bacteriophage in; 490

Essences, effect on bacteriophage; 303

Etiology of fowl typhosis; 479

Evaporation of bacteriophage; 286, 301

Exogenous autolysin hypothesis of bacteriophage; 314

Experimental disease vs. natural infection; 509

Feces, Cholera-bacteriophage from; 261

- Coli-bacteriophage in; 255,416

, Diphtheria-bacteriophage in; 258

_- Dysentery-bacteriophage in; 243

_- Enteritidis-bacteriophage in; 254

- - Enterococcus-bacteriophage in; 266

—, Flacherie-bacteriophage in; 256 
Feces, Friedländer-bacteriophage in; 256

- isolation of bacteriophage from; 39,137

- multiple races of bacteriophage in; 416

—, Paratyphoid-bacteriophage in; 252

-. Pestis-bacteriophage in; 504

_-, Pfaffi-bacteriophage in; 246

- , preservation of bacteriophage in; 285

—, Proteus-bacteriophage in; 257

-, Sanguinaria-bacteriophage in; 246

—, Streptococcus-bacteriophage in; 266

—, Subtilis-bacteriophage in; 261

-, Suipestifer-bacteriophage in; 254

-

—, Typhi murium-bacteriophage in; 254

—_, Typhoid-bacteriophage in; 251

Ferment secretion by bacteriophage; 373

Fetus, bacteriophage in; 423

Filter candles, adsorption of bacteriophage by; 289

Filtrable forms of bacteria; 226

Filtrability of bacteriophage; 21, 274

Filtration and speed of fixation; 107

Fixation of bacteriophage, effect of viscosity on; 108

— of bacteriophage to bacterium; 104

- of bacteriophage to heated bacteria; 108

— of bacteriophage to insusceptible bacteria; 107

- , rates of 120

Flacherie, bacteriophage races from; 256

Flacherie-bacteriophage in silkworms; 507

- , sources of ; 256

Flattenformen of Gildemeister; 11

Fleming phenomenon; 17

Flocculation of bacteriophage; 287, 301
Folliculitis, bacteriophage therapy of ; 559

Foods, bacteriophage in; 435

Formol, effect on bacteriophage; 304

Fowl-typhosis, bacteriophage from; 246

—, etiology of; 479

— organisms, homogeneity of; 245

Fowls, bacteriophage immunization of ; 511

- bacteriophage of ; $429,433,490$

Friedländer-bacteriophage, sources of ; 256

Furfurol, effect on bacteriophage; 304

Furunculosis, bacteriophage in; 483

- bacteriophage therapy of; 558

Gaertner-bacteriophage in horses; 432

Gallinarium-bacteriophage, immunization with; 511

— in avian typhosis; 481,490

_ in cattle; 431

__ in fowl; 429

_ in horses; 425

— in swine; 431

—. plaque formation by 79

Gelatin, adsorption of bacteriophage by; 291

__, effect on bacteriophagy ; 63,216

- effect on plaque formation; 90

Germicidal action of river water; 8

Glycerol, adaptation to; 343

—-, effect on bacteriophage; 286, 306

-, effect on Zoochlorella; 318

Goats, intestinal bacteriophage in; 431

Granule formation during bacteriophagy; 131

Gums, effect on bacteriophagy; 63

Heated bacteria, fixation by; 108

Hemorrhagic septicemia of buffaloes; 497

d'Herelle's hypothesis of bacteriophage; 326

- method of determining virulence; 150

Heterogeneous bacterial species; 168, 248 
Homogeneous bacterial species; 168 242

Horses, intestinal bacteriophage in; 425

— virulences of bacteriophage in; 432

Hydra viridis, parasitic phenomena in; 318

$\mathrm{H}$ ion concentration, effect on adsorption; 289

- — - effect on bacteriophagy; 43 , $69,184,187,300$

- - effect on plaque formation; 88

- tion; 126

$\mathrm{H}$ ions, flocculation of bacteriophage by; 287

Hypotheses concerning bacteriophage; 309

Immunization against avian typhosis; 510

—_ against barbone; 520

_- against dysentery; 533

— with bacteriophage; 509

Infants, appearance of bacteriophage in; 423

Infections, experimental and natural; 509

Infusorial earth, adsorption by; 289

Ingestion of bacteriophage; 416

Inhibition by antibacterial sera; 400

— by antibacteriophagic sera; 392

- by metabolic products of bacteriophage; 216

_- , relation to bacteriolysis; 51

Inorganic chemicals, effect on bacteriophage; 300

Intestinal bacteriophage, effect of diet on; 428

—— in avian typhosis; 481

—— in bacillary dysentery; 439

- in domestic animals; 424

—— in $\operatorname{man} ; 437$

— in typhoid fever; 451

disturbances, bacteriophage in; 437

— tract, bacteriophage of ; 416
Irradiation, effect on bacteriophage; 291

Isolation of bacteriophage; $21,38,137$

_ـ of pure races of bacteriophage; 156

Janzen and Wolff method of determining virulence; 146

Kabeshima hypothesis of bacterio-phage; 309

Kaolin, adsorption of bacteriophage by; 289

Kuttner hypothesis of bacteriophage; 313

Lactic acid, effect on bacteriophage; 304

Lead salts, effect on secondary cultures; 190

Leucocytes, effect of bacteriophage on; 408

Leucocytic origin of bacteriophage; 311

Life, criteria of ; 330

—, relation of bacteriophage to origin of ; 375

Limiting concentrations of the bacteriophage; 56

Lipoids, effect on bacteriophage; 306

Lisbonne and Carrère hypothesis of bacteriophage; 314

Locusts, Coccobacillus acridiorum in; 1

Lysis, definition of ; 18

Lysol, effect on bacteriophage; 304

Magnesium sulfate, effect on bacteriophagy; 71

Maitland method of determining virulence; 148

Mercuric chloride, adaptation of bacteriophage to; 348

——

Metabolic products, effect on bacteriophagy; 48

Methyl violet, effect on bacteriophagy; 72

Microscopic aspects of bacteriophagy; 130 
Mixed cultures, bacteriophagy in; 179

—_ - biochemistry of; 214

$\longrightarrow$ - constitution of; 209

- - - cultivation of; 208 - in cystitis; 232 in pyelocystitis; 233

- - in pyelor

—— natural occurrence of ; 230

- - - purification of ; 236

- - stability of ; 212

Mode of action of bacteriophage; 369

Monkey, intestinal bacteriophage of ; 430

Morphology of bacteria during bacteriophagy; 112, 130 of resistant bacteria; 219

Mutations and bacterial resistance; 219

— of B. coli; 221,255

of B. paratyphosus; 252

of $B$. proteus; 257

Multiple virulences of bacteriophage; 170,432

- —

Multiplication of bacteriophage; cause of arrest of; 127

— of bacteriophage corpuscles; 115 , 349

- of bacteriophage, effect of bacteria on; 124

of bacteriophage, effect of temperature on; 123

— of bacteriophage, intermittent course of ; 116

of bacteriophage, relation to number of bacteria dissolved; 125

of bacteriophage, relation to virulence; 140

Myxomycete, growth of ; 103

——, symbiosis of ; 318

Natural mixed cultures; 230

- resistance of bacteria; 193, 217

Nature of bacteriophage; 309, 329

Nuclease-immunoproteidine; 9

Nutritive vitiation hypothesis; 311

Opsonic action of bacteriophage; 408

Organic compounds, effect on bacteriophage; 302
Otto and Munter method of determining virulence; 147

Oxalic acid, effect on bacteriophage; 304

Oxygen, effect on bacteriophage; 302

Papaine, effect on bacteriophage; 282

Paramecium bursaria, parasitic phenomena in; 318

Parasitic phenomena with protozoa; 318

Paratyphoid-bacteriophage, effect of temperature on; 293

_ in domestic animals; 425

in $\operatorname{man} ; 438$

in stock cultures; 12

in typhoid fever; 452

in water; 436

-_- , sources of; 252

— therapy of paratyphoid fever; 550

- - virulence attenuation of ; 165

Paratyphoid fever, bacteriophage races from; 252

- bacteriophage therapy of; 550

Pasteurella bovis, homogeneity of ; 246

Pathogenicity of resistant bacteria; 219,484

Penetration of bacteria by bacteriophage; 112

Persistence of virulence in bacteriophage; 171

Pestis-bacteriophage, corpuscular nature of ; 85

$\longrightarrow$, filtrability of; 274

-, formation of ultrabacteria with; 226

- in plague; 504

__ in rats; 247,504

- in stock cultures; 12

— , plaque formation by; 79

- , sources of; 247

—-, spontaneous attenuation of; 167

—, temperature limits of; 59

- therapy of plague; 567

-

-

Pfaffi-bacteriophage immunization of fowl; 512 
Pfaffi-bacteriophage in avian typhosis; 492

- , sources of $; 246$

Pfreimbter, Sell and Pistorius method of determining virulence; 147

Phagocytosis, effect of bacteriophage on; 408

- - of bacteriophage; 383

Phenol, adaptation of bacteriophage to; 346

—, effect on bacteriophage; 303

Phosphates, effect on bacteriophagy ; 71

_-, effect on growth of secondary cultures; 190

Physical state of bacteriophage; 273

Plague, bacteriophage in; 503

- bacteriophage races from; 247

- bacteriophage therapy of; 567

Plants, bacteriophage in; 259,487

Plaque formation by bacteriophage; 76, 337

- - conditions controlling; 88

—— - effect of temperature on; 91

—— - index of multiplication; 116

—— inhibition of; 90

—— on gelatine; 90

—— relation to virulence; 139

method, purification by; 158

Plaques, bacteriophage in; 94

-, characters of; 92

- , enumeration of bacteriophage corpuscles by; 96

- limitation of size of; 95

—, size of; 92

- , source of ; 87

Potassium chloride, effect on bacteriophagy; 71

- cyanide, effect on bacteriophage; 301

Precipitation of bacteriophage; 287

Pressure, effect on bacteriophagy; 63

Prophylactic immunization with bacteriophage; 509

Proteus-bacteriophage, virulences of; 257

Protobe, meaning of; 360

Protobios bacteriophagus, a living virus; 355
Pseudobacteriophagy; 266

Purification of bacteriophage races; 156

Pus, bacteriophage in; 37, 248, 483

Pyelocystitis, bacteriophage therapy of ; 553

—, mixed cultures in; 233

Pyelonephritis, bacteriophage in; 484

Quinine derivatives, effect on bacteriophage; 71, 304

Rabbits, intestinal bacteriophage of; 431

Radicicola-bacteriophage, effect of irradiation on; 292

—, effect of temperature on; 297

— in plants; 487

- , sources of ; 259

Radium, effect on bacteriophage; 292

Rats, Pestis-bacteriophage in; 247, 504

Refractile index of bacteriophage; 134

Resistance, effect on agglutinability; 218

- in B. coli, loss of ; 421

- , loss of bacterial; 204

— - mode of acquisition; 194

— of bacteria, acquired; 217

- of bacteria, natural; 217

— of bacteria, significance of; 182

- - to antibacteriophagic sera; 386

Resistant $B$. coli in infections; 484

- B. typhi murium; 254

bacteria, biochemistry of ; 218

- - destruction of bacterio-

phage by ; 195, 211

203

——, effect on virulence; 196

_- f fixation to; 107

_— morphology of; 219

—— pathogenicity of ; 219

- , vitality of; 218

forms of staphylococci; 265

Rupture of bacteria during bacteriophagy; 113 
Salts, effect on bacteriophagy; 70

- - effect on secondary culture development; 190

Sanguinaria-bacteriophage, sources of ; 246

Sausage, bacteriophage in; 435

Secondary cultures, bacteria of; 206

—— behavior of bacteriophage in; 196

——, effect of salts on; 190

- - cause of ; 215

- - , origin of ; 188

——

Sedimentation of bacteriophage; 280

Seiffert hypothesis of bacteriophage; 314

Sensitization to toxin by bacteriophage; 404

Serial action, demonstration of ; 40

Serum, bacteriophage in; 427

—-, effect on bacteriophagy; 71

Sheep cells, adsorption of bacteriophage by ; 290

Silk-worms, bacteriophage races from; 256

—_ flacherie in; 507

Silver colloids, effect on bacteriophagy; 65

- salts, effect on growth of secondary cultures; 190

Size of bacteriophage corpuscles; 134 , 275

- of bacteriophage plaques; 92

Sodium chloride, effect on bacteriophage; 301

- fluoride, effect on bacteriophage; 71, 301

— sulfate, effect on bacteriophage; 301

195

Soil, bacteriophage in; $37,436,488$

- - Dysentery-bacteriophage in; 243

—-, Radicicola-bacteriophage in; 260

Solubility of bacteriophage; 282

Species, concept of ; 365

Specific therapy with bacteriophage; 540
Specificity of antibacteriophagic serum action; 389

- of opsonic action of bacteriophage; 411

Spontaneous attenuation of bacteriophage; 166

Stained preparations in bacteriophagy; 114,130

Staphylococci, bacterioclysis of; 14 , 264

- , colony form of ; 210

- , heterogeneity of; 265

- mutations of ; 225

- number dissolved by the bacteriophage; 54

Staphylococcus infections, bacteriophage in; 483

—_ bacteriophage therapy of; 558

Staphylo-bacteriophage, acquired resistances to; 192

—, action on dead bacteria; 49

—, adaptation of; 178

—, agglutination by; 74

—, antiphylaxis with; 405

—, attenuation by resistant bacteria; 198

—-, corpuscular nature of; 84

—-, effect of acetone on; 303

_-, effect of bacterial numbers on; 53

_-, effect of chloroform on; 303

—, effect of colloidal silver on; 65

—-, effect of dyestuffs on; 67

—-, effect of glycerol on; 306

- effect of $\mathrm{pH}$ on multiplication; 126

—-, effect of potassium cyanide on; 301

- , effect of temperature on ; 183, 294

—-, effect of viscosity on; 64

— 98

— , filtrability of; 274

- fixity of virulences of; 337

— in staphylococcus infections; $4 \$ 3$

- limiting concentration of; 56

- , maximum titre of ; 127

—, plaque formation by; 79 
Staphylo-bacteriophage plaques, size of ; 92

- , rate of fixation of ; 106, 120

_- , secondary cultures with; 183

- , specificity of ; 169

- , spontaneous attenuation of ; 167

—

_ therapy of wounds; 564

- , therapy with; 558

- , variations in virulence of; 159

- virulence attenuation of; 166

- , virulence determination of ; 144

-_, virulences of; 140,265

-

Sterile, definition; 19

Sterilization of ultrafilters; 32

Streptococcus-bacteriophage, source of ; 266

Streptococcus infections, bacteriophage in; 483

$-\ldots$, bacteriophage therapy of; 567

— of strangles, virulences in; 361

Subtilis-bacteriophage, sources of; 261

Successive dilutions method of determining virulence; 142

-_- purification of bacteriophage by; 157

Sugars, effect on bacteriophagy; 70

Suipestifer-bacteriophage in dogs; 434 - , source of ; 254

Sulfur colloids, effect on bacteriophagy; 67

Susceptible B. coli in feces; 420

Swine, intestinal bacteriophage of ; 431

Swine-fever, bacteriophage races from; 258

Sycosis, bacteriophage therapy of ; 562

Symbiosis between bacteria and bacteriophage; 211

— of Myxomycetes; 318

Synthetic media, bacteriophagy in ; 46

Temperature, effect on antibacteriophagic sera; 385

_- effect on bacterial resistance; 161

- effect on bacteriophage; 292
Temperature, effect on bacteriophage multiplication; 123

__ , effect on bacteriophagy; 183

_- effect on increase in virulence; 161

- , effect on opsonic action of bacteriophage; 409

_- effect on plaque formation; 91

_- limits of bacteriophagy; 59

- , relation to secondary cultures; 183

-. , virulence attenuation by; 165

Terminology; 18

Tetraline, effect on bacteriophage; 304

Therapy, bacteriophage; 540

- of $B$. coli infections with bacteriophage; 553

of bubonic plague with bacteriophage; 567

_ of chronic colitis with bacteriophage; 549

- of dysentery with bacteriophage; 540

- of paratyphoid fever with bacteriophage; 550

- of staphylococcus infections; 558 of streptococcus infections; 567

of typhoid fever with bacteriophage; 549

— of wound infections with bacteriophage; 564

Thermal death point of bacteriophage; 292

Tissues, bacteriophage in; 381

Toxicity, effect of bacteriophagy on; 403

Trypsin, effect on bacteriophage; 282

Twort phenomenon; 13

Typhi murium-bacteriophage, sources of; 254

Typhoid-bacteriophage, adaptation of ; 176

- , corpuscular nature of ; 85

-

—, effect of antisera on ; 389

— , effect of temperature on; 293

- filtrability of ; 274

- , fixation of ; 107 
Typhoid-bacteriophage, formation of ultrabacteria with; 226 in domestic animals; 425 in $\operatorname{man} ; 438$ in stock cultures; 11 in typhoid fever; 451 in water; 436

— , plaque formation by; 79

—, pure races of; 156

_ , rate of multiplication of; 124

- , specificity of; 168

—

therapy of typhoid fever; 549

-, virulence acquisition by; 176

- , virulence attenuation of; 165

—, virulences of; $248,334,352$

— , volatility of; 280

Typhoid fever, bacteriophage in; 451

— - bacteriophage in blood in; 470

248

— - bacteriophage therapy of; 549

Typhus fever, bacteriophage in; 257, 485

Ubiquity of bacteriophage; 37,416

Ultrabacteria, occurrence of; 226

- of B. typhosus; 478

Ultrafilters, denitrification of; 30

—, preparation of ; 26

- , sterilization of; 32

Ultrafiltration, methods of; 24

—of bacteriophage; 274

Ultrapure bacterial cultures; 207

Ultrasterile, definition; 19

Ultraviolet rays, effect on bacteriophage; 291

Unicity of bacteriophage; 358

Urine, bacteriophage in; $37,251,257$, 484

--, effect on bacteriophagy; 71

Vaccinated medium, effect on bacteriophagy; 127

Vaccinia, bacteriophage in; 16
Vaccinia, Twort phenomenon; 13

Variability in bacteriophage virulence; 137, 159

— of bacteriophage; 333,350

Variation in activity of antisera; 387

— in colony resistance; 207

— in mixed cultures; 210

Vibrio cholerae, action of river water on; 8

- , agglutinability of; 264

- cultures, bacteriophage from; 10 , 261

- fixation of bacteriophage to; 105

, heterogeneity of; 261

Virulence, acquisition of; 175

—, attenuation by antiseptics; 168

—, attenuation by time; 166

—, definition of ; 141

_- determination of ; 141

- determination,

Appelmans method; 142

- - Beckerich and Hauduroy method; 145

- _ d'Herelle method; 150

146

—— Maitland method; 148 - Otto and Munter method; 147

__ _ Pfreimbter, Sell and Pistorius method; 147

—— 142

- - effect of resistant bacteria on; 196

—-, expression of ; 141

__- expression of degrees of; 154

-

—

— of bacteriophage and colony type; 208

of bacteriophage and symptoms of dysentery; 440

— of bacteriophage in typhoid fever; 452

—_, persistence of; 171

_ range of bacteriophage; 168 
Virulence range of bacteriophage in fowl diseases; 246

—— of Dysentery-bacteriophage; 242

- , relation to multiplication of bacteriophage; 140

- relation to plaque formation; 139

- , relation to secondary cultures; 183

- , restoration of ; 165

- , specificity of; 168

- variations in bacteriophage; 137 , 159, 352, 437

- Werthemann expression of; 145

Virulences of bacteriophage in fowl; 429

- of bacteriophage in horses; 425 , 432

- of bacteriophage in man; 419

- of bacteriophage in normal animals; 431

- of bacteriophage, multiple; 170 , 432

__ of Cholera-bacteriophage; 263

of Diphtheria-bacteriophage; 259

— of Enteritidis-bacteriophage; 254 266

— of Paratyphoid-bacteriophage; 252

- of Pestis-bacteriophage; 247

— of Pfaffi-bacteriophage; 246 of Proteus-bacteriophage; 257
Virulences of Radicicola-bacteriophage; 260

— of Sanguinaria-bacteriophage; 246 of Staphylo-bacteriophage; 265 of strangles streptococci; 361 of Suipestifer-bacteriophage; 254

- of Typhi murium-bacteriophage; 254

- of Typhoid-bacteriophage; 248, 334

— , proof of multiple; 172

Virulences, retention of; 285

- variation in attenuation of ; 306

Virus nature of bacteriophage; 358

Viruses, adsorption of ; 291

Viscosity, effect on bacteriophage fixation; 108

- effect on bacteriophagy; 63,216

Vitality of bacteriophage; 166,283

- of resistant bacteria; 218

Volatility of bacteriophage; 8, 277

Water, antiseptic action of ; 8

- bacteriophage in; $12,37,243,251$, 436

- flocculation of bacteriophage by; 301

Werthemann method of expressing virulence; 145

Wound infections, bacteriophage therapy of; 564

Zoochlorella vulgaris, parasitic action of ; 318 


\section{.}




\section{Sans Tache}

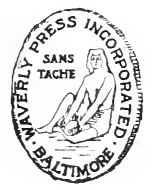




\section{Sans Tache}

T N THE "elder days of art" each artist or craftsman enjoyed the privilege of independent creation. He carried through a process of manufacture from beginning to end. The scribe of the days before the printing press was such a craftsman. So was the printer in the days before the machine process. He stood or fell, as a craftsman, by the merit or demerit of his finished product.

Modern machine production has added much to the worker's productivity and to his material welfare; but it has deprived him of the old creative distinctiveness. His work is merged in the work of the team, and lost sight of as something representing him and his personality.

Many hands and minds contribute to the manufacture of a book, in this day of specialization. There are seven distinct major processes in the making of a book: The type must first be set; by the monotype method, there are two processes, the "keyboarding" of the MS and the casting of the type from the perforated paper rolls thus produced. Formulas and other intricate work must be hand-set; then the whole brought together ("composed") in its true order, made into pages and forms. The results must be checked by proof reading at each stage. Then comes the "make-ready" and press-run and finally the binding into volumes.

All of these processes, except that of binding into cloth or leather covers, are carried on under our roof.

The motto of The Williams \& Wilkins Company is Sans Tache. Our ideal is to publish books "without blemish"-worthy books, worthily printed, with worthy typography - books to which we shall be proud to attach our imprint, made by craftsmen who are willing to accept 
open responsibility for their work, and who are entitled to credit for creditable performance.

The printing craftsman of today is quite as much a craftsman as, his predecessor. There is quite as much discrimination between poor work and good. We are of the opinion that the individuality of the worker should not be wholly lost. The members of our staff who have contributed their skill of hand and brain to this volume are:

Composing Room: Andrew Rassa, William Ǩoch, George Behr, Austin Uhland, John Crabill, Harry Harmeyer, 'Ernest Salgado, James Jackson, Edgar Simmons, Richard King, Nathan Miller, Walter Phillips, Herbert Leitch, Ray Kauffman, Anthony Wagner, Charles Wyatt, William Fite, James Armiger, John Flanagan, Theodore Nilson, Steve Simmons, Edward Rice, George Moss, Henry Shea.

Press Room: Fred Lucker, Clarence Ridgeway, August Hildebrand, John Ellis.

Proof Room: Sarah Katzin, Alice Reuter, Mary Reed, Ruth Trieshman, Lucille Bull, Edna Clark, Audrey Tanner, Angeline Eifert, Lillian Gilland, Ethel Strasinger, Dorothy Strasinger, Geraldine Browne, Arthur Baker.

Keyboard: Harry Susemihl, Katherine Kocent, Minnie Foard, Eleanor Luecke, Vera Taylor, Anna Kelly, Hannah Scott.

Casters: Kenneth Brown, Ernest Wann, Charles Aher, Frank Malanosky, Mahlon Robinson, Henry Lee, George Smith, Martin Griffen, Frederick Wall.

Folder: Laurence Krirug. 


\section{Another Book on the Bacteriophage}

Your library on the bacteriophage is not complete unless you have

\section{IMMUNITY IN NATURAL INFECTIOUS DISEASE}

In this book Dr. d'Herelle sets forth a new concept of the mechanism of immunity, involving a radical modification of some of the ideas which have hitherto dominated immunologic thought.

In four parts, as follows:

I The Reactions of Living Matter

II The Reactions Against Inanimate ObJects

III The Reaction Against Bacteria

IV The Ultraviruses and Immunity Against Them

Cloth, gold stamped $\quad 6 \times 9 \quad 400$ pages

$$
\text { Price } \$ 5.00
$$

\section{THE WILLIAMS \& WILKINS COMPANY}

Publishers of Scientific Books and Periodicals

BALTIMORE, U.S.A. 


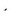




$$
\text { • }
$$


